

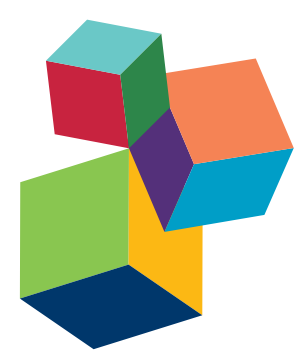

Frontiers Copyright Statement

(c) Copyright 2007-2016 Frontiers Media SA. All rights reserved.

All content included on this site, such as text, graphics, logos, button

icons, images, video/audio clips,

downloads, data compilations and

software, is the property of or is

licensed to Frontiers Media SA

("Frontiers") or its licensees and/or subcontractors. The copyright in the text of individual articles is the property of their respective authors, subject to a license granted to Frontiers.

The compilation of articles constituting

this e-book, wherever published,

as well as the compilation of all other

content on this site, is the exclusive property of Frontiers. For the conditions for downloading and copying of e-books from Frontiers' website, please see the Terms for Website Use. If purchasing Frontiers e-books from other websites or sources, the conditions of the website concerned apply.

Images and graphics not forming part of user-contributed materials may not be downloaded or copied without permission.

Individual articles may be downloaded and reproduced in accordance with the principles of the CC-BY licence subject to any copyright or other notices. They may not be re-sold as an e-book.

As author or other contributor you grant a $C C-B Y$ licence to others to reproduce your articles, including any graphics and third-party materials supplied by you, in accordance with the Conditions for Website Use and subject to any copyright notices which you include in connection with your articles and materials.

All copyright, and all rights therein, are protected by national and international copyright laws.

The above represents a summary only. For the full conditions see the Conditions for Authors and the Conditions for Website Use.

ISSN 1664-8714 ISBN 978-2-88919-936-5

DOI 10.3389/978-2-88919-936-5

\section{About Frontiers}

Frontiers is more than just an open-access publisher of scholarly articles: it is a pioneering approach to the world of academia, radically improving the way scholarly research is managed. The grand vision of Frontiers is a world where all people have an equal opportunity to seek, share and generate knowledge. Frontiers provides immediate and permanent online open access to all its publications, but this alone is not enough to realize our grand goals.

\section{Frontiers Journal Series}

The Frontiers Journal Series is a multi-tier and interdisciplinary set of open-access, online journals, promising a paradigm shift from the current review, selection and dissemination processes in academic publishing. All Frontiers journals are driven by researchers for researchers; therefore, they constitute a service to the scholarly community. At the same time, the Frontiers Journal Series operates on a revolutionary invention, the tiered publishing system, initially addressing specific communities of scholars, and gradually climbing up to broader public understanding, thus serving the interests of the lay society, too.

\section{Dedication to Quality}

Each Frontiers article is a landmark of the highest quality, thanks to genuinely collaborative interactions between authors and review editors, who include some of the world's best academicians. Research must be certified by peers before entering a stream of knowledge that may eventually reach the public - and shape society; therefore, Frontiers only applies the most rigorous and unbiased reviews.

Frontiers revolutionizes research publishing by freely delivering the most outstanding research, evaluated with no bias from both the academic and social point of view. By applying the most advanced information technologies, Frontiers is catapulting scholarly publishing into a new generation.

\section{What are Frontiers Research Topics?}

Frontiers Research Topics are very popular trademarks of the Frontiers Journals Series: they are collections of at least ten articles, all centered on a particular subject. With their unique mix of varied contributions from Original Research to Review Articles, Frontiers Research Topics unify the most influential researchers, the latest key findings and historical advances in a hot research area! Find out more on how to host your own Frontiers Research Topic or contribute to one as an author by contacting the Frontiers Editorial Office: researchtopics@frontiersin.org 


\section{AMINO ACIDS OF THE GLUTAMATE FAMILY: FUNCTIONS BEYOND PRIMARY METABOLISM}

Topic Editors:

Sakiko Okumoto, Virginia Tech, USA (presently at Texas A\&M University, USA*)

Dietmar Funck, University of Konstanz, Germany

Maurizio Trovato, Sapienza University of Rome, Italy

Giuseppe Forlani, University of Ferrara, Italy

Synthesis of Glutamate-derived Amino Acids

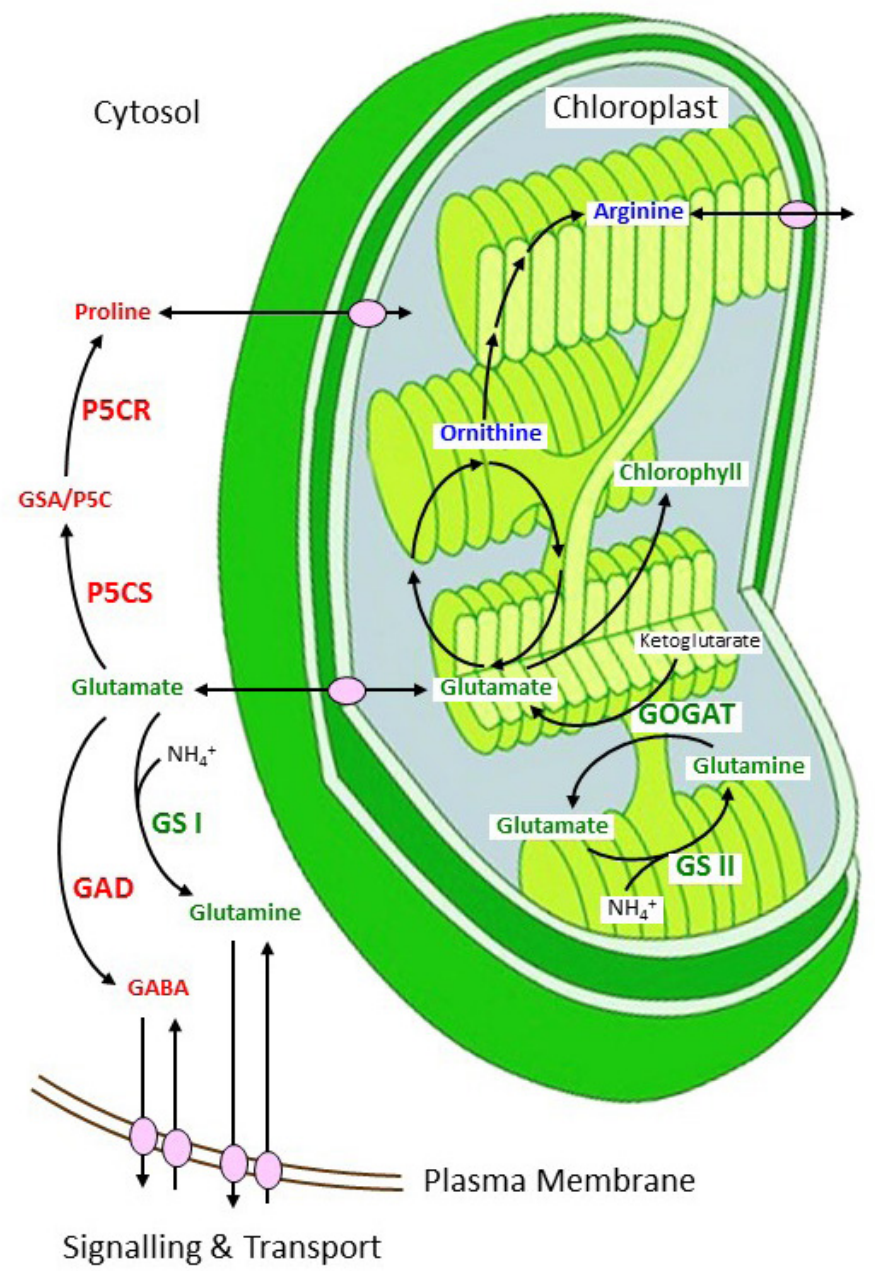

Anabolic connections between glutamate-derived amino acids. The key enzymes of nitrogen assimilation and synthesis of glutamate derived amino acids are localised in the cytosol and in plastids. GS: glutamine synthetase, GOGAT: glutamine-oxoglutarate aminotransferase, GSA: glutamate semialdehyde, P5C: pyrroline-5-carboxylate, P5CS: P5C synthetase, P5CR: P5C reductase, GAD: glutamate decarboxylase.

Figure by Dietmar Funck

*sokumoto@tamu.edu 
Cover image:

Anther with pollen of an Arabidopsis proline deficient $p 5 c s 1$ p5cs2/P5CS2 mutant with mixed populations of viable and non-viable pollen grains revealed by Alexander staining. Another version of this picture has originally been published in the article [Mattioli R, Biancucci M, Lonoce C, Costantino P, and Trovato M (2012) Proline is required for male gametophyte development in Arabidopsis. BMC Plant Biology, 12(1):236. Doi: 10.1186/1471-2229-12-236] under the terms of the Creative Commons Attribution License.

The life of proteins starts and ends as amino acids. In addition to the primary function as protein building blocks, amino acids serve multiple other purposes to make a plant's life worth living. This is true especially for the amino acids of the glutamate family, namely glutamate (Glu), glutamine (Gln), proline (Pro) and arginine (Arg), as well as the product of Glu decarboxylation, $\gamma$-aminobutyric acid (GABA). Synthesis, accumulation, interconversion and degradation of these five compounds contribute in many ways to the regulation of plant development and to responses to environmental challenges.

Glu and Gln hold key positions as entry points and master regulators of nitrogen metabolism in plants, and have a pivotal role in the regulatory interplay between carbon and nitrogen metabolism. Pro and GABA are among the best-studied compatible osmolytes that accumulate in response to water deficit, yet the full range of protective functions is still to be revealed. Arg, with its exceptionally high nitrogen-to-carbon ratio, has long been recognized as a major storage form of organic nitrogen.

Most of the enzymes involved in metabolism of the amino acids of the glutamate family in plants have been identified or can be predicted according to similarity with animal or microbial homologues. However, for some of these enzymes the detailed biochemical properties still remain to be determined in order to understand activities in vivo. Additionally, uncertainties regarding the subcellular localization of proteins and especially the lack of knowledge about intracellular transport proteins leave significant gaps in our understanding of the metabolic network connecting Glu, Gln, Pro, GABA and Arg. While anabolic reactions are distributed between the cytosol and chloroplasts, catabolism of the amino acids of the glutamate family takes place in mitochondria and has been implicated in fueling energy-demanding physiological processes such as root elongation, recovery from stress, bolting and pollen tube elongation.

Exceeding the metabolic functions, the amino acids of the glutamate family were recently identified as important signaling molecules in plants. Extracellular Glu, GABA and a range of other metabolites trigger responses in plant cells that resemble the actions of Glu and GABA as neurotransmitters in animals. Plant homologues of the Glu-gated ion channels from mammals and protein kinase signaling cascades have been implicated in these responses. Pollen tube growth and guidance depend on GABA signaling and the root architecture is specifically regulated by Glu. GABA and Pro signaling or metabolism were shown to contribute to the orchestration of defense and programmed cell death in response to pathogen attacks. Pro signaling was additionally proposed to regulate developmental processes and especially sexual reproduction. Arg is tightly linked to nitric oxide $(\mathrm{NO})$ production and signaling in plants, although Arg-dependent NO-synthases could still not be identified. Potentially Arg-derived polyamines constitute the missing link between Arg and NO signaling in response to stress.

Taken together, the amino acids of the glutamate family emerge as important signaling molecules that orchestrate plant growth and development by integrating the metabolic status of the plant with environmental signals, especially in stressful conditions.

This research topic collects contributions from different facets of glutamate family amino acid signaling or metabolism to bring together, and integrate in a comprehensive view the latest advances in our understanding of the multiple functions of Glu-derived amino acids in plants.

Citation: Okumoto, S., Funck, D., Trovato, M., Forlani, G., eds. (2016). Amino Acids of the Glutamate Family: Functions beyond Primary Metabolism. Lausanne: Frontiers Media.

doi: 10.3389/978-2-88919-936-5 


\section{Table of Contents}

06 Editorial: Amino Acids of the Glutamate Family: Functions beyond Primary Metabolism

Sakiko Okumoto, Dietmar Funck, Maurizio Trovato and Giuseppe Forlani

\section{Biochemistry of Glutamate Family Amino Acids}

\section{A. Proline}

09 Evolution of plant $\delta^{1}$-pyrroline-5-carboxylate reductases from phylogenetic and structural perspectives

Giuseppe Forlani, Kira S. Makarova, Milosz Ruszkowski, Michele Bertazzini and Boguslaw Nocek

24 Functional properties and structural characterization of rice $\delta^{1}$-pyrroline-5carboxylate reductase

Giuseppe Forlani, Michele Bertazzini, Marco Zarattini, Dietmar Funck, Milosz

Ruszkowski and Bogusław Nocek

37 Functional characterization and expression analysis of rice $\delta^{1}$-pyrroline-5carboxylate dehydrogenase provide new insight into the regulation of proline and arginine catabolism

Giuseppe Forlani, Michele Bertazzini, Marco Zarattini and Dietmar Funck

\section{B. Nitrogen Assimmilation by Glutamine Synthetase}

54 Glutamine synthetase in Medicago truncatula, unveiling new secrets of a very old enzyme

Ana R. Seabra and Helena G. Carvalho

61 Modulation of phenolic metabolism under stress conditions in a Lotus japonicus mutant lacking plastidic glutamine synthetase

Margarita García-Calderón, Teresa Pons-Ferrer, Anna Mrázova, Peter Pal'ove-Balang, Mária Vilková, Carmen M. Pérez-Delgado, José M. Vega, Adriana Eliášová, Miroslav Repčák, Antonio J. Márquez and Marco Betti

\section{Arginine/Ornithine}

77 Physiological implications of arginine metabolism in plants Gudrun Winter, Christopher D. Todd, Maurizio Trovato, Giuseppe Forlani and Dietmar Funck

91 Glutamate, Ornithine, Arginine, Proline, and Polyamine Metabolic Interactions: The Pathway Is Regulated at the Post-Transcriptional Level

Rajtilak Majumdar, Boubker Barchi, Swathi A. Turlapati, Maegan Gagne, Rakesh Minocha, Stephanie Long and Subhash C. Minocha 
Roles of Glutamate Family Amino Acids in Development

108 Role of proline in cell wall synthesis and plant development and its implications in plant ontogeny

Polavarapu B. Kavi Kishor, P. Hima Kumari, M. S. L. Sunita and Nese Sreenivasulu

125 Role of proline and GABA in sexual reproduction of angiosperms

Marco Biancucci, Roberto Mattioli, Giuseppe Forlani, Dietmar Funck, Paolo Costantino and Maurizio Trovato

136 How and why does tomato accumulate a large amount of GABA in the fruit? Mariko Takayama and Hiroshi Ezura

143 Connecting proline metabolism and signaling pathways in plant senescence Lu Zhang and Donald F. Becker

\section{Glutamate Family Amino Acids and Environmental Response}

A. Proline and Its Role in Stress Responses

151 Dynamic proline metabolism: importance and regulation in water limited environments

Govinal B. Bhaskara, Tsu-Hao Yang and Paul E. Verslues

158 P5CDH affects the pathways contributing to Pro synthesis after ProDH activation by biotic and abiotic stress conditions

Yanina S. Rizzi, Mariela I. Monteoliva, Georgina Fabro, Carola L. Grosso, Laura E. Laróvere and María E. Alvarez

169 Role of proline and pyrroline-5-carboxylate metabolism in plant defense against invading pathogens

Aarzoo Qamar, Kirankumar S. Mysore and Muthappa Senthil-Kumar

B. Roles of Amino Acids in Metabolism and Signaling

178 Closing the loop on the GABA shunt in plants: are GABA metabolism and signaling entwined?

Simon Michaeli and Hillel Fromm

185 The transporter GAT1 plays an important role in GABA-mediated carbonnitrogen interactions in Arabidopsis

Albert Batushansky, Menny Kirma, Nicole Grillich, Phuong A. Pham, Doris Rentsch, Gad Galili, Alisdair R. Fernie and Aaron Fait

195 Suppressor mutations in the Glutamine Dumper1 protein dissociate disturbance in amino acid transport from other characteristics of the Gdu1D phenotype

Shi Yu, Réjane Pratelli, Cynthia Denbow and Guillaume Pilot 


\title{
Editorial: Amino Acids of the Glutamate Family: Functions beyond Primary Metabolism
}

\author{
Sakiko Okumoto ${ }^{* *}$, Dietmar Funck ${ }^{2 *}$, Maurizio Trovato ${ }^{3 *}$ and Giuseppe Forlani ${ }^{4 *}$ \\ ${ }^{1}$ Department of Plant Pathology, Physiology and Weed Science, Virginia Tech, Blacksburg, VA, USA, ${ }^{2}$ Laboratory of Plant \\ Physiology and Biochemistry, Department of Biology, University of Konstanz, Konstanz, Germany, ${ }^{3}$ Department of Biology \\ and Biotechnology, Sapienza University of Rome, Rome, Italy, ${ }^{4}$ Laboratory of Plant Physiology and Biochemistry, Department \\ of Life Science and Biotechnology, University of Ferrara, Ferrara, Italy
}

Keywords: amino acids, glutamic acid, metabolism, abiotic stresses, plant biochemistry

\section{The Editorial on the Research Topic}

\section{Amino Acids of the Glutamate Family: Functions beyond Primary Metabolism}

\section{OPEN ACCESS}

Edited and reviewed by: Steven Carl Huber, United States Department of Agriculture - Agricultural Research Service, USA

*Correspondence: Sakiko Okumoto sokumoto@vt.edu; Dietmar Funck dietmar.funck@uni-konstanz.de; Maurizio Trovato maurizio.trovato@uniroma1.it; Giuseppe Forlani flg@unife.it

Specialty section: This article was submitted to Plant Physiology, a section of the journa Frontiers in Plant Science

Received: 15 February 2016 Accepted: 29 February 2016

Published: 21 March 2016

Citation: Okumoto S, Funck D, Trovato $M$ and Forlani G (2016) Editorial: Amino Acids of the Glutamate Family: Functions beyond Primary Metabolism.

Front. Plant Sci. 7:318. doi: 10.3389/fp/s.2016.00318
Amino acids in the plant body serve multiple purposes beyond being building blocks of proteins: They are major nitrogen carriers in the plants' long distance transport systems, precursors to important metabolites, nitrogen storage molecules, stress response molecules, and signaling molecules. Biochemically, the glutamine/glutamate family of amino acids (Gln, Glu, GABA, Pro, Arg) is close to the "entry point" of inorganic nitrogen (i.e., Glutamine Synthetase/Glutamateglutamine-oxoglutarate aminotransferase [GS/GOGAT] cycle) into organic nitrogen metabolism. Furthermore, the carbon skeleton of Glu and Gln is directly connected with primary energy metabolism (TCA cycle). Biosynthesis pathways for these amino acids are regulated at multiple levels, and in turn serve to communicate developmental and environmental cues.

This Research Topic presents some new studies on physiology and biochemistry of the Gln/Glu family of amino acids, as well as summarizes our current knowledge of their roles beyond protein synthesis. Even for an "old enzyme" such as GS, new understandings emerge by transcriptome analysis and metabolite profiling; for example, García-Calderón et al. found that, under stress conditions, a defect in GS2 activity affects phenolic metabolism in Lotus japonicus. These data indicate that the activity of GS impacts metabolism beyond amino acids and related primary metabolites. Also, not all isoforms of GS in plants are equally regulated by the same type of post-translational modifications. Recent studies on GS complexes from Medicago truncatula, which involve multiple regulatory molecules and are heavily compartmentalized, are summarized by Seabra and Carvalho. The function of Glu as the direct precursor for chlorophyll and heme biosynthesis in plants has recently been reviewed by Brzezowski et al. (2015) and is not included in this Research Topic.

GABA, which is synthesized from Glu by a single decarboxylation reaction, plays a versatile role in plants and, together with proline (see below), is one of the most common osmolytes accumulated by plant cells in response to stress. Accumulation of GABA also occurs under non-stress condition in specific tissues, e.g., growing tomato fruits, and functions in defense or taste development are being suggested (Takayama and Ezura). GABA is further catabolized within the mitochondria through a two-step pathway called the GABA shunt, which bypasses a part of the TCA cycle. The pathway plays a crucial role in carbon metabolism, especially at specific growth stages or under adverse conditions such as low light and water stress. Hence, as thoroughly discussed in Michaeli and Fromm, 
GABA levels could function as the key signal connecting metabolism to developmental or environmental responses. Corroborating this notion, a study using a loss-of-function mutant of a GABA transporter provides evidence that GABA transport acts in regulating $\mathrm{C} / \mathrm{N}$ balance signaling in Arabidopsis (Batushansky et al.). In some specific cases, the function of GABA as signal has been established, such as its role in pollen tube guidance (Biancucci et al.). In this context, a recent work showed that GABA acts as a genuine signaling molecule by directly modulating a membrane malate channel, ALMT (Ramesh et al., 2015). It remains to be seen whether some of the functions discussed in this Research Topic are mediated by the modulation of membrane transporter activity.

Another amino acid playing a pivotal role in stress responses is proline. It is well established that proline is accumulated in specific tissues at certain developmental stages such as in pollen grains (Biancucci et al.; Kavi Kishor et al.) and under stress conditions (i.e., salt, drought, pathogen attack) as a result of transcriptional and post-translational regulations. The biochemical mechanisms of the protective functions of proline in stress responses are starting to be understood. The role of $\mathrm{P} 5 \mathrm{C}$, a toxic intermediate in proline metabolism, in the production of reactive oxygen species (ROS) is reviewed by Qamar et al. Likewise, the role of proline-derived ROS production during senescence is also discussed in detail by Zhang and Becker.

Although the biochemical pathways of proline biosynthesis and catabolism are known, some aspects of enzymatic regulations had never been characterized in plants. In this Research Topic, Forlani et al. (number 565) report an extensive structural and biochemical characterization of $\delta^{1}$-pyrroline-5-carboxylate reductase (P5CR), and conclude that NADPH is the likely cofactor in vivo, and that stress-induced variations in redox and ion homeostasis could profoundly affect the enzymatic activity. A refined crystal structure and analysis of P5CR from Medicago truncatula indicated that these are common features of plant P5C reductases (Ruszkowski et al., 2015). Some of the properties, such as co-factor preferences, could be inferred by comparing the primary sequence between P5CRs from different organisms, suggesting evolutionarily conserved domains (Forlani et al., number 567). Post-translational mechanisms also seem to be important in regulating proline degradation, in particular $\delta^{1}$ pyrroline-5-carboxylate dehydrogenase ( $\mathrm{P} 5 \mathrm{CDH})$ activity. The activity of rice $\mathrm{P} 5 \mathrm{CDH}$ was strongly dependent on factors such as cation concentrations, co-factor availability, redox status, and free arginine pools, while modulation of transcript levels contributed little to the regulation of total enzyme activity, underscoring the importance of detailed biochemical analyses (Forlani et al., number 591). Another level of complexity is added by interactions between multiple steps that control proline levels; using a detailed metabolite and transcript analysis in a $p 5 c d h$ mutant, Rizzi et al. showed that $\mathrm{P} 5 \mathrm{CDH}$ modulates the flux through the pathways of proline biosynthesis by influencing the expression levels of $\delta^{1}$-pyrroline-5-carboxylate synthetase.

In addition to its metabolic roles, proline and its derivative, hydroxyproline, are also major constituent of cell wall proteins such as extensins. These polypeptides have cross-linking capability as cell wall components, and are regulated by developmental and environmental cues. Kavi Kishor et al. discuss the function of proline/hydroxyproline rich proteins in the cell wall in relation to proline metabolism.

Another group of amino acids within the Gln/Glu family, also linked to Pro metabolism through the common intermediate P5C, is composed of Arg and Orn. Arg biosynthesis is tightly regulated in plants by post-transcriptional modifications, feedback mechanisms, and interactions with signaling proteins to ensure appropriate Arg production for protein synthesis and nitrogen storage. As a link to signaling and stress defense, the conversion of Orn or Arg to polyamines is well documented, while the production of Nitric Oxide (NO) from amino acid precursors is mechanistically still unresolved in plants (Winter et al.). In addition, Orn is suggested to play a direct role as a signaling molecule, too. Increased consumption of Orn by overexpression of mouse Orn decarboxylase in Arabidopsis caused increased levels of polyamines while amino acid levels were not strongly altered, indicating that nitrogen assimilation and allocation to different metabolic pathways may be regulated by Orn. Interestingly, the regulatory changes seemed to happen predominantly at the post-transcriptional level (Majumdar et al.).

So where does the research on the Gln/Glu family of amino acids go from here? Although the scientific community has learned a lot in the past decade due to an increased amount of genetic resources and technological advances in mRNA profiling, some critical details are still unknown. For example, the metabolic pathways of the Gln/Glu family of amino acids involve three major cellular compartments (cytosol, mitochondria, and chloroplasts) that differ in critical parameters such as metabolite and ion concentrations and redox status. The biochemical studies presented in this issue clearly demonstrate that the conditions specific to the given cellular compartment need to be considered carefully, and that transcriptome data alone does not reflect the pathway activity correctly. Additionally, the transport mechanisms and kinetics between the compartments need to be characterized. Such efforts would eventually provide a predictive model of amino acid flux, which will inform us of critical factors behind phenotypically important traits. For example, a perspective article presented in this issue (Bhaskara et al.) proposes that the classic view of "more proline is better for stress resistance" might not be correct, and rather, it might be the flux through the pathway that matters. Such a hypothesis can be examined with a predictive flux model.

Another exciting recent development are the roles that the Gln/Glu family of amino acids play in signaling, either serving as signaling molecule themselves or as precursors. It is well established that the addition of $\mathrm{Gln} / \mathrm{Glu}$ induces a massive transcriptional change in plants (Gutiérrez et al., 2008), and amino acid imbalance causes stress responses in plants (Pilot et al., 2004; Hirner et al., 2006; Liu et al., 2010; Yu et al.). Also Pro has recently been identified as a signaling molecule regulating plant growth and development (Wang et al., 2014; Biancucci et al., 2015). What exactly is being sensed 
to initiate such responses? Are amino acids being sensed by the mechanisms we now know, such as glutamate-receptor like proteins (Price et al., 2012; Forde and Roberts, 2014) or ALMT (Ramesh et al., 2015), or are there additional mechanisms for recognition? Genetic tools to dissect amino acid sensing mechanisms, such as the ones presented by Yu et al. will help discern the key players in amino acid sensing and signaling pathways.

\section{AUTHOR CONTRIBUTIONS}

All authors listed have made substantial, direct, and intellectual contribution to the work, and approved it for publication.

\section{REFERENCES}

Biancucci, M., Mattioli, R., Moubayidin, L., Sabatini, S., Costantino, P., and Trovato, M. (2015). Proline affects the size of the root meristematic zone in Arabidopsis. BMC Plant Biol. 15:263. doi: 10.1186/s12870-0150637-8

Brzezowski, P., Richter, A. S., and Grimm, B. (2015). Regulation and function of tetrapyrrole biosynthesis in plants and algae. Biochim. Biophys. Acta 1847, 968-985. doi: 10.1016/j.bbabio.2015.05.007

Forde, B. G., and Roberts, M. R. (2014). Glutamate receptor-like channels in plants: a role as amino acid sensors in plant defence? F1000 Prime Rep. 6:37. doi: 10.12703/P6-37

Gutiérrez, R. A., Stokes, T. L., Thum, K., Xu, X., Obertello, M., Katari, M. S., et al. (2008). Systems approach identifies an organic nitrogenresponsive gene network that is regulated by the master clock control gene CCA1. Proc. Natl. Acad. Sci. U.S.A. 105, 4939-4944. doi: 10.1073/pnas.08002 11105

Hirner, A., Ladwig, F., Stransky, H., Okumoto, S., Keinath, M., Harms, A., et al. (2006). Arabidopsis LHT1 is a high-affinity transporter for cellular amino acid uptake in both root epidermis and leaf mesophyll. Plant Cell 18, 1931-1946. doi: 10.1105/tpc.106. 041012

Liu, G., Ji, Y., Bhuiyan, N. H., Pilot, G., Selvaraj, G., Zou, J., et al. (2010). Amino acid homeostasis modulates salicylic acid-associated redox status and defense responses in Arabidopsis. Plant Cell 22, 3845-3863. doi: 10.1105/tpc. 110.079392

Pilot, G., Stransky, H., Bushey, D. F., Pratelli, R., Ludewig, U., Wingate, V. P., et al. (2004). Overexpression of GLUTAMINE DUMPER1 leads to hypersecretion of

\section{FUNDING}

GF acknowledges support from AGER Foundation in the frame of the RISINNOVA project, grant \# 2010-2369. SO acknowledges support from NSF, grant MCB-1052048.

\section{ACKNOWLEDGMENTS}

As the topic editors, we would like to thank all our colleagues who contributed their articles. We would also like to express our gratitude to the numerous colleagues who contributed to the success of this Research topic by acting as reviewers or editors. We are especially grateful for excellent technical support provided by the editorial office and the chief editors.

glutamine from hydathodes of Arabidopsis leaves. Plant Cell 16, 1827-1840. doi: 10.1105/tpc.021642

Price, M. B., Jelesko, J., and Okumoto, S. (2012). Glutamate receptor homologs in plants: functions and evolutionary origins. Front. Plant Sci. 3:235. doi: 10.3389/fpls.2012.00235

Ramesh, S. A., Tyerman, S. D., Xu, B., Bose, J., Kaur, S., Conn, V., et al. (2015). GABA signalling modulates plant growth by directly regulating the activity of plant-specific anion transporters. Nat. Commun. 6:7879. doi: 10.1038/ncomms8879

Ruszkowski, M., Nocek, B., Forlani, G., and Dauter, Z. (2015). The structure of Medicago truncatula $\delta^{1}$-pyrroline-5-carboxylate reductase provides new insights into regulation of proline biosynthesis in plants. Front. Plant Sci. 6:869. doi: $10.3389 /$ fpls.2015.00869

Wang, G., Zhang, J., Fan, X., Sun, X., Qin, H., Xu, N., et al. (2014). Proline responding1 plays a critical role in regulating general protein synthesis and the cell cycle in maize. Plant Cell 26, 2582-2600. doi: 10.1105/tpc.114.1 25559

Conflict of Interest Statement: The authors declare that the research was conducted in the absence of any commercial or financial relationships that could be construed as a potential conflict of interest.

Copyright (c) 2016 Okumoto, Funck, Trovato and Forlani. This is an open-access article distributed under the terms of the Creative Commons Attribution License (CC $B Y)$. The use, distribution or reproduction in other forums is permitted, provided the original author(s) or licensor are credited and that the original publication in this journal is cited, in accordance with accepted academic practice. No use, distribution or reproduction is permitted which does not comply with these terms. 


\section{OPEN ACCESS}

Edited by: Irene Murgia,

Università degli Studi di Milano, Italy

Reviewed by:

Nabil I. Elsheery,

Tanta University, Egypt

Stefano Santabarbara,

Consiglio Nazionale delle Ricerche,

Italy

*Correspondence

Boguslaw Nocek,

The Bioscience Division, Argonne

National Laboratory, 9700 S. Cass

Avenue, Argonne, IL 60439, USA bnocek@anl.gov

Specialty section:

This article was submitted to

Plant Physiology,

a section of the journal

Frontiers in Plant Science

Received: 30 April 2015

Accepted: 09 July 2015

Published: 03 August 2015

Citation:

Forlani G, Makarova KS,

Ruszkowski M, Bertazzini M and Nocek B (2015) Evolution of plant $\delta^{1}$-pyrroline-5-carboxylate reductases

from phylogenetic and structural perspectives.

Front. Plant Sci. 6:567. doi: 10.3389/fp/s.2015.00567
Evolution of plant $\delta^{1}$-pyrroline-5-carboxylate reductases from phylogenetic and structural perspectives

\author{
Giuseppe Forlani', Kira S. Makarova ${ }^{2}$, Milosz Ruszkowski ${ }^{3}$, Michele Bertazzini ${ }^{1}$ and \\ Boguslaw Nocek ${ }^{4 *}$
}

${ }^{1}$ Department of Life Science and Biotechnology, University of Ferrara, Ferrara, Italy, ${ }^{2}$ National Center for Biotechnology Information, National Library of Medicine, National Institutes of Health, Bethesda, MD, USA, ${ }^{3}$ Synchrotron Radiation Research Section, Macromolecular Crystallography Laboratory, National Cancer Institute, Argonne National Laboratory, Argonne, IL, USA, ${ }^{4}$ The Bioscience Division, Argonne National Laboratory, Argonne, IL, USA

Proline plays a crucial role in cell growth and stress responses, and its accumulation is essential for the tolerance of adverse environmental conditions in plants. Two routes are used to biosynthesize proline in plants. The main route uses glutamate as a precursor, while in the other route proline is derived from ornithine. The terminal step of both pathways, the conversion of $\delta^{1}$-pyrroline-5-carboxylate (P5C) to L-proline, is catalyzed by $\mathrm{P} 5 \mathrm{C}$ reductase (P5CR) using NADH or NADPH as a cofactor. Since P5CRs are important housekeeping enzymes, they are conserved across all domains of life and appear to be relatively unaffected throughout evolution. However, global analysis of these enzymes unveiled significant functional diversity in the preference for cofactors (NADPH vs. NADH), variation in metal dependence and the differences in the oligomeric state. In our study we investigated evolutionary patterns through phylogenetic and structural analysis of P5CR representatives from all kingdoms of life, with emphasis on the plant species. We also attempted to correlate local sequence/structure variation among the functionally and structurally characterized members of the family.

Keywords: P5C reductase, phylogenetic analysis, 3-D structures of P5CRs, oligomer structure prediction, cofactor preference

\section{Introduction}

L-proline is a unique multifunctional amino acid that is increasingly being associated with many important aspects of biology (Nocek et al., 2005; Szabados and Savouré, 2010). Its cyclic side chain restricts the conformational flexibility of the backbone in the protein structure. Furthermore, proline shows geometric cis-trans isomerism, a phenomenon that plays a central role in the folding and function of proteins (Morgan and Rubenstein, 2013). Repetitive proline-rich sequences are found in many proteins, and in several cases they are believed to be signaling elements (Kay et al., 2000). Besides its structural role as a component of proteins, proline accumulation represents one of the major strategies used by plants as a response to various abiotic and biotic stress conditions (Lehmann et al., 2010; Szabados and Savouré, 2010; Funck et al., 2012). Typically, the accumulation occurs in the cytoplasm where it may also function as a molecular chaperon stabilizing the structure of proteins and buffering cellular redox potential (Maggio et al., 2002). Proline synthesis is directly linked to the $\mathrm{NAD}(\mathrm{P}) \mathrm{H} / \mathrm{NAD}(\mathrm{P})^{+}$redox pair, indicating that it might play a secondary role as a redox shuttle, used to transfer redox equivalents between mitochondria and the cytosol (Poolman et al., 1983; Phang, 1985). It was suggested that the cellular levels of proline are regulated by the 
rate of both synthesis and degradation. Due to the separation of these processes between cytoplasm and mitochondria, regulation of the intracellular proline transport is also possible (Lehmann et al., 2010).

Proline biosynthesis occurs via two routes: the glutamate and the ornithine pathway (Smith et al., 1980). The glutamate pathway is the primary route for proline biosynthesis in bacteria, whereas in eukaryotes it is predominantly used under stress and limited nitrogen availability. Higher plants use the pathway from ornithine, as the main route under normal conditions (Delauney and Verma, 1993). Four reaction steps, catalyzed by three enzymes are required to convert glutamate to proline. In the first step, glutamate is phosphorylated by $\gamma$-glutamyl kinase (EC 2.7.2.11) yielding $\gamma$-glutamyl phosphate. In the second step, $\gamma$-glutamyl phosphate is converted by the enzyme $\gamma$-glutamyl phosphate reductase (EC 1.2.1.41) to glutamate $\gamma$-semialdehyde. In plants a single bifunctional enzyme, namely P5C synthetase, catalyzes both reactions. Glutamate $\gamma$-semialdehyde undergoes a spontaneous cyclization to $\delta^{1}$-pyrroline-5-carboxylate (P5C). In the terminal step, that is catalyzed by $\mathrm{P} 5 \mathrm{C}$ reductase (P5CR; EC 1.5.1.2.), P5C is reduced by the cofactor NAD(P)H to yield L-proline and the oxidized cofactor $\mathrm{NAD}(\mathrm{P})^{+}$. The enzymes ornithine amino transferase (EC 2.6.1.13), and P5CR are required for the biosynthesis of proline from ornithine. Both pathways share the last enzymatic step, catalyzed by P5CR. This terminal step appears to be essential in some organisms such as Arabidopsis thaliana, where deletion of the P5CR gene was reported to be embryo-lethal (Funck et al., 2012). Similarly in fungi, the inhibition of the P5CR gene expression or activity leads to drastically reduced pathogenicity (Adachi et al., 2004). Also, specific inhibitors of P5CR exert cytotoxic effects, and could be potentially exploited for herbicide (Forlani et al., 2008) and antibiotic (Forlani et al., 2012) design. It was postulated that the enzymatic activity of $\mathrm{P} 5 \mathrm{CR}$ is regulated in various plant tissues at different developmental stages. In young, metabolically active tissues proline likely functions as an energy and/or nitrogen and carbon source, while it is mainly related to dehydration in mature tissues (Hua et al., 1997).

The P5CRs constitute a very interesting and large family of enzymes (over 37,000 representatives in the NCBI database), which in addition to their elementary cellular role, appear to be involved in many other biological functions. Even though proline metabolism has been studied for over 40 years, this important family remained enigmatic due to the lack of three-dimensional structures. In recent years several structures of bacterial and mammalian P5CRs have been determined. However, only a handful were analyzed and published. As a consequence, there is still a significant knowledge gap especially for plant representatives, which have not been structurally characterized to date. In order to address this problem, and improve understanding of these important enzymes, we analyzed sequences of plant P5CRs and correlated them with currently available structural information. Analysis of evolutionary origin and comparison of sequences and structures of known representatives reveal a number of important structural features, which indicate a global trend, for the plant P5CRs and the entire family.

\section{Materials and Methods}

\section{Sequence Analysis}

The sequences of P5CR proteins from plants and algae were retrieved from Refseq database (Pruitt et al., 2014; as of February 2015) as a result of PSIBLAST search (Altschul et al., 1997; one iteration with the $e$-value cutoff of 0.01 , and without either low complexity filtering or composition-based statistics). The additional eukaryotic sequences were retrieved from OrthoMCL database (Fischer et al., 2011; http://www.orthomcl. org/orthomcl/), the orthology group OG5_126801. Archaeal and bacterial sequences were retrieved from COG database for COG0345. The BLAST-Clust program (Wheeler and Bhagwat, 2007) set up with the length coverage cutoff of 0.95 and the score coverage threshold (bit score divided by alignment length) of 1.5 was used for clustering archaeal and bacterial sequences (Supplementary Material). One sequence was chosen for each cluster for further analysis. Multiple alignments were built for eukaryotic and prokaryotic sequences using MUSCLE program (Edgar, 2004). A few incomplete sequences were discarded, resulting in a set of 728 aligned protein sequences. The alignment was filtered to exclude sites with gap character fraction $>0.5$ and homogeneity $<0.1$ (Yutin et al., 2008). The resulting 298 informative positions of the alignment were used for maximum likelihood tree reconstruction using the FastTree program (Price et al., 2010) with default parameters: JTT evolutionary model, discrete gamma model with 20 rate categories. The FastTree software was also used to compute bootstrap values.

\section{Cloning, Overexpression, and Purification of AtP5CR and BcP5CR}

The coding genes of Arabidopsis thaliana P5CR (AtP5CR; Giberti et al., 2014) and Bacillus cereus P5CR (BcP5CR, Bc_2977; Q81C08) were cloned into vector pMCSG68 according to the standard protocol described previously (Eschenfeldt et al., 2013; Nocek et al., 2014). The pMCSG68 vector introduces a His ${ }_{6}$-tag followed by the Tobacco Etch Virus (TEV) protease cleavage site at the N-terminus of the expressed protein. The correctness of the insert was confirmed by DNA sequencing. Overexpression was carried out in BL21 Gold E. coli cells (Agilent Technologies). The bacteria were cultured with shaking at $210 \mathrm{rpm}$ in LB medium supplemented with $150 \mu \mathrm{g} / \mathrm{ml}$ ampicillin at $37^{\circ} \mathrm{C}$ until the $\mathrm{OD}_{600}$ reached 1.0. The temperature was lowered to $18^{\circ} \mathrm{C}$ and isopropyl-D-thiogalactopyranoside (IPTG) was added to a final concentration of $0.5 \mathrm{mM}$. The culture was grown for $18 \mathrm{~h}$ and then centrifuged at $4500 \mathrm{rpm}$ for $10 \mathrm{~min}$ at $4^{\circ} \mathrm{C}$. Cell pellet from $1 \mathrm{~L}$ culture was resuspended in $35 \mathrm{~mL}$ of lysis buffer (50 mM HEPES sodium salt $\mathrm{pH} 8.0,500 \mathrm{mM} \mathrm{NaCl}, 5 \%$ glycerol, $20 \mathrm{mM}$ imidazole, $10 \mathrm{mM} \beta$-mercaptoethanol) and stored at $-80^{\circ} \mathrm{C}$. The samples were thawed and the cells were disrupted by sonication using bursts of total duration of $5 \mathrm{~min}$, with appropriate intervals for cooling. Cell debris was pelleted by centrifugation at $15000 \mathrm{rpm}$ for $30 \mathrm{~min}$ at $4^{\circ} \mathrm{C}$. The supernatant 
was applied to a column packed with $10 \mathrm{~mL}$ of HisTrap HP resin (GE Healthcare), connected to VacMan (Promega) and the chromatographic process was accelerated with a vacuum pump (developed by R. Jedrzejczak). The column was washed with 20 bed volumes of lysis buffer and the $\mathrm{His}_{6}$-tagged P5CRs were eluted with $25 \mathrm{~mL}$ of elution buffer (50 mM HEPES pH 8.0, $500 \mathrm{mM} \mathrm{NaCl} ; 500 \mathrm{mM}$ imidazole; $2 \mathrm{mM}$ DTT). The His ${ }_{6}$-tag was cleaved with TEV protease ( $2 \mathrm{mg}$ of a $\mathrm{His}_{6}$-tagged form) overnight at $4^{\circ} \mathrm{C}$ and dialysis to remove the excess of imidazole was carried out simultaneously. The resulting solution was mixed with His-Trap HP resin to capture the cleaved $\mathrm{His}_{6}$-tag and the His $_{6}$-tagged TEV protease and the flow through containing the protein of interest was collected and concentrated.

\section{Determination of Molecular Weight of P5CRs (AtP5CR and BcP5CR)}

The molecular weights of $\mathrm{P} 5 \mathrm{CR}$ proteins were evaluated according to previously described protocol (Nocek et al., 2005), by size exclusion chromatography (SEC) on a HiLoad 16/600 Superdex 200 Prep Grade column (GE Healthcare). $1.5 \mathrm{~mL}$ aliquots of purified and concentrated proteins $(\sim 5 \mathrm{mg} / \mathrm{mL}$ ) were centrifuged for 5 min prior to the injection onto the column, which was equilibrated and run in lysis buffer. The column was calibrated with chymotrypsynogen A $(25 \mathrm{kDa})$; albumin $(67 \mathrm{kDa})$, Streptococcus pyogenes (SpP5CR, $275 \mathrm{kDa}$ decamer confirmed by SEC and X-ray crystallography methods; Nocek et al., 2005), and thyroglobulin $(669 \mathrm{kDa})$ as standards. The calibration curve of $K_{\mathrm{av}}$ versus log molecular weight was prepared by using the equation $K_{\mathrm{av}}=V_{\mathrm{e}}-V_{\mathrm{o}} / V_{\mathrm{t}}-V_{\mathrm{o}}$, where $V_{\mathrm{e}}=$ elution volume for the protein, $V_{\mathrm{o}}=$ column void volume, and $V_{\mathrm{t}}=$ total bed volume.

\section{3-D Structures of P5CRs}

A search in the Protein Data Bank archive revealed eleven models of six unique proteins representing P5CRs from the following organisms: Homo sapiens (PDB id: 2GER, 2GR9, 2GRA, 2IZZ- used for the most of analyses due to the highest resolution); Bacillus cereus PDBid: 3GT0 (NCBI taxonomy ID 226900); Coxiella burnetii PDBid: 3TRI (NCBI taxonomy ID 227377); Plasmodium falciparum PDBid: 2RCY (NCBI taxonomy ID 36329); Streptococcus pyogenes PDBid: 2AHR, 2AMF (NCBI taxonomy ID 301447); Neisseria meningitides PDBid: 1YQG, 2AG8 (NCBI taxonomy ID 122586). During the preparation of this manuscript we were able to determine a low-resolution (3.40 ̊) structure of rice Oryza sativa P5CR (OsP5CR; Forlani et al., 2015), and used some of the relevant information to better correlate sequence to structural features.

\section{Results and Discussion}

\section{Sequence and Phylogenetic Analysis of P5CR Family}

P5CRs (common synonyms: 1-pyrroline-5-carboxylate reductase, $\delta^{1}$-pyrroline-5-carboxylate reductase, $\mathrm{P} 5 \mathrm{C}$ reductase) are important housekeeping enzymes that are broadly distributed across all three domains of life. According to estimates from the OrthoMCL database (Fischer et al., 2011) they were identified in $\sim 80 \%$ of archaea and bacteria and in $88 \%$ of eukaryotes in the orthology group: OG5_126801. Typical plant P5CRs are composed of two domains [NAD(P)-binding Rossmann-like domain, CATH 3.40.50.720; and ProC C-terminal domain, CATH 1.10.3730.10] and are $\sim 280$ amino acid (a.a.) long. For example, AtP5CR has 274 a.a., OsP5CR has 284 a.a., while the longest P5CR (347 a.a. long) is found in the green alga Chlamydomonas reinhardtii. They can be recognized by the Prosite PA line pattern sequence signature [PALF]-x(2,3)-[LIV]-x(3)-[LIVM]-[STAC]-[STV]-x-[GANK]G-x-T-x(2)-[AG]-[LIV]-x(2)-[LMF]-[DENQK] (Sigrist et al., 2013). Most of the sequences with this signature are likely bona fide P5CRs.

Sequences of P5CR from all three domains of life were evaluated to reveal their evolutionary relationships, with the purpose of gaining more insights into evolution of plant orthologs. A detailed analysis of a very large group of proteins from the P5CR family (728 sequences), in which we included the most current and complete set of representatives from higher plants and algae, yielded the tree shown in Figure 1. The general topology of the tree is in agreement with a phylogenic analysis, which has been recently published based on a small subset of P5CRs (Fichman et al., 2014). In both analyses, metazoa are grouped with plants and algae apart from a large branch that includes most of bacteria, archaea, and other eukaryotic species. Remarkably, none of the three domains of life are monophyletic (i.e., they do not share a common ancestor). Majority of bacterial and archaeal lineages are not monophyletic either, with exception of cyanobacteria and Deinococcus-Thermus lineages, which are mostly monophyletic (Figure 1 and Supplementary Material File 1). A largely monophyletic clade of fungi and the remaining eukaryotes from different taxonomic groups are scattered among bacterial branches (Figure 1).

Since many of these species possess only one P5CR gene (or have lineage-specific duplications), the lack of clear concordance with organismal taxonomy could be explained by xenologous gene displacement (displacement of the ancestral gene by a horizontal gene transfer; Koonin et al., 2001). In several bacteria and archaea, however, there are distant and relatively fast-evolving paralogs of P5CR (such as ProG and ComER in Bacillus subtilis). These diverged paralogs are mostly grouped together (Supplementary Material File 1). As reported previously (Fichman et al., 2014), they are unlikely involved in proline biosynthesis and thus represent potential examples of subfunctionalization (Lynch and Force, 2000). It is noteworthy that there is at least one duplicate, which generally corresponds to mitochondrial pyrroline-5-carboxylate reductase 1 (P5CR1) and pyrroline-5-carboxylate reductase 3 (P5CR3) from Homo sapiens, that could be dated back to the metazoan ancestor (Fichman et al., 2014; Supplementary Material).

Genes from higher plants and algae are monophyletic and the respective branch is mostly concordant with organismal taxonomy (Figure 1). Although most algae have at least two genes for P5CR, higher plants seem to inherit only one gene. A few lineage-specific duplications in higher plants, such as 


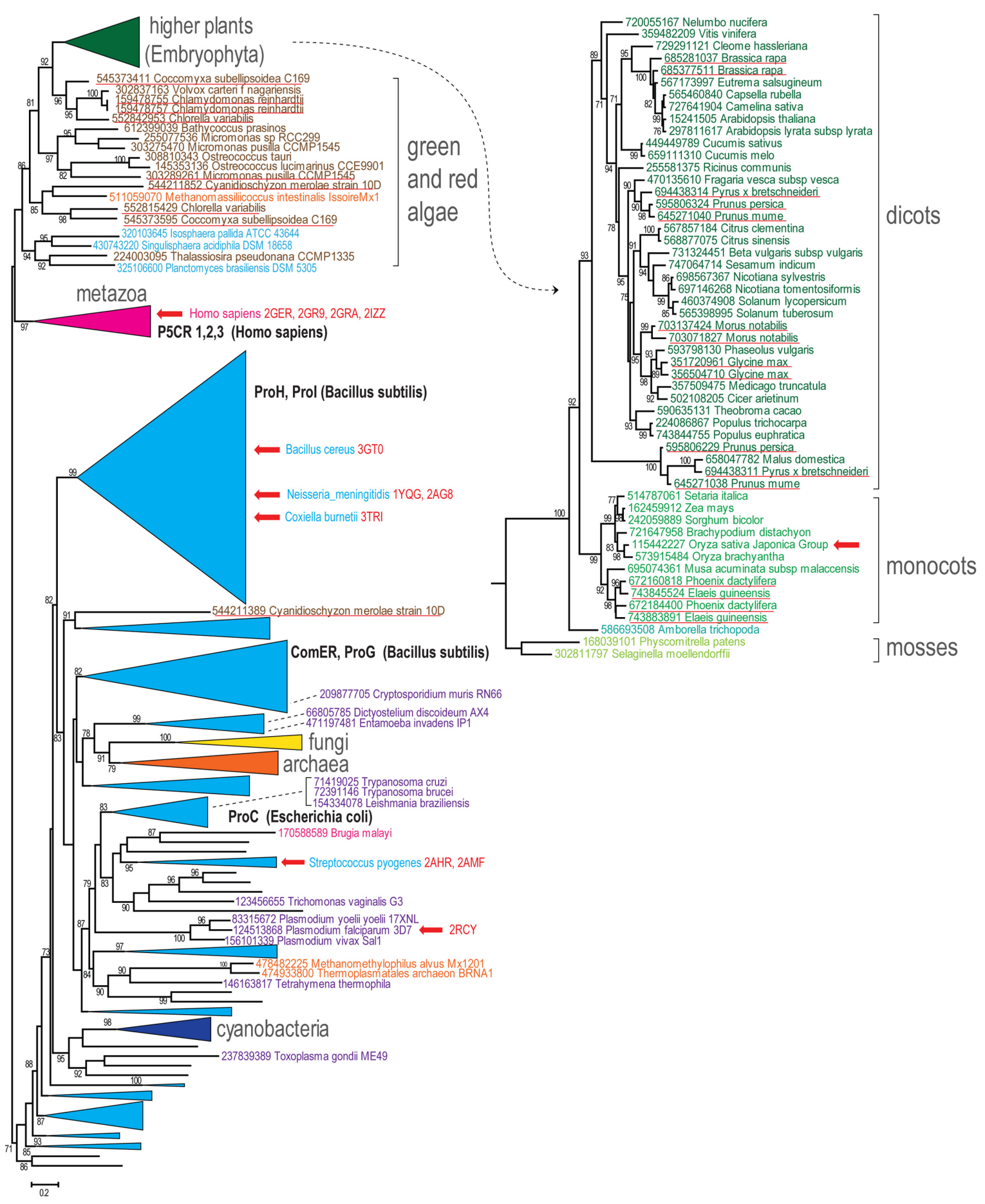

FIGURE 1 | Phylogenetic tree of predicted members of the pyrroline-5-carboxylate reductase (P5CR) family. Maximum-likelihood phylogenetic unrooted tree was built with the FastTree program using a multiple alignment for 728 P5CR amino acid sequences built by MUSCLE. FastTree program was also used to compute bootstrap values; only values $>70 \%$ are indicated. GenBank identifier number and systematic organism name marked the terminal nodes of the tree. Several bacterial terminal nodes are hidden for clarity and can be viewed in the Supplementary Material with complete tree data (Supplementary Material File 1). Several branches are collapsed and shown as triangles denoted by the respective lineage name. The branch corresponding to the higher plants is expanded and shown separately on the right side. Color code: bacteria, light blue; cyanobacteria, dark blue; archaea, orange; metazoan, purple; other eukaryotes, dark purple; fungi, yellow; plants, green shades; algae, dark brown. The characterized P5CR sequences are indicated by respective gene name highlighted by bold (for those that are within collapsed branches the organism name is also indicated). The organism names and PDB code are provided for P5CR proteins with solved crystallographic structure and red arrows show their location in the tree. Duplications in plants and algae are underlined. 
Glycine max, have been reported before (Shultz et al., 2006), but now many more cases of P5CR gene duplication can be observed (Figure 1, underlined in red). Some of them occurred relatively recently in narrow lineages, such as Brassica and Morus genera, but other examples include duplications that occurred earlier in evolution, at least in the common ancestor of Arecales (Elaeis and Phoenix genera) and in the common ancestor of Maloideae lineage (Pyrus, Malus, and Prunus genera). The latter case is quite notable, since it seems that the duplication was followed by an acceleration of the evolutionary rate. This fast evolution could have caused an erroneous placement of the Maloideae branch at the bottom of dicot branch. It could be expected that in these species P5CR gene underwent subfunctionalization. The generally accepted endosymbiosis transfer theory links chloroplasts found in plants and eukaryotic algae to cyanobacteria. However, our analysis does not reveal any evidence that P5CR genes in plants were acquired from cyanobacteria, which is an agreement with the results published before (Cullis et al., 2009; Fichman et al., 2014; Figure 1).

Thus, it appears that vertical descent dominates in evolution of P5CR genes in higher plants and algae. Some enzymes are able to function in both chloroplast and cytoplasm, whereas relatively rare cases of duplications might be related to differentiation of targeting affinities of the paralogs and are an interesting subject for further experimental study.

\section{A Minimalist P5CR Structure}

Pyrroline-5-carboxylate reductase enzymes characterized to date are composed of two unique domains: an N-terminal, dinucleotide binding domain (residues 1-175 in OsP5CR), and a C-terminal domain (residues 176-284 in OsP5CR, Figure 2).

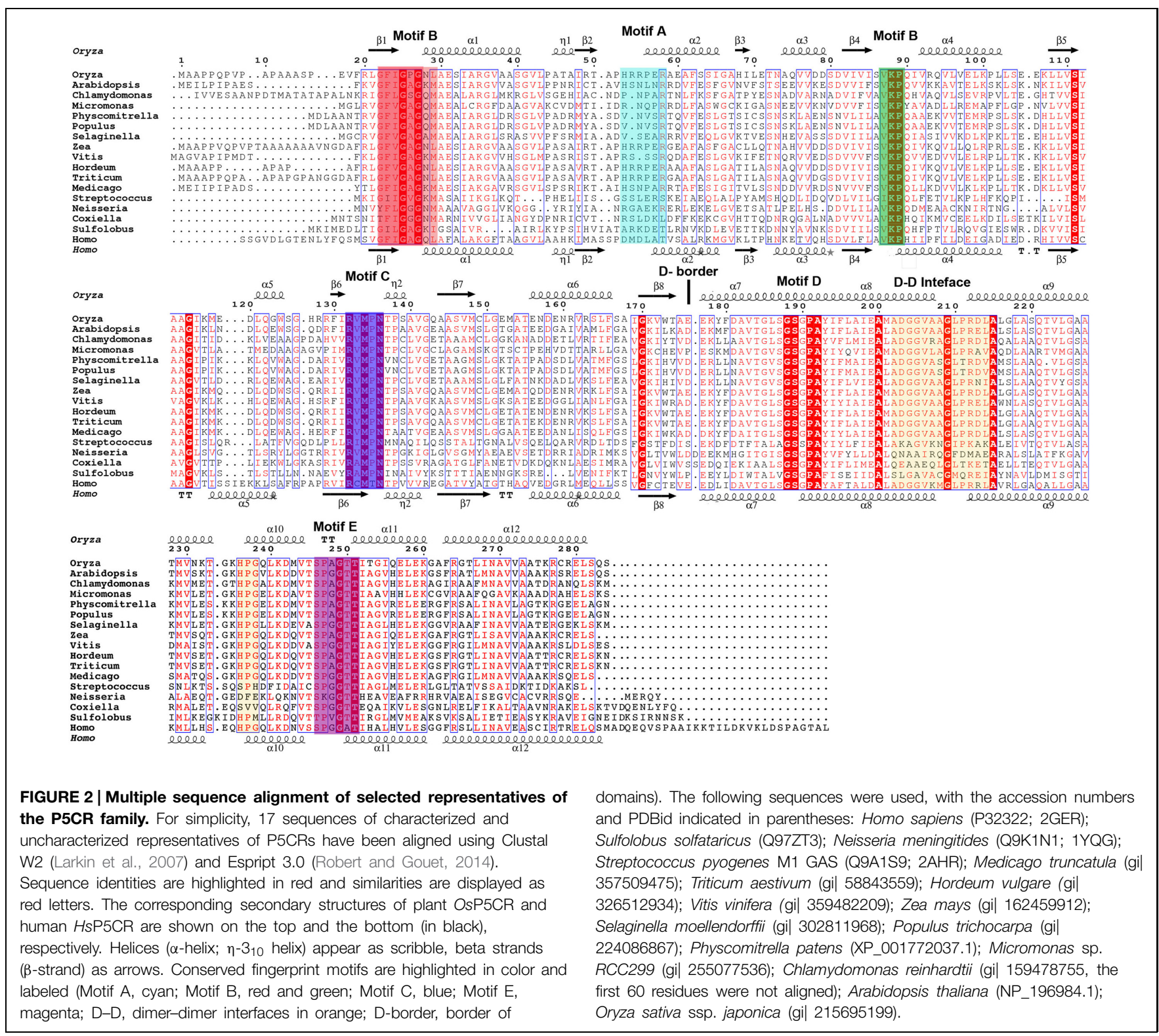


The typical N-terminal domain is comprised of a central core formed by parallel $\beta$-sheet $(\beta 3, \beta 2, \beta 1, \beta 4, \beta 5, \beta 6)$ surrounded by six alpha helices $(\alpha 1-\alpha 6)$ and two $3_{10}$ helices $(\eta 1, \eta 2)$ in the topological order $(\beta 1, \alpha 1, \eta 1, \beta 2, \alpha 2, \beta 3, \alpha 3, \beta 4, \alpha 4, \beta 5$, $\alpha 5, \beta 6, \eta 2, \beta 7, \alpha 6, \beta 8$ ), (Nocek et al., 2005; Meng et al., 2006). This domain is classified as a member of Pfam family PF03807 (F420_oxidored), and it is common amongst FAD or NAD(P)H dependent oxidoreductases (Carugo and Argos, 1997; Dym and Eisenberg, 2001; Kleiger and Eisenberg, 2002; Nocek et al., 2002). This fold is composed of three parallel $\beta$-strands linked by two $\alpha$-helices, founding the so-called Rossmann fold (Rossmann et al., 1974). The Rossmann fold can be identified by the short amino acid sequence motif (G-x-x-G-x-G, Motif $B$; Figure 2), which binds one nucleotide cofactor molecule. Since the $\mathrm{NAD}(\mathrm{P}) \mathrm{H}$ molecule has two nucleotides (adenosine and nicotinamide riboside), $\mathrm{NAD}(\mathrm{P}) \mathrm{H}$ dependent oxidoreductases, such as P5CR enzymes, use a 3-layer sandwich fold which consists of two fused mononucleotide-binding motifs. These motifs are structurally related by a pseudo-twofold rotation within one domain, yet they do not share any similarity in the sequence (Figure 2; Bottoms et al., 2002).
The C-terminal domain is entirely alpha helical (six helices: $\alpha 7-\alpha 12$ in SpP5CR) in typical size proteins such as plant P5CRs (Figure 2), while longer representatives such as Homo sapiens P5CR (HsP5CR) have extended C-terminus, which was missing in the structure (Meng et al., 2006; Pfam family: PF14748). The C-terminal domains are involved in the homodimer formation and arrangement of the active site. Based on structures, it is evident that dimerization is essential to the formation of a completely functional enzyme, indicating that the dimer is a basic biological unit utilized by the P5CR family. To date, no monomeric structures of P5CR were reported, and only dimers (Nocek et al., 2005) or higher oligomers with even number of subunits were observed (Nocek et al., 2005; Meng et al., 2006). Also, chemical treatment of $H s$ P5CR decamer with 1-4 M urea showed that it dissociates into homodimers, and no monomeric forms were observed (Meng et al., 2006). The requirement for a dimeric configuration is unambiguous based on the analysis of the structures of a typical P5CR monomer (Figures 3A,B). In the monomer, two parts of the active site are divided and separated by $\sim 30 \AA$, creating what would be an inactive enzyme. In contrast, in the dimer the fully functional active sites are

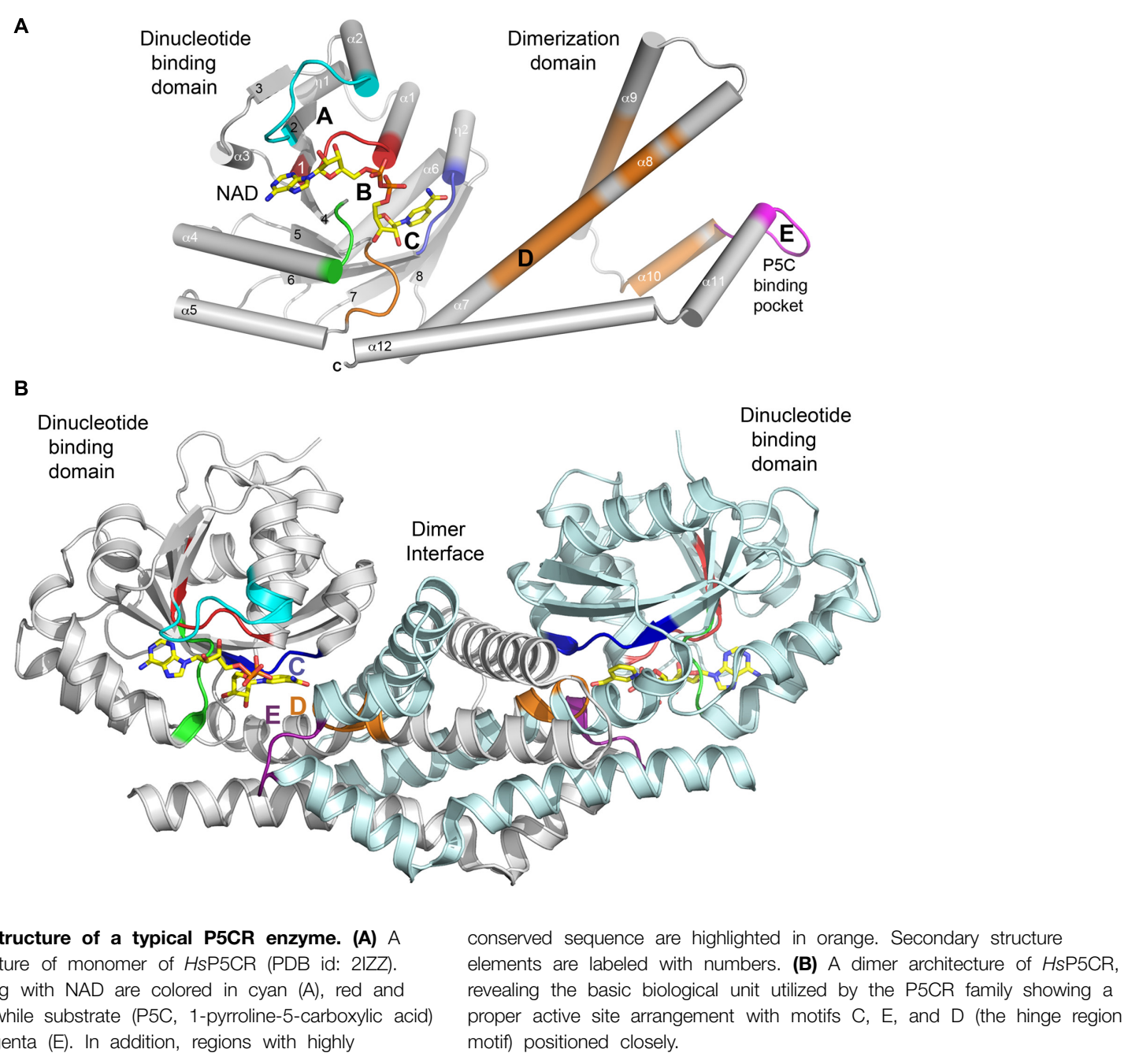

FIGURE 3 | Secondary structure of a typical P5CR enzyme. (A) A diagram showing architecture of monomer of HsP5CR (PDB id: 2IZZ). Parts of protein interacting with NAD are colored in cyan (A), red and green (B) and blue (C), while substrate (P5C, 1-pyrroline-5-carboxylic acid) pocket is colored in magenta $(E)$. In addition, regions with highly elements are labeled with numbers. (B) A dimer architecture of HsP5CR, revealing the basic biological unit utilized by the P5CR family showing a proper active site arrangement with motifs $C, E$, and $D$ (the hinge region 
assembled through contribution from the N-terminal domain of one subunit of the dimer and the dimerization domain of the opposite subunit, and vice-versa. This way, two welldefined active sites are formed, within $\sim 13 \AA$ distance between motif $\mathrm{C}$ of one subunit and motif $\mathrm{E}$ of the opposite subunit (Figures 2 and 3B).

\section{Dimer Interface}

Tight packing of hook-like dimerization domains between two neighboring molecules and swapping of the C-terminal helices ( $\alpha 9-\alpha 11)$ makes the intertwined dimer interface. A threedimensional domain swapping, in which a section of the monomeric protein is replaced by the matching part from a second monomer is one of the common structural adaptations used for protein oligomerization (Jones and Thornton, 1996; Bennett and Eisenberg, 2004). As there is no experimental evidence of reversible swapping of the domains (between monomer and dimer), it suggests that intertwined P5CR dimers might have evolved very early in evolution and became conserved. Sequence alignment analysis seems to support this hypothesis as almost complete conservation of the sequence for the first three helices of the C-terminal domain (residues 180-218 in OsP5CR) is observed in plant P5CRs, while 50\% of sequence identity (as estimated based on the alignment) is observed for all other representatives annotated as P5CR in Pfam database (PF14748.1). In fact, these three-conserved helices form a core that is sufficient to produce a stable dimer on its own, as shown in the structure of Bacillus cereus P5CR (BcP5CR, PDB id: 3GT0). The $B c$ P5CR structure model has residues 1-217 and is missing the last 55 residues (a full-length gene product of Bc_2977 has 272 residues), and yet it forms a stable dimeric structure. A hallmark of the dimerization core is a loop with a sequence motif G-S-XP-A (Motif D, Figure 2), which is predicted to be a hinge region according to both Hingeprot and StoneHinge softwares (Emekli et al., 2008; Keating et al., 2009). This loop contributes to the formation of the active site pocket together with residues from the motifs $\mathrm{C}$ and $\mathrm{E}$. It also stabilizes the active site core by a hydrogen bond formed between the conserved serine of the motif $\mathrm{D}$ with the last threonine of the motif E (S176, T238 in HsP5CR; S189, T251in OsP5CR).

\section{P5CR Decamers}

Examination of reported molecular weights $\left(M_{\mathrm{W}}\right)$ of P5CR enzymes reveals the presence of several multimeric forms. These were estimated, based on the SEC experiments under nondenaturating conditions and were ranging from dimers (Nocek et al., 2005), octamers (Kenklies et al., 1999), decamers (Deutch et al., 1982; Nocek et al., 2005), and dodecamers (Murahama et al., 2001; Giberti et al., 2014) to even higher polymers (Krueger et al., 1986; Meng et al., 2009). However, results obtained for full-length members of P5CR superfamily by crystallographic studies consistently showed two oligomeric forms: dimeric and decameric (Nocek et al., 2005; Meng et al., 2006). Despite the different types of crystallization conditions, crystal forms, and variation in the sequence, the decamer is the highest oligomeric species observed by crystallography [especially in eukaryotic representatives: HsP5CR (Meng et al., 2006), OsP5CR (Forlani et al., 2015) and AtP5CR (see below)].

Even though the SEC technique is a powerful method for size fractionation of biomolecules, it is highly dependent on the accurate and precise calibration curve, temperature and several other factors, which may cause an error of up to $10 \%$ in the estimated molecular weight (Folta-Stogniew and Williams, 1999; Moreira et al., 2007). Considering 10\% error, the forms estimated to be octamers and dodecamers could in fact be a decamers. In order to improve our SEC column calibration method, a representative of P5CRs from SpP5CR was utilized. SpP5CR has been experimentally characterized to be decamer by both SEC and X-ray crystallography methods and was used as one of the molecular weight markers (Nocek et al., 2005). Using this approach we were able to show that AtP5CR, recently reported to be a dodecamer (Giberti et al., 2014), under our experimental conditions is more likely a decamer with an estimated molecular weight of $275 \mathrm{kDa}$ (Supplementary Figure S1, 276 residues, theoretical $M w$ deduced from the sequence $28,624 \mathrm{kDa}$ and theoretical decamer $M \mathrm{w}=286,240 \mathrm{kDa})$.

A typical decameric structure of P5CR is described based on the structures of HsP5CR (Meng et al., 2006) and OsP5CR representatives (Forlani et al., 2015). It resembles an hourglassshaped assembly, which is formed by five closely interacting dimers, arranged around the fivefold symmetry axis. Monomers in each dimer are related by the twofold symmetry axis, and form a dimerization interface. The interacting dimerization domains form a very tight five-membered ring with a $25 \AA$ opening in the center, while the dinucleotide-binding domains are located on the top and the bottom of the ring, and do not interact with each other (Figure 4). In contrast to the strong interactions observed in the dimerization interface of HsP5CR and OsP5CR, the dimer-dimer interfaces forming the decamer are much weaker. In fact, analysis of these interactions using server PISA (Krissinel and Henrick, 2007) in HsP5CR shows that the dimer interface is contributed by all helices from the C-terminus and buries about $4100 \AA^{2}$ of the accessible surface area per dimer (which corresponds to about $25 \%$ of each subunit surface area). In contrast, the decamer interface is formed only by lateral interactions of the C-terminal $\alpha 8-$ $\alpha 12$ (residues located between 196-214 and 235-260) and its complementary symmetry mates. This leads to a substantially smaller interface between four contributing elements $\left(\sim 1100 \AA^{2}\right)$ from consecutive dimers, which accounts for less than $5 \%$ of each dimer surface (Figure 4B). Two pairs of loops from the dimers [from the top: the loop $\alpha 8-\alpha 9$ of molecule $A$ and the loop formed between $\alpha 10-\alpha 11$ (Motif D) of molecule D; and from the bottom, the loop $\alpha 8-\alpha 9$ of molecule $C$ and $\alpha 10-\alpha 11$ molecule $B$ (Motif $E$, the active site loop)] confine the interface that is localized around twofold symmetry axis. Interestingly, the pyrrolidine rings of conserved proline residues are located at the core of these loops at distances of $\sim 6 \AA$ and are antiparallel with respect to each other (Meng et al., 2006). Comparison of structures of $\mathrm{Hs} \mathrm{P} 5 \mathrm{CR}$ and OsP5CR (residue numbers are given in parentheses; see below) reveals a key set of highly conserved inter-unit salt bridges that stabilize the interface. These are formed by the interaction of R199 

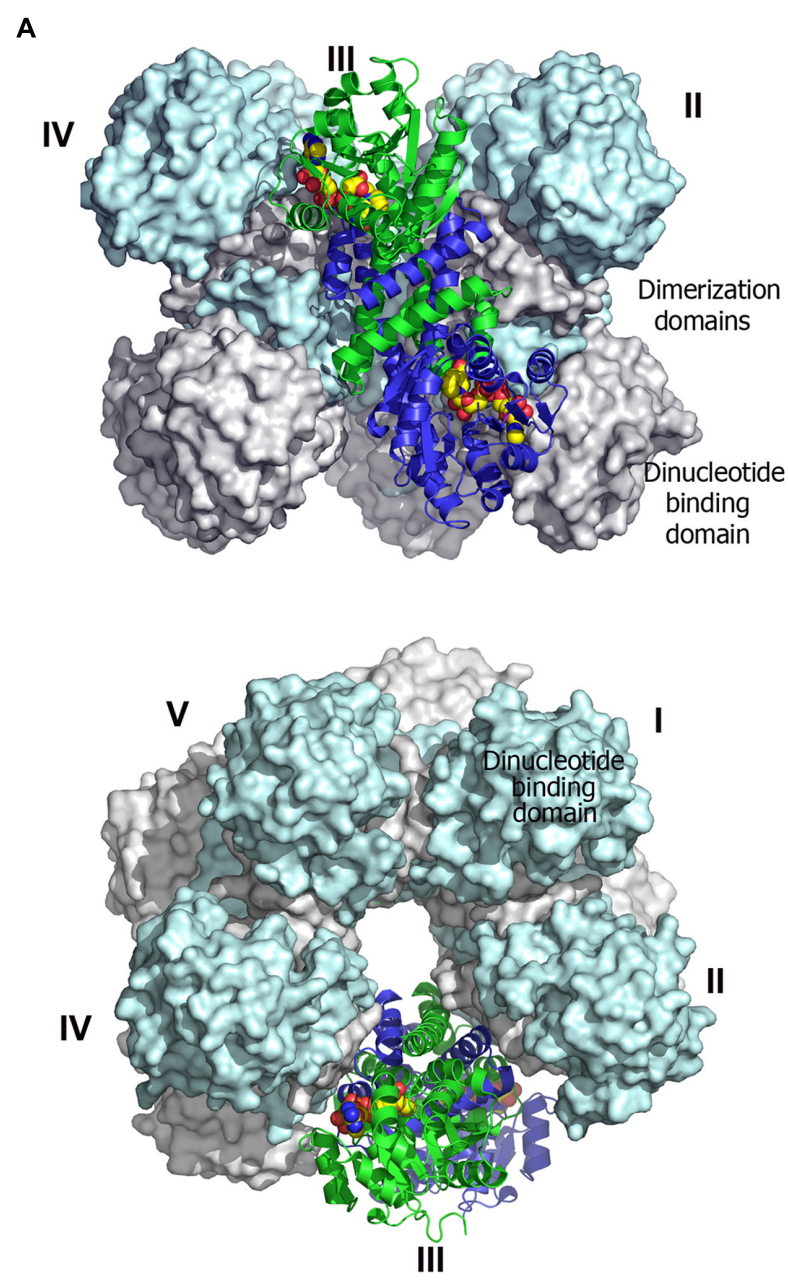

B

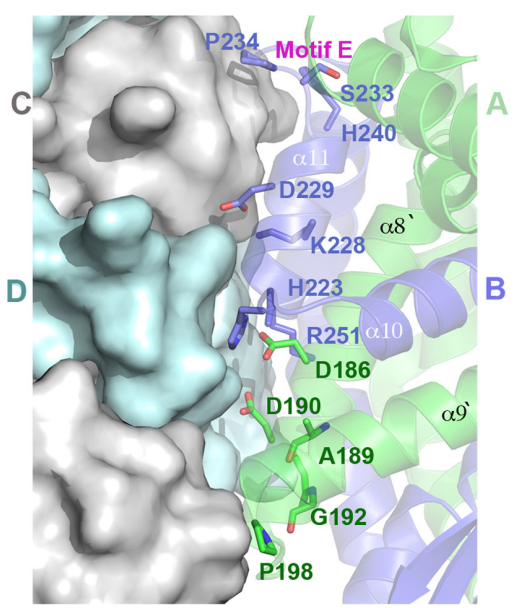

C

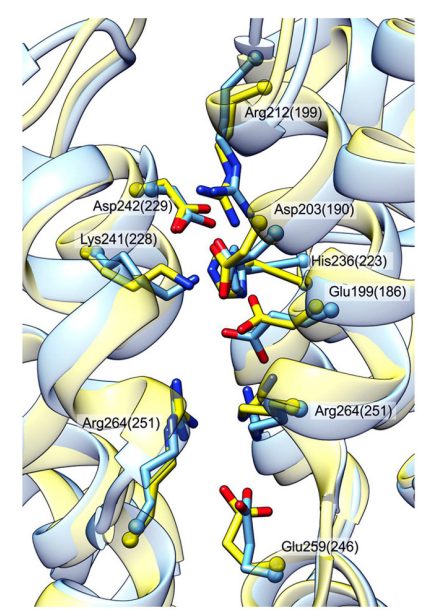

FIGURE 4 | Decameric structure of human P5CR. (A) Two views of the decamer of $\mathrm{HSP} 5 \mathrm{CR}$ (PDB id: $2 \mathrm{IZZ}$ ) related by $90^{\circ}$ rotation, displaying five dimers (numbered) arranged around the fivefold symmetry axis (surfaces of four dimers are shown in gray and cyan, while one dimer is show as cartoon in green and blue. The NAD molecules located on the side of the dinucleotide binding domains are shown as a yellow space filled models. (B) Close-up view of the dimer-dimer interface, revealing positions of proposed key residues involved in decamer interface formation. (C) The dimer-dimer interface network pattern appears to be similar and conserved between HsP5CR (yellow, the residues numbers in the brackets) and OsP5CR (blue).
(R212) with D229 (D242). Another set of salt bridges is created between D190 (D203) and K228 (K241), and H223 (H236) and D229 (D242), (Figure 4C). Additionally, a very interesting $\pi-\pi$ stacking interaction between two related R264 (R251) residues from the adjacent protein subunits is present in the middle of the dimer-dimer interface. All of the residues forming these salt bridges appear to be preserved in decameric P5CRs and are conserved in plants representatives displayed in Figure 2.

\section{Can Dimer/Decamer Determinants Be Predicted from the Sequence?}

The interfaces in proteins are formed by the interactions of favorable tight fitting regions. These regions are characterized by complementary areas distributed throughout the interface, which are enhanced by the presence of structurally conserved residues (Nooren and Thornton, 2003). These regions are considered "complementary" because they pair both their shapes and the collection of hydrophobic and hydrophilic residues within hydrophobic cores and "hot-spots" of charged residues (Moreira et al., 2007). The structural and sequence comparison of P5CRs shows that their complementary regions are located at the C-terminus, and they are extremely similar (for example in OsP5CR, 79 out of 107 residues are conserved or of similar character, Figure 2). Additional evidence of the importance of the C-terminal region to decamer formation comes from the analysis of the truncated and full-length forms of $B c$ P5CR. The full-length BcP5CR protein (Bc_2977, 272 residues) has been confirmed to form a decamer under our experimental conditions (Supplementary Figure S1, the experimental Mw of $275 \mathrm{kDa}$ vs. a deduced theoretical $M w$ of $293 \mathrm{kDa})$. On the contrary, the structure of the truncated $B c \mathrm{P} 5 \mathrm{CR}$ (PDB id: 3GT0; residues 1-217, missing the last 55 residues) was calculated by software PISA (Krissinel and Henrick, 2007) to be either a 
dimer or a tetramer. This strongly suggests that the last 3-4 helices of the dimerization domain (the last 55 residues for a typical-size P5CRs) are important for proper oligomer formation. An inspection of the sequences of selected representatives of P5CRs seems to corroborate this further as significant variation of sequences are observed between selected dimeric (Neisseria meningitides, NmP5CR and Coxiella burnetii, CbP5CR) and decameric members (HsP5CR and OsP5CR) at this region. Therefore, we propose that specific sequence differences may provide an explanation why some P5CRs form dimeric structures while others assemble into decamers.

The most noticeable differences are found in the sequences that span between the C-terminus of $\alpha 8$ and the N-terminal part of $\alpha 9$. The sequence motif (residues 202-214 in OsP5CR) that is conserved in plant $\mathrm{P} 5 \mathrm{CRs}$ and $H s \mathrm{P} 5 \mathrm{CR} \mathrm{A}-\mathrm{D}-\mathrm{G}-\mathrm{G}-$ V-A-A-G-L-P-R-D/R-L is replaced by Q-N-A-A-I-R-Q-G-F-DM-A-E in NmP5CR (dimer) and Q-E-A-A-E-Q-L-G-L-T-K$\mathrm{E}-\mathrm{T}$ in $\mathrm{CbP} 5 \mathrm{CR}$ (dimer). Mutation of glycine and proline residues located within this motif (G204, G205, P211 in plant decamers) to following residues (A204, A205, D211/T211 in dimers) likely changes the conformation of this region and disrupts its potential interface surface. In addition, replacement of conserved proline and glycine residues in HsP5CR (P198, P224, G225, and P234) with four different residues (D188, F214, E215, K224 in NmP5CR) and three residues (T198, V224, V225 in CbP5CR) might be even more disruptive. The proline residues are important in establishing complementary "sharp turns" of the loops between the dimer-dimer interfaces and bringing both interfaces closer together (Figure 4). Also, replacement of proline residues with bulkier residues increases the packing distance between molecules, and likely dislocates the interacting backbones. In addition, some of the residues that were shown to form the conserved salt bridges at the protein-protein interface of the decamer (Figures 2 and 4C) are missing in the sequence of dimers. For example, the symmetry related R264 residues were mutated to alanine in NmP5CR and to threonine in SpP5CR, while R212 (OsP5CR) was changed to methionine in NmP5CR. Our analysis shows that the dimer-dimer interface network pattern appears to be similar. Sequence comparison suggests that these replacements at the C-terminal region could be a reason, why certain representatives of the P5CR family do not form complementary dimer-dimer interfaces. Detailed biochemical and mutagenesis studies will be required to provide conclusive experimental evidence.

\section{Sequence Analysis of Dinucleotide Binding Domain (NADPH vs. NADH Preference)}

Analysis of the NADH/NADPH binding domains across the P5CR family showed that they can utilize both NADH and $\mathrm{NADPH}$ as a reducing agent, while their affinity for either one varies between the species and, sometimes, between different subcellular isoforms. The only difference between these cofactors is the presence of a phosphate group in NADPH, which replaces ribose 2-hydroxyl of adenosine in NADH. The phosphate group in NADPH does not influence the redox abilities of the molecule from the enzymatic standpoint. Both nucleotide cofactor pairs
$\left(\mathrm{NADH} / \mathrm{NAD}^{+}\right.$and NADPH/NADP ${ }^{+}$) serve as donors and/or acceptors of reducing equivalents quite efficiently in living cells, and have the same midpoint potential (-0.32 V). However, the additional phosphate group allows enzymes to discriminate between NADH and NADPH, which in turn allows the cell to regulate them both independently.

In the last 10 years, several complex structures of P5CR with cofactors have been determined, explaining the molecular basis of cofactors binding (Nocek et al., 2005; Meng et al., 2006). Superimposition of the structures of selected representatives of the P5CR family (PDB id: 2RCY, 2IZZ, 2GR9, 2AHR, 2AG8), including the recently determined low-resolution structure of OsP5CR (Forlani et al., 2015), showed virtually identical architecture of the $\mathrm{NAD}(\mathrm{P}) \mathrm{H}$ binding domain for all representatives. In addition, it appears that $\mathrm{NAD}(\mathrm{P}) \mathrm{H}$ binding modes are very similar in bacterial and human P5CRs, and is correlated by the high sequence similarity of the regions (Motifs $\mathrm{A}, \mathrm{B}$ and $\mathrm{C}$ ) involved in interaction with the cofactor. Therefore, a model of the cofactor binding was predicted and closely correlated with structures/sequences of eukaryotic representatives of $\mathrm{P} 5 \mathrm{CR}$ ( $\mathrm{Hs} \mathrm{P} 5 \mathrm{CR}$ and $\mathrm{OsP} 5 \mathrm{CR}$ ). Several structures have shown that the cofactor molecule binds in an extended conformation in a cavity between the $\mathrm{N}$-terminal domain and the dimerization domain burying the nicotinamide ring of the cofactor in the active center pocket (Nocek et al., 2005). The cofactor interactions with the protein are characterized by three fingerprint regions, as shown in Figure 3A (Motifs A, B, and C). Motif $\mathrm{A}$ is a loop formed between the second strand $(\beta 2)$ and the second helix $(\alpha 2)$ of the N-terminal domain. It provides positively charged residues for a direct interaction with the adenine moiety, and a 2 phosphate-binding region for NADPH. Three different modes of the NADPH phosphate group interaction can be inferred based on the two bacterial (SpP5CR and NmP5CR, Nocek et al., 2005) and Plasmodium falciparum P5CR (PfP5CR) structures, showing high propensity for the interactions involving serine and arginine (mode 1), lysine (mode 2 ) or asparagine (mode 3) (Figure 5A). Most of plant P5CRs has a sequence motif $\mathrm{H}-\mathrm{R}-\mathrm{R}-\mathrm{x}-\mathrm{x}-\mathrm{R}$ (residues 53-58 in OsP5CR) or its variation with $\mathrm{H}-\mathrm{x}-\mathrm{N}-\mathrm{x}-\mathrm{N}-\mathrm{R}$ (residues 53-58 in AtP5CR), (Figure 2). The preference for histidine and arginine residues in this region is especially interesting as the side chains of those residues are very often found to form preferable $\pi-\pi$ stacking interactions with the adenine ring of the cofactor (Mao et al., 2003; Pyrkov et al., 2009; Firoz et al., 2011). In addition, arginine residues are favorable residues for NADPH binding, and their guanidine side chains have been found to play a key role in binding of the $2^{\prime}$-adenosine phosphate, either alone [as it was observed in the NADPH-SpP5CR complex structure (Nocek et al., 2005)] or in concert with other arginines or lysines (Levy et al., 1996; Sabri et al., 2009). The presence of one or two positively charged amino acids in this region could be a good determinant of the enzyme's selectivity toward a phosphorylated version of $\mathrm{NADH}$, and mode 1 binding interaction.

Motif $B$, with the consensus sequence $G-x-x-G-x-G-x-M / L$ (a variant of the typical $G-x-G-x-x-G$ pattern motif of the Rossmann fold) is well conserved throughout the plant P5CRs as it forms a loop that can interact with charged groups of 


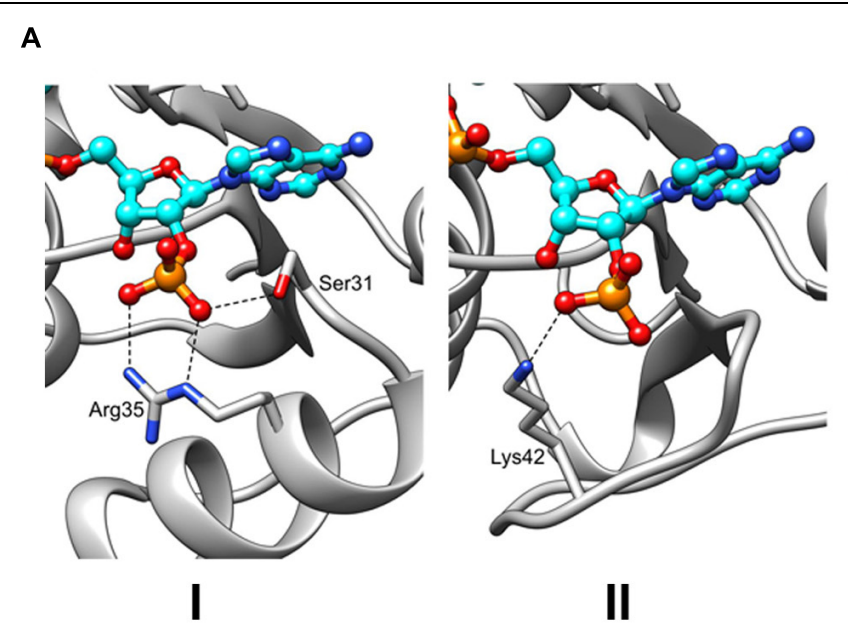

FIGURE 5 | The insights into the binding of NADPH and L-Proline.

(A) Three different types of interactions with the NADPH phosphate moieties can be hypothesized based on the previously characterized P5CR structures with the following residues: (Type 1) serine (S31) and arginine (R35), as observed in structure of SpP5CR (PDB id: 2AHR). (Type 2) lysine (K42) of PfP5CR (PDB
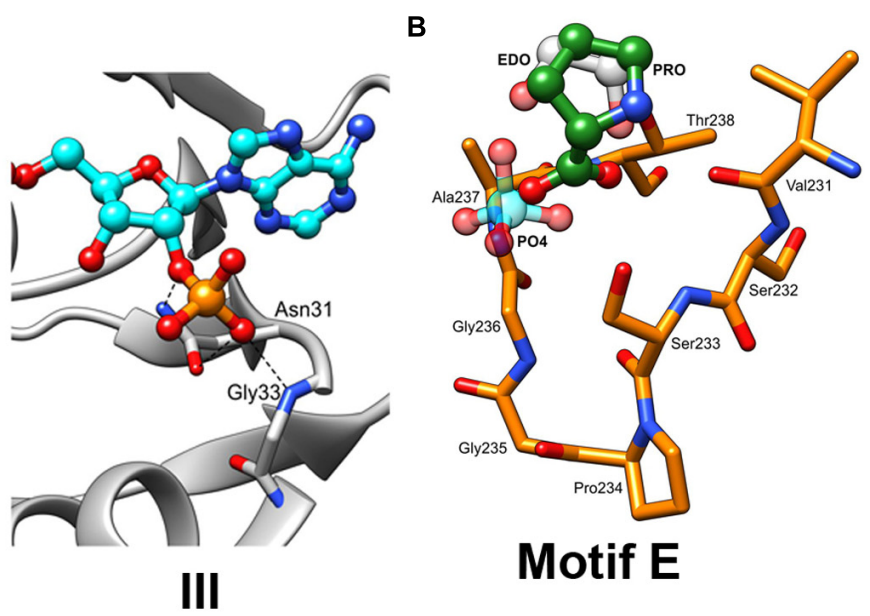

id: 2RCY). (Type 3) Asparagine (N31) of NmP5CR (PDB id: 2AG8). (B) Close-up view of the motif $\mathrm{E}$. Structures of enzymes with either buffer-derived molecules (phosphate ion shown in light blue and ethylene glycol molecule shown in gray) or L-proline bound (in green, as observed in the structure of SpP5CR) were superimposed. For clarity, only the structure of HsP5CR is displayed in orange. nearby pyrophosphate moieties of either of the cofactors. The glycine-rich loop is placed between the C-terminus of the strand $\beta 1$ and the $\mathrm{N}$-terminus of helix $\alpha 1$, which forms a dipol and provides charge stabilization of the phosphate group. The positioning of the glycine residues in this region, which due to their lack of the side chains have the lowest steric hindrance, is highly advantageous as it allows for close contact between the pyrophosphate group and the backbone of the adjacent helix. Mutations of glycine residues in the glycine rich region (Motif B) have been reported to reduce or completely eliminate enzyme activity (Wierenga et al., 1985)

There is another set of residues that should be included as a part of the motif $B$, as it influences the position of the pyrophosphate moiety of the cofactor, even though it is $\sim 60$ residues apart from the glycine-rich signature. This additional set of residues forms a loop positioned between $\beta 4$ and $\alpha 4$ and encompasses a consensus sequence $\mathrm{V}-\mathrm{K}-\mathrm{P}$. The conserved lysine residue in the middle of this motif (Figure 2), which is present in all representatives of P5CRs, acts as an anchor either directly through the interaction with one of the phosphate groups of the pyrophosphate, or indirectly by reducing the space around the pyrophosphate and pushing it toward the glycine-rich region on the other side of the cavity.

Finally, the last of nucleotide binding elements is motif C. It forms a conserved loop at the active site (132-136 in Os $\mathrm{P} 5 \mathrm{CR}$ ) with the $\mathrm{R}-\mathrm{x}-\mathrm{M}-\mathrm{x}-\mathrm{N}$ sequence. The methionine residue represents one of several non-polar residues that contribute to the active site pocket and is in proximity to the nicotinamide ring of the cofactor. This and another methionine, which is part of motif $\mathrm{B}$, surround the cofactor's nicotinamide ring and are major contributors to the hydrophobic environment, which is likely required in the active site to provide the essential hydride transfer step.

\section{Pyrroline-5-Carboxylate/Proline Binding Site}

In plants, sequence alignment of the P5CR family highlights a conserved consensus sequence motif S-P-A/G-G-T-T (Motif E) that is located at the C-terminal part of the protein (Figure 2). Very similar motifs are present in other organisms, demonstrating a common structural feature. This motif is located between helices $\beta 10$ and $\beta 11$ and creates a tight turn ( $\alpha$-turn), which reverses direction of main chain helices $\beta 11-$ $\beta 12$, and forms a small cavity (Nocek et al., 2005). Presence of proline (cyclic structure) and glycine (the most accommodating sterically) residues at the center of the cavity is ideally suited for the $\alpha$-turn as it allows for favorable positioning of serine and threonine at the boundaries of the cavity (Figure 5B). The backbones of these non-polar residues were observed to interact with the carboxylate group of L-proline (the product) in the structure of SpP5CR (Nocek et al., 2005), suggesting their essential role in positioning of the substrate in the active site. However, structure with L-P5C (the substrate) has not yet been reported, and it is a prediction based on the current structural results. In addition to binding the product of the enzymatic reaction, the same pocket was observed to bind a phosphate ion ( $\sim 2 \mathrm{M}$ potassium phosphate ion concentration was reported in crystallization condition for CbP5CR, PDB header) and 1,2ethanediol molecule, revealing affinity for small anions.

\section{Conformational Changes Observed in Selected P5CRs}

Pyrroline-5-carboxylate reductase enzymes have their dinucleotide domains loosely connected to the tightly packed dimerization domains via a predicted hinge region (Motif D; Figure 4A). Hinge regions are often placed between domains and are attributed the role of moderating the conformational movement of the domains. The structural superimposition of monomers of selected P5CRs revealed almost uniform 
arrangement of subunits in the decameric molecule (root-meansquare deviation (r.m.s.d.) of $0.3-\AA$ in SpP5CR and r.m.s.d. of $0.5-\AA$ in $H s \mathrm{P} 5 \mathrm{CR}$ between the most divergent regions). This indicates lack of significant structural changes and minimal differences in the relative orientation of the $\mathrm{N}$ - and C-terminal domains. This also allows us to believe that the previously proposed lock-and-key model, and the concept of enzyme undergoing only small rearrangements, is correct for some of the studied enzymes (Nocek et al., 2005). However, more prominent differences were observed in the dimeric structure of $C b P 5 C R$ (PDB id: 3TRI; Franklin et al., 2015) revealing a substantial r.m.s.d. of $2.5-\AA$ and indicating large changes in the relative orientation of the $\mathrm{N}$ - and the C-terminal domains (Figure 6A). In fact, pairwise structural alignment of both monomers in CbP5CR (with the C-terminal domains closely aligned) unveils $\sim 7$ - $\AA$ movement and the difference in the orientation of the respective $\mathrm{N}$-terminal domains. A close examination of domains in the dimer shows that both have a NADPH molecule bound; yet only one of the active sites has a phosphate ion present in the area of the motif E (L-proline binding motif; Figure 6C). The active site with both NADPH and the phosphate ion bound adopts a closed conformation, with the dinucleotide positioned in a narrow positively charged cleft and phosphate ion enclosed in the catalytic pocket (closed-conformation). The second active site, with only NADPH molecule bound, is wide and appears to be in an open-conformation (Figure 6B). Hence, the only difference between both sites is a presence of the phosphate ion bound in the active site. This suggests that the phosphate ion might trigger conformational changes and possibly acts as a mimic/inhibitor of L-proline/P5C in CbP5CR. It is also important to note that the open-to-closed motion in CbP5CR brings together critical residues from both domains, allowing the formation of a functionally competent active site.

The presence of the dynamic movement of the N-terminal domain and conformational changes in the active site may have their consequences for the mechanism of the ligands binding and the release of the products. In previous studies, the decameric structure of SpP5CR in complex with $\mathrm{NADP}^{+}$ revealed that the active site had insufficient opening for L-proline to enter (Nocek et al., 2005). This led to a hypothesis that the order of substrates binding might require L-P5C to bind ahead of the coenzyme, and was later confirmed experimentally (Petrollino and Forlani, 2012). In addition, experimental evidence supporting an ordered substrate binding in plant P5CRs has been obtained from the kinetic analysis of enzyme inhibition by some aminobisphosphonates (Forlani et al., 2007). The CbP5CR structure implies different possibilities in which the NADPH cofactor binds first ahead of the L-pyrroline, or both of them bind simultaneously as the active site seems to

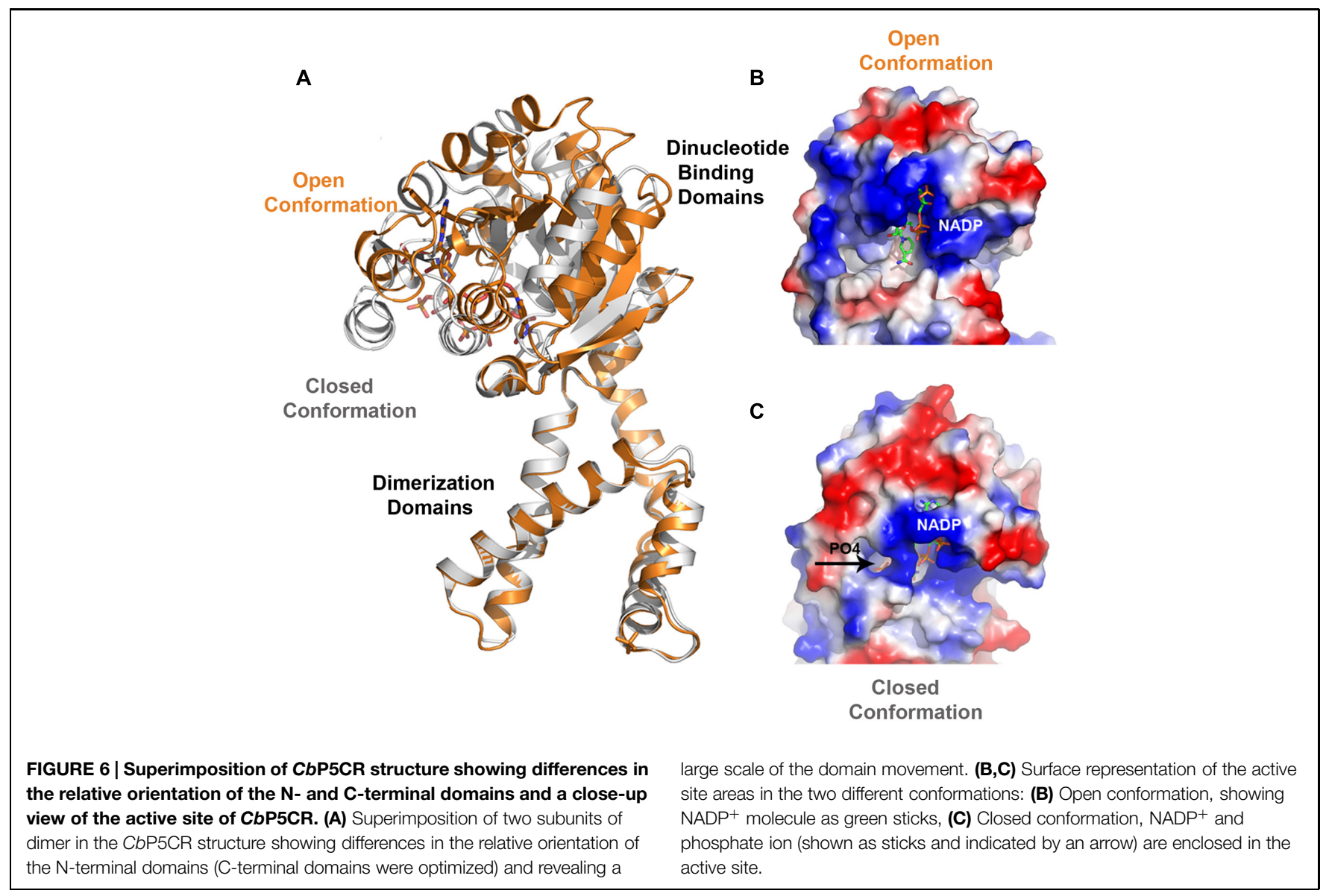


be fully open. However binding of one of the substrates triggers conformational changes as it has been observed in case of other $\mathrm{NAD}(\mathrm{P}) \mathrm{H}$-dependent reductase (Sanli et al., 2003).

It remains to be seen whether this mechanism is universal for other P5CR enzymes or is only limited to CbP5CR and/or reflects flexible nature of some representatives of the P5CR family. On this note, significant dynamic movements of the N-terminal domains have been observed in the OsP5CR structure, hindering modeling of these domains in the low-resolution decameric structure, and indicating that dynamic movement is also present in decameric plant P5CRs (Forlani et al., 2015).

\section{Feedback Inhibition and Metals Effect on Activity of P5CRs}

In enzymatic reactions where the product resembles the starting reactant, product inhibition can be often observed. This kind of feedback provides a very efficient mechanism of controlling concentration of the product of the reaction and regulating resources in the cell. The last step of the synthesis of proline, catalyzed by P5CR enzymes, was reported to be generally unaffected by feedback inhibition in plants (Szoke et al., 1992), in contrast to the first enzyme of the glutamate pathway (P5C synthetase; Aral and Kamoun, 1997; Kavi Kishor et al., 2005). However, recent studies on AtP5CR showed that this enzyme uses both NADPH and NADH, displaying a much higher affinity for the former (Giberti et al., 2014). When NADH was used as the electron donor, feedback inhibition by high yet physiological proline concentrations was reported. Structural studies of bacterial SpP5CR revealed that one of the possible mechanisms by which proline may inhibit P5CR enzymes is by partially blocking the access to the active site (Nocek et al., 2005). Analysis of proline and cofactor-bound structures of SpP5CR showed that one L-proline molecule was bound in the active site (Motif E), but the second L-proline molecule was found in the center of the active site pocket, at the position typically occupied by the nicotinamide ring moiety of the cofactor. It suggests that accumulation of product of the reaction in the active site might prevent $\mathrm{NAD}(\mathrm{P}) \mathrm{H}$ from binding of substrate and inhibit the reaction.

In addition, other studies on P5CRs have shown that metal ions may have either inhibitory or stimulatory effects on the enzymatic activity depending on the nature of the metal and its concentration. For instance, a stimulatory effect of $100 \mathrm{mM}$ $\mathrm{KCl}$ or $10 \mathrm{mM} \mathrm{MgCl}_{2}$ on the $\mathrm{NADH}$-dependent reaction for partially purified P5CR from Pisum sativum L. or Mycobacterium tuberculosis P5CR were reported (Rayapati et al., 1989; Yang et al., 2006). As metal binding sites in proteins differ in they coordination numbers and geometries, and their preference for certain environments, therefore sometimes their binding sites could be predicted. For example, the so-called 'alkali class' (Ca, K, $\mathrm{Na}, \mathrm{Mg}$ ) consists of metals that interact almost exclusively with oxygen atoms (Zheng et al., 2008). The ligands that often interact with alkaline class ions include side chains of aspartic, glutamic, serine, and threonine residues, or backbone carbonyl oxygen and water molecules. Occasionally, asparagine and glutamine side chains are also found to interact with metals. An inspection of P5CRs structures and sequences showed limited occurrence of the cluster of these residues within the active site and revealed only two potential locations. Close analysis of the OsP5CR structure shows that one of them is positioned close to the adenine moiety of the cofactor, and has only three suitable residues that potentially could interact with metal ion (S33, T59, and N56). The second one is the part of motif E (T184, S189, T250, and T251) that is involved in binding of the L-pyrroline mojety. Binding of the metal in the active site would certainly explain the inhibitory effect, however, it remains to be seen if metals could bind there.

\section{Summary}

The P5CR enzymes have been studied for several years, but remain relatively poorly understood from the structural perspective. Particular areas of interest include: structural details of cofactor preference and recognition, substrate binding site, oligomerization and metals effect on activity. The patterns that emerged from this comprehensive phylogenetic analysis suggest that a vertical descent dominated in evolution of P5CR genes, particularly in higher plants and algae. The plant P5CRs appear to be distant from the cyanobacterial and are much closer to the metazoan enzymes. At the sequence level, a close similarity of plant and human P5CRs ( $\sim 44 \%$ sequence identity between $H_{s} \mathrm{P} 5 \mathrm{CR}$ and $\mathrm{OsP} 5 \mathrm{CR}$ ) is somewhat visible revealing a consistent pattern of conserved residues. In contrast, a typical bacterial representative such as SpP5CR shares much lower $(\sim 30 \%)$ sequence similarity with OsP5CR, and shows reduced sequence conservations especially at the dimerization domain region. Despite evident sequence differences, X-ray crystallographic studies of $H s \mathrm{P} 5 \mathrm{CR}$ and SpP5CR enzymes unveiled very similar folds. Similar folds have been also observed in other representatives such as: NmP5CR, PfP5CR, and $\mathrm{CbP5CR}$. Analysis of these structures showed that dimer is the minimal form of P5CR required for activity (SpP5CR), while a decamer is another oligomeric form observed. Whether dimeric or decameric P5CRs exist likely depends on the specific sequence motifs. The presence of the conserved small proline and glycine residues in the loops and turns between the helices $\alpha 8-\alpha 9$ and $\alpha 10-\alpha 11$ increases the flexibility of the interface region and allows on the tight packing and the formation of the higher oligomers. Also, the preserved patterns of electrostatic interactions are present at the dimer-dimer interfaces of decamers of $H s \mathrm{P} 5 \mathrm{CR}$ and $O s P 5 C R$, likely contributing to the formation of the interface between molecules. The sequence alignment of plant P5CRs showed conservation of several functional motifs involved in the binding of substrates. Three sequence motifs (A, B, and C) are involved in the interaction with both cofactors NADH and NADPH. The presence of one or two positively charged amino acids within the motif A, especially arginine residues, could cause the preference toward the phosphorylated form of the cofactor. Lproline binds within the highly conserved motif $\mathrm{E}$ that is located at the C-terminus. This motif utilizes two conserved residues (serine and threonine), to interact with the carboxylate group of L-proline, as it was observed in the structure of bacterial enzymes ( $S p$ P5CR). In contrast to the $\mathrm{N}$-terminal parts of the 
P5CR enzymes (the dinucleotide-binding domains), the C-terminal domains (the dimerization domains) showed a remarkably high level of sequence similarity, especially at the regions predicted to be involved in a decamer formation. This suggests that plant P5CRs (shown in Figure 2) form higher oligomers, most likely decamers. This is certainly in agreement with the results of the most recent crystallographic studies of OsP5CR, which revealed a decameric arrangement (Forlani et al., 2015). The existence of the decameric structures in many of P5CRs is certainly interesting and brings up a question, what is a functional advantage of such arrangements? One of the possible explanations could be linked to the enzyme's function in osmotolerance. The ring structure has a structural stability that is required to operate in harsh environments such as high ion concentrations and low water content. Also, in order to control hydration the ring structure minimizes the area exposed to solvent vs. equivalent number of representatives, which would be required to perform the same function.

The modular design of P5CRs, and the presence of the conserved hinge region (Motif D) between domains suggest a dynamic behavior. In fact, the conformational rearrangements were observed in CbP5CR structure. The closed conformation of this enzyme is observed in the case when

\section{References}

Adachi, K., Covington, A. S., Darveaux, B. A., Dezwaan, T. M., Frank, S. A., Hamer, L., et al. (2004). Methods for the identification of inhibitors of pyrroline5-carboxylate reductase as antibiotics. Patent WO 2004078992 A2. Available at: http://www.google.com/patents/WO2004078992A2?cl=en

Altschul, S. F., Madden, T. L., Schaffer, A. A., Zhang, J., Zhang, Z., Miller, W., et al. (1997). Gapped BLAST and PSI-BLAST: a new generation of protein database search programs. Nucleic Acids Res. 25, 3389-3402. doi: 10.1093/nar/25. 17.3389

Aral, B., and Kamoun, P. (1997). The proline biosynthesis in living organisms. Amino Acids 13, 189-217. doi: 10.1007/BF01372588

Bennett, M. J., and Eisenberg, D. (2004). The evolving role of 3D domain swapping in proteins. Structure 12, 1339-1341. doi: 10.1016/j.str.2004.07.004

Bottoms, C. A., Smith, P. E., and Tanner, J. J. (2002). A structurally conserved water molecule in Rossmann dinucleotide-binding domains. Protein Sci. 11, 2125-2137. doi: 10.1110/ps.0213502

Carugo, O., and Argos, P. (1997). NADP-dependent enzymes. I: conserved stereochemistry of cofactor binding. Proteins 28, 10-28. doi: 10.1002/(SICI)1097-0134(199705)28:13.3.CO;2-R

Cullis, C. A., Vorster, B. J., Van Der Vyver, C., and Kunert, K. J. (2009). Transfer of genetic material between the chloroplast and nucleus: how is it related to stress in plants? Ann. Bot. 103, 625-633. doi: 10.1093/aob/mcn 173

Delauney, A. J., and Verma, D. P. S. (1993). Proline biosynthesis and osmoregulation in plants. Plant J. 4, 215-223. doi: 10.1046/j.1365313X.1993.04020215.x

Deutch, A. H., Smith, C. J., Rushlow, K. E., and Kretschmer, P. J. (1982). Escherichia coli $\delta^{1}$-pyrroline-5-carboxylate reductase: gene sequence, protein overproduction and purification. Nucleic Acids Res. 10, 7701-7714. doi: 10.1093/nar/10.23.7701

Dym, O., and Eisenberg, D. (2001). Sequence-structure analysis of FAD-containing proteins. Protein Sci. 10, 1712-1728. doi: 10.1110/ps.12801

Edgar, R. C. (2004). MUSCLE: multiple sequence alignment with high accuracy and high throughput. Nucleic Acids Res. 32, 1792-1797. doi: 10.1093/nar/ gkh340

Emekli, U., Schneidman-Duhovny, D., Wolfson, H. J., Nussinov, R., and Haliloglu, T. (2008). HingeProt: automated prediction of hinges in protein structures. Proteins 70, 1219-1227. doi: 10.1002/prot.21613 both NADPH and phosphate ion are bound in the active site, while the open conformation is seen for the site that contains only NADPH. This suggests that the phosphate ion might elicit conformational changes, and may suggest that similar changes occur when $\mathrm{P} 5 \mathrm{C}$ binds. It remains to be seen if similar dynamic movement and conformational changes are present in other representatives of the P5CR family.

\section{Acknowledgment}

We are grateful to Rory Mulligan, Cathy Hatzos-Skintges, Magdalena Makowska-Grzyska, Jurek Osipiuk, and Zbyszek Dauter for their comments and critical reading of the manuscript. Project was partially supported by the Intramural Research Program of the NCI, Center for Cancer Research.

\section{Supplementary Material}

The Supplementary Material for this article can be found online at: http://journal.frontiersin.org/article/10.3389/fpls.2015.00567

Eschenfeldt, W. H., Makowska-Grzyska, M., Stols, L., Donnelly, M. I., Jedrzejczak, R., and Joachimiak, A. (2013). New LIC vectors for production of proteins from genes containing rare codons. J. Struct. Funct. Genom. 14, 135-144. doi: 10.1007/s10969-013-9163-9

Fichman, Y., Gerdes, S. Y., Kovacs, H., Szabados, L., Zilberstein, A., and Csonka, L. N. (2014). Evolution of proline biosynthesis: enzymology, bioinformatics, genetics, and transcriptional regulation. Biol. Rev. Camb. Philos. Soc. doi: 10.1111/brv.12146 [Epub ahead of print].

Firoz, A., Malik, A., Joplin, K. H., Ahmad, Z., Jha, V., and Ahmad, S. (2011). Residue propensities, discrimination and binding site prediction of adenine and guanine phosphates. BMC Biochem. 12:20. doi: 10.1186/1471-209112-20

Fischer, S., Brunk, B. P., Chen, F., Gao, X., Harb, O. S., Iodice, J. B., et al. (2011). Using OrthoMCL to assign proteins to OrthoMCL-DB groups or to cluster proteomes into new ortholog groups. Curr. Protoc. Bioinformatics Chap. 6. Unit 6.12, 1-19. doi: 10.1002/0471250953.bi0612s35

Folta-Stogniew, E., and Williams, K. R. (1999). Determination of molecular masses of proteins in solution: implementation of an HPLC size exclusion chromatography and laser light scattering service in a core laboratory. J. Biomol. Tech. 10, 51-63.

Forlani, G., Bertazzini, M., Zarattini, M., Funck, D., Ruszkowski, M., and Nocek, B. P., (2015). Functional properties and structural characterization of rice $\delta^{1}$-pyrroline-5-carboxylate reductase. Front. Plant Sci. 6:565. doi: 10.3389/fpls.2015.00565

Forlani, G., Giberti, S., Berlicki, Ł., Petrollino, D., and Kafarski, P. (2007). Plant P5C reductase as a new target for aminomethylenebisphosphonates. J. Agric. Food Chem. 55, 4340-4347. doi: 10.1021/jf0701032

Forlani, G., Occhipinti, A., Berlicki, Ł., Dziêdzioła, G., Wieczorek, A., and Kafarski, P. (2008). Tailoring the structure of aminophosphonates to target plant P5C reductase. J. Agric. Food Chem. 56, 3193-3199. doi: 10.1021/jf80 $0029 t$

Forlani, G., Petrollino, D., Fusetti, M., Romanini, L., Nocek, B., Joachimiak, A., et al. (2012). $\Delta^{1}$-pyrroline-5-carboxylate reductase as a new target for therapeutics: inhibition of the enzyme from Streptococcus pyogenes and effects in vivo. Amino Acids 42, 2283-2291. doi: 10.1007/s00726-011-0970-7

Franklin, M. C., Cheung, J., Rudolph, M. J., Burshteyn, F., Cassidy, M., Gary, E., et al. (2015). Structural genomics for drug design against the pathogen Coxiella burnetii. Proteins. doi: 10.1002/prot.24841 [Epub ahead of print]. 
Funck, D., Winter, G., Baumgarten, L., and Forlani, G. (2012). Requirement of proline synthesis during Arabidopsis reproductive development. BMC Plant Biol. 12:191. doi: 10.1186/1471-2229-12-191

Giberti, S., Funck, D., and Forlani, G. (2014). $\Delta^{1}$-pyrroline-5-carboxylate reductase from Arabidopsis thaliana: stimulation or inhibition by chloride ions and feedback regulation by proline depend on whether NADPH or NADH acts as co-substrate. New Phytol. 202, 911-919. doi: 10.1111/nph.12701

Hua, X. J., Van de Cotte, B., Van Montagu, M., and Verbruggen, N. (1997). Developmental regulation of pyrroline-5-carboxylate reductase gene expression in Arabidopsis. Plant Physiol. 114, 1215-1224. doi: 10.1104/pp.114.4.1215

Jones, S., and Thornton, J. M. (1996). Principles of protein-protein interactions. Proc. Natl. Acad. Sci. U.S.A. 93, 13-20. doi: 10.1073/pnas.93.1.13

Kavi Kishor, P. B., Sangam, S., Amrutha, R. N., SriLaxmi, P., Naidu, K. R., Rao, K. R. S. S., et al. (2005). Regulation of proline biosynthesis, degradation, uptake and transport in higher plants: its implications in plant growth and abiotic stress tolerance. Curr. Sci. 88, 424-438.

Kay, B. K., Williamson, M. P., and Sudol, M. (2000). The importance of being proline: the interaction of proline-rich motifs in signaling proteins with their cognate domains. FASEB J. 14, 231-241.

Keating, K. S., Flores, S. C., Gerstein, M. B., and Kuhn, L. A. (2009). StoneHinge: hinge prediction by network analysis of individual protein structures. Protein Sci. 18, 359-371. doi: 10.1002/pro.38

Kenklies, J., Ziehn, R., Fritsche, K., Pich, A., and Andreesen, J. R. (1999). Proline biosynthesis from L-ornithine in Clostridium sticklandii: purification of $\delta^{1}$-pyrroline-5-carboxylate reductase, and sequence and expression of the encoding gene, proC. Microbiology 145, 819-826. doi: 10.1099/13500872-1454-819

Kleiger, G., and Eisenberg, D. (2002). GXXXG and GXXXA motifs stabilize FAD and $\mathrm{NAD}(\mathrm{P})$-binding Rossmann folds through $\mathrm{C}^{\alpha}-\mathrm{H} \cdots \mathrm{O}$ hydrogen bonds and van der Waals interactions. J. Mol. Biol. 323, 69-76. doi: 10.1016/S00222836(02)00885-9

Koonin, E. V., Makarova, K. S., and Aravind, L. (2001). Horizontal gene transfer in prokaryotes: quantification and classification. Annu. Rev. Microbiol. 55, 709-742. doi: 10.1146/annurev.micro.55.1.709

Krissinel, E., and Henrick, K. (2007). Inference of macromolecular assemblies from crystalline state. J. Mol. Biol. 372, 774-797. doi: 10.1016/j.jmb.2007.05.022

Krueger, R., Jäger, H. J., Hintz, M., and Pahlich, E. (1986). Purification to homogeneity of pyrroline-5-carboxylate reductase of barley. Plant Physiol. 80, 142-144. doi: 10.1104/pp.80.1.142

Larkin, M. A., Blackshields, G., Brown, N. P., Chenna, R., McGettigan, P. A., McWilliam, H., et al. (2007). Clustal W and Clustal X version 2.0. Bioinformatics 23, 2947-2948. doi: 10.1093/bioinformatics/btm404

Lehmann, S., Funck, D., Szabados, L., and Rentsch, D. (2010). Proline metabolism and transport in plant development. Amino Acids 39, 949-962. doi: 10.1007/s00726-010-0525-3

Levy, H. R., Vought, V. E., Yin, X., and Adams, M. J. (1996). Identification of an arginine residue in the dual coenzyme-specific glucose-6-phosphate dehydrogenase from Leuconostoc mesenteroides that plays a key role in binding $\mathrm{NADP}^{+}$but not $\mathrm{NAD}^{+}$. Arch. Biochem. Biophys. 326, 145-151. doi: 10.1016/abbi.1996.0058

Lynch, M., and Force, A. (2000). The probability of duplicate gene preservation by subfunctionalization. Genetics 154, 459-473.

Maggio, A., Miyazaki, S., Veronese, P., Fujita, T., Ibeas, J. I., Damsz, B., et al. (2002). Does proline accumulation play an active role in stress-induced growth reduction? Plant J. 31, 699-712. doi: 10.1046/j.1365-313X.2002.01389.x

Mao, L., Wang, Y., Liu, Y., and Hu, X. (2003). Multiple intermolecular interaction modes of positively charged residues with adenine in ATP-binding proteins. J. Am. Chem. Soc. 125, 14216-14217. doi: 10.1021/ja036096p

Meng, Z., Liu, Z., Lou, Z., Gong, X., Cao, Y., Bartlam, M., et al. (2009). Purification, characterization and crystallization of pyrroline-5-carboxylate reductase from the hyperthermophilic archeon Sulfolobus solfataricus. Protein Expres. Purif. 64, 125-130. doi: 10.1016/j.pep.2008.10.018

Meng, Z., Lou, Z., Liu, Z., Li, M., Zhao, X., Bartlam, M., et al. (2006). Crystal structure of human pyrroline-5-carboxylate reductase. J. Mol. Biol. 359, 1364-1377. doi: 10.1016/j.jmb.2006.04.053

Moreira, I. S., Fernandes, P. A., and Ramos, M. J. (2007). Hot spots-A review of the protein-protein interface determinant amino-acid residues. Proteins 68 , 803-812. doi: 10.1002/prot.21396
Morgan, A. A., and Rubenstein, E. (2013). Proline: the distribution, frequency, positioning, and common functional roles of proline and polyproline sequences in the human proteome. PLOS ONE 8:e53785. doi: 10.1371/journal.pone. 0053785

Murahama, M., Yoshida, T., Hayashi, F., Ichino, T., Sanada, Y., and Wada, K. (2001). Purification and characterization of $\delta^{1}$-pyrroline-5-carboxylate reductase isoenzymes, indicating differential distribution in spinach (Spinacia oleracea L.) leaves. Plant Cell Physiol. 42, 742-750. doi: 10.1093/pcp/pce093

Nocek, B., Chang, C., Li, H., Lezondra, L., Holzle, D., Collart, F., et al. (2005). Crystal structures of $\delta^{1}$-pyrroline-5-carboxylate reductase from human pathogens Neisseria meningitides and Streptococcus pyogenes. J. Mol. Biol. 354, 91-106. doi: 10.1016/j.jmb.2005.08.036

Nocek, B., Jang, S. B., Jeong, M. S., Clark, D. D., Ensign, S. A., and Peters, J. W. (2002). NADPH dependent 2-ketopropyl coenzyme M oxidoreductase/carboxylase. Biochemistry 41, 12907-12913. doi: $10.2210 / \mathrm{pdb} 1 \mathrm{mok} / \mathrm{pdb}$

Nocek, B., Starus, A., Makowska-Grzyska, M., Gutierrez, B., Sanchez, S., Jedrzejczak, R., et al. (2014). The dimerization domain in DapE enzymes is required for catalysis. PLoS ONE 9:e93593. doi: 10.1371/journal.pone. 0093593

Nooren, I. M., and Thornton, J. M. (2003). Structural characterisation and functional significance of transient protein-protein interactions. J. Mol. Biol. 325, 991-1018. doi: 10.1016/S0022-2836(02)01281-0

Petrollino, D., and Forlani, G. (2012). Coenzyme preference of Streptococcus pyogenes $\delta^{1}$-pyrroline-5-carboxylate reductase: evidence supporting NADPH as the physiological electron donor. Amino Acids 43, 493-497. doi: 10.1007/s00726-011-1077-x

Phang, J. M. (1985). The regulatory functions of proline and pyrroline-5-carboxylic acid. Curr. Top. Cell Regul. 25, 91-132. doi: 10.1016/B978-0-12-1528256.50008-4

Poolman, B., Konings, W. N., and Robillard, G. T. (1983). The location of redoxsensitive groups in the carrier protein of proline at the outer and inner surface of the membrane in Escherichia coli. Eur. J. Biochem. 135, 41-46. doi: 10.1111/j.1432-1033.1983.tb07615.x

Price, M. N., Dehal, P. S., and Arkin, A. P. (2010). FastTree 2 - approximately maximum-likelihood trees for large alignments. PLoS ONE 5:e9490. doi: 10.1371/journal.pone. 0009490

Pruitt, K. D., Brown, G. R., Hiatt, S. M., Thibaud-Nissen, F., Astashyn, A., Ermolaeva, O., et al. (2014). RefSeq: an update on mammalian reference sequences. Nucleic Acids Res. 42, 756-763. doi: 10.1093/nar/gkt1114

Pyrkov, T. V., Pyrkova, D. V., Balitskaya, E. D., and Efremov, R. G. (2009). The role of stacking interactions in complexes of proteins with adenine and guanine fragments of ligands. Acta Nat. 1, 124-127.

Rayapati, P. J., Stewart, C. R., and Hack, E. (1989). Pyrroline-5-carboxylate reductase is in pea (Pisum sativum L.) leaf chloroplasts. Plant Physiol. 91, 581-586. doi: 10.1104/pp.91.2.581

Robert, X., and Gouet, P. (2014). Deciphering key features in protein structures with the new ENDscript server. Nucleic Acids Res. 42, 320-324. doi: 10.1093/nar/gku316

Rossmann, M. G., Moras, D., and Olsen, K. W. (1974). Chemical and biological evolution of a nucleotide-binding protein. Nature 250, 194-199. doi: $10.1038 / 250194 \mathrm{a} 0$

Sabri, M., Dunford, A. J., McLean, K. J., Neeli, R., Scrutton, N. S., Leys, D., et al. (2009). Characterization of coenzyme binding and selectivity determinants in Mycobacterium tuberculosis FprA: analysis of Arg199 and Arg200 mutants at the $\operatorname{NADP}(\mathrm{H}) 2$ '-phosphate binding site. Biochem. J. 417, 103-112. doi: 10.1042/BJ20080466

Sanli, G., Dudley, J. I., and Blaber, M. (2003). Structural biology of the aldoketo reductase family of enzymes: catalysis and cofactor binding. Cell Biochem. Biophys. 38, 79-101. doi: 10.1385/CBB:38:1:79

Shultz, J. L., Kurunam, D., Shopinski, K., Iqbal, M. J., Kazi, S., Zobrist, K. et al. (2006). The soybean genome database (SoyGD): a browser for display of duplicated, polyploid, regions and sequence tagged sites on the integrated physical and genetic maps of Glycine max. Nucleic Acids Res. 34, 758-765. doi: 10.1093/nar/gkj050

Sigrist, C. J., de Castro, E., Cerutti, L., Cuche, B. A., Hulo, N., Bridge, A., et al. (2013). New and continuing developments at PROSITE. Nucleic Acids Res. 41, D344-D347. doi: 10.1093/nar/gks1067 
Smith, R. J., Downing, S. J., Phang, J. M., Lodato, R. F., and Aoki, T. T. (1980). Pyrroline-5-carboxylate synthase activity in mammalian cells. Proc. Natl. Acad. Sci. U.S.A. 77, 5221-5225. doi: 10.1073/pnas.77.9.5221

Szabados, L., and Savouré, A. (2010). Proline: a multifunctional amino acid. Trends Plant Sci. 15, 89-97. doi: 10.1016/j.tplants.2009.11.009

Szoke, A., Miao, G. H., Hong, Z., and Verma, D. P. S. (1992). Subcellular location of $\delta^{1}$-pyrroline-5-carboxylate reductase in root/nodule and leaf of soybean. Plant Physiol. 99, 1642-1649. doi: 10032-0889/92/99/1642/08

Wheeler, D., and Bhagwat, M. (2007). BLAST QuickStart: example-driven webbased BLAST tutorial. Methods Mol. Biol. 395, 149-176. doi: 10.1007/978-159745-514-5_9

Wierenga, R. K., De Maeyer, M. C. H., and Hol, W. G. J. (1985). Interaction of pyrophosphate moieties with $\alpha$-helixes in dinucleotide binding proteins. Biochemistry 24, 1346-1357. doi: 10.1021/bi00327a012

Yang, Y., Xu, S., Zhang, M., Jin, R., Zhang, L., Bao, J., et al. (2006). Purification and characterization of a functionally active Mycobacterium tuberculosis pyrroline-5-carboxylate reductase. Protein Expres. Purif. 45, 241-248. doi: 10.1016/j.pep.2005.08.007
Yutin, N., Makarova, K. S., Mekhedov, S. L., Wolf, Y. I., and Koonin, E. V. (2008). The deep archaeal roots of eukaryotes. Mol. Biol. Evol. 25, 1619-1630. doi: $10.1093 / \mathrm{molbev} / \mathrm{msn} 108$

Zheng, H., Chruszcz, M., Lasota, P., Lebioda, L., and Minor, W. (2008). Data mining of metal ion environments present in protein structures. J. Inorg. Biochem. 102, 1765-1776. doi: 10.1016/j.inorgbio.2008.05.006

Conflict of Interest Statement: The authors declare that the research was conducted in the absence of any commercial or financial relationships that could be construed as a potential conflict of interest.

Copyright (๑) 2015 Forlani, Makarova, Ruszkowski, Bertazzini and Nocek. This is an open-access article distributed under the terms of the Creative Commons Attribution License (CC BY). The use, distribution or reproduction in other forums is permitted, provided the original author(s) or licensor are credited and that the original publication in this journal is cited, in accordance with accepted academic practice. No use, distribution or reproduction is permitted which does not comply with these terms. 


\title{
Functional properties and structural characterization of rice $\delta^{1}$-pyrroline-5-carboxylate reductase
}

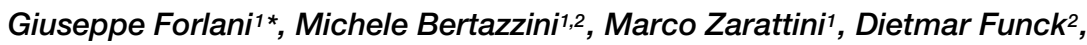 \\ Milosz Ruszkowski ${ }^{3}$ and Bogusław Nocek ${ }^{4}$
}

${ }^{1}$ Laboratory of Plant Physiology and Biochemistry, Department of Life Science and Biotechnology, University of Ferrara, Ferrara, Italy, ${ }^{2}$ Plant Physiology and Biochemistry, Department of Biology, University of Konstanz, Konstanz, Germany, ${ }^{3}$ Synchrotron Radiation Research Section, Macromolecular Crystallography Laboratory, National Cancer Institute, Argonne National Laboratory, Argonne, IL, USA, ${ }^{4}$ Biosciences Division, Argonne National Laboratory, Argonne, IL, USA

\section{OPEN ACCESS}

Edited by:

Hans-Peter Mock,

Institute of Plant Genetics and Crop

Plant Research, Germany

Reviewed by:

Omar Pantoja,

Universidad Nacional Autonoma

de Mexico, Mexico

Paul E. Vers/ues,

Academia Sinica, Taiwan

*Correspondence:

Giuseppe Forlani,

Laboratory of Plant Physiology and Biochemistry, Department of Life

Science and Biotechnology, University

of Ferrara, Via Luigi Borsari 46,

Ferrara 44121, Italy flg@unife.it

Specialty section This article was submitted to Plant Physiology, a section of the journal Frontiers in Plant Science

Received: 03 April 2015

Accepted: 09 July 2015

Published: 28 July 2015

Citation:

Forlani $G$, Bertazzini $M$, Zarattini $M$

Funck D, Ruszkowski $M$ and Nocek $B$

(2015) Functional properties and structural characterization of rice $\delta^{1}$-pyrroline-5-carboxylate reductase.

Front. Plant Sci. 6:565. doi: 10.3389/fpls.2015.00565
The majority of plant species accumulate high intracellular levels of proline to cope with hyperosmotic stress conditions. Proline synthesis from glutamate is tightly regulated at both the transcriptional and the translational levels, yet little is known about the mechanisms for post-translational regulation of the enzymatic activities involved. The gene coding in rice (Oryza sativa L.) for $\delta^{1}$-pyrroline-5-carboxylate (P5C) reductase, the enzyme that catalyzes the second and final step in this pathway, was isolated and expressed in Escherichia coli. The structural and functional properties of the affinitypurified protein were characterized. As for most species, rice P5C reductase was able to use in vitro either NADH or NADPH as the electron donor. However, strikingly different effects of cations and anions were found depending on the pyridine nucleotide used, namely inhibition of $\mathrm{NADH}$-dependent activity and stimulation of $\mathrm{NADPH}$-dependent activity. Moreover, physiological concentrations of proline and $\mathrm{NADP}^{+}$were strongly inhibitory for the NADH-dependent reaction, whereas the NADPH-dependent activity was mildly affected. Our results suggest that only NADPH may be used in vivo and that stress-dependent variations in ion homeostasis and $\mathrm{NADPH} / \mathrm{NADP}^{+}$ratio could modulate enzyme activity, being functional in promoting proline accumulation and potentially also adjusting NADPH consumption during the defense against hyperosmotic stress. The apparent molecular weight of the native protein observed in size exclusion chromatography indicated a high oligomerization state. We also report the first crystal structure of a plant P5C reductase at 3.40- $\AA$ resolution, showing a decameric quaternary assembly. Based on the structure, it was possible to identify dynamic structural differences among rice, human, and bacterial enzymes.

Keywords: proline synthesis, P5C reductase, enzyme properties, substrate ambiguity, cation and anion effects, product inhibition, oligomeric structure

\section{Introduction}

Among proteinogenic amino acids, proline plays an important role in protein structure, uniquely contributing to backbone folding and stability (Ge and Pan, 2009). Moreover, in most plants (Verbruggen and Hermans, 2008) and microorganisms (Empadinhas and da Costa, 2008; Takagi, 2008), a rapid and reversible increase of the intracellular concentration of free proline to high 
levels has been shown in response to either osmotic, oxidative, or temperature stress (Verslues and Sharma, 2010), implying a role in stress tolerance and osmoregulation (Szabados and Savouré, 2010; Hayat et al., 2012), redox balance (Liang et al., 2013), and apoptosis (Monteoliva et al., 2014). More recently, both the level of free proline and proline metabolism in plants were also hypothesized to influence the transition to flowering (Mattioli et al., 2009), as well as pollen and embryo development (Lehmann et al., 2010; Funck et al., 2012).

Although not conclusively analyzed experimentally (Funck et al., 2008), two metabolic routes leading to proline production have been proposed in higher plants. Under high nitrogen availability, the synthesis seems to proceed mainly through ornithine, an intermediate in the biosynthesis and degradation of arginine, which is converted to $\delta^{1}$-pyrroline-5-carboxylate (P5C) by a pyridoxal-dependent ornithine- $\delta$-aminotransferase (da Rocha et al., 2012). Conversely, under osmotic stress conditions and/or nitrogen starvation P5C is synthesized from glutamate by P5C synthetase (Kavi Kishor et al., 1995; TurchettoZolet et al., 2009). The two pathways share the last reaction, in which $\mathrm{P} 5 \mathrm{C}$ is reduced to proline by $\mathrm{P} 5 \mathrm{C}$ reductase (EC 1.5.1.2). Although both P5C synthetase (Székely et al., 2008) and P5C reductase (Verbruggen et al., 1993) transcripts are induced under osmotic stress conditions, only the former is believed to represent a rate-limiting step (Kesari et al., 2012). In the absence of a functional P5C reductase both routes for proline biosynthesis are blocked, and no alternative pathway has been described. Consistently, null mutations of $\mathrm{P} 5 \mathrm{C}$ reductase are embryo-lethal (Funck et al., 2012) and specific inhibitors of P5C reductase exert phytotoxic effects (Forlani et al., 2007), and may thus represent new active principles for weed control (Forlani et al., 2008).

As $\mathrm{P} 5 \mathrm{C}$ reductase occurs at the converging point of these two anabolic pathways, it should be subjected to fine regulation, even though it might not represent a rate-limiting step under most conditions. Indeed, when $\mathrm{P} 5 \mathrm{C}$ reductase protein levels and intracellular proline concentrations were measured in different tissues and in osmotically stressed seedlings, data were not in agreement with the corresponding mRNA levels (Hua et al., 1997). A complex pattern of regulation was postulated, in which differential mRNA stability, degree of polysome association and $5^{\prime}$ UTR effects on translation efficiency seem to play a role (Hua et al., 2001). Yet, trans-acting factors that can bind to the $P 5 C$ reductase promoter region or mRNA have not been identified. Moreover, a translation inhibition of Arabidopsis thaliana $\mathrm{P} 5 \mathrm{C}$ reductase was found under stress conditions (Hua et al., 2001), a result that seems inconsistent with a role in stress-induced proline accumulation. The occurrence of posttranslational regulative mechanisms was also proposed, but poorly investigated. In fact, plant $\mathrm{P} 5 \mathrm{C}$ reductase has been purified only from a few plant species, such as barley (Krueger et al., 1986), soybean (Chilson et al., 1991), and spinach (Murahama et al., 2001). These enzymes showed substrate ambiguity, being able to use either $\mathrm{NADH}$ or NADPH as the electron donor, even if the NADH-dependent activity was inhibited by equimolar concentrations of $\mathrm{NADP}^{+}$(Szoke et al., 1992). Moreover, a twofold stimulation of the $\mathrm{NADH}$-dependent reaction by $100 \mathrm{mM} \mathrm{KCl}$ or $10 \mathrm{mM} \mathrm{MgCl}_{2}$ was reported for partially purified pea P5C reductase (Rayapati et al., 1989), whereas the two isozymes purified from spinach were on the contrary inhibited by $\mathrm{NaCl}(100-500 \mathrm{mM})$ and $\mathrm{MgCl}_{2}(10-100 \mathrm{mM})$ when assayed using NADPH as the co-factor (Murahama et al., 2001).

Recently, we isolated and characterized P5C reductase from suspension-cultured cells of $A$. thaliana, where a single gene is present (Verbruggen et al., 1993; Funck et al., 2012). The purified protein was able to use either NADPH or NADH as the electron donor, with contrasting affinities, and maximum reaction rates. The presence of equimolar levels of $\mathrm{NADP}^{+}$ completely suppressed the NADH-dependent activity, whereas the NADPH-dependent reaction was only mildly affected. Proline inhibited only the NADH-dependent reaction. At physiological levels, increasing concentrations of salt steadily inhibited the $\mathrm{NADH}$-dependent activity, but were stimulatory of the NADPHdependent reaction (Giberti et al., 2014). These properties suggest a complex regulation of enzyme activity by the redox status of the pyridine nucleotide pools, and the levels of proline and chloride in the cytosol. However, also due to the above inconsistencies in the literature, it was not possible to conclude whether these features are shared or not by all plant P5C reductases. Similarly, although a clear-cut preference for NADPH was evident, it was unclear whether NADH could sustain at least in part the rate of $\mathrm{P} 5 \mathrm{C}$ reduction inside the plant cell under either physiological or stress conditions.

Besides the lack of a detailed biochemical characterization of the enzyme in a diverse array of plants, our knowledge of post-translational mechanisms regulating the activity of plant $\mathrm{P} 5 \mathrm{C}$ reductase is hampered also by the unavailability of its three-dimensional configuration. Crystal structures have been solved to date only for the enzyme of the bacterial pathogens Streptococcus pyogenes and Neisseria meningitides (Nocek et al., 2005) and for the human isozyme 1 (Meng et al., 2006). The plant and the bacterial sequences show similarity over their entire lengths, a fact that is suggestive of a similar tertiary structure. This notwithstanding, an alignment of the deduced amino acid sequences of $S$. pyogenes and $A$. thaliana $\mathrm{P} 5 \mathrm{C}$ reductase pointed out a moderate degree of conservation, with 33\% identities, $56 \%$ conserved residues, and 3\% gaps (Forlani et al., 2007). Consistently, the sensitivity of the bacterial enzyme to a group of aminobisphosphonate inhibitors (Forlani et al., 2012) was found to be strikingly higher than that of the plant enzyme (Forlani et al., 2013), showing IC $_{50}$ values 2-3 orders of magnitude lower. Therefore, significant differences may exist with respect to the substrate- and effector-binding protein domains. Moreover, a broad range of oligomeric states of $\mathrm{P} 5 \mathrm{C}$ reductase has been reported to date, extending from $125 \mathrm{kDa}$ (suggesting tetramer) to $200-340 \mathrm{kDa}$ (octamer-dodecamer; references in Nocek et al., 2005). For both the S. pyogenes (Nocek et al., 2005) and the human (Meng et al., 2006) enzyme a decameric architecture with five homodimer subunits and ten catalytic sites arranged around a peripheral circular groove has been described. Data obtained by gel permeation chromatography for plant P5C reductases were compatible with either a decameric (Murahama et al., 2001) or a dodecameric assembly (Giberti et al., 2014).

In the frame of a research project for integrated genetic and genomic approaches for new Italian rice breeding strategies, we 
aim at a better understanding of the biochemical mechanisms underlying salt tolerance and proline accumulation in rice. Here we describe the functional characterization of rice $\mathrm{P} 5 \mathrm{C}$ reductase. Our results confirmed in a monocotyledonous species the regulatory pattern previously found in A. thaliana. Taking one step further, the kinetic mechanisms for product inhibition were elucidated, and the regulatory effects of anions and cations were differentiated. On the whole, these results suggest that under physiological conditions only NADPH would act in vivo as the electron donor, and that a stress-induced increase in the cytosolic cation content and/or in the $\mathrm{NADPH} / \mathrm{NADP}^{+}$ratio would instantly enhance $\mathrm{P} 5 \mathrm{C}$ reductase activity, with no need of transcriptional control. A three-dimensional structure of rice $\mathrm{P} 5 \mathrm{C}$ reductase was also obtained, showing a homodecameric configuration.

\section{Materials and Methods}

\section{Cloning and Heterologous Expression}

The coding sequence of Oryza sativa $\mathrm{P} 5 \mathrm{C}$ reductase was amplified by PCR from cDNA clone J013104L18 (Rice Genome Resource Center, National Institute of Agrobiological Sciences DNA Bank, Japan) with the primers P5CR-fw (caccATGGCGGCGCCGCCTCA) and P5CRrev (gaggaTTAACTCTGAGAAAG), and inserted into the expression vector pET151 by directional TOPO cloning (Life Technologies, Carlsbad, CA, USA), yielding the vector pET151OsP5CR. For heterologous expression, E. coli BL21(DE3) pLysS cells (Invitrogen) were made competent by the calcium chloride method, transformed with the vector and selected on ampicillincontaining LB plates. After inducing the expression of P5C reductase by $1 \mathrm{mM}$ isopropyl-D-thiogalactopyranoside (IPTG) at $24^{\circ} \mathrm{C}$, the cells were lysed in a mortar with $2 \mathrm{~g} \mathrm{~g}^{-1}$ alumina and resuspended in $20 \mathrm{~mL} \mathrm{~g}^{-1}$ extraction buffer $(50 \mathrm{mM} \mathrm{Na}$ phosphate buffer, $\mathrm{pH} 7.5$, containing $200 \mathrm{mM} \mathrm{NaCl}, 0.5 \mathrm{mM}$ DTT, and $20 \mathrm{mM}$ imidazole). The His-tagged protein was purified from clarified extracts by affinity chromatography with a His-Select ${ }^{\mathrm{TM}}$ Nickel Affinity Gel column $(1.5 \mathrm{~mL}$ bed volume, Sigma H7788). Stepwise elution was achieved by increasing concentrations of imidazole in extraction buffer. For activity assays, the purified enzyme was diluted 1:1000 with water, and a proper aliquot $(2-5 \mu \mathrm{L})$ was added to the assay mixture. To remove the His-tag, aliquots $(100 \mu \mathrm{g})$ of the preparation were treated with $1 \mu \mathrm{g}$ of tobacco etch virus (TEV) protease (Sigma T4455), according to the cleavage protocol provided by the manufacturer.

\section{Enzyme Assay}

The physiological, forward reaction of $\mathrm{P} 5 \mathrm{C}$ reductase was measured at $35^{\circ} \mathrm{C}$ following the $\mathrm{P} 5 \mathrm{C}$-dependent oxidation of $\mathrm{NAD}(\mathrm{P}) \mathrm{H}$. Unless otherwise specified, the assay mixture contained $20 \mathrm{mM}$ Tris-HCl buffer, $\mathrm{pH} 7.75,1 \mathrm{mM} \mathrm{NADH}$ or $0.5 \mathrm{mM} \mathrm{NADPH}$, and $1 \mathrm{mM}$ DL-P5C (equivalent to $0.5 \mathrm{mM}$ L-P5C; Williams and Frank, 1975) in a final volume of $0.2 \mathrm{~mL}$. DL-P5C was synthesized by the periodate oxidation of $\delta$-allohydroxylysine (Sigma H0377) and purified by cation-exchange chromatography, as described previously (Forlani et al., 1997). A limiting amount of enzyme (from 8 to $16 \mathrm{ng}$ of the purified protein) was added to the pre-warmed mixture, and the decrease in absorbance at $340 \mathrm{~nm}$ was recorded at 20-s intervals and up to $5 \mathrm{~min}$ through an optical path of $0.5 \mathrm{~cm}$. Activity was calculated from the initial linear rate on the assumption of a molar extinction coefficient for $\mathrm{NAD}(\mathrm{P}) \mathrm{H}$ of $6,220 \mathrm{M}^{-1} \mathrm{~cm}^{-1}$. Linear regression analysis was computed by using Prism 6 (version 6.03, GraphPad Software, Inc., USA). Protein content was determined by the Coomassie Blue method (Bradford, 1976), using bovine serum albumin (BSA) as the standard. For the purified protein, direct absorbance at $280 \mathrm{~nm}$ was used instead, and the concentration was calculated on the basis of a deduced molar extinction coefficient for rice $\mathrm{P} 5 \mathrm{C}$ reductase of 14,000 $\mathrm{M}^{-1} \mathrm{~cm}^{-1}$ (http://web.expasy.org/cgi-bin/protparam/ protparam).

\section{Kinetic Analyses}

To evaluate substrate affinity, invariable substrates were fixed at the same levels as in the standard assay. The concentration of $\mathrm{L}-\mathrm{P} 5 \mathrm{C}$ ranged from 150 to $500 \mu \mathrm{M}$ with $\mathrm{NADH}$ as the electron donor, and from 100 to $225 \mu \mathrm{M}$ with NADPH. The concentration of NADH and NADPH ranged from 50 to $350 \mu \mathrm{M}$. To evaluate the mechanism of the inhibition brought about by proline and $\mathrm{NADP}^{+}$on the $\mathrm{NADH}$-dependent activity, $\mathrm{NADH}$ concentration ranged from 100 to $800 \mu \mathrm{M}$. When evaluating the effect of ions, L-P5C concentration was reduced to $200 \mu \mathrm{M}$ to minimize the carry-over of chloride anions. All assays were performed in triplicate. $K_{\mathrm{M}}$ and $V_{\max }$ values, as well as the concentrations causing $50 \%$ inhibition $\left(\mathrm{IC}_{50}\right)$ or $50 \%$ stimulation of $\mathrm{P} 5 \mathrm{C}$ reductase activity, $K_{\mathrm{I}}$ values and their confidence intervals were estimated by non-linear regression analysis using Prism 6. Catalytic constants were calculated from $V_{\max }$ values taking into account a homodecameric composition of the native holoenzyme, having each monomer a molecular mass of 29,670 Da.

\section{Determination of Isoelectric Point, Native, and Denatured Molecular Mass}

Discontinuous SDS-polyacrylamide gel electrophoresis was performed at $20^{\circ} \mathrm{C}$ by the method of Laemmli with a $4 \%$ stacking and a $12 \%$ separating gel, using a Minigel system (BioRad). Samples were denatured by boiling $5 \mathrm{~min}$ in $62 \mathrm{mM}$ Tris-HCl buffer ( $\mathrm{pH}$ 6.8), containing 2\% (w/v) SDS, $10 \%$ $(\mathrm{v} / \mathrm{v})$ glycerol, and $5 \%(\mathrm{v} / \mathrm{v}) \beta$-mercaptoethanol. Proteins were visualized after staining with $0.1 \%$ Coomassie brilliant blue R-250.

Gel permeation chromatography was performed by injecting $100 \mu \mathrm{L}$ aliquots of the purified protein onto a Superose 12 HR 10/30 (Pharmacia) column that had been equilibrated with $50 \mathrm{mM}$ Tris- $\mathrm{HCl}$ buffer, $\mathrm{pH} 7.75$, containing $250 \mathrm{mM} \mathrm{NaCl}$. Elution proceeded at the constant flow of $0.5 \mathrm{~mL} \mathrm{~min}-1$, for the collection of $0.5-\mathrm{mL}$ fractions, while monitoring the eluate at $280 \mathrm{~nm}$ (HPLC Detector 432, Kontron). Molecular weight markers for column calibration (Pharmacia) were bovine thyroid thyroglobulin $(669 \mathrm{kDa})$, horse spleen ferritin (440 and $960 \mathrm{kDa})$, bovine liver catalase $(232 \mathrm{kDa})$, rabbit muscle aldolase $(158 \mathrm{kDa})$, 
and BSA (67 and $268 \mathrm{kDa}$ ). Three runs were carried out for each marker, and six runs for the purified protein.

Isoelectric focusing was performed as described previously (Forlani et al., 1997), with ampholytes within the $\mathrm{pH}$ 3.510 range (Pharmacia); pI markers (Sigma) were bovine milk $\beta$-lactoglobulin A (pI 5.1), bovine erythrocyte carbonic anhydrase II (5.4 and 5.9), and bovine erythrocyte carbonic anhydrase I (6.6). After the run, individual tracks were cut from the gel and either sliced in 5-mm segments for the determination of $\mathrm{pH}$, or stained for protein as above.

\section{Protein Production for Crystallization}

The coding sequence of $O$. sativa $\mathrm{P} 5 \mathrm{C}$ reductase was subcloned into vector pMCSG68 according to the standard protocol described previously (Eschenfeldt et al., 2013). OsP5CR was overexpressed in BL21 Gold E. coli cells (Agilent Technologies). The bacteria were cultured with shaking at $210 \mathrm{rpm}$ in Lysogeny Broth supplemented with $150 \mu \mathrm{g} \mathrm{mL}^{-1}$ ampicillin at $37^{\circ} \mathrm{C}$ until the $\mathrm{OD}_{600}$ reached 1.0. The temperature was lowered to $18^{\circ} \mathrm{C}$ and IPTG was added to a final concentration of $0.5 \mathrm{mM}$. The culture was grown for $18 \mathrm{~h}$ and the cells were pelleted by centrifugation at $4^{\circ} \mathrm{C}$. Bacteria from $1 \mathrm{~L}$ culture were resuspended in $35 \mathrm{~mL}$ of binding buffer [ $50 \mathrm{mM}$ Tris- $\mathrm{HCl} \mathrm{pH} \mathrm{8.0,500} \mathrm{mM}$ $\mathrm{NaCl}, 20 \mathrm{mM}$ imidazole, $1 \mathrm{mM}$ Tris(2-carboxyethyl)phosphine (TCEP)] and stored at $-80^{\circ} \mathrm{C}$. The samples were thawed and the cells were disrupted by sonication using bursts of total duration of $5 \mathrm{~min}$, with appropriate intervals for cooling. Cell debris was pelleted by centrifugation at $18000 \mathrm{~g}$ for $30 \mathrm{~min}$ at $4^{\circ} \mathrm{C}$. The supernatant was applied to a column packed with $8 \mathrm{~mL}$ of HisTrap HP resin (GE Healthcare) connected to VacMan (Promega), and the chromatographic process was accelerated with a vacuum pump. After binding, the column was washed five times with $40 \mathrm{~mL}$ of binding buffer, and the $\mathrm{His}_{6}$-tagged protein was eluted with $20 \mathrm{~mL}$ of elution buffer $(50 \mathrm{mM}$ Tris$\mathrm{HCl} \mathrm{pH}$ 8.0, $500 \mathrm{mM} \mathrm{NaCl}, 300 \mathrm{mM}$ imidazole, $1 \mathrm{mM}$ TCEP). TEV protease $\left(2 \mathrm{mg}\right.$ ) was added to cleave the $\mathrm{His}_{6}$-tag, and the sample was immediately transferred to a dialysis tube. The dialysis was carried out overnight at $4^{\circ} \mathrm{C}$ against buffer lacking imidazole. The solution was again mixed with HisTrap HP resin to remove the $\mathrm{His}_{6}$-tag and the $\mathrm{His}_{6}$-tagged TEV protease. The flow-through, containing rice $\mathrm{P} 5 \mathrm{C}$ reductase, was concentrated to $4 \mathrm{~mL}$ and applied onto a HiLoad Superdex 200 16/60 column (GE Healthcare) equilibrated with $50 \mathrm{mM}$ Tris- $\mathrm{HCl}$ buffer, $\mathrm{pH}$ 8.0, containing $200 \mathrm{mM} \mathrm{NaCl}$ and $1 \mathrm{mM}$ TCEP. The size exclusion chromatography yielded a homogenous protein fraction.

\section{Crystallization, Data Collection, and Structure Solution}

The sample was concentrated using Amicon concentrators (Millipore) to $14 \mathrm{mg} \mathrm{mL}^{-1}$ as determined by measuring the absorbance at $280 \mathrm{~nm}$. Crystallization screening was performed using a Robotic Sitting Drop Vapor Diffusion setup (Mosquito). Manual optimization using hanging drops gave the following final conditions: $100 \mathrm{mM}$ Tris- $\mathrm{HCl}, \mathrm{pH} 8.5,200 \mathrm{mM} \mathrm{MgCl}_{2}, 18 \%$ polyethylene glycol 8000 . The crystallization drop was composed of $4 \mu \mathrm{L}$ of protein and $2 \mu \mathrm{L}$ of the reservoir solution. The needle-shaped crystals appeared after 3 days at $19^{\circ} \mathrm{C}$. The crystals were washed with the reservoir solution supplemented with $20 \%$ glycerol as a cryo-protectant and vitrified in liquid nitrogen.

The diffraction data were collected at 22-ID SER-CAT beamline at Advanced Photon Source, Argonne, USA. The diffraction images were processed with XDS (Kabsch, 2010). The structure was solved by molecular replacement in Phaser (McCoy et al., 2007) using a homology-based model of rice $\mathrm{P} 5 \mathrm{C}$ reductase prepared using Swiss-Model server (Biasini et al., 2014). The structure of human homolog withdrawn from Protein Data Bank (PDB, access ID: 2izz) served as the template. The protein model was built using Phenix AutoBuild (Terwilliger et al., 2008). Statistics of data collection, processing, and refinement are summarized in Table 1.

\section{Results}

\section{Functional Properties of Rice P5C Reductase}

The cDNA of the only gene coding for $\mathrm{P} 5 \mathrm{C}$ reductase in the japonica rice genome was subcloned into the expression vector pET151 and expressed in E. coli. The N-terminal addition of a stretch of six His residues (Supplementary Figure S1) and the adoption of a stepwise elution protocol allowed the attainment of homogeneous preparations in a single step (Supplementary Figure S2). The presence of the $\mathrm{His}_{6}$-tag did not affect the enzymatic activity, since virtually identical results were obtained in all experiments before and after the cleavage of the purified protein with TEV protease. Enzyme preparations were highly stable; if sterilized by filtration $(0.22 \mu \mathrm{m}$ pore size $)$, no detectable loss of activity was evident after 3 -month storage at $4^{\circ} \mathrm{C}$.

The maximal specific activity of the recombinant enzyme strongly depended on the electron donor used. With NADPH,

TABLE 1 | Data collection statistics.

\begin{tabular}{|c|c|c|c|}
\hline Data collection & & Refinement & \\
\hline Wavelength $(\AA ̊)$ & 0.97625 & $R_{\text {free }}$ reflections & 816 \\
\hline Temperature (K) & 100 & $\begin{array}{l}\text { Number of atoms } \\
\text { (non-H) }\end{array}$ & 38743 \\
\hline Space group & $P 2_{1}$ & $R_{\text {work }} / R_{\text {free }}(\%)$ & $24.6 / 33.8$ \\
\hline $\begin{array}{l}\text { Unit cell parameters } \\
\qquad \begin{array}{l}a, b, c(\AA) \\
\beta\left({ }^{\circ}\right)\end{array}\end{array}$ & $\begin{array}{l}84.6,192.4,212.1 \\
96.6\end{array}$ & $\begin{array}{l}\text { RMSD from ideal } \\
\text { geometry }\end{array}$ & \\
\hline Oscillation range $\left(^{\circ}\right)$ & 0.3 & Bond lengths $(\AA)$ & 0.018 \\
\hline Number of images & 330 & Bond angles $\left({ }^{\circ}\right)$ & 2.1 \\
\hline Resolution $(\AA)$ & $\begin{array}{l}40.0-3.40 \\
(3.60-3.40)\end{array}$ & $\begin{array}{l}\text { Ramachandran } \\
\text { statistics (\%) }\end{array}$ & \\
\hline $\begin{array}{l}\text { Reflections } \\
\text { collected/unique }\end{array}$ & 195821/90887 & Favored & 90.6 \\
\hline Completeness (\%) & $96.2(93.7)$ & Allowed & 6.6 \\
\hline Multiplicity & $2.1(2.1)$ & Outliers & 2.8 \\
\hline$R_{\text {meas }}{ }^{\mathrm{a}}(\%)$ & $14.7(77.0)$ & & \\
\hline$<\mid / \sigma(I)>$ & $9.2(2.0)$ & & \\
\hline
\end{tabular}


TABLE 2 | Properties of rice P5C reductase.

\begin{tabular}{|c|c|}
\hline Denatured molecular mass (by SDS-PAGE) & $30.1 \pm 0.4 \mathrm{kDa}$ \\
\hline Native molecular mass (by gel permeation) & $401 \pm 19 \mathrm{kDa}$ \\
\hline Isoelectric point & $6.58 \pm 0.04$ \\
\hline pH optimum & $8.75 \pm 0.21$ \\
\hline $\mathrm{V}_{\mathrm{Max}}(\mathrm{NADH})$ & $158.3 \pm 13.2 \mu$ kat $(\text { mg protein })^{-1}$ \\
\hline $\mathrm{V}_{\mathrm{Max} \text { (P5C, with NADH as the co-substrate) }}$ & $85.95 \pm 3.53 \mu$ kat $(\mathrm{mg} \text { protein })^{-1}$ \\
\hline $\mathrm{V}_{\mathrm{Max}}(\mathrm{NADPH})$ & $11.68 \pm 0.16 \mu$ kat $(\mathrm{mg} \text { protein })^{-1}$ \\
\hline $\mathrm{V}_{\mathrm{Max} \text { (P5C, with NADPH as the co-substrate) }}$ & $11.88 \pm 0.21 \mu$ kat $(\mathrm{mg} \text { protein })^{-1}$ \\
\hline $\mathrm{K}_{\mathrm{cat}}(\mathrm{NADH})$ per monomer & $4,697 \mathrm{~s}^{-1}$ \\
\hline $\mathrm{K}_{\text {cat }}(\mathrm{NADPH})$ per monomer & $350 s^{-1}$ \\
\hline $\mathrm{K}_{\mathrm{M}(\mathrm{app})}$ for L-P5C (NADH) & $283 \pm 25 \mu \mathrm{M}$ \\
\hline $\mathrm{K}_{\mathrm{M}(\mathrm{app})}$ for L-P5C $(\mathrm{NADPH})$ & $28.8 \pm 1.7 \mu \mathrm{M}$ \\
\hline $\mathrm{K}_{\mathrm{M}(\mathrm{app})}$ for $\mathrm{NADH}$ & $806 \pm 89 \mu \mathrm{M}$ \\
\hline $\mathrm{K}_{\mathrm{M}(\mathrm{app})}$ for NADPH & $49.4 \pm 1.8 \mu \mathrm{M}$ \\
\hline
\end{tabular}

a $V_{\max }$ of about $12 \mu \mathrm{mol} \mathrm{s}{ }^{-1}$ (mg protein) ${ }^{-1}$ was found, corresponding to a catalytic constant of $350 \mathrm{~s}^{-1}$ per monomer (Table 2). With $\mathrm{NADH}$ instead of $\mathrm{NADPH}$, a strikingly higher $\mathrm{V}_{\max }$ value was calculated, one that would result in more than 4,500 catalytic events in $1 \mathrm{~s}$ for a single subunit. However, the corresponding affinity was conversely lower (apparent $K_{\mathrm{M}}$ values of 49 and $806 \mu \mathrm{M}$ for NADPH or NADH, respectively), and saturating conditions were not obtained even at the highest
NADH concentration tested (Figure 1). As a consequence, the estimated $\mathrm{V}_{\max }$ with $\mathrm{P} 5 \mathrm{C}$ as the variable substrate was lower than that with NADH (Table 2), because the latter was still limiting under standard assay conditions. The use of NADH as the cosubstrate also resulted in a 10-fold higher apparent $\mathrm{K}_{\mathrm{M}}$ value for P5C.

While the NADPH-dependent activity was linear with time over the entire assay period, kinetics with $\mathrm{NADH}$ showed a progressive reduction of the catalytic rate as the reaction took place (data not shown). To verify whether this effect may be due to product inhibition, the impact of increasing levels of proline, $\mathrm{NADP}^{+}$and $\mathrm{NAD}^{+}$on the initial reaction velocity was assessed. In the range of concentrations tested, $\mathrm{NAD}^{+}$was substantially ineffective (results not presented). On the contrary, both proline and $\mathrm{NADP}^{+}$were able to inhibit the activity of P5C reductase (Figures 2A,B). However, a remarkably different sensitivity was found depending on the pyridine nucleotide used. With NADPH as the electron donor, proline was inhibitory only at concentrations exceeding $100 \mathrm{mM}$, and $\mathrm{NADP}^{+}$was effective if added at levels higher than that of NADPH in the assay mixture. On the contrary, the NADH-dependent activity was strongly reduced by the presence of either proline concentrations in the range from 5 to $100 \mathrm{mM}$, or micromolar levels of $\mathrm{NADP}^{+}$, with $\mathrm{IC}_{50}$ values of $48 \mathrm{mM}$ and $37 \mu \mathrm{M}$, respectively. To obtain further information, a thorough kinetic analysis was performed. When

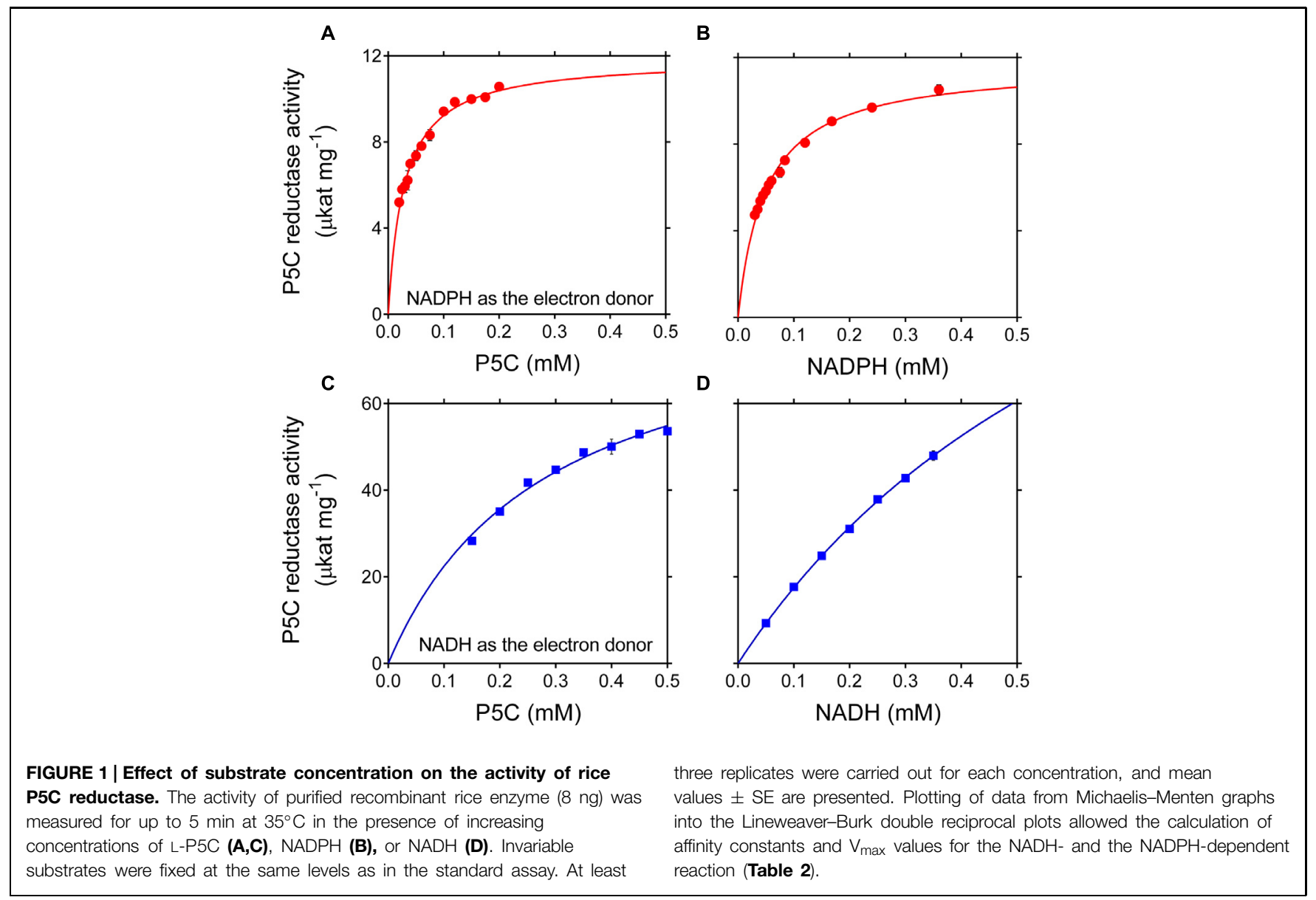


A

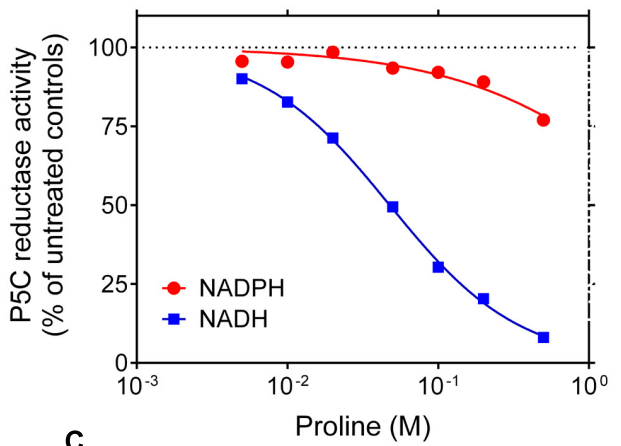

C

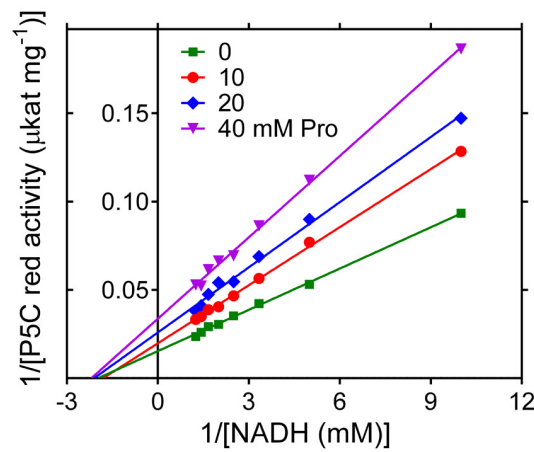

E

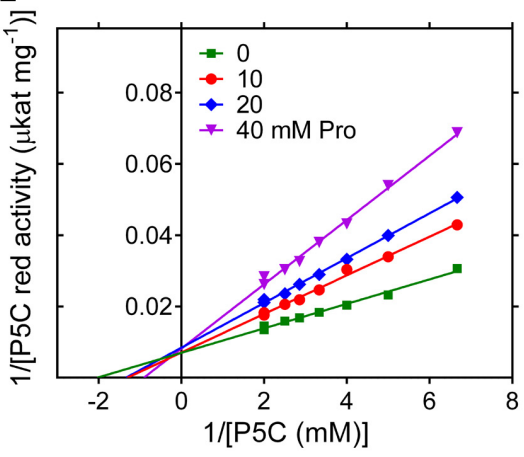

B
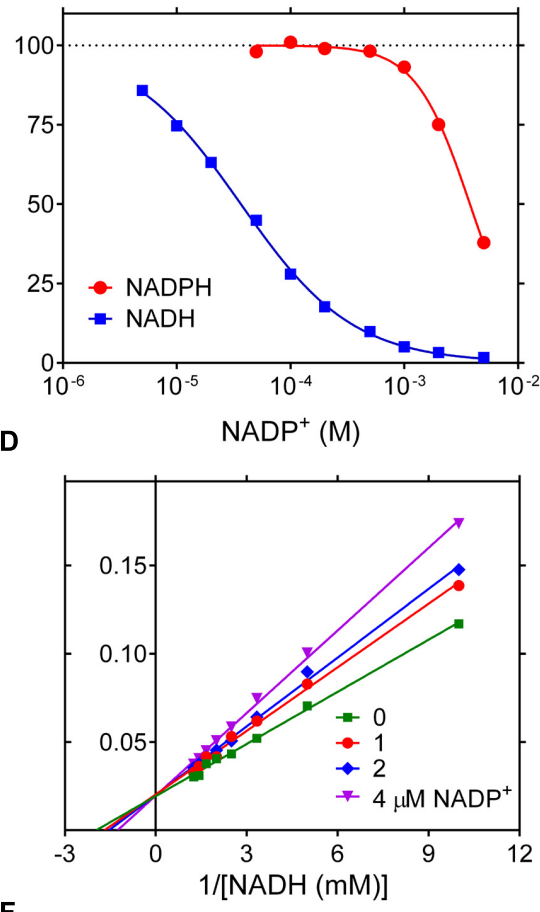

$\mathbf{F}$

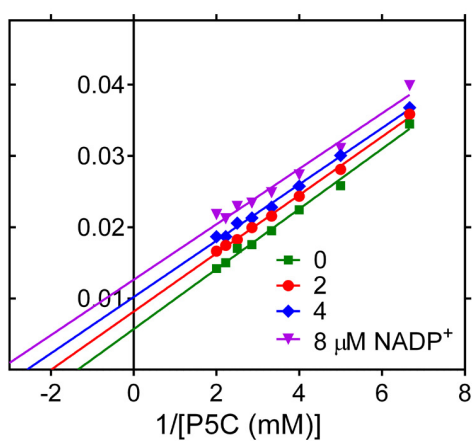

FIGURE 2 | Product inhibition of rice P5C reductase. The effect of increasing concentrations of proline $(\mathbf{A})$ and $\mathrm{NADP}^{+} \mathbf{( B )}$ on the activity of the enzyme was determined using either $\mathrm{NADH}$ or $\mathrm{NADPH}$ as the electron donor. Results were expressed as percent of the activity in the absence of supplements, and are mean $\pm \mathrm{SE}$ over three replicates. Non-linear regression analysis allowed the calculation of $\mathrm{IC}_{50}$ values, which in the case of the $\mathrm{NADH}$-dependent reaction were $47.7 \pm 1.6 \mathrm{mM}$ and $36.7 \pm 1.1 \mu \mathrm{M}$ for proline and $\mathrm{NADP}^{+}$, respectively. The NADPH-dependent activity was much less sensitive to proline and $\mathrm{NADP}^{+}$, with estimated $\mathrm{IC}_{50}$ values of $3.44 \pm 1.51 \mathrm{M}$ and $3.78 \pm 0.12 \mathrm{mM}$, respectively. To investigate the mechanisms of product inhibition, the affinity toward NADH (C,D) or L-P5C (E,F) was calculated in the

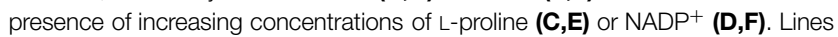
converging to the $y$ axis account for a mechanism of competitive type with respect to $\mathrm{P} 5 \mathrm{C}$ for the inhibition by proline $(\mathbf{E})$ and with respect to $\mathrm{NADH}$ by

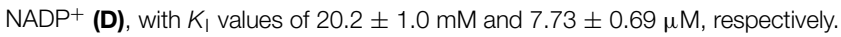
Lines converging to the $x$ axis suggest a mechanism of non-competitive type for proline with respect to $\mathrm{NADH}$ (C), $K_{1}$ being equal to $34.7 \pm 1.2 \mathrm{mM}$. On the contrary, parallel lines for different $\mathrm{NADP}^{+}$concentrations are consistent with an inhibition of uncompetitive type with respect to $\mathrm{P} 5 \mathrm{C}(\mathbf{F})$, with a $K_{\mathrm{I}}$ value of $8.48 \pm 0.56 \mu \mathrm{M}$. the effects of proline and $\mathrm{NADP}^{+}$were evaluated at varying the concentration of either substrate, Lineweaver-Burk plots were consistent with an inhibition mechanism of competitive type with respect to $\mathrm{P} 5 \mathrm{C}$ for proline (Figure 2E) and with respect to $\mathrm{NADH}$ for $\mathrm{NADP}^{+}$(Figure 2D), and of non-competitive type for proline with respect to $\mathrm{NADH}$ (Figure 2C). Interestingly, for $\mathrm{NADP}^{+}$an inhibition of uncompetitive type with respect to $\mathrm{P} 5 \mathrm{C}$ was found (Figure 2F), showing that P5C binding is required to allow NADH to enter the active site of the enzyme. $K_{\mathrm{I}}$ values with respect to $\mathrm{NADH}$ were $20 \mathrm{mM}$ and $8 \mu \mathrm{M}$ for proline and $\mathrm{NADP}^{+}$, respectively.

\section{Effects of Anions and Cations on the Activity of Rice P5C Reductase}

Because inconsistent results have been described in the literature about the sensitivity of plant $\mathrm{P} 5 \mathrm{C}$ reductase to salts, the effect of increasing concentrations of $\mathrm{NaCl}$ and $\mathrm{MgCl}_{2}$ on the catalytic rate of the rice enzyme was assessed. Once again, 
strikingly different properties were evident depending on the electron donor (Figures 3A,B). With NADH as the co-factor, an inhibition was found with both salts at levels exceeding 20-50 mM. With NADPH, a remarkable stimulation was on the contrary shown at concentrations in the range 5-200 $\mathrm{mM}$ $\mathrm{NaCl}$ and $0.1-70 \mathrm{mM} \mathrm{MgCl}_{2}$. Above these thresholds, the activity came back to control rates and, only in the case of $\mathrm{MgCl}_{2}$, was inhibited at levels exceeding $100 \mathrm{mM}$. To ascertain whether these effects may be ascribed to anions or cations, the effects of other chlorides or other sodium salts were also investigated. Results were plotted as a function of
$\mathrm{Cl}^{-}$(Figures 3C,D) or $\mathrm{Na}^{+}$(Figures 3E,F) concentration when NADH (Figures 3C,E) or NADPH (Figures 3D,F) was used as the co-factor. Almost overlapping patterns for several chlorides and dissimilar patterns for various sodium salts suggest that anions are the main cause of the inhibition of the NADH-dependent activity. Conversely, different patterns with different chlorides but substantially overlapping patterns with various sodium salts were consistent with a stimulation of the NADPH-dependent activity by cations, whereby divalent cations were remarkably more effective than monovalent cations (Figure 3D).

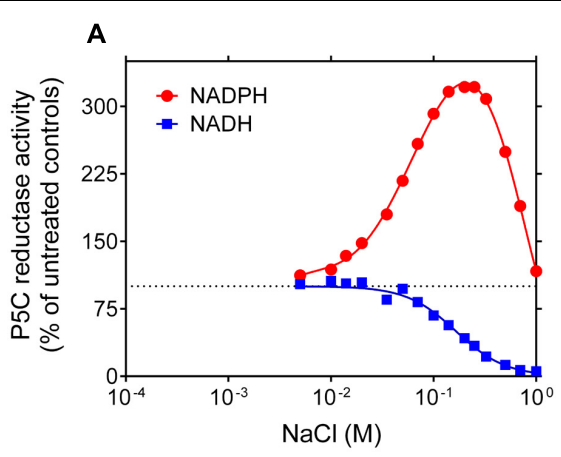

C

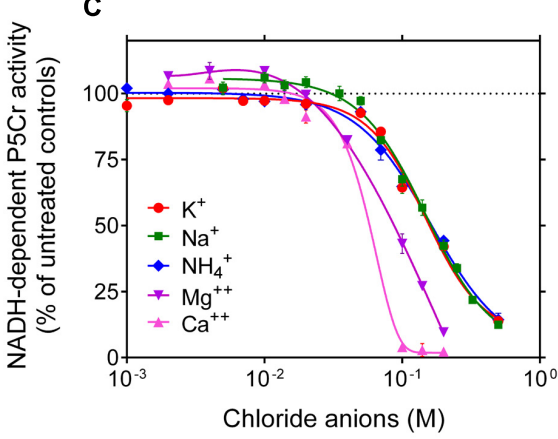

E

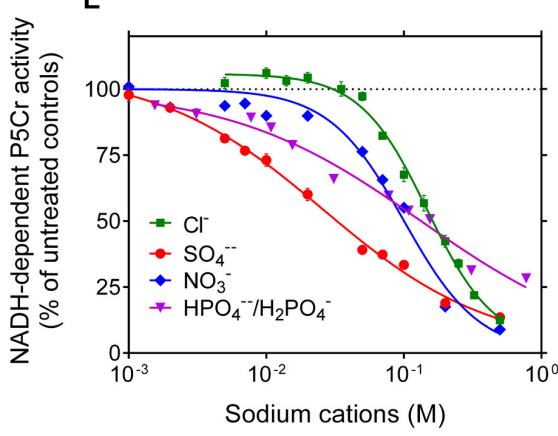

FIGURE 3 | Effect of anions and cations on the activity of rice P5C

reductase. The effects of the addition of increasing concentrations of $\mathrm{NaCl}$ (A) or $\mathrm{MgCl}_{2}$ (B) to the reaction mixture were assessed using either $1 \mathrm{mM} \mathrm{NADH}$ or $0.5 \mathrm{mM}$ NADPH as the electron donor. To minimize the carry-over of chloride anions from the purified preparation of the co-substrate, L-P5C levels were fixed at $0.2 \mathrm{mM}$, resulting in less than $10 \mathrm{mM} \mathrm{Cl}^{-1}$ in standard mixture. Results were expressed as percent of controls assayed in the absence of added salts, and are mean \pm SE over three replicates. Non-linear regression analysis allowed the calculation of $\mathrm{IC}_{50}$ values for the $\mathrm{NADH}$-dependent reaction, which were $165 \pm 13 \mathrm{mM}$ and $43.8 \pm 3.9 \mathrm{mM}$ for $\mathrm{NaCl}$ and $\mathrm{MgCl}_{2}$, respectively. To
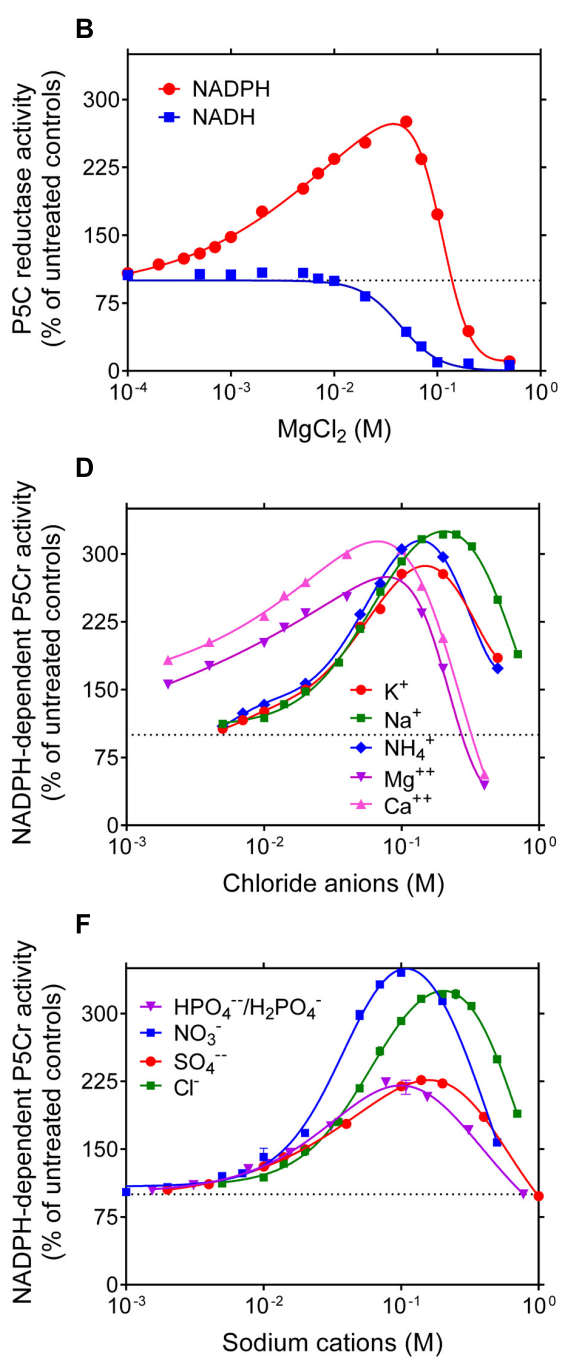

discriminate whether anions or cations were causing the striking stimulation of the NADPH-dependent activity and the inhibition of the NADH-dependent reaction, similar experiments were also performed with increasing concentrations of $\mathrm{KCl}, \mathrm{NH}_{4} \mathrm{Cl}$, and $\mathrm{CaCl}_{2}(\mathbf{C}, \mathbf{D})$, or with $\mathrm{NaNO}_{3}, \mathrm{Na}_{2} \mathrm{SO}_{4}$, and $\mathrm{NaH}_{2} \mathrm{PO}_{4} / \mathrm{Na}_{2} \mathrm{HPO}_{4}$ (in a molar ratio 0.292: 1, resulting in a $\mathrm{pH}$ value of 7.75 ) (E,F). Results were plotted together as a function of chloride or sodium ion concentration, respectively. Almost overlapping patterns suggest that cations in the range $10^{-3}-10^{-1} \mathrm{M}$ stimulate $\mathrm{P} 5 \mathrm{C}$ reductase activity when it uses NADPH as the substrate, whereas anions inhibit the reaction if $\mathrm{NADH}$ acts as the electron donor. 
Based on these data, P5C reductase activity seems therefore strongly dependent on both the use of NADH vs. NADPH as the electron donor, and the presence of reaction products and salts. To obtain further information, the activity of the purified enzyme was measured in the presence of substrate and effector concentrations similar to those reported to exist within plant cells. Results are summarized in Figure 4. Despite the fact that $\mathrm{V}_{\max }$ with $\mathrm{NADH}$ is more than 10 -fold higher than that with NADPH, very similar rates were obtained with either co-factor added to the reaction mixture at physiological levels. The activities were not additive, suggesting a preferential use of NADPH. This notwithstanding, the rate in the presence of both dinucleotides was slightly higher than that with NADPH alone, showing that also NADH may contribute to the overall velocity. However, when realistic levels of $\mathrm{NADP}^{+}$were also present, the NADPH-dependent activity was reduced to $50 \%$, whereas the $\mathrm{NADH}$-dependent reaction was almost abolished. The presence of $15 \mathrm{mM}$ proline was more inhibitory than previously observed under standard assay conditions, most likely because of the lower concentration of $\mathrm{P} 5 \mathrm{C}$. Interestingly, the presence of ion concentrations similar to those reported in the cell under normoosmotic conditions enhanced the NADPH-dependent activity while inhibiting that with $\mathrm{NADH}$. When all variables were

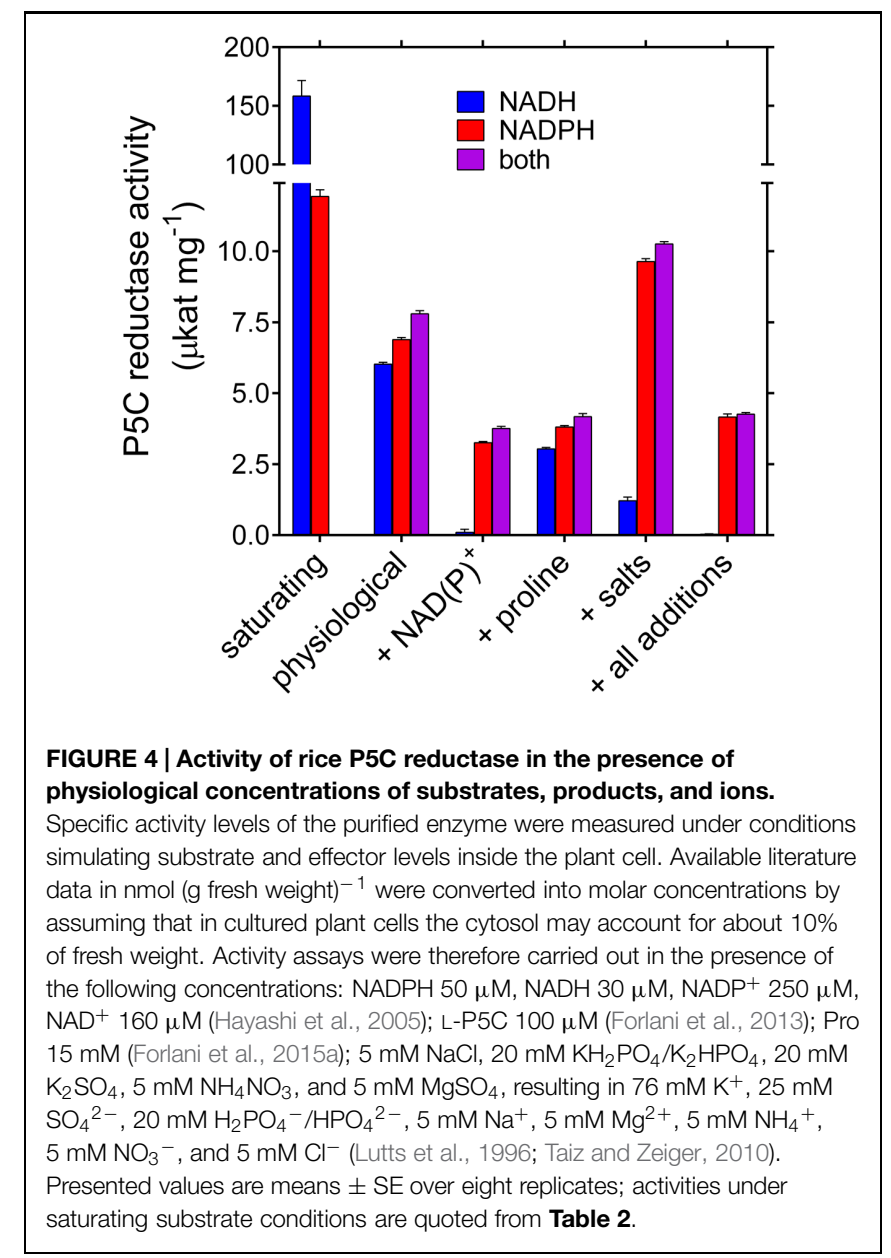

included, the enzymatic activity with NADPH corresponded to about $40 \%$ of the maximal rate, whereas that with $\mathrm{NADH}$ was completely abolished. Consistently, the activity level with both nucleotides was under these conditions not significantly different from that with NADPH alone.

\section{Structural Characterization of Rice P5C Reductase}

Under denaturing conditions, $\mathrm{P} 5 \mathrm{C}$ reductase migrated as a single band (Supplementary Figure S2) to a position corresponding to a molecular mass $(30.1 \pm 0.4 \mathrm{kDa})$ that is compatible with the deduced mass from the nucleotide sequence of the gene $(28,624$ Da; Supplementary Figure S1). To obtain an estimate of its relative mass under non-denaturing conditions, the TEVcleaved protein was subjected to gel filtration chromatography, and its retention pattern was compared with that of molecular weight markers (Figure 5). Results indicated a native molecular mass of $401 \pm 19 \mathrm{kDa}$, which would be consistent with an oligomer composed of 14 identical subunits. However, void volumes within the protein structure as well as any deviation from globular shape strongly affect protein retention, and molecular masses inferred from retention patterns may be subjected to significant errors (Erickson, 2009).

Indeed, a decameric composition was found when the crystal structure of $O$. sativa $\mathrm{P} 5 \mathrm{C}$ reductase was determined at a resolution of $3.40 \AA$. The structure was determined by the molecular replacement method with a search probe created by homology modeling based on the decameric human structure (PDB ID: 2izz; Forlani et al., 2015b). Analysis of the protein crystal solvent content, the so-called Matthew's coefficient calculation, indicated that two decameric assemblies are located in the asymmetric unit of the crystal lattice with $P 2_{1}$ space group symmetry. Rice P5C reductase can therefore be described as

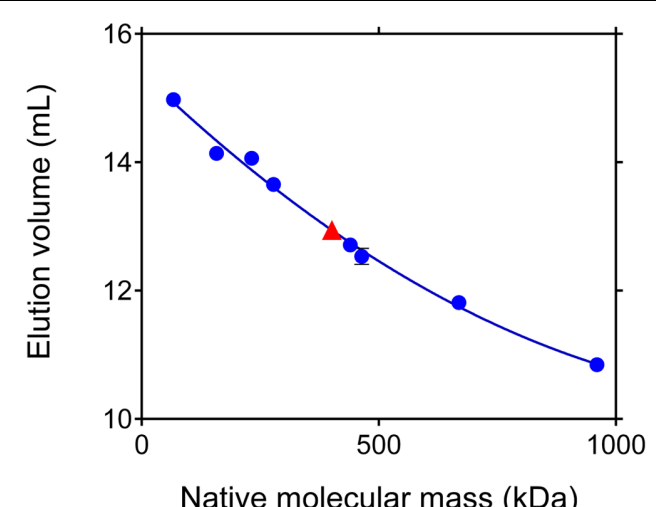

FIGURE 5 | Apparent molecular mass of rice P5C reductase under native conditions. Aliquots $(100 \mu \mathrm{L})$ of the purified protein, adjusted to $10 \mathrm{mg} \mathrm{mL}^{-1}$, were subjected to gel permeation chromatography on a Superose 12 HR 10/30 (Pharmacia) column that had been equilibrated with $50 \mathrm{mM}$ Tris- $\mathrm{HCl}$ buffer, $\mathrm{pH} 7.75$, containing $250 \mathrm{mM} \mathrm{NaCl}$. A molecular mass of $401 \pm 19 \mathrm{kDa}$ was estimated for P5C reductase. Based upon these results, the enzyme could consist of 13-14 subunits. Identical elution patterns were obtained at increasing $\mathrm{NaCl}$ concentration up to $1 \mathrm{M}$ (data not shown). 
a pentamer of dimers, which has a doughnut-like shape with the dimensions of $112 \AA \times 85 \AA$ (Figure 6). This arrangement is formed by a mutual exchange of the C-terminal domains between two neighboring protein subunits, while in contrast the nucleotide binding (N-terminal) domains do not interact with each other and are pointing away from the hollow core. The N-terminal domains are flexible, and only 11 out of 20 (in two decamers) were defined well enough in the electron density maps to be reliably modeled. The hinge that allows for independent movement of the $\mathrm{N}$-terminal domains is predicted to be around the residues 175-180. The protein chain for which the electron density maps were of the best quality was modeled and copied by non-crystallographic symmetry (NCS) operations, and a refinement was performed with secondary structure restraints for all $\mathrm{N}$-terminal domains. This approach allowed us to obtain the model of a full decamer (Figure 6). The electron density for nine (out of 20 in two decamers) N-terminal domains was poor, which is evident by the elevated $\mathrm{R}$ factors of model $\mathrm{R} / \mathrm{R}_{\text {free }}=25 / 34 \%$.

\section{Discussion}

\section{Plant P5C Reductases Share Peculiar Properties that Can be Functional to the Multiple Roles Hypothesized for Proline Metabolism in the Cell}

Here we report an exhaustive characterization of rice $\mathrm{P} 5 \mathrm{C}$ reductase, affinity-purified after heterologous expression in
E. coli. Under saturating conditions, the purified enzyme showed

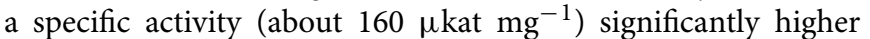
than those of the enzymes isolated from other plant sources [8.5 $\mu \mathrm{kat} \mathrm{mg}^{-1}$ for barley (Krueger et al., 1986), $4.4 \mu \mathrm{kat} \mathrm{mg}^{-1}$

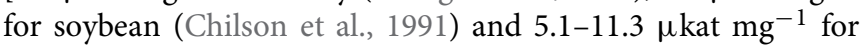
spinach (Murahama et al., 2001)]. However, this exceptionally high turnover rate depended on the use of NADH as the electron donor. With NADPH instead, a $V_{\max }$ value of about $12 \mu \mathrm{kat} \mathrm{mg}^{-1}$ was found. In any case, even with NADPH the resulting catalytic efficiency $\left(\mathrm{K}_{\mathrm{cat}} / \mathrm{K}_{\mathrm{M}}=7.09 \times 10^{6} \mathrm{M}^{-1} \mathrm{sec}^{-1}\right)$ is significantly higher than that reported for other enzymes in amino acid metabolism. For instance, a $\mathrm{K}_{\mathrm{cat}} / \mathrm{K}_{\mathrm{M}}=4.2 \times 10^{4} \mathrm{M}^{-1}$ $\mathrm{s}^{-1}$ has been found for $E$. coli $\gamma$-glutamyl kinase, the enzyme that catalyzes the first step in proline biosynthesis in bacteria (PérezArellano et al., 2006). That of P5C dehydrogenase, the enzyme that oxidizes $\mathrm{P} 5 \mathrm{C}$ back to glutamate, ranged from $3.4 \times 10^{5}$ to $4.2 \times 10^{5} \mathrm{M}^{-1} \mathrm{sec}^{-1}$ in rat (Small and Jones, 1990) and potato (Forlani et al., 1997), respectively. No information is available regarding this parameter for plant $\mathrm{P} 5 \mathrm{C}$ reductases other than the similarly high value $\left(26.7 \times 10^{6} \mathrm{M}^{-1} \mathrm{sec}^{-1}\right)$ found for the enzyme from $A$. thaliana (Giberti et al., 2014). The enzyme from the human pathogen $S$. pyogenes also showed a high $\mathrm{K}_{\mathrm{cat}} / \mathrm{K}_{\mathrm{M}}$ value $\left(28 \times 10^{6} \mathrm{M}^{-1} \mathrm{sec}^{-1}\right.$; Petrollino and Forlani, 2012). The extremely high efficiency for the reaction catalyzed by $\mathrm{P} 5 \mathrm{C}$ reductase may be a consequence of the need to rapidly convert even small amounts of $\mathrm{P} 5 \mathrm{C}$ into proline, in order to avoid possible cytotoxic effects caused by P5C accumulation (Deuschle et al., 2004; Miller et al., 2009; Senthil-Kumar and Mysore, 2012). This seems consistent with the relatively high $K_{\mathrm{M}(\mathrm{app})}$ values found

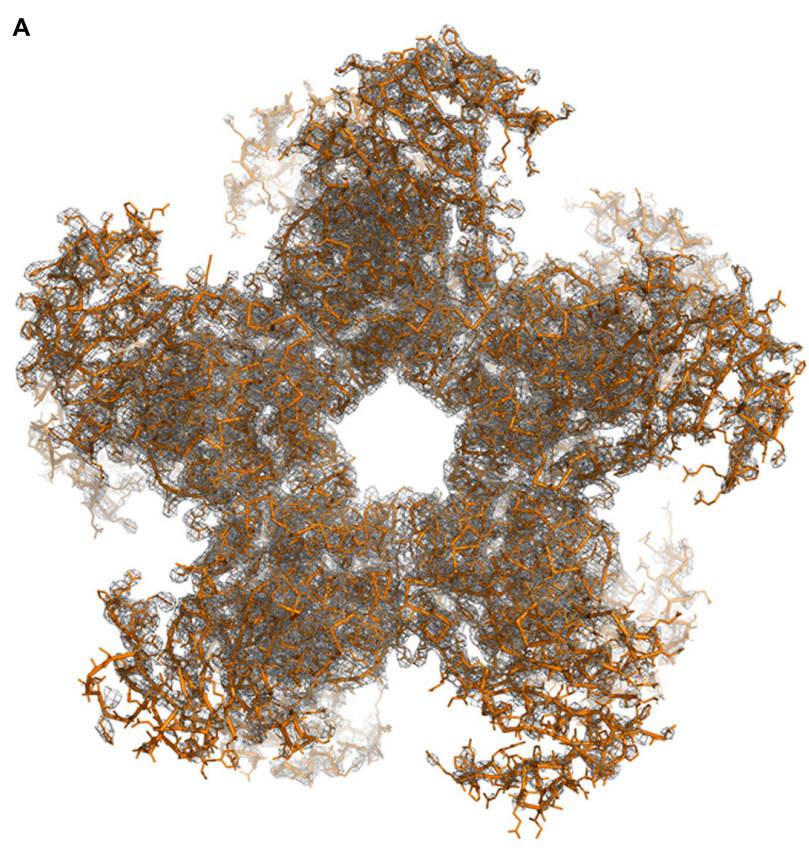

B

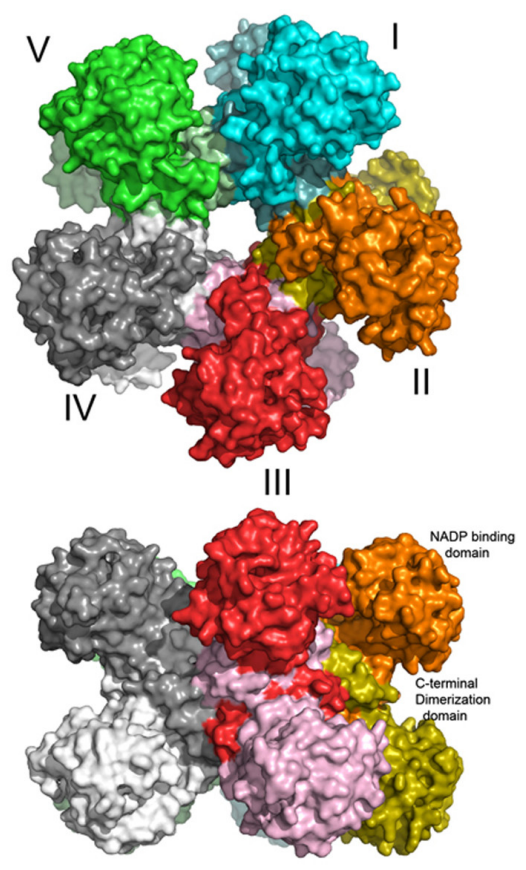

FIGURE 6 | The decameric structure of of rice P5C reductase. The top view (A) along the fivefold NCS axis reveals a pentamer of dimers, shown in orange. The two $F_{\text {obs }}-F_{\text {calc }}$ density maps contour at $1 \sigma$ level covering the entire decamer is shown in gray. Top and side views (B) of the decamer in surface-representation. The subunits are shown in different colors. 
for L-P5C (Table 2), suggesting that the activity of the enzyme is far from being saturated at physiological concentrations of the substrate: any increase in P5C concentration would therefore result in an equivalent increase of the rate of its utilization by $\mathrm{P} 5 \mathrm{C}$ reductase.

The significantly higher maximal reaction rate observed with $\mathrm{NADH}$ most likely depends on a faster $\mathrm{NAD}^{+}$release from the active site. Consistently, the NADH-dependent activity was strikingly inhibited by micromolar concentrations of $\mathrm{NADP}^{+}$. If both pyridine nucleotides were made available, the reaction proceeded at rates only slightly higher than those in the presence of NADPH alone, confirming a preferential use of $\mathrm{NADPH}$ but also suggesting that both co-factors could be used alternately. Such a substrate ambiguity, together with the inhibition by oxidized pyridine dinucleotides, has been interpreted as functional to the proposed role of proline synthesis in maintaining a favorable $\mathrm{NADP}^{+} / \mathrm{NADPH}$ ratio under stress conditions, as well as in regenerating $\mathrm{NADP}^{+}$ in photosynthetic tissues (Sharma et al., 2011). From this perspective, in humans the non-allosterically regulated isozyme specifically expressed in erythrocytes, PYCR1, would serve primarily for $\mathrm{NADP}^{+}$generation, whereas the other, prolinesensitive isozyme ubiquitously expressed in the other tissues would be devoted to proline synthesis (Merrill et al., 1989). Since only a single enzyme form is present in most plants, both functions might be ensured through the emerging complex pattern of substrate preference and product inhibition, where proline and $\mathrm{NADP}^{+}$inhibit only the $\mathrm{NADH}$-dependent reaction.

Patterns in Lineweaver-Burk plots (Figure 2F) accounted for an inhibition by $\mathrm{NADP}^{+}$of uncompetitive type with respect to $\mathrm{P} 5 \mathrm{C}$. This strengthens earlier results obtained with phosphonate inhibitors of plant P5C reductase (Forlani et al., 2007, 2008) and provides experimental evidence supporting an ordered substrate binding, previously hypothesized only on the basis of the crystal structure of the bacterial enzyme (Nocek et al., 2005). The present data therefore suggest that P5C binds before NADPH. Since in the case of most $\mathrm{NAD}(\mathrm{P}) \mathrm{H}$-dependent reductases the coenzyme binds before the substrate (Sanli et al., 2003), this is an unusual feature that might depend on the cyclic structure of both substrate and product.

Most interestingly, the use of either co-factor had drastic effects on the susceptibility of $\mathrm{P} 5 \mathrm{C}$ reductase to the presence of salts. A twofold stimulation by $100 \mathrm{mM} \mathrm{KCl}$ or $10 \mathrm{mM}$ $\mathrm{MgCl}_{2}$ had been reported for the enzyme partially purified from pea (Rayapati et al., 1989), but in that case activity had been evaluated with $\mathrm{NADH}$ as the electron donor. Conversely, the two isozymes purified from spinach were inhibited by $\mathrm{NaCl}$ in the 100-500 $\mathrm{mM}$ range, and by $\mathrm{MgCl}_{2}$ at lower concentrations, when assayed using NADPH (Murahama et al., 2001). These contrasting results could imply a functional diversity among plant P5C reductases. However, the experiments performed in this study on the enzyme from rice, a monocot, yielded patterns overall similar to those previously obtained with the enzyme from the $\operatorname{dicot} A$. thaliana (Giberti et al., 2014). Therefore, it appears more likely that such differences may depend on nonuniform experimental conditions, e.g., the inclusion of different $\mathrm{MgCl}_{2}$ levels in the standard assay mixture. Most importantly, in previous studies the possibility that significant levels of chloride and $\mathrm{Na}^{+}$or $\mathrm{K}^{+}$ions may be present in the standard reaction mixture as a consequence of P5C buffering has been largely underestimated. $\mathrm{P} 5 \mathrm{C}$ is a labile compound; when synthesized by the periodate oxidation of hydroxylysine, it is purified by cation-exchange chromatography in $1 \mathrm{M} \mathrm{HCl}$ (Williams and Frank, 1975). The resulting low $\mathrm{pH}$ values stabilize the compound that is routinely stored under these conditions and neutralized just before the enzymatic assay. If neutralization were achieved with sodium or potassium hydroxide, concentrations as high as $100 \mathrm{mM} \mathrm{NaCl}$ or $\mathrm{KCl}$ would be present in "untreated controls." In the present study, the adoption of strictly controlled assay conditions ensured that chloride ion concentration in controls was never higher than $15 \mathrm{mM}$, and no inorganic cations were present.

Making one step forward, the effect of various cations and anions was investigated. Our results clearly show that anions are the cause of the inhibition of the NADH-dependent reaction of $\mathrm{P} 5 \mathrm{C}$ reductase, with sulfates being inhibitory at concentrations as low as $1 \mathrm{mM}$. On the contrary, the stimulation of the $\mathrm{NADPH}$-dependent activity seems to depend on the presence of cations, and divalent cations were effective at lower doses than monovalent ions (Figure 3D). Divalent anions at high concentration had detrimental effects, since the $\mathrm{Na}^{+}$-dependent stimulation of enzyme activity was lower with sodium salts of divalent anions than that obtained with sodium salts of monovalent anions (Figure 3F). If the concentrations at which these effects were evident in vitro are considered, in several cases it seems likely that they can occur in vivo and influence the resulting rate of proline synthesis.

\section{Substrate Affinity, Product Inhibition, and Ion Effects May Unravel Substrate Ambiguity, and Represent a Likely Mechanism for in vivo Modulation of P5C Reductase Activity}

Taking into account the main, if not exclusive, cytosolic localization of P5C reductase (Funck et al., 2012), the question remains as to which may be the physiological electron donor. The mechanisms for substrate inhibition that have been shown in the present study for the NADH-dependent activity seem to substantially prevent the use of the non-phosphorylated cofactor. Indeed, in the presence of $\operatorname{NAD}(\mathrm{P})(\mathrm{H})$ and proline concentrations similar to those reported for rice cells, the activity with both electron donors did not significantly differ from that with NADPH alone, probably because the inhibitory effect of proline was amplified by the low physiological concentration of P5C (Forlani et al., 2013), and that of $\mathrm{NADP}^{+}$by the high $\mathrm{NADP}^{+} / \mathrm{NADH}$ ratio (Hayashi et al., 2005; Figure 4). Notwithstanding this, the ability of NADH utilization could be useful in special circumstances.

The activation of proline synthesis may maintain a favorable redox balance inside the cell. On the other side, the complex pattern of co-factor preference, substrate inhibition, and salt effects may contribute to a fast activation of proline synthesis under salt stress conditions. Following the exposure to hyperosmotic stress, the oxidative pentose phosphate pathway 
(OPPP) is rapidly induced, leading to cytosolic NADPH production (Baxter et al., 2007). Moreover, stress-induced inward $\mathrm{Ca}^{2+}$-fluxes are able to activate calmodulin-modulated NAD kinase isozymes (Hashida et al., 2009) that in turn increase the $\mathrm{NADP}(\mathrm{H}) / \mathrm{NAD}(\mathrm{H})$ ratio. A higher $\mathrm{NADPH}$ availability would enhance the activity of $\mathrm{P} 5 \mathrm{C}$ reductase, which shows an $\mathrm{K}_{\mathrm{M} \text { (app) }}$ for NADPH very close to the intracellular concentration of the dinucleotide, and raise the carbon flux within the proline biosynthetic route. Consistently, A. thaliana plants overexpressing NAD kinase 2 showed increased levels of free proline (Takahashi et al., 2009). In the meantime, the activity of the OPPP would lower the $\mathrm{NADP}^{+} / \mathrm{NADH}$ ratio, relieving in part the inhibition of the NADH-dependent activity. Over a longer period, an increase of the cytosolic $\mathrm{Na}^{+}$concentration, which can reach $60 \mathrm{mM}$ (Anil et al., 2007), would enhance further the NADPH-fueled reaction. In this way, $\mathrm{P} 5 \mathrm{C}$ reductase would be able to respond to wide fluctuations of P5C synthesis by P5C synthetase isozymes without the need of a transcriptional control.

Also changes in the levels of other ions could modulate P5C reductase activity. For instance, magnesium ion concentration in rice cells is estimated to range between 1 and $10 \mathrm{mM}$ (Hayatsu et al., 2014), and it was found to increase up to three-fold in leaves of salt-stressed rice plants (Bertazzini et al., 2012). Such fluctuations would positively affect the catalytic rate of rice $\mathrm{P} 5 \mathrm{C}$ reductase using $\mathrm{NADPH}$ as the electron donor. In any case, the overall picture supporting the occurrence of differential effects of salts on the activity of P5C reductase depending on the electron donor used (Table 3) allows to explain previous contradictory findings. For instance, the translation inhibition of AtP5CR under stress conditions (Hua et al., 2001), and the inhibition of spinach $\mathrm{P} 5 \mathrm{C}$ reductase by salts in the $10^{-2}-10^{-1} \mathrm{M}$ range (Murahama et al., 2001), results that in the absence of post-translational mechanisms modulating enzyme activity and a strong preference in vivo for NADPH, respectively, would be inconsistent with stress-induced proline accumulation.

\section{The Three-Dimensional Structure of P5C Reductase is Conserved Across all Kingdoms of Life, but the Rice Enzyme Reveals Dynamic Movements}

Here, we also report the first crystal structure of a plant P5C reductase. Our analysis was limited to basic structural studies due

TABLE 3 | Effect of selected factors on the activity of rice P5C reductase depending on whether NADPH or NADH acts as co-substrate.

\begin{tabular}{lcc}
\hline & With NADH & with NADPH \\
\hline NAD $^{+}$ & $\Leftrightarrow$ & $\Leftrightarrow$ \\
NADP $^{+}\left(10^{-5}-10^{-3} \mathrm{M}\right)$ & $\Omega \Omega$ & $\Leftrightarrow$ \\
Proline $\left(10^{-2}-10^{-1} \mathrm{M}\right)$ & $\Omega$ & $\Leftrightarrow$ \\
Monovalent anions $\left(10^{-2}-10^{-1} \mathrm{M}\right)$ & $\Omega$ & $\Leftrightarrow$ \\
Divalent anions $\left(10^{-3}-10^{-1} \mathrm{M}\right)$ & $\Omega ु$ & $\Omega$ \\
Monovalent cations $\left(10^{-2}-10^{-1} \mathrm{M}\right)$ & $\Leftrightarrow$ & $\Leftrightarrow$ \\
Divalent cations $\left(10^{-3}-10^{-1} \mathrm{M}\right)$ & $\Omega$ & 仓
\end{tabular}

to the fact that only low-resolution $(3.40 \AA)$ data were obtained, and some problems hindered the refinement of the structure influencing the quality of the final model. Due to high R-factors and lack of the electron density for a substantial part of the protein, we decided not to deposit the structure in the PDB. Nevertheless, the structural results are solid enough to solve the uncertainties about the oligomeric state of plant $\mathrm{P} 5 \mathrm{C}$ reductase. In previous studies the migration of the native protein during gel permeation chromatography led to a rough estimate of 1012 monomers in spinach (Murahama et al., 2001), and of 12-14 monomers in A. thaliana (Giberti et al., 2014). Also the rice enzyme showed an elution profile that is consistent with a 14mer (Figure 5). Based on the structural information obtained, a decameric arrangement might therefore be assumed for all three plant proteins.

However, even though the bacterial, the human, and the plant enzymes form very similar decameric arrangements, the structure of rice $\mathrm{P} 5 \mathrm{C}$ reductase reveals dynamic movements of the domains. During model building and refinement, several of the dinucleotide binding domains had unclear or missing density and could not be reliably modeled, which suggests the presence of mobile elements in the crystal (Figure 6). This is contrasting with previous studies on bacterial ( $S$. pyogenes and $N$. meningitides) representatives that have shown almost identical conformation of the subunits in their substrate-free and substrate-bound structures. Because no conformational changes were observed in these proteins, it was hypothesized that they operate by the lock and key mechanism (Nocek et al., 2005). Similarly, no significant conformational changes were reported for the extensively studied human enzyme (Meng et al., 2006). One possible explanation of lack of movement could be the formation of crystal lattice contacts between domains and their stabilization. In fact, an inspection of crystal contacts in the case of the human $\mathrm{P} 5 \mathrm{C}$ reductase confirmed the presence of interactions between molecules that lock the previously mentioned hinge between the two domains of monomers (Salemme et al., 1988). The presence of a conformational plasticity implies the existence of regulation mechanisms. Many allosteric systems contain semi-rigid domains or subunits interacting via flexible regions. This design allows for the propagation of local events over a long distance to affect activities elsewhere (Cui and Karplus, 2008). Further biochemical and biophysical studies are required to investigate the dynamic nature of $\mathrm{P} 5 \mathrm{C}$ reductases. However, it appears that these enzymes might be more dynamic than it has been previously thought.

\section{Acknowledgments}

This work was funded by AGER Foundation in the frame of the RisinnovA project, grant \# 2010-2369. Partial support from the University of Konstanz and the Intramural Research Program of the NCI, Center for Cancer Research, is also gratefully acknowledged. Diffraction data were collected at the SER-CAT beamline 22-ID at the Advanced Photon Source, Argonne National Laboratory. Use of the Advanced Photon Source was 
funded by the US Department of Energy, Office of Science, Office of Basic Energy Sciences under Contract No. W-31-109-Eng-38. $\mathrm{MB}$ was the recipient of a DAAD (German Academic Exchange Service) fellowship supporting a stage in DF's laboratory.

\section{References}

Anil, V. S., Krishnamurthy, H., and Mathew, M. K. (2007). Limiting cytosolic $\mathrm{Na}^{+}$confers salt tolerance to rice cells in culture: a two-photon microscopy study of SBFI-loaded cells. Physiol. Plant. 129, 607-621. doi: 10.1111/j.13993054.2006.00854.x

Baxter, C. J., Redestig, H., Schauer, N., Repsilber, D., Patil, K. R., Nielsen, J., et al. (2007). The metabolic response of heterotrophic Arabidopsis cells to oxidative stress. Plant Physiol. 143, 312-325. doi: 10.1104/pp.106.090431

Bertazzini, M., Lucchini, G., Sacchi, G. A., and Forlani, G. (2012). "Cation homeostasis in Italian rice cultivars showing differential tolerance to salt stress," in Proceedings of the Plant Biology Congress 2012, Freiburg im Breisgau, 302.

Biasini, M., Bienert, S., Waterhouse, A., Arnold, K., Studer, G., Schmidt, T., et al. (2014). SWISS-MODEL: modelling protein tertiary and quaternary structure using evolutionary information. Nucleic Acids Res. 42, W252-W258. doi: 10.1093/nar/gku340

Bradford, M. M. (1976). A rapid and sensitive method for the quantitation of microgram quantities of protein utilizing the principle of protein-dye binding. Anal. Biochem. 72, 248-254. doi: 10.1016/0003-2697(76)90527-3

Chilson, O. P., Kelly-Chilson, A. E., and Siegel, N. R. (1991). Pyrroline5-carboxylate reductase in soybean nodules: isolation/partial primary structure/evidence for isozymes. Arch. Biochem. Biophys. 288, 350-357. doi: 10.1016/0003-9861(91)90206-X

Cui, Q., and Karplus, M. (2008). Allostery and cooperativity revisited. Protein Sci. 17, 1295-1307. doi: 10.1110/ps.03259908

da Rocha, I. M., Vitorello, V. A., Silva, J. S., Ferreira-Silva, S. L., Viégas, R. A., Silva, E. N., et al. (2012). Exogenous ornithine is an effective precursor and the $\delta$-ornithine amino transferase pathway contributes to proline accumulation under high $\mathrm{N}$ recycling in salt-stressed cashew leaves. J. Plant Physiol. 169, 41-49. doi: 10.1016/j.jplph.2011.08.001

Deuschle, K., Funck, D., Forlani, G., Stransky, H., Biehl, A., Leister, D., et al. (2004) The role of $\delta^{1}$-pyrroline-5-carboxylate dehydrogenase in proline degradation. Plant Cell 16, 3413-3425. doi: 10.1105/tpc.104.023622

Diederichs, K., and Karplus, P. A. (1997). Improved R-factors for diffraction data analysis in macromolecular crystallography. Nat. Struct. Biol. 4, 269-275. doi: $10.1038 /$ nsb0497-269

Empadinhas, N., and da Costa, M. S. (2008). Osmoadaptation mechanisms in prokaryotes: distribution of compatible solutes. Int. Microbiol. 11, 151-161. doi: $10.2436 / 20.1501 .01 .55$

Erickson, H. P. (2009). Size and shape of protein molecules at the nanometer level determined by sedimentation, gel filtration, and electron microscopy. Biol. Proced. Online 11, 32-51. doi: 10.1007/s12575-009-9008-x

Eschenfeldt, W. H., Makowska-Grzyska, M., Stols, L., Donnelly, M. I., Jedrzejczak, R., and Joachimiak, A. (2013). New LIC vectors for production of proteins from genes containing rare codons. J. Struct. Funct. Genomics 14, 135-144. doi: 10.1007/s10969-013-9163-9

Forlani, G., Berlicki, Ł., Duò, M., Dziędzioła, G., Giberti, S., Bertazzini, M., et al. (2013). Synthesis and evaluation of effective inhibitors of plant $\delta^{1}$ pyrroline-5-carboxylate reductase. J. Agric. Food Chem. 61, 6792-6798. doi: $10.1021 /$ jf $401234 \mathrm{~s}$

Forlani, G., Bertazzini, M., Zarattini, M., and Funck, D. (2015a). Functional characterization and expression analysis of rice $\delta^{1}$-pyrroline-5-carboxylate dehydrogenase provide new insight into the regulation of proline and arginine catabolism. Front. Plant Sci. 6:591. doi: 10.3389/fpls.2015.00591

Forlani, G., Makarova, K., Ruszkowski, M., Bertazzini, M., and Nocek, B. (2015b). Evolution of plant $\delta^{1}$-pyrroline-5-carboxylate reductases from phylogenic and structural perspectives. Front. Plant Sci. 6:567. doi: 10.3389/fpls.2015.00567

Forlani, G., Giberti, S., Berlicki, Ł., Petrollino, D., and Kafarski, P. (2007). Plant $\mathrm{P} 5 \mathrm{C}$ reductase as a new target for aminomethylenebisphosphonates. J. Agric. Food Chem. 55, 4340-4347. doi: 10.1021/jf0701032

\section{Supplementary Material}

The Supplementary Material for this article can be found online at: http://journal.frontiersin.org/article/10.3389/fpls.2015.00565

Forlani, G., Occhipinti, A., Berlicki, Ł., Dziędzioła, G., Wieczorek, A., and Kafarski, P. (2008). Tailoring the structure of aminophosphonates to target plant P5C reductase. J. Agric. Food Chem. 56, 3193-3199. doi: 10.1021/jf800029t

Forlani, G., Petrollino, D., Fusetti, M., Romanini, L., Nocek, B., Joachimiak, A., et al. (2012). $\Delta^{1}$-pyrroline-5-carboxylate reductase as a new target for therapeutics: inhibition of the enzyme from Streptococcus pyogenes and effects in vivo. Amino Acids 42, 2283-2291. doi: 10.1007/s00726-011-0970-7

Forlani, G., Scainelli, D., and Nielsen, E. (1997). $\Delta^{1}$-pyrroline-5-carboxylate dehydrogenase from cultured cells of potato. Purification and properties. Plant Physiol. 113, 1413-1418.

Funck, D., Stadelhofer, B., and Koch, W. (2008). Ornithine- $\delta$-aminotransferase is essential for arginine catabolism but not for proline biosynthesis. BMC Plant Biol. 8:40. doi: 10.1186/1471-2229-8-40

Funck, D., Winter, G., Baumgarten, L., and Forlani, G. (2012). Requirement of proline synthesis during Arabidopsis reproductive development. BMC Plant Biol. 12:191. doi: 10.1186/1471-2229-12-191

Ge, M., and Pan, X. M. (2009). The contribution of proline residues to protein stability is associated with isomerization equilibrium in both unfolded and folded states. Extremophiles 13, 481-489. doi: 10.1007/s00792-009-0233-7

Giberti, S., Funck, D., and Forlani, G. (2014). $\Delta^{1}$-pyrroline-5-carboxylate reductase from Arabidopsis thaliana: stimulation or inhibition by chloride ions and feedback regulation by proline depend on whether $\mathrm{NADPH}$ or $\mathrm{NADH}$ acts as co-substrate. New Phytol. 202, 911-919. doi: 10.1111/nph.12701

Hashida, S. N., Takahashi, H., and Uchimiya, H. (2009). The role of NAD biosynthesis in plant development and stress responses. Ann. Bot. 103, 819-824. doi: $10.1093 / \mathrm{aob} / \mathrm{mcp} 019$

Hayashi, M., Takahashi, H., Tamura, K., Huang, J., Yu, L. H., Kawai-Yamada, M., et al. (2005). Enhanced dihydroflavonol-4-reductase activity and NAD homeostasis leading to cell death tolerance in transgenic rice. Proc. Natl. Acad. Sci. U.S.A. 102, 7020-7025. doi: 10.1073/pnas.0502556102

Hayat, S., Hayat, Q., Alyemeni, M. N., Wani, A. S., Pichtel, J., and Ahmad, A. (2012). Role of proline under changing environments: a review. Plant Signal. Behav. 7, 1456-1466. doi: 10.4161/psb.21949

Hayatsu, M., Suzuki, S., Hasegawa, A., Tsuchiya, S., and Sasamoto, H. (2014). Effect of $\mathrm{NaCl}$ on ionic content and distribution in suspension-cultured cells of the halophyte Sonneratia alba versus the glycophyte Oryza sativa. J. Plant Physiol. 171, 1385-1391. doi: 10.1016/j.jplph.2014.06.008

Hua, X. J., Van de Cotte, B., Van Montagu, M., and Verbruggen, N. (1997). Developmental regulation of pyrroline-5-carboxylate reductase gene expression in Arabidopsis. Plant Physiol. 114, 1215-1224. doi: 10.1104/pp.114.4.1215

Hua, X. J., Van de Cotte, B., Van Montagu, M., and Verbruggen, N. (2001). The 5 ' untranslated region of the At-P5R gene is involved in both transcriptional and post-transcriptional regulation. Plant J. 26, 157-169. doi: 10.1046/j.1365313x.2001.01020.x

Kabsch, W. (2010). XDS. Acta Crystallogr. D Biol. Crystallogr. 66, 125-132. doi: 10.1107/S0907444909047337

Kavi Kishor, P. B., Hong, Z., Miao, G.-H., Hu, C.-A. A., and Verma, D. P. S. (1995). Overexpression of $\delta^{1}$-pyrroline-5-carboxylate synthetase increases proline production and confers osmotolerance in transgenic plants. Plant Physiol. 108, 1387-1394. doi: 10.1104/pp.108.4.1387

Kesari, R., Lasky, J. R., Villamor, J. G., Des Marais, D. L., Chen, Y. J., Liu, T. W., et al. (2012). Intron-mediated alternative splicing of Arabidopsis P5CS1 and its association with natural variation in proline and climate adaptation. Proc. Natl. Acad. Sci. U.S.A. 109, 9197-9202. doi: 10.1073/pnas.1203433109

Krueger, R., Jäger, H. J., Hintz, M., and Pahlich, E. (1986). Purification to homogeneity of pyrroline-5-carboxylate reductase of barley. Plant Physiol. 80, 142-144. doi: 10.1104/pp.80.1.142

Liang, X., Zhang, L., Natarajan, S. K., and Becker, D. F. (2013). Proline mechanisms of stress survival. Antioxid. Redox Signal. 19, 998-1011. doi: 10.1089 /ars.2012.5074 
Lehmann, S., Funck, D., Szabados, L., and Rentsch, D. (2010). Proline metabolism and transport in plant development. Amino Acids 39, 949-962. doi: 10.1007/s00726-010-0525-3

Lutts, S., Kinet, J. M., and Bouharmont, J. (1996). Effects of various salts and of mannitol on ion and proline accumulation in relation to osmotic adjustment in rice (Oryza sativa L.) callus cultures. J. Plant Physiol. 149, 186-195. doi: 10.1016/S0176-1617(96)80193-3

Mattioli, R., Costantino, P., and Trovato, M. (2009). Proline accumulation in plants: not only stress. Plant Signal. Behav. 4, 1016-1018. doi: 10.4161/psb.4.11.9797

McCoy, A. J., Grosse-Kunstleve, R. W., Adams, P. D., Winn, M. D., Storoni, L. C., and Read, R. J. (2007). Phaser crystallographic software. J. Appl. Crystallogr. 40, 658-674. doi: 10.1107/S0021889807021206

Meng, Z., Lou, Z., Liu, Z., Li, M., Zhao, X., Bartlam, M., et al. (2006). Crystal structure of human pyrroline-5-carboxylate reductase. J. Mol. Biol. 359, 1364-1377. doi: 10.1016/j.jmb.2006.04.053

Merrill, M. J., Yeh, G. C., and Phang, J. M. (1989). Purified human erythrocyte pyrroline-5-carboxylate reductase. Preferential oxidation of NADPH. J. Biol. Chem. 264, 9352-9358.

Miller, G., Honig, A., Stein, H., Suzuki, N., Mittler, R., and Ziberstein, A. (2009). Unraveling $\delta^{1}$-pyrroline-5-carboxylate-proline cycle in plants by uncoupled expression of proline oxidation enzymes. J. Biol. Chem. 284, 26482-26492. doi: 10.1074/jbc.M109.009340

Monteoliva, M. I., Rizzi, Y. S., Cecchini, N. M., Hajirezaei, M. R., and Alvarez, M. E. (2014). Context of action of proline dehydrogenase (ProDH) in the hypersensitive response of Arabidopsis. BMC Plant Biol. 14:21. doi: 10.1186/1471-2229-14-21

Murahama, M., Yoshida, T., Hayashi, F., Ichino, T., Sanada, Y., and Wada, K. (2001). Purification and characterization of $\delta^{1}$-pyrroline-5-carboxylate reductase isoenzymes, indicating differential distribution in spinach (Spinacia oleracea L.) leaves. Plant Cell Physiol. 42, 742-750. doi: 10.1093/pcp/pce093

Nocek, B., Chang, C., Li, H., Lezondra, L., Holzle, D., Collart, F., et al. (2005). Crystal structures of $\delta^{1}$-pyrroline-5-carboxylate reductase from human pathogens Neisseria meningitides and Streptococcus pyogenes. J. Mol. Biol. 354, 91-106. doi: 10.1016/j.jmb.2005.08.036

Pérez-Arellano, I., Rubio, V., and Cervera, J. (2006). Mapping active site residues in glutamate-5-kinase. The substrate glutamate and the feed-back inhibitor proline bind at overlapping sites. FEBS Lett. 580, 6247-6253. doi: 10.1016/j.febslet.2006.10.031

Petrollino, D., and Forlani, G. (2012). Coenzyme preference of Streptococcus pyogenes $\delta^{1}$-pyrroline-5-carboxylate reductase: evidence supporting NADPH as the physiological electron donor. Amino Acids 43, 493-497. doi: 10.1007/s00726-011-1077-x

Rayapati, P. J., Stewart, C. R., and Hack, E. (1989). Pyrroline-5-carboxylate reductase is in pea (Pisum sativum L.) leaf chloroplasts. Plant Physiol. 91, 581-586. doi: 10.1104/pp.91.2.581

Salemme, F. R., Genieser, L., Finzel, B. C., Hilmer, R. M., and Wendoloski, J. J. (1988). Molecular factors stabilizing protein crystals. J. Crystal Growth 90, 273-282. doi: 10.1016/0022-0248(88)90323-5

Sanli, G., Dudley, J. I., and Blaber, M. (2003). Structural biology of the aldoketo reductase family of enzymes: catalysis and cofactor binding. Cell Biochem. Biophys. 38, 79-101. doi: 10.1385/CBB:38:1:79

Senthil-Kumar, M., and Mysore, K. S. (2012). Ornithine- $\delta$-aminotransferase and proline dehydrogenase genes play a role in non-host disease resistance by regulating pyrroline-5-carboxylate metabolism-induced hypersensitive response. Plant Cell Environ. 35, 1329-1343. doi: 10.1111/j.1365-3040.2012.02492.x

Sharma, S., Villamor, J. G., and Verslues, P. E. (2011). Essential role of tissuespecific proline synthesis and catabolism in growth and redox balance at low water potential. Plant Physiol. 157, 292-304. doi: 10.1104/pp.111. 183210

Small, W. C., and Jones, M. E. (1990). Pyrroline 5-carboxylate dehydrogenase of the mitochondrial matrix of rat liver. Purification, physical and kinetic characteristics. J. Biol. Chem. 265, 18668-18672.

Szabados, L., and Savouré, A. (2010). Proline: a multifunctional amino acid. Trends Plant Sci. 15, 89-97. doi: 10.1016/j.tplants.2009.11.009

Székely, G., Abrahám, E., Cséplo, A., Rigó, G., Zsigmond, L., Csiszár, J., et al. (2008). Duplicated P5CS genes of Arabidopsis play distinct roles in stress regulation and developmental control of proline biosynthesis. Plant J. 53, 11-28. doi: 10.1111/j.1365-313X.2007.03318.x

Szoke, A., Miao, G. H., Hong, Z., and Verma, D. P. S. (1992). Subcellular location of $\delta^{1}$-pyrroline-5-carboxylate reductase in root/nodule and leaf of soybean. Plant Physiol. 99, 1642-1649. doi: 10.1104/pp.99.4.1642

Taiz, L., and Zeiger, E. (2010). Plant Physiology. Sunderland: Sinauer Associates.

Takagi, H. (2008). Proline as a stress protectant in yeast: physiological functions, metabolic regulations, and biotechnological applications. Appl. Microbiol. Biotechnol. 81, 211-223. doi: 10.1007/s00253-008-1698-5

Takahashi, H., Takahara, K., Hashida, S. N., Hirabayashi, T., Fujimori, T., KawaiYamada, M., et al. (2009). Pleiotropic modulation of carbon and nitrogen metabolism in Arabidopsis plants overexpressing the NAD kinase2 gene. Plant Physiol. 151, 100-113. doi: 10.1104/pp.109.140665

Terwilliger, T. C., Grosse-Kunstleve, R. W., Afonine, P. V., Moriarty, N. W., Zwart, P. H., Hung, L. W., et al. (2008). Iterative model building, structure refinement and density modification with the PHENIX AutoBuild wizard. Acta Crystallogr. D Biol. Crystallogr. 64, 61-69. doi: 10.1107/S090744490705024X

Turchetto-Zolet, A. C., Margis-Pinheiro, M., and Margis, R. (2009). The evolution of pyrroline-5-carboxylate synthase in plants: a key enzyme in proline synthesis. Mol. Genet. Genomics 281, 87-97. doi: 10.1007/s00438-008-0396-4

Verbruggen, N., and Hermans, C. (2008). Proline accumulation in plants: a review. Amino Acids 35, 753-759. doi: 10.1007/s00726-008-0061-6

Verbruggen, N., Villarroel, R., and Van Montagu, M. (1993). Osmoregulation of a pyrroline-5-carboxylate reductase gene in Arabidopsis thaliana. Plant Physiol. 103, 771-781. doi: 10.1104/pp.103.3.771

Verslues, P. E., and Sharma, S. (2010). Proline metabolism and its implications for plant-environment interaction. Arabidopsis Book 8:e0140. doi: 10.1199/tab.0140

Williams, I., and Frank, L. (1975). Improved chemical synthesis and enzymatic assay of $\delta^{1}$-pyrroline-5-carboxylic acid. Anal. Biochem. 64, 85-97. doi: 10.1016/0003-2697(75)90408-X

Conflict of Interest Statement: The authors declare that the research was conducted in the absence of any commercial or financial relationships that could be construed as a potential conflict of interest.

Copyright (c) 2015 Forlani, Bertazzini, Zarattini, Funck, Ruszkowski and Nocek. This is an open-access article distributed under the terms of the Creative Commons Attribution License (CC BY). The use, distribution or reproduction in other forums is permitted, provided the original author(s) or licensor are credited and that the original publication in this journal is cited, in accordance with accepted academic practice. No use, distribution or reproduction is permitted which does not comply with these terms. 
OPEN ACCESS

Edited by: Irene Murgia,

Università degli Studi di Milano, Italy

Reviewed by:

Greg Moorhead,

University of Calgary, Canada

Vittoria Locato,

Università Campus Bio-Medico, Italy

*Correspondence:

Giuseppe Forlani, Laboratory of Plant Physiology and

Biochemistry, Department of Life Science and Biotechnology, University of Ferrara, Via L. Borsari 46, Ferrara,

44121, Italy

flg@unife.it

Specialty section:

This article was submitted to

Plant Physiology,

a section of the journal

Frontiers in Plant Science

Received: 30 April 2015

Accepted: 16 July 2015

Published: 05 August 2015

Citation:

Forlani G, Bertazzini M, Zarattini $M$ and

Funck D (2015) Functional

characterization and expression

analysis of rice

$\delta^{1}$-pyrroline-5-carboxylate

dehydrogenase provide new insight

into the regulation of proline and

arginine catabolism.

Front. Plant Sci. 6:591

doi: 10.3389/fpls.2015.00591
Functional characterization and expression analysis of rice $\delta^{1}$-pyrroline-5-carboxylate dehydrogenase provide new insight into the regulation of proline and arginine catabolism

\author{
Giuseppe Forlani ${ }^{1 *}$, Michele Bertazzini ${ }^{1,2}$, Marco Zarattini ${ }^{1}$ and Dietmar Funck ${ }^{2}$ \\ ${ }^{1}$ Department of Life Science and Biotechnology, University of Ferrara, Ferrara, Italy, ${ }^{2}$ Biology Section, Department of Plant \\ Physiology and Biochemistry, University of Konstanz, Konstanz, Germany
}

While intracellular proline accumulation in response to various stress conditions has been investigated in great detail, the biochemistry and physiological relevance of proline degradation in plants is much less understood. Moreover, the second and last step in proline catabolism, the oxidation of $\delta^{1}$-pyrroline-5-carboxylic acid (P5C) to glutamate, is shared with arginine catabolism. Little information is available to date concerning the regulatory mechanisms coordinating these two pathways. Expression of the gene coding for P5C dehydrogenase was analyzed in rice by real-time PCR either following the exogenous supply of amino acids of the glutamate family, or under hyperosmotic stress conditions. The rice enzyme was heterologously expressed in $E$. coli, and the affinity-purified protein was thoroughly characterized with respect to structural and functional properties. A tetrameric oligomerization state was observed in size exclusion chromatography, which suggests a structure of the plant enzyme different from that shown for the bacterial P5C dehydrogenases structurally characterized to date. Kinetic analysis accounted for a preferential use of $\mathrm{NAD}^{+}$as the electron acceptor. Cations were found to modulate enzyme activity, whereas anion effects were negligible. Several metal ions were inhibitory in the micromolar range. Interestingly, arginine also inhibited the enzyme at higher concentrations, with a mechanism of uncompetitive type with respect to P5C. This implies that millimolar levels of arginine would increase the affinity of P5C dehydrogenase toward its specific substrate. Results are discussed in view of the involvement of the enzyme in either proline or arginine catabolism.

Keywords: proline and arginine catabolism, enzyme properties, cation and anion effects, gene expression, plant response to stress conditions 


\section{Introduction}

Intracellular accumulation of high proline levels as a stress protectant has long been reported in many plant species in response to a wide array of abiotic stress conditions (Verbruggen and Hermans, 2008), ranging from drought, excess salt, and cold (Hayat et al., 2012) to the treatment with heavy metals (Sharma and Dietz, 2006). Besides acting as a compatible osmolyte to counteract water withdrawal from the cytoplasm caused by either hyperosmotic or freezing stress, proline has been reported to stabilize proteins, DNA and membranes, to reduce lipid peroxidation in plants subjected to stress (Kavi Kishor and Sreenivasulu, 2014) and to act as a radical scavenger (Signorelli et al., 2014). On the other hand, high concentrations of proline in the cell increase in turn reactive oxygen species (ROS) formation in the mitochondrion through the activity of a proline dehydrogenase [EC 1.4.3] that is believed to transfer electrons directly to the respiratory chain (Liang et al., 2013). The need to balance ROS production and scavenging to maintain optimal signaling levels for reinstating metabolic homeostasis during stress situations has been well-established (Türkan and Demiral, 2009). The product of proline oxidation, $\delta^{1}$-pyrroline5-carboxylic acid (P5C), is non-enzymatically linearized to glutamate semialdehyde (GSA) and further oxidized by a P5C dehydrogenase [EC 1.5.1.12], yielding glutamate (Forlani et al., 1997a). During the recovery from stress, this short catabolic pathway provides energy, reducing power and precursors for nucleotide synthesis to allow the resumption of cell division (Hare and Cress, 1997).

In enterobacteria, a proline oxidase associated with the cytoplasmic membrane was shown to possess both proline dehydrogenase and $\mathrm{P} 5 \mathrm{C}$ dehydrogenase activity, allowing substrate channeling of $\mathrm{P} 5 \mathrm{C}$ between the two active sites of the bifunctional enzyme (Arentson et al., 2012). For all other species tested so far, these activities depended on two distinct proteins. This discrepancy could reflect differences in arginine catabolism, which takes place via ornithine and $\mathrm{P} 5 \mathrm{C}$ in most eukaryotes, while some bacteria, namely $E$. coli, use predominantly the arginine succinyltransferase pathway, which does not use P5C as an intermediate (Schneider et al., 1998; Winter et al., 2015). The presence of a distinct P5C dehydrogenase may enable better, simultaneous regulation of the two convergent pathways of arginine and proline catabolism (Figure 1).

Measurements of proline-dependent oxygen uptake of mitochondria isolated from water-stressed barley and maize plants suggested that the modulation of proline channeling into the respiratory chain could play a role in stress-induced proline accumulation (Stewart et al., 1977; Sells and Koeppe, 1981). When proline dehydrogenase and P5C dehydrogenase activities were measured separately in mitochondria from osmotically-stressed maize seedlings, the former was found to be strongly reduced, whereas $\mathrm{P} 5 \mathrm{C}$ dehydrogenase activity was unaffected. The occurrence of stress-driven regulating mechanism(s) was thus inferred exclusively for the first enzyme in proline catabolism (Rayapati and Stewart, 1991). More recently, molecular data confirmed a strong induction of proline dehydrogenase following release from hyperosmotic stress or the

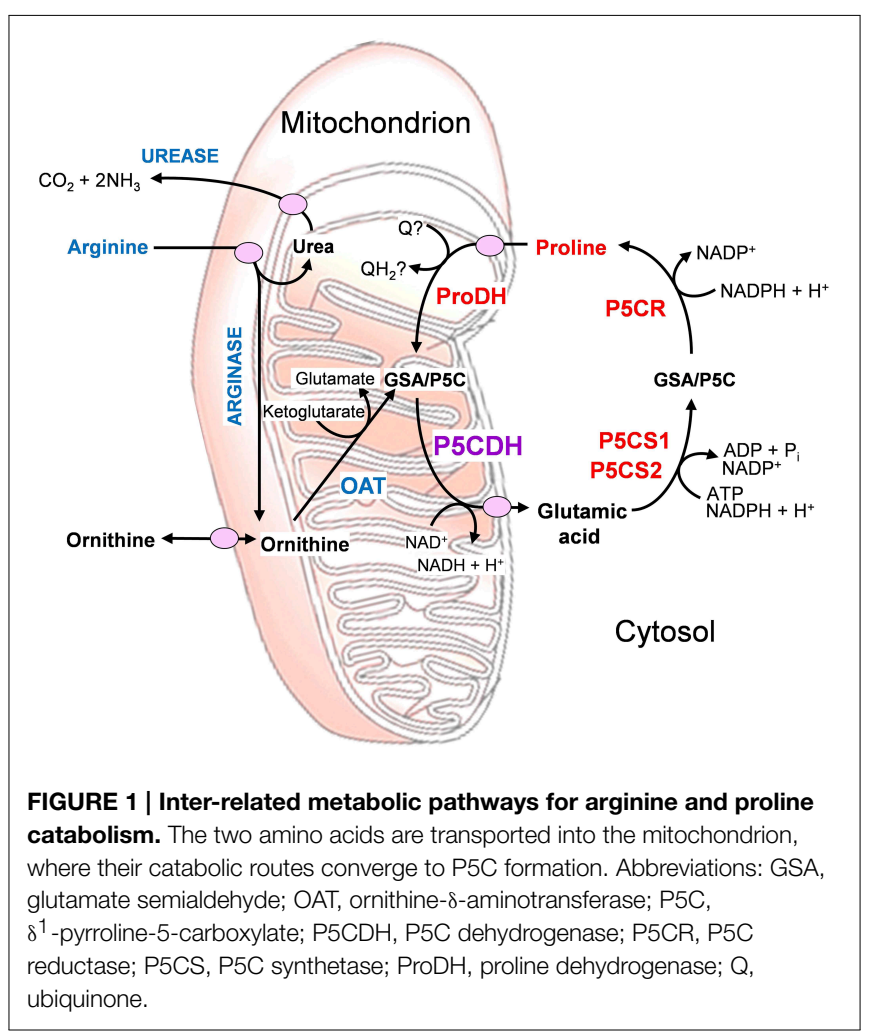

treatment with exogenous proline (Funck et al., 2010; Szabados and Savouré, 2010), and showed its connections within the mitochondrial metabolism in plants (Schertl et al., 2014).

Despite the protective role under stress, exogenously supplied proline was found to exert phytotoxic effects and trigger the activation of programmed cell death (PCD) (Deuschle et al., 2004). An early induction of the gene for P5C dehydrogenase was shown in several crops infected by virulent fungal strains (Mitchell et al., 2006). Moreover, induction of proline oxidation was reported in Arabidopsis thaliana during incompatible plantpathogen interactions (Cecchini et al., 2011). Therefore the possibility exists that proline metabolism is also involved in the process leading to PCD during the hypersensitive defense reaction (Senthil-Kumar and Mysore, 2012). However, it is still unclear which may be the active molecule, whether proline, $\mathrm{P} 5 \mathrm{C}$ or ROS produced during proline catabolism. Interestingly, the early activation of proline dehydrogenase during pathogen attack was accompanied by an increase in $\mathrm{P} 5 \mathrm{C}$ reductase but not in $\mathrm{P} 5 \mathrm{C}$ dehydrogenase transcripts, apparently with few changes occurring in proline and P5C levels (Cecchini et al., 2011). Therefore, some authors postulated that $\mathrm{P} 5 \mathrm{C}$ might trespass the mitochondrial membrane, and that a $\mathrm{P} 5 \mathrm{C} /$ proline cycle would occur as a consequence, leading to reactive oxygen species production (Miller et al., 2009). This cycle would be mediated by the activities of proline dehydrogenase in the mitochondrion and $\mathrm{P} 5 \mathrm{C}$ reductase in the cytosol (Figure 1). Indeed, the analysis of wild-type $A$. thaliana plants and $p 5 c d h$ mutants showed that the absence of $\mathrm{P} 5 \mathrm{C}$ dehydrogenase does not reduce ROS production, cell death, or pathogen resistance, and suggested that the enzyme 
does not act synergistically with proline dehydrogenase in the potentiation of such defense responses (Monteoliva et al., 2014). In any case, the exact mechanisms underlying the role of proline metabolism under biotic stress conditions still await full elucidation.

Interestingly, the gene coding for $\mathrm{P} 5 \mathrm{C}$ dehydrogenase in A. thaliana was found regulated by endogenous siRNAs coordinating the cell response to salt stress conditions. AtP5CDH was shown to partially overlap on opposite strands with SRO5, a gene of unknown function. The former seems constitutively expressed, whereas the latter is salt-stress inducible. When both genes are transcribed, a 24-nt SRO5-P5CDH siRNA is formed, promoting the degradation of $\mathrm{P} 5 \mathrm{CDH}$ transcripts (Borsani et al., 2005). This mechanism could be functional to fuel the P5C/Pro cycle, thereby increasing ROS production (Miller et al., 2009). However, this would apparently conflict with the proposed role for SRO5, which is believed to counteract ROS generation (Borsani et al., 2005).

Moreover, when the mRNA levels of the aldehyde dehydrogenase gene superfamily were measured in rice (Oryza sativa L.) seedlings under different experimental conditions, the expression of some genes was found up-regulated by drought and salinity and the exogenous application of abscisic acid (ABA), indicating that the products of these genes are potentially involved in rice osmotic stress adaptation. Quite surprisingly, the gene encoding $\mathrm{P} 5 \mathrm{C}$ dehydrogenase $(\mathrm{OsP} 5 \mathrm{CDH}$, also referred to as OsALDH12, locus name OsJ_19352) was unaffected by salinity stress, and induced (rather than repressed) by drought and ABA treatments (Gao and Han, 2009). However, in that study neither the corresponding intracellular levels of proline nor a time course of gene expression during the stress were shown.

In addition to the uncertainties on the modulation at the transcriptional level in response to environmental signals, very little is known about the occurrence of posttranslational regulation mechanisms for P5C dehydrogenase. In early studies, maximal activity was detected in crude mitochondrial preparations after detergent treatment. Biochemical characterization pointed out a clear-cut preference for $\mathrm{NAD}^{+}$as the electron acceptor, and $K_{\mathrm{M}}$ values in the range of $10^{-4}$ to $10^{-3} \mathrm{M}$ for both $\mathrm{P} 5 \mathrm{C}$ and $\mathrm{NAD}^{+}$(Stewart and Lai, 1974; Boggess et al., 1975). Biochemical analyses supported the occurrence of two distinct enzyme forms, each one specifically involved in either proline or arginine oxidation in maize and wild tobacco (Elthon and Stewart, 1981; Forlani et al., 1997b). However, in most diploid genomes analyzed so far, including A. thaliana and $O$. sativa, only a single $P 5 C D H$ gene seems to exist (Deuschle et al., 2001; Ayliffe et al., 2005). To date, plant P5C dehydrogenase has been purified only from a Solanum tuberosum cell culture (Forlani et al., 1997a). The characterization of the potato enzyme showed that $\mathrm{NADP}^{+}$can also serve as electron acceptor, and that $\mathrm{NaCl}$ inhibits enzyme activity in a concentration-dependent manner. A subsequent analysis of protein levels in plant tissues and in response to salt stress and exogenous proline suggested a developmental, but not an environmental control of P5C dehydrogenase expression. This notwithstanding, protein levels showed an overall positive correlation with free proline concentration inside the cell (Forlani et al., 2000).

In the frame of a research project for integrated genetic and genomic approaches for new Italian rice breeding strategies, we aim at a better understanding of the biochemical mechanisms underlying salt tolerance in rice. To achieve this goal, the regulative switches responsible for the intracellular accumulation of proline under hyperosmotic stress conditions need to be identified. Here, we report a thorough characterization of rice $\mathrm{P} 5 \mathrm{C}$ dehydrogenase, affinity-purified after heterologous expression in E. coli. Activity was inhibited by cations and arginine in the millimolar range, whereas some heavy metals were effective in the micromolar range. In cultured rice cells, mRNA levels were measured by real-time PCR, showing a constitutive expression of $\mathrm{OsP} 5 \mathrm{CDH}$, as well as slightly enhanced transcript levels in response to PEG treatments.

\section{Materials and Methods}

\section{Plant Materials and Growth Conditions}

Suspension cultured cells of rice (Oryza sativa L. ssp. japonica, cultivars Vialone nano and Loto) were grown in MS medium (Murashige and Skoog, 1962) supplemented with $30 \mathrm{~g} \mathrm{~L}^{-1}$ sucrose and $2 \mathrm{mg} \mathrm{L}^{-1}$ 2,4-dichlorophenoxyacetic acid, as previously described (Rożkowicz et al., 2003). Cultures were maintained in continuous balanced growth by subculturing $12 \mathrm{~mL}$-aliquots to $50 \mathrm{~mL}$ of fresh medium in $250 \mathrm{~mL}$ Erlenmeyer flasks every $7 \mathrm{~d}$.

Hyperosmotic stress conditions were obtained either by adding to the culture medium a suitable volume of a $5 \mathrm{M}$ solution of $\mathrm{NaCl}$ in water, or by resuspending cells in MS medium containing PEG 6000 at concentrations ranging from 15 to $25 \%(\mathrm{w} / \mathrm{v})$. Exogenous supply of amino acids was performed through the addition of proper volumes of $1 \mathrm{M}$ solutions, sterilized by filtration $(0.22 \mu \mathrm{m})$. To avoid $\mathrm{pH}$-dependent nonspecific effects, arginine was used as its hydrochloride. Abscisic acid was prepared as a $50 \mathrm{mM}$ solution in pure ethanol. All treatments were applied directly at subculturing, and were carried out in triplicate. Cell viability was measured by the Evans' blue uptake assay, as described (Rożkowicz et al., 2003).

\section{Reverse Transcriptase Real-time PCR Analysis}

Total RNA was extracted from $50 \mathrm{mg}$ of plant material by using the Plant RNA Isolation kit (Agilent Technologies), according to the manufacturer's instructions. Following spectrophotometric quantification and assessment of RNA integrity, RNA $(1 \mu \mathrm{g})$ was incubated at $30^{\circ} \mathrm{C}$ for $30 \mathrm{~min}$ with $5 \mathrm{U}$ of amplification grade DNase I (Sigma AMPD1), treated for $10 \mathrm{~min}$ at $70^{\circ} \mathrm{C}$ to denature both the DNase I and the RNA, chilled on ice and immediately used for single strand cDNA synthesis by reverse transcription in a $20 \mu \mathrm{L}$ reaction mixture using the iScript cDNA Synthesis Kit (Bio-Rad). First strand cDNA was used as template for PCR amplifications using specific primers for target and housekeeping genes (Table 1). For semi-quantitative PCR analysis, the amplification was carried out in a Mastercycler Personal thermal cycler (Eppendorf) in a $20 \mu \mathrm{L}$ reaction mixture containing $10 \mu \mathrm{L}$ iQTMSupermix (Bio-Rad), 20 pmol forward 
TABLE 1 | Specific primers used for RT-PCR and qRT-PCR.

\begin{tabular}{|c|c|c|c|}
\hline Gene & Primers & cDNA product size & Genomic DNA product size \\
\hline P5C synthetase 1 (protein ID Q941T1) & $\begin{array}{l}\text { fwd CGCAGGATCAATTCGTGAAATCGCA } \\
\text { rev GCAATCTGTACCAAGGCATCAGGA }\end{array}$ & 162 & 291 \\
\hline P5C synthetase 2 (protein ID 004226) & $\begin{array}{l}\text { fwd GATTGGGTGCTGAGGTTGGCATAA } \\
\text { rev CGACATCCTTGTCACCATTCACCA }\end{array}$ & 132 & - \\
\hline P5C reductase (protein ID Q8GT01) & $\begin{array}{l}\text { fwd ATCGTAAGGCAGGTACTGGTTGAG } \\
\text { rev AGCTGAACGGGTGTITGGCATT }\end{array}$ & 150 & 377 \\
\hline Ornithine- $\delta$-aminotransferase (protein ID Q10G56) & $\begin{array}{l}\text { fwd GAGGGGTITGCTTAATGCAGTGGA } \\
\text { rev CAGCGGAGGCGCTAATCTGATTAT }\end{array}$ & 142 & 142 \\
\hline Proline dehydrogenase (protein ID Q336U3) & $\begin{array}{l}\text { fwd ACGGTCCAGTGGAGCAGATCAT } \\
\text { rev AGCATTGCAGCCTTGAACCT }\end{array}$ & 133 & 261 \\
\hline P5C dehydrogenase (protein ID B9FLB9) & $\begin{array}{l}\text { fwd ATGCTTGCAGTGGTCAGAAGTG } \\
\text { rev TGGGCCAATGGTCAAGTCTTCA }\end{array}$ & 134 & 487 \\
\hline Actin-1 (protein ID Q10DV7) & $\begin{array}{l}\text { fwd CTGCGGGTATCCATGAGACT } \\
\text { rev GCAATGCCAGGGAACATAGT }\end{array}$ & 118 & 118 \\
\hline Elongation factor (EF) $1 \alpha$ (protein ID 064937) & $\begin{array}{l}\text { fwd TCTCTGGGTITGAGGGTGACAACA } \\
\text { rev TTGATCTGGTCAAGAGCCTCAAGC }\end{array}$ & 94 & 94 \\
\hline
\end{tabular}

Sequences were designed using the Primer 3 software (http://bioinfo.ut.ee/primer3-0.4.0/).

and reverse primers, and $50 \mathrm{ng}$ cDNA. Cycling conditions consisted of an initial $5 \mathrm{~min}$ at $95^{\circ} \mathrm{C}$, followed by $60 \mathrm{~s}$ denaturing at $94^{\circ} \mathrm{C}, 30 \mathrm{~s}$ annealing at $61^{\circ} \mathrm{C}$, and $60 \mathrm{~s}$ elongation at $72^{\circ} \mathrm{C}$ repeated for a given number of cycles, and with $5 \mathrm{~min}$ final extension at $72^{\circ} \mathrm{C}$. The exponential phase of amplification was determined by analyzing gel (1.5\% agarose) electrophoresis pattern of the PCR products generated with different numbers of cycles. qPCR was carried out with $25 \mathrm{ng}$ cDNA in a volume of $15 \mu \mathrm{L}$ on a CFX96 Real-Time PCR Detection System (BioRad) using SsoAdvanced ${ }^{\mathrm{TM}}$ Universal SYBR ${ }^{\circledR}$ Green Supermix (Bio-Rad) with the following thermal profile: enzyme activation at $98^{\circ} \mathrm{C}$ for $30 \mathrm{~s}$, followed by 40 cycles of denaturation for $5 \mathrm{~s}$ at $95^{\circ} \mathrm{C}$ and annealing and extension for $15 \mathrm{~s}$ at $61^{\circ} \mathrm{C}$. Melting curve analysis was performed after cycle completion to validate amplicon identity. The expression levels of both housekeeping genes were used to obtain the relative expression of each target gene by the $\Delta \Delta_{\mathrm{Ct}}$ method (Pfaffl, 2001) using the Gene Expression Module of CFX Manager software ${ }^{\mathrm{TM}}$ (Version 3.1, Bio-Rad). For each treatment, three biological replications were performed, and two technical replicates were run for each sample in qPCR analysis.

\section{Amino Acid Extraction and Analysis}

Cells were harvested by vacuum filtration on nylon filters $(50 \mu \mathrm{m}$ mesh), weighed and ground in a mortar with $2 \mathrm{~mL} \mathrm{~g}^{-1}$ of a $3 \%$ $(w / v)$ solution of 5-sulphosalicylic acid. Following centrifugation for $10 \mathrm{~min}$ at $12,000 \mathrm{~g}$, the supernatant was mixed with the same volume of $o$-phthaldialdehyde solution $[0.5 \mathrm{M}$ in $0.5 \mathrm{M}$ sodium borate buffer, $\mathrm{pH} 10.0$, containing $0.5 \mathrm{M} \beta$-mercaptoethanol, and $10 \%(\mathrm{v} / \mathrm{v})$ methanol]. After exactly $60 \mathrm{~s}, 20 \mu \mathrm{L}$ of derivatized samples were injected onto a $4.6 \times 250 \mathrm{~mm}$ Zorbax ODS column (Agilent Technologies) equilibrated with 59\% solvent A [ $50 \mathrm{mM}$ sodium phosphate- $50 \mathrm{mM}$ sodium acetate buffer, $\mathrm{pH}$ 7.5 , containing $2 \%(\mathrm{v} / \mathrm{v})$ of both methanol and tetrahydrofuran] and $41 \%$ solvent B $(65 \%$ methanol). Elution proceeded at a flow rate of $60 \mathrm{~mL} \mathrm{~h}^{-1}$ using a computer-controlled (Data System
450; Kontron, Munich, Germany) complex gradient from 41 to $100 \%$ solvent B, as described (Forlani et al., 2014), monitoring the eluate at $340 \mathrm{~nm}$. Proline and total amino acid content were quantified by a modification of the acid ninhydrin method (Williams and Frank, 1975). Data were expressed on a fresh weight basis.

\section{Cloning of OsP5CDH and Heterologous Expression in E. coli}

The coding sequence of $O$. sativa $\mathrm{P} 5 \mathrm{C}$ dehydrogenase was amplified by PCR from cDNA clone J033091016 (Rice Genome Resource Center, National Institute of Agrobiological Sciences DNA Bank, Japan) with the primers P5CDHfwd (caccTCAGGtCCaGCCGCACTC) and P5CDH-rev (GTCAAGGCTAGATTAGCTTGTAGC), and inserted into the expression vector pET151 by directional TOPO cloning (Life Technologies, Carlsbad, CA, USA), yielding the vector pET151-OsP5CDH. The same procedure carried out with the primers ntP5CDH-fwd (caccATGAGCCTCATCCTTTCCCG) and $\mathrm{P} 5 \mathrm{CDH}-\mathrm{rev}$ yielded the vector pET151-ntOsP5CDH coding the non-truncated form of the protein bearing its mitochondrial transit peptide at the amino terminus (Supplementary Figure 1).

For heterologous expression, E. coli BL21(DE3) pLysS cells (Invitrogen) were made competent by the calcium chloride method, transformed with either vector and selected on ampicillin-containing LB plates. After inducing the expression of P5C dehydrogenase by $1 \mathrm{mM}$ isopropyl-D-thiogalactopyranoside (IPTG) at $24^{\circ} \mathrm{C}$, the cells were lysed in a mortar with $2 \mathrm{~g} \mathrm{~g}^{-1}$ alumina and resuspended in $20 \mathrm{~mL} \mathrm{~g}^{-1}$ extraction buffer (50 mM Na phosphate buffer, $\mathrm{pH} 7.5$, containing $200 \mathrm{mM} \mathrm{NaCl}$, $0.5 \mathrm{mM}$ DTT, and $20 \mathrm{mM}$ imidazole). The His-tagged protein was purified from clarified extracts by affinity chromatography with a His-Select ${ }^{\mathrm{TM}}$ Nickel Affinity Gel column $(1.5 \mathrm{~mL}$ bed volume, Sigma H7788). Stepwise elution was achieved by increasing concentrations of imidazole in extraction buffer. For activity assays, the purified enzyme was diluted 1:100 with water, and 
a proper aliquot $(2$ to $5 \mu \mathrm{L}$ ) was added to the assay mixture. To remove the His-tag, aliquots $(50 \mu \mathrm{g})$ of the preparation were treated with $0.5 \mu \mathrm{g}$ of tobacco etch virus (TEV) protease (Sigma T4455), according to the cleavage protocol provided by the manufacturer.

\section{P5C Dehydrogenase Assay}

Enzyme activity was measured at $35^{\circ} \mathrm{C}$ as the $\mathrm{P} 5 \mathrm{C}$-dependent reduction of $\mathrm{NAD}(\mathrm{P})^{+}$. The assay mixture contained $50 \mathrm{mM}$ Hepes-KOH buffer, $\mathrm{pH} 7.5,10 \mathrm{mM} \mathrm{NAD}^{+}$or $20 \mathrm{mM} \mathrm{NADP}^{+}$, and $1 \mathrm{mM} \mathrm{L-P5C}$ in a final volume of $0.2 \mathrm{~mL}$. A limiting amount of enzyme (from 0.2 to $1 \mu \mathrm{g}$ of the purified protein) was added to the pre-warmed mixture and the increase in absorbance was determined at $340 \mathrm{~nm}$ for up to $30 \mathrm{~min}$ at 1min intervals against blanks from which P5C had been omitted. Activity was determined from the initial linear rate, based on the assumption of an extinction coefficient for $\mathrm{NAD}(\mathrm{P}) \mathrm{H}$ of $6220 \mathrm{M}^{-1} \mathrm{~cm}^{-1}$. Linear regression analysis was computed by using Prism 6 (version 6.03, GraphPad Software, Inc., USA). DL-P5C was synthesized by the periodate oxidation of $\delta$-allohydroxylysine (Sigma H0377) and purified by cation-exchange chromatography, as described previously (Forlani et al., 1997a). L-P5C was assumed to represent $50 \%$ of the added P5C concentration, and D-P5C was neglected because it has been shown to be biologically inactive in the assay (Williams and Frank, 1975). Therefore, throughout this study, P5C refers to L-P5C only. The protein concentration was determined by the method of Bradford (1976), using bovine serum albumin (BSA) as the standard. For the purified protein, direct absorbance at $280 \mathrm{~nm}$ was used instead, and the concentration was calculated on the basis of a deduced molar extinction coefficient for rice P5C dehydrogenase of $67,630 \mathrm{M}^{-1} \mathrm{~cm}^{-1}$ (http://web.expasy.org/ protparam).

\section{Kinetic Analyses}

To evaluate substrate affinity, unvariable substrates were fixed at the same levels as in the standard assay. The concentration of $\mathrm{L}-\mathrm{P} 5 \mathrm{C}$ ranged from 100 to $1000 \mu \mathrm{M} ; \mathrm{NAD}^{+}$or $\mathrm{NADP}^{+}$ were added in the range from 0.5 to $10 \mathrm{mM}$ and from 1 to $20 \mathrm{mM}$, respectively. To evaluate the mechanism of the inhibition brought about by arginine, $\mathrm{NAD}^{+}$and $\mathrm{P} 5 \mathrm{C}$ concentration ranged from 1 to $7 \mathrm{mM}$ and from 100 to $700 \mu \mathrm{M}$, respectively. When evaluating the effect of ions, $\mathrm{L}-\mathrm{P} 5 \mathrm{C}$ concentration was reduced to $250 \mu \mathrm{M}$ to minimize the carry-over of chloride anions, and Hepes buffer was used at $12.5 \mathrm{mM}$ to reduce the content of potassium cations; in this case, $\mathrm{pH}$ was adjusted with $\mathrm{NH}_{3}$, which at concentrations below $100 \mathrm{mM}$ had no effect on enzyme activity. All assays were performed in triplicate. $K_{\mathrm{M}}$ and $\mathrm{V}_{\max }$ values, as well as the concentrations causing $50 \%$ inhibition $\left(\mathrm{IC}_{50}\right)$ of $\mathrm{P} 5 \mathrm{C}$ dehydrogenase activity, $K_{\mathrm{I}}$-values and their confidence intervals were estimated by non-linear regression analysis using Prism 6 . Activation energy was obtained by the replotting (Arrhenius plot) of data from two independent experiments in which the catalytic rate was measured as a function of temperature in the range 25 to $42.5^{\circ} \mathrm{C}$, at $2.5^{\circ} \mathrm{C}$-intervals. Thermostability of the enzyme was determined by incubating the purified protein for various intervals of time at 40 to $50^{\circ} \mathrm{C}$, followed by determination of the remaining activity under standard assay conditions. Catalytic constants were calculated from $\mathrm{V}_{\max }$ values taking into account a homotetrameric composition of the native holoenzyme, with each monomer having a molecular mass of 57,670 Da.

\section{Determination of Isoelectric Point, Native, and Denatured Molecular Mass}

Discontinuous SDS-polyacrylamide gel electrophoresis was performed at $20^{\circ} \mathrm{C}$ by the method of Laemmli with a $4 \%$ stacking and a $8 \%$ separating gel, using a Minigel system (BioRad). Samples were denatured by boiling $5 \mathrm{~min}$ in $62 \mathrm{mM}$ Tris- $\mathrm{HCl}$ buffer ( $\mathrm{pH} 6.8$ ), containing 2\% (w/v) SDS, 10\% (v/v) glycerol, and $5 \%(\mathrm{v} / \mathrm{v}) \beta$-mercaptoethanol. Proteins were visualized after staining with $0.1 \%$ Coomassie brilliant blue R-250.

Gel permeation chromatography was performed by injecting $100-\mu \mathrm{L}$ aliquots of the purified protein onto a Superose 12 HR 10/30 (Pharmacia) column that had been equilibrated with $50 \mathrm{mM}$ Tris- $\mathrm{HCl}$ buffer, $\mathrm{pH} 7.75$, containing $250 \mathrm{mM} \mathrm{NaCl}$. Elution proceeded at the constant flow of $0.5 \mathrm{~mL} \mathrm{~min} \mathrm{~m}^{-1}$, for the collection of $0.5-\mathrm{mL}$ fractions, while monitoring the eluate at $280 \mathrm{~nm}$ (HPLC Detector 432, Kontron). Molecular weight markers for column calibration (Pharmacia) were bovine thyroid thyroglobulin $(669 \mathrm{kDa})$, horse spleen ferritin $(440 \mathrm{kDa})$, bovine liver catalase $(232 \mathrm{kDa})$, rabbit muscle aldolase $(158 \mathrm{kDa})$, and BSA (67 and $268 \mathrm{kDa}$ ). Three runs were carried out for each marker, and six runs for the purified protein.

Isoelectric focusing was performed as described previously (Forlani et al., 1997a), with ampholytes within the $\mathrm{pH}$ 3.5-10 range (Pharmacia). pI markers (Sigma) were bovine milk $\beta$ lactoglobulin A (pI 5.1), bovine erythrocyte carbonic anhydrase II (5.4 and 5.9), and bovine erythrocyte carbonic anhydrase I (6.6). After the run, individual tracks were cut from the gel and either sliced in 5-mm segments for the determination of $\mathrm{pH}$, or stained for protein as above.

\section{Results}

\section{Expression of Rice P5CDH under Hyperosmotic Stress Conditions, and in Response to the Exogenous Supply of Metabolically Related Amino Acids}

To investigate the regulation of $\mathrm{OsP} 5 \mathrm{CDH}$ during stress-induced proline accumulation, rice suspension cultured cells were treated with increasing concentrations of either $\mathrm{NaCl}$ or polyethylene glycol (PEG) to induce salt or osmotic stress conditions, respectively. Cell growth was progressively reduced by both stress treatments, but not completely suppressed (not shown). As expected, free proline content in salt-stressed cells increased with time in a dose-responsive manner (Figure 2A). However, also total amino acid content showed a similar pattern (Figure 2C), and if proline content was expressed as percentage of total amino acids, only minor variations, if any, were evident in response to salt treatment (Figure 2E). A remarkably different picture was obtained with PEG. In this case only a slight but reproducible increase of intracellular proline levels was found soon after the exposure to osmotic stress conditions, which at 

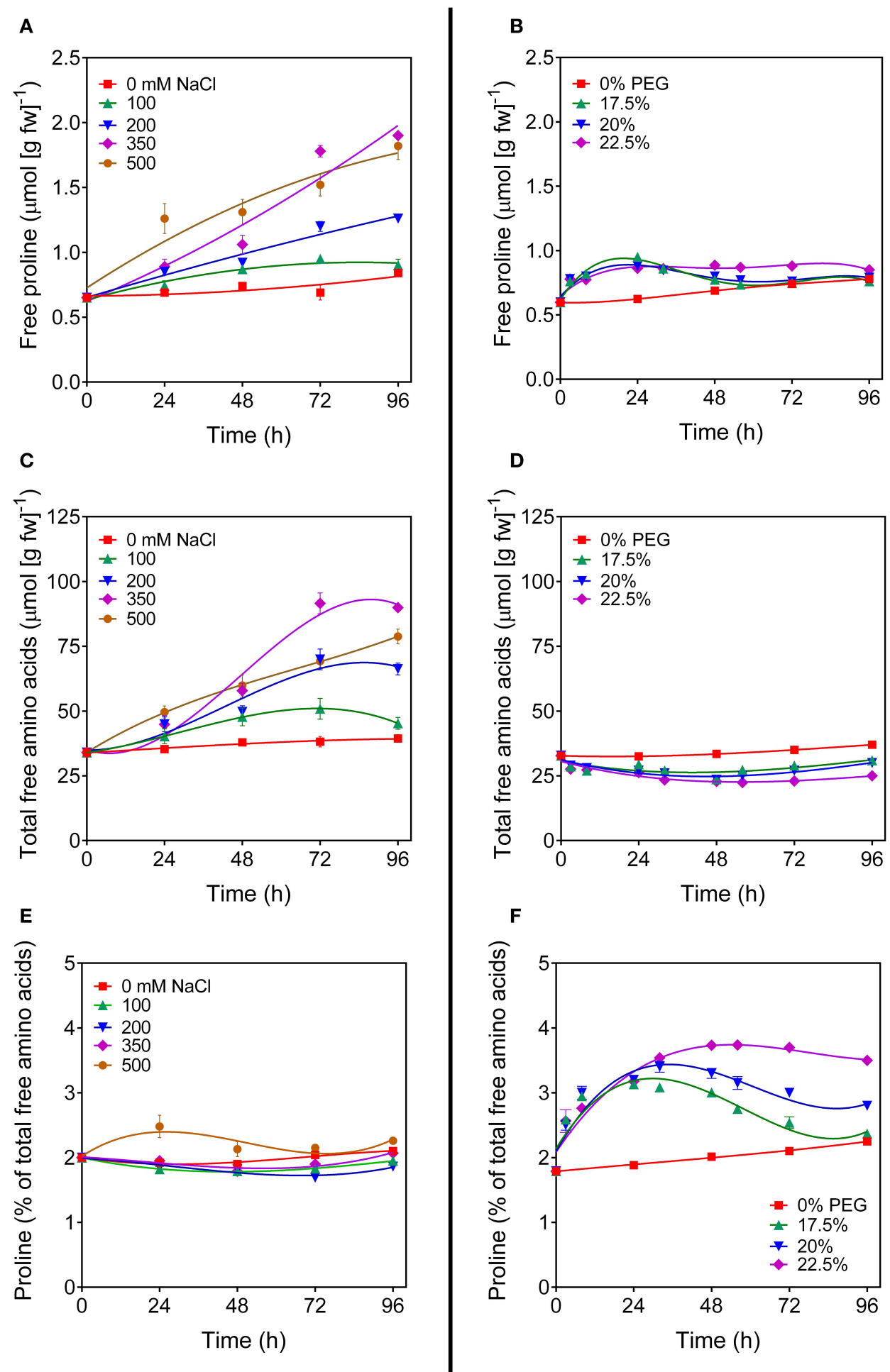

FIGURE 2 | Proline levels in salt- and PEG-treated rice cells. Suspension cultured cells of rice ( $C V$ Vialone nano) were treated with different, growth inhibitory concentrations of either $\mathrm{NaCl}$ (A,C,E) or PEG 6000 (B,D,F). At increasing time after the treatments, free proline levels $(\mathbf{A}, \mathbf{B})$ and total amino acid

concentrations (C,D) were determined on a fresh weight basis. Proline concentration was also expressed as percent of total amino acids (E,F). All treatments were carried out in triplicate, and means \pm SE are depicted. Very similar results were obtained also with cell cultures of the $\mathrm{cr}$ Loto. 
low PEG doses came back to control levels after a couple of days (Figure 2B). The concentration of free amino acids did not increase, but showed on the contrary a slight decrease (Figure 2D). Because the treatment caused a 20 to $35 \%$-loss of cell viability (data not presented), such a decrease most likely depended on the presence of a significant amount of dead cells that influences the amino acid content, as expressed on a fresh weight basis. Interestingly, when proline levels were expressed as percentage of total amino acids, a significant increase was evident that was proportional to the severity of the stress. At 22.5\% PEG, this increase was maintained over the entire culture cycle (Figure 2F). The effects of higher PEG concentrations were not tested because of the resulting undesirable consequence on cell viability. Because the plant response to hyperosmotic stress conditions is at least in part mediated by abscisic acid, similar experiments were performed also by treating cells with $50 \mu \mathrm{M}$ ABA, either alone or in combination with PEG. Results were identical to those obtained for untreated cells or with cell treated with PEG alone, respectively (data not shown). To rule out the possibility that these results could be cultivar-specific, or depend on some mutation that may have occurred with time at the undifferentiated tissue level, where mutations that are lethal in planta can be maintained within the cell population, the experiments were carried out with independent cell cultures of two japonica rice cultivars (Loto and Vialone nano). Virtually overlapping patterns of amino acid profile changes were found in both cell cultures (data not shown).

Based on these pieces of evidence, the effect of the treatment with $22.5 \%$ PEG on the expression level of the genes coding for the enzymes involved in proline synthesis and catabolism was investigated. In unstressed cells, P5C synthetase 2 and P5C reductase mRNAs were present at significantly higher levels than that of OsP5CDH, whereas P5C synthetase 1 and Proline dehydrogenase transcripts were almost undetectable (Figure 3A). In PEG-stressed cells, despite the rapid and relevant increase of free proline, mRNA levels of $P 5 C$ reductase did not change at all, and only minor variations were found for the two $P 5 C$ synthetases, with a two-fold increase $48 \mathrm{~h}$ after the start of the treatment. Concerning the mRNA for P5C dehydrogenase, a twofold increase was also evident, and the same trend was shared by the transcript of Proline dehydrogenase (Figure 3B).

Even under the best conditions found, the increase of proline concentration in the cells appeared too slight to allow the investigation of its utilization during a subsequent relief from the stress. As an alternative, the fate of exogenously-supplied proline was studied. Rice cells were treated with millimolar concentrations of proline. The addition did not significantly affect cell viability up to 4 days after the treatment (results not shown). Under the experimental conditions adopted, exogenous proline was rapidly and actively taken up by the cells, whereas no significant decrease of proline concentration was found in the absence of cells during the time of the experiment (Figure 4A). Intracellular proline levels reached their maximum $24 \mathrm{~h}$ after addition of proline to the medium (Figure 4E). Thereafter, intracellular concentrations slowly came back to control levels. On the contrary, no variations were found with respect to total free amino acid content (Figure 4C).

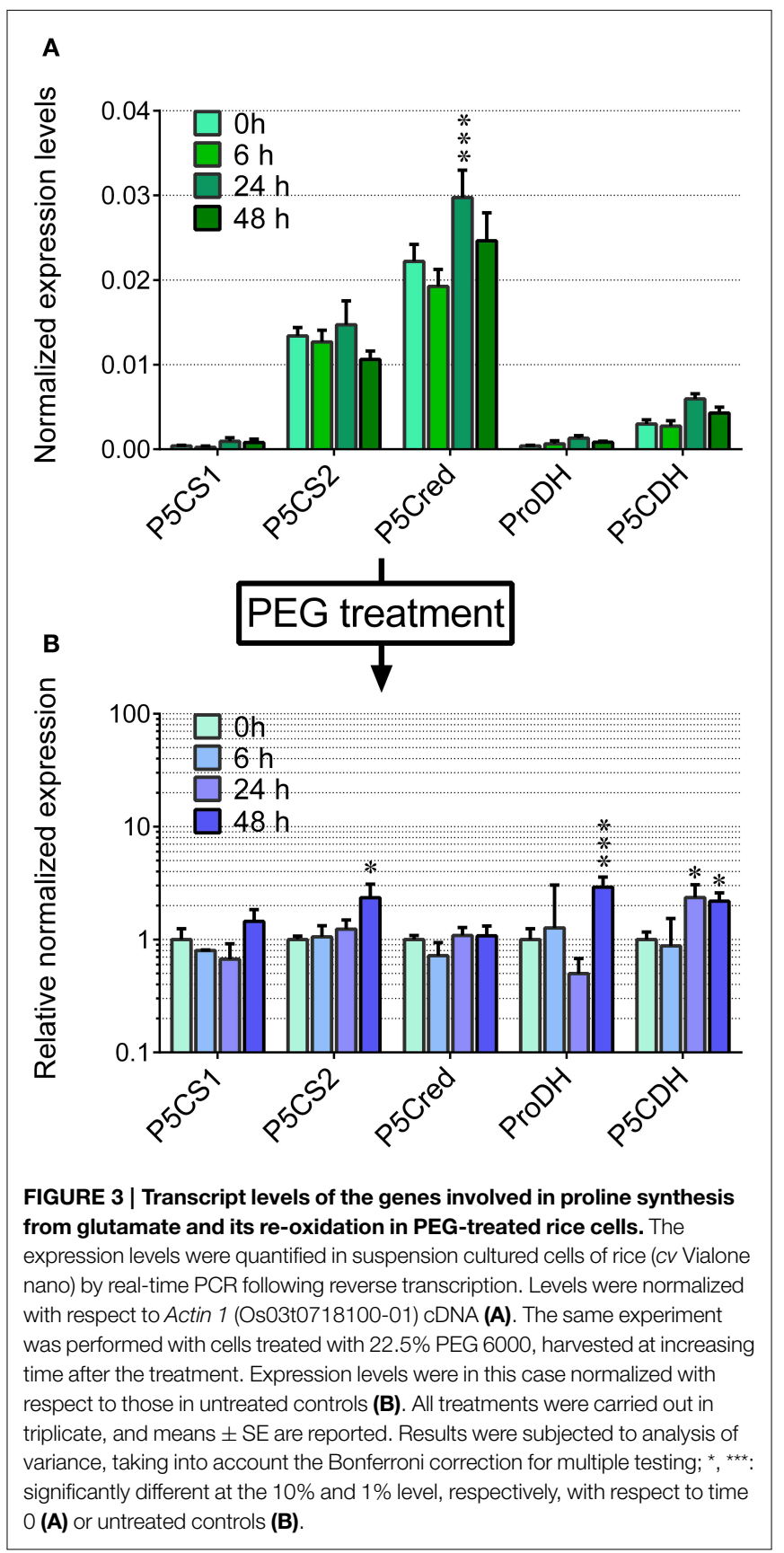

Because the absolute amount of proline utilized was higher in cells treated with $5 \mathrm{mM}$ proline than in cells treated with $2 \mathrm{mM}$ proline (Figure 4E), it is likely that proline is not simply used for protein synthesis for growth. Indeed, transcript level analysis of the genes involved in $\mathrm{P} 5 \mathrm{C}$ metabolism in proline-fed cells revealed that $24 \mathrm{~h}$ after proline supply Proline dehydrogenase transcript levels were 5-fold higher than those of OsP5CDH and Ornithine- $\delta$-aminotransferase (OAT), the gene encoding for the enzyme catalyzing the conversion of ornithine into P5C. This corresponds to a 60-fold increase of Proline dehydrogenase transcripts over basal levels (Figure 5A). At $48 \mathrm{~h}$ after proline treatment the Proline dehydrogenase transcript came 

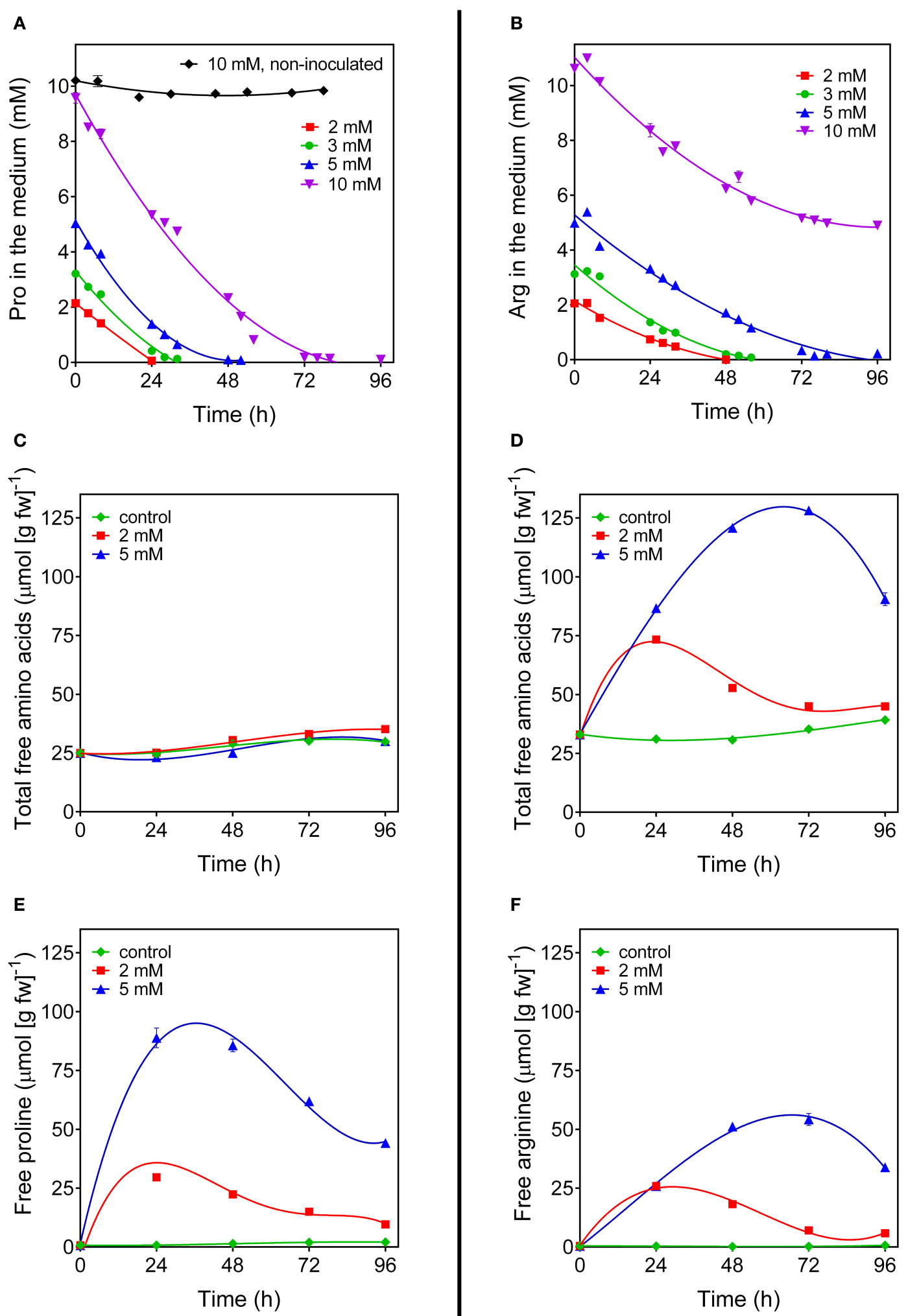

FIGURE 4 | Arginine and proline utilization in amino acid-fed rice cells. Suspension cultured cells of rice ( $C V$ Loto) were supplied with different concentrations of either proline (A,C,E) or arginine (B,D,F). At increasing time after the addition of the exogenous amino acid, its residual level in the medium was determined (A,B). Cells from the same samples were harvested

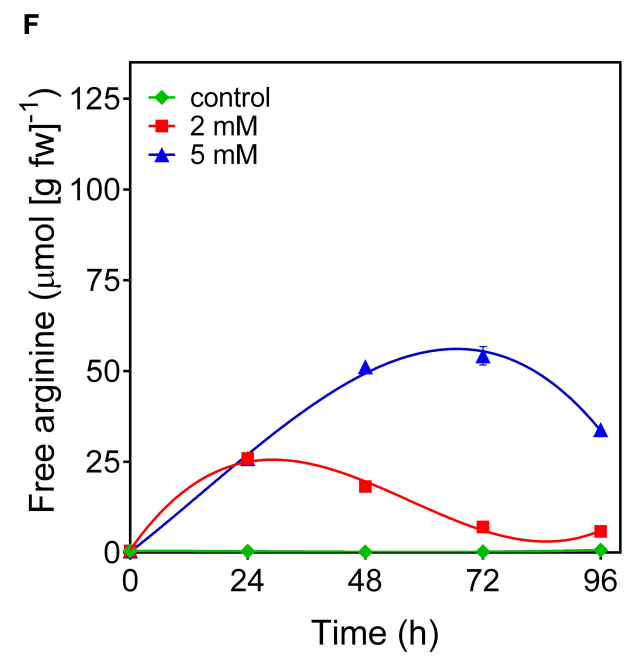

and washed thoroughly before intracellular levels of total amino acid (C,D), free proline $(\mathbf{E})$ and free arginine $(\mathbf{F})$ were determined on a fresh weight basis. All treatments were carried out in triplicate, and means \pm SE are reported. Virtually overlapping patterns were obtained with cell cultures of the $\mathrm{CV}$ Vialone nano. 

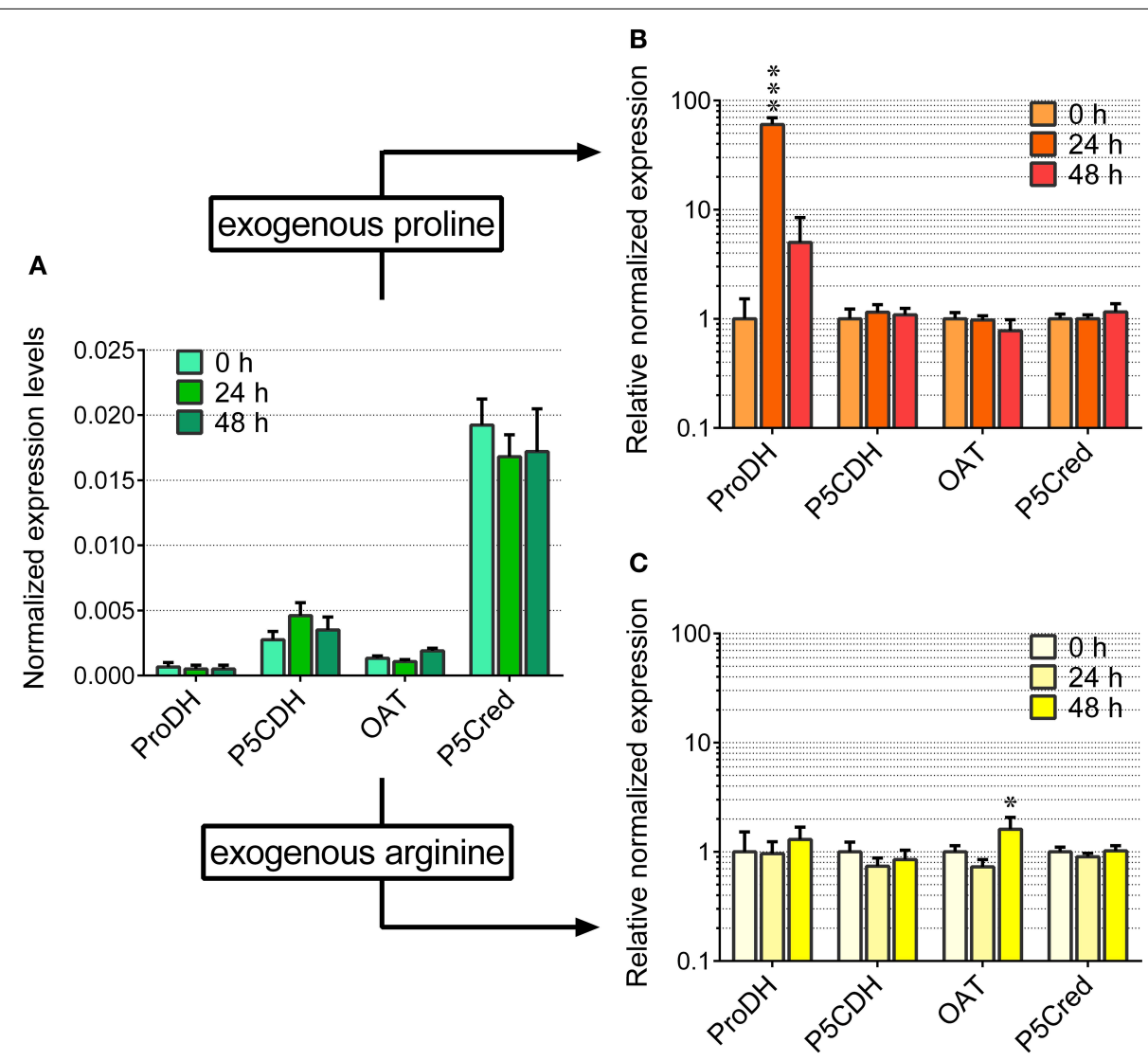

FIGURE 5 | Transcript levels of the genes involved in proline and arginine catabolism in arginine- and proline-fed rice cells. The expression of the genes involved in proline and ornithine oxidation to glutamate was quantified in suspension cultured cells of rice ( $C V$ Loto) by real-time PCR following reverse transcription. Levels were normalized with respect to Actin 1 cDNA (A). The same experiment was performed with cells treated with either $2 \mathrm{mM}$ proline (B) or $2 \mathrm{mM}$ arginine (C), harvested at increasing time after the addition of the exogenous amino acid. Expression levels were in this case normalized with respect to those in untreated controls. All treatments were carried out in triplicate, and means \pm SE are reported. Results were subjected to analysis of variance, taking into account the Bonferroni correction for multiple testing; *, ${ }^{* * *}$ : significantly different at the $10 \%$ and $1 \%$ level, respectively, from time 0 (A) or untreated controls $(\mathbf{B}, \mathbf{C})$. back to control levels. Notwithstanding this, the mRNA levels of $\mathrm{OsP} 5 \mathrm{CDH}$ were unaffected at both analyzed time points (Figure 5B).

Since P5C dehydrogenase is potentially involved also in arginine catabolism, the same approach was carried out with exogenous arginine. In this case amino acid uptake proceeded more slowly, with significant levels of the amino acid still present in the culture medium 2 days after the treatment with $5 \mathrm{mM}$ arginine (Figure 4B). Consistently, intracellular concentrations of free arginine increased more slowly, and reached lower values (Figure 4F). In this case the increase of total free amino acids exceeded that of arginine alone (Figure 4D vs. Figure 4F), suggesting the rapid conversion of arginine into other amino acids. Strikingly, no significantly higher levels of ornithine, the immediate degradation product of arginine, were found in cell extracts (data not presented), although OAT transcript levels did not show remarkable induction, with only a two-fold increase $48 \mathrm{~h}$ after arginine feeding (Figure 5C). Again, also transcript levels of $\mathrm{OsP} 5 \mathrm{CDH}$ remained largely constant, indicating that enhanced conversion of arginine into other amino acids does not require transcriptional up-regulation of either $O A T$ or $\mathrm{OsP} 5 \mathrm{CDH}$.

\section{Cloning, Heterologous Expression in E. coli and Affinity Purification of Rice P5C Dehydrogenase}

The cDNA of the only recognizable gene coding for $\mathrm{P} 5 \mathrm{C}$ dehydrogenase in the japonica rice genome, OsP5CDH, was subcloned into the expression vector pET151. Because the $\mathrm{OsP} 5 \mathrm{CDH}$ open reading frame codes for a pre-protein including a mitochondrial transit peptide, two constructs were prepared producing the full-length pre-protein or a version truncated by 16 amino acids at the $\mathrm{N}$-terminus, corresponding to the predicted transit peptide (Supplementary Figure 1A). Both constructs yielded strong inducible expression of rice P5C dehydrogenase in E. coli (Figure 6A, lanes 2 and 4). However, most of the recombinant protein was sequestered in inclusion bodies, and the corresponding protein band was barely detectable in the soluble protein fraction (Figure 6B, lane 5). As a consequence, following affinity chromatography the final yield was quite low, with an average of about $390 \mu \mathrm{g}$ protein ( $\mathrm{g}$ of induced cells) ${ }^{-1}$ for the 


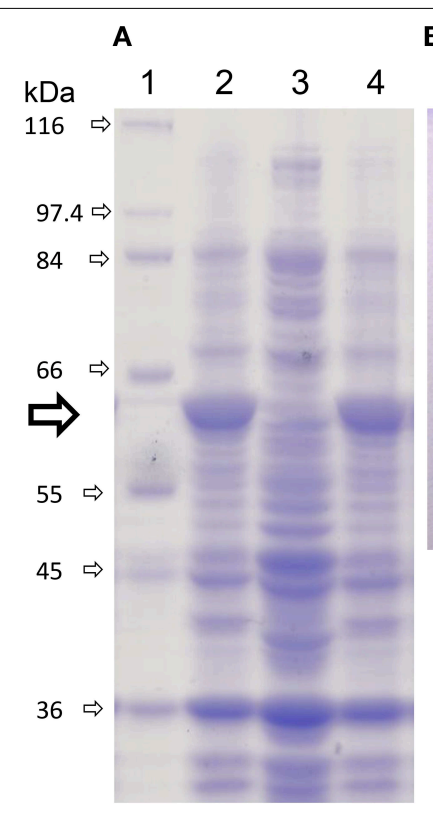

B

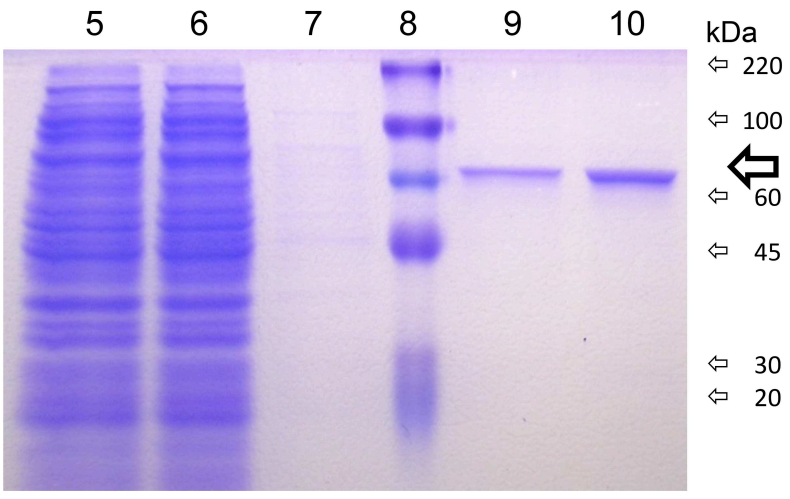

$1 \& 8$, molecular weight markers

3 , total protein from non-induced cells

$2 \& 4$, total protein from induced cells

5 , soluble protein from induced cells

6 , column flow-through

7 , column washing with $25 \mathrm{mM}$ imidazole

$9 \& 10$, elution with 50 and $100 \mathrm{mM}$ imidazole, respectively

FIGURE 6 | Expression of rice P5C dehydrogenase in E. coli and affinity purification. A truncated version of rice $\mathrm{P} 5 \mathrm{C}$ dehydrogenase lacking the predicted mitochondrial transit peptide (Supplementary Figure 1) was expressed in E. coli, strain BL21 DE3 pLysS. Cells were analyzed by
SDS-PAGE before and after the induction with $1 \mathrm{mM}$ IPTG (A). Six hours after IPTG addition, cells were harvested and extracted, and the plant protein $(\Rightarrow)$ was purified by affinity chromatography on a His-Select ${ }^{\mathrm{TM}}$ Nickel Gel column (B). The molecular mass of protein standards is indicated. truncated version, and only $26 \mu \mathrm{g}$ protein ( $\mathrm{g}$ of induced cells) ${ }^{-1}$ for the non-truncated form of $\mathrm{P} 5 \mathrm{C}$ dehydrogenase. The latter was found catalytically active, with an activity rate corresponding to about $32 \%$ of that of the protein without the predicted transit peptide. However, only the truncated protein was fully characterized. Identification of peptic and tryptic peptides by LCMS/MS analysis confirmed that the purified protein corresponds to the deduced amino acid sequence of the pET151-OsP5CDH expression construct (Supplementary Figure 1B).

The presence of the $\mathrm{His}_{6}$-tag did not affect the enzymatic activity, since virtually identical results were obtained in all experiments before and after the cleavage of the purified protein with TEV protease. Enzyme preparations were relatively stable at low temperatures; if sterilized by filtration $(0.22 \mu \mathrm{m}$ pore size), more than $70 \%$ of activity was retained after 1-month storage at $4^{\circ} \mathrm{C}$.

\section{Functional and Structural Characterization of Rice P5C Dehydrogenase}

The recombinant enzyme was thoroughly characterized with respect to functional and structural properties (Table 2). Interestingly, maximal activity was found at $\mathrm{pH} \mathrm{6.72,} \mathrm{an}$ unusual feature for an enzyme that is functionally located in the mitochondrial matrix. However, the curve for the $\mathrm{pH}$ dependence of the specific activity was much better fitted as a superimposition of two Gaussian distribution curves with maxima at $\mathrm{pH} 6.70$ and $\mathrm{pH} 7.79$ than by a curve with a single maximum (Figure 7C). An isoelectric point of 5.60 was experimentally determined, in good agreement with that calculated in silico (5.84). The inclusion of $\mathrm{Mg}^{2+}$ ions into
TABLE 2 | Properties of rice P5C dehydrogenase.
Denatured molecular mass (by SDS-PAGE)

Native molecular mass (by gel permeation)

Isoelectric point

pH optimum

Temperature optimum

Activation energy

$\mathrm{V}_{\max \left(\mathrm{NAD}^{+}\right)}$

$\mathrm{V}_{\max }\left(\mathrm{P} 5 \mathrm{C}\right.$, with $\mathrm{NAD}^{+}$as the co-substrate)

$\mathrm{V}_{\max }\left(\mathrm{NADP}^{+}\right)$

$\mathrm{V}_{\text {max }}\left(\mathrm{P} 5 \mathrm{C}\right.$, with $\mathrm{NADP}^{+}$as the co-substrate)

$\mathrm{K}_{\text {cat }}\left(\mathrm{NAD}^{+}\right)$

$\mathrm{K}_{\text {cat }}\left(\mathrm{NADP}^{+}\right)$

$\mathrm{K}_{\mathrm{M}(\mathrm{app})}$ for L-P5C $\left.{ }_{(\mathrm{NAD}+}{ }\right)$

$\mathrm{K}_{\mathrm{M}(\mathrm{app})}$ for L-P5C ${ }_{(\mathrm{NADP}+)}$

$\mathrm{K}_{\mathrm{M}(\mathrm{app})}$ for $\mathrm{NAD}^{+}$

$\mathrm{K}_{\mathrm{M}(\mathrm{app})}$ for $\mathrm{NADP}^{+}$

$$
\begin{gathered}
58.2 \pm 1.3 \mathrm{kDa} \\
259 \pm 14 \mathrm{kDa} \\
5.60 \pm 0.05 \\
6.72 \\
46 \pm 1^{\circ} \mathrm{C}
\end{gathered}
$$

$54.6 \pm 3.7{\text { kjoules } \mathrm{mol}^{-1}}^{-1}$

$196.9 \pm 2.1$ nkat (mg protein) $)^{-1}$

$207.7 \pm 3.3$ nkat $\left(\mathrm{mg}\right.$ protein) ${ }^{-1}$

$49.2 \pm 1.7$ nkat (mg protein) $)^{-1}$

$51.0 \pm 1.0$ nkat (mg protein) $)^{-1}$

$12 s^{-1}$

$3 s^{-1}$

$358 \pm 14 \mu \mathrm{M}$

$265 \pm 13 \mu \mathrm{M}$

$644 \pm 30 \mu \mathrm{M}$

$4695 \pm 462 \mu \mathrm{M}$ the reaction mixture at millimolar concentrations only slightly influenced enzyme activity, with a mild stimulation when $\mathrm{NADP}^{+}$was used as the electron acceptor, and a slight inhibition if $\mathrm{NAD}^{+}$was the co-factor (Figure 7D).

\section{Rice P5C Dehydrogenase Can Use either NAD $\mathrm{NADP}^{+}$as the Electron Acceptor, but its Properties Suggest a Preferential Use of $\mathrm{NAD}^{+}$in Vivo}

The maximal specific activity of the recombinant enzyme depended on the electron acceptor used. With $\mathrm{NADP}^{+}$, a $\mathrm{V}_{\max }$ 

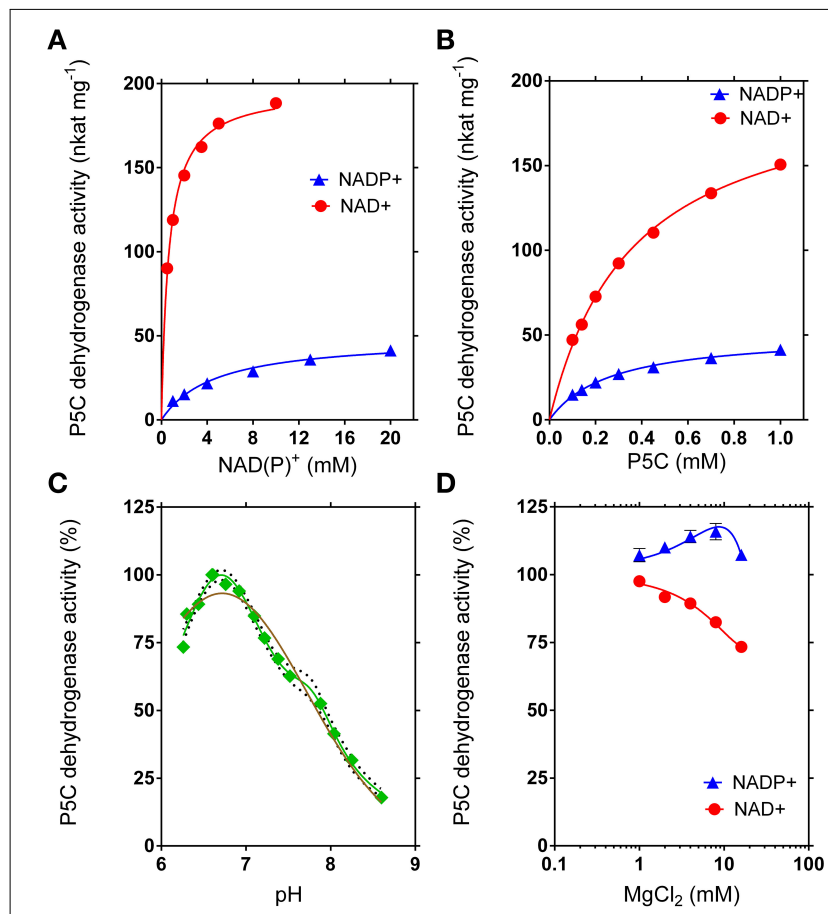

FIGURE 7 | Kinetic features of rice P5C dehydrogenase. The activity of purified recombinant rice $\mathrm{P} 5 \mathrm{C}$ dehydrogenase $(0.5 \mu \mathrm{g})$ was measured for up to $30 \mathrm{~min}$ at $35^{\circ} \mathrm{C}$ in the presence of increasing concentrations of $\mathrm{NAD}(\mathrm{P})^{+}(\mathbf{A})$ or L-P5C (B). Invariable substrates were fixed at the same levels as in the standard assay. Plotting of data from Michaelis-Menten graphs into the Lineweaver-Burk double reciprocal plots allowed the calculation of affinity constants and $\mathrm{V}_{\max }$ values for the $\mathrm{NAD}^{+}$- and the $\mathrm{NADP}^{+}$-dependent reaction (Table 2). The $\mathrm{pH}$-dependence of enzyme activity was determined using $\mathrm{NAD}^{+}$as the electron acceptor (C). Better fiting of the experimental results was obtained assuming the sum of two gaussian distributions (green line $r^{2}=0.9834$ ) than a single gaussian (brown line; $\left.r^{2}=0.9504\right)$. The $95 \%$-confidence intervals of the adopted interpolating curve are indicated by a dotted line. The effect of $\mathrm{Mg}^{2+}$ ions in the range from 1 to $10 \mathrm{mM}$ was evaluated by adding $\mathrm{MgCl}_{2}$ to the standard reaction mixture (D). In all cases, at least three replicates were carried out for each treatment, and mean values \pm SE are presented.

of about 50 nkat (mg protein $)^{-1}$ was found, corresponding to a catalytic constant of only 3 catalytic events $\mathrm{s}^{-1}$ per monomer (Table 2). With $\mathrm{NAD}^{+}$instead of $\mathrm{NADP}^{+}$, a remarkably higher $\mathrm{V}_{\max }$ value was obtained, corresponding to about 12 catalytic events $s^{-1}$ for a single subunit. The use of either cofactor did not significantly change the affinity for the specific substrate, P5C, with $K_{\mathrm{M}}$ values of about $0.3 \mathrm{mM}$ (Figure 7B). However, the affinity for $\mathrm{NADP}^{+}$was strikingly lower than that for $\mathrm{NAD}^{+}$(apparent $K_{\mathrm{M}}$ values of $0.6 \mathrm{mM}$ and $5 \mathrm{mM}$ for $\mathrm{NAD}^{+}$or $\mathrm{NADP}^{+}$, respectively) (Figure 7A). Taking all these characteristics together, results clearly suggest that $\mathrm{NAD}^{+}$most likely represents the preferred, if not the only co-factor used under in vivo conditions inside mitochondria.

\section{Contrary to the Bacterial Protein, the Native P5C Dehydrogenase from Rice is a Homotetramer}

Recent data suggested that bacterial P5C dehydrogenases from Thermus thermophilus and Deinococcus radiodurans form trimer-of-dimers hexamers in solution, whereas the enzyme from Bacillus spp. forms dimers but does not assemble into higherorder oligomers (Luo et al., 2013). To verify whether the same may apply to rice P5C deydrogenase, its relative molecular mass was determined by gel filtration chromatography. Results were consistent with a native mass of $259 \pm 14 \mathrm{kDa}$ (Figure 8). The same pattern was obtained at different ionic strengths of the eluent in the range from $250 \mathrm{mM}$ to $1 \mathrm{M} \mathrm{NaCl}$, suggesting that the rice protein consists of 4 identical subunits, and does not disassemble into dimers.

\section{P5C Dehydrogenase is Progressively Inactivated by Treatment at Temperatures above $40^{\circ} \mathrm{C}$, Showing a Biphasic Profile of Thermal Inactivation}

When the purified P5C dehydrogenase was assayed as a function of the temperature, maximal initial activity was obtained at a temperature as low as $46^{\circ} \mathrm{C}$ (Figure 9A). The corresponding activation energy, calculated by the Arrhenius plot (Figure 9B), was $54.6 \pm 3.7 \mathrm{~kJ} \mathrm{~mol}^{-1}$. Moreover, the prolonged incubation of the rice protein at temperatures in the 40 to $50^{\circ} \mathrm{C}$ range in the absence of its substrates caused a dramatic and rapid loss of activity (Figure 9C). A treatment at $47.5^{\circ} \mathrm{C}$ for $5 \mathrm{~min}$ resulted in a 50\%-inactivation of the enzyme. Inactivation curves did not follow the conventional one-phase exponential decay. The best fit of data was obtained assuming a two phase-decay with a very short (fast) and a moderate (slow) half-life. At $40^{\circ} \mathrm{C}$, halflife (fast) and half-life (slow) were $5.2 \pm 0.9 \mathrm{~min}$ and $507 \pm$ $219 \mathrm{~min}$, whereas at $45^{\circ} \mathrm{C}$ they were $1.8 \pm 0.6 \mathrm{~min}$ and $49 \pm$ 4 min, respectively.

\section{P5C Dehydrogenase Activity is Slightly Sensitive to Anions, whereas a Variety of Cations are Inhibitory and Appear to Modulate the Catalytic Rate at Physiological Concentrations}

Since P5C dehydrogenase from potato cultured cells had been found to be inhibited by $\mathrm{NaCl}$ concentrations above $100 \mathrm{mM}$ (Forlani et al., 1997a), the possibility that high solute levels may reduce the activity of $\mathrm{P} 5 \mathrm{C}$ dehydrogenase (and proline oxidation in turn) was considered. Because P5C neutralization and reaction mixture buffering may increase both anion and cation concentration in the assay environment, assay conditions were modified to limit the carry-over of ions. Under these conditions, the addition of non-ionic solutes up to $1 \mathrm{M}$ to the reaction mixture was ineffective (Figure 10E). Anions exerted quite slight effects, and only bicarbonate and nitrate (but not chloride) were inhibitory at concentrations lower than $200 \mathrm{mM}$ (Figure 10D). Interestingly, cations were much more potent inhibitors of P5C dehydrogenase. $\mathrm{Li}^{+}$and $\mathrm{Na}^{+}$progressively inhibited enzyme activity above $10 \mathrm{mM}$, whereas $\mathrm{K}^{+}$and $\mathrm{NH}_{4}^{+}$ were ineffective below $200 \mathrm{mM}$ (Figure 10A). Divalent cations, also not considering the non-physiological $\mathrm{Be}^{2+}$ ions, were even more inhibitory, with a $32 \%$ reduction of the catalytic rate by $\mathrm{Mg}^{2+}$ at a concentration of $10 \mathrm{mM}$ (Figure 10B). Very similar data were obtained when using $\mathrm{NADP}^{+}$instead of $\mathrm{NAD}^{+}$as the electron acceptor (not shown).

Interestingly, when the effect of a variety of other divalent cations was investigated, most were found to severely affect the 


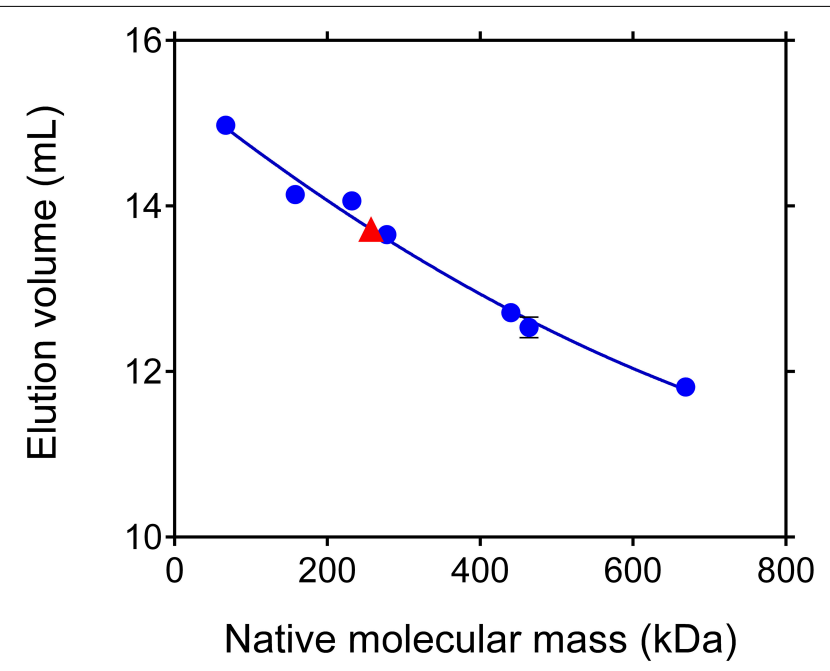

FIGURE 8 | Apparent molecular mass of rice P5C dehydrogenase under native conditions. Aliquots $(100 \mu \mathrm{L})$ of purified recombinant rice $\mathrm{P} 5 \mathrm{C}$ dehydrogenase, adjusted to $0.5 \mathrm{mg} \mathrm{mL}^{-1}$, were subjected to gel permeation chromatography on a Superose 12 HR 10/30 (Pharmacia) column that had been equilibrated with $50 \mathrm{mM}$ Tris- $\mathrm{HCl}$ buffer, $\mathrm{pH} 7.75$, containing $250 \mathrm{mM}$ $\mathrm{NaCl}$. A molecular mass of $259 \pm 14 \mathrm{kDa}$ was estimated for P5C dehydrogenase, suggesting a homotetrameric composition of the enzyme. Molecular weight markers used (Pharmacia 17-0442-01 and 17-0441-01) are specified in Materials and Methods.

activity of rice $\mathrm{P} 5 \mathrm{C}$ dehydrogenase (Figure 10C). Some of them, as $\mathrm{Cd}^{2+}\left(\mathrm{IC}_{50}\right.$ of $\left.2.2 \times 10^{-7} \mathrm{M}\right)$, may owe this ability to their oxidative properties, or are not of physiological significance. However, for others it does not seem the case, as they are normally present inside the plant cell and play a relevant role as micro-nutrients. Mainly for $\mathrm{Zn}^{2+}\left(\mathrm{IC}_{50}\right.$ of $\left.1.5 \times 10^{-6} \mathrm{M}\right), \mathrm{Mn}^{2+}$ $\left(\mathrm{IC}_{50}\right.$ of $\left.2.4 \times 10^{-6} \mathrm{M}\right)$, as well as $\mathrm{Cu}^{2+}\left(\mathrm{IC}_{50}\right.$ of $\left.4.0 \times 10^{-6} \mathrm{M}\right)$, the possibility therefore exists that local concentrations in the mitochondria may be high enough to influence the catalytic rate of the enzyme.

\section{Arginine Inhibits P5C Dehydrogenase Activity with a Mechanism of Uncompetitive Type with Respect to P5C}

To evaluate whether the rice enzyme may be subjected to further post-translational regulative mechanisms, the activity of the purified protein was measured in the presence of increasing concentrations of proline, arginine, ornithine or glutamic acid. Since enzyme activity is strongly influenced by the $\mathrm{pH}$ in the 7 to 9 range (Figure 7C), special attention was paid to rule out the possibility that changes in the catalytic rate may depend on $\mathrm{pH}$ fluctuation induced by the addition of a given amino acid to the reaction mixture. Glutamate, proline and ornithine were substantially ineffective at concentrations ranging from 1 to $100 \mathrm{mM}$ (data not presented). On the contrary, arginine was found to progressively inhibit the enzyme, with an $\mathrm{IC}_{50}$ value of $87 \pm 11 \mathrm{mM}$. Citrulline was also inhibitory, but to a lower extent (not shown). Interestingly, a kinetic analysis showed that the inhibition by arginine is of non-competitive type with

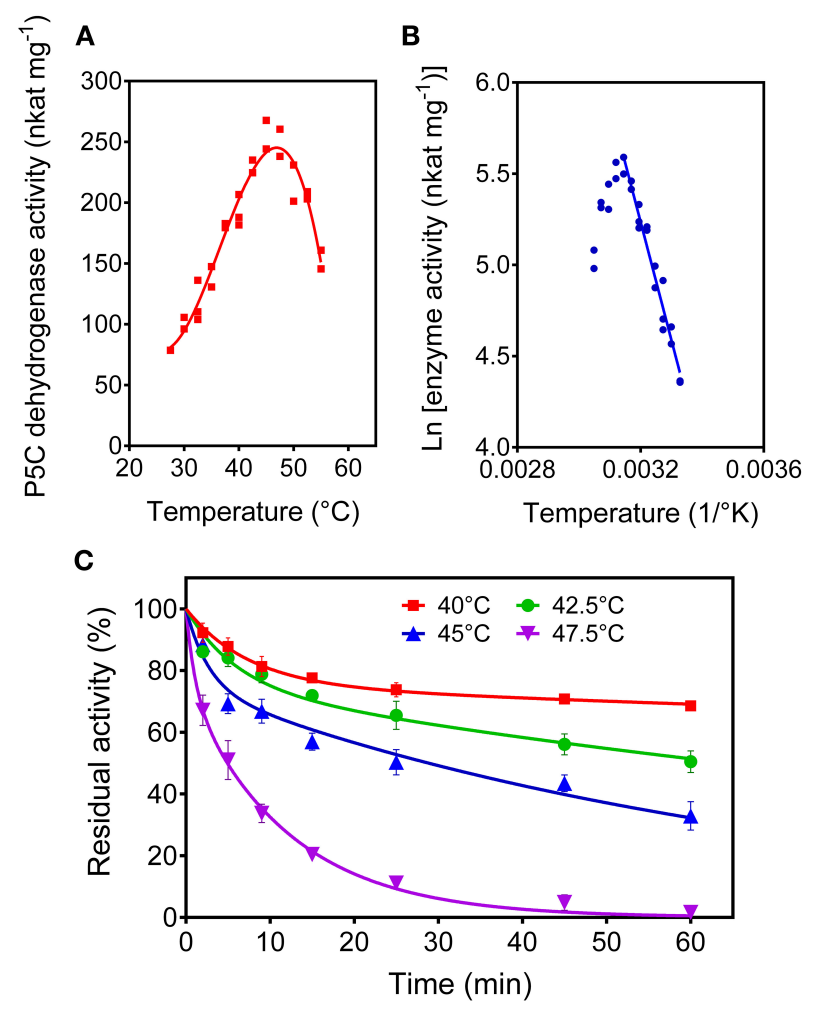

FIGURE 9 | Thermal stability of rice P5C dehydrogenase. The activity rate of the purified enzyme was measured for up to 5 min under standard assay conditions at increasing temperatures (A). Replotting data in the so-called Arrhenius plot (B) allowed the calculation of the activation energy (Table 2). Thermal stability of the enzyme was determined by incubating aliquots for increasing time at increasing temperature in the absence of substrates (C). After the indicated times, the aliquots were immediately re-equilibrated on ice and the residual activity was then measured at $35^{\circ} \mathrm{C}$, and expressed as percentage of activity in untreated controls. Three replicates were carried out for each treatment, and means \pm SE over replicates are shown.

respect to $\mathrm{NAD}^{+}$, but of uncompetitive type with respect to P5C (Figure 11). The latter feature, quite uncommon, implies that in the presence of millimolar levels of arginine the $\mathrm{K}_{M}$ of P5C dehydrogenase for its specific substrate would be lowered, enhancing the binding of $\mathrm{P} 5 \mathrm{C}$ even in the presence of low concentrations of the substrate.

\section{Discussion}

\section{Rice P5C Dehydrogenase is Constitutively Expressed and High Intracellular Levels of Proline Increase only Slightly its mRNA Abundance, whereas its Transcript Level Seems not Subject to Environmental Control}

Proline oxidation to glutamate takes place in the mitochondrion by means of a short pathway in which a membrane-bound proline dehydrogenase converts proline into $\mathrm{P} 5 \mathrm{C}$, which in turn is further oxidized by a soluble P5C dehydrogenase (Forlani et al., 1997a). P5C can be alternatively produced from 

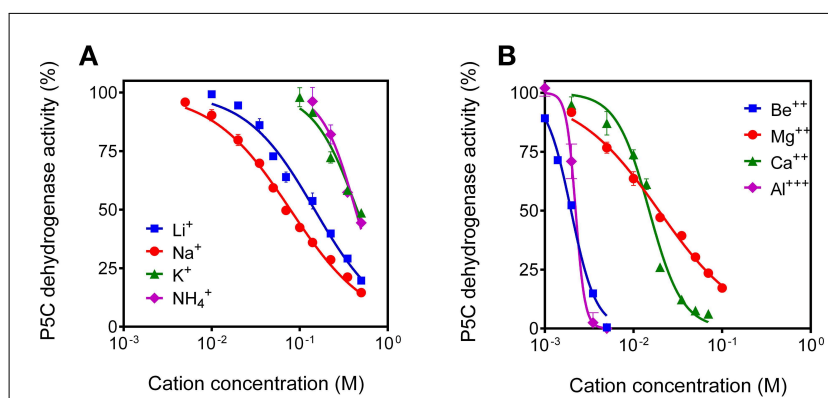

C

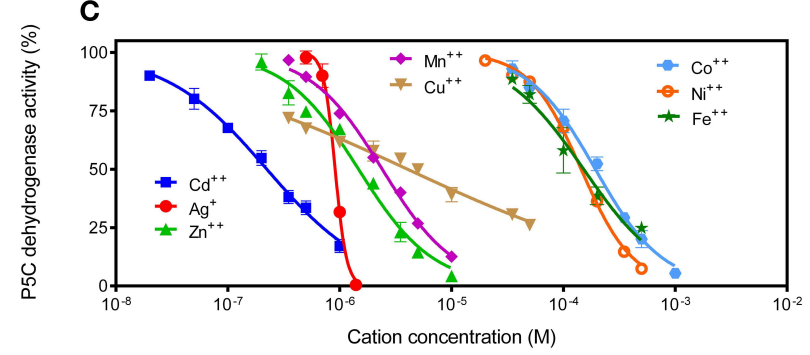

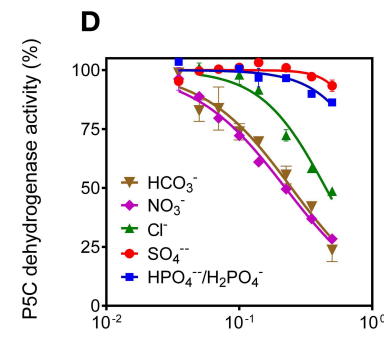

Anion concentration $(\mathrm{M})$

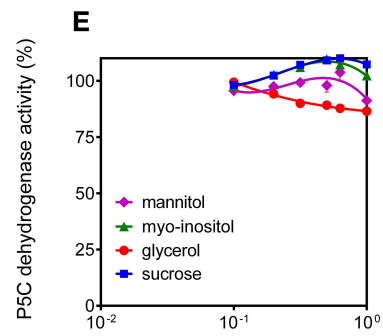

Non-ionic solute concentration (M)
FIGURE 10 | Effect of anions and cations on the activity of P5C dehydrogenase. The effects of the addition of increasing concentrations of monovalent cations (A), divalent cations (B), heavy metals (C), various anions (D), and non-ionic solutes (E) to the reaction mixture were assessed using $\mathrm{NAD}^{+}$as the electron acceptor. Cations were added as chlorides, with the exception of $\mathrm{Ca}^{2+}, \mathrm{Mg}^{2+}, \mathrm{Be}^{2+}, \mathrm{Cu}^{2+}, \mathrm{Zn}^{2+}$, and $\mathrm{Fe}^{2+}$, which were added as sulfates. Anions were added as potassium salts. To minimize the carry-over of chloride anions, L-P5C concentration was reduced to $250 \mu \mathrm{M}$, and the P5C solution was neutralized just before use with $1 \mathrm{M} \mathrm{NH}_{3}$. Additionally, Hepes buffer was used at $12.5 \mathrm{mM}$ to reduce the presence of potassium cations in the standard reaction mixture. In all cases, three replicates were carried out for each treatment. Data were expressed as percent of untreated controls, and mean values \pm SE are presented.

ornithine by OAT, thus P5C dehydrogenase is involved also in arginine catabolism (Winter et al., 2015). Export of P5C from mitochondria in a proline-P5C cycle has also been postulated (Miller et al., 2009). In this case, the balance between export and $\mathrm{P} 5 \mathrm{C}$ dehydrogenase activity would be decisive about the further fate of organic nitrogen and carbon derived from proline and arginine catabolism. Although P5C dehydrogenase is usually considered as non-rate-limiting in proline and arginine catabolism, its fine control may be important to balance the two pathways and to determine the utilization of $\mathrm{P} 5 \mathrm{C}$. Very little is known about the occurrence of post-translational regulatory mechanisms for plant $\mathrm{P} 5 \mathrm{C}$ dehydrogenases, whereas some studies described fluctuations of transcript levels in response to either exogenous proline, with maximal expression $48 \mathrm{~h}$ after the treatment (Deuschle et al., 2001), or to ABA

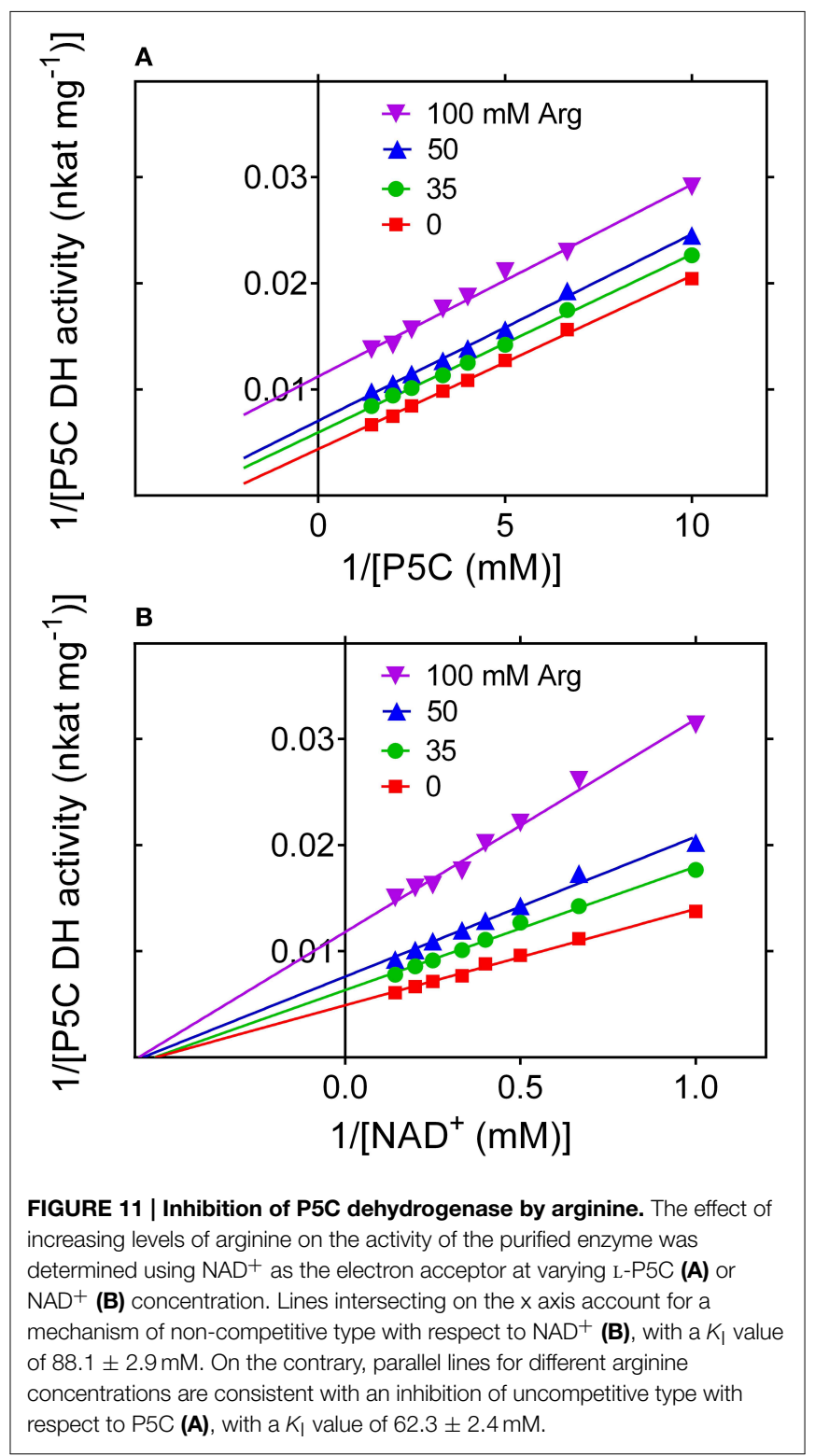

treatment and hyperosmotic stress conditions induced by PEG (but not by salt; Gao and Han, 2009). However, in the latter case a 2-fold induction of the transcript level was in apparent contrast with the need to accumulate proline to withstand stress conditions. In all cases, intracellular free proline content had not been determined and related to expression levels.

The results obtained in the present study showed the presence of transcripts of $\mathrm{Os} P \mathrm{CDDH}$, the apparently only gene coding for a $\mathrm{P} 5 \mathrm{C}$ dehydrogenase in rice, in cell cultures under all the conditions tested. OsP5CDH transcript levels were generally low in comparison to those of the anabolic P5C reductase and $P 5 C$ synthetase 2, and no significant variations were found in response to either salt or drought stress, ABA treatment or exogenous administration of proline (or arginine), despite significant fluctuations of endogenous free 
proline (or arginine) levels during some of these treatments. Merely $24 \mathrm{~h}$ after the exposure to high PEG concentrations, when proline content had already doubled over control levels, a two-fold increase of the $\mathrm{OsP} 5 \mathrm{CDH}$ transcript level was evident. Interestingly, also the relative normalized expression of the genes coding for the enzymes involved in proline biosynthesis did not show remarkable variations in response to PEG treatments, suggesting that some fluctuation of free proline content may arise without the need of transcriptional control. Also the detailed characterization of $\mathrm{P} 5 \mathrm{C}$ reductase activity patterns recently suggested post-translational control of fluxes in proline metabolism (Giberti et al., 2014; Forlani et al., 2015). Nevertheless, normalized expression levels of Proline dehydrogenase were found almost undetectable in controls, but showed a strong increase in response to exogenous proline. On the contrary, OAT transcript levels were similarly low under control conditions but were not enhanced by treatment with exogenous arginine. On the whole, it seems therefore that P5C dehydrogenase is expressed constitutively at levels high enough to allow the utilization of even highest concentrations of P5C generated by proline and arginine degradation. However, in this overall picture it is unclear which may be the role of P5C dehydrogenase in actively proliferating cells under normoosmotic conditions, when proline dehydrogenase is virtually unexpressed and arginine accounts for less than $0.5 \%$ of total free amino acids. In several cereals, the expression of the genes coding for P5C dehydrogenase was almost undetectable in leaves, but showed hundredfold induction following the infection with a compatible rust strain (Ayliffe et al., 2002). On the contrary, a pathogen-induced early induction of Proline dehydrogenase transcript levels in $A$. thaliana was accompanied by an increase in P5C reductase, but not in AtP5CDH transcripts (Cecchini et al., 2011). These apparently inconsistent data may reflect differences among plant species, or depend on the interaction with specific pathogens. In any case, the results obtained by RT-PCR for $O s P 5 C D H$ expression levels in rice cell cultures are in agreement with previous Northern blot data on $A$. thaliana seedlings (Deuschle et al., 2001) and Western blot analysis of potato tissues (Forlani et al., 2000). In the latter study, protein levels were also suggestive of a developmental, but not of an environmental control of $\mathrm{P} 5 \mathrm{C}$ dehydrogenase expression in response to abiotic stress.

Taken together, all these elements could imply that an inverse transcriptional control of proline dehydrogenase and $\mathrm{P} 5 \mathrm{C}$ synthetase is sufficient to determine the suitable intracellular concentration of proline. However, it has been shown that in A. thaliana the need to limit proline oxidation under salt and drought stress conditions requires an at least partial silencing of AtP5CDH expression via naturally occurring siRNAs derived from induction of the overlapping SRO5 gene (Borsani et al., 2005). Nevertheless, this does not seem to be a universal system, since no genes partially overlapping with $\mathrm{OsP} 5 \mathrm{CDH}$ are annotated in rice, and a BLAST search with the A. thaliana SRO5 (either nucleotide or amino acid) sequence did not show the presence of any ortholog ( $E$-value $>0.2$ in BLAST-N) in the rice genome. Even in the absence of control mechanisms at both the transcriptional and the translational level, a modulation of the rate of $\mathrm{P} 5 \mathrm{C}$ oxidation may derive from post-translational regulation of enzyme activity.

\section{Rice P5C Dehydrogenase is a Quite Labile, Homotetrameric Enzyme with a Low Apparent Catalytic Efficiency and a Clear-cut Preference for $\mathrm{NAD}^{+}$as the Electron Acceptor}

The assessment of post-translational regulation of $\mathrm{P} 5 \mathrm{C}$ dehydrogenase activity has been hampered to date by a substantial lack of information about the biochemical properties of the enzyme from higher plants. In early studies, enzyme activity was detected in crude mitochondrial extracts, and a preliminary characterization carried out with partially purified preparations pointed out a preference for $\mathrm{NAD}^{+}$over $\mathrm{NADP}^{+}$ as the electron acceptor and $K_{\mathrm{M}}$ values in the micromolar to millimolar range for both substrates (Stewart and Lai, 1974; Boggess et al., 1975). So far, the plant enzyme has been purified and kinetically characterized only from potato (Forlani et al., 1997a). The results obtained with the recombinant rice P5C dehydrogenase provide some confirmation, and add important new information on activity modulation according to the physiological status of the cell. Similarly to the potato enzyme, rice $\mathrm{P} 5 \mathrm{C}$ dehydrogenase showed affinity constants between $10^{-4} \mathrm{M}$ and $10^{-3} \mathrm{M}$ for both $\mathrm{P} 5 \mathrm{C}$ and $\mathrm{NAD}^{+}$, i.e., values very close to the intracellular concentrations of $\mathrm{P} 5 \mathrm{C}(10 \mathrm{nmol}$ to $20 \mathrm{nmol}$ [g fresh weigh $]^{-1}$; Forlani et al., 2013) and the preferred electron acceptor $\mathrm{NAD}^{+}\left(16 \mathrm{nmol}\right.$ [g fresh weigh] ${ }^{-1}$; Hayashi et al., 2005). This implies that inside the cell the catalytic rate may be much lower than the $\mathrm{V}_{\max }$ measured in vitro. The catalytic constant of $12 \mathrm{~s}^{-1}$ suggests that P5C dehydrogenase is an enzyme with a rather poor efficiency. However, $K_{\mathrm{M}}$ values in the range of the actual substrate concentrations facilitate the attainment of homeostatic levels, since any increase of substrate concentrations would immediately cause a corresponding increase of enzyme activity. Moreover, the evaluation of the temperature-activity relationship pointed out a poor thermal stability of $\mathrm{P} 5 \mathrm{C}$ dehydrogenase, which in the absence of its substrates was rapidly and irreversibly inactivated at temperatures exceeding $40^{\circ} \mathrm{C}$, and lost more than $25 \%$ activity in 1 month even when stored at $4{ }^{\circ} \mathrm{C}$. As a term of comparison, A. thaliana $\mathrm{P} 5 \mathrm{C}$ reductase showed a catalytic constant of about $700 \mathrm{~s}^{-1}$ (Giberti et al., 2014), and retained more than 50\% activity after 3 weeks at room temperature (Funck et al., 2012). In the presence of substrates, the effect was slightly milder, but temperatures exceeding $46^{\circ} \mathrm{C}$ rapidly led to activity loss of rice $\mathrm{P} 5 \mathrm{C}$ dehydrogenase. A protective effect of substrates against thermal inactivation has been reported for other enzymes in amino acid metabolism (e.g., Forlani, 1997). The low catalytic efficiency and fast turn-over rate of $\mathrm{P} 5 \mathrm{C}$ dehydrogenase may explain at least in part the need to maintain relatively high constitutive transcript levels.

On the other hand, size exclusion chromatography experiments clearly supported a homotetrameric composition of recombinant rice $\mathrm{P} 5 \mathrm{C}$ dehydrogenase. This confirms previous data on the potato enzyme, and indicates a different oligomeric composition of plant $\mathrm{P} 5 \mathrm{C}$ dehydrogenases compared to the corresponding bacterial enzymes. The latter have been recently shown to form dimers that do not assemble into higher-order 
oligomers, or form trimer-of-dimers hexamers in solution (Luo et al., 2013). No evidence supporting the existence of free dimers has been obtained for the rice protein, since elution patterns upon gel filtration were unaffected by the addition of up to $1 \mathrm{M} \mathrm{NaCl}$ to the eluent. The enzyme from plants seems therefore to possess a structure significantly different from either the bifunctional proline oxidase present in enterobacteria and bearing both proline- and $\mathrm{P} 5 \mathrm{C}$-dehydrogenase activities, or the monofunctional P5C dehydrogenase from archea and Gram positive eubacteria.

\section{Several Parameters, Including pH and Cation Concentrations, Regulate the Activity of Rice P5C Dehydrogenase}

Interestingly, both the presence of salts and changes of $\mathrm{pH}$ in the physiological range were found to greatly influence the catalytic rate of purified rice $\mathrm{P} 5 \mathrm{C}$ dehydrogenase. Maximal activity was shown between $\mathrm{pH} 6.6$ and $\mathrm{pH}$ 7, and a progressive loss of efficiency was evident along with the alkalinization of the reaction mixture, with about $50 \%$ activity at $\mathrm{pH} 8.0$ and almost no activity at $\mathrm{pH}$ 9.0. This activity pattern is most probably a superimposition of the intrinsic enzyme properties and the equilibrium in solution between P5C and GSA, which seems to be the actual substrate of $\mathrm{P} 5 \mathrm{C}$ dehydrogenase (Arentson et al., 2012). The GSA/P5C ratio shows a very sharp transition around $\mathrm{pH}$ 6.2, with GSA being the prevalent form in acidic conditions (Bearne and Wolfenden, 1995). The peak of P5C dehydrogenase activity between $\mathrm{pH} 6.8$ and $\mathrm{pH} 7$ may thus reflect the optimum between GSA concentration and protonation dependent inactivation of the enzyme, while the shoulder at $\mathrm{pH}$ 8 may reflect the optimal $\mathrm{pH}$ for catalysis. Recently, transient partial depolarization of the inner mitochondrial membrane has been reported to occur in $A$. thaliana in response to high temperature, $\mathrm{H}_{2} \mathrm{O}_{2}$, or cadmium treatments. Membrane potential pulses were coincident with a pronounced transient alkalinization (up to $>1 \mathrm{pH}$ unit) of the matrix (Schwarzländer et al., 2012). The authors proposed a role for pulsing as a transient uncoupling mechanism to counteract mitochondrial dysfunction and reactive oxygen species production. However, this might also reduce $\mathrm{P} 5 \mathrm{C}$ (and proline) oxidation under oxidative stress conditions, when proline may serve as a radical scavenger.

Moreover, several cations were found to inhibit rice $\mathrm{P} 5 \mathrm{C}$ dehydrogenase, whereas anions were effective only at highest, mostly unphysiological levels. Previous data accounting for a strong inhibition by chlorides (e.g., Boggess et al., 1975) could most likely be a consequence of the carry-over of $\mathrm{Cl}^{-}$ions from $\mathrm{P} 5 \mathrm{C}$ solution to the standard assay mixture. Based on our results, a salt stress-driven increase of the intracellular concentration of $\mathrm{Na}^{+}$cations from 10 to $100 \mathrm{mM}$ would result in a progressive reduction of $\mathrm{P} 5 \mathrm{C}$ oxidation, possibly contributing to proline accumulation. The same seems true for $\mathrm{Mg}^{2+}$ : since $\mathrm{Mg}^{2+}$ concentration has been found remarkably constant and low in the cytosol but tenfold higher in the mitochondrial matrix, where its fluctuation might regulate cell respiration (Gout et al., 2014), also this feature might be of physiological significance. Even more interestingly, rice P5C dehydrogenase showed severe inhibition by a wide array of divalent cations.
In some cases, cation inhibition is unlikely to occur in vivo, since ions like $\mathrm{Cd}^{2+}$ are not usually present inside living cells. In other cases, on the contrary, these effects could allow environmental factors to modulate P5C oxidation. For instance, iron, copper, and manganese have been detected at micromolar levels in higher plant mitochondria, and some evidence has been described supporting the possibility that their fluctuations may modulate the activity of several mitochondrial enzymes (Tan et al., 2010). Once again, such variations were found following in vivo and in vitro oxidative stresses, conditions that could prompt proline accumulation and therefore require modulation of the degradation pathway.

\section{High Concentrations of Free Arginine may Increase the Affinity of P5C Dehydrogenase for its Specific Substrate, Possibly Influencing the Balance between P5C Export into the Cytosol and its Oxidation to Glutamate}

The presence of amino acids of the glutamate family at physiological concentrations did not significantly influence P5C dehydrogenase activity, except for arginine. In the 10 to $100 \mathrm{mM}$ range, arginine was found to progressively inhibit the activity of the purified enzyme. Inhibition of P5C dehydrogenase by high concentrations of arginine seems inconsistent with a role of the enzyme in arginine utilization. High intracellular levels of arginine have been described in germinating seeds of various species, where arginine degradation supplies organic and $\mathrm{NH}_{4}^{+}$ nitrogen for anabolic processes (Winter et al., 2015). To allow the synthesis of nitrogen-containing compounds needed for growth, arginine is converted into urea and glutamate, the latter serving as the main donor of amino mojeties. However, the mechanism of enzyme inhibition shed some light on this apparent discrepancy. Being of uncompetitive type with respect to $\mathrm{P} 5 \mathrm{C}$, the inhibition brought about by arginine would be most effective in the presence of saturating levels of $\mathrm{P} 5 \mathrm{C}$, which on the contrary is maintained at very low levels inside the cell to avoid cytotoxic effects (Deuschle et al., 2004). In the presence of limiting P5C concentrations, the interaction with arginine results in a higher affinity for P5C. For instance, in the presence of 35 or $50 \mathrm{mM}$ arginine, the $\mathrm{K}_{\mathrm{M}(\mathrm{app})}$ for $\mathrm{L}-\mathrm{P} 5 \mathrm{C}\left(\mathrm{NAD}^{+}\right)$is reduced from $358 \mu \mathrm{M}$ (Table 2) to 270 and $235 \mu \mathrm{M}$, respectively. Since P5C levels of no more than $10-20 \mathrm{nmol}$ ( $\mathrm{g}$ fresh weigh $)^{-1}$ have been found in plant cell cultures (Forlani et al., 2013), this would result in a stimulation of $\mathrm{P} 5 \mathrm{C}$ oxidation to glutamate over its transfer into the cytosol to fuel the $\mathrm{P} 5 \mathrm{C} /$ proline cycle. Further data will be required to obtain conclusive evidence supporting the occurrence of this mechanism in vivo. However, because of the structural difference between arginine and $\mathrm{P} 5 \mathrm{C}$, and the lack of a similar effect in the case of ornithine, it seems unlikely that the presence of allosteric effects of arginine (and citrulline, although to a lower extent) on P5C dehydrogenase activity may be purely casual.

The occurrence of two enzyme forms, as reported for $N$. plumbaginifolia P5C dehydrogenase (Forlani et al., 1997b), would provide an efficient way to regulate separately arginine and proline catabolism. The discrimination between proline- and arginine-derived $\mathrm{P} 5 \mathrm{C}$ could in this case be achieved in vivo by protein-protein interactions, with $\mathrm{P} 5 \mathrm{C}$ dehydrogenases being 
associated with either proline dehydrogenase or OAT (Elthon and Stewart, 1982). However, in several if not in most diploid plant species only a single gene seems to exist, and so far no direct evidence for different splicing variants or protein modifications was reported (e.g., Deuschle et al., 2001). As a consequence, multiple regulative mechanisms are required to modulate the activity of a single P5C dehydrogenase in response to either the activation of proline or arginine catabolism, or the need to limit proline oxidation to cope with abiotic stress conditions.

The results described in this work indicate that $\mathrm{P} 5 \mathrm{C}$ dehydrogenase activity might be highly sensitive to metabolite or ion concentration changes in the micro-environment of the enzyme, and may well have a regulatory impact on the rates or routes of $\mathrm{P} 5 \mathrm{C}$ metabolism. For instance, during seed germination, the hydrolysis of arginine-rich seed proteins increases free arginine in the cell to millimolar levels: this in turn would increase the affinity of P5C dehydrogenase to P5C, favoring its oxidation to glutamate. On the other hand, under either oxidative or salt stress conditions, increased mitochondrial levels of sodium ions and heavy metals might limit P5C oxidation, possibly facilitating the P5C/Pro cycle (Miller et al., 2009). Conversely, their lowering to homeostatic concentrations would favor proline oxidation under the subsequent recovery

\section{References}

Arentson, B. W., Sanyal, N., and Becker, D. F. (2012). Substrate channeling in proline metabolism. Front. Biosci. 17, 375-388. doi: 10.2741/3932

Ayliffe, M. A., Mitchell, H. J., Deuschle, K., and Pryor, A. J. (2005). Comparative analysis in cereals of a key proline catabolism gene. Mol. Gen. Genomics 274, 494-505. doi: 10.1007/s00438-005-0048-x

Ayliffe, M. A., Roberts, J. K., Mitchell, H. J., Zhang, R., Lawrence, G. J., Ellis, J. G., et al. (2002). A plant gene up-regulated at rust infection sites. Plant Physiol. 129, 169-180. doi: 10.1104/pp.010940

Bearne, S. L., and Wolfenden, R. (1995). Glutamate $\gamma$-semialdehyde as a natural transition state analogue inhibitor of Escherichia coli glucosamine-6-phosphate synthase. Biochemistry 34, 11515-11520. doi: 10.1021/bi00036a026

Boggess, S. F., Paleg, L. G., and Aspinall, D. (1975). $\Delta^{1}$-Pyrroline-5-carboxylic acid dehydrogenase in barley, a proline-accumulating species. Plant Physiol. 56, 259-262. doi: 10.1104/pp.56.2.259

Borsani, O., Zhu, J., Verslues, P. E., Sunkar, R., and Zhu, J. K. (2005). Endogenous siRNAs derived from a pair of natural cis-antisense transcripts regulate salt tolerance in Arabidopsis. Cell 123, 1279-1291. doi: 10.1016/j.cell.2005.11.035

Bradford, M. M. (1976). A rapid and sensitive method for the quantitation of microgram quantities of protein utilizing the principle of protein-dye binding. Anal. Biochem. 72, 248-254. doi: 10.1016/0003-2697(76)90527-3

Cecchini, N. M., Monteoliva, M. I., and Alvarez, M. E. (2011). Proline dehydrogenase contributes to pathogen defense in Arabidopsis. Plant Physiol. 155, 1947-1959. doi: 10.1104/pp.110.167163

Deuschle, K., Funck, D., Forlani, G., Stransky, H., Biehl, A., Leister, D., et al. (2004). The role of $\delta^{1}$-pyrroline-5-carboxylate dehydrogenase in proline degradation. Plant Cell 16, 3413-3425. doi: 10.1105/tpc.104.023622

Deuschle, K., Funck, D., Hellmann, H., Daeschner, K., Binder, S., and Frommer, W. B. (2001). A nuclear gene encoding mitochondrial $\delta^{1}$-pyrroline-5-carboxylate dehydrogenase and its potential role in protection from proline toxicity. Plant J. 27, 345-356. doi: 10.1046/j.1365-313X.2001.01101.x

Elthon, T. E., and Stewart, C. R. (1981). Submitochondrial location and electron transport characteristics of enzymes involved in proline oxidation. Plant Physiol. 67, 780-784. doi: 10.1104/pp.67.4.780

Elthon, T. E., and Stewart, C. R. (1982). Proline oxidation in corn mitochondria: involvement of $\mathrm{NAD}$, relationship to ornithine metabolism, and sidedness on the inner membrane. Plant Physiol. 70, 567-572. doi: 10.1104/pp.70.2.567 from stress conditions. In the next future we plan to verify our results at the plant level, on both salt stress sensitive and tolerant rice genotypes, and see whether different expression levels and/or properties of $\mathrm{P} 5 \mathrm{C}$ dehydrogenase may correlate with a differential susceptibility to excess salt.

\section{Acknowledgments}

This work was funded by AGER Foundation in the frame of the RISINNOVA project, grant \# 2010-2369. Support from the University of Konstanz is also gratefully acknowledged. MB was the recipient of a DAAD (German Academic Exchange Service) fellowship supporting a stage in DF's laboratory. The authors thank Dr. Andreas Marquardt (Proteomics Facility of the University of Konstanz) for peptide mapping of the purified protein.

\section{Supplementary Material}

The Supplementary Material for this article can be found online at: http://journal.frontiersin.org/article/10.3389/fpls.2015. 00591

Forlani, G., Berlicki, Ł., Duò, M., Dziędzioła, G., Giberti, S., Bertazzini, M., et al. (2013). Synthesis and evaluation of effective inhibitors of plant $\delta^{1}$ pyrroline-5-carboxylate reductase. J. Agric. Food Chem. 61, 6792-6798. doi: $10.1021 /$ jf401234s

Forlani, G., Bertazzini, M., and Giberti, S. (2014). Differential accumulation of $\gamma$-aminobutyric acid in elicited cells of two rice cultivars showing contrasting sensitivity to the blast pathogen. Plant Biol. 16, 1127-1132. doi: $10.1111 /$ plb. 12165

Forlani, G., Bertazzini, M., Zarattini, M., Funck, D., Ruszkowski, M., and Nocek, B. P., (2015). Functional properties and structural characterization of rice $\delta^{1}$ pyrroline-5-carboxylate reductase. Front. Plant Sci. 6:565. doi: 10.3389/fpls. 2015.00565

Forlani, G., Mangiagalli, A., Pinter, C., and Nielsen, E. (2000). Expression of $\delta^{1}$-pyrroline-5-carboxylate dehydrogenase and proline/arginine homeostasis in Solanum tuberosum. Physiol. Plant. 110, 22-27. doi: 10.1034/j.13993054.2000.110103.x

Forlani, G., Scainelli, D., and Nielsen, E. (1997a). $\Delta^{1}$-pyrroline-5carboxylate dehydrogenase from cultured cells of potato. Purification and properties. Plant Physiol. 113, 1413-1418. doi: 10.1007/s004250 050125

Forlani, G., Scainelli, D., and Nielsen, E. (1997b). Two $\delta^{1}$-pyrroline-5-carboxylate dehydrogenase isoforms are expressed in cultured Nicotiana plumbaginifolia cells and are differentially modulated during the culture growth cycle. Planta 202, 242-248. doi: 10.1007/s004250050125

Forlani, G. (1997). Properties of the 5-enol-pyruvyl-shikimate-3-phosphate synthase isoforms isolated from maize cultured cells. J. Plant Physiol. 150, 369-375. doi: 10.1016/S0176-1617(97)80084-3

Funck, D., Eckard, S., and Müller, G. (2010). Non-redundant functions of two proline dehydrogenase isoforms in Arabidopsis. BMC Plant Biol. 10:70. doi: 10.1186/1471-2229-10-70

Funck, D., Winter, G., Baumgarten, L., and Forlani, G. (2012). Requirement of proline synthesis during Arabidopsis reproductive development. BMC Plant Biol. 12:191. doi: 10.1186/1471-2229-12-191

Gao, C., and Han, B. (2009). Evolutionary and expression study of the aldehyde dehydrogenase (ALDH) gene superfamily in rice (Oryza sativa). Gene. 431, 86-94. doi: 10.1016/j.gene.2008.11.010

Giberti, S., Funck, D., and Forlani, G. (2014). $\Delta^{1}$-pyrroline-5-carboxylate reductase from Arabidopsis thaliana: stimulation or inhibition by chloride ions 
and feed-back regulation by proline depend on whether NADPH or NADH acts as co-substrate. New Phytol. 202, 911-919. doi: 10.1111/nph.12701

Gout, E., Rébeillé, F., Douce, R., and Bligny, R. (2014). Interplay of $\mathrm{Mg}^{2+}$, ADP, and ATP in the cytosol and mitochondria: unravelling the role of $\mathrm{Mg}^{2+}$ in cell respiration. Proc. Natl. Acad. Sci. U.S.A. 111, E4560-E4567. doi: 10.1073/pnas.1406251111

Hare, P. D., and Cress, W. A. (1997). Metabolic implications of stressinduced proline accumulation in plants. Plant Growth Regul. 21, 79-102. doi: 10.1023/A:1005703923347

Hayashi, M., Takahashi, H., Tamura, K., Huang, J., Yu, L. H., Kawai-Yamada, M., et al. (2005). Enhanced dihydroflavonol-4-reductase activity and NAD homeostasis leading to cell death tolerance in transgenic rice. Proc. Natl. Acad. Sci. U.S.A. 102, 7020-7025. doi: 10.1073/pnas. 0502556102

Hayat, S., Hayat, Q., Alyemeni, M. N., Wani, A. S., Pichtel, J., and Ahmad, A. (2012). Role of proline under changing environments: a review. Plant Signal. Behav. 7, 1456-1466. doi: 10.4161/psb.21949

Kavi Kishor, P. B., and Sreenivasulu, N. (2014). Is proline accumulation per se correlated with stress tolerance or is proline homeostasis a more critical issue? Plant Cell Environ. 37, 300-311. doi: 10.1111/pce. 12157

Liang, X, Zhang, L., Natarajan, S. K., and Becker, D. F. (2013). Proline mechanisms of stress survival. Antioxid. Redox Signal. 19, 998-1011. doi: 10.1089/ars.2012.5074

Luo, M., Singh, R. K., and Tanner, J. J. (2013). Structural determinants of oligomerization of $\delta^{1}$-pyrroline-5-carboxylate dehydrogenase: identification of a hexamerization hot spot. J. Mol. Biol. 425, 3106-3120. doi: 10.1016/j.jmb. 2013.05.027

Miller, G., Honig, A., Stein, H., Suzuki, N., Mittler, R., and Ziberstein, A. (2009). Unraveling $\delta^{1}$-pyrroline-5-carboxylate-proline cycle in plants by uncoupled expression of proline oxidation enzymes. J. Biol. Chem. 284, 26482-26492. doi: 10.1074/jbc.M109.009340

Mitchell, H. J., Ayliffe, M. A., Rashid, K. Y., and Pryor, A. J. (2006). A rust-inducible gene from flax (fis1) is involved in proline catabolism. Planta 223, 213-222. doi: 10.1007/s00425-005-0079-x

Monteoliva, M. I., Rizzi, Y. S., Cecchini, N. M., Hajirezaei, M. R., and Alvarez, M. E. (2014). Context of action of proline dehydrogenase (ProDH) in the hypersensitive response of Arabidopsis. BMC Plant Biol. 14:21. doi: 10.1186/1471-2229-14-21

Murashige, T., and Skoog, F. (1962). A revised medium for rapid growth and bio assays with tobacco tissue cultures. Physiol. Plant. 15, 473-497. doi: 10.1111/j.1399-3054.1962.tb08052.x

Pfaffl, M. W. (2001). A new mathematical model for relative quantification in real-time RT-PCR. Nucl. Acids Res. 29:e45. doi: 10.1093/nar/29.9.e45

Rayapati, P. J., and Stewart, C. R. (1991). Solubilization of a proline dehydrogenase from maize (Zea mays L.) mitochondria. Plant Physiol. 95, 787-791. doi: 10.1104/pp.95.3.787

Rożkowicz, A., Picco, A. M., Rodolfi, M., Pinamonti, S., and Forlani, G. (2003). Differential responses in vitro of rice cultivars to Italian lineages of the blast pathogen Pyricularia grisea (Cooke) Sacc. 1. Oxidative burst. J. Plant Physiol. 160, 1033-1040. doi: 10.1078/0176-161700941

Schertl, P., Cabassa, C., Saadallah, K., Bordenave, M., Savouré, A., and Braun, H. P. (2014). Biochemical characterization of proline dehydrogenase in Arabidopsis mitochondria. FEBS J. 281, 2794-2804. doi: 10.1111/febs. 12821
Schneider, B. L., Kiupakis, A. K., and Reitzer, L. J. (1998). Arginine catabolism and the arginine succinyltransferase pathway in Escherichia coli. J Bacteriol. 180, 4278-4286.

Schwarzländer, M., Logan, D. C., Johnston, I. G., Jones, N. S., Meyer, A. J., Fricker, M. D., et al. (2012). Pulsing of membrane potential in individual mitochondria: a stress-induced mechanism to regulate respiratory bioenergetics in Arabidopsis. Plant Cell 24, 1188-1201. doi: 10.1105/tpc.112.096438

Sells, G. D., and Koeppe, D. E. (1981). Oxidation of proline by mitochondria isolated from water-stressed maize shoots. Plant Physiol. 68, 1058-1063. doi: 10.1104/pp.68.5.1058

Senthil-Kumar, M., and Mysore, K. S. (2012). Ornithine- $\delta$-aminotransferase and proline dehydrogenase genes play a role in non-host disease resistance by regulating pyrroline-5-carboxylate metabolism-induced hypersensitive response. Plant Cell Environ. 35, 1329-1343. doi: $10.1111 / j .1365-3040.2012 .02492 . x$

Sharma, S. S., and Dietz, K. J. (2006). The significance of amino acids and amino acid-derived molecules in plant responses and adaptation to heavy metal stress. J. Exp. Bot. 57, 711-726. doi: 10.1093/jxb/erj073

Signorelli, S., Coitiño, E. L., Borsani, O., and Monza, J. (2014). Molecular mechanisms for the reaction between (.) OH radicals and proline: insights on the role as reactive oxygen species scavenger in plant stress. J. Phys. Chem. $B$ 118, 37-47. doi: 10.1021/jp407773u

Stewart, C. R., Boggess, S. F., Aspinall, D., and Paleg, G. (1977). Inhibition of proline oxidation by water stress. Plant Physiol. 59, 930-932. doi: $10.1104 /$ pp.59.5.930

Stewart, C. R., and Lai, E. Y. (1974). $\Delta^{1}$-pyrroline-5-carboxylic acid dehydrogenase in mitochondrial preparations from plant seedlings. Plant Sci. Lett. 3, 173-181. doi: 10.1016/0304-4211(74)90073-X

Szabados, L., and Savouré, A. (2010). Proline: a multifunctional amino acid. Trends Plant Sci. 15, 89-97. doi: 10.1016/j.tplants.2009.11.009

Tan, Y. F., O’Toole, N., Taylor, N. L., and Millar, A. H. (2010). Divalent metal ions in plant mitochondria and their role in interactions with proteins and oxidative stress-induced damage to respiratory function. Plant Physiol. 152, 747-761. doi: 10.1104/pp.109.147942

Türkan, I., and Demiral, T. (2009). Recent developments in understanding salinity tolerance. Environ. Exp. Bot. 67, 2-9. doi: 10.1016/j.envexpbot.2009.05.008

Verbruggen, N., and Hermans, C. (2008). Proline accumulation in plants: a review. Amino Acids 35, 753-759. doi: 10.1007/s00726-008-0061-6

Williams, I., and Frank, L. (1975). Improved chemical synthesis and enzymatic assay of $\delta^{1}$-pyrroline-5-carboxylic acid. Anal. Biochem. 64, 85-97. doi: 10.1016/0003-2697(75)90408-X

Winter, G., Todd, C. D., Trovato, M., Forlani, G., and Funck, D. (2015). Physiological implications of arginine metabolism in plants. Front. Plant Sci. 6:534. doi: $10.3389 /$ fpls.2015.00534

Conflict of Interest Statement: The authors declare that the research was conducted in the absence of any commercial or financial relationships that could be construed as a potential conflict of interest.

Copyright (๑) 2015 Forlani, Bertazzini, Zarattini and Funck. This is an open-access article distributed under the terms of the Creative Commons Attribution License (CC $B Y)$. The use, distribution or reproduction in other forums is permitted, provided the original author(s) or licensor are credited and that the original publication in this journal is cited, in accordance with accepted academic practice. No use, distribution or reproduction is permitted which does not comply with these terms. 


\section{OPEN ACCESS}

Edited by:

Sakiko Okumoto,

Virginia Polytechnic Institute and State

University, USA

Reviewed by:

Uener Kolukisaoglu,

University of Tuebingen, Germany

Vasileios Fotopoulos,

Cyprus University of Technology,

Cyprus

Jorge E. Mayer,

fAB Consult (freelance AgBiotech),

Australia

*Correspondence:

Helena G. Carvalho,

Laboratory of Molecular Biology of

Nitrogen Assimilation, Instituto de

Biologia Molecular e Celular,

Universidade do Porto, Rua do

Campo Alegre, 823, 4150-180 Porto,

Portugal

mhcarval@ibmc.up.p

Specialty section:

This article was submitted to

Plant Physiology,

a section of the journal

Frontiers in Plant Science

Received: 30 April 2015

Accepted: 13 July 2015

Published: 27 July 2015

Citation:

Seabra AR and Carvalho HG (2015)

Glutamine synthetase in Medicago truncatula, unveiling new secrets of a

very old enzyme.

Front. Plant Sci. 6:578.

doi: 10.3389/fpls.2015.00578

\section{Glutamine synthetase in Medicago truncatula, unveiling new secrets of a very old enzyme}

\author{
Ana R. Seabra and Helena G. Carvalho*
}

Laboratory of Molecular Biology of Nitrogen Assimilation, Instituto de Biologia Molecular e Celular, Universidade do Porto, Porto, Portugal

Glutamine synthetase (GS) catalyzes the first step at which nitrogen is brought into cellular metabolism and is also involved in the reassimilation of ammonium released by a number of metabolic pathways. Due to its unique position in plant nitrogen metabolism, GS plays essential roles in all aspects of plant development, from germination to senescence, and is a key component of nitrogen use efficiency (NUE) and plant yield. Understanding the mechanisms regulating GS activity is therefore of utmost importance and a great effort has been dedicated to understand how GS is regulated in different plant species. The present review summarizes exciting recent developments concerning the structure and regulation of GS isoenzymes, using the model legume Medicago truncatula. These include the understanding of the structural determinants of both the cytosolic and plastid located isoenzymes, the existence of a seed-specific GS gene unique to $M$. truncatula and closely related species and the discovery that GS isoenzymes are regulated by nitric oxide at the post-translational level. The data is discussed and integrated with the potential roles of the distinct GS isoenzymes within the whole plant context.

Keywords: glutamine synthetase, Medicago truncatula, nitrogen metabolism, post-translational regulation, seed metabolism

\section{Introduction}

Glutamine synthetase (GS, E.C. 6.3.1.2) is a tightly controlled enzyme located at the core of nitrogen metabolism. GS catalyzes the first step in nitrogen assimilation, the ATP-dependent condensation of ammonium with glutamate. This reaction yields the readily metabolizable glutamine, which is used for the synthesis of all other essential nitrogenous compounds contained in the cells. Besides this key role in primary nitrogen assimilation, GS is crucial for the reassimilation of the $\mathrm{NH}_{4}{ }^{+}$ constantly released in large quantities via processes such as photorespiration, lignin biosynthesis, and protein catabolism (Lea and Miflin, 2010). The enzyme is thus essential for the initial incorporation of inorganic nitrogen into an organic composition but also fulfills a broad spectrum of functions beyond primary metabolism in nitrogen reassimilation and amino acid metabolism.

Being involved in all aspects of nitrogen metabolism, GS is a key component of nitrogen use efficiency (NUE) and plant yield, justifying the extensive amount of studies that have been dedicated to understand how GS is regulated and how it regulates nitrogen metabolism in plants (for recent reviews see, Bernard and Habash, 2009; Lea and Miflin, 2010; Thomsen et al., 2014). To achieve its multiple roles in plant metabolism, GS belongs to a small multigene family encoding 4-6 isoenzymes located in the cytosol (GS1) and in the plastids (GS2). The GS genes follow a complex pattern of expression, influenced by developmental and environmental cues, and their encoded enzymes 


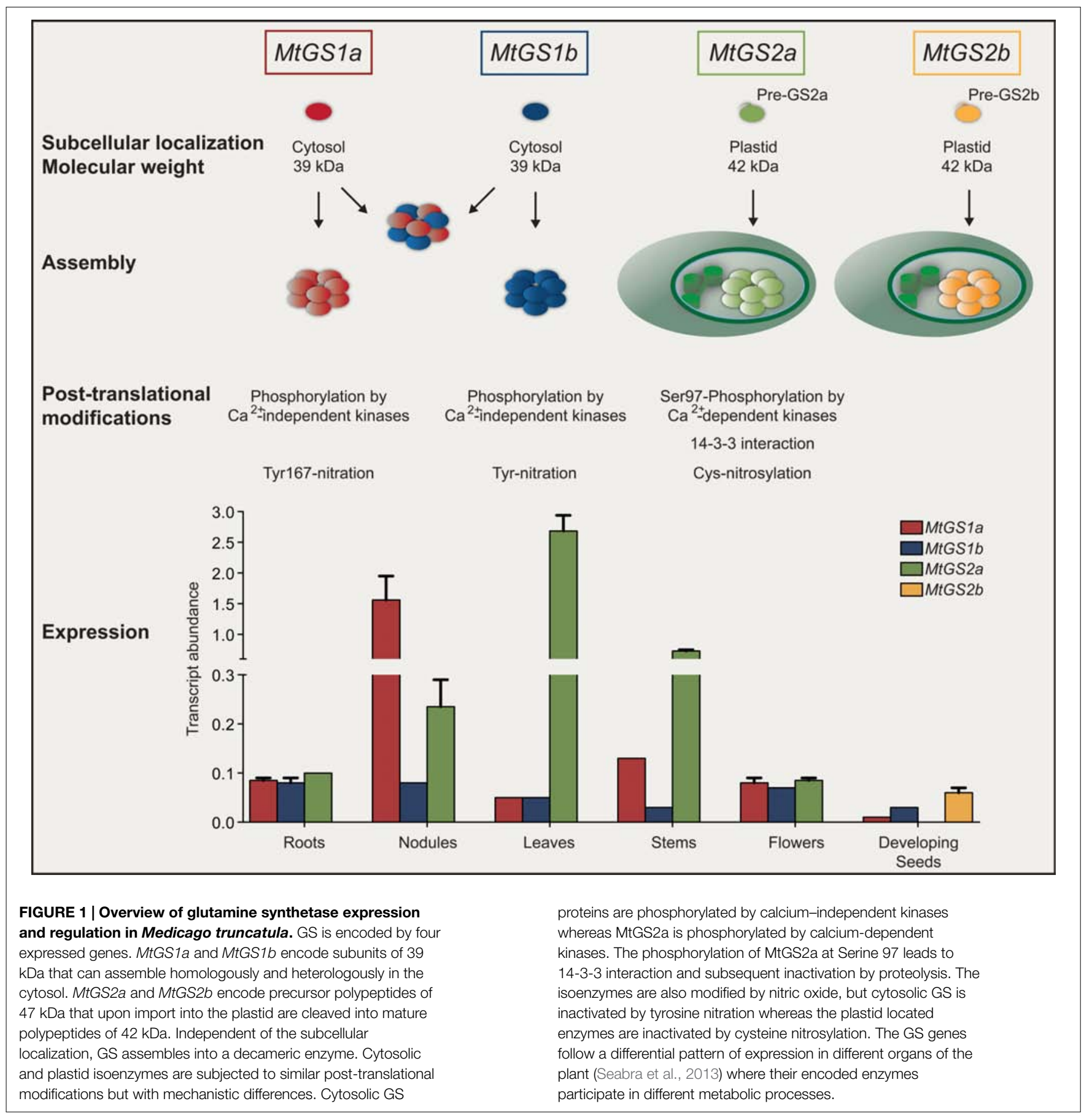

participate in different metabolic processes. Some genes show an organ/tissue specific expression and others are ubiquitously expressed. GS2 is highly expressed in photosynthetic tissues and plays a major role in the reassimilation of $\mathrm{NH}_{4}{ }^{+}$released during photorespiration and in the assimilation of $\mathrm{NH}_{4}{ }^{+}$deriving from $\mathrm{NO}_{3}{ }^{-}$reduction in plastids (Wallsgrove et al., 1987; Orea et al., 2002). GS1 is important for primary $\mathrm{NH}_{4}{ }^{+}$assimilation in roots and for reassimilation of $\mathrm{NH}_{4}{ }^{+}$generated by several different processes occurring in the cytosol, including aminoacid catabolism and the activities of phenylalanine ammonia lyase and asparaginase (Bernard and Habash, 2009; Lea and Miflin, 2010).

Owing the vital role and GS involvement in many aspects of the complex matrix of nitrogen metabolism, the enzyme must be tightly controlled. The first control occurs at the level of gene expression, an aspect that has been most intensively studied, but recent advances in molecular biology and protein biochemistry are revealing an impressive array of novel regulatory controls operating at many different levels to coordinate in vivo GS activity. It has been reported that GS is controlled at the level of 
mRNA processing and stability (Ortega et al., 2006; Stanulovic et al., 2006; Simon and Sengupta-Gopalan, 2010), translation initiation (Ortega et al., 2012) and at the post-translational level by phosphorylation and interaction with 14-3-3 (Moorhead et al., 1999; Finnemann and Schjoerring, 2000; Riedel et al., 2001; Lima et al., 2006a,b), interaction with other proteins (Masalkar et al., 2010; Seabra et al., 2013) protein turnover (Ortega et al., 1999, 2001) and by the nitric oxide (NO) induced post-translational modifications (PTMs) cysteine nitrosylation (Lindermayr et al., 2005; Melo et al., 2011) and tyrosine nitration (Cecconi et al., 2009; Lozano-Juste et al., 2011; Melo et al., 2011). Each isoenzyme appears to be regulated by a combination of mechanisms, coordinated to adapt the enzyme to operate at best performance under a specific metabolic context. Since GS isoenzymes cover a broad range of metabolic functions and the proteins share a high degree of amino-acid similarity, it is not simple to understand the specific metabolic function and the regulatory controls of individual members. This is especially difficult for the cytosolic enzymes, which are often simultaneously expressed in the same cell. Furthermore, the function of orthologous genes is not necessarily conserved in different plant species, and thus the function of a specific isoenzyme can hardly be inferred from one species to another. Recent studies covered the whole GS family in a single species, most of them on crop cereals due to the importance of GS for productivity (Swarbreck et al., 2011; Thomsen et al., 2014). In the case of legumes, an additional interest exists due to the strategic role that GS plays in the assimilation of ammonium produced by symbiotic nitrogen fixing rhizobia in root nodules. We have chosen the model legume Medicago truncatula to try to obtain a holistic view of GS regulation in a single legume species. This plant provides an excellent model system to study GS because it contains a simple GS gene family, with only four expressed genes, MtGS1a and MtGS1b encoding cytosolic polypeptides of $39 \mathrm{kDa}$, and MtGS2a and MtGS2b, encoding plastid-located polypeptides of $42 \mathrm{kDa}$, the latter of which is seed specific and unique to $M$. truncatula and closely related species (Seabra et al., 2010; Figure 1). The other three GS genes are expressed in almost all organs of the plant, but in a cellspecific manner. MtGS2a is highly expressed in all photosynthetic tissues, where it functions to assimilate the ammonia derived from photorespiration and nitrate reduction (Melo et al., 2003). The cytosolic MtGS1a is highly expressed in root nodules, where its main function is the assimilation the $\mathrm{NH}_{4}{ }^{+}$produced by nitrogen fixation (Carvalho et al., 2000b), but it is also strongly expressed in the vascular bundles of all plant organs and probably involved in supplying glutamine for transport (Carvalho et al., 2000a). MtGS1b is ubiquitously expressed in all organs of the plant, functioning as a housekeeping gene, but its expression is increased during senescence and presumably it encodes the isoenzyme responsible for the reassimilation of the ammonia derived from protein catabolism (Carvalho et al., 2000a; Carvalho and Cullimore, 2003). Besides the discovery of a second plastid GS gene, our studies in $M$. truncatula revealed a number of new unsuspected mechanisms of GS regulation (Figure 1). This mini review summarizes exciting recent developments concerning the structure and regulation of GS isoenzymes, using the model legume M. truncatula.

\section{Dynamics of Enzyme Catalysis Revealed by the Structure Analysis of Cytosolic and Plastid-located GS}

Determining the three-dimensional structures of closely related GS isoenzymes and their catalytic properties is critical to establish structure-function relationships. However, and in spite of the importance of GS in plants, the structure of the plant enzyme has only recently been elucidated (Unno et al., 2006; Torreira et al., 2014). For a long time it was believed that eukaryotic GS had a octameric architecture, based on extrapolation from the best-studied prokaryotic enzymes (Eisenberg et al., 1987). The determination of the atomic structure of the cytosolic GS1a from maize (ZmGS1a) and M. truncatula (MtGS1a) by X-ray crystallography revealed a decameric organization (Unno et al., 2006; Seabra et al., 2009; Torreira et al., 2014) that is common to the human and dog (Krajewski et al., 2008) and also to the yeast enzymes (He et al., 2009). Eukaryotic GS is arranged in two stacked (face-to-face) pentameric rings with extensive contacts established between subunits of the same ring and limited interactions with the subunits of the opposite ring. All monomers adopt a very similar conformation with each of the 10 active sites formed between two adjacent monomers from the same pentamer.

Here, we highlight some interesting features related to the dynamics of enzyme catalysis revealed by the structural analysis of the cytosolic (MtGS1a) and plastid-located GSs (MtGS2a) from M. truncatula (Torreira et al., 2014). The three-dimensional structure of unliganded MtGS1a was determined at $2.35 \AA$ resolution by X-ray crystallography (Figure 2; Seabra et al., 2009), and a comparison with the maize ortholog, whose structure was determined in complex with GS inhibitors (Unno et al., 2006) revealed some critical differences: The Trp141-Gly152 surface segment displays a different arrangement in the two enzymes, resulting in a $15^{\circ}$ rotation in the relative position of the two rings in the free MtGS1a enzyme, when compared to the inhibited maize enzyme, suggesting that substrate binding and release could be related to the rotation of the pentameric rings (Torreira et al., 2014). The plastid-located MtGS2a is also a decamer composed of two superposed pentameric rings, as revealed by cryo-electron microscopy, but the association between the double-stacked rings appears to be more flexible than in MtGS1a (Torreira et al., 2014; Figure 2). The detection of two oligomeric states of MtGS2a by mass spectrometry under non-denaturing conditions, consistent with decamers and pentamers, argues in favor of the higher flexibility of inter-ring connections, suggesting a zipper-like mechanism for MtGS2a ring-ring assembly (Torreira et al., 2014). The funnel-shaped GS active site is structurally conserved between eukaryotic and the well-studied prokaryotic protein (Eisenberg et al., 2000), comprising the $\mathrm{N}$-terminal $\beta$-grasp domain of one subunit and the highly curved $\beta$-sheet in the C-terminal catalytic domain of the neighboring subunit. Because MtGS1a was crystallized in the absence of substrates or inhibitors, the region that comprises the glutamate-binding loop (Thr293-Ala299) is disordered, leaving the entrance to the glutamate-binding site open at the narrower end of the funnel-shaped cavity. Interestingly, this flexibility of 


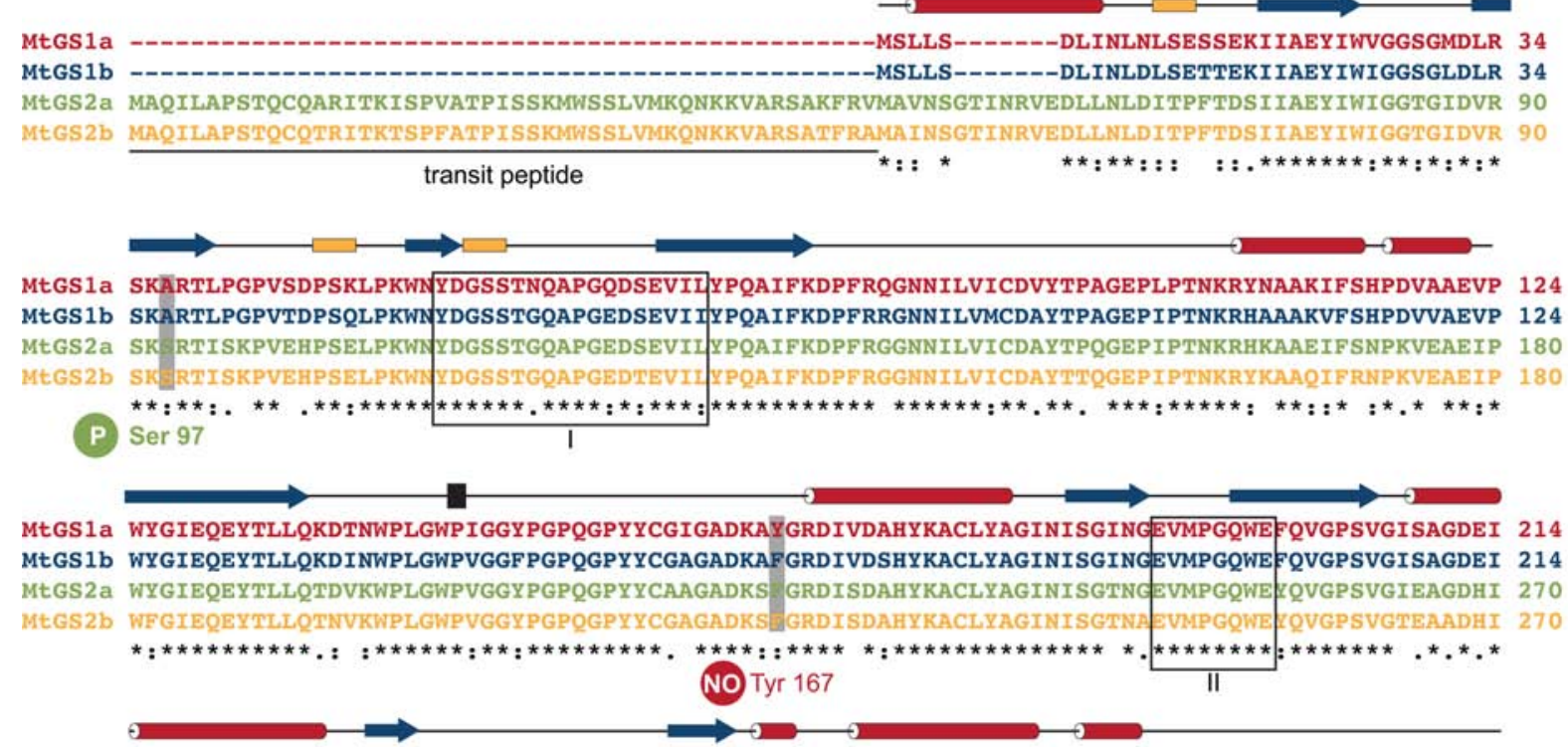

MTGS1a WAARYILERITEIAGVVVSFDPKPIPGDWNGAGAHTNYSTKSMRENGGYEIIKKAIEKLGLRHKEHIAAYGEGNERRLT̈G̈K̈HETAMIDINTF 304 MtGS1b WVARYILERITEIADVVLSFDPKPIKGDWNGAGAHTNYSTKSMREDGGYEVILKAIEKLGKKHKEHIAAYGEGNERRLTGRHETADINTF 304 MLGS2a WASRYILERITEQAGVVLTLDPKPIEGDWNGAGCHTNYSTKSMREDGGFEVIKKAILNLSLRHKIHIEAYGEGNERRLITGKHETA'SI TF 390 MUGS2b WASRYILERITEOAGVVLSEDPKPIEGDWNGAGCHTNYSTKSMREDGGFEVIKKAILNLSLRHKVHMEAYGEGNERRITGKHETR'SI TFF 390

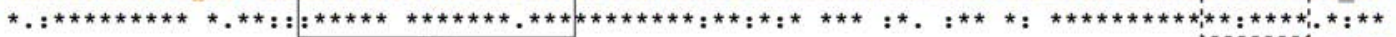
III GBL Ásp 358

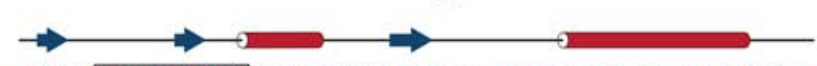

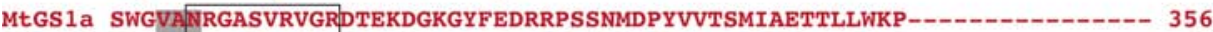

MTGS1b LWGVANRGASIRVGRDTEKAGKGYFEDRRPSSNMDPYVVTSMIADTTILWKP----:-- 356

MLGS2a SWG RGCSIRVGRTEKNGKGYLEDRRPASNMDPYVVTALLAESTLLWEPTLEAEALAAOKIALKV 428 MUGS2b SWG RGCSIRVGR TEKMGKGYLEDRRPASMMPYVVTALLAESTLLWEPTLEAEALAAQKLALKV 428

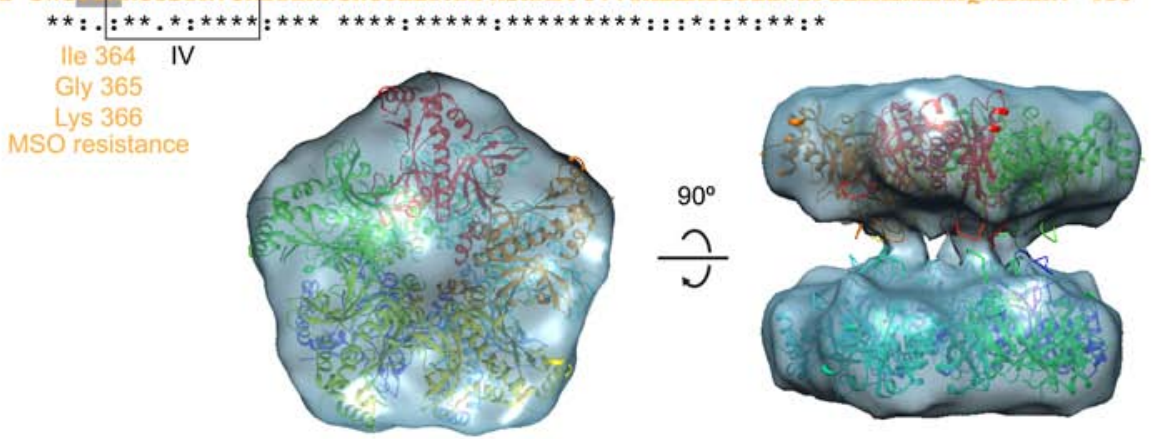

FIGURE 2 | Structural features of $\boldsymbol{M}$. truncatula GSs. The four $M$. truncatula GS polypeptides, MtGS1a, MtGS1b, MtGS2a, and MtGS2b share a high degree of amino-acid conservation. The secondary structure elements above the alignment, $\alpha$-helices in red, $3_{10}$ helices in yellow and $\beta$-strands in blue correspond to the crystal structure of MtGS1a. Serine 97 is the regulatory phosphorylation site of MtGS2a (Lima et al., 2006a), and tyrosine 167 is the regulatory nitration site of MtGS1a (Melo et al., 2011). Boxed fragments I to IV represent the GS conserved regions involved in the active site, which were defined by Eisenberg et al. (1987). GBL corresponds to the conserved glutamate-binding loop referred in the text. The four MtGS2b amino-acid substitutions, presumably responsible for the resistance to the GS inhibitor methionine-S-sulfoximine (MSO), are also indicated. The bottom part of the figure shows a top and side view of the crystal structure of MtGS1a, in which each monomer is colored differently, fitting the electron cryo-microscopy reconstruction of MtGS2a (Torreira et al., 2014). the glutamate-binding loop observed in the crystal structure of MtGS1a is predicted to be essential for the catalytic activity of the enzyme, allowing the exchange of newly synthesized glutamine for glutamate.

Understanding the three-dimensional structures of GS provides useful knowledge to develop new herbicides. Significant progress was achieved in M. truncatula with the determination of the catalytic properties of the four GS isoenzymes (Seabra et al., 2013) and their susceptibility to inhibition by phosphinothricin (PPT) and methionine-S-sulfoximine (MSO; Torreira et al., 2014). These are the active components of known herbicides, which have been shown to compete with glutamate binding. Interestingly, MtGS2b is extremely resistant to MSO inhibition. This significant functional difference likely arises from a few amino-acid changes 
that occur in the vicinity of the glutamate-binding site and are conserved in all other GS proteins (Asp358; Ile364-Gly365-Lys366 in MtGS2b and Asn302; Val308-Ala309-Asn310 in MtGS1a and other GS proteins; Figure 2). In its closed conformation, the glutamate-binding loop shields the catalytic intermediates (Liaw and Eisenberg, 1994) and the current available structural models indicate that these amino-acid modifications could stabilize the open conformation of the glutamate-binding loop, resulting in increased resistance to inhibition by MSO. These insights into the structural determinants of GS inhibition, open new possibilities to engineer GS for selective herbicide resistance in crop plants.

\section{GS Isoenzymes are Regulated by Multiple Post-translational Mechanisms}

Post-translational modifications play an essential function in the regulation of metabolic pathways as they can, reversibly or irreversibly, alter the activity and function of enzymes, providing a short-term response to sudden metabolic fluctuations. GS proteins are known to suffer various PTMs, including phosphorylation, oxidative turnover, and nitration by NO. Yet, because of its complexity, a full understanding of the posttranslational regulation of GS in plants remains elusive. Multiple isoenzymes, are often simultaneously expressed in the same organ, in some cases sharing similar size and isoelectric point, and a single GS isoenzyme can suffer more than one PTM. Thus, it is not easy to understand the effect of a specific PTM modification on individual GS proteins. In vitro studies using recombinant enzymes are useful to investigate the effect of a particular PTM on enzyme activity, but the physiological significance of PTMs requires studies in planta. $M$. truncatula is a useful model to study GS PTMs, because it contains only two cytosolic isoenzymes that can be separated by isoelectric focusing.

Our studies using recombinant enzymes from $M$. truncatula revealed that a single isoenzyme can suffer multiple PTMs, but the mechanisms of action and the effects on GS activity are isoenzyme specific. The picture is complex, with the three major M. truncatula GS isoenzymes MtGS1a, MtGS1b, and MtGS2a being regulated by phosphorylation (Lima et al., 2006a,b) and also by NO mediated PTMs, but by different mechanisms and with different outcomes on enzyme activity (Melo et al., 2011; Silva and Carvalho, 2013; Figure 1). The effect of NO is radical, leading to total enzyme inactivation, but with significant differences in the mechanisms of action for the three major GS proteins. The cytosolic enzymes MtGS1a and MtGS1b are irreversibly inactivated by tyrosine nitration, whereas MtGS2 appears to be reversibly inhibited by cysteine-nitrosylation. The intricacy of this regulation is further evidenced by the observation that the regulatory MtGS1a nitration residue, tyrosine 167 (Melo et al., 2011), is not conserved in MtGS1b (Figure 2), illustrating that in spite of the high amino-acid similarity between the two cytosolic isoenzymes, they are sufficiently different to be differentially affected by NO. The physiological significance of the differential NO-mediated PTMs in the different isoenzymes is still not fully understood, but the nodule enzyme MtGS1a has been shown to be nitrated in vivo and its nitration status was found to be related to higher NO content in the root nodules and reduced nitrogen fixation activity, leading to the proposal that the NO-mediated post-translational inactivation of GS is related to metabolite channeling to boost the antioxidant defenses (Melo et al., 2011; Silva and Carvalho, 2013).

Similarly, all three major $M$. truncatula isoenzymes are regulated by phosphorylation, but by different kinases and with different outcomes for GS activity. MtGS1a and MtGS1b are phosphorylated by calcium-independent kinase(s) (Lima et al., 2006b), whereas the plastid-located GS is phosphorylated by calcium-dependent kinase(s) (Lima et al., 2006a). Phosphorylation of GS2 at Serine 97 creates a 14-3-3 binding site and the complex is recognized by an unknown plant protease that cleaves the enzyme near the C-terminal causing enzyme inactivation (Lima et al., 2006a). 14-3-3 binding to phosphorylated GS has also been reported for GS2 in tobacco (Riedel et al., 2001) and GS1 in Brassica napus (Finnemann and Schjoerring, 2000), but with contrasting effects, as in these species 14-3-3s appears to act as GS activity activators, suggesting that either there are species-specific differences in the way GS is modulated or that senescence influences GS1 phosphorylation as the studies in Brassica used senescing leaves whereas the work in M. truncatula used mature leaves. The physiological effect of phosphorylation on the $M$. truncatula enzymes is still unclear, but cytosolic GS phosphorylation was found to be affected by light in leaves and by nitrogen fixation in root nodules, whereas GS2 phosphorylation was unaffected by these conditions (Lima et al., 2006b). Thus, it is clear that the enzymes are differentially phosphorylated under different physiological conditions, but understanding the physiological significance of phosphorylation will require further studies that must include quantitative measurements of phosphorylated versus non-phosphorylated GS.

There is also compelling evidence for the regulation of GS by protein-protein interactions in $M$. truncatula. The integration of GS proteins into organ-specific protein complexes with different molecular mass (Seabra et al., 2013) implies additional PTMs occurring under defined physiological conditions, denoting that there is still much to be discovered regarding the posttranslational regulation of GS. The identification of the GS phosphorylating kinases, of the effect of phosphorylation on the activities of the enzymes and the characterization of proteins binding to each individual GS isoenzyme will be critical to understanding the molecular and physiological consequences of those PTMs for GS and for nitrogen metabolism.

\section{Medicago truncatula Contains a Second GS2 Gene, which is Seed-specific}

Plants typically have a single gene encoding the plastid-located GS per haploid genome, M. truncatula is an exception to this rule as it contains an additional gene encoding GS2, which is expressed exclusively in the seeds. Seeds are essential elements for the dispersal of plant species and due to their high nutrient content are also important components of human diet. Seed yield is strongly dependent on efficient nitrogen remobilization from vegetative tissues, a process in which GS is determinant 
(Tabuchi et al., 2005; Martin et al., 2006). Nitrogen is remobilized mainly as asparagine and glutamine, which are subsequently deaminated in the developing seeds, implying that the resulting ammonia is re-assimilated by GS. Legume seeds are protein rich and it is predictable that GS activity is critical to achieve this high protein content. The existence of a seed-specific GS gene in M. truncatula (MtGS2b) with unique features, strongly suggests a specific role related to legume seed metabolism (Seabra et al., 2010).

The gene emerged from a recent duplication event estimated to have occurred 10 million years ago and hence after legume speciation. Thus, unlike the majority of plant GS gene families, $M$. truncatula and its closely-related species of the Vicioide clade have two genes encoding a plastid-located enzyme. Contrasting with the general expression of plastid-located GS in all organs of the plant, $M t G S 2 b$ is exclusively expressed in developing seeds. Even thought both $M$. truncatula GS2 genes are transcribed during the initial stages of seed development, $M t G S 2 a$ is repressed at the onset of seed filling, whereas $M t G S 2 b$ is continuously expressed. This pattern of expression suggests that MtGS2b represents a functional adaptation to ammonium assimilation in legume seeds. Consistent with these observations are the unique kinetic properties of MtGS2b, which has the highest turnover rate and the lowest affinity for the three substrates, glutamate, ammonium and ATP of the four M. truncatula GS isoenzymes (Seabra et al., 2013). The low ammonium affinity of MtGS2b is in line with the predicted affinity of grain cytosolic GS isoforms (Goodall et al., 2013) pointing to the existence of special physiological conditions for ammonium assimilation in seeds.

In legumes, seed reserves are synthesized and stored in the embryo cotyledons rather than in the endosperm and it is conceivable that an increased plastid GS activity in embryos benefits a metabolism directed to the production of storage proteins. M. truncatula, as other legumes, have photoheterotrophic embryos, which ensures that the high energy required for the synthesis of storage compounds is fulfilled. High protein content also implies that resources are diverted from carbohydrate synthesis and channeled to amino-acid metabolism. By funneling glutamine into glutamate and aspartate production, GS plays a pivotal role in providing the building blocks for the biosynthesis of all amino-acids whose pathways are fully plastidbased (reviewed, in Wise and Hoober, 2007). It is also known that amino-acid metabolism contributes to the energy status of developing seeds by providing energy donors through the TCA

\section{References}

Bernard, S. M., and Habash, D. Z. (2009). The importance of cytosolic glutamine synthetase in nitrogen assimilation and recycling. New Phytol. 182, 608-620. doi: 10.1111/j.1469-8137.2009.02823.x

Carvalho, H., and Cullimore, J. (2003). "Regulation of glutamine synthetase isoenzymes and genes in the model legume Medicago truncatula," in Recent Research Development in Plant Molecular Biology, Vol. 1, ed. S. G. Pandalai (Trivandrum: Research Signpost), 157-175.

Carvalho, H., Lima, L., Lescure, N., Camut, S., Salema, R., and Cullimore, J. (2000a). Differential expression of the two cytosolic glutamine synthetase genes in various organs of Medicago truncatula. Plant Sci. 159, 301-312. doi: 10.1016/S0168-9452(00)00360-5 cycle, a pathway also completely located in the plastid (reviewed, in Galili et al., 2014).

Given the important contribution of the metabolic pathways occurring inside the plastids of developing seeds to the synthesis of storage compounds, in particular to the synthesis of storageproteins, we postulate that MtGS2b participates in the partitioning of assimilates into seed storage compounds. Plastid GS could also be involved in the remobilization of nitrogen released during the differentiation of embryonic chloroplasts into storage plastids. In such a role MtGS2b would be crucial to alleviate ammonium toxicity resulting from the degradation of the photosynthetic machinery, thereby affecting embryo development and consequently the whole plant life cycle.

\section{Concluding Remarks}

This mini review emphasizes novel developments regarding the structure and regulation of GS isoenzymes, which were unveiled using the model legume Medicago truncatula. Major breakthroughs include the disclosure of the three-dimensional structure of the cytosolic and plastid-located enzymes which revealed a surprisingly dynamic molecule; the discovery of multiple PTMs such as phosphorylation, 14-3-3 interaction, tyrosine nitration and cysteine nitrosylation, which appear to be isoenzyme specific; and the existence of a second gene encoding a seed-specific GS2 with unique kinetic properties, which reflects a recent evolutionary functional specialization probably related to the production of seed storage proteins.

\section{Author Contributions}

ARS and HC contributed equally to the content, drafting, and editing of the manuscript.

\section{Acknowledgments}

We gratefully acknowledge Pedro Pereira for the structural analysis of MtGS1a. We also acknowledge funding from Fundo Europeu de Desenvolvimento Regional (FEDER) through the Operational Competitiveness Programme (COMPETE), and national funds through Fundação para a Ciência e a Tecnologia (Portugal) under projects FCOMP-01-0124-FEDER-037277 (PEst-C/SAU/LA0002/2013) and FCOMP-01-0124-FEDER028335 (PTDC/BIA-PLA/2291/2012).

Carvalho, H., Lescure, N., de Billy, F., Chabaud, M., Lima, L., Salema, R., et al. (2000b). Cellular expression and regulation of the Medicago truncatula cytosolic glutamine synthetase genes in root nodules. Plant Mol. Biol. 42, 741-756. doi: 10.1023/A:1006304003770

Cecconi, D., Orzetti, S., Vandelle, E., Rinalducci, S., Zolla, L., and Delledonne, M. (2009). Protein nitration during defense response in Arabidopsis thaliana. Electrophoresis 30, 2460-2468. doi: 10.1002/elps.200800826

Eisenberg, D., Almassy, R. J., Janson, C. A., Chapman, M. S., Suh, S. W., Cascio, D., et al. (1987). Some evolutionary relationships of the primary biological catalysts glutamine synthetase and RuBisCO. Cold. Spring Harb. Symp. Quant. Biol. 52, 483-490. doi: 10.1101/SQB.1987.052.01.055

Eisenberg, D., Gill, H. S., Puegl, G. M. U., and Rotstein, S. H. (2000). Structurefunction relationships of glutamine synthetases. Biochim. Biophys. Acta 1477, 122-145. doi: 10.1016/S0167-4838(99)00270-8 
Finnemann, J., and Schjoerring, J. K. (2000). Post-translational regulation of cytosolic glutamine synthetase by reversible phosphorylation and 14-33 protein interaction. Plant J. 24, 171-181. doi: 10.1046/j.1365-313x.2000. 00863.x

Galili, G., Avin-Wittenberg, T., Angelovici, R., and Fernie, A. R. (2014). The role of photosynthesis and amino acid metabolism in the energy status during seed development. Front. Plant Sci. 5:447. doi: 10.3389/fpls.2014.00447

Goodall, A. J., Kumar, P., and Tobin, A. K. (2013). Identification and expression analyses of cytosolic glutamine synthetase genes in barley (Hordeum vulgare $\mathrm{L}$.). Plant Cell Physiol. 54, 492-505. doi: 10.1093/pcp/pct006

He, Y.-X., Gui, L., Liu, Y.-Z., Du, Y., Zhou, Y., Li, P., et al. (2009). Crystal structure of Saccharomyces cerevisiae glutamine synthetase Gln1 suggests a nanotube-like supramolecular assembly. Proteins 76, 249-254. doi: 10.1002/prot.22403

Krajewski, W. W., Collins, R., Holmberg-Schiavone, L., Jones, T. A., Karlberg, T., and Mowbray, S. L. (2008). Crystal structures of mammalian glutamine synthetases illustrate substrate-induced conformational changes and provide opportunities for drug and herbicide design. J. Mol. Biol. 375, 217-228. doi: 10.1016/j.jmb.2007.10.029

Lea, P. J., and Miflin, B. J. (2010). Nitrogen assimilation and its relevance to crop improvement. Annu. Plant Rev. 42, 1-40. doi: 10.1002/9781444328608.ch1

Liaw, S. H., and Eisenberg, D. (1994). Structural model for the reaction mechanism of glutamine synthetase, based on five crystal structures of enzyme-substrate complexes. Biochemistry 33, 675-681. doi: 10.1021/bi00169a007

Lima, L., Seabra, A., Melo, P., Cullimore, J., and Carvalho, H. (2006a). Phosphorylation and subsequent interaction with 14-3-3 proteins regulate plastid glutamine synthetase in Medicago truncatula. Planta 223, 558-567. doi: 10.1007/s00425-005-0097-8

Lima, L., Seabra, A., Melo, P., Cullimore, J., and Carvalho, H. (2006b). Post-translational regulation of cytosolic glutamine synthetase of Medicago truncatula. J. Exp. Bot. 57, 2751-2761. doi: 10.1093/jxb/erl036

Lindermayr, C., Saalbach, G., and Durner, J. (2005). Proteomic identification of S-nitrosylated proteins in Arabidopsis. Plant Physiol. 137, 921-930. doi: 10.1104/pp.104.058719

Lozano-Juste, J., Colom-Moreno, R., and Leon, J. (2011). In vivo protein tyrosine nitration in Arabidopsis thaliana. J. Exp. Bot. 62, 3501-3517. doi: $10.1093 / \mathrm{jxb} / \mathrm{err} 042$

Martin, A., Lee, J., Kichey, T., Gerentes, D., Zivy, M., Tatout, C., et al. (2006). Two cytosolic glutamine synthetase isoforms of maize are specifically involved in the control of grain production. Plant Cell 18, 3252-3274. doi: $10.1105 /$ tpc.106.042689

Masalkar, P., Wallace, I. S., Hwang, J. H., and Roberts, D. M. (2010). Interaction of cytosolic glutamine synthetase of soybean root nodules with the C-terminal domain of the symbiosome membrane nodulin 26 aquaglyceroporin. J. Biol. Chem. 285, 23880-23888. doi: 10.1074/jbc.M110.135657

Melo, P. M., Lima, L. M., Santos, I. M., Carvalho, H. G., and Cullimore, J. V. (2003). Expression of the plastid-located glutamine synthetase of Medicago truncatula. Accumulation of the precursor in root nodules reveals an in vivo control at the level of protein import into plastids. Plant Physiol. 132, 390-399. doi: 10.1104/pp.102.016675

Melo, P. M., Silva, L. S., Ribeiro, I., Seabra, A. R., and Carvalho, H. G. (2011). Glutamine synthetase is a molecular target of nitric oxide in root nodules of Medicago truncatula and is regulated by tyrosine nitration. Plant Physiol. 157, 1505-1517. doi: 10.1104/pp.111.186056

Moorhead, G., Douglas, P., Cotelle, V., Harthill, J., Morrice, N., Meek, S., et al. (1999). Phosphorylation-dependent interactions between enzymes of plant metabolism and 14-3-3 proteins. Plant J. 18, 1-12. doi: 10.1046/j.1365313X.1999.00417.x

Orea, A., Pajuelo, P., Pajuelo, E., Quidiello, C., Romero, J. M., and Marquez, A. J. (2002). Isolation of photorespiratory mutants from Lotus japonicus deficient in glutamine synthetase. Physiol. Plant. 115, 352-361. doi: 10.1034/j.13993054.2002.1150304.x

Ortega, J. L., Moguel-Esponda, S., Potenza, C., Conklin, C. F., Quintana, A., and Sengupta-Gopalan, C. (2006). The $3^{\prime}$ untranslated region of a soybean cytosolic glutamine synthetase (GS1) affects transcript stability and protein accumulation in transgenic alfalfa. Plant J. 45, 832-846. doi: 10.1111/j.1365313X.2005.02644.x

Ortega, J. L., Roche, D., and Sengupta-Gopalan, C. (1999). Oxidative turnover of soybean root glutamine synthetase. In vitro and in vivo studies. Plant Physiol. 119, 1483-1496. doi: 10.1104/pp.119.4.1483
Ortega, J. L., Temple, S. J., and Sengupta-Gopalan, C. (2001). Constitutive overexpression of cytosolic glutamine synthetase (GS1) gene in transgenic alfalfa demonstrates that GS1 may be regulated at the level of RNA stability and protein turnover. Plant Physiol. 126, 109-121. doi: 10.1104/pp.126.1.109

Ortega, J. L., Wilson, O. L., and Sengupta-Gopalan, C. (2012). The $5^{\prime}$ untranslated region of the soybean cytosolic glutamine synthetase beta(1) gene contains prokaryotic translation initiation signals and acts as a translational enhancer in plants. Mol. Genet. Genomics 287, 881-893. doi: 10.1007/s00438-012-0724-6

Riedel, J., Tischner, R., and Mack, G. (2001). The chloroplastic glutamine synthetase (GS-2) of tobacco is phosphorylated and associated with 14-3-3 proteins inside the chloroplast. Planta 213, 396-401. doi: 10.1007/s004250000509

Seabra, A. R., Carvalho, H., and Pereira, P. J. B. (2009). Crystallization and preliminary crystallographic characterization of glutamine synthetase from Medicago truncatula. Acta Crystallogr. Sect. F Struct. Biol. Cryst. Commun. 65, 1309-1312. doi: 10.1107/S1744309109047381

Seabra, A. R., Silva, L. S., and Carvalho, H. G. (2013). Novel aspects of glutamine synthetase (GS) regulation revealed by a detailed expression analysis of the entire GS gene family of Medicago truncatula under different physiological conditions. BMC Plant Biol. 13:137. doi: 10.1186/1471-2229-13-137

Seabra, A. R., Vieira, C. P., Cullimore, J. V., and Carvalho, H. G. (2010). Medicago truncatula contains a second gene encoding a plastid located glutamine synthetase exclusively expressed in developing seeds. BMC Plant Biol. 10:183. doi: 10.1186/1471-2229-10-183

Silva, L., and Carvalho, H. (2013). Possible role of glutamine synthetase in the NO signaling response in root nodules by contributing to the antioxidant defenses. Front. Plant Sci. 4:372. doi: 10.3389/fpls.2013.00372

Simon, B., and Sengupta-Gopalan, C. (2010). The $3^{\prime}$ untranslated region of the two cytosolic glutamine synthetase $\left(\mathrm{GS}_{1}\right)$ genes in alfalfa (Medicago sativa) regulates transcript stability in response to glutamine. Planta 232, 1151-1162. doi: 10.1007/s00425-010-1247-1

Stanulovic, V. S., Garcia de Veas Lovillo, R. M., Labruyere, W. T., Ruijter, J. M., Hakvoort, T. B., and Lamers, W. H. (2006). The $3^{\prime}$-UTR of the glutaminesynthetase gene interacts specifically with upstream regulatory elements, contains mRNA-instability elements and is involved in glutamine sensing. Biochimie 88, 1255-1264. doi: 10.1016/j.biochi.2006.05.012

Swarbreck, S. M., Defoin-Platel, M., Hindle, M., Saqi, M., and Habash, D. Z. (2011). New perspectives on glutamine synthetase in grasses. J. Exp. Bot. 62, 1511-1522. doi: 10.1093/jxb/erq356

Tabuchi, M., Sugiyama, K., Ishiyama, K., Inoue, E., Sato, T., Takahashi, H., et al. (2005). Severe reduction in growth rate and grain filling of rice mutants lacking OsGS1;1, a cytosolic glutamine synthetase 1;1. Plant J. 42, 641-651. doi: 10.1111/j.1365-313X.2005.02406.x

Thomsen, H. C., Eriksson, D., Moller, I. S., and Schjoerring, J. K. (2014). Cytosolic glutamine synthetase: a target for improvement of crop nitrogen use efficiency? Trends Plant Sci. 19, 656-663. doi: 10.1016/j.tplants.2014.06.002

Torreira, E., Seabra, A. R., Marriott, H., Zhou, M., Llorca, Ó., Robinson, C. V., et al. (2014). The structures of cytosolic and plastid-located glutamine synthetases from Medicago truncatula reveal a common and dynamic architecture. Acta Cryst. Sect. D70, 981-993. doi: 10.1107/S1399004713034718

Unno, H., Uchida, T., Sugawara, H., Kurisu, G., Sugiyama, T., Yamaya, T., et al. (2006). Atomic structure of plant glutamine synthetase: a key enzyme for plant productivity. J. Biol. Chem. 281, 29287-29296. doi: 10.1074/jbc.M601497200

Wallsgrove, R. M., Turner, J. C., Hall, N. P., Kendall, A. C., and Bright, S. W. (1987). Barley mutants lacking chloroplast glutamine synthetase-biochemical and genetic analysis. Plant Physiol. 83, 155-158.

Wise, R. R., and Hoober, J. K. (eds). (2007). "Amino acid synthesis in plastids," in The Structure and Function of Plastids, Vol. 23, eds R. R. Wise and J. K. Hoober (Dordrecht: Springer), 355-385.

Conflict of Interest Statement: The authors declare that the research was conducted in the absence of any commercial or financial relationships that could be construed as a potential conflict of interest.

Copyright (c) 2015 Seabra and Carvalho. This is an open-access article distributed under the terms of the Creative Commons Attribution License (CC BY). The use, distribution or reproduction in other forums is permitted, provided the original author(s) or licensor are credited and that the original publication in this journal is cited, in accordance with accepted academic practice. No use, distribution or reproduction is permitted which does not comply with these terms. 


\section{Modulation of phenolic metabolism under stress conditions in a Lotus japonicus mutant lacking plastidic glutamine synthetase}

\section{OPEN ACCESS}

Edited by:

Giuseppe Forlani,

University of Ferrara, Italy

Reviewed by:

Roy Navarre,

United Stated Department of

Agriculture, USA

Marco Zancani,

University of Udine, Italy

Sonia Osorio,

Málaga University, Spain

*Correspondence:

Marco Betti,

Departamento de Bioquímica Vegetal y Biología Molecular, Facultad de Química, Universidad de Sevilla, C/Profesor García González, 1 ,

Sevilla 41012, Spain mbetti@us.es

${ }^{\dagger}$ These authors have contributed equally to this work.

Specialty section:

This article was submitted to

Plant Physiology,

a section of the journal

Frontiers in Plant Science

Received: 30 March 2015 Accepted: 04 September 2015 Published: 25 September 2015

Citation:

García-Calderón M, Pons-Ferrer T, Mrázova A, Pal'ove-Balang P, Vilková M, Pérez-Delgado CM, Vega JM, Eliášová $A$, Repčák $M$, Márquez AJ and Betti M (2015) Modulation of phenolic metabolism under stress conditions in a Lotus japonicus mutant lacking plastidic

glutamine synthetase.

Front. Plant Sci. 6:760.

doi: 10.3389/fpls.2015.00760

\begin{abstract}
Margarita García-Calderón ${ }^{1 \dagger}$, Teresa Pons-Ferrer ${ }^{1 \dagger}$, Anna Mrázova ${ }^{2}$, Peter Pal'ove-Balang ${ }^{2}$, Mária Vilková ${ }^{3}$, Carmen M. Pérez-Delgado ${ }^{1}$, José M. Vega ${ }^{1}$, Adriana Eliášová ${ }^{4}$, Miroslav Repčák ${ }^{2}$, Antonio J. Márquez ${ }^{1}$ and Marco Betti ${ }^{\text {* }}$

'Departamento de Bioquímica Vegetal y Biología Molecular, Facultad de Química, Universidad de Sevilla, Seville, Spain, ${ }^{2}$ Faculty of Science, Institute of Biology and Ecology, P. J. Šafárik University, Košice, Slovakia, ${ }^{3}$ Faculty of Natural Sciences, Institute of Chemistry, P. J. Šafárik University, Košice, Slovakia, ${ }^{4}$ Department of Ecology, Faculty of Humanities and Natural Sciences, University of Prešov, Prešov, Slovakia
\end{abstract}

This paper was aimed to investigate the possible implications of the lack of plastidic glutamine synthetase $\left(\mathrm{GS}_{2}\right)$ in phenolic metabolism during stress responses in the model legume Lotus japonicus. Important changes in the transcriptome were detected in a GS 2 mutant called Ljgln2-2, compared to the wild type, in response to two separate stress conditions, such as drought or the result of the impairment of the photorespiratory cycle. Detailed transcriptomic analysis showed that the biosynthesis of phenolic compounds was affected in the mutant plants in these two different types of stress situations. For this reason, the genes and metabolites related to this metabolic route were further investigated using a combined approach of gene expression analysis and metabolite profiling. A high induction of the expression of several genes for the biosynthesis of different branches of the phenolic biosynthetic pathway was detected by qRT-PCR. The extent of induction was always higher in Ljgln2-2, probably reflecting the higher stress levels present in this genotype. This was paralleled by accumulation of several kaempferol and quercetine glycosides, some of them described for the first time in $L$. japonicus, and of high levels of the isoflavonoid vestitol. The results obtained indicate that the absence of $\mathrm{GS}_{2}$ affects different aspects of phenolic metabolism in $L$. japonicus plants in response to stress.

Keywords: Lotus japonicus, phenolic compounds, glutamine synthetase, flavonoids, isoflavonoids

\section{Introduction}

Glutamine synthetase (GS, EC 6.3.1.2) is the key enzyme in charge of glutamine biosynthesis in nature. This enzyme catalyzes the incorporation into one molecule of glutamate of the ammonium derived either from primary nitrogen assimilation (nitrate reduction, $\mathrm{N}_{2}$ fixation) or nitrogen reassimilation in the plants. Two types of GS isoenzymes exist in plants: cytosolic (called $\mathrm{GS}_{1}$ ) or plastidic (called $\mathrm{GS}_{2}$ ). $\mathrm{GS}_{1}$ is localized in the vascular tissue and plays an important role in the assimilation of external ammonium, the ammonia derived from $\mathrm{N}_{2}$ fixation and other sources of nitrogen, as well as in the remobilization of nitrogen during senescence. On the other hand, $\mathrm{GS}_{2}$ is 
predominantly expressed in green tissues, and it has been demonstrated that this particular isoform has an essential role in the reassimilation of the ammonium released by photorespiration and it has also implications in the general metabolism of the plant, and also in nodulation (Betti et al., 2014). The two major isoforms of GS seem to play important roles during stress. $\mathrm{GS}_{1}$ has been implicated in the production of proline in the phloem (Brugière et al., 1999) and/or in the remobilization of nitrogen during chronic water stress (Bauer et al., 1997) whereas $\mathrm{GS}_{2}$ was also associated with stress tolerance (Kozaki and Takeba, 1996; Hoshida et al., 2000). More recent work of our group has also demonstrated an involvement of $\mathrm{GS}_{2}$ in proline production of L. japonicus plants in the response to drought stress (Díaz et al., 2010). However, little is known yet on the implications of the different isoforms of GS in relation with other aspects of the stress responses in plants, particularly phenolic metabolism.

Plant phenolics are the most widely distributed secondary metabolites that are involved in the response to stress (Cheynier et al., 2013). This family comprehends a huge array of compounds that arise from phenylalanine with either monomeric or polymeric combinations of the original phenolic ring (for comprehensive reviews of the genetic and biochemistry of plant phenolics metabolism see Davies and Schwinn, 2006 and Saito et al., 2013). Flavonoids are one of the most important classes of plant phenolics in the response to stress, since they respond to almost all kinds of adverse environmental conditions and play several protective roles. Flavonoids can provide protections against UV light and pathogens (Winkel-Shirley, 2002) and exhibit high antioxidative activity in vitro, reason why they are also assumed to function as antioxidants in vivo (Nakabayashi and Saito, 2015). In agreement with this hypothesis, recent reports showed an increased tolerance to oxidative stress and drought stress in transgenic plants that overaccumulated flavonoids (Nakabayashi et al., 2014). Moreover, isoflavonoids, a distinct class of flavonoids, are also produced by legume plants. These compounds are the typical legume phytoalexins that defend the plant against pathogens, but also participate in the establishment of symbiosis with nitrogen fixing bacteria (Shelton et al., 2012). Therefore, phenolic metabolism is an attractive topic of study in the response to stress, particularly in legume plants.

Our research group has been working for more than 20 years with Lotus japonicus, a plant that has been used as a model for the study of several other legumes (Handberg and Stougaard, 1992), and, particularly, of other cultivated Lotus species that are widely used as pastures, and whose productivity is seriously hampered by abiotic stress conditions like drought (Pal'oveBalang et al., 2014). Recent works have been carried out in our laboratory using functional genomic approaches in order to study the response to different kinds of stress in L. japonicus. This has been carried out using both WT plants and a mutant called Ljgln2-2 that lacks of the plastidic isoform of glutamine synthetase $\left(\mathrm{GS}_{2}\right)$, the enzyme responsible of the reassimilation of the ammonium produced by the photorespiratory cycle. This mutant has been particularly useful in the identification of several stress-responsive genes in L. japonicus (Betti et al., 2012).
Two different stress situations have been previously analyzed in these mutant plants. On the one hand, the response to drought stress under $\mathrm{CO}_{2}$-enriched atmosphere $\left(0.7 \% \mathrm{CO}_{2}\right.$, where photorespiration is suppressed; Díaz et al., 2010). On the other hand, the response of the mutant plants under nondrought conditions when transferred from a $\mathrm{CO}_{2}$-enriched atmosphere to normal $\mathrm{CO}_{2}$ conditions (about $0.04 \% \mathrm{CO}_{2}$ ) where the photorespiratory cycle is active (Pérez-Delgado et al., 2013). In the case of drought, Ljgln2-2 accumulated lower levels of proline compared to the WT, a molecule that plays several protective roles under drought and osmotic stress. On the other hand, active photorespiration (PR) produced in Ljgln2-2 a completely different stress situation due to the impairment of the photorespiratory cycle and the corresponding accumulation of photorespiratory ammonium (Pérez-Delgado et al., 2013). Transcriptomic profiling indicated that these two different stress conditions caused massive transcriptomic changes in the mutant genotype and, to a lower extent, in the WT (Díaz et al., 2010; Pérez-Delgado et al., 2013).

The present work was aimed to investigate if plastidic glutamine synthetase, a key enzyme for nitrogen and photorespiratory metabolism, may also play a role beyond primary metabolism; more specifically, if the lack of this enzyme may affect the response of phenolic metabolism under stress. For this purpose, a comparative transcriptomic analysis of the response of L. japonicus WT and Ljgln2-2 mutant plants to either drought or active PR was carried out. An ample transcriptional modulation of several genes related to phenolic metabolism was observed in these two different types of stress situations. The different genes and metabolites related with the biosynthesis of phenolic compounds, particularly flavonoid and isoflavonoids, have been further analyzed by measurements of gene expression and metabolite profiling.

\section{Materials and Methods}

\section{Plant Growth Conditions and Harvesting of Plant Material}

The model legume L. japonicus (Regel) K. Larsen ecotype "Gifu” was used in all the experiments carried out. Seeds were initially obtained from Jens Stougaard (Aarhus University) and selfpropagated at the University of Seville. The Ljgln2-2 mutant that lacks of plastidic $\mathrm{GS}_{2}$ protein and activity, was isolated from photorespiratory mutants screening carried out using ethyl methanesulfonate as previously described (Orea et al., 2002). The mutant offspring of two consecutive backcrosses into the WT background were employed. WT and mutant seeds were scarified and surface sterilized and germinated in $1 \%(\mathrm{w} / \mathrm{v})$ agar in Petri dishes. Later on they were transferred to pots using vermiculite as solid support for the active PR experiment and a mixture of sand:vermiculite $(1: 1, \mathrm{v} / \mathrm{v})$ for the drought stress experiment. Five seedlings were planted in each pot and grown during 35 days in a growth chamber under 16 -h-day $\left(20^{\circ} \mathrm{C}\right)$ and 8-h-night $\left(18^{\circ} \mathrm{C}\right)$ conditions with a photosynthetic photon flux density of $250 \mu \mathrm{mol} \mathrm{m}^{-2} \mathrm{~s}^{-1}$ and a constant humidity of $70 \% . \mathrm{CO}_{2}$ was automatically injected to a final concentration of $0.7 \%(\mathrm{v} / \mathrm{v})$ to 
allow normal growth of the Ljgln2-2 mutant in an atmosphere where PR was suppressed. Plants were irrigated with "Hornum" nutrient solution containing $5 \mathrm{mM} \mathrm{NH}_{4} \mathrm{NO}_{3}$ and $3 \mathrm{mM} \mathrm{KNO}_{3}$ (Handberg and Stougaard, 1992). After 35 days of growth under high $\mathrm{CO}_{2}$ atmosphere the plants had an average number of 7 trefoils. At this time, total leaf tissue was harvested for each plant genotype, constituting the control condition (zero time) for both the active PR experiments and for the drought stress experiments (see Supplemental Figure S1). For the active PR treatment, plants were then transferred for 2 days from high $\mathrm{CO}_{2}$ $\left(0.7 \% \mathrm{CO}_{2} \mathrm{v} / \mathrm{v}\right)$ to normal $\mathrm{CO}_{2}$ conditions $\left(0.04 \% \mathrm{CO}_{2} \mathrm{v} / \mathrm{v}\right)$ and harvested, as also described by Pérez-Delgado et al. (2013). For the drought treatment plants were grown all the time under high $\mathrm{CO}_{2}\left(0.7 \% \mathrm{CO}_{2} \mathrm{v} / \mathrm{v}\right)$ conditions and drought was imposed by withholding watering for 4 days, after which both WT and Ljgln22 mutant genotypes showed a relative water content of about 55\% (see Díaz et al., 2010 for more details), and the plants were subsequently harvested. All the leaf samples used in this work were harvested $4 \mathrm{~h}$ after the beginning of the light period. For the active PR treatment, no visible symptoms of the air sensitivity phenotype of the Ljgln2-2 mutant plants were observed after 2 days under active photorespiratory conditions.

The leaf tissue for RNA extraction was pooled and flash-frozen in liquid $\mathrm{N}_{2}$, grinded with a pestle in a mortar that was precooled with liquid $\mathrm{N}_{2}$ and the powder was stored at $-80^{\circ} \mathrm{C}$ until use. Leaf tissue for metabolite analysis was dried for 2 days at $100^{\circ} \mathrm{C}$ using plastic weighing bottles and stored in the dark at room temperature until use. Previous studies showed that incubation at temperatures between 80 and $100^{\circ} \mathrm{C}$ for long period of time did not change significantly the main flavonoid profiles (Pal'ove-Balang et al., unpublished results), in agreement with previous reports from other groups (Heigl and Franz, 2003). Three independent biological replicates were harvested for each genotype and condition. A biological replicate consisted of tissue pooled from the five plants grown in the same pot.

\section{RNA Extraction and Quantitative Real-time RT-PCR}

Total RNA was isolated from leaf tissues stored at $-80^{\circ} \mathrm{C}$ using the hot borate method (Sánchez et al., 2008). The integrity and concentration of the RNA preparations were checked using an Experion bioanalyzer (Bio-Rad) with RNA StdSens chips and a Nano-Drop 2000 (Nano-Drop Technologies), respectively. For real-time qRT-PCR analysis, total RNA was treated with the TURBO DNA-free DNase (Ambion). Reverse transcription was carried out using the SuperScript III reverse transcriptase (Invitrogen), oligo(dT), and RNAsin RNase inhibitor (Ambion). DNA contamination was checked by carrying out quantitative real-time RT-PCR reactions with oligonucleotides that amplified an intron in the L. japonicus Hypernodulation Aberrant Root (LjHAR1; chr3.CM0091.1690.r2.m) gene. The efficiency of cDNA synthesis was checked by amplifying the $3^{\prime}$ and $5^{\prime}$ ends of the gene encoding for $L$. japonicus glyceraldehyde-3-P dehydrogenase (LjGAPDH; chr4.CM1854.510.r2.a). qRT-PCR reactions were carried out in $10 \mu \mathrm{L}$ final volume with a 384 -well PCR plate in a LightCycler 480 thermal cycler (Roche) using a SensiFAST SYBR No-ROX Kit (Bioline). Expression data were normalized using the geometric mean of three housekeeping genes: L. japonicus protein phosphatase 2A ( LjPp2A; chr2.CM0310.22), L. japonicus ubiquitin carrier protein 10 ( LjUbc10; chr1.TM0487.4), and L. japonicus polyubiquitin 4 (LjUbq4; chr5.CM0956.27), that were selected among the most stably expressed genes in plants (Czechowski et al., 2004). A list of all the oligonucleotides used is provided in Supplemental Table S1.

\section{Transcriptomic Data Analysis}

The datasets generated by Díaz et al. (2010) for drought stress and Pérez-Delgado et al. (2013) for active PR were further analyzed in this work. Both transcriptomic experiments were carried out using the Affymetrix GeneChip Lotus1a520343 and the corresponding "Minimum Information about a Microarray Experiment" (MIAME)-compliant data are deposited at Array Express with the accession codes E-MEXP-2690 and EMEXP-3603 for the drought and active PR experiments respectively.

The differentially expressed genes between control conditions and stress treatments were identified by the analysis of the different Affychip gene probesets using a significance-based comparison at a false discovery rate (FDR) of $<0.05$. In the L. japonicus Affymetrix GeneChip Lotus1a520343 several gene probesets may recognize the same gene. For this reason the number of gene probesets that changes under each comparison may be higher than the real number of genes that are actually modulated in the plant. The different gene probesets that changed in the different comparisons were visualized using the MapMan program (Usadel et al., 2005) and analyzed according to the corresponding metabolic pathways. Since different probesets from the gene chip may recognize the same transcript, homology search was carried out using the Blast program at the Kazusa database (http://www.kazusa.or.jp/lotus/) in order to assign with more accuracy each probeset to the corresponding gene. For the determination of the significantly overrepresented pathways within a group of gene probesets the Pathexpress software was used (Goffard and Weiller, 2007) with a statistical cut-off of $p<0.05$.

\section{Metabolites Profiling Analysis}

For analytical HPLC analysis, phenolic compounds were extracted from $50 \mathrm{mg}$ of dry leaves with $1 \mathrm{ml}$ of $50 \%(\mathrm{v} / \mathrm{v})$ methanol or $1 \mathrm{ml}$ of $100 \%(\mathrm{v} / \mathrm{v})$ methanol (for determination of isoflavonoids and tannins), spinned shortly and filtered using a $0.2 \mu \mathrm{m}$ membrane. Acid hydrolysis was performed for $15 \mathrm{~min}$ at $95^{\circ} \mathrm{C}$ in a water bath after addition of $\mathrm{HCl} 2.9 \mathrm{~N}$ (volume ratio of the methanol extract to $\mathrm{HCl} 1: 1$ ). Both the hydrolyzed and nonhydrolyzed samples were analyzed by gradient reversed phase high-performance liquid chromatography (HPLC) on Agilent 1260 Infinity Quaternary LC System with 1260 Infinity DAD detector and Kromasil $\mathrm{C}_{18} 250 \times 5 \mu \mathrm{m}$ I. D. reversed phase column. The mobile phases were 5\% acetonitrile (A) and $90 \%$ acetonitrile (B). The solvent was delivered to the column at a flow rate of $0.7 \mathrm{ml} / \mathrm{min}$ as follows: $0 \mathrm{~min}, \mathrm{~A} / \mathrm{B}$ (75:25); 0-25 min, linear gradient to $\mathrm{A} / \mathrm{B}(50: 50) ; 25-30 \mathrm{~min}$, linear gradient to $\mathrm{A} / \mathrm{B}$ (0:100); 30-40 min A/B (0:100); 40-50 min, linear gradient to $\mathrm{A} / \mathrm{B}$ (75:25); detection at 280 and $370 \mathrm{~nm}$. For the analysis of 
vestitol the mobile phases were $60 \%$ acetonitrile $(\mathrm{A})$ and $90 \%$ acetonitrile (B) and the gradient protocol was $0-5 \mathrm{~min}, \mathrm{~A} / \mathrm{B}$ (100:0); 5-15 min, linear gradient to $A / B(50: 50) ; 15-20$ linear gradient to $\mathrm{A} / \mathrm{B}(0: 100) ; 20-25 \mathrm{~min} \mathrm{~A} / \mathrm{B}(0: 100) ; 25-30$ linear gradient to $\mathrm{A} / \mathrm{B}$ (100:0).

The peaks were identified based on their retention times and UV-VIS spectra measurements carried out during the analysis in comparison to commercially available standards of quercetine, kaempferol, p-coumaric acid, p-ferulic acid (Sigma-Aldrich) and upon the data of Suzuki et al. (2008) and Lanot and Morris (2005). LC/ESI-MS analyses of flavonoid glycosides were carried out on Dionex UltiMate 3000 Quarternary Analytical LC System with diode array detector (Germering, Germany) interfaced to a Varian 310 MS, Triple Quadrupole mass spectrometer with electrospray ionization (ESI) source (Walnut Creek, CA, USA). The source parameters were as follows: positive-ion mode, capillary voltage of $4 \mathrm{kV}$, nebulization with nitrogen at $50.0 \mathrm{psi}$, drying with nitrogen at $30.0 \mathrm{psi}$ and $300^{\circ} \mathrm{C}$. Mass spectra were recorded between $\mathrm{m} / z 200$ and 1000 .

For total tannin measurements, methanol extracts were centrifuged for $5 \mathrm{~min}$ at $3000 \times \mathrm{g}$ and then $1 \mathrm{ml}$ aliquots of supernatant were assayed with $5 \mathrm{ml}$ of $1 \%$ vanillin containing 1.2 $\mathrm{N} \mathrm{HCl}$ in absolute methanol. Each sample was left in a water bath at $30^{\circ} \mathrm{C}$ for exactly $20 \mathrm{~min}$ together with the corresponding blank containing $1 \mathrm{ml}$ of supernatant and $5 \mathrm{ml} 1.2 \mathrm{~N} \mathrm{HCl}$ (without vanillin). Absorbance was registered at $500 \mathrm{~nm}$ and calculated to catechin equivalents.

In order to collect enough material to unambiguously identify compounds by NMR, kaempferol-glycosides were isolated by combining glass column chromatography and preparative HPLC. Dry tissue from plants leaves (60 g approximately) grown under control conditions was subsequently extracted with chloroform, methanol, 50\% methanol, and finally with acetic acid. The methanol extracts were shaken 3 times with chloroform to remove the rest of chlorophylls, the methanol and chloroform layer was separated each time by addition of some drops of distilled water. Glass column ( $90 \mathrm{~cm}$ long, $5.5 \mathrm{~cm}$ in diameter) chromatography on silica gel was used for both methanol and $50 \%$ methanol fractions with chloroform-methanol (20:1-5:2) solution as a mobile phase. About 400 fractions were collected by automatic collector, $15 \mathrm{ml}$ of each. The compounds in fractions were followed during the glass-column separation by analytical HPLC (in the same analytical conditions as described above for crude extract analyses). The step-by step changes of chloroformmethanol ratio in mobile phase in the glass column was decided according to the flavonoid content of the collected fractions. Fractions were joined according to their content, evaporated in Rotation vacuum evaporator and re-dissolved in methanol. The abundant peaks were then further purified using preparative HPLC system consisting of a Tessek SGX C18 column $(7 \mu \mathrm{m}$ $8 \times 250 \mathrm{~mm}$ ), a ECOM LCP 4100 pump and a UV-VIS detector LCD 2040 and a flow rate of $4 \mathrm{ml} / \mathrm{min}$, to be suitable for NMR analysis. The compounds identities were verified by NMR spectra at room temperature on NMR spectrometer Varian VNMRS 600 (Palo Alto, CA, USA) operating at $599.868 \mathrm{MHz}$ for ${ }^{1} \mathrm{H}$ and $150.836 \mathrm{MHz}$ for ${ }^{13} \mathrm{C}$. Spectra were recorded in $\mathrm{CD}_{3} \mathrm{OD}-\mathrm{d}_{4}$. The 2D gCOSY, 290 TOCSY, NOESY, gHSQC, gHMBC (optimized for a long-range coupling of $8 \mathrm{~Hz}$ ), 1D291 sel TOCSY, gH2BC, and gTOXY-HSQC methods were employed.

\section{Statistical Data Analysis}

Significant changes in gene expression levels in the microarray experiments were analyzed using a significance-based comparison applying a false discovery rate $($ FRD $)<0.05$. Other details as described by Pérez-Delgado et al. (2013). Significant differences in gene expression levels (qRT-PCR) between control and stress conditions were determined for each genotype according to Student's $t$-test $(p<0.05)$. Data from the metabolite measurements were analyzed according to the Student's $t$-test with $p<0.05$ or $p<0.01$.

\section{Results}

\section{Comparative Transcriptomic Analysis of the Response of $L$. japonicus to Drought and Active Photorespiration}

In order to investigate the role of plastidic $\mathrm{GS}_{2}$ in the response to stress in L. japonicus, a mutant called Ljgln2-2 that lacks of this enzyme was submitted to two different stress treatments: drought or active PR. The gene probesets significantly modulated by each stress treatment in leaves of the mutant plants were identified by comparing the transcriptome obtained under stress conditions with the transcriptome of the plants in control conditions (normal watering and suppressed PR). For comparative purposes, WT plants were also submitted to the same treatments. In total, 2608 and 1480 gene probesets were modulated by drought or active PR respectively in the WT compared to the control conditions, while 7915 and 6610 gene probesets changed in the mutant plants under the same conditions (Table 1). Interestingly, 2173 gene probesets were commonly affected by both types of stress situations in Ljgln22, which corresponded to 27.5 and $41.0 \%$ of the total number of gene probesets modulated by drought or active PR respectively (Table 1). This suggested the existence of a common response to different stress conditions in the Ljgln2-2 mutant. In contrast, in the case of the WT, only 187 gene probesets were found to

TABLE 1 | Total number of gene probesets modulated by drought stress or active PR in WT and LjgIn2-2 mutant plants.

\begin{tabular}{lccc}
\hline & $\begin{array}{c}\text { Experiment 1 } \\
\text { Drought }\end{array}$ & $\begin{array}{c}\text { Experiment 2 } \\
\text { Active PR }\end{array}$ & Commonly affected \\
\hline WT & 2608 & 1480 & 187 \\
Ljgln2-2 & 7915 & 6610 & 2173 \\
\hline
\end{tabular}

The total number of gene probesets that changed significantly $(p<0.05)$ by drought was calculated by comparing the gene expression levels under drought conditions with the expression levels under control conditions (high $\mathrm{CO}_{2}$ atmosphere, normal watering) as described by Diaz et al. (2010). The gene probesets that changed significantly $(p<0.05)$ by active PR conditions were identified by comparing the gene expression levels with a control under photorespiratory suppressed conditions (high $\mathrm{CO}_{2}$ atmosphere, normal watering) as described by Pérez-Delgado et al. (2013). The column on the right shows the total number of gene probesets that were commonly elicited in the drought stress experiment and in the active PR experiment. 
be commonly modulated in drought or active PR (Table $\mathbf{1}$ ). The genes that were commonly modulated in the response to drought or active PR are further analyzed below in this paper separately for WT and the Ljgln2-2 mutant plants.

\section{Analysis of the Gene Probesets Modulated By Drought or Active PR}

The 187 gene probesets that changed under the two types of stress situation in WT plants were represented in the context of general metabolism using the MapMan software (Supplemental Figure S2, see Supplemental Table S2 for a list of these gene probesets). The MapMan program allows the visualization of the changes observed in transcriptomic data by providing an overview of metabolic pathway responses. Each significantly modulated gene is indicated with a square within a box that represents the corresponding metabolic pathway. MapMan visualization indicated that the genes commonly affected by drought or active PR in the WT were quite dispersed through the metabolic map and no particular pathway seemed to be highly regulated. In contrast, some interesting correlations were observed in the analysis the group of 2173 gene probesets that were commonly modulated by drought or active PR in Ljgln2-2 mutant plants. First of all, it was shown that the great majority of these gene probesets (2030) changed in the same direction (i.e., were commonly induced or repressed) in response to either drought or active PR (Supplemental Figure S3). Of these, 1073 gene probesets were commonly induced while 957 were commonly repressed. Linear regression analysis indicated that the gene probesets that changed in the same direction under both types of stress conditions were slightly more modulated by drought compared to active PR (Supplemental Figure S3).

In order to get further insight into the convergent response of Ljgln2-2 to drought and active PR, the 1073 gene probesets that were commonly induced by drought or active PR treatments were visualized in the context of general metabolism using the MapMan software (Figure 1A). The 957 gene probesets that were repressed by both types of stress are represented in Figure 1B. Since the extent of change for these commonly induced and repressed gene probesets was different under either drought or active PR, a fold-change value of 2 and -2 was arbitrarily assigned for the MapMan representation of Figure 1. Several genes related to ascorbate and glutathione metabolism, sucrose degradation and phenylpropanoid metabolism were highly induced by both stress treatments (Figure 1A). On the other hand, the analysis of the genes commonly repressed by these two types of stress treatments showed a general repression of several pathways related to photosynthesis like the biosynthesis of the structural components of the photosystems, the Calvin cycle, photorespiration and genes related to carbohydrate degradation (Figure 1B). To determine which metabolic pathways were significantly over-represented among these groups of genes, the common gene probesets induced or repressed under these two types of stress treatments were further analyzed using Pathexpress (Goffard and Weiller, 2007). Pathexpress is a webbased tool that allows the identification of the most relevant sub-networks (metabolic pathways, sub-pathways, and enzyme neighborhood) associated with a set number of genes. An overrepresented pathway in a query list of gene probesets is identified by comparing the number of enzymes reactions of this route represented within the query list compared to the total number of enzyme reactions present in the pathway. The significance of this comparison is then tested using a hypergeometric distribution test as developed in the BlastSets system (Goffard and Weiller, 2007 and references therein). The analysis carried out confirmed that the most significantly repressed pathways were mainly related to photosynthesis and carbon metabolism. Among the most repressed routes were glycolysis, starch and sucrose metabolism, carbon fixation (essentially the Calvin cycle) as well as the biosynthesis of chlorophyll and photosynthetic pigments (Table 2). On the other hand, the most induced pathway by both stress treatments in the $\operatorname{Ljgln2-2}$ mutant was stilbene/lignin/coumarin biosynthesis, which comprises several branches of the complex route for the biosynthesis of phenolic compounds (Table 2). Flavonoid biosynthesis, another branch of plant phenolics metabolism, was also found among the most over-represented routes, as well as phenylalanine metabolism, where genes encoding for the enzymes for the first steps of the biosynthesis of phenolic compounds like phenylalanine ammonia lyase (PAL) and cinnamate 4-hydroxylase $(\mathrm{C} 4 \mathrm{H})$ were induced. In addition, it was also noted that one particular gene for phenolic metabolism, corresponding to isoflavone $2^{\prime}$ hydroxylase, was among the top 20 commonly induced genes under drought stress conditions in the Ljgln2-2 mutants, and was the most induced genes by active $\mathrm{PR}$ in this genotype (Supplemental Table S3).

Previous works have analyzed the consequences of the lack of $\mathrm{GS}_{2}$ in primary metabolism (Pérez-Delgado et al., 2013). Interestingly, the results obtained now in the present paper indicated that the biosynthesis of phenolic compounds was also greatly modulated in response to stress as a result of the absence of plastidic $\mathrm{GS}_{2}$ in the Ljgln2-2 mutant. The following sections of this paper analyze further in detail by qRT-PCR and metabolic profiling the different changes in phenolic metabolism associated with the lack of $\mathrm{GS}_{2}$ in response to drought or active PR stress conditions.

\section{Modulation of the Expression of Gene Probesets for the Biosynthesis of Phenolic Compounds}

A real-time qRT-PCR analysis was carried out to further investigate and validate the changes in the expression of genes for the biosynthesis of phenolic compounds that were found in the transcriptomics studies. Given the great complexity of the biosynthetic route of plant phenolics a focus was made on the key genes encoding for enzymes that catalyze the first common steps of the route, and also several ones for the central flavonoid biosynthetic pathway, for isoflavonoid biosynthesis as well as for the branch that lead to anthocyanins and protoanthocyanidins. Several of the enzyme activities of this pathway are encoded by large gene families like in the case of phenylalanine-ammonia lyase, encoded by 10 genes, and chalcone synthase encoded by 12 (Supplemental Table S4). Moreover, some of these gene families were organized in tandem repeats on the same chromosome like in the case of chalcones isomerase (Shimada et al., 2003), 

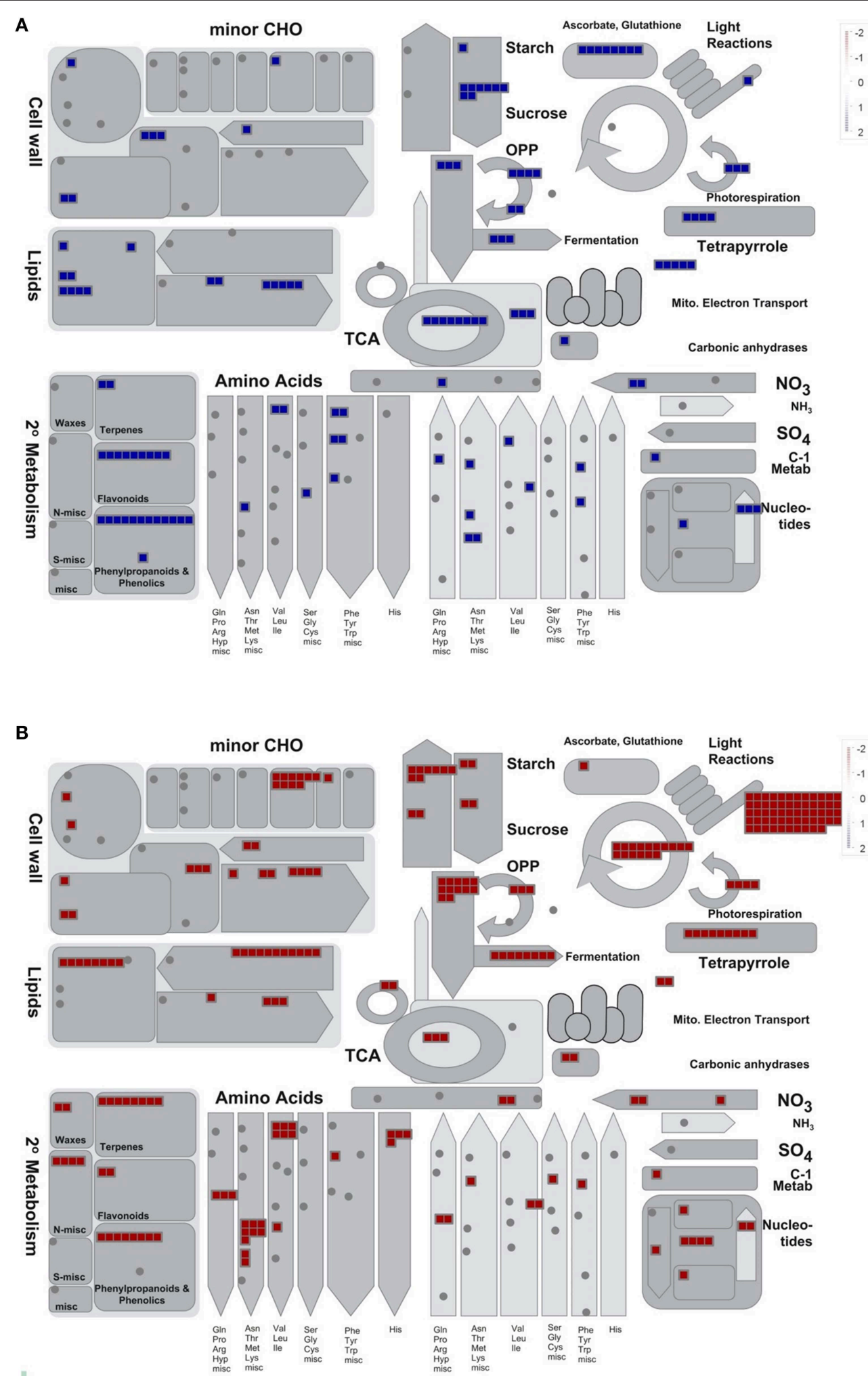

FIGURE 1 | MapMan overview of general metabolism for the gene probesets that were commonly induced or repressed in Ljgln2-2 mutant plants either in drought or in active PR stress conditions. (A) Shows the 1073 gene probesets that were commonly induced by the two types of stress situations and (B) the 957 gene probesets that were commonly repressed analyzed in the context of general metabolism using the MapMan software. Each square represent a gene probeset that changed significantly $(p<0.05)$ under both types of stress situation. The gene probesets are within a figure that represents the correspondent metabolic 
be commonly modulated in drought or active PR (Table $\mathbf{1}$ ). The genes that were commonly modulated in the response to drought or active PR are further analyzed below in this paper separately for WT and the Ljgln2-2 mutant plants.

\section{Analysis of the Gene Probesets Modulated By Drought or Active PR}

The 187 gene probesets that changed under the two types of stress situation in WT plants were represented in the context of general metabolism using the MapMan software (Supplemental Figure S2, see Supplemental Table S2 for a list of these gene probesets). The MapMan program allows the visualization of the changes observed in transcriptomic data by providing an overview of metabolic pathway responses. Each significantly modulated gene is indicated with a square within a box that represents the corresponding metabolic pathway. MapMan visualization indicated that the genes commonly affected by drought or active PR in the WT were quite dispersed through the metabolic map and no particular pathway seemed to be highly regulated. In contrast, some interesting correlations were observed in the analysis the group of 2173 gene probesets that were commonly modulated by drought or active PR in Ljgln2-2 mutant plants. First of all, it was shown that the great majority of these gene probesets (2030) changed in the same direction (i.e., were commonly induced or repressed) in response to either drought or active PR (Supplemental Figure S3). Of these, 1073 gene probesets were commonly induced while 957 were commonly repressed. Linear regression analysis indicated that the gene probesets that changed in the same direction under both types of stress conditions were slightly more modulated by drought compared to active PR (Supplemental Figure S3).

In order to get further insight into the convergent response of Ljgln2-2 to drought and active PR, the 1073 gene probesets that were commonly induced by drought or active PR treatments were visualized in the context of general metabolism using the MapMan software (Figure 1A). The 957 gene probesets that were repressed by both types of stress are represented in Figure 1B. Since the extent of change for these commonly induced and repressed gene probesets was different under either drought or active PR, a fold-change value of 2 and -2 was arbitrarily assigned for the MapMan representation of Figure 1. Several genes related to ascorbate and glutathione metabolism, sucrose degradation and phenylpropanoid metabolism were highly induced by both stress treatments (Figure 1A). On the other hand, the analysis of the genes commonly repressed by these two types of stress treatments showed a general repression of several pathways related to photosynthesis like the biosynthesis of the structural components of the photosystems, the Calvin cycle, photorespiration and genes related to carbohydrate degradation (Figure 1B). To determine which metabolic pathways were significantly over-represented among these groups of genes, the common gene probesets induced or repressed under these two types of stress treatments were further analyzed using Pathexpress (Goffard and Weiller, 2007). Pathexpress is a webbased tool that allows the identification of the most relevant sub-networks (metabolic pathways, sub-pathways, and enzyme neighborhood) associated with a set number of genes. An overrepresented pathway in a query list of gene probesets is identified by comparing the number of enzymes reactions of this route represented within the query list compared to the total number of enzyme reactions present in the pathway. The significance of this comparison is then tested using a hypergeometric distribution test as developed in the BlastSets system (Goffard and Weiller, 2007 and references therein). The analysis carried out confirmed that the most significantly repressed pathways were mainly related to photosynthesis and carbon metabolism. Among the most repressed routes were glycolysis, starch and sucrose metabolism, carbon fixation (essentially the Calvin cycle) as well as the biosynthesis of chlorophyll and photosynthetic pigments (Table 2). On the other hand, the most induced pathway by both stress treatments in the $\operatorname{Ljgln2-2}$ mutant was stilbene/lignin/coumarin biosynthesis, which comprises several branches of the complex route for the biosynthesis of phenolic compounds (Table 2). Flavonoid biosynthesis, another branch of plant phenolics metabolism, was also found among the most over-represented routes, as well as phenylalanine metabolism, where genes encoding for the enzymes for the first steps of the biosynthesis of phenolic compounds like phenylalanine ammonia lyase (PAL) and cinnamate 4-hydroxylase $(\mathrm{C} 4 \mathrm{H})$ were induced. In addition, it was also noted that one particular gene for phenolic metabolism, corresponding to isoflavone $2^{\prime}$ hydroxylase, was among the top 20 commonly induced genes under drought stress conditions in the Ljgln2-2 mutants, and was the most induced genes by active $\mathrm{PR}$ in this genotype (Supplemental Table S3).

Previous works have analyzed the consequences of the lack of $\mathrm{GS}_{2}$ in primary metabolism (Pérez-Delgado et al., 2013). Interestingly, the results obtained now in the present paper indicated that the biosynthesis of phenolic compounds was also greatly modulated in response to stress as a result of the absence of plastidic $\mathrm{GS}_{2}$ in the Ljgln2-2 mutant. The following sections of this paper analyze further in detail by qRT-PCR and metabolic profiling the different changes in phenolic metabolism associated with the lack of $\mathrm{GS}_{2}$ in response to drought or active PR stress conditions.

\section{Modulation of the Expression of Gene Probesets for the Biosynthesis of Phenolic Compounds}

A real-time qRT-PCR analysis was carried out to further investigate and validate the changes in the expression of genes for the biosynthesis of phenolic compounds that were found in the transcriptomics studies. Given the great complexity of the biosynthetic route of plant phenolics a focus was made on the key genes encoding for enzymes that catalyze the first common steps of the route, and also several ones for the central flavonoid biosynthetic pathway, for isoflavonoid biosynthesis as well as for the branch that lead to anthocyanins and protoanthocyanidins. Several of the enzyme activities of this pathway are encoded by large gene families like in the case of phenylalanine-ammonia lyase, encoded by 10 genes, and chalcone synthase encoded by 12 (Supplemental Table S4). Moreover, some of these gene families were organized in tandem repeats on the same chromosome like in the case of chalcones isomerase (Shimada et al., 2003), 


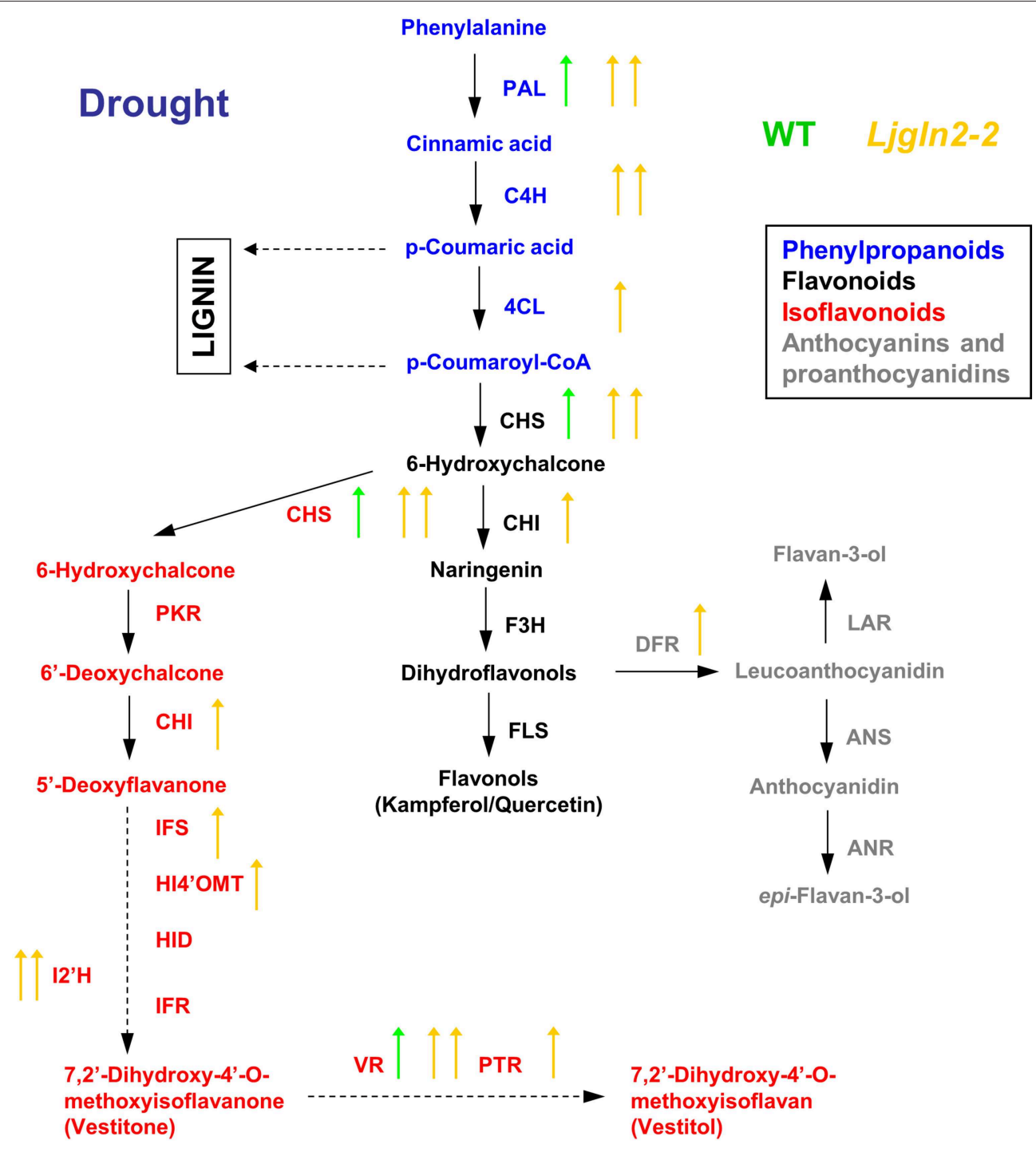

FIGURE 2 | Graphical summary of the changes in the expression of genes for the biosynthesis of phenolic compounds under drought conditions. qRT-PCR data are from Table 3. Arrows highlight significant changes in gene expression according to the analysis of Table 1; green and yellow represent WT and Ljgln2-2 plants respectively. Arrows pointing up mean induction and arrows pointing down mean repression; one arrow means moderate ( $<6$ times, up or down) modulation while two arrows mean high (>6 times, up or down) modulation. A color code has been used to emphasize different branches of the pathway: blue for the common initial steps for phenylpropanoid biosynthesis, black for some enzymes of the central flavonoid biosynthetic pathway, gray for some enzymes for anthocyanins and protoanthocyanidins and red for some enzymes for isoflavonoid biosynthesis. For space reasons several intermediates in the biosynthesis of isoflavonoids have been omitted. The genes measured encode for enzymes that belongs to: (1) the common "entry" to the pathway, that comprehends the genes encoding for PAL, C4H and 4CL; (2) the central flavonoid biosynthetic pathways including the two key enzymes for the formation of the cyclic flavonoid scaffold: CHS, $\mathrm{CHI}$, F3H, and FLS for the branch that leads to flavonols; (3) the first committed reactions for the biosynthesis of anthocyanins: DFR and ANS and for the biosynthesis of protoanthocyanidins (also known as condensed tannins): LAR and ANR; 4) the branch for the biosynthesis of isoflavonoids, that shares the enzyme activities CHS and $\mathrm{CHI}$ with the common flavonoid pathway, but also needs the activities of PKR, IFS, HI4'OMT, HID and I2' $\mathrm{H}$ and the "late" activities of this branch that lead to the formation of the phytoalexins vestitone: IFR and of the isoflavan vestitol: VR and PTR. For a detailed description of these steps see Davies and Schwinn (2006) and Shelton et al. (2012).

by active PR in both genotypes. Finally, several genes for isoflavonoids biosynthesis were induced under the two types of stress conditions that were examined. This induction was dramatic in the case of active PR, where all the genes for isoflavonoid biosynthesis were induced at least 9 times in the mutants and several of them were also induced, although to a lesser extent, in the WT. On the other hand, different genes for isoflavonoid biosynthesis were also induced by drought stress but exclusively in the mutant genotype.

Taken together, these results strongly suggest that the two types of stress situations analyzed in this paper may stimulate in different ways the production of phenolic compounds, 


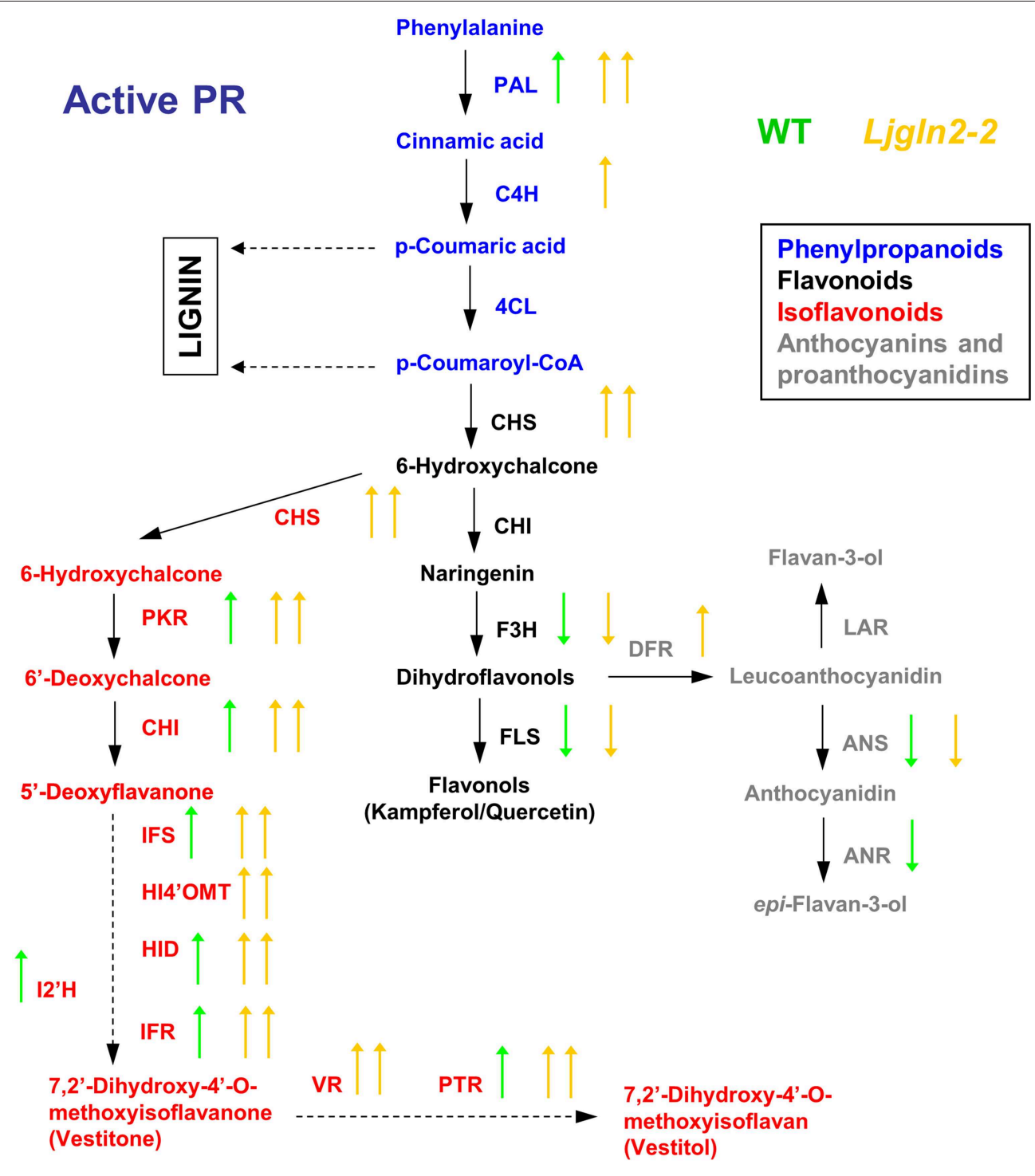

FIGURE 3 | Graphical summary of the changes in the expression of genes for the biosynthesis of phenolic compounds under active photorespiratory conditions. Other details as in the legend of Figure 2.

particularly in the Ljgln2-2 mutant genotype. Noteworthy, a much higher induction of specific genes for isoflavonoid biosynthesis was observed in the mutant plants compared to the WT, particularly under photorespiratory active conditions.

\section{Metabolite Profiling of Phenolic Compounds under Control and Stress Conditions}

To further explore the possible changes in phenolic metabolism in WT and Ljgln2-2 mutants under stress conditions a metabolic profiling of phenolic compounds was carried out in leaves from L. japonicus plants. In order to show the different metabolites identified, we present first, as an example, the analysis carried out by analytical HPLC for WT plants under control conditions and under drought stress conditions (Figure 4). Several peaks corresponding to phenolic metabolites were detected in the chromatograms obtained, which was in good agreement with previous reports from L. japonicus leaves (Suzuki et al., 2008). The analysis carried out allowed detecting all classes of phenolic compounds that may play a role in the response to stress: phenylpropanoids, flavonoids, isoflavonoids, and tannins, while anthocyanins were undetectable. The identity of the compounds was assigned based on their retention times and UV-VIS spectra and confirmed by LC/ESI-MS detection (Supplemental Table S5). Moreover, preparative HPLC was also used in order to purify the most abundant compounds of unknown chemical structure for their identification by NMR. A description of the NMR spectra and of their interpretation can be found online (Supplemental Figure S5). The most abundant flavonoids detected were kaempferol and quercetin, both belonging to the family of flavonols. Total kaempferol 
TABLE 3 | Changes in the relative expression levels of selected genes for the biosynthesis of phenolic compounds measured by qRT-PCR.

\begin{tabular}{|c|c|c|c|c|}
\hline & \multicolumn{4}{|c|}{ Fold change in gene expression } \\
\hline & \multicolumn{2}{|c|}{ Drought } & \multicolumn{2}{|c|}{ Active photorespiration } \\
\hline & WT & LjgIn2-2 & WT & LjgIn2-2 \\
\hline \multicolumn{5}{|c|}{ GENE PRODUCT } \\
\hline PAL & $3.06 \pm 1.15$ & $7.86 \pm 2.43$ & $1.90 \pm 0.59$ & $10.78 \pm 2.50$ \\
\hline $\mathrm{C} 4 \mathrm{H}$ & $2.39 \pm 0.78$ & $10.7 \pm 4.72$ & $1.21 \pm 1.16$ & $16.12 \pm 3.22$ \\
\hline $4 \mathrm{CL}$ & $1.09 \pm 0.62$ & $5.14 \pm 1.67$ & $1.29 \pm 0.41$ & $1.93 \pm 0.79$ \\
\hline $\mathrm{CHS}$ & $3.76 \pm 0.45$ & $9.59 \pm 2.04$ & $2.54 \pm 1.70$ & $13.85 \pm 5.12$ \\
\hline $\mathrm{CHI}$ & $1.52 \pm 0.51$ & $2.66 \pm 0.65$ & $2.02 \pm 0.40$ & $9.65 \pm 2.53$ \\
\hline $\mathrm{F} 3 \mathrm{H}$ & $0.86 \pm 0.36$ & $0.68 \pm 0.33$ & $0.58 \pm 0.08$ & $0.38 \pm 0.09$ \\
\hline FLS & $0.74 \pm 0.40$ & $0.78 \pm 0.40$ & $0.63 \pm 0.02$ & $0.55 \pm 0.01$ \\
\hline DFR & $1.70 \pm 1.12$ & $3.21 \pm 1.63$ & $0.83 \pm 0.20$ & $8.16 \pm 1.34$ \\
\hline ANS & $1.16 \pm 0.39$ & $1.28 \pm 0.58$ & $0.27 \pm 0.15$ & $0.41 \pm 0.18$ \\
\hline LAR & $1.06 \pm 0.21$ & $0.87 \pm 031$ & $0.40 \pm 0.22$ & $0.36 \pm 0.24$ \\
\hline ANR & $1.21 \pm 1.03$ & $1.34 \pm 0.96$ & $0.26 \pm 0.19$ & $0.71 \pm 0.33$ \\
\hline PKR & $1.16 \pm 0.77$ & $1.75 \pm 0.44$ & $2.68 \pm 1.58$ & $13.04 \pm 3.30$ \\
\hline IFS & $1.59 \pm 0.64$ & $5.06 \pm 2.13$ & $2.11 \pm 0.49$ & $8.85 \pm 2.45$ \\
\hline $\mathrm{HI} 4^{\prime} \mathrm{OMT}$ & $1.17 \pm 0.72$ & $2.08 \pm 0.42$ & $1.87 \pm 1.22$ & $13.42 \pm 6.61$ \\
\hline HID & $1.38 \pm 1.01$ & $1.64 \pm 1.10$ & $1.98 \pm 0.69$ & $13.67 \pm 5.74$ \\
\hline $12^{\prime} \mathrm{H}$ & $1.26 \pm 0.60$ & $8.16 \pm 3.12$ & $3.24 \pm 0.79$ & $17.47 \pm 7.16$ \\
\hline IFR & $1.69 \pm 0.98$ & $2.50 \pm 0.65$ & $1.19 \pm 0.38$ & $28.99 \pm 17.53$ \\
\hline VR & $2.95 \pm 1.54$ & $18.82 \pm 7.49$ & $2.50 \pm 0.89$ & $16.38 \pm 9.98$ \\
\hline PTR & $1.85 \pm 0.86$ & $2.94 \pm 1.01$ & $3.32 \pm 1.04$ & $22.25 \pm 11.32$ \\
\hline
\end{tabular}

The levels of transcript for each gene under control conditions in each genotype were taken as 1.00. Numbers in red mean significant difference between control and stress conditions for each genotype according to Student's $t$ test $(p<0.05)$. Transcript levels have been measured for three independent biological replicates. The gene products highlighted in blue belong to the entry of the pathway, black highlights the enzymes of the central flavonoid biosynthetic pathway, gray highlights enzymes for anthocyanins and protoanthocyanidins biosynthesis and in red are highlighted enzymes for isoflavonoid biosynthesis. The abbreviations for the enzyme activities are: $P A L$, phenylalanine-ammonia lyase; $4 C L$, 4-Coumarate:CoA ligase; $C 4 H$, cinnamate-4dehydrogenase; $\mathrm{CHS}$, chalcone synthase; $\mathrm{CHI}$, chalcone isomerase; $\mathrm{F} 3 \mathrm{H}$, flavanone $3 \beta$ hydroxylase; FLS, flavonol synthase; DFR, dihydroflavonol reductase; ANS, anthocyanin synthase; LAR, leucoanthocyanidin reductase; ANR, anthocyanidin reductase; PKR, polyketide reductase also called chalcone reductase; IFS, isoflavone synthase; HIL'OMT, 2,4,7'-Hydroxyisoflavanone 4'-O-methyltransferase; HID, isoflavanone dehydratase; $12^{\prime} \mathrm{H}$, isoflavone 2'-hydroxylase; IFR, isoflavone reductase; VR, vestitone reductase; and PTR, pterocarpan reductase. $\mathrm{CHS}$ and $\mathrm{CHI}$ are reported as belonging to the flavonoid biosynthetic pathway but are also fundamental for the biosynthesis of isoflavonoids. Expression data were normalized using the geometric mean of three housekeeping genes: L. japonicus protein phosphatase 2A (LjPp2A; chr2.CM0310.22), L. japonicus ubiquitin carrier protein 10 (LjUbc10; chr1.TM0487.4), and L. japonicus polyubiquitin 4 (LjUbq4; chr5.CM0956.27), that were selected among the most stably expressed genes in plants (Czechowski et al., 2004). Data are the mean \pm S.D. of three independent biological replicates.

and quercetin levels were determined by removing the sugar moieties from the flavonoid scaffold by acid hydrolysis. On the other hand, metabolite profiling without acid hydrolysis permitted the detection of several different kaempferol and quercetin glycosides (Figure 4). Two triple glycosides were found for the first time in L. japonicus leaves: kaempferol 3-O-glucosyl (1-2)-glucoside-7-O-rhamnoside and kaempferol 3-O-glucosyl (1-2)-galactoside-7-O-rhamnoside (Figure 5). Moreover, this is, to our knowledge, the first ever detection of kaempferol 3-O-glucosyl (1-2)-galactoside-7-O-rhamnoside in plants.

Interestingly, some differences in the basal levels of phenolic compounds were detected in control (non-stress) conditions between the WT and the Ljgln2-2 mutant plants (Table 4). The levels of kaempferol glycosides were generally lower in the mutant compared to the WT while quercetin glycosides tended to be higher in the mutant; however these differences were not usually higher than 2-fold (Table 4). These data indicate that the absence of plastidic $\mathrm{GS}_{2}$ has some minor effects in phenolic metabolism in L. japonicus plants under non-stress conditions.

Very remarkable changes in the levels of phenolic compounds were observed among WT and Ljgln2-2 mutant plants submitted to either drought or active PR conditions (Table 5). Representative elution profiles for WT under control and drought stress conditions are presented in Figure 4, while the elution profiles for WT under active PR and for the mutant plants under both types of stress situations are available online (Supplemental Figures S6-S8). The total kaempferol content was significantly increased in both genotypes by drought stress but not by active PR. Among the most abundant kaempferol-glycosides there was a significant increase in kaempferol-3,7-di-O-rhamnoside and of the triple glycoside kaempferol-3-O-glucosyl (1-2)-glucoside-7-O-rhamnoside in drought conditions in both genotypes, while no significant changes in total kaempferol or in specific kaempferol-glycosides were observed under active photorespiratory conditions. On the other hand, changes in quercetin levels were also observed under the two different stress conditions examined (Table 5). Total quercetin and different quercetin glycosides were dramatically increased in WT plants under drought stress but not on active PR. In addition, significant, although lower changes in total quercetin and in some quercetin glycosides were observed under both stress conditions in the case of $\operatorname{Lggln} 2-2$. A third, minor flavonol-like 6-deoxyglycoside was also found in leaves, and its levels did not change in any of the conditions considered. pcoumaric acid, the product of the $\mathrm{C} 4 \mathrm{H}$ enzyme, was substantially reduced by drought in the WT genotype. Therefore, under the same conditions massive increases in quercetin levels, as well as a smaller increase in kaempferol, were observed for WT plants.

Regarding isoflavonoids, vestitol was the most abundant isoflavan phytoalexin in leaves of Lotus as also reported by Lanot and Morris (2005). Important differences were observed among WT and mutant plants regarding vestitol. Vestitol levels were increased particularly in the mutant genotype under drought, and much more (around 9-fold) under active photorespiratory conditions (Table 5). This was in very good agreement with the high induction of the genes for isoflavonoids biosynthesis observed in Ljgln2-2 mutant plants under active PR, as described above. Nevertheless, a very slight but significant increase in vestitol was also observed in the WT as a consequence of the transfer to active photorespiratory conditions.

While many of the genes for the biosynthesis of anthocyanins and tannins were modulated (Table 3), no detectable levels of anthocyanins were found in the leaves of the samples used in this study (data not shown). This confirms previous reports that failed to detect anthocyanins in L. japonicus leaves despite of a 

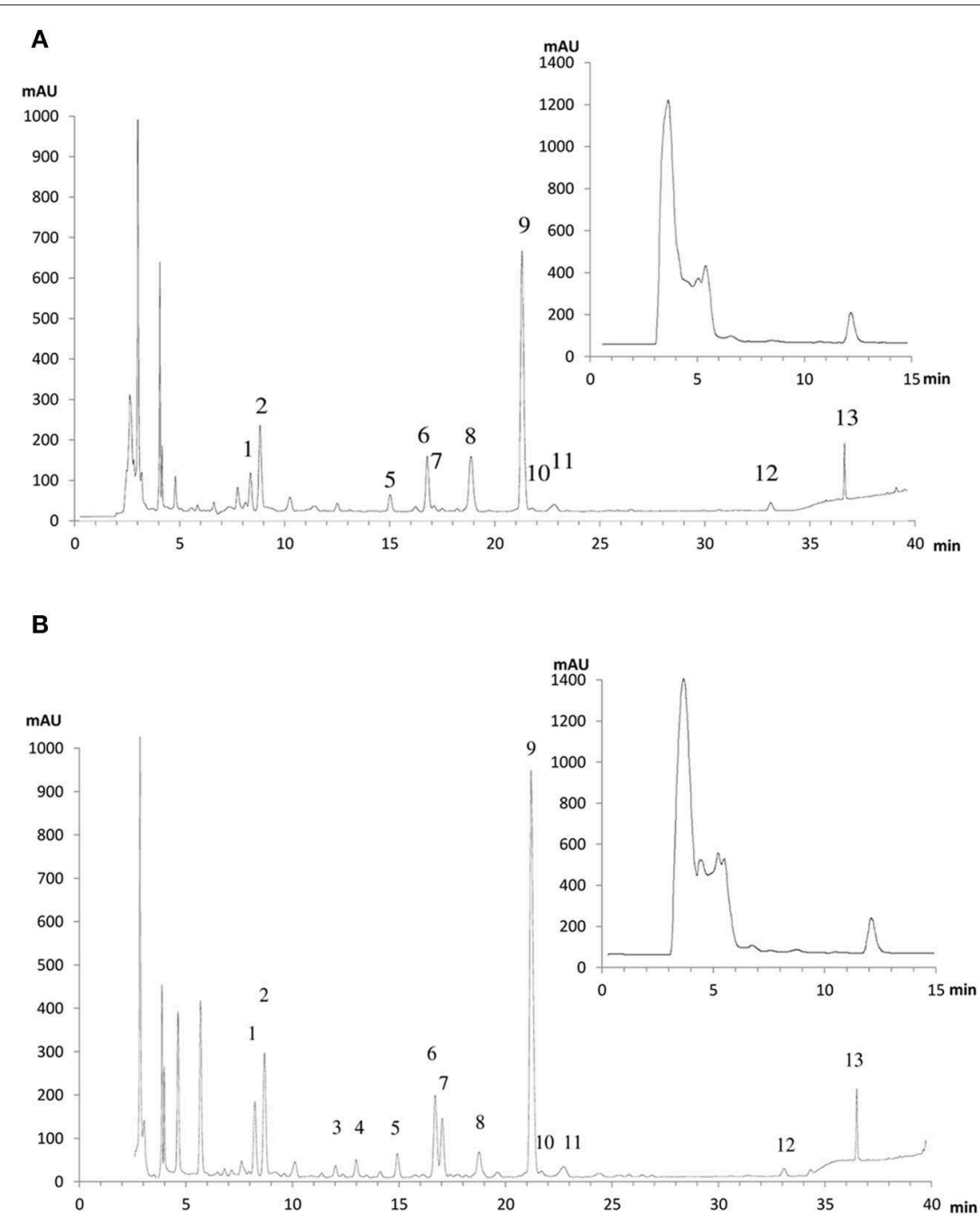

FIGURE 4 | HPLC elution profiles of flavonoids and isoflavonoidsfrom L. japonicus leaves of (A) WT plants under normal watering conditions and (B) WT plants under drought stress conditions. A typical chromatographic profile for $50 \%$ methanol leaf extracts from WT plants grown under either control conditions or drought stress conditions is presented. The compounds were detected according to their absorbance at $280 \mathrm{~nm}$. The peak numbers correspond to the metabolites: 1: kaempferol-3-O-glucosyl (1-2)-glucoside-7-O-rhamnoside; 2: kaempferol-3-O-glucosyl (1-2)-galactoside-7-O-rhamnoside; 3: quercetin

6-deoxyhexose-hexose; 4: quercetin 6-deoxyhexose-hexose; 5: kaempferol-3-O-galactosyl-7-O-rhamnoside; 6: kaempferol-3-O-glucosyl-7-O-rhamnoside; 7: quercetin 6-deoxyhexose-6-deoxyhexose; 8: p-coumaric acid; 9: kaempferol-3,7-di-O-rhamnoside; 10: p-ferulic acid; 11: simple phenylpropanoid; 12:

kaempferol-6-deoxyhexose; 13: flavonol-6-deoxyhexose. The chromatograms shown here are for only one of the six different biological replicates used in this work. For the relative quantification of metabolite levels presented in Table $\mathbf{5}$ the mean of all the six replicates was used. In the inset is the chromatographic profile for the $100 \%$ methanol leaf extracts, where the peak corresponding to vestitol can be seen, eluting at min 12.

high expression of the corresponding biosynthetic gene probesets (Shimada et al., 2005). The total tannin content did not change significantly in any condition (Table 5).

\section{Discussion}

The present work has shown the existence of important changes in phenolic metabolism as a result of $\mathrm{GS}_{2}$ deficiency in L. japonicus plants in response to stress. These changes were first detected from a transcriptomic analysis of the plants combined with further qRT-PCR validation, followed by phenolic metabolites profiling and characterization. Two different kinds of stress situations were examined: on the one hand, drought stress in the absence of active photorespiration, and, on the other hand, the stress produced as a result of the impairment of the photorespiratory cycle due to the lack of $\mathrm{GS}_{2}$, under normal watering. Transcriptomic analysis indicated the existence of a pattern of convergent responses in L. japonicus mutants lacking $\mathrm{GS}_{2}$ when comparing both types of stress situations, in spite of the fact that they are very different stress conditions. Therefore, the lack of $\mathrm{GS}_{2}$ must be associated with the common changes that were observed in both types of stress. Bioinformatics analysis indicated that the genes for the biosynthesis of phenolic compounds were over-represented 


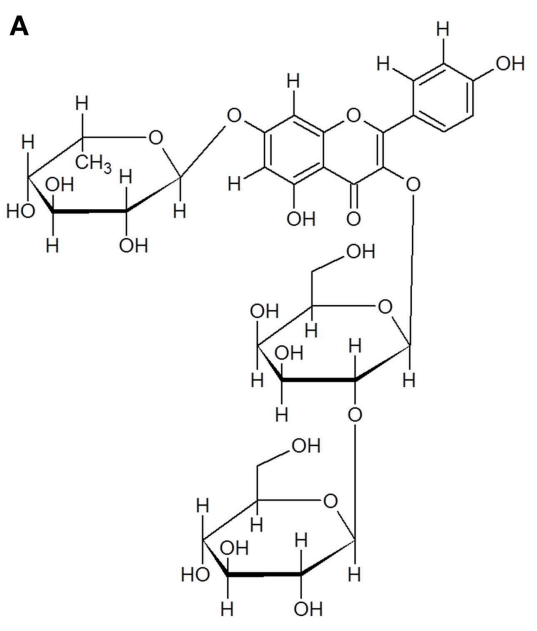

Kaempferol - glu-gal-rha

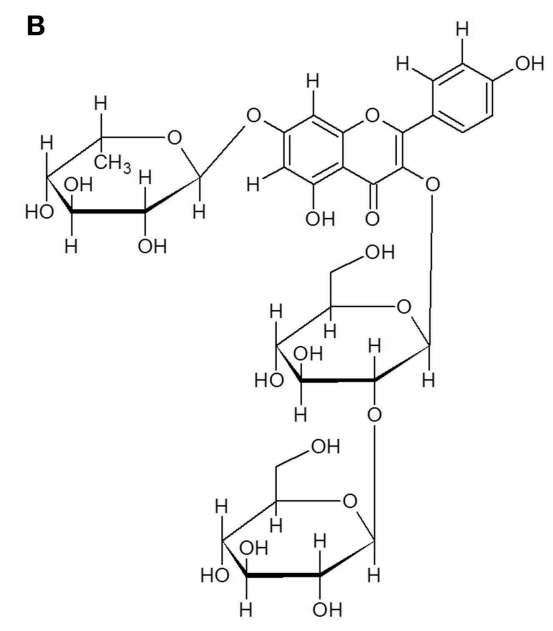

Kaempferol - glu-glu-rha

FIGURE 5 | Chemical structures of (A) kaempferol 3-O-glucosyl (1-2)-galactoside-7-O-rhamnoside and (B) kaempferol 3-O-glucosyl (1-2)-glucoside-7-O-rhamnoside. The compounds were purified from crude extracts of WT L. japonicus leaves from plants grown under control conditions by glass column chromatography followed by preparative HPLC. NMR determination of the chemical structure of the compounds was carried out as described in materials and methods.

among the group of genes that changed under both types of stress situations.

Phenylpropanoid and flavonoid biosynthesis were found to be generally stimulated in leaves of $L$. japonicus in stress, particularly as a result of $\mathrm{GS}_{2}$ deficiency. qRT-PCR analysis indicated that several genes for the biosynthesis of phenolic compounds were highly induced by both stress conditions, especially in the mutant. In fact, the gene probesets encoding for the enzymes that catalyze the "entry" reactions of the pathway $(\mathrm{PAL}, \mathrm{C} 4 \mathrm{H}$, and $4 \mathrm{CL}$ ) were highly induced in the mutant under both stress conditions.

Flavonols are probably the most important flavonoids participating in stress response and show a wide range of biological activities (Falcone Ferreyra et al., 2012). An increasing body of evidence is indicating that flavonols, especially those with dihydroxy-subsituted B-ring, play an antioxidant role in plant stress response (Pollastri and Tattini, 2011). In this paper it was observed that kaempferol and quercetine, the main flavonols detected in L. japonicus leaves, tended to accumulate in response to stress. In contrast, in control conditions, the levels of phenolic compounds was similar in the mutant plants than in the WT, except for some slight increase in quercetine glycosides in the mutant plants, that could be compensating for the also slight decrease observed in the mutants in some kaempferol glycosides. Flavonol accumulation may represent a defense against the increased oxidative stress produced by drought (that would affect both genotypes) or active PR (that affects mainly the Ljgln2-2 mutant). In fact, previous works have shown an increased level of oxidative stress in L. japonicus plants as a result of drought (Díaz et al., 2010) or active photorespiration in the mutant plants (Pérez-Delgado et al., 2013). Consistent with this theory,
WT plants under active photorespiratory conditions did not accumulate flavonoids, with the exception of a minor increase in vestitol levels. It is important to note that active photorespiration should not mean any stress situation for the WT plants since they have a normal operation of the photorespiratory cycle, in contrast with the Ljgln2-2 mutant plants, which have an impairment of the photorespiratory cycle due to the lack of $\mathrm{GS}_{2}$. However, drought stress produced a stress situation that was clearly apparent both in WT and mutant plants.

Several kaempferol glycosides were identified in the $L$. japonicus leaf extracts used in this work. Some of these compounds have been described in previous reports like Suzuki et al. (2008), including kaempferol-3,7-di-O-rhamnoside that was the most abundant kaempferol glycoside in L. japonicus leaves under control conditions. Suzuki et al. (2008) also detected kaempferol-3-O-glucosyl-7-O-rhamnoside and kaempferol-3O-galactosyl-7-O-rhamnoside. On the other hand, two triple glycosides of kaempferol were detected in this work and one of them, kaempferol-3-O-glucosyl (1-2)-glucoside-7-Orhamnoside, showed the higher change in relative metabolite content under stress. This particular triple glycoside has been also identified in Arabidopsis leaves (Kachlicki et al., 2008). The other kaempferol triple glycoside detected, kaempferol-3-Oglucosyl (1-2)-galactoside-7-O-rhamnoside, did not accumulate significantly under stress conditions and, to our knowledge, was detected for the first time in plants.

We call attention now to the fact that a massive increase of quercetine and a slight increase of kaempferol compounds were detected in the WT plants under stress conditions, whereas in the mutant, the main phenolic pathway that was altered was the biosynthesis and accumulation of isoflavonoids, 
TABLE 4 | Relative levels of phenolic compounds in WT and LjgIn2-2 leaves under control conditions (normal watering and suppressed PR).

Compound
kaempferol-3,7-di-O-rhamnoside ${ }^{a, b}$
kaempferol-3-O-glucosyl-7-O-rhamnoside ${ }^{a}$,
kaempferol-3-O-galactosyl-7-O-rhamnoside
kaempferol-3-O-glucosyl (1-2)-galactoside-7
kaempferol-3-O-glucosyl (1-2)-glucoside-7-O-r
kaempferol-6-deoxyhexose ${ }^{\text {b }}$
Total kaempferol (after acid hydrolysis)
Quercetin-6-deoxyhexose, -hexose ${ }^{b}$
Quercetin-6-deoxyhexose, -hexose
Quercetin-6-deoxyhexose, 6-deoxyhexose ${ }^{b}$
total quercetine (after acid hydrolysis)
flavonol-6-deoxyhexoseb
p-coumaric acid
p-ferulic acid
Simple phenylpropanoid
vestitol

LjgIn 2-2/WT

$0.82 \pm 0.28$

$0.65 \pm 0.20^{*}$

$0.70 \pm 0.23^{*}$

$0.69 \pm 0.23^{*}$

$1.10 \pm 0.62$

$0.56 \pm 0.33^{*}$

$0.97 \pm 0.34$

$2.46 \pm 1.53^{*}$

$1.57 \pm 1.10$

$1.44 \pm 0.98$

$1.87 \pm 0.91^{*}$

$0.60 \pm 0.25^{*}$

$0.64 \pm 0.41$

$0.94 \pm 0.51$

$0.76 \pm 0.48$

$1.12 \pm 0.34$

Total tannins

$1.05 \pm 0.15$

The differences in the relative levels of phenolic compounds between WT and Ljgln22 mutant plants are reported as the ratio between metabolite levels in Ljgln2-2 and the levels of the same metabolite in WT plants. Total crude extracts from leaves were obtained in 50\% methanol and analyzed by HPLC as described in materials and methods, with the exception of vestitol, that due to its low polarity was quantified in leaf extracts obtained in $100 \%$ methanol. The different compounds were identified in HPLC chromatograms employing DAD detector. Tannins were determined spectrophotometrically. Identities of compounds were verified by ${ }^{a} \mathrm{NMR}$ or ${ }^{b} \mathrm{LC} / \mathrm{ESI}-\mathrm{MS}$. For the compounds in italics it was not possible to determine their accurate chemical structure (e.g., for the two different quercetin-6-deoxyhexose,-hexoses isomers, that showed different retention times). Significant difference between levels in mutant and WT at ${ }^{*} p<0.05(n=4)$ according to Student's $t$-test. Data are the mean \pm S.D. of six independent biological replicates.

particularly under photorespiratory active conditions. This is in good agreement with the gene-expression data showing a strong induction of both PAL, which is the primary mediator of the flux toward the phenylpropanoid pathway under all stress conditions and of several genes encoding for enzymes of isoflavonoid pathway, mainly in the $\mathrm{GS}_{2}$ deficient mutant plants. The data obtained indicate that the increased flux toward the phenylpropanoid pathway is probably directed toward vestitol production in the mutant plants while in the WT this increased flux is probably aimed to increased flavonols production. In fact, accumulation of vestitol, the main isoflavonoid normally present in L. japonicus leaves (Lanot and Morris, 2005) was substantially increased in the mutant plants under the two types of stress conditions examined, most particularly under active photorespiration. Vestitol is a typical phytoalexin, mainly active in the response to pathogen attack (Shimada et al., 2000). However, there are also reports showing that, in legumes, accumulation of isoflavonoid phytoalexins and induction of their biosynthesis may also occur in different types of abiotic stresses such as UV-irradiation, drought or presence of heavy metals (Parry et al., 1994; Yamaguchi et al., 2010; Zavala et al., 2014). Interestingly, the previous work of Pérez-Delgado et al.
(2013) showed that active PR caused, in the Ljgln2-2 mutant, an increased production of $\mathrm{H}_{2} \mathrm{O}_{2}$ and an increase in transcript levels and enzyme activity of a glycolate oxidase isoform that was positively co-expressed with gene probesets involved in the response to biotic stress. Therefore, it is possible that the stress perceived by the mutant under active PR may mimic some stages of the signal transduction pathway that is elicited by biotic stress thus stimulating the biosynthesis of isoflavonoids over the flavonols. For soybean, a strong bias toward increasing the expression of gene probesets for isoflavonoid phytoalexin synthesis was documented after infection with Pseudomonas syringae, that was concomitant with some down regulation of gene probesets involved in the synthesis of other groups of flavonoids comprising flavonols, anthocyanins, and tannins (Zabala et al., 2006). Moreover, a high induction of several gene probesets for isoflavonoids biosynthesis was reported also by Shimada et al. (2007) and Shelton et al. (2012) when isoflavonoids production was elicited in L. japonicus leaves using reduced glutathione, a treatment that induces mainly gene probesets associated with the response to biotic challenges like the ones for phytoalexin production (Shimada et al., 2000; Foyer and Noctor, 2005; Shelton et al., 2012).

In summary, the results shown in the present paper indicate that the presence or absence of $\mathrm{GS}_{2}$ produce important differences in the different pathways for phenolics biosynthesis in L. japonicus in response to stress. While the presence of $\mathrm{GS}_{2}$ results in a high increase in flavonols (quercetine and/or kaempherol), as detected in the WT, the absence of $\mathrm{GS}_{2}$ results in a high increase in isoflavonoids (vestitol) in the mutant plants. Thus, it can be concluded a clear implication of $\mathrm{GS}_{2}$ in phenolic metabolism and in the different stress responses L. japonicus plants. The fact that isoflavonoid metabolism is particularly important in legume plants, in difference with other plants species such as Arabidopsis, makes the results obtained in L. japonicus of special interest. It is possible that $\mathrm{GS}_{2}$ may be connected with some type of regulatory network related to phenolic metabolism in L. japonicus plants. Previous works have established the crucial role of $\mathrm{GS}_{2}$ in the $\mathrm{C} / \mathrm{N}$ balance of L. japonicus plants (García-Calderón et al., 2012; Betti et al., 2014). On the other hand, different lines of evidence suggested that the response of Ljgln2-2 mutants to either drought stress or active photorespiration involve oxidative stress. The increase in thiobarbituric acid-reactive species observed in the mutant under drought conditions was significantly higher than in the WT (Díaz et al., 2010), indicating higher levels of oxidative stress in this genotype. In addition, the reactivation of the photorespiratory cycle was paralleled by accumulation of $\mathrm{H}_{2} \mathrm{O}_{2}$ exclusively in the mutant (Pérez-Delgado et al., 2013). Different kinds of abiotic stress are known to induce the accumulation of reactive oxygen species, and as a consequence of that, trigger several antioxidant defenses in the plant (Nakabayashi and Saito, 2015). Moreover, reactive oxygen species are also part of signaling cascades that modulate several signal transduction pathways related to abiotic stress (Jaspers and Kangasjärvi, 2010). Further work would be still required to analyze the regulatory networks and transcription factors that may regulate the different branches of the flavonoid pathway, a very actual 
TABLE 5 | Relative changes of phenolic compounds under drought or active photorespiratory conditions.

\begin{tabular}{|c|c|c|c|c|}
\hline Compound & \multicolumn{2}{|c|}{ Drought } & \multicolumn{2}{|c|}{ Active photorespiration } \\
\hline kaempferol-3,7-di-O-rhamnoside ${ }^{a, b}$ & $1.35 \pm 0.27^{\star}$ & $1.61 \pm 0.60^{*}$ & $1.05 \pm 0.11$ & $1.10 \pm 0.38$ \\
\hline kaempferol-3-O-glucosyl-7-O-rhamnoside ${ }^{a, b}$ & $1.22 \pm 0.19$ & $1.55 \pm 0.78$ & $0.96 \pm 0.18$ & $1.01 \pm 0.30$ \\
\hline kaempferol-3-O-galactosyl-7-O-rhamnoside ${ }^{\mathrm{b}}$ & $1.36 \pm 0.36^{\star}$ & $1.48 \pm 0.59$ & $1.14 \pm 0.28$ & $0.97 \pm 0.31$ \\
\hline kaempferol-3-O-glucosyl (1-2)-galactoside-7-O-rhamnoside ${ }^{a, b}$ & $1.09 \pm 0.28$ & $1.54 \pm 0.68$ & $0.96 \pm 0.20$ & $0.73 \pm 0.29$ \\
\hline Total kaempferol (after acid hydrolysis) & $1.37 \pm 0.31^{\star}$ & $1.24 \pm 0.21^{*}$ & $1.07 \pm 0.30$ & $1.31 \pm 0.53$ \\
\hline Quercetin-6-deoxyhexose, -hexose ${ }^{b}$ & $20.81 \pm 9.13^{\star \star}$ & $1.76 \pm 0.85^{\star}$ & $0.99 \pm 0.65$ & $1.08 \pm 0.51$ \\
\hline Quercetin-6-deoxyhexose, -hexose ${ }^{b}$ & $15.33 \pm 9.49^{\star \star}$ & $1.62 \pm 1.31$ & $1.23 \pm 0.83$ & $3.29 \pm 1.92^{\star}$ \\
\hline Quercetin-6-deoxyhexose, 6-deoxyhexose ${ }^{\mathrm{b}}$ & $8.78 \pm 6.24^{\star *}$ & $1.45 \pm 0.87$ & $0.96 \pm 0.64$ & $1.19 \pm 0.70$ \\
\hline Total quercetine (after acid hydrolysis) & $11.68 \pm 4.02^{\star *}$ & $2.63 \pm 0.50^{\star \star}$ & $0.86 \pm 0.45$ & $1.56 \pm 0.54^{*}$ \\
\hline Simple phenylpropanoid & $0.44 \pm 0.25^{\star \star}$ & $0.96 \pm 0.56$ & $1.03 \pm 0.38$ & $1.26 \pm 0.47$ \\
\hline Vestitol & $1.20 \pm 0.35$ & $2.08 \pm 0.52^{\star \star}$ & $1.24 \pm 0.15^{\star}$ & $8.94 \pm 4.13^{\star \star}$ \\
\hline Total tannins & $1.02 \pm 0.10$ & $1.20 \pm 0.28$ & $1.24 \pm 0.23$ & $1.19 \pm 0.10$ \\
\hline
\end{tabular}

The changes in the relative levels of phenolic compounds in the two genotypes as a consequence of drought or active PR are reported as the ratio between metabolite levels under control conditions (high $\mathrm{CO}_{2}$, normal watering) and metabolite levels under stress conditions. Total crude extracts from leaves were obtained in $50 \%$ methanol and analyzed by HPLC as described in materials and methods, with the exception of vestitol, that due to its low polarity was quantified in leaf extracts obtained in 100\% methanol. The different compounds were identified in HPLC chromatograms employing DAD detector, tannins were determined spectrophotometrically. Identities of compounds were verified by ${ }^{a} \mathrm{NMR}$ or ${ }^{b} \mathrm{LC} / \mathrm{ES} / \mathrm{-MS}$. For the compounds in italics it was not possible to determine their accurate chemical structure. Significant difference between levels in mutant and $W T$ at ${ }^{*} p<0.05$ or ${ }^{* *} p<0.01$ ( $n=4$ ) according to Student's t-test. Data are the mean \pm S.D. of six independent biological replicates.

and interesting topic that needs further investigation especially in legumes.

The results presented here constitute a substantial advance in the study of the response of the model legume L. japonicus to different stress situations, making use for this purpose of Ljgln22 photorespiratory mutants deficient in $\mathrm{GS}_{2}$. Transcriptomic analysis and qRT-PCR studies revealed the stimulation of the expression of several genes for the biosynthesis of phenolic compounds under stress conditions, particularly in the mutant plants. A differential response among WT and mutant plants was observed regarding the biosynthesis and accumulation of different branches of flavonoids such as flavonols (quercetin/kaempferol) or isoflavonoids (vestitol). In addition, accumulation of several flavonol glycosides, some of them described for the first time in here, was also observed. Therefore, the results obtained constitute very novel and interesting findings which point out a crucial relevance of $\mathrm{GS}_{2}$ in relation to phenolic metabolism and stress responses of $L$. japonicus plants, thus providing a very nice example of functions beyond primary metabolism of the amino acids of the glutamate family, which is the research topic that was analyzed.

\section{Author Contributions}

The experiments were conceived and designed by PP, JV, MR, $\mathrm{AM}$, and MB. The experiments were carried out by MG, TP, AM, $\mathrm{CP}, \mathrm{MV}$, and AE. The data were analyzed by PP, MR, CP, MV, and $\mathrm{MB}$. The paper was written by $\mathrm{PP}, \mathrm{AM}$, and MB.

\section{Acknowledgments}

We acknowledge financial support given by projects AGL2014-54413-R from FEDER-Ministerio de Economía y Competitividad, Spain, and P1O-CVI-6368 and BIO-163, from Consejería de Economía, Innovación y Ciencia, Junta de Andalucía, as well as the European project EXPERT (ITMS code 26110230056), VEGA project 1/0046/4 from Ministry of Education, Science, Research and Sport of the Slovak Republic, and a V Plan Propio fellowship to CP from the University of Seville. TP acknowledges the Educational, Audiovisual and Cultural Executive Agency (EACEA) of the European Commission for a postgraduate fellowship for the Master Erasmus Mundus in Forensic Science.

\section{Supplementary Material}

The Supplementary Material for this article can be found online at: http://journal.frontiersin.org/article/10.3389/fpls.2015. 00760

Supplemental Figure S1 | Experimental design used in this work. WT and Ljgln2-2 mutant plants were grown under high $\mathrm{CO}_{2}(0.7 \% \mathrm{v} / \mathrm{v})$ conditions and watered with Hornum medium. After 35 days leaf samples were harvested from both genotypes, constituting the control condition for both experiments. For drought treatment, plants were kept under high $\mathrm{CO}_{2}$ conditions and watering was withheld for 4 days, after which leaf samples were harvested. For active PR treatment, plants were transferred for 2 days to a normal $\mathrm{CO}_{2}(0.04 \% \mathrm{v} / \mathrm{v})$ atmosphere and leaf samples were harvested. More details in materials and methods. 
Supplemental Figure S2 | Mapman overview of general metabolism of the gene probesets that changed under both types of stress situations in WT plants. Since the change in gene expression levels for these gene probesets was different in the response to drought and active PR, an arbitrary fold-change of 2 was imposed. More details about the graphical representation of genes in the context of metabolic pathways used by the MapMan software can be found in the legend of Figure 1.

Supplemental Figure S3 | Relationship of the fold-change in gene expression levels produced by drought or active photorespiratory conditions for the different gene probesets modulated exclusively in the LjgIn2-2 mutant. (A) Graphical representation of the $\log _{2}$ of the fold-change for all the 2173 gene probesets that were modulated exclusively in the mutant under drought or active PR conditions. (B) Gene probesets that changed in the same direction (commonly induced or repressed) under drought or active photorespiratory conditions. The linear regression analysis carried out gave a $\mathrm{r}^{2}$ value of 0.80 and a slope of 0.60 . (C) Gene probesets that changed in opposite directions in response to drought and active PR. The linear regression analysis carried out gave $\mathrm{r}^{2}$ value of 0.77 and a slope of -0.52 .

Supplemental Figure S4 | Comparison of microarray and qRT-PCR data for genes involved in the biosynthesis of phenolic compounds. The points plotted in this graph represent the $\log _{2}$ of the fold-change for different gene probesets involved in the biosynthesis of phenolic compounds that were significantly modulated according to both techniques. The data points plotted are from both WT and Ljgln2-2 genotypes and both drought or active photorespiratory conditions. Linear regression analysis of microarray data versus QRT-PCR data gave a regression coefficient of $r^{2}=0.92$ and a slope of 0.81 .

Supplemental Figure S5 | Data of the NMR spectra used for the identification of the kaempferol glycosides detected in this work.

Supplemental Figure S6 | HPLC elution profiles of flavonoids from L. japonicus leaves of (A) WT plants under high $\mathrm{CO}_{2}$ conditions and (B) WT plants after 2 days under normal $\mathrm{CO}_{2}$ conditions. The compounds were detected according to their absorbance at $280 \mathrm{~nm}$. Correspondence between peak numbers and metabolites as described in the legend of Figure 4 . The chromatograms shown here are for only one of the six different biological replicates used in this work. For the relative quantification of metabolite levels presented in Table $\mathbf{5}$ the mean of all the six replicates was used.

Supplemental Figure S7 | HPLC elution profiles of flavonoids from L. japonicus leaves of (A) Ljgln2-2 plants under normal watering conditions and (B) LjgIn2-2 plants under drought stress conditions. The compounds

\section{References}

Bauer, D., Biehler, K., Fock, H., Carrayol, E., Hirel, B., Miggie, A., et al. (1997). A role for cytosolic glutamine synthetase in the remobilization of leaf nitrogen during water stress in tomato. Physiol. Plant. 99, 241-247. doi: 10.1111/j.13993054.1997.tb05408.x

Betti, M., García-Calderón, M., Pérez-Delgado, C. M., Credali, A., Estivill, G., Galván, F., et al. (2012). Glutamine synthetase in legumes: recent advances in enzyme structure and functional genomics. Int. J. Mol. Sci. 13, 7994-8024. doi: 10.3390/ijms13077994

Betti, M., García-Calderón, M., Pérez-Delgado, C. M., Credali, A., Pal'ove-Balang, P., Estivill, G., et al. (2014). Reassimilation of ammonium in Lotus japonicus. J. Exp. Bot. 65, 5557-5566. doi: 10.1093/jxb/eru260

Brugière, N., Dubois, F., Limami, A. M., Lelandais, M., Roux, Y., Sangwan, R. S., et al. (1999). Glutamine synthetase in the phloem plays a major role in controlling proline production. Plant Cell 11, 1995-2011. doi: 10.1105/tpc.11.10.1995

Cheynier, V., Comte, G., Davies, K. M., Lattanzio, V., and Martens, S. (2013). Plant phenolics: recent advances on their biosynthesis, genetics, and ecophysiology. Plant Physiol. Biochem. 72, 1-20. doi: 10.1016/j.plaphy.2013. 05.009

Czechowski, T., Bari, R. P., Stitt, M., Scheible, W. R., and Udvardi, M. K. (2004). Real-time RT-PCR profiling of over 1400 Arabidopsis transcription factors: were detected according to their absorbance at $280 \mathrm{~nm}$. Correspondence between peak numbers and metabolites as described in the legend of Figure 4. The chromatograms shown here are for only one of the six different biological replicates used in this work. For the relative quantification of metabolite levels presented in Table 5 the mean of all the six replicates was used.

\section{Supplemental Figure S8 | HPLC elution profiles of flavonoids from} L. japonicus leaves of (A) Ljgln2-2 plants under high $\mathrm{CO}_{2}$ conditions and (B) LjgIn2-2 plants after 2 days under normal $\mathrm{CO}_{2}$ conditions. The compounds were detected according to their absorbance at $280 \mathrm{~nm}$. Correspondence between peak numbers and metabolites as described in the legend of Figure 4. The chromatograms shown here are for only one of the six different biological replicates used in this work. For the relative quantification of metabolite levels presented in Table $\mathbf{5}$ the mean of all the six replicates was used.

Supplemental Table S1 | Oligonucleotides used for qRT-PCR analysis. The gene copy amplified by each oligonucleotide pair is indicated. In some cases, more than one gene copy was amplified by the oligonucleotides due the high homology among different members of the gene family; in this case the different gene copies recognized are specified in the column "Gene(s) amplified." For an exhaustive list of the gene copies encoding for each of the enzymes considered in this work see Supplemental Table S4.

Supplemental Table S2 | List of the gene probesets that changed under the two types of stress situations in WT and mutant plants. The list of the 187 gene probesets that changed under both types of stress treatments in the WT plants and of the 2173 that changed under both types of stress treatments in the mutant are presented together with a description of the product of each gene.

Supplemental Table S3 | Top 20 genes that were induced bydrought or active photorespiration in the LjgIn2-2 mutant. The highlighted genes are those that were in common among the the list of 20 most induced genes that were observed in the drought stress or active PR treatments.

Supplemental Table S4 | List of the available gene sequences for the biosynthesis of phenolic compounds found in the current release of the L. japonicus genome. Data mining was carried out in the 2.5 release of L. japonicus genome at the Kazusa institute database (http://www.kazusa.or.jp/ lotus/). The gene names for the members of each gene family were defined in this work or, when available, taken from the bibliography.

Supplemental Table S5 | LC/ESI-MS data of flavonol glycosides identified in $\mathbf{5 0} \%$ methanol extracts from $L$. japonicus leaves. Correspondence between peak numbers and metabolites as described in the legend of Figure 5. unprecedented sensitivity reveals novel root- and shoot-specific genes. Plant J. 38, 366-379. doi: 10.1111/j.1365-313X.2004.02051.x

Davies, K. M., and Schwinn, K. E. (2006). "Molecular biology and biotechnology of flavonoid biosynthesis," in Flavonoids: Chemistry, Biochemistry and Applications, ed Ø. M. Andersen and K. R. Markham (Boca Ratón, FL: Taylor \& Francis Group), 143-218.

Díaz, P., Betti, M., Sánchez, D. H., Udvardi, M. K, Monza, J., and Márquez, A. J. (2010). Deficiency in plastidic glutamine synthetase alters proline metabolism and transcriptomic response in Lotus japonicus under drought stress. New Phytol. 188, 1001-1013. doi: 10.1111/j.1469-8137.2010.03440.x

Falcone Ferreyra, M. L., Rius, S. P., and Casati, P. (2012). Flavonoids: biosynthesis, biological functions, and biotechnological applications. Front. Plant Sci. 3:222. doi: $10.3389 /$ fpls.2012.00222

Foyer, C. H., and Noctor, G. (2005). Oxidant and antioxidant signalling in plants: a re-evaluation of the concept of oxidative stress in a physiological context. Plant Cell Environ. 28, 1056-1071. doi: 10.1111/j.1365-3040.2005.01327.x

García-Calderón, M., Chiurazzi, M., Espuny, M. R., and Márquez, A. J. (2012). Photorespiratory metabolism and nodule function: behavior of Lotus japonicus mutants deficient in plastid glutamine synthetase. Mol. Plant-Microbe Interact. 25, 211-219. doi: 10.1094/MPMI-07-11-0200

Goffard, N., and Weiller, G. (2007). PathExpress: a web-based tool to identify relevant pathways in gene expression data. Nucleic Acids Res. 35, W176-W818. doi: $10.1093 / \mathrm{nar} / \mathrm{gkm} 261$ 
Handberg, K., and Stougaard, J. (1992). Lotus japonicus, an autogamous, diploid legume species for classical and molecular genetics. Plant J. 2, 487-496. doi: 10.1111/j.1365-313X.1992.00487.x

Heigl, D., and Franz, G. (2003). Stability testing on typical flavonoid containing herbal drugs. Parmazie 58, 881-885.

Hoshida, H., Tanaka, Y., Hibino, T., Hayashi, Y., Tanaka, A., Takabe, T., et al. (2000). Enhanced tolerance to salt stress in transgenic rice that overexpress chloroplast glutamine synthetase. Plant Mol. Biol. 43, 103-111. doi: 10.1023/A:1006408712416

Jaspers, P., and Kangasjärvi, J. (2010). Reactive oxygen species in abiotic stress signalling. Physiol. Plant. 138, 405-413. doi: 10.1111/j.1399-3054.2009.01321.x

Kachlicki, P., Einhorn, J., Muth, D., Kerhoas, L., and Stobiecki, M. (2008). Evaluation of glycosylation and malonylation patterns in flavonoid glycosides during LC/MS/MS metabolite profiling. J. Mass Spectrom. 43, 572-586. doi: 10.1002/jms.1344

Kozaki, A., and Takeba, G. (1996). Photorespiration protects C3 plants from photooxidation. Nature 384, 557-560. doi: 10.1038/384557a0

Lanot, A., and Morris, P. (2005). "Elicitation of isoflavan phytoalexins," in Lotus japonicus Handbook, ed A. J. Márquez (Dordrecht: Springer), 355-361. doi: 10.1007/1-4020-3735-X_35

Nakabayashi, R., Yonekura-Sakakibara, K., Urano, K., Suzuki, M., Yamada, Y., Nishizawa, T., et al. (2014). Enhancement of oxidative and drought tolerance in Arabidopsis by overaccumulation of antioxidant flavonoids. Plant J. 77, 367-379. doi: $10.1111 /$ tpj. 12388

Nakabayashi, R., and Saito, K. (2015). Integrated metabolomics for abiotic stress responses in plants. Curr. Opin. Plant Biol. 24, 10-16. doi: 10.1016/j.pbi.2015.01.003

Orea, A., Pajuelo, P., Pajuelo, E., Quidiello, C., Romero, J. M., and Márquez, A. J. (2002). Isolation of photorespiratory mutants from Lotus japonicus deficient in glutamine synthetase. Physiol. Plant. 115, 352-361. doi: 10.1034/j.13993054.2002.1150304.x

Pal'ove-Balang, P., Betti, M., Díaz, P., Pérez-Delgado, C. M., García-Calderón, M., Monza, J., et al. (2014). "Abiotic stress in Lotus: aluminium and drought," in Molecular Approaches in Plant Abiotic Stress, eds R. K. Gaur and P. Sharma (Boca Raton, FL: CRC Press), 284-303.

Parry, A. D., Tiller, S. A., and Edwards, R. (1994). The effects of heavy metals and root immersion on isoflavonoid metabolism in alfalfa (Medicago sativa). Plant Physiol. 106, 195-202.

Pérez-Delgado, C. M., García-Calderón, M., Sánchez, D. H., Udvardi, M. K., Kopka, J., Márquez, A. J., et al. (2013). Transcriptomic and metabolic changes associated with photorespiratory ammonium accumulation in the model legume Lotus japonicus. Plant Physiol. 162, 1834-1848. doi: $10.1104 /$ pp.113.217216

Pollastri, S., and Tattini, M. (2011). Flavonols: old compounds for old roles. Ann. Bot. 108, 1225-1233. doi: 10.1093/aob/mcr234

Sánchez, D. H., Lippold, F., Redestig, H., Hannah, M. A., Erban, A., Krämer, U., et al. (2008). Integrative functional genomics of salt acclimatation in the model legume Lotus japonicus. Plant J. 53, 973-987. doi: 10.1111/j.1365313X.2007.03381.x

Saito, K., Yonekura-Sakakibara, K., Nakabayashi, R., Higashi, Y., Yamazaki, M., Tohge, T., et al. (2013). The flavonoid biosynthetic pathway in Arabidopsis: structural and genetic diversity. Plant Physiol. Biochem. 72, 21-34. doi: 10.1016/j.plaphy.2013.02.001

Shelton, D., Stranne, M., Mikkelsen, L., Pakseresht, N., Welham, T., Hiraka, H., et al. (2012). Transcription Factors of Lotus: regulation of isoflavonoid biosynthesis requires coordinated changes in transcription factor activity. Plant Physiol. 159, 531-547. doi: 10.1104/pp.112.194753
Shimada, N., Akashi, T., Aoki, T., and Ayabe, S.-I. (2000). Induction of isoflavonoids pathway in the model legume Lotus japonicus: molecular characterization of enzymes involved in phytoalexin biosynthesis. Plant Sci. 160, 37-47. doi: 10.1016/S0168-9452(00)00355-1

Shimada, N., Aoki, T., Sato, S., Nakamura, Y., Tabata, S., and Ayabe, S.-I. (2003). A cluster of genes encodes the two types of chalcone isomerase involved in the biosynthesis of general flavonoids and legume-specific 5-deoxy(iso)flavonoids in Lotus japonicus. Plant Physiol. 131, 941-951. doi: 10.1104/pp. 004820

Shimada, N., Sasaki, R., Sato, S., Kaneko, T., Tabata, S., Aoki, T., et al. (2005). A comprehensive analysis of six dihydroflavonol 4-reductases encoded by a gene cluster of the Lotus japonicus genome. J. Exp. Bot. 419, 2573-2585. doi: $10.1093 / \mathrm{jxb} / \mathrm{eri} 251$

Shimada, N., Sato, S., Akashi, T., Nakamura, Y., Tabata, S., Ayabe, S.-I., et al. (2007). Genome-wide analyses of the structural gene families involved in the legume-specific 5-deoxyisoflavonoid biosynthesis of Lotus japonicus. DNA Res. 14, 25-36. doi: 10.1093/dnares/dsm004

Suzuki, H., Sasaki, R., Ogata, Y., Nakamura, Y., Sakurai, N., Kitajima, M., et al. (2008). Metabolic profiling of flavonoids in Lotus japonicus using liquid chromatography Fourier transform ion cyclotron resonance mass spectrometry. Phytochemistry 69, 99-111. doi: 10.1016/j.phytochem.2007.06.017

Usadel, B., Nagel, A., Thimm, O., Redestig, H., Blaesing, O. E., Palacios-Rojas, N., et al. (2005). Extension of the visualization tool MapMan to allow statistical analysis of arrays, display of corresponding genes, and comparison with known responses. Plant Physiol. 138, 1195-1204. doi: 10.1104/pp.105.060459

Winkel-Shirley, B. (2002). Biosynthesis of flavonoids and effect of stress. Curr. Opin. Plant Biol. 5, 218-223. doi: 10.1016/S1369-5266(02)00256-X

Yamaguchi, M., Valliyodan, B., Zhang, J., Lenoble, M. E., Yu, O., Rogers, E. E., et al. (2010). Regulation of growth response to water stress in the soybean primary root. I. Proteomic analysis reveals region-specific regulation of phenylpropanoid metabolism and control of free iron in the elongation zone. Plant Cell Environ. 33, 223-243. doi: 10.1111/j.1365-3040.2009.02073.x

Zabala, G., Zou, J., Tuteja, J., Gonzalez, D. O., Clough, S. J., and Vodkin, L. O. (2006). Transcriptome changes in the phenylpropanoid pathway of Glycine max in response to Pseudomonas syringae infection. BMC Plant Biol. 6:26. doi: 10.1186/1471-2229-6-26

Zavala, J. A., Mazza, C. A., Dillon, F. M., Chludil, H. D., and Ballaré, C. L. (2014). Soybean resistance to stink bugs (Nezara viridula and Piezodorus guildinii) increases with exposure to solar UV-B radiation and correlates with isoflavonoids content in pods under field conditions. Plant Cell Environ. 38, 920-928. doi: 10.1111/pce.12368

Zhang, X., and Liu, C.-J. (2015). Multifaceted regulations of gateway enzyme phenylalanine-ammonia lyase in the biosynthesis of phenylpropanoids. Mol. Plant 8, 17-27. doi: 10.1016/j.molp.2014.11.001

Conflict of Interest Statement: The authors declare that the research was conducted in the absence of any commercial or financial relationships that could be construed as a potential conflict of interest.

Copyright (C) 2015 García-Calderón, Pons-Ferrer, Mrázova, Pal'ove-Balang, Vilková, Pérez-Delgado, Vega, Eliášová, Repčák, Márquez and Betti. This is an open-access article distributed under the terms of the Creative Commons Attribution License (CC $B Y)$. The use, distribution or reproduction in other forums is permitted, provided the original author(s) or licensor are credited and that the original publication in this journal is cited, in accordance with accepted academic practice. No use, distribution or reproduction is permitted which does not comply with these terms. 


\section{OPEN ACCESS}

Edited by:

Wim Van den Ende, KU Leuven, Belgium

Reviewed by:

Iwona M. Morkunas, Poznań University of Life Sciences,

Poland

Pierre Carol,

Université Pierre et Marie Curie,

France

*Correspondence: Dietmar Funck,

Laboratory of Plant Physiology

and Biochemistry, Department of Biology, University of Konstanz,

Universitätsstraße 10,

78457 Konstanz, Germany dietmar.funck@uni-konstanz.de

Specialty section:

This article was submitted to Plant Physiology,

a section of the journal Frontiers in Plant Science

Received: 30 April 2015 Accepted: 29 June 2015 Published: 30 July 2015

Citation:

Winter G, Todd CD, Trovato M, Forlani $G$ and Funck D (2015) Physiological implications of arginine metabolism

in plants.

Front. Plant Sci. 6:534. doi: 10.3389/fpls.2015.00534

\section{Physiological implications of arginine metabolism in plants}

\author{
Gudrun Winter ${ }^{1}$, Christopher D. Todd ${ }^{2}$, Maurizio Trovato ${ }^{3}$, Giuseppe Forlani ${ }^{4}$ and \\ Dietmar Funck ${ }^{1 *}$
}

1 Laboratory of Plant Physiology and Biochemistry, Department of Biology, University of Konstanz, Konstanz, Germany, ${ }^{2}$ Department of Biology, University of Saskatchewan, Saskatoon, SK, Canada, ${ }^{3}$ Department of Biology and Biotechnology, Sapienza University of Rome, Rome, Italy, ${ }^{4}$ Laboratory of Plant Physiology and Biochemistry, Department of Life Science and Biotechnology, University of Ferrara, Ferrara, Italy

Nitrogen is a limiting resource for plant growth in most terrestrial habitats since large amounts of nitrogen are needed to synthesize nucleic acids and proteins. Among the 21 proteinogenic amino acids, arginine has the highest nitrogen to carbon ratio, which makes it especially suitable as a storage form of organic nitrogen. Synthesis in chloroplasts via ornithine is apparently the only operational pathway to provide arginine in plants, and the rate of arginine synthesis is tightly regulated by various feedback mechanisms in accordance with the overall nutritional status. While several steps of arginine biosynthesis still remain poorly characterized in plants, much wider attention has been paid to inter- and intracellular arginine transport as well as arginine-derived metabolites. A role of arginine as alternative source besides glutamate for proline biosynthesis is still discussed controversially and may be prevented by differential subcellular localization of enzymes. Apparently, arginine is a precursor for nitric oxide (NO), although the molecular mechanism of NO production from arginine remains unclear in higher plants. In contrast, conversion of arginine to polyamines is well documented, and in several plant species also ornithine can serve as a precursor for polyamines. Both NO and polyamines play crucial roles in regulating developmental processes as well as responses to biotic and abiotic stress. It is thus conceivable that arginine catabolism serves on the one hand to mobilize nitrogen storages, while on the other hand it may be used to finetune development and defense mechanisms against stress. This review summarizes the recent advances in our knowledge about arginine metabolism, with a special focus on the model plant Arabidopsis thaliana, and pinpoints still unresolved critical questions.

Keywords: arginine, arginine biosynthesis, arginase, ornithine aminotransferase, urease, polyamines, nitric oxide

\section{Introduction}

Plant growth is often limited by the availability of nutrients. In many cases nitrogen is the limiting essential element. Nitrogen shortage causes detrimental effects on agricultural productivity, yet excessive nitrogen fertilization accounts for negative economic and environmental impacts. Improving nitrogen use efficiency represents a main challenge for agriculture, and it becomes increasingly important to investigate the mechanisms of nitrogen uptake, storage and recycling and to understand the interplay of these processes with the regulation of plant development and stress defense. 
Due to the highest nitrogen to carbon ratio among the 21 proteinogenic amino acids, arginine is a major storage and transport form for organic nitrogen in plants in addition to its role as an amino acid for protein synthesis, a precursor for polyamines and nitric oxide (NO) and an essential metabolite for many cellular and developmental processes. In seed proteins of different plant species $40-50 \%$ of the total nitrogen reserve is represented by arginine (VanEtten et al., 1963; King and Gifford, 1997), and this amino acid accounts for $50 \%$ of the nitrogen in the free amino acid pool in developing embryos of soybean (Micallef and Shelp, 1989) and pea (de Ruiter and Kollöffel, 1983). Arginine is often a major nitrogen storage form also in underground storage organs and roots of trees and other plants (Nordin and Näsholm, 1997; Bausenwein et al., 2001; Rennenberg et al., 2010). Therefore, arginine metabolism plays a key role in nitrogen distribution and recycling in plants (Slocum, 2005).

Slocum (2005) reviewed those genes that have been identified as encoding enzymes involved in arginine synthesis in Arabidopsis (Arabidopsis thaliana) and presented the current state of their characterization, including subcellular targeting, gene expression, available mutants and cDNAs of each enzyme. Over the past 10 years, research mainly on Arabidopsis as model plant has generated significant progress in our understanding of arginine metabolism, whereas several crucial questions remain unanswered. The present review highlights challenges for future research on plant arginine metabolism by summarizing recent advances about biosynthesis, distribution and catabolism of arginine and its contribution to polyamine and NO synthesis.

\section{Arginine Biosynthesis}

The biosynthetic pathway of arginine can be divided in two processes. First, ornithine is synthesized from glutamate either in a cyclic or a linear pathway, followed by the synthesis of arginine from ornithine.

\section{Cyclic and Linear Pathways for Ornithine Synthesis}

Ornithine is synthesized from glutamate via several acetylated intermediates (Figure 1). In the first step, $N$-acetylglutamate synthase (NAGS) uses acetyl-coenzyme A (Acetyl-CoA) to transfer an acetyl moiety to glutamate forming $N$-acetylglutamate (Slocum, 2005). $\mathrm{N}$-acetylglutamate is then phosphorylated at the C5 position by $\mathrm{N}$-acetylglutamate kinase (NAGK). The next step, the formation of $N$-acetylglutamate-5-semialdehyde (NAcGSA), is catalyzed by $N$-acetylglutamate-5-P reductase (NAGPR). In the fourth step, an amino group is transferred from a second glutamate molecule to $N$-acetylglutamate5-semialdehyde by $N^{2}$-acetylornithine aminotransferase (NAOAT), yielding $N^{2}$-acetylornithine. Subsequently, ornithine is released by transferring the acetyl residue to glutamate by $N$-acetylornithine: $N$-acetylglutamate acetyltransferase (NAOGAcT), giving this enzyme the key role of conserving the acetyl group for the next cycle of ornithine synthesis. NAOGAcT is found in non-enteric bacteria (Cunin et al., 1986), fungi (Davis, 1986), and plants (Shargool et al., 1988).
Escherichia coli and other enterobacteria, as well as yeast, synthesize ornithine in a linear pathway due to the presence of $\mathrm{N}$-acetylornithine deacetylase (NAOD), which hydrolyses $\mathrm{N}$-acetylornithine to ornithine and acetate as the final step (Vogel and Bonner, 1956; Meinnel et al., 1992; Crabeel et al., 1997). Plants were considered as unable to use this pathway, since NAOD activity has not been demonstrated in plants so far (Slocum, 2005; Page et al., 2012; Frémont et al., 2013). Recently, Molesini et al. (2015) revealed the first hint of NAOD activity in Arabidopsis using T-DNA insertion lines (see below).

Glutamate is a precursor for both proline and ornithine biosynthesis in plants and other organisms (Morris et al., 1969; Cunin et al., 1986; Davis, 1986; Caldovic and Tuchman, 2003). After acetylation of glutamate, the NAcGSA intermediate is incapable of undergoing cyclisation. Thus, acetylation of glutamate commits it to ornithine synthesis. In contrast, in proline biosynthesis the corresponding non-acetylated glutamate-5-semialdehyde (GSA) spontaneously forms pyrroline-5-carboxylate (P5C) by cyclisation (Slocum, 2005).

\section{Arginine Synthesis from Ornithine}

Arginine is synthesized from ornithine by the enzymes of the linear "arginine pathway" (Micallef and Shelp, 1989; Slocum, 2005). Ornithine transcarbamoylase (OTC) delivers the third $\mathrm{N}$-atom by carbamoylation of the $\delta$-amino group of ornithine, forming citrulline. This reaction requires carbamoyl phosphate, which is generated from ATP, bicarbonate and the $\delta$-amino group of glutamine by carbamoyl phosphate synthetase (CPS). The fourth $\mathrm{N}$-atom of arginine is derived from aspartate, which is ligated to citrulline by argininosuccinate synthase (ASSY). As substrates of OTC and ASSY, the amino acids aspartate and glutamine are additional essential precursors for arginine synthesis. Finally, argininosuccinate lyase (ASL) splits off fumarate, generating the final product arginine (Slocum, 2005; Figure 1).

The enzymes of plant arginine biosynthesis have been partly characterized biochemically (Shargool et al., 1988), but still little is known about the genes encoding these enzymes, and many steps of arginine biosynthesis remain poorly characterized in plants.

\section{Genes and Enzymes of Arginine Biosynthesis and their Regulation NAGS}

The first, and presently the only, characterized plant NAGS was isolated from tomato (Kalamaki et al., 2009). SlNAGS1 is a single copy gene and the SlNAGS protein shows a high level of similarity to two predicted Arabidopsis NAGS proteins, NAGS1 (At2g22910) and NAGS2 (At4g37670). A plastid transit peptide is predicted for SINAGS1 and the plastid localization is supported by the expression of SINAGS1 in all aerial organs, whereas no expression was detected in roots (Kalamaki et al., 2009). Transgenic Arabidopsis plants overexpressing SINAGS1 showed a significant accumulation of ornithine in the leaves, and a higher tolerance to salt and drought stress compared to wild type plants. The improved tolerance to salt stress of SINAGS1 overexpressions was attributed to the elevated levels of ornithine, 


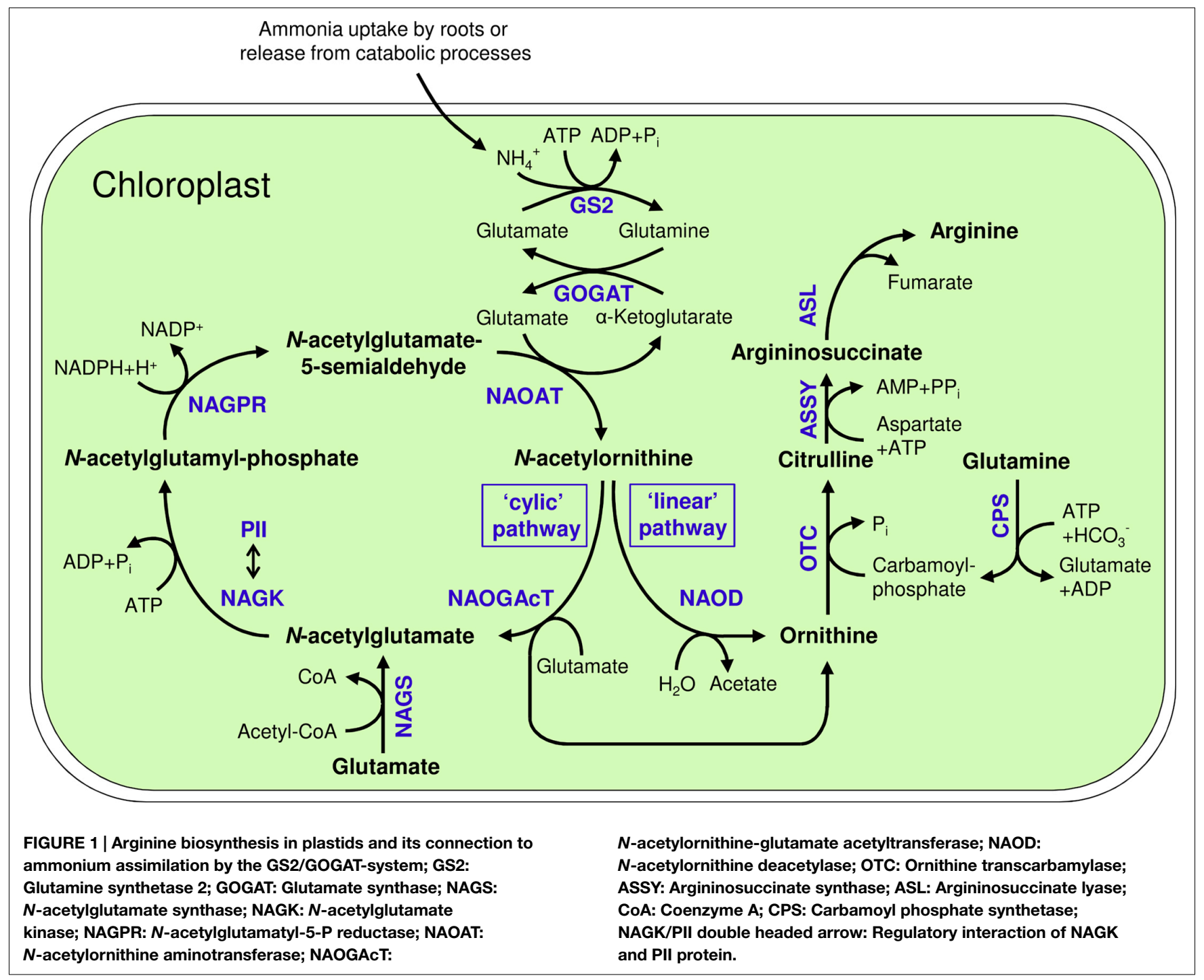

citrulline and arginine, since these amino acids have been reported to accumulate together with proline in higher plants under salinity stress (Mansour, 2000; Ashraf and Harris, 2004).

NAGS activity is a target of feedback regulation by arginine in prokaryotes and a similar mechanism is proposed for plant NAGS (Kalamaki et al., 2009; Sancho-Vaello et al., 2009). Sancho-Vaello et al. (2009) showed regulation of Pseudomonas aeruginosa NAGS activity by arginine, being an activator at low arginine concentration as well as an inhibitor at higher arginine concentration. The effects of arginine on NAGS activity were mediated by altering domain interactions within NAGS.

\section{NAGK}

The localization of Arabidopsis NAGK (At3g57560) in chloroplasts was predicted by sequence analysis and was experimentally demonstrated by Chen et al. (2006). Feedback regulation of NAGK mediated by the plastidic PII protein was first described in the cyanobacterium Synechococcus elongatus (Heinrich et al., 2004; Maheswaran et al., 2004) and in Arabidopsis (Burillo et al., 2004). PII proteins are among the most highly conserved, widely distributed and ancient signal transduction proteins known in bacteria, archaebacteria, cyanobacteria, eukaryotic algae and higher plants. They are involved in sensing the carbon/nitrogen balance and the energy status of the cell. Targets include signal transduction proteins, key metabolic enzymes and transporters involved in nitrogen assimilation and uptake (Slocum, 2005; Feria Bourrellier et al., 2009). The PII protein has been shown to interact tightly with NAGK, inducing a conformational change of its T-loop and leading to decreased feedback inhibition of the enzyme complex by arginine (Heinrich et al., 2004; Slocum, 2005; Llacer et al., 2008; Feria Bourrellier et al., 2009). Interaction of NAGK and the PII protein under conditions of high nitrogen availability strongly increases the catalytic efficiency of NAGK and decreases significantly the sensitivity of the enzyme complex to arginine, resulting in high arginine production. Limitation of nitrogen prevents the formation of the NAGK/PII complex, resulting in decreased enzyme activity of NAGK and increasing the feedback 
inhibition of the complex by arginine (Heinrich et al., 2004; Maheswaran et al., 2004; Slocum, 2005; Chen et al., 2006; Llacer et al., 2008). Feria Bourrellier et al. (2009) demonstrated that the interaction of NAGK and the PII protein was counteracted by $\alpha$-ketoglutarate/2-oxoglutarate, the carbon skeleton used to form glutamate during nitrogen assimilation and a lownitrogen abundance signal in plants (Lancien et al., 2000), as well as by arginine and glutamate. The flux through the arginine biosynthetic pathway depends on the balance between energy status and nitrogen and carbon availability for nitrogen assimilation via the glutamine synthetase (GS)/glutamate synthase (GOGAT) pathway in the plastids. Schneidereit et al. (2006) showed a threefold decrease in the intracellular $\alpha$-ketoglutarate level induced by high nitrogen conditions in plants, due to a rapid $\mathrm{NH}_{4}{ }^{+}$assimilation by the GS/GOGAT cycle. This suggests that a high nitrogen status will be sensed by the PII protein through a low level of $\alpha$-ketoglutarate, and thus under these conditions PII-NAGK complex formation will be favored leading to arginine synthesis and nitrogen storage, as well as an increase in arginine and glutamate concentrations, which are expected to limit arginine accumulation by inhibition of NAGK (Feria Bourrellier et al., 2009). Chellamuthu et al. (2014) identified an additional PII-mediated regulatory mechanism, by which high nitrogen availability activates NAGK and thus promotes arginine synthesis. Glutamine binding alters PII conformation, promoting the interaction with and activation of NAGK. This mechanism appears to be conserved from algae to flowering plants with the exception of the Brassicaceae, including Arabidopsis.

\section{NAGPR, NAOAT, and NAOGAcT}

Since Slocum (2005) NAGPR has not been characterized further in Arabidopsis. Rice NAGPR was crystallized and characterized (Nonaka et al., 2005). The crystal structure of a putative NAGPR from Arabidopsis (At2g19940) was deposited in the protein data bank (www.rcsb.org/pdb; PDB accession number \#1XYG; Levin et al., 2007). However, the details of these structures have not been reported so far.

The NAOAT encoding gene Arg9 was identified and characterized in the green alga Chlamydomonas reinhardtii (Remacle et al., 2009). Plastidial localization of NAOAT was demonstrated by complementation studies as well as immunoblot analysis. The TUMOR PRONE5 (TUP5, At1g80600) gene of Arabidopsis was demonstrated to encode a NAOAT (Frémont et al., 2013). Characterization of the gene and its mutant lines showed a strongly reduced free arginine content in the chemically-induced recessive mutant tup5, suggesting that the biosynthesis of amino acids that are produced downstream of the NAOAT enzymatic reaction is impaired in this mutant. Consistently, tup5 showed a short root growth phenotype, restorable by supplementation with arginine and its metabolic precursors. A yeast NAOAT mutant was complemented by TUP5. Two null alleles of TUP5 showed a reduced viability of gametes and embryo lethality, possibly caused by insufficient arginine supply from maternal tissue. A TUP5-green fluorescent protein was localized in chloroplasts (Frémont et al., 2013). TUP5 expression is positively regulated by light, and tup 5 showed a unique light-dependent short root phenotype. The roots of tup5 seedlings of different ages cultivated in darkness immediately stopped growth when they were shifted into light. Frémont et al. (2013) attributed this phenotype to a blue light-dependent switch from indeterminate growth to determinate growth with arresting cell production and an exhausted root apical meristem and, thus, a critically dependence of root growth on arginine in the presence of light.

No experimental analysis of the putative Arabidopsis NAOGAcT (At2g37500) has been described yet. Expression of the poplar NAOGAcT homolog was not altered in response to putrescine overproduction in a transgenic line (Page et al., 2012).

\section{NAOD}

NAOD activity has never been demonstrated in plants, although many putative NAOD-like genes have been identified (Slocum, 2005). Molesini et al. (2015) analyzed the NAOD-activity in Arabidopsis after downregulation of the putative NAOD gene (At4g17830) by using RNA silencing and T-DNA insertion mutants. All analyzed NAOD-suppressed plants showed consistently reduced ornithine content compared with wild-type plants, suggesting that in addition to NAOGAcT action, NAOD contributes to the regulation of ornithine levels in plant cells. Ornithine depletion was associated with increased putrescine and decreased spermine concentrations, and the reduced AtNAOD expression resulted in developmental alterations, namely early flowering and impaired seed setting. A connection between ornithine levels or metabolism and reproductive development had already been proposed by Trovato et al. (2001), who observed early flowering and enhanced flower formation in tobacco plants overexpressing ornithine cyclodeaminase (RolD) from Agrobacterium rhizogenes (see below).

\section{OTC, CPS, ASSY, and ASL}

Quesada et al. (1999) described a transfer DNA (T-DNA) insertion mutant of Arabidopsis with an insertion downstream of the OTC (At1g75330) open reading frame. The mutant plants showed an increased sensitivity to exogenous ornithine, which was attributed to reduced OTC expression, potentially due to problems in mRNA $3^{\prime}$-end formation (Quesada et al., 1999). The chemically-induced Arabidopsis mutants ven 3 and ven6, where the small subunit (At3g27740) and the large subunit (At1g29900) of CPS were affected, showed increased ornithine and decreased citrulline levels, respectively, suggesting a disrupted conversion of ornithine to citrulline because of reduced carbamoyl phosphate availability (Mollá-Morales et al., 2011). We could not find any further recent publication reporting on the characterization of plant OTC or ASSY (At4g24830).

A characterization of the Arabidopsis ASL (At5g10920) is also still missing. The rice ASL mutant osred 1 showed a short root phenotype like the Arabidopsis NAOAT mutant tup5-1, supporting the suggestion that arginine is essential for normal root growth in different plant species (Frémont et al., 2013; Xia et al., 2014a,b). Expression analysis revealed two alternatively spliced transcripts of OsASL1, OsASL1.1, and OsASL1.2, coding for two ASL isoforms with slightly different N-termini. OsASL1.1 was expressed throughout the entire growth period in most 
organs, whereas OsASL1.2 was expressed mainly in the roots. In contrast to the plastid-localized OsASL1.1, OsASL1.2 was localized in the cytosol and nucleus. Only OsASL1.1 showed ASL activity in a yeast complementation study. The short-root phenotype of the osred 1 mutant was rescued by external arginine supply but not by a NO donor, supporting the hypothesis that arginine is required for normal root growth independently of its function as putative NO precursor (Xia et al., 2014a,b). The poplar ASL homolog was the only gene, among 17 analyzed genes of arginine metabolism in poplar, whose expression was higher in response to putrescine overproduction in a transgenic line. Page et al. (2012) hypothesized a biochemical regulation of arginine biosynthesis involving substrate concentrations or co-factors rather than a regulation at the transcriptional level.

\section{Arginine Transport}

\section{Long Distance Transport}

Long distance transport of arginine to nitrogen storing organs or seeds occurs probably in the vascular tissue and is presumably dependent on amino acid transporters of the AAP family of amino acid/proton co-transporters. Especially important for long distant arginine transport seem to be AAP3 (At1g77380) and AAP5 (At1g44100), which are involved in loading and unloading the vascular tissue (Fischer et al., 1995, 2002; Okumoto et al., 2004; Svennerstam et al., 2008; Tegeder, 2014). AAP5 transports arginine and lysine with high affinity (Svennerstam et al., 2008) and seems to have an important role in the uptake of basic amino acids by roots (Svennerstam et al., 2011). An additional function of AAP5 in the transport of arginine within plants is supported by its expression throughout the entire vascular system of Arabidopsis (Fischer et al., 1995, 2002; Svennerstam et al., 2008). AAP3 also displays high affinity for basic amino acids (Fischer et al., 2002; Taylor et al., 2015) and was shown to be expressed in the phloem, predominantly in roots (Okumoto et al., 2004). AAP transporters have been localized to the collection phloem of legumes and they are predicted to play a major role in amino acid loading of this tissue. In Arabidopsis, the assignment of clear-cut physiological function to individual AAPs has not been reported so far (Tegeder, 2014).

Dündar and Bush (2009) identified and characterized a bidirectional amino acid transporter (BAT1, At2g01170) in Arabidopsis. Both direct measurement of amino acid transport and yeast growth experiments demonstrated transport activity of BAT1 for alanine, arginine, glutamate and lysine. BAT1 is a single copy gene in the Arabidopsis genome and its mRNA is ubiquitously produced in all organs. Promoter-GUS analysis localized BAT1 expression in the vascular tissue, suggesting that BAT1 may function in amino acid export from the phloem into sink tissues (Dündar and Bush, 2009).

\section{Intracellular Transport}

Arginine metabolism is distributed over the three cellular compartments cytosol, plastids and mitochondria. Newly synthesized arginine can be used for protein synthesis directly in plastids or, after intracellular transport, in the cytosol and mitochondria. This generates a need for transport systems for arginine, as well as for synthesis and degradation intermediates. Very little is known about the transport of amino acids into or out of chloroplasts. Members of the preprotein and amino acid transporter (PRAT) family were proposed to mediate transport of amino acids across the inner envelope membrane (Murcha et al., 2007; Pudelski et al., 2010). So far, experimental evidence is only available for the function of PRATs in protein import (Rossig et al., 2013).

The prevalent group of carrier proteins in mitochondria is the mitochondrial carrier family (MCF) with 58 putative members in Arabidopsis (Picault et al., 2004; Haferkamp and Schmitz-Esser, 2012). Two members of the MCF were identified as basic amino acid transporters (BAC1 and BAC2) which mediate the transport of arginine, ornithine and lysine with decreasing affinity and were postulated to be localized in the mitochondrial inner membrane (Hoyos et al., 2003; Palmieri et al., 2006). BAC1 (At2g33820) and BAC2 (At1g79900), together with BOU (a bout de souffle, Lawand et al., 2002), form a sub-group of MCF proteins distinct from other Arabidopsis mitochondrial carriers regarding sequences and function (Catoni et al., 2003; Hoyos et al., 2003; Picault et al., 2004; Toka et al., 2010).

BAC1 and BAC2 were identified as basic amino acid transporters by complementation of the yeast mutant arg11. This mutant is defective in mitochondrial ornithine/arginine transport due to a loss-of-function mutation in the ORT1 carrier (Catoni et al., 2003; Hoyos et al., 2003; Palmieri et al., 2006). ORT1 is an antiporter for ornithine, arginine or lysine and is important for ornithine export from mitochondria, an essential step for arginine biosynthesis in Saccharomyces cerevisiae (Palmieri et al., 1997, 2006). The transport characteristics of BAC1 and BAC2 resemble each other, they were inactivated by the same inhibitors and their $\mathrm{K}_{m}$ and $\mathrm{V}_{\max }$ values were very similar for their most efficiently transported and preferred substrate arginine (Palmieri et al., 2006).

Arabidopsis bac2 mutants showed a conditional phenotype as they grew more slowly than the wild-type on arginine as sole source of nitrogen, while $B A C 2$ overexpressing plants showed the opposite phenotype. Presumably, the expression of BAC2 is a limiting factor for mitochondrial arginine transport in vivo and therefore for the mobilization of nitrogen from arginine (Toka et al., 2010). This is consistent with the higher expression levels of BAC2 in wild type seedlings growing on arginine as sole source of nitrogen (Catoni et al., 2003).

Since bac2 mutants did not show any phenotypical difference to the wild type when growing on soil, other pathways or transporters seem to compensate the lack of BAC2 during vegetative growth (Toka et al., 2010). BAC2 expression was induced during stress and senescence (Toka et al., 2010) and bac2 mutant seedlings recovering from hyperosmotic stress showed significantly reduced leaf growth (Planchais et al., 2014). Probably BAC2-dependent arginine import into mitochondria is required during stress conditions and for recovery of growth after stress (Planchais et al., 2014). The functional redundancy between BAC1 and $\mathrm{BAC} 2$ and the expression patterns indicate that BAC1 is sufficient for mitochondrial arginine import during normal plant growth (Catoni et al., 2003; Hoyos et al., 2003; Toka et al., 2010). So 
far, no information about bac1 knock-out mutants or phenotypes of $\mathrm{BACl}$ overexpressing plants is available, leaving this point speculative.

Like in many other plant species, storage of nitrogen in the form of arginine in seeds is also likely in Arabidopsis, since the total arginase activity, which initiates the release of nitrogen from arginine, increased strongly up to 6 days after germination accompanied by increases in free arginine and urea levels (Zonia et al., 1995). In order to degrade arginine stored in seeds for nitrogen remobilization, large amounts of arginine have to be transported into mitochondria by the BAC carriers during early seedling development making it accessible to the mitochondrial localized arginase (Flores et al., 2008). However, only low levels or no expression of $\mathrm{BAC} 2$ were found in seeds and seedlings of Arabidopsis (Hoyos et al., 2003; Toka et al., 2010). This finding argues against a prominent function of BAC2 in storage mobilization, indicating that another transporter, probably BAC1, mediates the import of arginine into mitochondria during early seedling development. This suggestion is supported by RT-PCR analysis of $\mathrm{BAC1}$, which is highly expressed in seedlings, contrary to BAC2, which is mostly expressed in stamens and pollen grains of flowers (Hoyos et al., 2003; Palmieri et al., 2006; Toka et al., 2010; Monné et al., 2015).

Amino acid analysis revealed accumulation of proline and alanine in bac2 mutants (Planchais et al., 2014). BAC2 overexpressing plants showed low arginine levels and simultaneously high levels of ornithine, urea and citrulline, all products of arginine catabolism. Thus, BAC2 is able to increase arginine availability for degradation inside mitochondria, especially under stress conditions (Toka et al., 2010; Planchais et al., 2014; Monné et al., 2015).

\section{Arginine Catabolism and Arginine-Derived Metabolites}

\section{Arginine Catabolism}

After the import of arginine by $\mathrm{BAC} 1$ and $\mathrm{BAC} 2$ into mitochondria, arginine catabolism starts with degradation of arginine to ornithine and urea by arginase (Figure 2). Urea is exported to the cytosol, where it is further degraded to ammonia by urease (Witte, 2011; Polacco et al., 2013). Ornithine could be transported back into plastids to re-enter arginine biosynthesis as in the mammalian urea cycle. However, cycling between ornithine and arginine is unlikely to occur in a single cell or tissue in plants, as it would constitute a waste of energy and assimilated nitrogen. Ornithine degradation proceeds by transfer of the $\delta$-amino group to $\alpha$-ketoglutarate, catalyzed by ornithine $\delta$-aminotransferase ( $\delta \mathrm{OAT})$, yielding GSA/P5C, and glutamate. GSA/P5C is subsequently converted to a second molecule of glutamate by $\mathrm{P} 5 \mathrm{C}$ dehydrogenase $(\mathrm{P} 5 \mathrm{CDH})$. Glutamate is either exported from mitochondria as an anabolic precursor for multiple pathways, or it is further degraded inside mitochondria to $\alpha$-ketoglutarate, ammonium and $\mathrm{NADH}$ by glutamate dehydrogenase $(\mathrm{GDH})$. NADH can be used to fuel respiratory ATP production, while $\alpha$-ketoglutarate can be fed into the citric acid cycle or can be used to re-assimilate ammonium in the GS/GOGAT system.

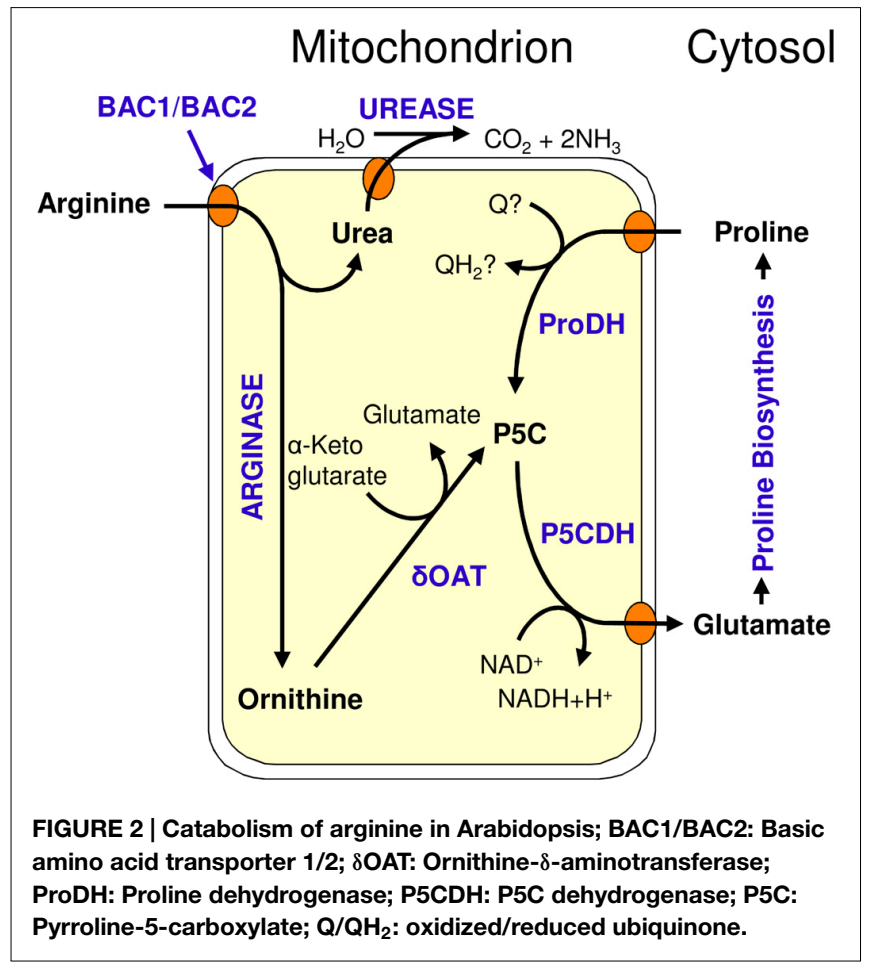

Like arginine synthesis, arginine catabolism is also regulated in accordance with the overall nutritional status of the plant cell. Arginine utilization seems to be coordinated with the availability of carbohydrates, since sugar starvation caused a substantial increase of enzyme activities of arginase and urease, as well as arginine decarboxylase (ADC), starting polyamine synthesis (see below) in yellow lupin (Lupinus luteus L.; Borek et al., 2001).

In the following sections, we will firstly summarize the available data on the single steps in the degradation of arginine to glutamate and secondly we will discuss alternative metabolic routes using arginine as a precursor.

\section{Arginase}

Arginase (L-arginine ureahydrolase or amidohydrolase) catalyzes the initial step of arginine degradation and is a binuclear manganese metalloenzyme (Goldraij and Polacco, 2000; Todd et al., 2001). Crystal structures of arginases revealed a homotrimeric quaternary structure for rat arginase 1 (Kanyo et al., 1996), human arginase 1 (Di Costanzo et al., 2007) and human arginase 2 (Cama et al., 2003), as well as homo-hexameric structures of Bacillus caldovelox (Bewley et al., 1999) and Thermus thermophilus (Dowling et al., 2008) arginases. Arginases have been purified and characterized from different plant species such as soybean (Kang and Cho, 1990), iris bulbs (Boutin, 1982), peanut seedlings (Desai, 1983), oakmoss (Martín-Falquina and Legaz, 1984) and ginseng (Hwang et al., 2001), showing variable sizes of monomers and oligomers. For arginase purified from Catjang (Vigna unguiculata var. cylindrica), a native molecular weight of $210 \mathrm{kDa}$ was estimated, while the monomer had an apparent molecular weight of $52 \mathrm{kDa}$ (Dabir et al., 2005). This supports a tetrameric structure of arginase from Catjang. In contrast, 
arginase purified from ginseng was estimated to be decameric (Hwang et al., 2001).

Arabidopsis contains two arginase genes, ARGAH1 (At4g08900) and ARGAH2 (At4g08870), which probably arose by a recent gene duplication (Krumpelman et al., 1995; Brownfield et al., 2008). The predicted mature proteins show $91 \%$ sequence identity, whereas the predicted mitochondrial transit peptide share only $39 \%$ sequence identity. GFP-fusion proteins showed that both ARGAH1 and ARGAH2 are mitochondrial proteins (Palmieri et al., 2006; Flores et al., 2008). The formation of homoand hetero-oligomers of the two Arabidopsis arginase isoforms has been demonstrated, while the precise oligomeric state and three-dimensional structure remain to be resolved (Winter, 2013).

Seedling arginase activity increases sharply during germination in Arabidopsis (Zonia et al., 1995), loblolly pine (King and Gifford, 1997) and other plant species (Splittstoesser, 1969; Kollöffel and van Dijke, 1975; Kang and Cho, 1990; Goldraij and Polacco, 1999). The analysis of T-DNA insertion mutants demonstrated that roughly $85 \%$ of the arginase activity in Arabidopsis seedlings depends on ARGAH2 whereas no developmental defects of argah2 or argah1 mutants were reported (Flores et al., 2008). Infection of mature Arabidopsis plants with the necrotrophic fungus Botrytis cinerea or the protist Plasmodiophora brassicae, causing the agriculturally important clubroot disease, resulted in an upregulation of ARGAH2 expression. Consistently, argah2 mutants showed an increased sensitivity toward clubroot disease (Brauc et al., 2012; Gravot et al., 2012).

Conversely to increased pathogen susceptibility, argah1-1 and argah2-1 T-DNA insertion mutants as well as the double mutant argah1argah 2 showed increased tolerance to abiotic stress. Higher tolerance to water deficit, salt stress and freezing was accompanied by increased NO and polyamine accumulation (Flores et al., 2008; Shi et al., 2013). Consistently, overexpression of arginase in Arabidopsis decreased the resistance and defense against abiotic stress (Shi et al., 2013). No developmental defects were reported for the argahlargah2 double mutants under normal growth conditions.

Interestingly, mutation of the single copy arginase gene in rice caused a strong decrease in growth and fertility, affecting both grain size and the rate of seed setting, whereas overexpression of arginase improved yield under nitrogen-limiting conditions ( $\mathrm{Ma}$ et al., 2012).

\section{Urea and Urease}

Arginase activity is the main source for endogenous urea in higher plants, and recycling of urea seems to be especially important under stress conditions. The available information about metabolism and transport of urea in plants has recently been reviewed by Witte (2011) and Polacco et al. (2013). Urease (urea amidohydrolase) is the only known Ni-containing enzyme in plants (Dixon et al., 1975) and catalyzes the hydrolysis of urea to ammonia and carbamic acid; the latter spontaneously hydrolyzes to ammonia and bicarbonate (Figure 2). The functional assembly of Arabidopsis urease (At1g67550) requires at least three accessory proteins (Witte et al., 2005; Witte, 2011). In Arabidopsis, the production of urea is induced by jasmonic acid by upregulation of ARGAH2 expression (Brownfield et al., 2008).
The rapid hydrolysis of urea by urease could cause localized alkalinisation, which, in turn, could further stimulate arginase which has a $\mathrm{pH}$ optimum $\geq 9.5$ (Jenkinson et al., 1996; Polacco et al., 2013). The alkalinization of the cytosol by induction of arginase and urease activity might constitute an active component of pathogen defense mechanisms (Polacco et al., 2013).

There is a large flux of nitrogen from arginine to ammonia pools due to arginase and urease activity, especially during germination. The increases in free urea levels during germination are generally rather moderate, indicating that urea export from mitochondria and urease are not limiting for nitrogen re-mobilization (Polacco et al., 2013). In addition to germination, urease plays a key role in recycling of nitrogen stored as arginine during senescence or during seasonal changes.

\section{Ornithine $\delta$-Aminotransferase}

The second product of arginine hydrolysis is ornithine, which is catabolized by $\delta \mathrm{OAT}$ to GSA and glutamate (Figure 2). $\delta \mathrm{OAT}$ transfers the $\delta$-amino group of ornithine to $\alpha$-ketoglutarate and the equilibrium of the $\delta \mathrm{OAT}$ reaction has been found far on the GSA + glutamate side (Adams and Frank, 1980).

A direct contribution of $\delta \mathrm{OAT}$ to stress-induced proline accumulation, which would require an unknown exit route of mitochondrial GSA or P5C to the cytosol, where $\mathrm{P} 5 \mathrm{C}$ reductase (P5CR) is localized, is controversial (Stránská et al., 2008). Proline production by the reverse reaction of proline dehydrogenase (ProDH) is energetically unfavorable. Furthermore, due to the chemical instability of GSA/P5C (Williams and Frank, 1975) and its toxicity when accumulating (Deuschle et al., 2004), export from mitochondria to the cytosol and thus contribution to proline synthesis seems unlikely, but cannot be fully excluded. Roosens et al. (1998) hypothesized that Arabidopsis SOAT (At5g46180) plays an important role in proline accumulation during osmotic stress in plants, because of increased free proline content, $\delta$ OAT activity and $\delta$ OAT mRNA in young plantlets under salt stress conditions. This hypothesis was supported by the analysis of transgenic Nicotiana plumbaginifolia plants overexpressing Arabidopsis $\delta \mathrm{OAT}$, which synthesized more proline than the control plants and showed a higher biomass and a higher germination rate under osmotic stress conditions (Roosens et al., 2002). The exclusive targeting of $\delta$ OAT to mitochondria in Arabidopsis and unchanged proline accumulation in salt-stressed Soat knockout mutants provided strong evidence against a direct contribution of $\delta \mathrm{OAT}$ to stress-induced proline accumulation (Funck et al., 2008). However, $\delta$ OAT activity was correlated to proline accumulation in salt-stressed cashew plants and ornithine application strongly enhanced proline accumulation (da Rocha et al., 2012). Overexpression of $\delta$ OAT in rice resulted in higher proline levels and activated the antioxidant defense, rendering the plants more stress-tolerant (You et al., 2012).

Silencing or deletion of $\delta O A T$ in tobacco and Arabidopsis, respectively, compromised non-host pathogen defense and pathogen-induced ROS formation (Senthil-Kumar and Mysore, 2012). Further research is needed to understand how SOAT contributes to stress defense and whether GSA produced by $\delta \mathrm{OAT}$ can be used directly for proline synthesis or is obligatorily converted to glutamate by $\mathrm{P} 5 \mathrm{CDH}$. 


\section{P5C Dehydrogenase}

GSA produced by $\delta \mathrm{OAT}$ inside mitochondria is most probably further converted to glutamate by mitochondrial $\mathrm{P} 5 \mathrm{CDH}$ (Deuschle et al., 2001; Funck et al., 2008). Due to the mentioned chemical instability and toxicity of GSA/P5C, the formation of a reversible enzyme complex of $\delta \mathrm{OAT}$ and $\mathrm{P} 5 \mathrm{CDH}$ seems likely, channeling GSA to P5CDH without releasing it to the mitochondrial matrix (Elthon and Stewart, 1982; Funck et al., 2008). Substrate channeling from ProDH to P5CDH has been reported for the bifunctional enzyme PutA from Geobacter sulfurreducens and recently also for the monofunctional enzymes from Thermus thermophilus (Singh et al., 2014; Sanyal et al., 2015). A similar co-operation between ProDH, $8 \mathrm{OAT}$ and $\mathrm{P} 5 \mathrm{CDH}$ in plants might explain the relatively low affinity of isolated $\mathrm{P} 5 \mathrm{CDH}$ for P5C (around $0.5 \mathrm{mM}$ ) as opposed to the fivefold higher affinity of P5C reductase (Forlani et al., 1997a,b; Giberti et al., 2014). Consistently, two P5CDH isoforms have been detected in Nicotiana plumbaginifolia, that may be specifically involved in the oxidation of $\mathrm{P} 5 \mathrm{C}$ deriving from either proline or arginine (Forlani et al., 1997a). The Arabidopsis genome contains a single P5CDH gene (At5g62530) and knockout mutants were hypersensitive to external supply of arginine or ornithine (Deuschle et al., 2004). In tissues with a high energy demand, glutamate produced by $\delta \mathrm{OAT}$ and $\mathrm{P} 5 \mathrm{CDH}$ may be further degraded by GDH to fuel mitochondrial energy production. However, since GDH also releases ammonia, which needs to be re-assimilated in an energy-demanding process, a direct recycling of glutamate for anabolic pathways seems more likely.

\section{Arginine as Precursor for Proline Biosynthesis}

Feeding of plants with arginine or ornithine resulted in elevated proline levels and radiotracer experiments demonstrated that both ${ }^{3} \mathrm{H}$ and ${ }^{14} \mathrm{C}$ from arginine can be recovered as proline (Adams and Frank, 1980; da Rocha et al., 2012). The physiological relevance and the biochemical pathway of the conversion of arginine to proline in plants remain unclear. The most prominent hypothesis is that ornithine, derived from arginine catabolism, is converted by $8 \mathrm{OAT}$ to $\mathrm{GSA} / \mathrm{P} 5 \mathrm{C}$, which then serves as substrate for proline synthesis by P5CR. This model has been doubted, since Arabidopsis $\delta \mathrm{OAT}$ was found to be exclusively localized in mitochondria, while P5CR is localized in the cytosol (Funck et al., 2008, 2012). Isolated corn mitochondria incubated with proline or ornithine released very little P5C and this release was strongly $\mathrm{pH}$ dependent and stimulated upon swelling of the mitochondria (Elthon and Stewart, 1982). Direct export of P5C from mitochondria and conversion to proline by $\mathrm{P} 5 \mathrm{C}$ reductase were postulated as part of a reactive oxygen species-producing proline-P5C cycle (Miller et al., 2009). Direct evidence for the transport of $\mathrm{P} 5 \mathrm{C}$ and the operation of this cycle under physiological conditions is still missing. Inside mitochondria, GSA/P5C is further converted to glutamate by P5CDH (see above). Export of glutamate from mitochondria has been demonstrated and could be the basis for proline synthesis via the glutamate pathway (Linka and Weber, 2005; Di Martino et al., 2006).

Removal of the $\alpha$-amino group of ornithine and conversion of the resulting pyrroline-2-carboxylate to proline has also been proposed (Mestichelli et al., 1979), but the required enzymes were not described in plants to date. Another alternative pathway from ornithine to proline would be via ornithine cyclodeaminase (OCD), which is found in bacteria and is transferred into plants with the T-DNA of Agrobacterium rhizogenes (Trovato et al., 2001; Mattioli et al., 2008). In the Arabidopsis genome, a homolog (At5g52810) of bacterial OCDs and mammalian $\mu$-crystallins has been identified. However, plants with a decreased expression of the putative OCD had higher rather than lower proline levels and the analysis of the recombinant protein yielded no evidence for OCD activity (Sharma et al., 2013).

\section{Polyamine Synthesis from Arginine}

Polyamines (putrescine, spermidine, and spermine) are essential for development and stress responses of plants. Embryogenesis, organogenesis, particularly flower initiation and development, fruit setting and ripening, as well as leaf senescence all require polyamines (Page et al., 2012; Majumdar et al., 2013; Pathak et al., 2014). In addition, the role of polyamines for abiotic stress tolerance and the regulation of nitrogen assimilation is well established in plants. Accumulation of polyamines in large amounts in the cell points toward roles in metabolic regulation of ammonia toxicity, $\mathrm{NO}$ production, and balancing organic nitrogen metabolism in the cell (Maiale et al., 2004; Marco et al., 2011; Gupta et al., 2013; Guo et al., 2014; Minocha et al., 2014; Pathak et al., 2014; Tiburcio et al., 2014).

Arginine is a precursor for the synthesis of polyamines (Bagni and Tassoni, 2001; Illingworth et al., 2003) and polyamine biosynthesis in Arabidopsis begins with arginine, which is converted by arginine decarboxylase (ADC1: At2g16500 and ADC2: At4g34710) to agmatine. ADC was found to be localized in the chloroplast of oat (Avena sativa, Borrell et al., 1995), which is consistent with the biosynthesis of arginine in the chloroplasts. The enzymatic reaction of ADC is followed by agmatine iminohydrolase (AIH, At5g08170, Janowitz et al., 2003) and $N$-carbamoylputrescine amidase (NLP, At2g27450, Piotrowski et al., 2003) producing putrescine. Subsequently spermidine and spermine are formed by two different aminopropyltransferases, spermidine synthase (SPDS, Atlg23820, Atlg70310) and spermine synthase (SPMS, At1g23820, At1g70310, At5g53120; reviewed in, Shao et al., 2012) with so far unknown localization (Figure 3).

Ornithine decarboxylase (ODC) represents an alternative way for putrescine synthesis by decarboxylation of ornithine. ODC homologs were identified and analyzed in different plant species including datura (Michael et al., 1996), tomato (Alabadí and Carbonell, 1998), tobacco (Lee and Cho, 2001), and chilli (Zainal et al., 2002). The Arabidopsis genome probably lacks a recognizable ODC (Hanfrey et al., 2001). This is in apparent contradiction to the findings of Molesini et al. (2015), who demonstrated that a lowered ornithine level in Arabidopsis NAOD insertion mutants did not influence arginine content but affected the levels of polyamines. In turn, this is consistent with the findings of Majumdar et al. (2013), who analyzed the regulation of ornithine and ornithine-related pathways (arginine and polyamines) in Arabidopsis by diversion of ornithine 


\section{Chloroplast or mitochondria \\ Chloroplast \\ Chloroplast or cytosol}

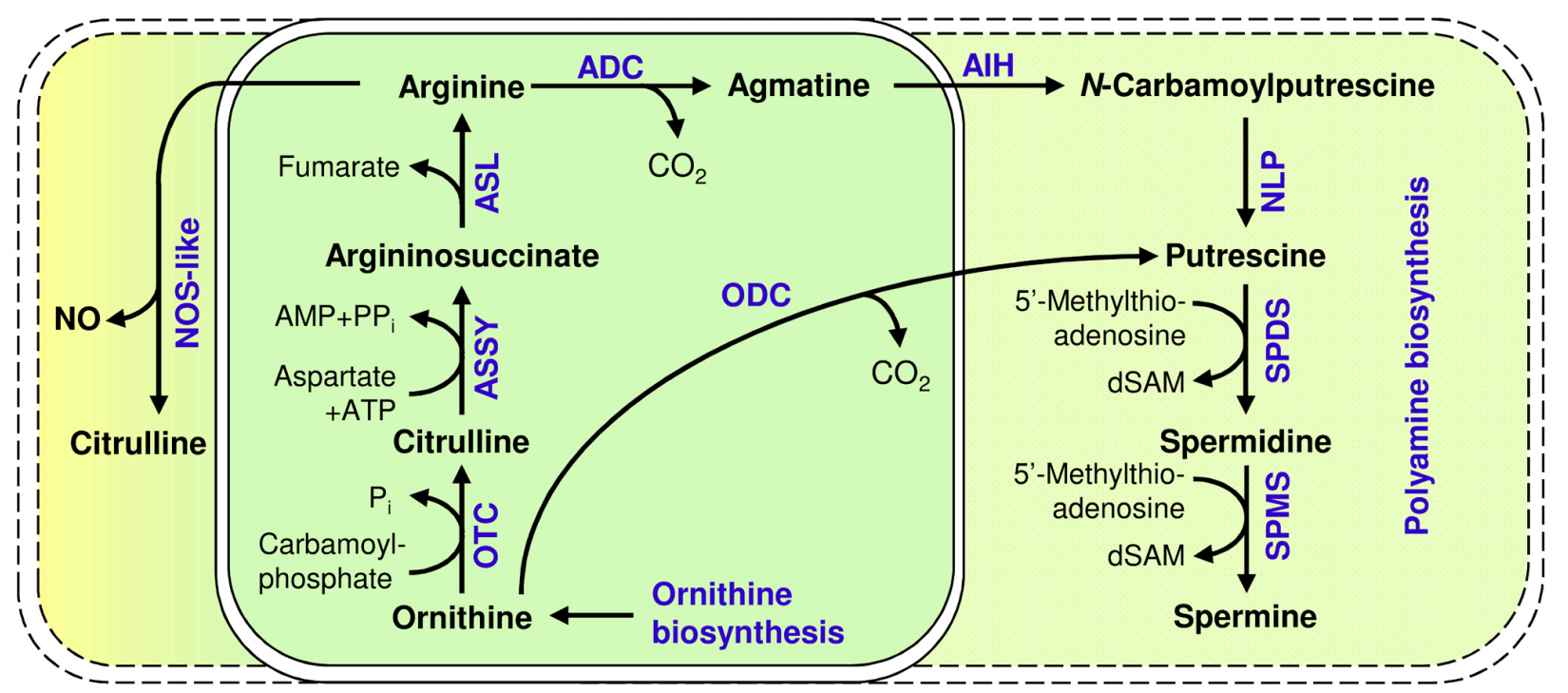

FIGURE 3 | Metabolism of arginine and its utilization for polyamine synthesis and NO generation in Arabidopsis; ODC: Ornithine decarboxylase; OTC: Ornithine transcarbamylase; ASSY: Argininosuccinate synthase; ASL: Argininosuccinate lyase; ADC:
Arginine decarboxylase; AlH: Agmatine iminohydrolase; NLP: $\mathrm{N}$-carbamoylputrescine amidase; SPDS: Spermidine synthase; SPMS: Spermine synthase; dSAM: decarboxylated S-Adenosyl-L-methionine; NOS-like: Nitric oxide synthase like; NO: Nitric oxide. from arginine biosynthesis, via the overexpression of a mouse ODC. The removal of large amounts of ornithine did not negatively impact arginine biosynthesis itself or the production of polyamines from arginine by ADC, neither by limiting the availability of its substrate arginine nor via feedback inhibition of ADC by excess putrescine. Furthermore, arginine levels were not altered differently in Arabidopsis arginase overexpressing and arginase insertion mutant lines (Shi et al., 2013), pointing toward multiple mechanisms regulating arginine and polyamine biosynthesis.

Arginase T-DNA insertion mutants of Arabidopsis showed much higher expression levels of polyamine biosynthesis enzymes ( $A D C 1$ and $A D C 2, A I H, N L P 1)$ as well as higher putrescine and spermine contents, whereas arginase overexpressing lines had significantly lower mRNA levels of the analyzed enzymes compared to the wild type (Shi et al., 2013). This is consistent with another report demonstrating that reduced arginase activity in Arabidopsis transgenic lines led to a significant increase in putrescine concentration (Brauc et al., 2012).

Polyamines were found to be non-competitive inhibitors for SOAT (Stránská et al., 2010) in Pisum sativum. It seems that increased polyamine concentrations can significantly reduce the activity of pea $\delta \mathrm{OAT}$ in vivo and Stránská et al. (2010) hypothesized that this would result in slowing down arginine catabolism. Since polyamines are involved in diverse physiological responses, it could be advantageous for plants to slow down arginine catabolism in favor of polyamine synthesis if necessary.

\section{NO Production Involving Arginine}

As a neutral and lipophilic gaseous molecule of small dimensions, NO can easily cross membranes, diffuse into the cytosol and bind to its soluble targets to act as a multifunctional signaling molecule. Beside implication of NO in plant growth and development from germination to fruit ripening and flowering, it is also generated in response to a wide range of biotic stresses, such as biotrophic and necrotrophic pathogens, as well as to a number of abiotic stresses, such as heavy metal, drought and salt stress (Neill et al., 2008; Moreau et al., 2010; Yu et al., 2014; Zhang et al., 2014; Domingos et al., 2015).

In plants, there are several sources of $\mathrm{NO}$, including arginine and nitrite-dependent pathways, as well as non-enzymatic NO generation (Leitner et al., 2009; Froehlich and Durner, 2011). The role of arginine as a precursor for NO is increasingly apparent, although the molecular mechanism of NO production from arginine remains elusive. Biochemical assays and the effects of inhibitors of animal NO synthase (NOS) support the existence of NOS-like enzymes in plants, converting arginine into NO and citrulline (Zhang et al., 2014; Domingos et al., 2015). However, all attempts to identify such enzymes in higher plants failed so far, whereas a NOS protein was described in the green alga Ostreococcus tauri (Foresi et al., 2010). Based on enzymatic assays or staining techniques, NOS-like activity in plants has been associated with mitochondria, chloroplasts and peroxisomes (Froehlich and Durner, 2011).

Flores et al. (2008) proposed that mitochondrial arginase activity competes with arginine-dependent NO production in 
Arabidopsis, while Shi et al. (2013) showed that both Arabidopsis arginase isoenzymes are able to negatively regulate polyamine synthesis as well as NO synthesis. Leaving the existence of a NOSlike activity in plants speculative, other researches showed that polyamines could induce rapid biosynthesis of NO in root tips and primary leaves of Arabidopsis seedlings (Tun et al., 2006; Siddiqui et al., 2011; Wimalasekera et al., 2011; Tiburcio et al., 2014).

Both polyamines and NO function in regulation of plant development and as signaling molecules mediating a range of responses to biotic and abiotic stresses (Zhao et al., 2007; Neill et al., 2008; Lozano-Juste and León, 2010; Gupta et al., 2011; Wang et al., 2011). Further research is needed to decipher how arginine, arginine degradation and arginine-derived NO and polyamines influence each other to orchestrate development and plant defense response to stress.

\section{Conclusion}

The present review gives an update on the recent advances in research on arginine metabolism in higher plants, mainly derived from work with Arabidopsis. Experimental evidence indicated that both pathways for ornithine biosynthesis, the well characterized cyclic pathway and the linear pathway using NAOD, are present in plants. Arginine levels in plant tissues seem to be regulated by a multitude of mechanisms, since most of the experimental manipulations of arginine biosynthesis

\section{References}

Adams, E., and Frank, L. (1980). Metabolism of proline and the hydroxyprolines. Annu. Rev. Biochem. 49, 1005-1061. doi: 10.1146/annurev.bi.49.070180.005041

Alabadí, D., and Carbonell, J. (1998). Expression of ornithine decarboxylase is transiently increased by pollination, 2,4-dichlorophenoxyacetic acid, and gibberellic acid in tomato ovaries. Plant Physiol. 118, 323-328. doi: 10.1104/pp.118.1.323

Ashraf, M., and Harris, P. J. (2004). Potential biochemical indicators of salinity tolerance in plants. Plant Sci. 166, 3-16. doi: 10.1016/j.plantsci.2003.10.024

Bagni, N., and Tassoni, A. (2001). Biosynthesis, oxidation and conjugation of aliphatic polyamines in higher plants. Amino Acids 20, 301-317. doi: $10.1007 / \mathrm{s} 007260170046$

Bausenwein, U., Millard, P., Thornton, B., and Raven, J. A. (2001). Seasonal nitrogen storage and remobilization in the forb Rumex acetosa. Funct. Ecol. 15, 370-377. doi: 10.1046/j.1365-2435.2001.00524.x

Bewley, M. C., Jeffrey, P. D., Patchett, M. L., Kanyo, Z. F., and Baker, E. N. (1999). Crystal structures of Bacillus caldovelox arginase in complex with substrate and inhibitors reveal new insights into activation, inhibition and catalysis in the arginase superfamily. Structure 7, 435-448. doi: 10.1016/S0969-2126(99)800562

Borek, S., Morkunas, I., Ratajczak, W., and Ratajczak, L. (2001). Metabolism of amino acids in germinating yellow lupin seeds-III. Breakdown of arginine in sugar-starved organs cultivated in vitro. Acta Physiol. Plant 23, 141-148. doi: 10.1007/s11738-001-0001-5

Borrell, A., Culianez-Macia, F. A., Altabella, T., Besford, R. T., Flores, D., and Tiburcio, A. F. (1995). Arginine decarboxylase is localized in chloroplasts. Plant Physiol. 109, 771-776.

Boutin, J. P. (1982). Purification, properties and subunit structure of arginase from Iris bulbs. Eur. J. Biochem. 127, 237-243. doi: 10.1111/j.1432-1033.1982.tb06861.x

Brauc, S., de Vooght, E., Claeys, M., Geuns, J. M. C., Höfte, M., and Angenon, G. (2012). Overexpression of arginase in Arabidopsis thaliana influences defence responses against Botrytis cinerea. Plant. Biol. (Stuttg.) 14(Suppl. 1), 39-45. doi: 10.1111/j.1438-8677.2011.00520.x or catabolism did not alter arginine concentrations. The role of arginine as important amino acid for nitrogen storage in plants is complemented by arginine catabolism mobilizing stored nitrogen and fine-tuning the production of NO, polyamines and potentially proline. While several regulatory mechanisms of arginine biosynthesis were identified, understanding of the regulation of the different pathways for arginine utilization requires further research.

Detailed biochemical and physiological characterization of the enzymes mediating arginine metabolism and the regulatory mechanisms allocating arginine-derived nitrogen to signaling, growth, reproduction or defense to stress will provide a better understanding of the role of arginine metabolism in nitrogen use efficiency in plants. Knowledge about essential intermediates and developmental switches between nitrogen storage and remobilization may help to improve crop plants or cultivation conditions. Optimized nitrogen use efficiency of crop plants will be crucial to reduce detrimental effects of nitrogen shortage on productivity and will help to avoid negative economic and environmental impact of excessive nitrogen fertilization in agriculture.

\section{Author Contributions}

All authors have contributed significantly and approved the final manuscript.

Brownfield, D. L., Todd, C. D., and Deyholos, M. K. (2008). Analysis of Arabidopsis arginase gene transcription patterns indicates specific biological functions for recently diverged paralogs. Plant Mol. Biol. 67, 429-440. doi: 10.1007/s11103008-9336-2

Burillo, S., Luque, I., Fuentes, I., and Contreras, A. (2004). Interactions between the nitrogen signal transduction protein PII and $\mathrm{N}$-acetyl glutamate kinase in organisms that perform oxygenic photosynthesis. J. Bacteriol. 186, 3346-3354. doi: 10.1128/JB.186.11.3346-3354.2004

Caldovic, L., and Tuchman, M. (2003). N-acetylglutamate and its changing role through evolution. Biochem. J. 372, 279-290. doi: 10.1042/BJ20030002

Cama, E., Colleluori, D. M., Emig, F. A., Shin, H., Kim, S. W., Kim, N. N., et al. (2003). Human arginase II: crystal structure and physiological role in male and female sexual arousal. Biochemistry 42, 8445-8451. doi: 10.1021/bi034340j

Catoni, E., Desimone, M., Hilpert, M., Wipf, D., Kunze, R., Schneider, A., et al. (2003). Expression pattern of a nuclear encoded mitochondrial arginineornithine translocator gene from Arabidopsis. BMC Plant Biol. 3:1. doi: 10.1186/1471-2229-3-1

Chellamuthu, V.-R., Ermilova, E., Lapina, T., Lüddecke, J., Minaeva, E., Herrmann, C., et al. (2014). A widespread glutamine-sensing mechanism in the plant kingdom. Cell 159, 1188-1199. doi: 10.1016/j.cell.2014.10.015

Chen, Y. M., Ferrar, T. S., Lohmeir-Vogel, E., Morrice, N., Mizuno, Y., Berenger, B., et al. (2006). The PII signal transduction protein of Arabidopsis thaliana forms an arginine-regulated complex with plastid $\mathrm{N}$-acetyl glutamate kinase. J. Biol. Chem. 281, 5726-5733. doi: 10.1074/jbc.M510945200

Crabeel, M., Abadjieva, A., Hilven, P., Desimpelaere, J., and Soetens, O. (1997). Characterization of the Saccharomyces cerevisiae ARG7 gene encoding ornithine acetyltransferase, an enzyme also endowed with acetylglutamate synthase activity. Eur. J. Biochem. 250, 232-241. doi: 10.1111/j.1432-1033.1997. 0232a.x

Cunin, R., Glansdorff, N., Piérard, A., and Stalon, V. (1986). Biosynthesis and metabolism of arginine in bacteria. Microbiol. Rev. 50, 314-352.

Dabir, S., Dabir, P., and Somvanshi, B. (2005). Purification, properties and alternate substrate specificities of arginase from two different sources: Vigna catjang cotyledon and buffalo liver. Int. J. Biol. Sci. 1, 114-122. doi: 10.7150/ ijbs. 1.114 
da Rocha, I. M. A., Vitorello, V. A., Silva, J. S., Ferreira-Silva, S. L., Viégas, R. A., Silva, E. N., et al. (2012). Exogenous ornithine is an effective precursor and the $\delta$ ornithine amino transferase pathway contributes to proline accumulation under high N recycling in salt-stressed cashew leaves. J. Plant Physiol. 169, 41-49. doi: 10.1016/j.jplph.2011.08.001

Davis, R. H. (1986). Compartmental and regulatory mechanisms in the arginine pathways of Neurospora crassa and Saccharomyces cerevisiae. Microbiol. Rev. 50, 280-313.

de Ruiter, H., and Kollöffel, C. (1983). Arginine catabolism in the cotyledons of developing and germinating pea seeds. Plant Physiol. 73, 525-528. doi: 10.1104/pp.73.3.525

Desai, H. V. (1983). Purification and properties of arginase from Arachis hypogea L. seedlings. Indian J. Biochem. Biophys. 20, 236-237.

Deuschle, K., Funck, D., Forlani, G., Stransky, H., Biehl, A., Leister, D., et al. (2004). The role of $\Delta^{1}$-pyrroline-5-carboxylate dehydrogenase in proline degradation. Plant Cell 16, 3413-3425. doi: 10.1105/tpc.104.023622

Deuschle, K., Funck, D., Hellmann, H., Däschner, K., Binder, S., and Frommer, W. B. (2001). A nuclear gene encoding mitochondrial $\Delta^{1}$-pyrroline-5-carboxylate dehydrogenase and its potential role in protection from proline toxicity. Plant J. 27, 345-356. doi: 10.1046/j.1365-313X.2001.01101.x

Di Costanzo, L., Pique, M. E., and Christianson, D. W. (2007). Crystal structure of human arginase I complexed with thiosemicarbazide reveals an unusual thiocarbonyl $\mu$-sulfide ligand in the binuclear manganese cluster. J. Am. Chem. Soc. 129, 6388-6389. doi: 10.1021/ja071567j

Di Martino, C., Pizzuto, R., Pallotta, M. L., de Santis, A., and Passarella, S. (2006). Mitochondrial transport in proline catabolism in plants: the existence of two separate translocators in mitochondria isolated from durum wheat seedlings. Planta 223, 1123-1133. doi: 10.1007/s00425-005-0166-Z

Dixon, N. E., Gazzola, T. C., Blakeley, R. L., and Zermer, B. (1975). Letter: jack bean urease (EC 3.5.1.5). A metalloenzyme. A simple biological role for nickel? J. Am. Chem. Soc. 97, 4131-4133. doi: 10.1021/ja00847a045

Domingos, P., Prado, A. M., Wong, A., Gehring, C., and Feijo, J. A. (2015). Nitric oxide: a multitasked signaling gas in plants. Mol. Plant 8, 506-520. doi: 10.1016/j.molp.2014.12.010

Dowling, D. P., Di Costanzo, L., Gennadios, H. A., and Christianson, D. W. (2008). Evolution of the arginase fold and functional diversity. Cell. Mol. Life Sci. 65, 2039-2055. doi: 10.1007/s00018-008-7554-Z

Dündar, E., and Bush, D. R. (2009). BAT1, a bidirectional amino acid transporter in Arabidopsis. Planta 229, 1047-1056. doi: 10.1007/s00425-009-0892-8

Elthon, T. E., and Stewart, C. R. (1982). Proline oxidation in corn mitochondria: involvement of NAD, relationship to ornithine metabolism, and sidedness on the inner membrane. Plant Physiol. 70, 567-572. doi: 10.1104/pp.70.2.567

Feria Bourrellier, A. B., Ferrario-Méry, S., Vidal, J., and Hodges, M. (2009). Metabolite regulation of the interaction between Arabidopsis thaliana PII and $\mathrm{N}$-acetyl-l-glutamate kinase. Biochem. Biophys. Res. Commun. 387, 700-704. doi: 10.1016/j.bbrc.2009.07.088

Fischer, W. N., Kwart, M., Hummel, S., and Frommer, W. B. (1995). Substrate specificity and expression profile of amino acid transporters (AAPs) in Arabidopsis. J. Biol. Chem. 270, 16315-16320. doi: 10.1074/jbc.270.27.16315

Fischer, W.-N., Loo Donald, D. F., Koch, W., Ludewig, U., Boorer, K. J., Tegeder, M., et al. (2002). Low and high affinity amino acid $\mathrm{H}^{+}$-cotransporters for cellular import of neutral and charged amino acids. Plant J. 29, 717-731. doi: 10.1046/j.1365-313X.2002.01248.x

Flores, T., Todd, C. D., Tovar-Mendez, A., Dhanoa, P. K., Correa-Aragunde, N., Hoyos, M. E., et al. (2008). Arginase-negative mutants of Arabidopsis exhibit increased nitric oxide signaling in root development. Plant Physiol. 147, 1936-1946. doi: 10.1104/pp.108.121459

Foresi, N., Correa-Aragunde, N., Parisi, G., Caló, G., Salerno, G., and Lamattina, L. (2010). Characterization of a nitric oxide synthase from the plant kingdom: NO generation from the green alga Ostreococcus tauri is light irradiance and growth phase dependent. Plant Cell 22, 3816-3830. doi: 10.1105/tpc.109. 073510

Forlani, G., Scainelli, D., and Nielsen, E. (1997a). Two $\Delta^{1}$-pyrroline-5-carboxylate dehydrogenase isoforms are expressed in cultured Nicotiana plumbaginifolia cells and are differentially modulated during the culture growth cycle. Planta 202, 242-248. doi: 10.1007/s004250050125

Forlani, G., Scainelli, D., and Nielsen, E. (1997b). $\Delta^{1}$-Pyrroline-5-carboxylate dehydrogenase from cultured cells of potato (purification and properties). Plant Physiol. 113, 1413-1418.
Frémont, N., Riefler, M., Stolz, A., and Schmülling, T. (2013). The Arabidopsis TUMOR PRONE5 gene encodes an acetylornithine aminotransferase required for arginine biosynthesis and root meristem maintenance in blue light. Plant Physiol. 161, 1127-1140. doi: 10.1104/pp.112.210583

Froehlich, A., and Durner, J. (2011). The hunt for plant nitric oxide synthase (NOS): is one really needed? Plant Sci. 181, 401-404. doi: 10.1016/j.plantsci.2011.07.014

Funck, D., Stadelhofer, B., and Koch, W. (2008). Ornithine- $\delta$-aminotransferase is essential for arginine catabolism but not for proline biosynthesis. BMC Plant Biol. 8:40. doi: 10.1186/1471-2229-8-40

Funck, D., Winter, G., Baumgarten, L., and Forlani, G. (2012). Requirement of proline synthesis during Arabidopsis reproductive development. BMC Plant Biol. 12:191. doi: 10.1186/1471-2229-12-191

Giberti, S., Funck, D., and Forlani, G. (2014). $\Delta^{1}$-Pyrroline-5-carboxylate reductase from Arabidopsis thaliana: stimulation or inhibition by chloride ions and feedback regulation by proline depend on whether NADPH or NADH acts as co-substrate. New Phytol. 202, 911-919. doi: 10.1111/nph.12701

Goldraij, A., and Polacco, J. C. (1999). Arginase is inoperative in developing soybean embryos. Plant Physiol. 119, 297-304. doi: 10.1104/pp.119.1.297

Goldraij, A., and Polacco, J. C. (2000). Arginine degradation by arginase in mitochondria of soybean seedling cotyledons. Planta 210, 652-658. doi: 10.1007/s004250050056

Gravot, A., Deleu, C., Wagner, G., Lariagon, C., Lugan, R., Todd, C., et al. (2012). Arginase induction represses gall development during clubroot infection in Arabidopsis. Plant Cell Physiol. 53, 901-911. doi: 10.1093/pcp/pcs037

Guo, Z., Tan, J., Zhuo, C., Wang, C., Xiang, B., and Wang, Z. (2014). Abscisic acid, $\mathrm{H}_{2} \mathrm{O}_{2}$ and nitric oxide interactions mediated cold-induced $\mathrm{S}$ adenosylmethionine synthetase in Medicago sativa subsp. falcata that confers cold tolerance through up-regulating polyamine oxidation. Plant Biotechnol. J. 12, 601-612. doi: 10.1111/pbi.12166

Gupta, K., Dey, A., and Gupta, B. (2013). Plant polyamines in abiotic stress responses. Acta Physiol. Plant 35, 2015-2036. doi: 10.1007/s11738-013-1239-4

Gupta, K. J., Igamberdiev, A. U., Manjunatha, G., Segu, S., Moran, J. F., Neelawarne, B., et al. (2011). The emerging roles of nitric oxide (NO) in plant mitochondria. Plant Sci. 181, 520-526. doi: 10.1016/j.plantsci.2011.03.018

Haferkamp, I., and Schmitz-Esser, S. (2012). The plant mitochondrial carrier family: functional and evolutionary aspects. Front. Plant Sci. 3:2. doi: 10.3389/fpls.2012.00002

Hanfrey, C., Sommer, S., Mayer, M. J., Burtin, D., and Michael, A. J. (2001). Arabidopsis polyamine biosynthesis: absence of ornithine decarboxylase and the mechanism of arginine decarboxylase activity. Plant J. 27, 551-560. doi: 10.1046/j.1365-313X.2001.01100.x

Heinrich, A., Maheswaran, M., Ruppert, U., and Forchhammer, K. (2004). The Synechococcus elongatus PII signal transduction protein controls arginine synthesis by complex formation with $\mathrm{N}$-acetyl-L-glutamate kinase. Mol. Microbiol. 52, 1303-1314. doi: 10.1111/j.1365-2958.2004.04058.x

Hoyos, M. E., Palmieri, L., Wertin, T., Arrigoni, R., Polacco, J. C., and Palmieri, F. (2003). Identification of a mitochondrial transporter for basic amino acids in Arabidopsis thaliana by functional reconstitution into liposomes and complementation in yeast. Plant J. 33, 1027-1035. doi: 10.1046/j.1365313X.2003.01685.X

Hwang, H. J., Kim, E. H., and Cho, Y. D. (2001). Isolation and properties of arginase from a shade plant, ginseng (Panax ginseng C.A. Meyer) roots. Phytochemistry 58, 1015-1024. doi: 10.1016/S0031-9422(01)00392-2

Illingworth, C., Mayer, M. J., Elliott, K., Hanfrey, C., Walton, N. J., and Michael, A. J. (2003). The diverse bacterial origins of the Arabidopsis polyamine biosynthetic pathway. FEBS Lett. 549, 26-30. doi: 10.1016/S0014-5793(03) 00756-7

Janowitz, T., Kneifel, H., and Piotrowski, M. (2003). Identification and characterization of plant agmatine iminohydrolase, the last missing link in polyamine biosynthesis of plants. FEBS Lett. 544, 258-261. doi: 10.1016/S0014-5793(03)00515-5

Jenkinson, C. P., Grody, W. W., and Cederbaum, S. D. (1996). Comparative properties of arginases. Comp. Biochem. Physiol. B Biochem. Mol. Biol. 114, 107-132. doi: 10.1016/0305-0491(95)02138-8

Kalamaki, M. S., Alexandrou, D., Lazari, D., Merkouropoulos, G., Fotopoulos, V., Pateraki, I., et al. (2009). Over-expression of a tomato N-acetyl-L-glutamate synthase gene (SINAGS1) in Arabidopsis thaliana results in high ornithine levels and increased tolerance in salt and drought stresses. J. Exp. Bot. 60, 1859-1871. doi: $10.1093 /$ jxb/erp072 
Kang, J. H., and Cho, Y. D. (1990). Purification and properties of arginase from soybean, glycine max, axes. Plant Physiol. 93, 1230-1234. doi: 10.1104/pp.93.3.1230

Kanyo, Z. F., Scolnick, L. R., Ash, D. E., and Christianson, D. W. (1996). Structure of a unique binuclear manganese cluster in arginase. Nature 383, 554-557. doi: $10.1038 / 383554 \mathrm{a} 0$

King, J. E., and Gifford, D. J. (1997). Amino acid utilization in seeds of loblolly pine during germination and early seedling growth (I. arginine and arginase activity). Plant Physiol. 113, 1125-1135.

Kollöffel, C., and van Dijke, H. D. (1975). Mitochondrial arginase activity from cotyledons of developing and germinating seeds of Vicia faba L. Plant Physiol. 55, 507-510. doi: 10.1104/pp.55.3.507

Krumpelman, P. M., Freyermuth, S. K., Cannon, J. F., Fink, G. R., and Polacco, J. C. (1995). Nucleotide sequence of Arabidopsis thaliana arginase expressed in yeast. Plant Physiol. 107, 1479-1480. doi: 10.1104/pp.107.4.1479

Lancien, M., Gadal, P., and Hodges, M. (2000). Enzyme redundancy and the importance of 2-oxoglutarate in higher plant ammonium assimilation. Plant Physiol. 123, 817-824. doi: 10.1104/pp.123.3.817

Lawand, S., Dorne, A.-J., Long, D., Coupland, G., Mache, R., and Carol, P. (2002). Arabidopsis A BOUT DE SOUFFLE, which is homologous with mammalian carnitine acyl carrier, is required for postembryonic growth in the light. Plant Cell 14, 2161-2173. doi: 10.1105/tpc.002485

Lee, Y. S., and Cho, Y. D. (2001). Identification of essential active-site residues in ornithine decarboxylase of Nicotiana glutinosa decarboxylating both L-ornithine and L-lysine. Biochem. J. 360, 657-665. doi: 10.1042/0264-6021:3600657

Leitner, M., Vandelle, E., Gaupels, F., Bellin, D., and Delledonne, M. (2009). NO signals in the haze: nitric oxide signaling in plant defence. Curr. Opin. Plant Biol. 12, 451-458. doi: 10.1016/j.pbi.2009.05.012

Levin, E. J., Kondrashov, D. A., Wesenberg, G. E., and Phillips, G. N. (2007). Ensemble refinement of protein crystal structures: validation and application. Structure 15, 1040-1052. doi: 10.1016/j.str.2007.06.019

Linka, M., and Weber, A. P. M. (2005). Shuffling ammonia between mitochondria and plastids during photorespiration. Trends Plant Sci. 10, 461-465. doi: 10.1016/j.tplants.2005.08.002

Llacer, J. L., Fita, I., and Rubio, V. (2008). Arginine and nitrogen storage. Curr. Opin. Struct. Biol. 18, 673-681. doi: 10.1016/j.sbi.2008.11.002

Lozano-Juste, J., and León, J. (2010). Enhanced abscisic acid-mediated responses in nia1 nia2noa1-2 triple mutant impaired in NIA/NR- and AtNOA1-dependent nitric oxide biosynthesis in Arabidopsis. Plant Physiol. 152, 891-903. doi: 10.1104/pp.109.148023

Ma, X., Cheng, Z., Qin, R., Qiu, Y., Heng, Y., Yang, H., et al. (2012). OsARG encodes an arginase that plays critical roles in panicle development and grain production in rice. Plant J. 73, 190-200. doi: 10.1111/j.1365-313X.2012.05122.x

Maheswaran, M., Urbanke, C., and Forchhammer, K. (2004). Complex formation and catalytic activation by the PII signaling protein of N-acetyl-L-glutamate kinase from Synechococcus elongatus strain PCC 7942. J. Biol. Chem. 279, 55202-55210. doi: 10.1074/jbc.M410971200

Maiale, S., Sánchez, D. H., Guirado, A., Vidal, A., and Ruiz, O. A. (2004). Spermine accumulation under salt stress. J. Plant Physiol. 161, 35-42. doi: 10.1078/01761617-01167

Majumdar, R., Shao, L., Minocha, R., Long, S., and Minocha, S. C. (2013). Ornithine: the overlooked molecule in the regulation of polyamine metabolism. Plant Cell Physiol. 54, 990-1004. doi: 10.1093/pcp/pct053

Mansour, M. M. (2000). Nitrogen containing compounds and adaptation of plants to salinity stress. Biol. Plantarum 43, 491-500. doi: 10.1023/A:1002873531707

Marco, F., Alcázar, R., Tiburcio, A. F., and Carrasco, P. (2011). Interactions between polyamines and abiotic stress pathway responses unraveled by transcriptome analysis of polyamine overproducers. OMICS 15, 775-781. doi: 10.1089/omi.2011.0084

Martín-Falquina, A., and Legaz, M. E. (1984). Purification and properties of the constitutive arginase of Evernia prunastri. Plant Physiol. 76, 1065-1069. doi: 10.1104/pp.76.4.1065

Mattioli, R., Marchese, D., D’Angeli, S., Altamura, M. M., Costantino, P., and Trovato, M. (2008). Modulation of intracellular proline levels affects flowering time and inflorescence architecture in Arabidopsis. Plant Mol. Biol. 66, 277-288. doi: 10.1007/s11103-007-9269-1

Meinnel, T., Schmitt, E., Mechulam, Y., and Blanquet, S. (1992). Structural and biochemical characterization of the Escherichia coli $\arg E$ gene product. $J$. Bacteriol. 174, 2323-2331.
Mestichelli, L. J., Gupta, R. N., and Spenser, I. D. (1979). The biosynthetic route from ornithine to proline. J. Biol. Chem. 254, 640-647.

Micallef, B. J., and Shelp, B. J. (1989). Arginine metabolism in developing soybean cotyledons: I. Relationship to nitrogen nutrition. Plant Physiol. 90, 624-630. doi: 10.1104/pp.90.2.624

Michael, A. J., Furze, J. M., Rhodes, M. J., and Burtin, D. (1996). Molecular cloning and functional identification of a plant ornithine decarboxylase cDNA. Biochem. J. 314, 241-248.

Miller, G., Honig, A., Stein, H., Suzuki, N., Mittler, R., and Zilberstein, A. (2009). Unraveling $\Delta^{1}$-pyrroline-5-carboxylate-proline cycle in plants by uncoupled expression of proline oxidation enzymes. J. Biol. Chem. 284, 26482-26492. doi: 10.1074/jbc.M109.009340

Minocha, R., Majumdar, R., and Minocha, S. C. (2014). Polyamines and abiotic stress in plants: a complex relationship. Front. Plant Sci. 5:175. doi: 10.3389/fpls.2014.00175

Molesini, B., Mennella, G., Martini, F., Francese, G., and Pandolfini, T. (2015). Involvement of the putative $\mathrm{N}$-acetylornithine deacetylase from Arabidopsis thaliana in flowering and fruit development. Plant Cell Physiol. 56, 1084-1096. doi: $10.1093 / \mathrm{pcp} / \mathrm{pcv030}$

Mollá-Morales, A., Sarmiento-Mañús, R., Robles, P., Quesada, V., Pérez-Pérez, J. M., González-Bayón, R., et al. (2011). Analysis of ven3 and ven6 reticulate mutants reveals the importance of arginine biosynthesis in Arabidopsis leaf development. Plant J. 65, 335-345. doi: 10.1111/j.1365-313X.2010. 04425.x

Monné, M., Miniero, D. V., Daddabbo, L., Palmieri, L., Porcelli, V., and Palmieri, F. (2015). Mitochondrial transporters for ornithine and related amino acids: a review. Amino Acids doi: 10.1007/s00726-015-1990-5 [Epub ahead of print].

Moreau, M., Lindermayr, C., Durner, J., and Klessig, D. F. (2010). NO synthesis and signaling in plants-where do we stand? Physiol. Plantarum 138, 372-383. doi: 10.1111/j.1399-3054.2009.01308.x

Morris, C. J., Thompson, J. F., and Johnson, C. M. (1969). Metabolism of glutamic acid and $\mathrm{N}$-acetylglutamic acid in leaf discs and cell-free extracts of higher plants. Plant Physiol. 44, 1023-1026. doi: 10.1104/pp.44.7.1023

Murcha, M. W., Elhafez, D., Lister, R., Tonti-Filippini, J., Baumgartner, M., Philippar, K., et al. (2007). Characterization of the preprotein and amino acid transporter gene family in Arabidopsis. Plant Physiol. 143, 199-212. doi: 10.1104/pp.106.090688

Neill, S., Barros, R., Bright, J., Desikan, R., Hancock, J., Harrison, J., et al. (2008). Nitric oxide, stomatal closure, and abiotic stress. J. Exp. Bot. 59, 165-176. doi: $10.1093 / \mathrm{jxb} / \mathrm{erm} 293$

Nonaka, T., Kita, A., Miura-Ohnuma, J., Katoh, E., Inagaki, N., Yamazaki, T., et al. (2005). Crystal structure of putative N-acetyl-gamma-glutamyl-phosphate reductase (AK071544) from rice (Oryza sativa). Proteins 61, 1137-1140. doi: 10.1002/prot.20679

Nordin, A., and Näsholm, T. (1997). Nitrogen storage forms in nine boreal understorey plant species. Oecologia 110, 487-492. doi: 10.1007/s004420050184

Okumoto, S., Koch, W., Tegeder, M., Fischer, W. N., Biehl, A., Leister, D., et al. (2004). Root phloem-specific expression of the plasma membrane amino acid proton co-transporter AAP3. J. Exp. Bot. 55, 2155-2168. doi: 10.1093/jxb/erh233

Page, A. F., Minocha, R., and Minocha, S. C. (2012). Living with high putrescine: expression of ornithine and arginine biosynthetic pathway genes in high and low putrescine producing poplar cells. Amino Acids 42, 295-308. doi: 10.1007/s00726-010-0807-9

Palmieri, L., de Marco, V., Iacobazzi, V., Palmieri, F., Runswick, M. J., and Walker, J. E. (1997). Identification of the yeast $A R G-11$ gene as a mitochondrial ornithine carrier involved in arginine biosynthesis. FEBS Lett. 410, 447-451. doi: 10.1016/S0014-5793(97)00630-3

Palmieri, L., Todd, C. D., Arrigoni, R., Hoyos, M. E., Santoro, A., Polacco, J. C., et al. (2006). Arabidopsis mitochondria have two basic amino acid transporters with partially overlapping specificities and differential expression in seedling development. Biochim. Biophys. Acta 1757, 1277-1283. doi: 10.1016/j.bbabio.2006.03.025

Pathak, M. R., Teixeira da Silva, J. A., and Wani, S. H. (2014). Polyamines in response to abiotic stress tolerance through transgenic approaches. GM Crops Food 5, 87-96. doi: 10.4161/gmcr.28774

Picault, N., Hodges, M., Palmieri, L., and Palmieri, F. (2004). The growing family of mitochondrial carriers in Arabidopsis. Trends Plant Sci. 9, 138-146. doi: 10.1016/j.tplants.2004.01.007 
Piotrowski, M., Janowitz, T., and Kneifel, H. (2003). Plant C-N hydrolases and the identification of a plant $\mathrm{N}$-carbamoylputrescine amidohydrolase involved in polyamine biosynthesis. J. Biol. Chem. 278, 1708-1712. doi: 10.1074/jbc.M205699200

Planchais, S., Cabassa, C., Toka, I., Justin, A.-M., Renou, J.-P., Savouré, A., et al. (2014). BASIC AMINO ACID CARRIER 2 gene expression modulates arginine and urea content and stress recovery in Arabidopsis leaves. Front. Plant Sci. 5:330. doi: $10.3389 /$ fpls.2014.00330

Polacco, J. C., Mazzafera, P., and Tezotto, T. (2013). Opinion: nickel and urease in plants: still many knowledge gaps. Plant Sci. 199-200, 79-90. doi: 10.1016/j.plantsci.2012.10.010

Pudelski, B., Kraus, S., Soll, J., and Philippar, K. (2010). The plant PRAT proteins-preprotein and amino acid transport in mitochondria and chloroplasts. Plant Biol. (Stuttg.) 12(Suppl. 1), 42-55. doi: 10.1111/j.1438-8677.2010.00357.x

Quesada, V., Ponce, M. R., and Micol, J. L. (1999). OTC and AUL1, two convergent and overlapping genes in the nuclear genome of Arabidopsis thaliana. FEBS Lett. 461, 101-106. doi: 10.1016/S0014-5793(99)01426-X

Remacle, C., Cline, S., Boutaffala, L., Gabilly, S., Larosa, V., Barbieri, M. R., et al. (2009). The ARG9 gene encodes the plastid-resident $\mathrm{N}$-acetyl ornithine aminotransferase in the green alga Chlamydomonas reinhardtii. Eukaryot. Cell 8, 1460-1463. doi: 10.1128/EC.00108-09

Rennenberg, H., Wildhagen, H., and Ehlting, B. (2010). Nitrogen nutrition of poplar trees. Plant Biol. (Stuttg.) 12, 275-291. doi: 10.1111/j.1438-8677.2009. 00309.x

Roosens, N. H., Al Bitar, F., Loenders, K., Angenon, G., and Jacobs, M. (2002). Overexpression of ornithine- $\delta$-aminotransferase increases proline biosynthesis and confers osmotolerance in transgenic plants. Mol. Breed. 9, 73-80. doi: 10.1023/A:1026791932238

Roosens, N. H., Thu, T. T., Iskandar, H. M., and Jacobs, M. (1998). Isolation of the ornithine- $\delta$-aminotransferase cDNA and effect of salt stress on its expression in Arabidopsis thaliana. Plant Physiol. 117, 263-271. doi: 10.1104/pp.117. 1.263

Rossig, C., Reinbothe, C., Gray, J., Valdes, O., von Wettstein, D., and Reinbothe, S. (2013). Three proteins mediate import of transit sequence-less precursors into the inner envelope of chloroplasts in Arabidopsis thaliana. Proc. Natl. Acad. Sci. U.S.A. 110, 19962-19967. doi: 10.1073/pnas.1319648110

Sancho-Vaello, E., Fernández-Murga, M. L., and Rubio, V. (2009). Mechanism of arginine regulation of acetylglutamate synthase, the first enzyme of arginine synthesis. FEBS Lett. 583, 202-206. doi: 10.1016/j.febslet.2008.12.001

Sanyal, N., Arentson, B. W., Luo, M., Tanner, J. J., and Becker, D. F. (2015). First evidence for substrate channeling between proline catabolic enzymes: a validation of domain fusion analysis for predicting protein-protein interactions. J. Biol. Chem. 290, 2225-2234. doi: 10.1074/jbc.M114.625483

Schneidereit, J., Häusler, R. E., Fiene, G., Kaiser, W. M., and Weber, A. P. M. (2006). Antisense repression reveals a crucial role of the plastidic 2-oxoglutarate/malate translocator DiT1 at the interface between carbon and nitrogen metabolism. Plant J. 45, 206-224. doi: 10.1111/j.1365-313X.2005.02594.x

Senthil-Kumar, M., and Mysore, K. S. (2012). Ornithine- $\delta$-aminotransferase and proline dehydrogenase genes play a role in non-host disease resistance by regulating pyrroline-5-carboxylate metabolism-induced hypersensitive response. Plant Cell Environ. 35, 1329-1343. doi: 10.1111/j.1365-3040.2012.02492.x

Shao, L., Majumdar, R., and Minocha, S. C. (2012). Profiling the aminopropyltransferases in plants: their structure, expression and manipulation. Amino Acids 42, 813-830. doi: 10.1007/s00726-011-0998-8

Shargool, P. D., Jain, J. C., and McKay, G. (1988). Ornithine biosynthesis, and arginine biosynthesis and degradation in plant cells. Phytochemistry 27, 1571-1574. doi: 10.1016/0031-9422(88)80404-7

Sharma, S., Shinde, S., and Verslues, P. E. (2013). Functional characterization of an ornithine cyclodeaminase-like protein of Arabidopsis thaliana. BMC Plant Biol. 13:182. doi: $10.1186 / 1471-2229-13-182$

Shi, H., Ye, T., Chen, F., Cheng, Z., Wang, Y., Yang, P., et al. (2013). Manipulation of arginase expression modulates abiotic stress tolerance in Arabidopsis: effect on arginine metabolism and ROS accumulation. J. Exp. Bot. 64, 1367-1379. doi: $10.1093 / \mathrm{jxb} / \mathrm{ers} 400$

Siddiqui, M. H., Al-Whaibi, M. H., and Basalah, M. O. (2011). Role of nitric oxide in tolerance of plants to abiotic stress. Protoplasma 248, 447-455. doi: 10.1007/s00709-010-0206-9
Singh, H., Arentson, B. W., Becker, D. F., and Tanner, J. J. (2014). Structures of the PutA peripheral membrane flavoenzyme reveal a dynamic substratechanneling tunnel and the quinone-binding site. Proc. Natl. Acad. Sci. U.S.A. 111, 3389-3394. doi: 10.1073/pnas.1321621111

Slocum, R. D. (2005). Genes, enzymes and regulation of arginine biosynthesis in plants. Plant Physiol. Biochem. 43, 729-745. doi: 10.1016/j.plaphy.2005.06.007

Splittstoesser, W. E. (1969). Metabolism of arginine by aging and 7 day old pumpkin seedlings. Plant Physiol. 44, 361-366. doi: 10.1104/pp.44.3.361

Stránská, J., Kopecný, D., Tylichová, M., Snégaroff, J., and Sebela, M. (2008). Ornithine- $\delta$-aminotransferase: an enzyme implicated in salt tolerance in higher plants. Plant Signal. Behav. 3, 929-935. doi: 10.4161/psb.6771

Stránská, J., Tylichová, M., Kopecný, D., Snégaroff, J., and Sebela, M. (2010). Biochemical characterization of pea ornithine- $\delta$-aminotransferase: substrate specificity and inhibition by di- and polyamines. Biochimie 92, 940-948. doi: 10.1016/j.biochi.2010.03.026

Svennerstam, H., Ganeteg, U., and Näsholm, T. (2008). Root uptake of cationic amino acids by Arabidopsis depends on functional expression of amino acid permease 5. New Phytol. 180, 620-630. doi: 10.1111/j.1469-8137.2008.02589.x

Svennerstam, H., Jämtgård, S., Ahmad, I., Huss-Danell, K., Näsholm, T., and Ganeteg, U. (2011). Transporters in Arabidopsis roots mediating uptake of amino acids at naturally occurring concentrations. New Phytol. 191, 459-467. doi: $10.1111 /$ j.1469-8137.2011.03699.x

Taylor, M. R., Reinders, A., and Ward, J. M. (2015). Transport function of rice amino acid permeases (AAPs). Plant Cell Physiol. doi: 10.1093/pcp/pcv053 [Epub ahead of print].

Tegeder, M. (2014). Transporters involved in source to sink partitioning of amino acids and ureides: opportunities for crop improvement. J. Exp. Bot. 65, 1865-1878. doi: 10.1093/jxb/eru012

Tiburcio, A. F., Altabella, T., Bitrian, M., and Alcazar, R. (2014). The roles of polyamines during the lifespan of plants: from development to stress. Planta 240, 1-18. doi: 10.1007/s00425-014-2055-9

Todd, C. D., Cooke, J. E., Mullen, R. T., and Gifford, D. J. (2001). Regulation of loblolly pine (Pinus taeda L.) arginase in developing seedling tissue during germination and post-germinative growth. Plant Mol. Biol. 45, 555-565. doi: 10.1023/A:1010645616920

Toka, I., Planchais, S., Cabassa, C., Justin, A.-M., de Vos, D., Richard, L., et al. (2010). Mutations in the hyperosmotic stress-responsive mitochondrial BASIC AMINO ACID CARRIER2 enhance proline accumulation in Arabidopsis. Plant Physiol. 152, 1851-1862. doi: 10.1104/pp.109.152371

Trovato, M., Maras, B., Linhares, F., and Costantino, P. (2001). The plant oncogene rolD encodes a functional ornithine cyclodeaminase. Proc. Natl. Acad. Sci. U.S.A. 98, 13449-13453. doi: 10.1073/pnas.231320398

Tun, N. N., Santa-Catarina, C., Begum, T., Silveira, V., Handro, W., Floh, E. I. S., et al. (2006). Polyamines induce rapid biosynthesis of nitric oxide (NO) in Arabidopsis thaliana seedlings. Plant Cell Physiol. 47, 346-354. doi: $10.1093 / \mathrm{pcp} / \mathrm{pci} 252$

VanEtten, C. H., Wolff, I. A., Jones, Q., and Miller, R. W. (1963). Amino acid composition of seeds from 200 angiospermous plant species. J. Agric. Food Chem. 11, 399-410. doi: 10.1021/jf60129a016

Vogel, H. J., and Bonner, D. M. (1956). Acetylornithinase of Escherichia coli: partial purification and some properties. J. Biol. Chem. 218, 97-106.

Wang, B.-Q., Zhang, Q.-F., Liu, J.-H., and Li, G.-H. (2011). Overexpression of PtADC confers enhanced dehydration and drought tolerance in transgenic tobacco and tomato: effect on ROS elimination. Biochem. Biophys. Res. Commun. 413, 10-16. doi: 10.1016/j.bbrc.2011.08.015

Williams, I., and Frank, L. (1975). Improved chemical synthesis and enzymatic assay of $\Delta^{1}$-pyrroline-5-carboxylic acid. Anal. Biochem. 64, 85-97. doi: 10.1016/00032697(75) $90408-\mathrm{X}$

Wimalasekera, R., Tebartz, F., and Scherer, G. F. E. (2011). Polyamines, polyamine oxidases and nitric oxide in development, abiotic and biotic stresses. Plant Sci. 181, 593-603. doi: 10.1016/j.plantsci.2011.04.002

Winter, G. (2013). Molecular and Physiological Characterization of Arginine and Proline Catabolism in Arabidopsis. Ph.D. thesis, University of Konstanz, Konstanz.

Witte, C.-P. (2011). Urea metabolism in plants. Plant Sci. 180, 431-438. doi: 10.1016/j.plantsci.2010.11.010

Witte, C.-P., Rosso, M. G., and Romeis, T. (2005). Identification of three urease accessory proteins that are required for urease activation in Arabidopsis. Plant Physiol. 139, 1155-1162. doi: 10.1104/pp.105.070292 
Xia, J., Yamaji, N., Che, J., Shen, R. F., and Ma, J. F. (2014a). Normal root elongation requires arginine produced by argininosuccinate lyase in rice. Plant J. 78, 215-226. doi: $10.1111 /$ tpj.12476

Xia, J., Yamaji, N., and Ma, J. F. (2014b). An appropriate concentration of arginine is required for normal root growth in rice. Plant Signal. Behav. 9, e28717. doi: $10.4161 / \mathrm{psb} .28717$

You, J., Hu, H., and Xiong, L. (2012). An ornithine $\delta$-aminotransferase gene $O s O A T$ confers drought and oxidative stress tolerance in rice. Plant Sci. 197, 59-69. doi: 10.1016/j.plantsci.2012.09.002

Yu, M., Lamattina, L., Spoel, S. H., and Loake, G. J. (2014). Nitric oxide function in plant biology: a redox cue in deconvolution. New Phytol. 202, 1142-1156. doi: 10.1111/nph.12739

Zainal, Z., Sajari, R., and Ismail, I. (2002). Molecular cloning and sequence analysis of a cDNA encoding ornithine decarboxylase cDNA from chilli (Capsicum annuum). J. Biochem. Mol. Biol. Biophys. 6, 415-419. doi: $10.1080 / 1025814021000036151$

Zhang, J.-J., Li, X.-Q., Sun, J.-W., and Jin, S.-H. (2014). Nitric oxide functions as a signal in ultraviolet-B-induced baicalin accumulation in Scutellaria baicalensis suspension cultures. Int. J. Mol. Sci. 15, 4733-4746. doi: 10.3390/ijms15034733
Zhao, M.-G., Tian, Q.-Y., and Zhang, W.-H. (2007). Nitric oxide synthasedependent nitric oxide production is associated with salt tolerance in Arabidopsis. Plant Physiol. 144, 206-217. doi: 10.1104/pp.107. 096842

Zonia, L. E., Stebbins, N. E., and Polacco, J. C. (1995). Essential role of urease in germination of nitrogen-limited Arabidopsis thaliana seeds. Plant Physiol. 107, 1097-1103. doi: 10.1104/pp.107.4.1097

Conflict of Interest Statement: The authors declare that the research was conducted in the absence of any commercial or financial relationships that could be construed as a potential conflict of interest.

Copyright (๑) 2015 Winter, Todd, Trovato, Forlani and Funck. This is an open-access article distributed under the terms of the Creative Commons Attribution License (CC $B Y)$. The use, distribution or reproduction in other forums is permitted, provided the original author(s) or licensor are credited and that the original publication in this journal is cited, in accordance with accepted academic practice. No use, distribution or reproduction is permitted which does not comply with these terms. 


\section{Glutamate, Ornithine, Arginine, Proline, and Polyamine Metabolic Interactions: The Pathway Is \\ OPEN ACCESS \\ Edited by: \\ Dietmar Funck, \\ University of Konstanz, Germany Regulated at the Post-Transcriptional Level}

Reviewed by:

Barry J. Shelp,

University of Guelph, Canada

Manchikatla Venkat Rajam,

University of Delhi South Campus,

India

*Correspondence:

Subhash C. Minocha

sminocha@unh.edu

${ }^{\dagger}$ Present Address:

Rajtilak Majumdar,

United States Department of

Agriculture-Agricultural Research

Service, Southern Regional Research

Center, New Orleans, LA, USA

Maegan Gagne,

Department of Botany, University of

Wisconsin, Madison, WI, USA

Specialty section:

This article was submitted to

Plant Physiology,

a section of the journal

Frontiers in Plant Science

Received: 17 July 2015 Accepted: 16 January 2016

Published: 16 February 2016

Citation:

Majumdar R, Barchi B, Turlapati SA,

Gagne M, Minocha R, Long S and

Minocha SC (2016) Glutamate,

Ornithine, Arginine, Proline, and

Polyamine Metabolic Interactions: The

Pathway Is Regulated at the

Post-Transcriptional Level.

Front. Plant Sci. 7:78.

doi: 10.3389/fp/s.2016.00078

\author{
Rajtilak Majumdar ${ }^{1 \dagger}$, Boubker Barchi ${ }^{1}$, Swathi A. Turlapati ${ }^{1,2}$, Maegan Gagne ${ }^{1 \dagger}$, \\ Rakesh Minocha ${ }^{2}$, Stephanie Long ${ }^{2}$ and Subhash C. Minocha ${ }^{1 *}$ \\ ${ }^{1}$ Department of Biological Sciences, University of New Hampshire, Durham, NH, USA, ${ }^{2}$ United States Department of \\ Agriculture Forest Service, Northern Research Station, Durham, NH, USA
}

The metabolism of glutamate into ornithine, arginine, proline, and polyamines is a major network of nitrogen-metabolizing pathways in plants, which also produces intermediates like nitric oxide, and $\gamma$-aminobutyric acid (GABA) that play critical roles in plant development and stress. While the accumulations of intermediates and the products of this network depend primarily on nitrogen assimilation, the overall regulation of the interacting sub-pathways is not well understood. We tested the hypothesis that diversion of ornithine into polyamine biosynthesis (by transgenic approach) not only plays a role in regulating its own biosynthesis from glutamate but also affects arginine and proline biosynthesis. Using two high putrescine producing lines of Arabidopsis thaliana (containing a transgenic mouse ornithine decarboxylase gene), we studied the: (1) effects of exogenous supply of carbon and nitrogen on polyamines and pools of soluble amino acids; and, (2) expression of genes encoding key enzymes in the interactive pathways of arginine, proline and GABA biosynthesis as well as the catabolism of polyamines. Our findings suggest that: (1) the overall conversion of glutamate to arginine and polyamines is enhanced by increased utilization of ornithine for polyamine biosynthesis by the transgene product; (2) proline and arginine biosynthesis are regulated independently of polyamines and GABA biosynthesis; (3) the expression of most genes (28 that were studied) that encode enzymes of the interacting sub-pathways of arginine and GABA biosynthesis does not change even though overall biosynthesis of Orn from glutamate is increased several fold; and (4) increased polyamine biosynthesis results in increased assimilation of both nitrogen and carbon by the cells.

Keywords: Arabidopsis thaliana, arginine, $\gamma$-aminobutyric acid, glutamate, ornithine, polyamines, proline, quantitative polymerase chain reaction 


\section{INTRODUCTION}

The glutamate (Glu) to proline (Pro), ornithine (Orn), arginine (Arg), polyamines (PAs), and $\gamma$-aminobutyric acid (GABA) group of reactions constitutes one of the major interactive pathways for carbon $(\mathrm{C})$ and nitrogen $(\mathrm{N})$ assimilation and partitioning (Figure 1). The products of this pathway have a wide range of physiological functions in plants. In addition to the production of amino acids and signal molecules like GABA and nitric oxide (NO), this group of sub-pathways is the primary source of putrescine (Put) biosynthesis, which in turn produces the other two common PAs, spermidine (Spd) and spermine (Spm). The three PAs are obligatory requirements for cell survival and growth through their molecular interactions with nucleic acids (transcription and translation) and cellular membranes (Kusano et al., 2007; Minocha et al., 2014); they are also a source of GABA, which plays critical roles in diverse cellular functions in plants (Shelp et al., 2012a). Interactions of PAs with polyanionic macromolecules and cellular membranes (Wallace et al., 2003), and with hydroxycinnamic acids, fatty acids or alkaloids underlie some of their roles in abiotic and biotic stress responses (Flores and Filner, 1985; Martin-Tanguy, 1997; Ghosh, 2000; Bagni and Tassoni, 2001; Subramanyam et al., 2015).

Cellular accumulation of PAs is the net result of their biosynthesis, inter-conversions, catabolism and conjugation (the last one particularly in mature tissues and organs); their biosynthesis is controlled by enzyme activity as well as the availability of substrates. The two pathways of Put biosynthesis (Figure 1) appear to be independently regulated by separate enzymes, namely Arg decarboxylase (ADC - EC: 4.1.1.19) and Orn decarboxylase (ODC - EC: 4.1.1.17). Spermidine and Spm biosynthesis is controlled by a combination of Sadenosylmethionine decarboxylase (SAMDC, a.k.a. AdoMet DC - EC: 4.1.1.50) and two aminopropyltransferases, namely Spd synthase (SPDS - EC 2.5.1.16) and Spm synthase (SPMS EC 2.5.1.22; reviewed in Shao et al., 2014). Additionally, it is known that the diversion of SAM toward PAs (e.g., via transgenic expression of yeast SPDS and SAMDC genes in tomato fruits) can enhance the metabolic interactions (cf. competition) of PAs and ethylene $\left(\mathrm{C}_{2} \mathrm{H}_{4}\right)$ biosynthesis, and delay fruit ripening and senescence, thereby increasing the shelf life of the fruit (Nambeesan et al., 2010; Lasanajak et al., 2014). The catabolism of PAs, which produces GABA (a metabolite of great significance for its positive role in the oxidative stress response-Shi et al., 2010; Vergara et al., 2012), and $\mathrm{H}_{2} \mathrm{O}_{2}$ in the apoplast (for cell wall lignin biosynthesis), is also involved in maintaining the balance of C:N in plants (Bouché and Fromm, 2004; Fait et al., 2008). Further complexity of cellular PA functions involves their interactions with plasma membrane cellular $\mathrm{H}^{+}$pumps (Garufi et al., 2007) and the transport of $\mathrm{Ca}^{2+}$ and $\mathrm{K}^{+}$across root membranes in a species-specific manner (Zepeda-Jazo et al., 2011).

Due to their pleiotropic functions, regulation of PA homeostasis is complex (Agostinelli, 2014). Several recent studies have shown that homeostatic up-regulation of Put biosynthesis (e.g., via transgenic approaches) leads to widespread metabolic consequences affecting several amino acids, sugars, sugar alcohols, phytochelatins, organic acids and inorganic ions
(Minocha et al., 2004; Mattoo et al., 2010; Mohapatra et al., 2010a,b; Page et al., 2012; Majumdar et al., 2013). Arginine, Pro, GABA and Put concentrations in plants are among the known indicators of various forms of abiotic stress in herbaceous annuals, as well as woody perennials (Ericsson et al., 1993, 1995; Näsholm et al., 1994, 1997, 2000; Wargo et al., 2002; Mohapatra et al., 2010b; Minocha et al., 2013, 2015).

Glutamate $\rightarrow$ Orn $\rightarrow$ Arg, Glu $\rightarrow$ Pro, and Orn $\rightarrow$ Pro are largely reversible linear pathways, while Put production is a branched irreversible pathway using Orn and Arg as substrates; this pathway also leads to the production of Spd, Spm, and GABA (Figure 1). In addition, GABA is synthesized directly from Glu by the enzyme Glu decarboxylase (GAD - EC: 4.1.1.15). Although there is abundant literature on GABA biosynthesis and its physiological functions, specific contributions of the direct (Glu $\rightarrow \mathrm{GABA})$ vis-a-vis indirect $(\mathrm{Glu} \rightarrow \mathrm{Orn} / \mathrm{Arg} \rightarrow \mathrm{Put} \rightarrow \mathrm{GABA})$ pathways of its biosynthesis are not known (Shelp et al., 2012a; Trobacher et al., 2013; $\mathrm{Hu}$ et al., 2015). Likewise, regulation of the flux of Glu into $\mathrm{Orn} / \mathrm{Arg} / \mathrm{Put}$ and Pro under conditions of increased need for the biosynthesis of Put (e.g., due to abiotic stress response or experimental up-regulation of Put production via transgenic approaches) is still enigmatic. Equally puzzling is the mechanism by which the multi-step process of Glu $\rightarrow$ Orn/Arg is regulated. Our previous studies with genetically engineered poplar (Populus nigra $x$ maximowiczii-clone NM6) cell cultures for high Put production [via transgenic expression of a mouse $O D C(\mathrm{mODC})$, which produces Put from Orn] have suggested that the need for increased Orn production may be met without induction (i.e., transcription) of most of the enzymes of the Glu $\rightarrow$ Orn pathway (Page et al., 2007, 2012).

The research presented here was aimed at investigating the effects of perturbation of the Glu $\rightarrow$ Orn $\rightarrow$ Arg $\rightarrow$ Put, $\mathrm{Glu} \rightarrow \underline{\mathrm{Orn}} \rightarrow$ Pro, Arg $\rightarrow \underline{\mathrm{Orn}} \rightarrow$ Glu and Pro pathway into PAs by creating a new path (a short cut) for redirecting Orn toward Put, something that $A$. thaliana does not naturally do. The transgenic production of a mODC enzyme, which has a rather low $\mathrm{Km}$ $(<100 \mu \mathrm{M})$ for Orn (Coleman et al., 1993), efficiently converts large amounts of Orn into Put (Descenzo and Minocha, 1993; Bastola and Minocha, 1995; Bhatnagar et al., 2001; Majumdar et al., 2013), which can be stored in plants in relatively large (mM) concentrations.

We report here the results of our study involving: (1) the effects of supplementary $\mathrm{N}$ and $\mathrm{C}$ application on cellular PAs and amino acids in the wild type (WT) and transgenic plants of Arabidopsis thaliana, which produce several-fold higher concentrations of Put via the constitutive (2x35S CaMV promoter) expression of $\mathrm{mODC}$ gene (cDNA); and (2) the effects of inducible expression of $\mathrm{mODC}$ (transient increase in Put production) on the expression of genes encoding several enzymes of the Orn $\rightarrow$ Arg, Orn $\Leftrightarrow$ Pro, Glu/PAs $\rightarrow$ GABA pathways, as well as those involved with initial steps in the PA catabolism pathway. The aim was to assess if one or more specific step (or steps) in the pathway is (are) transcriptionally triggered in response to increased consumption of Orn (or increased production of Put) via constitutive or inducible production of $m O D C$. We 


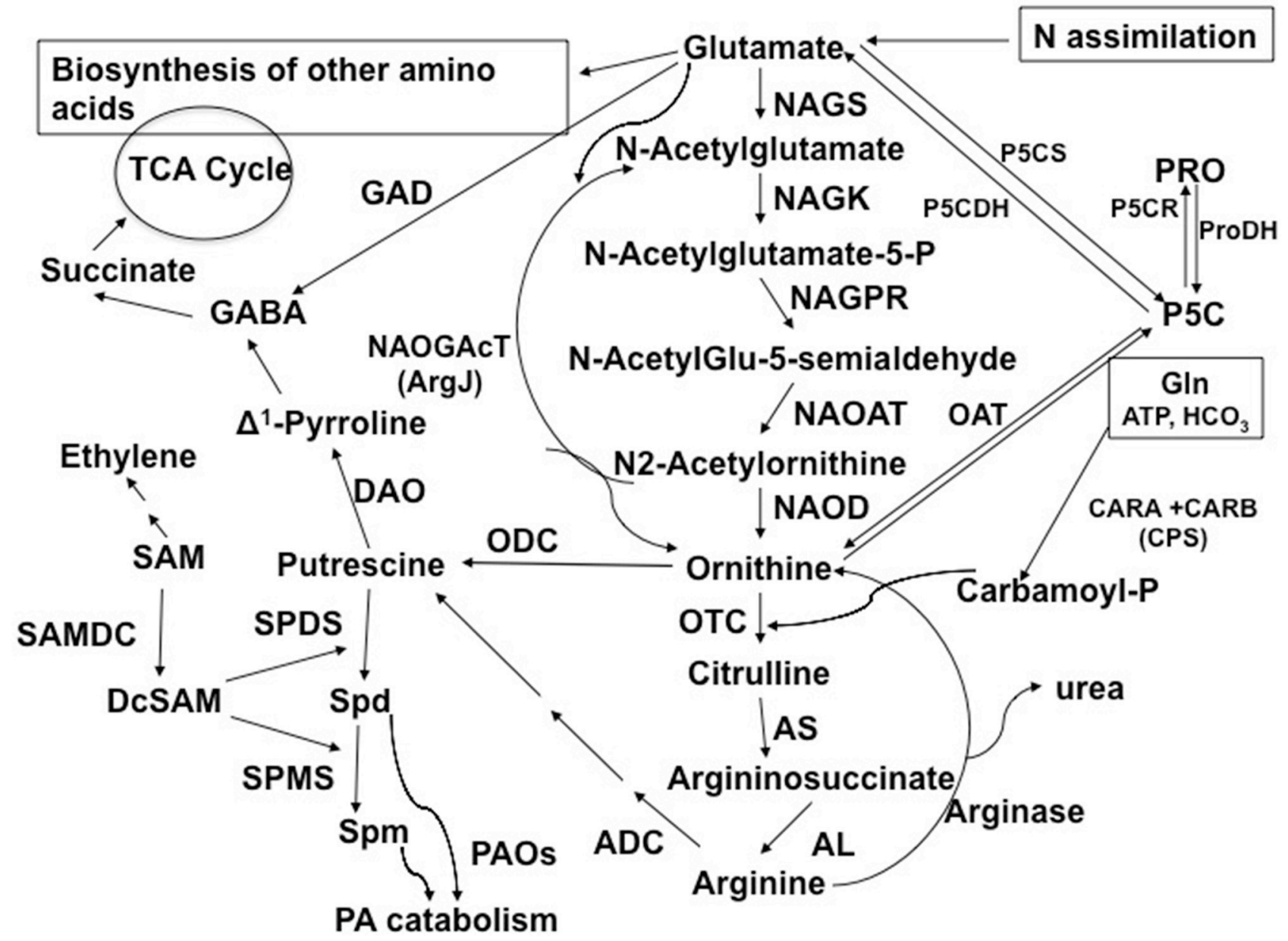

FIGURE 1 | Abbreviated pathway for the biosynthesis and metabolism of Orn in plants in connection to polyamines, amino acids, TCA cycle metabolites, and alkaloids. Abbreviations and $E C$ numbers of the enzymes: ADC, arginine decarboxylase (EC 4.1.1.19); AL, argininosuccinate lyase (EC 4.3.2.1); AS, argininosuccinate synthase (EC 6.3.4.5); CPS (CARA and CARB, carbamoylphosphate synthetase (EC 6.3.5.5); DAO, diamine oxidase (EC 1.4.3.22); GAD, glutamate decarboxylase (EC 4.1.1.15); NAGK, N-acetylglutamate kinase (EC 2.7.2.8); NAGPR, N-acetylglutamate-5-phosphate reductase (EC 1.2.1.38); NAGS, $N$-acetylglutamate synthase (EC 2.3.1.1); NAOAT, N-acetylornithine aminotransferase (EC 2.6.1.11); NAOD, N-acetylornithine deacetylase (EC 3.5.1.16); NAOGAcT, $\mathrm{N}_{2}$-Acetylornithine:Glu-acetyl transferase or ArgJ (EC 2.3.1.35); OAT, ornithine $\delta$-aminotransferase (EC 2.6.1.13); ODC, ornithine decarboxylase (EC 4.1.1.17); OTC, ornithine transcarbamoylase (EC 2.1.3.3); ProDH, proline dehydrogenase (EC 1.5.99.8); P5CDH, $\Delta^{1}$-pyrroline-5-carboxylate dehydrogenase (EC 1.5.1.12); P5CR, $\Delta^{1}$-pyrroline-5-carboxylate reductase (EC 1.5.1.2); P5CS, $\Delta^{1}$-pyrroline-5-carboxylate synthetase (EC 2.7.2.11/1.2.1.41); SAMDC, S-adenosylmethionine decarboxylase (EC 4.1.1.50); SPDS, spermidine synthase (EC 2.5.1.16); PAO, polyamine oxidase (EC 1.5.3.11); SPMS, spermine synthase (EC 2.5.1.22). Modified from Majumdar et al. (2015).

also report on the accumulation of biomass and changes in chlorophyll and total $\mathrm{N}$ and $\mathrm{C}$ per plant in response to additional supply of $\mathrm{N}$ and $\mathrm{C}$ to the plants.

\section{MATERIALS AND METHODS}

\section{Transgenic Arabidopsis thaliana Plants}

The mODC cDNA that was earlier used for poplar cell transformation (Bhatnagar et al., 2001) was PCR amplified and cloned into pCR8.0/GW/TOPO or $\mathrm{pENTR}^{\mathrm{TM}} / \mathrm{D}-\mathrm{TOPO}^{\circledR}$ vector (Invitrogen, Carlsbad, CA), and subsequently transferred into Gateway-compatible pMDC32 vector (containing 2x35S promoter) and pMDC7 vector (containing estradiol inducible
promoter-Curtis and Grossniklaus, 2003; Majumdar et al., 2013), respectively. The resultant vectors with the mODC coding sequence were used to transform $A$. thaliana (Columbia-0) plants to obtain constitutive and inducible mODC transgenic lines (Majumdar et al., 2013). These lines were characterized for the presence of the transgenes (hygromycin resistanceHPTII for selection of transformants and the mODC gene) by Polymerase Chain Reaction (PCR), their transcription (by Reverse Transcriptase PCR), and for their PA contents by High Performance Liquid Chromatography (HPLC). Third or fourth generation $\left(\mathrm{T}_{3}\right.$ or $\mathrm{T}_{4}$ homozygous for the $\mathrm{mODC}$ gene) seeds homozygous for the two transgenes were used for all experiments. 


\section{Growth Conditions and Treatments}

Arabidopsis seeds $\left(\mathrm{T}_{3}\right)$ were surface sterilized with ethanol, dried under the laminar flow hood, and grown on Petri dishes with solid germination medium (GM) containing 4.3 g. $\mathrm{L}^{-1}$ of Murashige and Skoog (1962) premix salt powder (MS) plus Gamborg's organics (Gamborg et al., 1968), 0.5 g.L $\mathrm{L}^{-1}$ of 2-(N-Morpholino) ethanesulfonic acid (MES), $1 \%(\sim 30 \mathrm{mM})$ sucrose, and $0.8 \%$ type A agar (Sigma Aldrich, St. Louis, MO), and adjusted to $\mathrm{pH}$ 5.7. For $\mathrm{N}$ and sucrose treatments, seeds were germinated on: (i) GM supplemented with either $30 \mathrm{mM}$ or $60 \mathrm{mM}$ additional $\mathrm{KNO}_{3}$ (regular GM contains $39.43 \mathrm{mM}$ $\mathrm{NO}_{3}$ ), (ii) modified GM without $\mathrm{N}$ (i.e., $\mathrm{MS}$ without $\mathrm{NH}_{4} \mathrm{NO}_{3}$ and $\mathrm{KNO}_{3}$ but with additional $\mathrm{KCl}$ to maintain equimolar concentration of K), and (iii) GM without sucrose or containing 20 or $70 \mathrm{mM}$ additional concentration of sucrose. Following cold $\left(4^{\circ} \mathrm{C}\right)$ treatment (in the dark) for 2 days, the seeds in Petri dishes were transferred to a walk-in growth chamber at $25 \pm 1^{\circ} \mathrm{C}$ under $12 / 12 \mathrm{~h}$ photoperiod $\left(80 \pm 10 \mu \mathrm{E} \mathrm{m}^{-2} \cdot \mathrm{s}^{-1}\right)$. At 12 days, batches of seedlings (7-8 per batch) were collected for fresh weight (FW) and dry weight (DW) analysis in glass vials, and for PA and amino acids analyses in $5 \%$ perchloric acid (PCA, $\sim 0.77 \mathrm{~N}-9 \mu \mathrm{L} \cdot \mathrm{mg}^{-1}$ $\mathrm{FW})$. The former were dried at constant temperature $\left(70^{\circ} \mathrm{C}\right)$ in a drying oven and the latter were stored at $-20^{\circ} \mathrm{C}$ prior to analysis of PAs and amino acids (AAs).

For short-term mODC induction experiments, 2 week-old seedlings (from $\mathrm{T}_{4}$ seeds) of an inducible $\mathrm{mODC}$ line germinated on solid GM were transferred to 12 well $(4$ columns $\times 3$ rows) culture plates. Each well contained $15-20$ seedlings (3 replicate wells for each treatment) resting in $1 \mathrm{~mL}$ of liquid GM plus $5 \mu \mathrm{M}$ estradiol (inducer; Sigma-Aldrich) or no estradiol (control treatment). Replicate wells of the same treatment were arranged in a column and induced vs. un-induced plants of the same genotype were kept in adjacent wells. Thus, the WT and transgenic plants were kept in different 12 -well culture plates. All plates were kept in the walk-in growth chamber under conditions described above. Seedlings were collected at 24 and $48 \mathrm{~h}$ after induction/treatment. At the time of collection, seedlings were placed on paper towels for a few seconds to remove excess liquid, and $\sim 50 \mathrm{mg}$ FW (8-10 whole seedlings) samples were collected in triplicate in microfuge tubes and mixed with 5\% PCA $(9 \mu \mathrm{L} 5 \%$ PCA per mg FW seedlings), and stored at $-20^{\circ} \mathrm{C}$ for PA analyses.

\section{Plant Growth in Soil}

Arabidopsis seeds ( $\mathrm{T}_{4}$ generation) were sown in moist soil mix containing 3 parts Scott's 360 Metro-Mix (Scotts Company, Marysville, $\mathrm{OH}$ ) and 1 part perlite in $3^{\prime \prime}$ pots. In each pot, 1015 seeds were planted and the pots were placed in a plastic tray covered with a clear plastic lid; the trays were kept for $48 \mathrm{~h}$ in the dark at $4^{\circ} \mathrm{C}$. Thereafter, the trays were moved to a walk-in growth chamber at $21^{\circ} \mathrm{C}$ under $18 \mathrm{~h}$ photoperiod $\left(80 \pm 10 \mu \mathrm{E} \mathrm{m}^{-2} \mathrm{~s}^{-1}\right)$. Plants were watered on alternate days and supplied with the addition of $1 / 4$ strength Miracle-Gro (Scotts Company) synthetic fertilizer in the irrigation water every 5th day. Two weeks after germination, the plants were thinned to two plants per pot.

The foliage of 5 week-old WT and mODC-1-7 transgenic (constitutive) plants grown in pots $(3$ pots/treatment for both WT and mODC plants; each pot containing 2 plants) were sprayed with two different $\mathrm{N}$ fertilizers (each at $2 \%$ concentration): urea (20-0-0) or Nitamin ${ }^{\circledR}$ (30-0-0; http://www. kochagronomicservices.com/downloads/d682.aspx?type=view) or pure water, all containing 0.05\% Silwet surfactant (http:// www.helenachemical.com/products/utility/silwet-177/). For PA analysis, leaves (3-4 leaves to yield $\sim 100 \mathrm{mg} \mathrm{FW}$ ) were collected in 5\% PCA (3 replicates) at 1, 2, 5, and 8 days after treatment. For DW analysis, whole plants were collected (three replicates) from each treatment after 8 days, weighed, oven dried $\left(\right.$ at $80^{\circ} \mathrm{C}$ for $48 \mathrm{~h}$ ) and weighed again.

\section{Quantification of Soluble Protein, Chlorophyll and Total $\mathbf{C}$ and $\mathbf{N}$}

Chlorophyll analysis was performed using a modified protocol from Gitelson et al. (2009). Two to three healthy rosette leaves from outer whorls of 4 week-old-plants were weighed and homogenized in $\mathrm{MeOH}\left(200 \mu \mathrm{L} \mathrm{mg}^{-1} \mathrm{FW}\right)$ using a mortar and pestle. Ten to twenty mg of $\mathrm{CaCO}_{3}$ was added to the samples while grinding to prevent pheophytization of chlorophyll. Homogenates were centrifuged for $5 \mathrm{~min}$ at 10,000 $\mathrm{xg}$ and the $\mathrm{A}_{665}$ and $\mathrm{A}_{652}$ of the supernatants were measured (Spectronic Instruments Inc., Rochester, NY). Chlorophyll a and chlorophyll b concentrations were calculated as per Lichtenthaler and Buschmann (1987).

For the analyses of total $\mathrm{C}$ and $\mathrm{N}$, individual plants were collected separately, dried at $70^{\circ} \mathrm{C}$, and analyzed using a $\mathrm{CE}$ Elantech Flash EA1112 combustion NC Soil analyzer (Thermo Scientific, Lakewood, NJ) according to EPA method 440.0 using NIST (National Institute of Standards and Technology, Gaithersburg, MD, USA) standard reference materials 1515 (apple leaves) and 1547 (peach leaves) for procedure verification (Mohapatra et al., 2010a).

Total soluble protein concentration was analyzed in tissue extracts in potassium phosphate buffer $(0.1 \mathrm{M} ; \mathrm{pH} 7.0)$ by the Bradford (1976) method using bovine serum albumin as standard.

\section{Quantification of Polyamines and Amino Acids}

Polyamines and amino acids were analyzed in samples stored in 5\% PCA by dansylation and HPLC. Plant samples in PCA were subjected to three cycles of freezing at $-20^{\circ} \mathrm{C}$ and thawing at room temperature before dansylation. The extracts, after the final thawing, were vortexed for $1 \mathrm{~min}$ and centrifuged for $5 \mathrm{~min}$ at $14,000 \mathrm{xg}$. One hundered $\mu \mathrm{L}$ of the supernatant from each sample and 5 standards (mixture of 3 PAs) with $20 \mu \mathrm{L}$ of $0.1 \mathrm{mM}$ heptanediamine as the internal standard were dansylated as per Minocha and Long (2004) using $50 \mu \mathrm{L}$ of $20 \mathrm{mg} \mathrm{mL}^{-1}$ Asn (in water) to remove the unreacted dansyl chloride. The dansyl-PAs were extracted in $400 \mu \mathrm{L}$ of toluene by partitioning. Aliquots of $200 \mu \mathrm{L}$ from the toluene fraction were transferred into new microfuge tubes and vacuum dried. Dansyl-PAs were dissolved in $500 \mu \mathrm{L}$ of methanol and transferred into autosampler vials for analysis by HPLC. The HPLC system included a series 200 autosampler (PerkinElmer Inc., Waltham, MA), quaternary pump and fluorescence detector fitted with a Pecosphere $\mathrm{C}_{18}$ 
reversed phase cartridge column $(4.6 \times 33 \mathrm{~mm}, 3 \mu \mathrm{m})$. The detector was set at excitation and emission wavelengths of 340 and $515 \mathrm{~nm}$, respectively. Ten or $20 \mu \mathrm{L}$ of standards and samples were injected and separated using a $40 \%$ acetonitrile (in $10 \mathrm{mM}$ heptane sulfonic acid) to $100 \%$ acetonitrile in a linear gradient at a flow rate of $2.5 \mathrm{~mL} \mathrm{~min}{ }^{-1}$. The data were integrated using Perkin Elmer TotalChrom software (version 6.2.1). Amino acids were analyzed by HPLC as per Minocha and Long (2004).

\section{Statistical Analysis of Biochemical Data}

For all experiments, typically three biological replicates were used per treatment. Each experiment was repeated at least twice and data from a single representative experiment are presented here. The data were analyzed using one-way ANOVA and Tukey's test for comparison between the treatment and the respective control as indicated specifically in Figure legends. Analyses were done using SYSTAT Version 10.2 for Windows (Systat Software, Inc., San Jose, CA 95131 USA) and Microsoft Excel (Version 2010); significant difference between treatment and control were analyzed at $P \leq 0.05$.

\section{Analysis of Gene Expression by qPCR - Preparation of RNA and cDNA}

Two separate experiments were conducted for analysis of gene expression, which involved slightly different procedures. In one experiment, 28 genes were tested using a single set of homozygous plants ( $\mathrm{T}_{3}$ generation) from which RNA was isolated once and cDNA was prepared twice. The procedures for RNA isolation, cDNA preparation and qPCR analysis data for this experiment are presented as Supplemental Material. The second experiment involved the following procedure, but was used only for 15 genes (including $\mathrm{mODC}$ ); the same transgenic line was used for this experiment.

At 14 days post germination, batches of $12-15$ seedlings were transferred from the germination plates into 12-well plates. Each batch of seedlings placed in a well was considered one replicate and each 12-well plate contained all treatments. Treatment position was randomly allocated within each block following a randomized complete block design with 4 replicates. Each well contained $1 \mathrm{~mL}$ of liquid GM. The seedlings were allowed to acclimate overnight in the growth chamber. Induction of mODC was accomplished by adding a final concentration of $5 \mu \mathrm{M}$ estradiol (from $10 \mathrm{mM}$ stock dissolved in dimethyl sulfoxide). Samples were collected at 24 and $48 \mathrm{~h}$ after induction for PA quantification (by HPLC) as well as for RNA extraction (frozen in liquid nitrogen and at $-80^{\circ} \mathrm{C}$ ).

RNA extractions were performed maintaining the block structure of the experiment. Frozen tissue was homogenized in liquid nitrogen using disposable RNase-free pestles and a cordless pestle motor (Thermo-Fisher Scientific, Waltham, MA). While tissue was still frozen, Tri reagent (Thermo-Fisher) was added at a ratio of $1 \mathrm{~mL}$ to $100 \mathrm{mg}$ of tissue. Samples were incubated at room temperature for $5 \mathrm{~min}$, then centrifuged at 12,000 $\mathrm{xg}$ for $10 \mathrm{~min}$ at $4^{\circ} \mathrm{C}$. The clear homogenate was transferred to a new tube and chloroform was added at a ratio of $0.2 \mathrm{~mL}$ for $1 \mathrm{~mL}$ of Tri reagent originally added. Tubes were shaken aggressively, incubated at room temperature for $3 \mathrm{~min}$, and centrifuged at
$12,000 \mathrm{xg}$ for $15 \mathrm{~min}$ at $4^{\circ} \mathrm{C}$. The upper aqueous phase was removed and mixed with half a volume of isopropanol. Samples were inverted 10 times and frozen at $-20^{\circ} \mathrm{C}$ overnight. RNA was pelleted by centrifuging at $12,000 \mathrm{xg}$ for $10 \mathrm{~min}$ at $4^{\circ} \mathrm{C}$. The pellets was washed with $75 \%$ ethanol by centrifugation, dried for $10 \mathrm{~min}$ at room temperature, re-suspended in RNase-free water, and analyzed on the Agilent 2200 TapeStation with the RNA ScreenTape kit (Agilent Technologies, Santa Clara, CA). Three high quality samples with clear $18 \mathrm{~S}, 28 \mathrm{~S}, 16 \mathrm{~S}$, and $23 \mathrm{~S}$ peaks were used for CDNA preparation.

First strand cDNA synthesis was done using High-Capacity RNA to cDNA kit (Applied Biosystems, Grand Island, NY) following the manufacturer's protocol. The final reaction volume of $20 \mu \mathrm{L}$ contained $10 \mu \mathrm{L}$ of $2 \mathrm{X}$ RT Master Mix and $10 \mu \mathrm{L}$ of RNA sample (up to $2 \mu \mathrm{g}$ of total RNA). The reaction was set up in an Eppendorf Mastercycler ${ }^{\circledR}$ Nexus PCR thermal cycler (Eppendorf NA, Hauppauge, NY) with the following conditions: $60 \mathrm{~min}$ at $37^{\circ} \mathrm{C}$ and $5 \mathrm{~min}$ at $95^{\circ} \mathrm{C}$, hold at $4^{\circ} \mathrm{C}$. The cDNA was stored at $-20^{\circ} \mathrm{C}$ for later use in $\mathrm{qPCR}$ analysis. All gene expression analyses reported here were done from the same RNA preparation and one set of cDNA preparation.

\section{Quantitative Polymerase Chain Reaction (qPCR)}

RT-PCRs were conducted by using $1 \mu \mathrm{l}$ of cDNA as initial template, $200 \mathrm{nM}$ concentration of each gene specific forward and reverse primer combination (Supplemental Table 1), and One-Taq ${ }^{\circledR}$ Quick-Load ${ }^{\circledR}$ 2X Mastermix with standard Buffer (New England Biolabs, Ipswich, MA) in Eppendorf Mastercycler (Eppendorf Corp, Enfield, CT) following standard cycling conditions (denaturation at $94^{\circ} \mathrm{C}$ for $30 \mathrm{~s}$ followed by 35 cycles of denaturation at $94^{\circ} \mathrm{C}$ for $30 \mathrm{~s}$, annealing for $30 \mathrm{~s}$ at $58^{\circ} \mathrm{C}$ and extension step at $68^{\circ} \mathrm{C}$ for $1 \mathrm{~min}$ ). The amplified products were electrophoresed on $1 \%$ agarose gels and the size of the amplicons was verified with the published literature.

The qPCR standards were made as follows: mix $2 \mu \mathrm{L}$ aliquot from each cDNA sample, and make seven 4-fold serial dilutions. Primer efficiencies and dissociation curve analyses were conducted by using Fast SYBR ${ }^{\circledR}$ Green Master Mix (Applied Biosystems, Grand Island, NY) following the manufacturer's protocol. Briefly, $5.5 \mu \mathrm{L}$ of Fast SYBR green, and a final $300 \mathrm{nM}$ concentration of each of the gene-specific forward and reverse primers (Supplemental Table 1), and $4 \mu \mathrm{L}$ volume from each standard were mixed in a final reaction volume of $10 \mu \mathrm{L}$. The reactions were run with two technical replicates per biological replicate in non-skirted natural 96-well reaction plates (MIDSCI, Valley Park, MO), using Quantitative PCR, SYBR green dissociation curve method (Stratagene MxPro 3000 qPCR Thermocycler). The qPCR reactions were conducted with the following conditions: initial denaturation at $95^{\circ} \mathrm{C}$ for $15 \mathrm{~s}$, and 40 cycles of denaturation at $95^{\circ} \mathrm{C}$ for $15 \mathrm{~s}$ and annealing for $30 \mathrm{~s}$ at $60^{\circ} \mathrm{C}$ followed by a dissociation step of denaturation at $95^{\circ} \mathrm{C}$ for $1 \mathrm{~min}, 55^{\circ} \mathrm{C}$ for $30 \mathrm{~s}$ and $95^{\circ} \mathrm{C}$ for $30 \mathrm{~s}$ to confirm the single specific amplicon.

For all primer sets, the dissociation curve resulted in a single peak and the resulting standard curve showed an $R^{2}$ value > 
0.9. The primer efficiencies were within $10 \%$ difference with the internal control gene. Those gene primers that worked under standard conditions were used further for comparative quantitation using $20 \mathrm{ng}$ of each induced and un-induced sample with the latter referred to as calibrator. The standard thermal cycling conditions were imported to conduct comparative quantitation assays. Non-template controls were included for each reaction, and they showed no amplification and had no $\mathrm{Ct}$ value. The data were analyzed using MxPro Software which follows standard Pfaffl (2001) method based on the gene primer efficiencies. All gene expression data were analyzed for relative fold changes using AtTIP41 (At4g34270-Czechowski et al., 2005; Han et al., 2013) as normalizer or internal control gene run within each plate for both 24 and $48 \mathrm{~h}$ induced and uninduced samples. The fold changes in the induced samples were calculated in the comparative quantitation assay based on the calibrator average. Statistical comparison between induced and un-induced samples was done using one-way ANOVA and Tukey's test for comparison between the induced and the uninduced plants. Analyses were done using SYSTAT Version 10.2 for Windows; significant difference between treatment and control were analyzed at $P \leq 0.05$. However, only differences that were greater than 2 -fold are marked with an * in the Figures.

\section{RESULTS}

\section{Increased Polyamine Biosynthesis Draws Extra Nitrogen and Carbon in Young Seedlings}

Since Orn is a limiting metabolite for continued production of Put by the transgenic $\mathrm{mODC}$, and since it is present in relatively small concentrations, and is largely produced from Glu whose biosynthesis requires the continued supply/assimilation of $\mathrm{N}$ and C, two specific questions were addressed: (1) Will increased supply of $\mathrm{N}$ or $\mathrm{C}$ in the growth medium allow extra Put to be produced in the WT and/or the transgenic plants? (2) Will the increased availability of either $\mathrm{N}$ or $\mathrm{C}$ in the medium affect the amino acids pool in the WT and/or the transgenic plants?

With a few exceptions, the FW as well as DW of seedlings of both genotypes were higher in the presence of additional $\mathrm{NO}_{3}$ or sucrose in the medium (over and above the normal concentration in the GM) as compared to those growing in control GM (Figures 2A,B). Additional sucrose at $70 \mathrm{mM}$ had the greatest effect on DW in both genotypes (Figure 2B). Absence of $\mathrm{N}$ in the medium caused a significant reduction (vs. GM) in FW and DW of both genotypes.

When compared to the WT, the mODC-1-7 transgenic seedlings had up to 50 fold higher concentration of Put (Figures 3A,B) with only small changes in the concentrations of Spd and Spm (Figures 3C,D). In the absence of N, Put was reduced by $>90 \%$ as compared to the control GM for both genotypes, and Spd and Spm were reduced by up to $40 \%$. The seedlings of both genotypes appeared unhealthy (i.e., yellowish) in the absence of N. Supplementation of GM with $30 \mathrm{mM} \mathrm{NO}_{3}$ had no effect on Put in either genotype; however, Spd was slightly lower and Spm slightly higher in the transgenic seedlings.
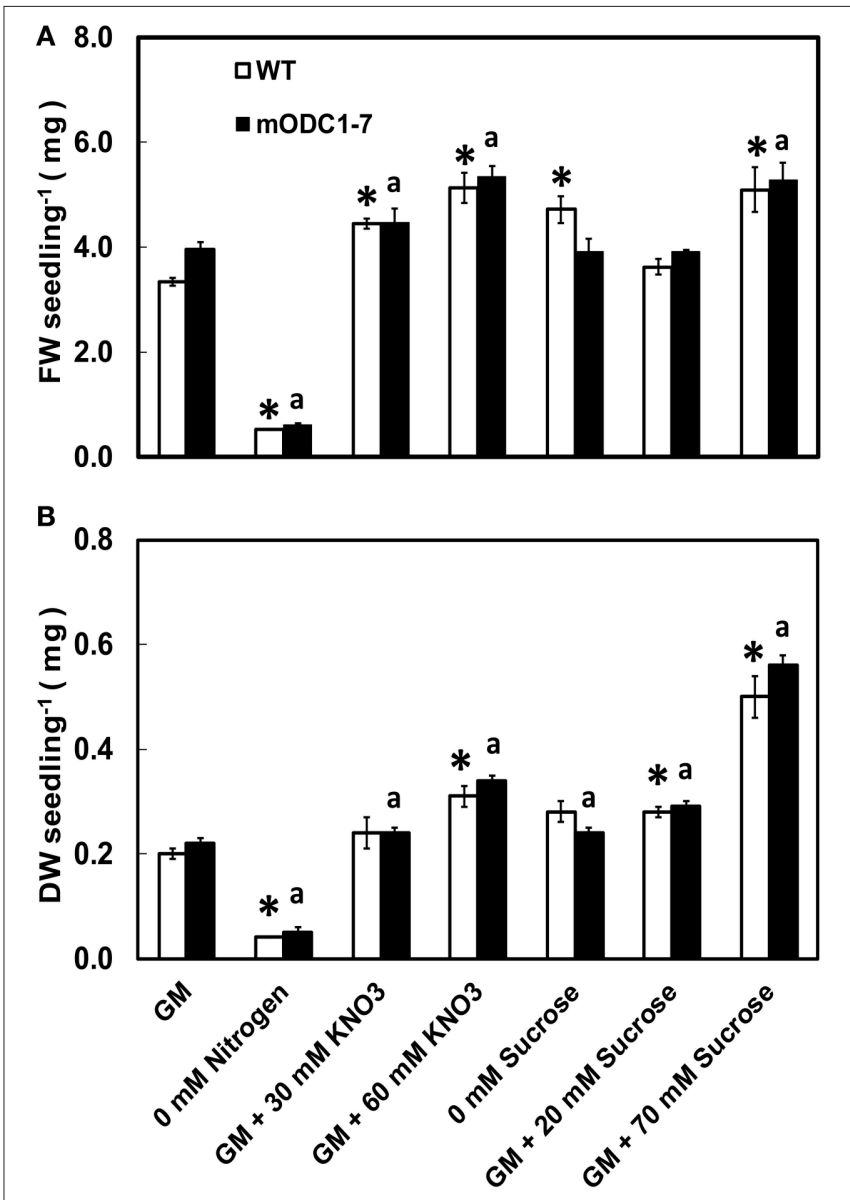

FIGURE 2 | Effects of different concentrations of nitrate and sucrose on (A) fresh weight (FW) and (B) dry weight (DW) of 12 day old WT and 2x35S::mODC-1-7 transgenic Arabidopsis thaliana seedlings grown on solid GM. Data are mean of $4 \pm$ SE; each replicate consists of 40-50 seedlings. *Denotes significant difference between treatments and control in WT seedlings and ${ }^{a}$ denotes significant difference between treatments and control in mODC seedlings $(P \leq 0.05)$.

At $60 \mathrm{mM}$ additional $\mathrm{NO}_{3}$, there was a negative effect on Put and Spd in both genotypes but a small increase in Spm was observed in the transgenic seedlings; the effect on Put was more pronounced in transgenic plants (Figure 3D).

In the absence of sucrose, concentrations of the three PAs were affected differently; the differences were not always significant (Figure 3). In the WT seedlings, additional sucrose caused a concentration-dependent increase in Put, but in the transgenic plants the greatest increase was in response to $20 \mathrm{mM}$ additional sucrose; $70 \mathrm{mM}$ additional sucrose actually having a deleterious effect on Put in these plants. Additional sucrose caused a significant increase in Spd concentration in the WT plants, but less so in the transgenic plants; Spm was higher than the control $\mathrm{GM}$ in the transgenic plants given $70 \mathrm{mM}$ additional sucrose.

Significant differences were observed in cellular concentrations of several amino acids in the two genotypes in response to different $\mathrm{NO}_{3}$ and sucrose treatments. The abundant ( $>500 \mathrm{nmol} \mathrm{g}^{-1} \mathrm{FW}$ ) amino acids in both genotypes included Glu, Gln, Arg (+Thr+Gly), serine (Ser), alanine 

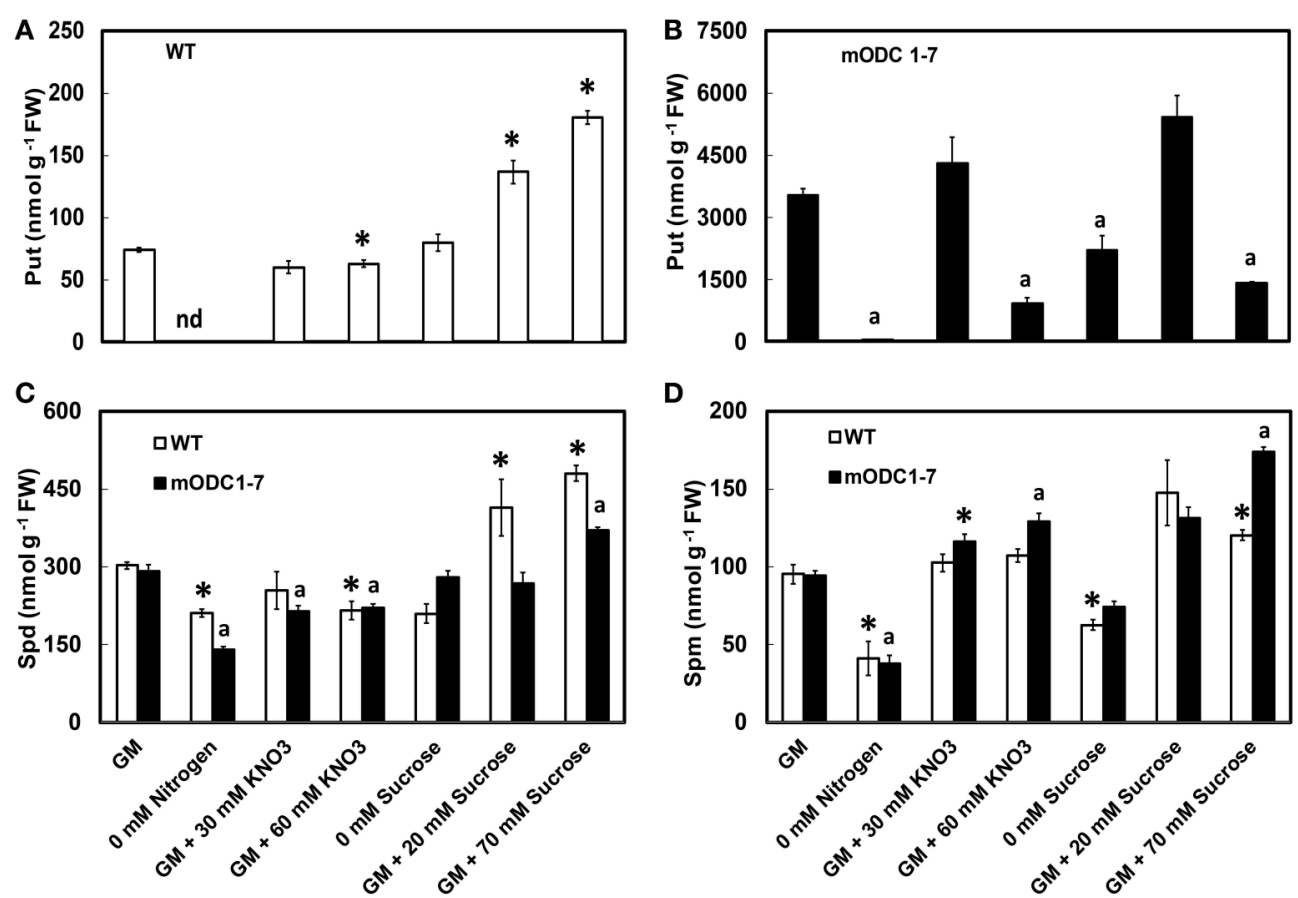

FIGURE 3 | Effects of different concentrations of nitrate and sucrose on PCA soluble polyamines in 12 day old WT and 2x35S::mODC Arabidopsis thaliana seedlings grown on solid GM. Cellular concentrations of (A,B) putrescine, (C) spermidine, and (D) spermine. Data are mean of $4 \pm$ SE; each replicate $=$ 7-8 seedlings. For symbols, see Figure 2.

(Ala), and Pro; those present in the group of relatively low concentrations $\left(<100 \mathrm{nmol} \mathrm{g}^{-1} \mathrm{FW}\right)$ were Leu, Met, Lys, His, and Trp (Figure 4 and Supplemental Figure 1). Ornithine, which was always present in the WT plants, was below detection limits in the transgenic plants under almost all conditions (Figure 4F). As mentioned above, plants in the $\mathrm{N}$-free medium appeared unhealthy and their cellular amino acid concentrations were highly reduced.

There was no significant difference in the cellular concentrations of Glu or Gln in either genotype in response to additional $\mathrm{N}$ or sucrose with the exceptions of increase in both these amino acids in the transgenic plants grown on GM with $70 \mathrm{mM}$ additional sucrose (Figures 4A,B). While the absence of $\mathrm{N}$ caused a significant reduction in the two amino acids in both genotypes, the absence of sucrose had no such effect. In the control GM, Pro concentration was similar in the two genotypes and its cellular concentration increased with increasing concentrations of both $\mathrm{NO}_{3}$ and sucrose in the medium. In response to $70 \mathrm{mM}$ additional sucrose, Pro concentrations were almost twice in the mODC seedlings vs. the WT seedlings (Figure 4C). The absence of either N or sucrose in the medium significantly reduced Pro in both genotypes.

The combined concentrations of Arg+Thr+Gly (peaks inseparable in most cases by the HPLC method used) were similar in the WT and transgenic seedlings. This group of amino acids was generally unaffected by additional sucrose and both the absence of $\mathrm{N}$ as well as additional $\mathrm{N}$ at $60 \mathrm{mM}$ caused a reduction in these amino acids (Figure 4D). Whereas, the absence of $\mathrm{N}$ resulted in lower cellular concentration of GABA in the transgenic plants, the absence of sucrose had little effect on GABA. Higher sucrose resulted in elevated GABA concentrations in both genotypes (Figure 4E).

In the transgenic plants, Orn was below the detection limit of our technique (Minocha and Long, 2004) in all treatments except for supplementation of the medium with $30 \mathrm{mM} \mathrm{NO}_{3}$ (Figure 4F). In the WT plants, higher $\mathrm{NO}_{3}$ in the medium resulted in a decrease in Orn concentration. Extra sucrose $(\mathrm{GM}+20 \mathrm{mM})$ caused an increase, but $+70 \mathrm{mM}$ caused a decrease in Orn in the WT plants; there was no detectable effect of increased sucrose on Orn in the transgenic plants.

Changes in cellular concentrations of other amino acids that are not direct intermediates/products of the $\mathrm{Glu} \rightarrow \mathrm{Orn} \rightarrow \mathrm{Arg} / \mathrm{Pro} / \mathrm{Put}$ pathway are described in the Supplemental Material section (Supplemental Figure 1).

\section{Constitutive Expression of $\mathrm{mODC}$ in Mature Plants}

Two of the transgenic mODC lines (mODC1-7 and mODC411; constitutive expression), which were tested for Put at the seedling stage (Majumdar et al., 2013), showed a delay (generally 7-8 days) in flowering as compared to the WT plants (data not shown). While $\sim 90 \%$ of the WT plants were flowering by the end of 5 weeks, $<50 \%$ of the (mODC constitutive) transgenic plants were flowering at that time. The initial delay in flowering was eventually overcome in the course of time and the transgenic lines showed a significant increase in vegetative 


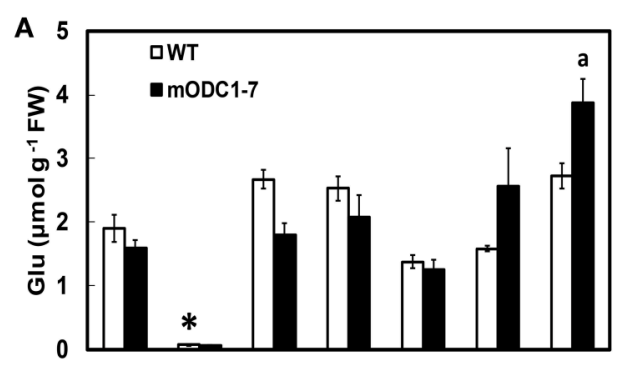

C

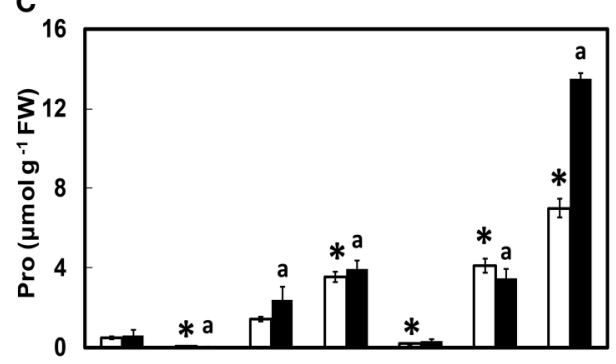

E

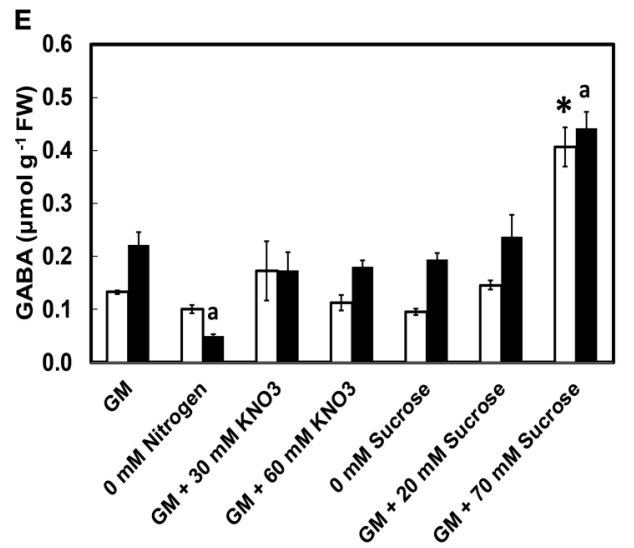

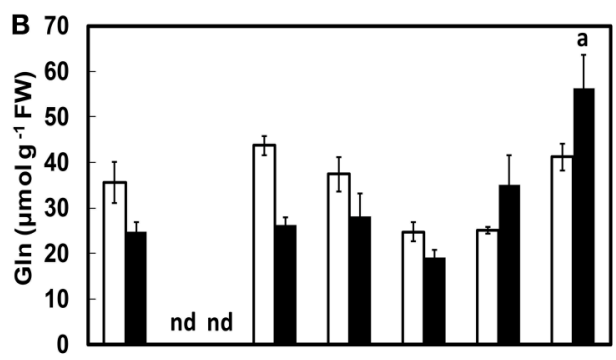

D

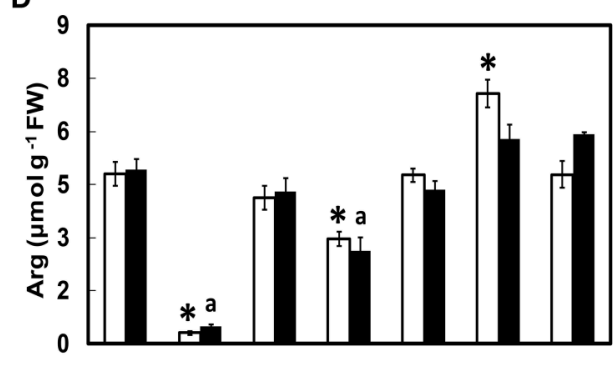

F

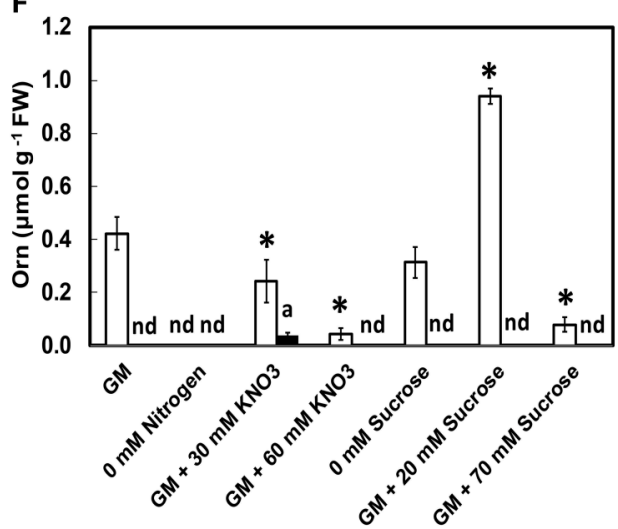

FIGURE 4 | Effects of different concentrations of nitrate and sucrose on PCA soluble amino acids in 12 day old WT and 2x35S::mODC-1-7 Arabidopsis thaliana seedlings. Cellular concentrations of (A) glutamate, (B) glutamine, (C) proline, (D) arginine+threonine+glycine, (E) $\gamma$-aminobutyric acid, and (F) ornithine. Data are mean of $4 \pm$ SE; each replicate $=7-8$ seedlings. For symbols, see Figure 2.

as well as reproductive growth. Both FW and DW per plant (at 6 weeks post germination) were significantly higher in the transgenic line mODC4-11 (Figures 5A,B). At full maturity, the number of branches and the number of siliques per plant were greater in both transgenic lines vs. the WT plants (Figures 5C,D).

As compared to the WT, both mODC transgenic lines had significantly higher chlorophyll $a$ and chlorophyll $b$ concentrations on per mg protein basis (Figure $5 \mathrm{E}$ ). The contents of both total $\mathrm{C}$ and total $\mathrm{N}$ were slightly, yet significantly, higher in $\mathrm{mODC}-4-11$ plants vs. the WT plants (Figure $5 \mathbf{F}$ ).

Since the mODC-1-7 line had consistently higher concentration of Put vs. the mODC-4-11 line at the seedling stage, the plants of this line were grown to maturity and various plant organs were analyzed for their PA and amino acid concentrations (Figure 6; Supplemental Figures 2, 3). Significantly higher Put was observed in the cauline leaves and silique tissues of the transgenic plants as compared to the WT plants (Supplemental Figure 2A). The only notable difference in Spd was in the rosette leaves, which was significantly lower in the $\mathrm{mODC}$ plants (Supplemental Figure 2B). Spermine was higher in the rosette leaves, buds and flowers of the mODC line as compared with the WT plants (Supplemental Figure 2C). Cadaverine was always present in all organs of transgenic plants, but was never detected in the WT plants (Supplemental Figure 2D).

In mature plants the amino acids of Glu family, whose concentrations were higher in the transgenic plants (vs. WT plants) were Glu and Gln in buds, and Pro in rosette leaves (Figures 6A-C). Conversely, Glu in the leaves, Orn in the flowers, and Arg and Orn in the siliques were somewhat higher in the WT vs. the transgenic plants (Figures 6A,D,F). In all plant parts tested, GABA was not significantly different in the two genotypes (Figure 6E). The remaining amino acids are discussed in the Supplemental Material (Supplemental Figure 3). 


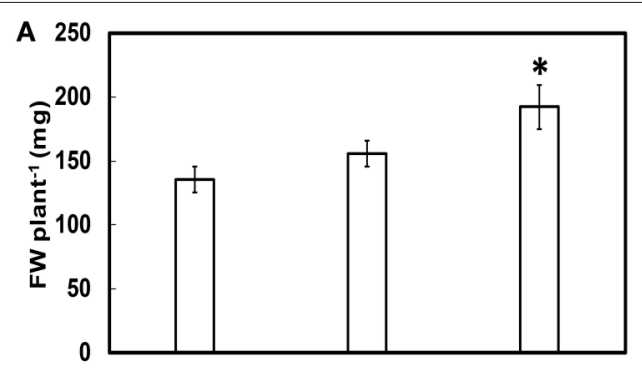

C

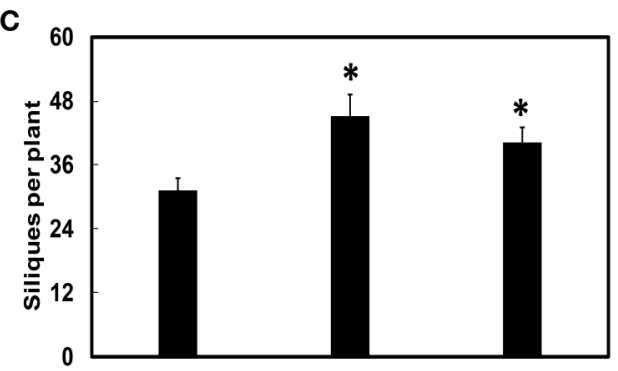

E

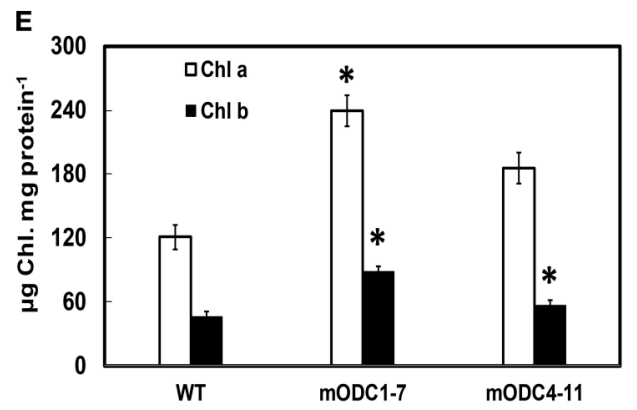

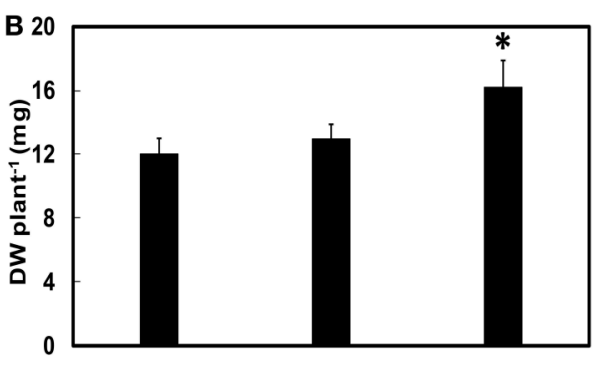

D

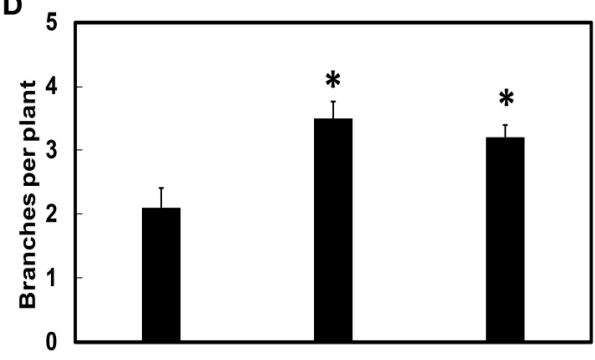

$\mathbf{F}$

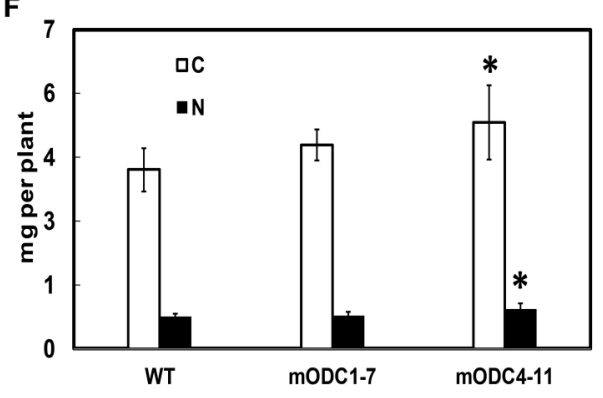

FIGURE 5 | Comparison of different phenotypic and related biochemical parameters between WT and mODC transgenic Arabidopsis thaliana plants for (A) fresh weight ( $n=9)$, (B) dry weight $(n=9)$, (C) silique number $(n=7)$, (D) number of branches $(n=10)$, (E) leaf chlorophyll concentration ( $n=4)$, and $(\mathbf{F})$ total carbon and nitrogen concentrations $(\boldsymbol{n}=\mathbf{1 0})$. Data are mean \pm SE. Plants for $(\mathbf{A}-\mathbf{E})$ were 6 week-old and near full maturity. ${ }^{*}$ Denotes significant difference between $\mathrm{WT}$ and $\mathrm{mODC}$ transgenic plants $(P \leq 0.05)$.

\section{Effect of Foliar Nitrogen Application on Polyamines and Biomass}

In order to investigate if mature plants producing constitutively high Put were able to assimilate leaf-applied N, WT and constitutive transgenic plants (mODC-1-7) were sprayed with either $2 \%$ urea $(20: 0: 0)$ or $2 \%$ Nitamin (30:0:0), and tested for their PA concentration in the rosette leaves, and also for total above-ground plant biomass. As compared to the water sprayed (control) plants, the application of either urea or Nitamin to the foliage resulted in a 2.5 to 3 -fold increase in cellular Put within 1 to 2 days after spray in the leaves of transgenic plants (Figures 7A,B); this increase was not sustained after 2 days. On the other hand, there was no significant change in Put concentration in WT plants in response to $\mathrm{N}$ treatments as compared to the control (water) treatment. It should be noted that Put concentration of mature leaves of transgenic plants was only 2 to 3 -fold higher than the WT plants, whereas at the seedling stage, the transgenic plants often accumulated as much as 40-fold higher concentrations of Put (Figures 3, 7). Cellular concentrations of Spd and Spm were similar in the $\mathrm{WT}$ and the transgenic plants, and generally decreased at $24 \mathrm{~h}$ in response to Nitamin treatments (Figures 7C-F). Nitamin treatment caused a significant decrease in Spm in both genotypes at all times of analysis. There was no significant increase in DW of transgenic plants in response to foliar $\mathrm{N}$ application (Supplemental Figure 4).

\section{Gene Expression of the \\ Glu-Orn-Pro/Arg-PA-GABA Pathway Enzymes in Response to Increased Put Biosynthesis}

Our experimental manipulation of one step (i.e., Orn $\rightarrow$ Put) in the interactive pathways shown in Figure 1, using a transgene that has no sequence homology to a native gene in A. thaliana, obviously resulted in overutilization of Orn, which must be replenished via its increased biosynthesis from Glu to serve the demands of $\mathrm{mODC}$ as well as the other products of these pathways. The pathways of Glu to Arg, Pro, PAs, and GABA involve about 20 enzymes encoded by $>30$ genes that have been identified in A. thaliana (Supplemental Table 1). In order to understand the transcriptional regulation of $\mathrm{Glu} \rightarrow \mathrm{Orn} \rightarrow \mathrm{Arg}$, 

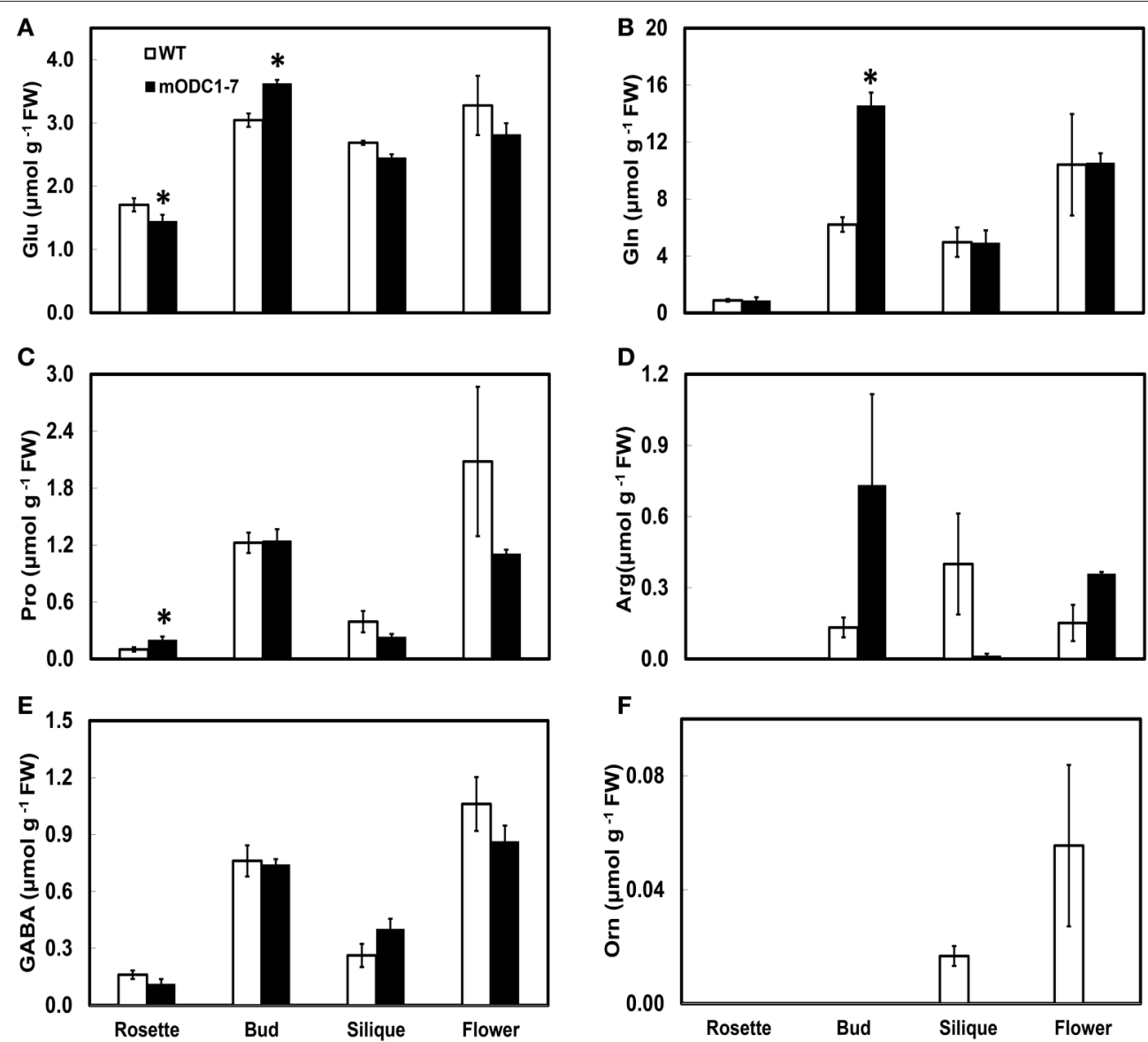

FIGURE 6 | Cellular concentrations of amino acids (A) Glu, (B) GIn, (C) Pro, (D) Arg, (E) GABA, and (F) Orn, in different tissues of mature (6 week-old) WT and 2x35S::mODC-1-7 transgenic Arabidopsis thaliana plants. Data are mean of $3 \pm$ SE; each replicate consists of 2-3 individual plants. ${ }^{\star}$ Denotes significant difference between WT and mODC transgenic plants for each particular organ $(P \leq 0.05)$.

$\mathrm{Glu} \Leftrightarrow \operatorname{Pro} \Leftrightarrow$ Orn, Put/PA catabolism, and $\mathrm{Glu} \rightarrow \mathrm{GABA}$ in response to short term $(24-48 \mathrm{~h})$ increase in Put via induction (by estradiol) of the inducible $\mathrm{mODC}$ transgene, we addressed three complementary questions in the present study: (i) Does the increased biosynthesis of Orn from Glu require the induction of genes encoding various enzymes involved in Orn biosynthesis? (ii) Does the overutilization of Orn affect gene expression of the enzymes involved in the interacting pathways of Arg, Pro and GABA biosynthesis? (iii) Does the expression of genes involved in PA catabolism (some that produce GABA) change in response to overproduction of Put? The technique of qPCR using primers specific for each of the known $A$. thaliana genes of these sub-pathways was used with cDNA made from mRNA isolated at 24 and $48 \mathrm{~h}$ after the induction of $\mathrm{mODC}$ in young seedlings. The results presented in Figure 8 and Supplemental Figure 5 are the results of two separate experiments using the same inducible transgenic cell line but slightly different approaches for qPCR setup and analyses of results (See Materials and Methods, Figure legends and Supplemental Material for details). The results of relative expression of various genes are grouped according to sub-pathways of Glu $\rightarrow$ Orn, Orn $\rightarrow$ Arg,
$\mathrm{Glu} \Leftrightarrow \mathrm{Pro} \Leftrightarrow$ Orn, $\mathrm{Put} / \mathrm{PA} \quad$ catabolism and $\mathrm{Glu} \rightarrow \mathrm{GABA}$ production.

The mODC transgenic seedlings used for qPCR typically showed several-hundred-fold increase in mODC transcripts upon induction, with very little $\mathrm{mODC}$ transcript being detected in the un-induced plants (data not shown). There was a concomitant 10 - to 20 -fold increase in Put in the induced seedlings vs. the control (un-induced) seedlings at 24 and $48 \mathrm{~h}$, with only small or no change in Spd and Spm (data published earlier - Majumdar et al., 2013).

\section{Glutamate to Orn-Arg-Pro Pathway Genes}

Of the six enzymes involved in Glu to Orn subpathway, cDNA sequences for all genes except NAOD were available in the literature (Supplemental Table 1). Six of the seven total genes were tested for change in their relative expression (vs. uninduced) following induction at 24 and/or $48 \mathrm{~h}$; none showed a significant change that was greater than 2-fold (Figure 8A and Supplemental Figure 5A). Only one gene of the Orn $\rightarrow$ Arg pathway (i.e., CARB) encoding the large subunit of CPS (Gln-dependent carbamoyl-P synthetase) showed a significant 

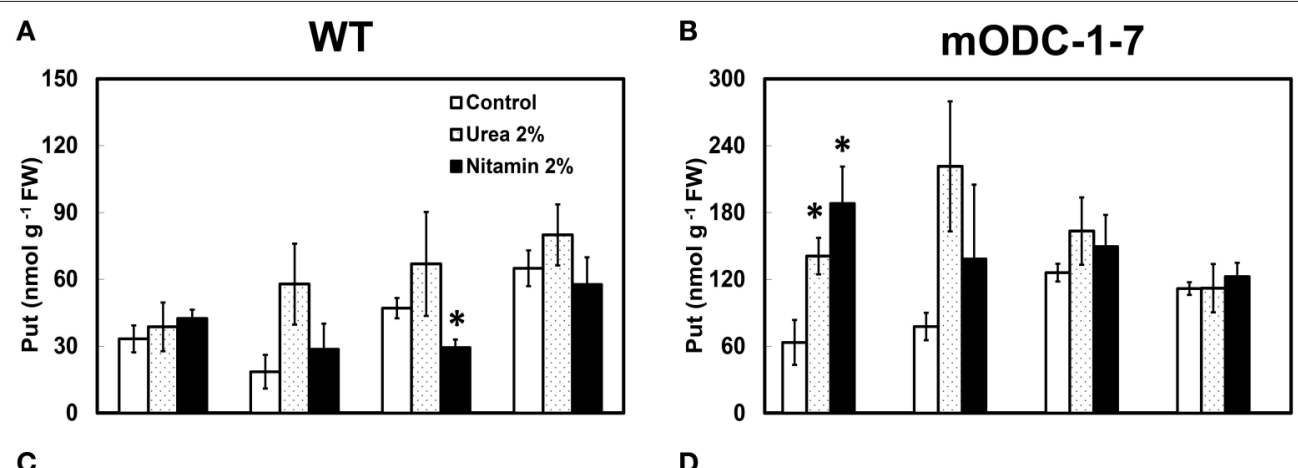

C
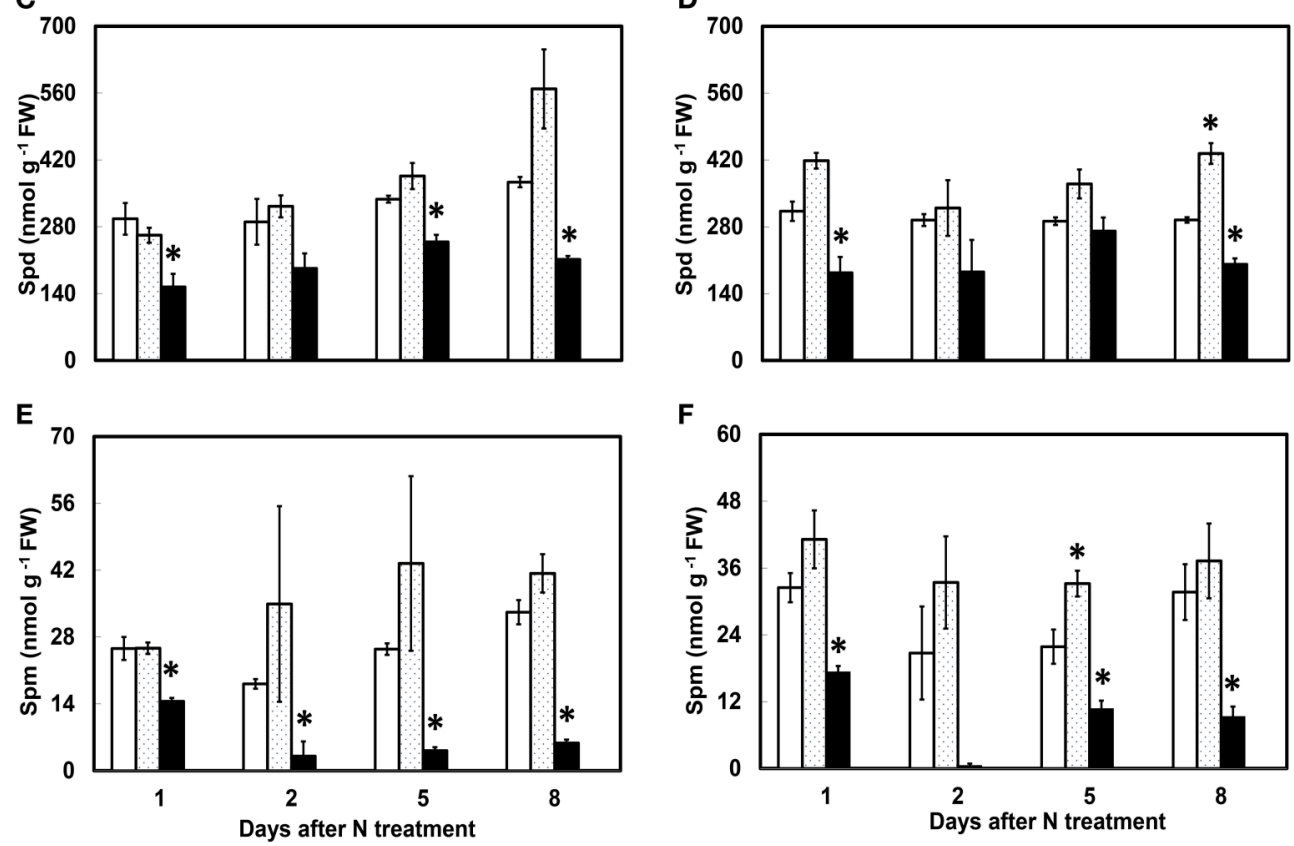

FIGURE 7 | Effect of foliar nitrogen treatments on PCA soluble polyamines in the rosette leaves of 6 week-old WT and 2x35S::mODC Arabidopsis thaliana plants. Cellular concentrations of (A,B) putrescine, (C,D) spermidine, and (E,F) spermine. Data are mean of $3 \pm$ SE. ${ }^{*}$ Denotes significant difference between treatment and the corresponding control for each time period for WT and for mODC transgenic plants $(P \leq 0.05)$.

increase in relative expression on induction at $24 \mathrm{~h}$ in one experiment (Supplemental Figure 5B); a repeat of this experiment did not show such a large change in expression at either 24 or $48 \mathrm{~h}$ of induction (Supplemental Table 2, Figure 8B).

There are seven genes that encode for five enzymes involved in $\mathrm{Glu} \Leftrightarrow \operatorname{Pro} \Leftrightarrow$ Orn interconversion (Supplemental Table 1); the expression of all of these genes was similar in the induced and uninduced plants at 24 as well as $48 \mathrm{~h}$ (Figure $8 \mathrm{C}$ and Supplemental Figure 5C). The gene P5CR has two splice variants in A. thaliana - P5CR - NM_001085115.1, and P5CR.1 - NM_121484.4; the primers designed for qPCR (Supplemental Table 1) were able to distinguish between the two splice variants.

\section{Genes of Polyamine Catabolism and GABA Biosynthesis Related Genes}

Of the five PAO and two DAO genes involved in PA catabolism, none showed a consistent change greater than 2-fold on induction of mODC (Figure 8C, Supplemental Table 2 and
Supplemental Figure 5D). Only one gene for Glu catabolism into GABA (GAD2) was successfully amplified (others showed multiple bands in endpoint PCR); its expression was not different in the induced plants vs. the un-induced control.

\section{DISCUSSION}

Understanding the regulation of PA and amino acid metabolism in plants is of major interest (Sinclair et al., 2004; Slocum, 2005; Kalamaki et al., 2009; Rees et al., 2009) because these two groups of metabolites occupy key positions in connecting $\mathrm{N}$ metabolism, $\mathrm{C}$ fixation, and several pathways associated with secondary metabolism. In this regard, Orn is a key metabolite sitting at the crossroads of several interactive pathways involving major amino acids: Glu $\rightarrow$ Orn $\rightarrow$ Arg $\rightarrow$ Put, $\mathrm{Glu} \rightarrow \underline{\mathrm{Orn}} \rightarrow$ Pro, Arg $\rightarrow \underline{\text { Orn }} \rightarrow$ Glu and Pro (Figure 1). In earlier reports (Majumdar et al., 2013, 2015; Minocha et al., 2014), we have argued that adding the transgenic mODC shunt in 


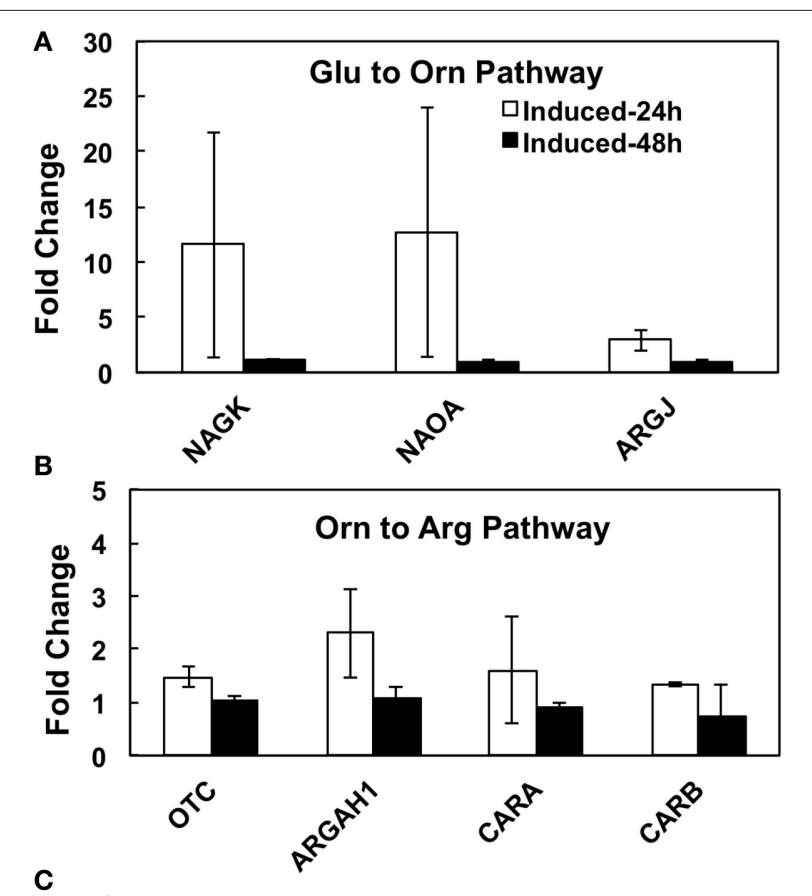

C

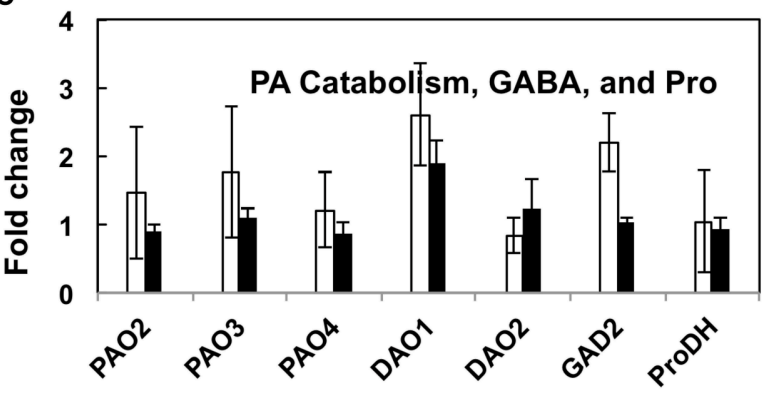

FIGURE 8 | The effects of inducible increase in putrescine production on changes in the relative expression of various genes of (A) Glu to Orn, (B) Orn to Arg, and (C) PA catabolism and GABA biosynthesis pathways in Arabidopsis thaliana. cDNA from transgenic mODC induced and un-induced seedlings collected at 24 and $48 \mathrm{~h}$ after induction with estradiol were used with gene specific primers for qPCR. The qPCR assays were conducted with two technical replicates per biological replicate (see details in Supplemental Table 2). Data are Mean \pm SE of 3 biological replicates. For explanation of enzymes names refer to legend to Figure $\mathbf{1}$ and for gene names refer to Supplemental Table 1. The bars represent fold change in gene expression in the mODC induced as compared to the un-induced control seedlings. *Denotes difference between mODC induced seedlings and the corresponding un-induced control plants at the same time of analysis where the difference is significant $(P \leq 0.05)$ and at least 2 fold; differences that were significant but $\leq 2$ fold are not shown.

A. thaliana would significantly disturb the homeostatic flux of Glu to PAs, Pro, Arg and GABA by diverting large amounts of Orn (the substrate for $\mathrm{mODC}$ as well these metabolites) to the production of Put. Further, it was postulated that increased metabolic conversion of Glu/Orn into Put may considerably affect the pool of other amino acids in the cell, thus leading to $\mathrm{N}$ deficiency and decrease in protein synthesis.

For this study, we postulated that in the mODC transgenic plants, the depletion of Orn caused by its increased use by $\mathrm{mODC}$ will result in increased conversion of Glu to Orn, which would be compensated partially by increased biosynthesis of Glu from assimilated $\mathrm{N}$ and $\mathrm{C}$. Furthermore, we surmised that the production of Pro may be affected by increased utilization of Glu into Orn/Arg part of the pathway.

Glutamate can be considered as the "Center of the Universe" for $\mathrm{N}$ metabolism in plants since most of the assimilated $\mathrm{N}$ passes through this step before it is re-distributed to major $\mathrm{N}$ metabolites (reviewed by Forde and Lea, 2007). While, it is the primary source of amide group for all protein amino acids via transamination reactions, it is also a direct precursor of several other $\mathrm{N}$-rich metabolites, which play important physiological roles in plant development and stress response; PAs and GABA being among them (Minocha et al., 2014; Majumdar et al., 2015). Furthermore, Pro and Arg, for which Glu is the direct source in plants, also play important roles in stress response (reviewed in Minocha et al., 2014; Majumdar et al., 2015) and in the production of signal molecules like NO (Morris, 2007, 2009). Thus, the distribution of Glu into different competing but complementary pathways must be intricately regulated to achieve homeostatic levels of various products whose biosynthesis depends on this amino acid. At the same time, it is well established that overall $\mathrm{N}$ assimilation in plants has a complex dependence on the availability of $\mathrm{C}$ and vice versa. It is therefore not surprising that major rechanneling of $\mathrm{N}$ has wide-ranging effects on the reallocation of cellular C. Under conditions of "threat to survival" (e.g., abiotic stress due to non-toxic conditions/treatments), the plants respond by reallocating $\mathrm{N}$ as well as $\mathrm{C}$ into metabolites like Pro, GABA, PAs, glycinebetaine and $\beta$-Ala (together referred to as compatible solutes), partly because they play protective roles under stress and perhaps also help alleviate toxicity of $\mathrm{NH}_{3}$ produced within the cells. The Glu-Pro-Arg-PA-GABA pathway is thus central to the biosynthesis of these metabolites to achieve a balance between assimilated $\mathrm{N}$ and $\mathrm{C}$ in the plant. In the present study we explored wider effects of altering the flow of Glu to PAs by creating a shunt for redirecting Orn toward Put.

\section{Ornithine as a Gatekeeper in Controlling PAs, Pro, and GABA Biosynthesis}

In plants, Orn is usually present in very small concentrations as compared to its products Arg, Pro, and Glu; its biosynthesis from Glu involves several enzymes (Shargool et al., 1988; Slocum, 2005; Figure 1). The first (regulatory?) step in Orn biosynthesis in plants is believed to be Glu $\rightarrow$ NAG via NAGS; this is in contrast to animals where nutritional Arg is the primary source of Orn, and the reaction is controlled by arginase (Morris, 2006, 2007). Morris (2009) has argued for the role of charged tRNA ${ }^{\text {Arg }}$ in regulating cellular Arg levels in mammals. As an extension of the argument of Ramos et al. (1970) about the role of Orn and citrulline (Cit) in regulating Arg-Orn pathway, we argue in favor of the importance of cellular Orn in regulating the Glu $\rightarrow$ Orn flux, particularly in response to increased demand (or depletion) of Orn by $\mathrm{mODC}$ in the transgenic plants. It was shown earlier in poplar cell cultures that this increased flux apparently does not involve increased expression of most of the genes encoding enzymes of this pathway, except perhaps NAGS (Page et al., 2012). 
Kalamaki et al. (2009) demonstrated that constitutive overexpression of a tomato NAGS1 gene in Arabidopsis led to higher accumulation of Orn and Cit in leaves without significant increase in Arg concentration. In the present study, we contend that significant changes in protein- and non-protein amino acids that accompany the increase in Put via mODC shunt must cause increased flux of Glu $\rightarrow$ Orn proportionate to its demand imposed by the transgenic mODC. Additionally, since Orn biosynthesis is distributed in several cellular compartments (Shargool et al., 1988) but its consumption by mODC is entirely cytoplasmic, it can be argued that Orn transport across organelles must also be co-regulated with its consumption in order to ensure its continued availability for its multiple usage. Another interesting aspect of Orn metabolism revealed by this study is that the amount of Put accumulating in mature transgenic plants was several-fold lower than that in the corresponding seedlings. This suggests two possibilities: either the mODC production (transcription or translation) was lower in mature tissues, or the flux of Glu $\rightarrow$ Orn/Arg or its transport to the cytoplasm was low for $\mathrm{mODC}$ to work optimally. This is consistent with the presence (in mature plants) of large amounts of Cad, apparently the product of $\mathrm{mODC}$ using Lys as a substrate.

\section{$\gamma$-Aminobutyric Acid Production from Gad and Put Catabolism}

Regulation of GABA metabolism in plants is complex since various enzymes associated with GABA metabolism are spatially compartmentalized in the cell (Shelp et al., 2012b). Whether or not its biosynthesis and catabolism are regulated at the transcription level is not known. Our results on the production of GABA via Put catabolism in $\mathrm{mODC}$ transgenic cells of poplar (Quan et al., 2002; Lasanajak et al., 2014) and seedlings of Arabidopsis (data presented here) do not indicate the induction of DAO even though the production of GABA through this pathway is increased several-fold. However, since there are additional $D A O$ genes as well as the GAD genes that we did not analyze, these conclusions may be tentative. Our findings that higher Put degradation can occur without increase in $D A O$ expression are consistent with those of Shelp et al. (2012a). However, the question of relative contributions of the anabolic (GAD) and the catabolic (DAO) pathways for GABA production to maintain its homeostasis in plants still remains unanswered.

The lack of change in Glu concentration in both genotypes by extra $\mathrm{NO}_{3}^{-}$in the medium with/or without concomitant change in either GABA or PA concentration (Figures 3, 4) are consistent with the suggestion of Fait et al. (2008), who emphasized the role of GABA in repartitioning of $\mathrm{C}$ and $\mathrm{N}$ during seed development and germination in GAD transgenic seedlings of Arabidopsis. In line with these findings, we observed that greater availability of $\mathrm{N}$ in the medium could enhance $\mathrm{N}$ metabolism and growth (biomass?) in the $\mathrm{mODC}$ transgenic plants without $\mathrm{C}$ becoming limited. A similar outcome was observed for $\mathrm{C}$ supplementation of the medium; however, at higher concentrations of $\mathrm{C}, \mathrm{N}$ appeared to become limited for Put production in the transgenic plants but not for GABA production. These results strongly suggest that the redirection of Orn into Put and then into GABA shunt could enhance $C$ uptake and assimilation through increased $\mathrm{N}$ assimilation, thus restoring Glu loss due to Orn depletion.

\section{Gene Expression and the Regulation of Glu $\rightarrow$ Orn $\rightarrow$ Arg Pathway}

Nabais et al. (2005) studied the role of Orn in N remobilization in evergreen Quercus ilex and found that in early spring Orn was the first detectable amino acid in the xylem sap (presumably resulting from Arg degradation via arginase). Similar increases in Orn have been reported in germinating seeds when protein reserves are used to generate Glu, Gln, and Pro from Arg (Cañas et al., 2008). This situation is analogous to mammalian Glu and Gln biosynthetic pathways where nutritional Arg is metabolized into other amino acids and PAs, and for $\mathrm{NH}_{3}$ detoxification via the combined actions of Arginase and urease (Boon et al., 1999; Levillain et al., 2004). In young Arabidopsis seedlings, the primary source of Orn was Glu (and not Arg), which was produced from assimilation of $\mathrm{N}$ from the medium. While OAT is involved in Arg conversion to Glu following Arginase-urease actions, the regulatory enzyme for Orn (and hence Arg and Put) production from Glu is considered to be NAGS (Figure 1). Our results indicate that neither of the two NAGS genes was upregulated on induction of $\mathrm{mODC}$, which is inconsistent with its presumed role as a potential regulatory step in Glu $\rightarrow$ Orn biosynthesis (Kalamaki et al., 2009). Regulation of the remaining set of reactions involved in Glu $\rightarrow$ Orn $\rightarrow$ Arg flux is not known. Our past qPCR analysis with high Put poplar cells did not show major changes in the expression of most genes encoding enzymes of this pathway in poplar (Page et al., 2012); the results with $A$. thaliana presented here further corroborate these observations. Surprisingly, despite several-folds increase in demand for Orn by $\mathrm{mODC}$, there was no major effect on either the expression of genes coding for enzymes of the Orn $\rightarrow$ Arg or those coding for $\mathrm{Glu} \rightarrow$ Pro parts of the pathway. Concurrently, the resulting large decrease in Glu was compensated at least partly by increased $\mathrm{N}$ assimilation into Glu, especially under conditions of abundant $\mathrm{N}$ and $\mathrm{C}$ in the medium. Unfortunately, the current study did not involve expression of the genes encoding either enzymes of the $\mathrm{N}$ assimilation or those involved in $\mathrm{C}$ uptake/assimilation pathways. An important conclusion from these results is that a significant proportion of $\mathrm{N}$ assimilation is driven by downstream $\mathrm{N}$ utilization as long as $\mathrm{C}$ is not limiting. Furthermore, it can be argued that the limitation of $\mathrm{C}$ under these conditions may also be alleviated (at least partially) by its enhanced uptake and assimilation from the medium. Whether or not such an increase in the metabolic flux of inorganic $\mathrm{N}$ into this pathway would lead to increase in $\mathrm{C}$ fixation through photosynthesis has not yet been tested in mature transgenic plants.

\section{Regulation of Glu $\Leftrightarrow$ Pro $\Leftrightarrow$ Arg}

Sucrose concurrently affected Pro and GABA (both being products of Glu), but the responses were different in the transgenic vs. the WT seedlings. The results suggest that in the transgenic plants, re-routing of Glu into PAs might have caused a limitation of $\mathrm{N}$ for Pro production, which was reversed by additional $\mathrm{N}$ in the medium. Based on observations that limitation of $\mathrm{C}$ in the medium affected Pro, Arg, and GABA, 
and the addition of sucrose to the medium enhanced the accumulation of all three of these amino acids, it can be argued that: (1) the $\mathrm{N}$ reserves of transgenic cells producing high Put are more rapidly diluted than the $\mathrm{C}$ reserves, and (2) its reversal by adding extra $\mathrm{N}$ does not lead to the uptake and assimilation of $\mathrm{C}$. However, having extra $\mathrm{C}$ available to the plants leads to additional $\mathrm{N}$ uptake, a situation if translated in the field grown plants should result in enhancement of total biomass through this increased $\mathrm{N}$ use in the plants. The response of WT and high Put transgenic plants to additional $\mathrm{C}$ in the medium was much less pronounced for Gln and Arg, indicating a different level of regulation for these two amino acids. Ornithine still remained undetectable in the transgenic plants regardless of the addition of $\mathrm{N}$ or $\mathrm{C}$, suggesting that this amino acid was still limited for the transgenic mODC.

Proline biosynthesis from Orn in plants is apparently not regulated by feedback inhibition of the enzymes $\delta$-OAT and P5CR (Larosa et al., 1991). Induction of mODC resulted in up-regulation of Pro (as also seen in high-Put poplar cells by Mohapatra et al. (2010b). Higher accumulation of GABA and Pro both under inducible and constitutive mODC expression conditions in A. thaliana seedlings and mature plants indicate that these plants might be useful tools to study a wide range of stress responses. Whereas significant changes in Pro, Glu, GABA and Put were seen in response to additional $C$, changes in the accumulation of Spd and Spm were relatively small; reconfirming our earlier evidence of an independent and tight regulation of their biosynthesis and accumulation.

\section{Phenotypic Changes in mODC Transgenic Plants}

While the transgenic plants constitutively over-expressing mODC were phenotypically similar to the WT plants, there were subtle developmental differences between the two genotypes. The transgenic plants had higher fresh biomass as well as dry mass per plant just before bolting, which was delayed by at least a week as compared to the WT plants. Higher chlorophyll concentration in the transgenic plants, and greater number of branches and siliques, all seem to indicate positive effects of high Put production in the present study. These effects are consistent with the observed increase in total $\mathrm{C}$ and $\mathrm{N}$ in the transgenic plants, which may be related to higher chlorophyll (photosynthesis?) in the leaves. The diamine Put has been shown to promote light reactions of photosynthesis through increased photophosphorylation (Ioannidis et al., 2006), and it is also the best stimulator of ATP synthesis as compared to Spd and Spm (Ioannidis and Kotzabasis, 2007). If this turns out to be the case, we would have discovered an alternate way to manipulate photosynthesis via $\mathrm{N}$ assimilation into PAs to increase biomass in bioenergy crops.

\section{CONCLUSIONS AND FUTURE PERSPECTIVES}

Over the years, metabolic engineering in plants has gained considerable attention in transgenic research to enhance the nutritional value of food/feed crops, to improve the abiotic stress tolerance of plants (Hussain et al., 2011; Mattoo et al., 2011), improved quality of flowers and fruits (Lücker et al., 2001; Goff and Klee, 2006), increased production of secondary metabolites (Pilate et al., 2002; Verhoeyen et al., 2002), and increase in essential amino acids (e.g., Lys, Met, Trp) in food and forage crops (Galili and Höfgen, 2002). Various mechanisms have been postulated in controlling metabolic fluxes of connected pathways that share a common substrate (Allen et al., 2009; Palsson, 2009; Grüning et al., 2010). One mechanism of increasing the cellular production/concentration of a metabolite without increases in transcription or translation of the related enzymes can be through increasing the demand for utilization of the product (Zhu and Galili, 2003), i.e., the metabolic pull (or a sink). While most studies have lacked experimental evidence involving detailed analysis of the pleiotropic effects of such metabolic manipulations, several reports from our lab (Page et al., 2007, 2012; Mohapatra et al., 2010a,b; Page et al., unpublished) have demonstrated that manipulation of a single step in the PA biosynthetic pathway (i.e., increased Put production via transgenic $O D C$ ) can cause a major redirection of the cell's metabolome. Thus, for plants to be used for industrial purposes (e.g., bioenergy, biofuels, pharmaceuticals, enzyme catalysts, etc.), metabolic engineering to increase $\mathrm{N}$ assimilation, and the resulting/accompanying $\mathrm{C}$ sequestration via manipulation of $\mathrm{PA}$ biosynthesis may provide a useful tool in not only producing greater biomass under conditions of optimal growth but also under stress because of the beneficial effects of higher PAs in improving stress response.

Some of the specific conclusions from this study are: (1) pathway from Glu to Orn and from Orn to Arg is regulated primarily at the enzymatic level (vs. gene transcriptional level) and it involves tight regulation of cellular Orn concentration; (2) production of Pro is regulated independently of the $\mathrm{Glu} \rightarrow$ Orn $\rightarrow$ Arg part of the pathway; and (3) depletion of Glu caused by increased flux toward Orn/Arg and Pro may be compensated partially by its increased biosynthesis from assimilated $\mathrm{N}$.

\section{FUNDING}

Partial funding was provided by the New Hampshire Agricultural Experiment Station. This is Scientific Contribution Number 2608. This work was supported by the USDA National Institute of Food and Agriculture (McIntire-Stennis) Project (NH00062 and NH00076-M).

\section{ACKNOWLEDGMENTS}

The authors acknowledge the contribution of Gloria Quigley for help with the analysis of amino acids, Ms. Kara Sarver for providing RNA of inducible $\mathrm{mODC}$ seedlings used for a part of the qPCR analysis, and Dr. Louis Tisa for help with the analysis of qPCR results.

\section{SUPPLEMENTARY MATERIAL}

The Supplementary Material for this article can be found online at: http://journal.frontiersin.org/article/10.3389/fpls.2016. 00078 


\section{REFERENCES}

Agostinelli, E. (2014). Polyamines and transglutaminases: biological, clinical, and biotechnological perspectives. Amino Acids 46, 475-485. doi: 10.1007/s00726014-1688-0

Allen, D. K., Libourel, I. G. L., and Shachar-Hill, Y. (2009). Metabolic flux analysis in plants: coping with complexity. Plant Cell Environ. 32, 1241-1257. doi: 10.1111/j.1365-3040.2009.01992.x

Bagni, N., and Tassoni, A. (2001). Biosynthesis, oxidation and conjugation of aliphatic polyamines in higher plants. Amino Acids 20, 301-317. doi: 10.1007/s007260170046

Bastola, D. R., and Minocha, S. C. (1995). Increased putrescine biosynthesis through transfer of mouse ornithine decarboxylase cDNA in carrot promotes somatic embryogenesis. Plant Physiol. 109, 63-71.

Bhatnagar, P., Glasheen, B. M., Bains, S. K., Long, S. L., Minocha, R., Walter, C., et al. (2001). Transgenic manipulation of the metabolism of polyamines in poplar cells. Plant Physiol. 125, 2139-2153. doi: 10.1104/pp.125.4.2139

Boon, L., Geerts, W. J. C., Jonker, A., Lamers, W. H., and Van Noorden, C. J. F. (1999). High protein diet induces pericentral glutamate dehydrogenase and ornithine aminotransferase to provide sufficient glutamate for pericentral detoxification of ammonia in rat liver lobules. Histochem. Cell Biol. 111, 445-452. doi: 10.1007/s004180050380

Bouché, N., and Fromm, H. (2004). GABA in plants: just a metabolite? Trends Plant Sci. 9, 110-115. doi: 10.1016/j.tplants.2004.01.006

Bradford, M. M. (1976). A rapid and sensitive method for the quantitation of microgram quantities of protein utilizing the principle of protein-dye binding. Anal. Biochem. 72, 248-254. doi: 10.1016/0003-2697(76)90527-3

Cañas, R. A., Villalobos, D. P., Díaz-Moreno, S. M., Cánovas, F. M., and Cantón, F. R. (2008). Molecular and functional analyses support a role of ornithine$\delta$-aminotransferase in the provision of glutamate for glutamine biosynthesis during pine germination. Plant Physiol. 148, 77-88. doi: 10.1104/pp.108.122853

Coleman, C. S., Stanley, B. A., and Pegg, A. E. (1993). Effect of mutations at active site residues on the activity of ornithine decarboxylase and its inhibition by active site-directed irreversible inhibitors. J. Biol. Chem. 268, 24572-24579.

Curtis, M. D., and Grossniklaus, U. (2003). A Gateway cloning vector set for highthroughput functional analysis of genes in planta. Plant Physiol. 133, 462-469. doi: $10.1104 /$ pp.103.027979

Czechowski, T., Stitt, M., Altmann, T., Udvardi, M. K., and Scheible, W.-R. (2005). Genome-wide identification and testing of superior reference genes for transcript normalization in Arabidopsis. Plant Physiol. 139, 5-17. doi: 10.1104/pp.105.063743

Descenzo, R. A., and Minocha, S. C. (1993). Modulation of cellular polyamines in tobacco by transfer and expression of mouse ornithine decarboxylase cDNA. Plant Mol. Biol. 22, 113-127. doi: 10.1007/BF00039000

Ericsson, A., Nordén, L.-G., Näsholm, T., and Walheim, M. (1993). Mineral nutrient imbalances and arginine concentrations in needles of Picea abies (L.) Karst. from two areas with different levels of airborne deposition. Trees 8, 67-74. doi: 10.1007/BF00197683

Ericsson, A., Walheim, M., Nordén, L.-G., and Näsholm, T. (1995). Concentrations of mineral nutrients and arginine in needles of Picea Abies trees from different areas in Southern Sweden in relation to nitrogen deposition and humus form. Ecol. Bull. 44, 147-157. Available online at: http://www.jstor.org/stable/ 20113158

Fait, A., Fromm, H., Walter, D., Galili, G., and Fernie, A. R. (2008). Highway or byway: the metabolic role of the GABA shunt in plants. Trends Plant Sci. 13, 14-19. doi: 10.1016/j.tplants.2007.10.005

Flores, H. E., and Filner, P. (1985). "Metabolic relationships of putrescine, GABA and alkaloids in cell and root cultures of Solanaceae," in Primary and Secondary Metabolism of Plant Cell Cultures, eds K.-H. Neumann, W. Barz, and E. Reinhard (Berlin; Heidelberg: Springer), 174-185. doi: 10.1007/978-3-64270717-9_17

Forde, B. G., and Lea, P. J. (2007). Glutamate in plants: metabolism, regulation, and signalling. J. Exp. Bot. 58, 2339-2358. doi: 10.1093/jxb/erm 121

Galili, G., and Höfgen, R. (2002). Metabolic engineering of amino acids and storage proteins in plants. Metab. Eng. 4, 3-11. doi: 10.1006/mben.2001.0203

Gamborg, O. L., Miller, R. A., and Ojima, K. (1968). Nutrient requirements of suspension cultures of soybean root cells. Exp. Cell Res. 50, 151-158. doi: 10.1016/0014-4827(68)90403-5
Garufi, A., Visconti, S., Camoni, L., and Aducci, P. (2007). Polyamines as physiological regulators of 14-3-3 interaction with the plant plasma membrane H+-ATPase. Plant Cell Physiol. 48, 434-440. doi: 10.1093/pcp/pcm010

Ghosh, B. (2000). Polyamines and plant alkaloids. Indian J. Exp. Biol. 38, 1086-1091.

Gitelson, A. A., Chivkunova, O. B., and Merzlyak, M. N. (2009). Nondestructive estimation of anthocyanins and chlorophylls in anthocyanic leaves. Am. J. Bot. 96, 1861-1868. doi: 10.3732/ajb.0800395

Goff, S. A., and Klee, H. J. (2006). Plant volatile compounds: sensory cues for health and nutritional value? Science 311, 815-819. doi: 10.1126/science.1112614

Grüning, N.-M., Lehrach, H., and Ralser, M. (2010). Regulatory crosstalk of the metabolic network. Trends Biochem. Sci. 35, 220-227. doi: 10.1016/j.tibs.2009. 12.001

Han, B., Yang, Z., Samma, M., Wang, R., and Shen, W. (2013). Systematic validation of candidate reference genes for qRT-PCR normalization under iron deficiency in Arabidopsis. Biometals 26, 403-413. doi: 10.1007/s10534-0139623-5

Hu, X., Xu, Z., Xu, W., Li, J., Zhao, N., and Zhou, Y. (2015). Application of $\gamma$ aminobutyric acid demonstrates a protective role of polyamine and GABA metabolism in muskmelon seedlings under $\mathrm{Ca}(\mathrm{NO} 3) 2$ stress. Plant Physiol. Biochem. 92, 1-10. doi: 10.1016/j.plaphy.2015.04.006

Hussain, S. S., Ali, M., Ahmad, M., and Siddique, K. H. M. (2011). Polyamines: natural and engineered abiotic and biotic stress tolerance in plants. Biotechnol. Adv. 29, 300-311. doi: 10.1016/j.biotechadv.2011.01.003

Ioannidis, N. E., and Kotzabasis, K. (2007). Effects of polyamines on the functionality of photosynthetic membrane in vivo and in vitro. Biochim. Biophys. Acta 1767, 1372-1382. doi: 10.1016/j.bbabio.2007.10.002

Ioannidis, N. E., Sfichi, L., and Kotzabasis, K. (2006). Putrescine stimulates chemiosmotic ATP synthesis. Biochim. Biophys. Acta 1757, 821-828. doi: 10.1016/j.bbabio.2006.05.034

Kalamaki, M. S., Alexandrou, D., Lazari, D., Merkouropoulos, G., Fotopoulos, V., Pateraki, I., et al. (2009). Over-expression of a tomato N-acetyl-L-glutamate synthase gene (SINAGS1) in Arabidopsis thaliana results in high ornithine levels and increased tolerance in salt and drought stresses. J. Exp. Bot. 60, 1859-1871. doi: $10.1093 / \mathrm{jxb} / \mathrm{erp} 072$

Kusano, T., Yamaguchi, K., Berberich, T., and Takahashi, Y. (2007). Advances in polyamine research in 2007. J. Plant Res. 120, 345-350. doi: 10.1007/s10265007-0074-3

Larosa, P. C., Rhodes, D., Rhodes, J. C., Bressan, R. A., and Csonka, L. N. (1991). elevated accumulation of proline in $\mathrm{NaCl}$-adapted tobacco cells is not due to altered Delta-pyrroline-5-carboxylate reductase. Plant Physiol. 96, 245-250. doi: 10.1104/pp.96.1.245

Lasanajak, Y., Minocha, R., Minocha, S., Goyal, R., Fatima, T., Handa, A., et al. (2014). Enhanced flux of substrates into polyamine biosynthesis but not ethylene in tomato fruit engineered with yeast S-adenosylmethionine decarboxylase gene. Amino Acids 46, 729-742. doi: 10.1007/s00726-013-1624-8

Levillain, O., Hus-Citharel, A., Garvi, S., Peyrol, S., Reymond, I., Mutin, M., et al. (2004). Ornithine metabolism in male and female rat kidney: mitochondrial expression of ornithine aminotransferase and arginase II. Am. J. Physiol Renal Physiol. 286, F727-F738. doi: 10.1152/ajprenal.00315.2003

Lichtenthaler, H. K., and Buschmann, C. (1987). Chlorophylls and carotenoids: measurement UNIT F4.3 and characterization by UV-VIS spectroscopy. Curr. Protoc. Food Anal. Chem. 4, 3.1-3.8.

Lücker, J., Bouwmeester, H. J., Schwab, W., Blaas, J., Van Der Plas, L. H., and Verhoeven, H. A. (2001). Expression of Clarkia S-linalool synthase in transgenic petunia plants results in the accumulation of S-linalyl-betaD-glucopyranoside. Plant J. 27, 315-324. doi: 10.1046/j.1365-313x.2001. 01097.x

Majumdar, R., Minocha, R., and Minocha, S. C. (2015). "Ornithine: at the crossroads of multiple paths to amino acids and polyamines," in Amino Acids in Higher Plants, ed J. P. F. D’Mello (Boston, MA: CABI Publishers), 156-176.

Majumdar, R., Shao, L., Minocha, R., Long, S., and Minocha, S. C. (2013). Ornithine: the overlooked molecule in regulation of polyamine metabolism. Plant Cell Physiol. 54, 990-1004. doi: 10.1093/pcp/pct053

Marina, M., Sirera, F. V., Rambla, J. L., Gonzalez, M. E., Blázquez, M. A., Carbonell, J., et al. (2013). Thermospermine catabolism increases Arabidopsis thaliana resistance to Pseudomonas viridiflava. J. Exp. Bot. 64, 1393-1402. doi: $10.1093 /$ jxb/ert012 
Martin-Tanguy, J. (1997). Conjugated polyamines and reproductive development: biochemical, molecular and physiological approaches. Physiol. Plant. 100, 675-688. doi: 10.1111/j.1399-3054.1997.tb03074.x

Mattioli, R., Costantino, P., and Trovato, M. (2009). Proline accumulation in plants: not only stress. Plant Signal. Behav. 4, 1016-1018. doi: 10.4161/psb.4.11.9797

Mattoo, A. K., Minocha, S. C., Minocha, R., and Handa, A. K. (2010). Polyamines and cellular metabolism in plants: transgenic approaches reveal different responses to diamine putrescine versus higher polyamines spermidine and spermine. Amino Acids 38, 405-413. doi: 10.1007/s00726-009-0399-4

Mattoo, A. K., Shukla, V. T. F., Handa, A. K., and Yachha, S. K. (2011). "Genetic engineering to enhance crop-based phytonutrients (nutraceuticals) to alleviate diet-related diseases," in In Bio-Farms for Nutraceuticals: Functional Food and Safety Control by Biosensors, eds M. T. Giardi, G. Rea, and B. Berra (Austin, TX: Springer and Landes Biosciences), 122-143.

Minocha, R., Lee, J. S., Long, S., Bhatnagar, P., and Minocha, S. C. (2004). Physiological responses of wild type and putrescine-overproducing transgenic cells of poplar to variations in the form and concentration of nitrogen in the medium. Tree Physiol. 24, 551-560. doi: 10.1093/treephys/24.5.551

Minocha, R., and Long, S. (2004). Simultaneous separation and quantitation of amino acids and polyamines of forest tree tissues and cell cultures within a single high-performance liquid chromatography run using dansyl derivatization. J. Chromatogr. A 1035, 63-73. doi: 10.1016/j.chroma.2004.02.026

Minocha, R., Majumdar, R., and Minocha, S. C. (2014). Polyamines and abiotic stress in plants: a complex relationship. Front. Plant Sci. 5:175. doi: $10.3389 /$ fpls.2014.00175

Minocha, R., Turlapati, S. A., Long, S., McDowell, W. H., and Minocha, S. C. (2015). Long-term trends of changes in pine and oak foliar nitrogen metabolism in response to chronic nitrogen amendments at the Harvard Forest, MA. Tree Physiol. 35, 894-909. doi: 10.1093/treephys/tpv044

Minocha, R., Turlapati, S. A., Long, S., and North, M. (2013). Fuel treatment effects on soil chemistry and foliar physiology of three coniferous species at the Teakettle Experimental Forest, California, USA. Trees Struct. Funct. 27, 1101-1113. doi: 10.1007/s00468-013-0860-6

Mohapatra, S., Cherry, S., Minocha, R., Majumdar, R., Thangavel, P., Long, S., et al. (2010a). The response of high and low polyamine producing cell lines to aluminum and calcium stress. Plant Physiol. Biochem. 48, 612-620. doi: 10.1016/j.plaphy.2010.04.010

Mohapatra, S., Minocha, R., Long, S., and Minocha, S. C. (2010b). Transgenic manipulation of a single polyamine in poplar cells affects the accumulation of all amino acids. Amino Acids 38, 1117-1129. doi: 10.1007/s00726-009-0322-z

Morris, S. M. Jr. (2006). Arginine: beyond protein. Am. J. Clin. Nutr. 83, 508S-512S.

Morris, S. M. Jr. (2007). Arginine metabolism: boundaries of our knowledge. Am. J. Clin. Nutr. 137, 1602S-1609S.

Morris, S. M. Jr. (2009). Recent advances in arginine metabolism: roles and regulation of the arginases. Br. J. Pharmacol. 157, 922-930. doi: 10.1111/j.14765381.2009.00278.x

Murashige, T., and Skoog, F. (1962). A revised medium for rapid growth and bio assays with tobacco tissue culture. Physiol. Plant. 15, 473-497. doi: 10.1111/j.1399-3054.1962.tb08052.x

Nabais, C., Hagemeyer, J., and Freitas, H. (2005). Nitrogen transport in the xylem sap of Quercus ilex: the role of ornithine. J. Plant Physiol. 162, 603-606. doi: 10.1016/j.jplph.2004.11.004

Nambeesan, S., Datsenka, T., Ferruzzi, M. G., Malladi, A., Mattoo, A. K., and Handa, A. K. (2010). Overexpression of yeast spermidine synthase impacts ripening, senescence and decay symptoms in tomato. Plant J. 63, 836-847. doi: 10.1111/j.1365-313X.2010.04286.x

Näsholm, T., Edfast, A.-B., Ericsson, A., and Nordén, L.-G. (1994). Accumulation of amino acids in some boreal forest plants in response to increased nitrogen availability. New Phytol. 126, 137-143. doi: 10.1111/j.1469-8137.1994. tb07539.x

Näsholm, T., Huss-Danell, K., and Högberg, P. (2000). Uptake of organic nitrogen in the field by four agriculturally important plant species. Ecology 81, 1155-1161. doi: 10.1890/0012-9658(2000)081[1155:UOONIT]2.0.CO;2

Näsholm, T., Nordin, A., Edfast, A. B., and Hogberg, P. (1997). Identification of coniferous forests with incipient nitrogen saturation through arginine and nitrogen-15 abundance in trees. J. Environ. Qual. 26, 302-309. doi: $10.2134 /$ jeq1997.00472425002600010042x

Page, A. F., Minocha, R., and Minocha, S. C. (2012). Living with high putrescine: expression of ornithine and arginine biosynthetic pathway genes in high and low putrescine producing poplar cells. Amino Acids 42, 295-308. doi: 10.1007/ s00726-010-0807-9

Page, A. F., Mohapatra, S., Minocha, R., and Minocha, S. C. (2007). The effects of genetic manipulation of putrescine biosynthesis on transcription and activities of the other polyamine biosynthetic enzymes. Physiol. Plant. 129, 707-724. doi: 10.1111/j.1399-3054.2007.00860.x

Palsson, B. (2009). Metabolic systems biology. FEBS Lett. 583, 3900-3904. doi: 10.1016/j.febslet.2009.09.031

Pfaffl, M. W. (2001). A new mathematical model for relative quantification in real-time RT-PCR. Nucleic Acids Res. 29, e45-e45. doi: 10.1093/nar/29.9.e45

Pilate, G., Guiney, E., Holt, K., Petit-Conil, M., Lapierre, C., Leplé, J. C., et al. (2002). Field and pulping performances of transgenic trees with altered lignification. Nat. Biotechnol. 20, 607-612. doi: 10.1038/nbt0602-607

Quan, Y., Minocha, R., and Minocha, S. C. (2002). Genetic manipulation of polyamine metabolism in poplar II: effects on ethylene biosynthesis. Plant Physiol. Biochem. 40, 929-937. doi: 10.1016/S0981-9428(02)01456-0

Ramos, F., Thuriaux, P., Wiame, J. M., and Bechet, J. (1970). The participation of ornithine and citrulline in the regulation of arginine metabolism in Saccharomyces cerevisiae. Eur. J. Biochem. 12, 40-47. doi: 10.1111/j.14321033.1970.tb00818.x

Rees, J. D., Ingle, R. A., and Smith, J. A. C. (2009). Relative contributions of nine genes in the pathway of histidine biosynthesis to control of free histidine concentrations in Arabidopsis thaliana. Plant Biotechnol. J. 7, 499-511. doi: 10.1111/j.1467-7652.2009.00419.x

Shao, L., Bhatnagar, P., Majumdar, R., Minocha, R., and Minocha, S. C. (2014). Putrescine overproduction does not affect the catabolism of spermidine and spermine in poplar and Arabidopsis. Amino Acids 46, 743-757. doi: 10.1007/s00726-013-1581-2

Shargool, D., Jain, J. C., and McKay, G. (1988). Ornithine biosynthesis, and arginine biosynthesis and degradation in plant cells. Phytochemistry 27, 1571-1574. doi: 10.1016/0031-9422(88)80404-7

Shelp, B. J., Bozzo, G. G., Trobacher, C. P., Zarei, A., Deyman, K. L., and Brikis, C. J. (2012a). Hypothesis/review: Contribution of putrescine to 4-aminobutyrate (GABA) production in response to abiotic stress. Plant Science 193-194, 130-135. doi: 10.1016/j.plantsci.2012.06.001

Shelp, B. J., Mullen, R. T., and Waller, J. C. (2012b). Compartmentation of GABA metabolism raises intriguing questions. Trends Plant Sci. 17, 57-59. doi: 10.1016/j.tplants.2011.12.006

Shi, S.-Q., Shi, Z., Jiang, Z.-P., Qi, L.-W., Sun, X.-M., Li, C.-X., et al. (2010). Effects of exogenous GABA on gene expression of Caragana intermedia roots under $\mathrm{NaCl}$ stress: regulatory roles for $\mathrm{H}_{2} \mathrm{O}_{2}$ and ethylene production. Plant Cell Environ. 33, 149-162. doi: 10.1111/j.1365-3040.2009.02065.x

Sinclair, T. R., Purcell, L. C., and Sneller, C. H. (2004). Crop transformation and the challenge to increase yield potential. Trends Plant Sci. 9, 70-75. doi: 10.1016/j.tplants.2003.12.008

Slocum, R. D. (2005). Genes, enzymes and regulation of arginine biosynthesis in plants. Plant Physiol. Biochem. 43, 729-745. doi: 10.1016/j.plaphy.2005.06.007

Subramanyam, S., Sardesai, N., Minocha, S. C., Zheng, C., Shukle, R. H., and Williams, C. E. (2015). Hessian fly larval feeding triggers enhanced polyamine levels in susceptible but not resistant wheat. BMC Plant Biol. 15:3. doi: 10.1186/s12870-014-0396-y

Takahashi, Y., Cong, R., Sagor, G. H. M.,Niitsu, M., Berberich, T., and Kusano, T. (2010). Characterization of five polyamine oxidase isoforms in Arabidopsis thaliana. Plant Cell Rep. 29, 955-965. doi: 10.1007/s00299-010-0881-1

Trobacher, C. P., Zarei, A., Liu, J., Clark, S. M., Bozzo, G. G., and Shelp, B. J. (2013). Calmodulin-dependent and calmodulin-independent glutamate decarboxylases in apple fruit. BMC Plant Biol. 13:144. doi: 10.1186/1471-222913-144

Vergara, R., Parada, F., Rubio, S., and Pérez, F. J. (2012). Hypoxia induces $\mathrm{H}(2) \mathrm{O}(2)$ production and activates antioxidant defence system in grapevine buds through mediation of $\mathrm{H}(2) \mathrm{O}(2)$ and ethylene. J. Exp. Bot. 63, 4123-4131. doi: 10.1093/jxb/ers094

Verhoeyen, M. E., Bovy, A., Collins, G., Muir, S., Robinson, S., De Vos, C. H. R., et al. (2002). Increasing antioxidant levels in tomatoes through modification 
of the flavonoid biosynthetic pathway. J. Exp. Bot. 53, 2099-2106. doi: 10.1093/jxb/erf044

Wallace, H. M., Fraser, A. V., and Hughes, A. (2003). A perspective of polyamine metabolism. Biochem. J. 376, 1-14. doi: 10.1042/bj20031327

Wargo, P. M., Minocha, R., Wong, B. L., Long, R. P., Horsley, S. B., and Hall, T. J. (2002). Measuring changes in stress and vitality indicators in limed sugar maple on the Allegheny Plateau in north-central Pennsylvania. Can. J. For. Res. 32, 629-641. doi: 10.1139/x02-008

Zepeda-Jazo, I., Velarde-Buendía, A. M., Enriquez-Figueroa, R., Bose, J., Shabala, S., Muniz-Murguia, J., et al. (2011). Polyamines interact with hydroxyl radicals in activating $\mathrm{Ca}^{2+}$ and $\mathrm{K}^{+}$transport across the root epidermal plasma membranes. Plant Physiol. 157, 2167-2180. doi: 10.1104/pp.111.179671

Zhu, X., and Galili, G. (2003). Increased lysine synthesis coupled with a knockout of its catabolism synergistically boosts lysine content and also transregulates the metabolism of other amino acids in Arabidopsis seeds. Plant Cell 15, 845-853. doi: $10.1105 /$ tpc.009647

Conflict of Interest Statement: The authors declare that the research was conducted in the absence of any commercial or financial relationships that could be construed as a potential conflict of interest.

Copyright (C) 2016 Majumdar, Barchi, Turlapati, Gagne, Minocha, Long and Minocha. This is an open-access article distributed under the terms of the Creative Commons Attribution License (CC BY). The use, distribution or reproduction in other forums is permitted, provided the original author(s) or licensor are credited and that the original publication in this journal is cited, in accordance with accepted academic practice. No use, distribution or reproduction is permitted which does not comply with these terms. 


\section{OPEN ACCESS}

Edited by:

Sakiko Okumoto,

Virginia Polytechnic Institute and State

University, USA

Reviewed by:

Naser A. Anjum,

University of Aveiro, Portugal

Santiago Signorelli,

Universidad de la República, Uruguay

Maurizio Trovato

Sapienza - Università di Roma, Italy

${ }^{\star}$ Correspondence:

Polavarapu B. Kavi Kishor, Department of Genetics, Osmania University, Hyderabad 500007, India

pbkavi@yahoo.com

Specialty section:

This article was submitted to Plant Physiology, a section of the journal Frontiers in Plant Science

Received: 17 April 2015

Accepted: 06 July 2015

Published: 20 July 2015

Citation:

Kavi Kishor PB, Hima Kumari $P$, Sunita MSL and Sreenivasulu N (2015) Role of proline in cell wall synthesis and plant development and its implications in plant ontogeny.

Front. Plant Sci. 6:544.

doi: $10.3389 / f p / s .2015 .00544$

\section{Role of proline in cell wall synthesis and plant development and its implications in plant ontogeny}

\author{
Polavarapu B. Kavi Kishor ${ }^{1 *}$, P. Hima Kumari' ${ }^{1}$, M. S. L. Sunita ${ }^{1}$ and Nese Sreenivasulu ${ }^{2,3}$ \\ ${ }^{1}$ Department of Genetics, Osmania University, Hyderabad, India, ${ }^{2}$ Leibniz Institute of Plant Genetics and Crop Plant \\ Research, Gatersleben, Germany, ${ }^{3}$ Grain Quality and Nutrition Center, International Rice Research Institute, Metro Manila, \\ Philippines
}

Proline is a proteogenic amino acid and accumulates both under stress and non-stress conditions as a beneficial solute in plants. Recent discoveries point out that proline plays an important role in plant growth and differentiation across life cycle. It is a key determinant of many cell wall proteins that plays important roles in plant development. The role of extensins, arabinogalactan proteins and hydroxyproline- and proline-rich proteins as important components of cell wall proteins that play pivotal roles in cell wall signal transduction cascades, plant development and stress tolerance is discussed in this review. Molecular insights are also provided here into the plausible roles of proline transporters modulating key events in plant development. In addition, the roles of proline during seed developmental transitions including storage protein synthesis are discussed.

Keywords: proline, plant ontogeny, proline-rich proteins, hydroxylproline-rich glycoproteins, hybrid proline-rich proteins

\section{Introduction}

Glutamate is an important amino acid and acts as a precursor for the biosynthesis of $\gamma$-aminobutyric acid, arginine, glutamine, and proline in plants as well as in other eukaryotes (Rhodes et al., 1986; Szekely et al., 2008; Sharma and Verslues, 2010). Proline can be distinguished among all other amino acids due to its unique structure with its $\alpha$-amino group as a secondary amine and possesses distinctive cyclic structure which causes exceptional conformational rigidity to the protein structure (MacArthur and Thornton, 1991). It has also specific chemical properties like zwitterionic nature, neutral $\mathrm{pH}$, high compatibility with cell milieu and extreme solubility as $14 \mathrm{kgs}$ of it can be dissolved in $1 \mathrm{~kg}$ of water (LeRudulier et al., 1984). Though evidence has been presented for the synthesis of proline from glutamate and ornithine in higher plants (Delauney and Verma, 1993; Sharma et al., 2013), the role of ornithine as proline precursor is doubtful as pointed out by Funck et al. (2008). The pathway for proline biosynthesis in higher plants differs from that of bacteria (Kishor et al., 2005; Verbruggen and Hermans, 2008; Szabados and Savoure, 2010). The genes that encode the enzymes involved in proline biosynthesis like pyrroline-5-carboxylate synthetase (P5CS), pyrroline-5-carboxylate reductase (P5CR), and ornithine- $\delta$-aminotransferase $(O A T)$ and proline catabolism such as proline dehydrogenase $(P D H)$ and pyrroline-5-carboxylate dehydrogenase $(\mathrm{P} 5 \mathrm{CDH})$ have been identified and isolated (Delauney and Verma, 1990; $\mathrm{Hu}$ et al., 1992; Delauney et al., 1993; Kiyosue et al., 1996; Strizhov et al., 1997; Deuschle et al., 2001; Funck et al., 2010). P5CS1 was first cloned from Vigna aconitifolia by complementation technique ( $\mathrm{Hu}$ et al., 1992), and found to be a novel bifunctional enzyme (with activities 
of both $\gamma$-glutamy kinase and glutamic $\gamma$-semialdehyde dehydrogenase) that catalyzes glutamate to pyrroline-5 carboxylate, an intermediate in proline biosynthesis. This is a rate limiting step in proline biosynthesis. P5CS is encoded by two differentially regulated genes (P5CS1 and P5CS2) and P5CS2 was first cloned from Arabidopsis thaliana (Strizhov et al., 1997). While P5CS1 is abundantly expressed in most plant parts but not in dividing cells, P5CS2 is highly expressed at the transcriptional level in dividing cells (Strizhov et al., 1997). Besides acting as a proteogenic amino acid, it accumulates in large quantities and plays a role during abiotic stress tolerance in plants (Kishor et al., 1995, 2005; Sharma and Verslues, 2010). Thus, it is known that proline participates in the biosynthesis of primary metabolism, but also has special functions to carry as a metabolite during growth and development (Hare and Cress, 1997; Hare et al., 1999; Trovato et al., 2001; Mattioli et al., 2009a; Funck et al., 2012). Proline not only accumulates during abiotic stress, but also in different tissues of plants under non-stress conditions too (Kishor and Sreenivasulu, 2014). The role of proline as osmoprotectant in stress tolerance has been extensively reviewed (Verbruggen and Hermans, 2008; Szabados and Savoure, 2010). Though many amino acids constitute a major source of the components used for cellular growth and differentiation in higher plants, proline is distinct from others. The emerging roles of proline in plant growth and development from the perspective of proline homeostasis in mediating growth and development has been reviewed (Kishor and Sreenivasulu, 2014). Over and above, proline is an important source of cell wall matrix. As a component of cell wall proteins, it plays pivotal role in plant development but understanding of its diverse functions appears to be enigmatic. Cell walls contain hydroxyproline-rich O-glycoproteins (HRGPs) as complex macromolecules with varying structures and functions and encompass a broad category of extracellular proteins. HRGPs contain variations of Pro-Pro repeats (Cassab and Varner, 1988; Showalter, 1993) and are lightly glycosylated. Their classification is based on proline residue-containing proteins (Cassab and Varner, 1988; Showalter, 1993). It is a superfamily that is classified into extensins (EXTs) that are moderately glycosylated, arabinogalactan-proteins (AGPs) that are hyperglycosylated (Henrissat et al., 2001; Lamport et al., 2011) and Hyp/Pro-rich proteins (H/PRPs) that may not be glycosylated at all, or either weakly- or highly glycosylated. HRGPs undergo post-translational modifications like conversion of proline to hydroxyproline (Hyp) by a membrane-bound prolyl 4-hydroxylases (P4Hs) or glycosylation of HRGPs by glycosyltransferases (Rhee et al., 2003; Mayer and Jurgens, 2004; Wang and He, 2004). This sub-family is characterized by $O$-Hyp linked arabinosides and arabinogalactan polysaccharides (Lamport, 1967, 1977; Pope, 1977) which define the molecular surface. Arabinosylation and arabinogalactosylation would depend upon contiguous Hyp and clustered non-contiguous Hyp. What directs the Hyp glycosylation is a code based on peptide sequence. While Ser-Hyp 4 motifs of EXTs are preferred sites for the addition of arabinoside, Ser-Hyp and Ala-Hyp repeats of the AGPs are the usual sites of arabinogalactan heteropolysaccharide addition (Kieliszewski and Shpak, 2001;
Zhao et al., 2002; Held et al., 2004). Studies carried out by many suggest that species variation and tissue specific Hyp glycosylation occurs (Kieliszewski and Shpak, 2001; Zhao et al., 2002; Held et al., 2004). HRGPs appear to be not solubilized in cell walls as a response to the stress (Showalter, 1993; Fowler et al., 1999). They are expressed during wounding and pathogen attack in some tissues and implicated in different stages of plant growth and development, nodule formation, fertilization, cytokinesis, apoptosis, senescence, and cell wall lignification (Ye et al., 1991; Showalter, 1993, 2001; Nothnagel, 1997; Wu et al., 2001; Hall and Cannon, 2002; Lamport et al., 2006). Different roles of proline in plant ontogeny and during key transitions are summarized in Table 1. In the present endeavor, we aim at reviewing the progress of multidimensional functions of proline in plant ontogeny which is contributing to cell wall modifications and also during key transitional events especially reproductive development. In addition, the implications of proline during seed development and seed storage metabolism has been highlighted.

\section{Significance of Proline in Developing Seeds and in Protein Synthesis}

It is known that proline plays a vital role in regulating general protein synthesis in plants. Seed mutants with opaque phenotypes have been discovered in maize (Schmidt et al., 1987; Coleman et al., 1995; Kim et al., 2006). These mutants have been found to be associated with zein (a prolamin protein in seeds) synthesis (Holding et al., 2010; Wang et al., 2011). The auxotrophic recessive opaque mutant in maize, named as Proline responding1 (pro1), displays not only collapsed starchy endosperm morphology but also seedling lethality (Gavazzi et al., 1975; Ma and Nelson, 1975). Later, it was found out that mutants can be restored as normal phenotypes with the supply of exogenous proline (Racchi et al., 1978; Tonelli et al., 1984) and they have predicted that these mutants might have a defect in the biosynthesis of proline (Tonelli et al., 1986). Recently, Wang et al. (2014) reported positional cloning and functional characterization of proline responding1 gene (pro1). The conclusion of Wang et al. (2014) that maize Pro1 encodes a P5CS2 is based on the phylogenetic tree built on the P5CS full-length protein sequences taken from the genomes of A. thaliana, rice and maize. It has been found that this protein is localized in cytoplasm and expressed in roots, stems, silk, tassels, and kernels in these mutants and external feeding of proline rescued the viability of prol seedlings. They also unraveled the mechanism related to this mutation. In these pro1 mutants, lack of proline results in enhanced accumulation of uncharged tRNA ${ }^{\text {pro AGG }}$. But, such an accumulation leads to a sequence of consequences, first the phosphorylation of eukaryotic initiation factor $2 \alpha(\mathrm{elF} 2 \alpha)$ in the mutant pro1 and subsequently a reduction in general protein synthesis. Thus, in pro1 mutants, lack of proline affects protein accumulation. Interestingly, Wang et al. (2014) further revealed that proline deficiency also leads to downregulation of cyclin genes at the 
TABLE 1 | Multidimensional roles of proline in different cell organelles, tissues, organs, and whole plants.

\begin{tabular}{|c|c|c|c|}
\hline $\begin{array}{l}\text { Cell organelle/ } \\
\text { tissue/organ }\end{array}$ & $\begin{array}{l}\text { Proline/proline-rich/hydroxyproline } \\
\text { (Hyp)-rich proteins }\end{array}$ & Function & Reference \\
\hline Cell wall & PRPs & Wall components & McCann et al. (2001) \\
\hline Cell & PRPs & Cell elongation & Ito et al. (1998) \\
\hline Cell & PRPs & Root hair development & Bernhardt and Tierney (2000) \\
\hline Whole plant & PRPs & Abscission and senescence & Merkouropoulos and Shirsat (2003) \\
\hline Whole plant & PRPs & Development and abiotic stress tolerance & Zhan et al. (2012) \\
\hline Floral buds & PRPs & Structural integrity of the style & Cheung et al. (1993) \\
\hline Flower & PRPs & Flower development & Fowler et al. (1999) \\
\hline Flower & PRPs & Flower development & Gothandam et al. (2010) \\
\hline Flower & PRPs & Cotton fiber development & Xu et al. (2013) \\
\hline Phloem & PRPs & Expression in phloem in response to drought stress & Battaglia et al. (2007) \\
\hline Cell wall & PRPs & Drought stress & $\begin{array}{l}\text { Creelman and Mullet (1991), } \\
\text { Colmenero-Flores et al. (1997) }\end{array}$ \\
\hline Plasma membrane & Repetitive proline-rich proteins (PRPs) & Sensitivity of roots to ABA & Tseng et al. (2013) \\
\hline Plasma membrane & HRGPs & Plasma membrane-cytoskeleton continuum & Showalter (1993), Showalter et al. (2010) \\
\hline Cell & HRGPs & Wall assembly and remodeling during cell growth & Doblin et al. (2010), Ellis et al. (2010) \\
\hline Cell & HRGPs & Cell-cell integrations and communications & Wu et al. (2001) \\
\hline Pollen and style & HRGPs & Pollen tube and style growth & Zhang et al. (2014) \\
\hline Callus/suspensions & HRGPs & $\begin{array}{l}\text { Somatic embryogenesis, germination of embryos } \\
\text { during plant regeneration }\end{array}$ & Xu et al. (2011) \\
\hline Callus & Hybrid PRPs (HyPRPs) & Cell elongation, enhanced size of callus & Dvorakova et al. (2012) \\
\hline Whole plants & Proline transporters & Xylogenesis & Grallath et al. (2005) \\
\hline Transgenic plants & Proline over-production & Increased root biomass & Kishor et al. (1995) \\
\hline Transgenic plants & Proline over accumulation & $\begin{array}{l}\text { Seed germination and stimulation of seedling } \\
\text { growth }\end{array}$ & Mattioli et al. (2009b) \\
\hline Transgenic plants & Proline over accumulation & Inhibition of stem growth & Nanjo et al. (1999) \\
\hline Transgenic plants & Proline over accumulation & Affects flowering time & Schwacke et al. (1999), Mattioli et al. (2009b) \\
\hline Transgenic plants & Proline accumulation & Flower initiation & Mattioli et al. (2008), Ghanti et al. (2011) \\
\hline Transgenic plants & Proline accumulation & Male fertility & Funck et al. (2012) \\
\hline Untransformed plant & Proline accumulation & More seed yield under non-stress & Bo et al. (2000) \\
\hline Untransformed plant & Proline accumulation & $\begin{array}{l}\text { Improves final grain production under non-stress } \\
\text { conditions }\end{array}$ & Spoljarevic et al. (2011) \\
\hline Flower buds & Proline accumulation & Bud-break & Xue et al. (2009) \\
\hline Floral nectars & Proline accumulation & Attraction of pollinators & $\begin{array}{l}\text { Alm et al. (1990), Carter et al. (2006), } \\
\text { Thornburg (2007) }\end{array}$ \\
\hline Whole plants & Defect in proline biosynthesis & $\begin{array}{l}\text { Reduction in protein synthesis. Downregulation of } \\
\text { cyclin genes }\end{array}$ & Wang et al. (2014) \\
\hline Embryo & Defect in proline biosynthesis & Embryo lethality and defective seed development & $\begin{array}{l}\text { Szekely et al. (2008), Mattioli et al. (2009b), } \\
\text { Funck et al. (2012) }\end{array}$ \\
\hline Leaf/grain/flower & Normal proline levels & Flavor compound in leaf/flower/grain & $\begin{array}{l}\text { Buttery et al. (1982), Romanczyk et al. (1995), } \\
\text { Chen et al. (2008) }\end{array}$ \\
\hline Fruit & Proline accumulation & Enhances fermentability in grapevine & Stines et al. (2000) \\
\hline Seed & Normal proline levels & Seed germination & Botha et al. (1992) \\
\hline
\end{tabular}

transcriptional level and thus the cell cycle in the pro1 mutants is significantly affected. RT-PCR analysis revealed that CDC6 and $M C M 7$ genes encoding proteins involved in the formation of prereplicative complex are downregulated in pro1 mutants, but external supply of proline induces the expression of $A, B$, and $\mathrm{D}$ cyclins, $\mathrm{H} 2, \mathrm{H} 3$, and $\mathrm{H} 4$ histones and DNA replication related genes in pro1 mutants (Wang et al., 2014). These studies point out that lack of proline affects cyclin genes at the transcriptional level and ultimately protein accumulation in seeds.

\section{Proline Accumulation and Root Biomass}

Though proline is produced at low levels in all tissues in unstressed conditions, it is actively transported to the roots under stress conditions. Further, it is compartimentalized into the mitochondria where it is degraded back to glutamate releasing energy in the form of $\mathrm{FADH}_{2}$ and $\mathrm{NAD}(\mathrm{P}) \mathrm{H}$ by two different enzymes (Szabados and Savoure, 2010). At low water potentials, maize primary roots have been shown to accumulate proline (Ober and Sharp, 1994). Maintenance of root elongation at 
low water potential depends on enhanced ABA content. When endogenous $\mathrm{ABA}$ levels were decreased either by using fluridone or vp5 mutant, proline concentrations also decreased in the root elongation zone. But, proline concentrations in fluridone treated roots were restored back by the addition of $7 \mu \mathrm{M}$ $\mathrm{ABA}$. These experiments demonstrate that increased $\mathrm{ABA}$ is necessary for the deposition of proline in maize primary root growth zone. Voetberg and Sharp (1991) also demonstrated that proline levels are high toward the root apex $(120 \mathrm{~m} . \mathrm{mol}$ growing at a water potential of $-1.6 \mathrm{mpa}$ ), where elongation rates of roots are maintained under a range of water deficit conditions. They suggested that proline deposition in the growing root zone is important for osmotic adjustment. But, how proline deposition plays a role in the maintenance of root elongation at low water potentials is not clearly known. Verslues and Sharp (1999) found out that proline synthesis in maize root tips from $[3 \mathrm{H}]$ glutamate and $[14 \mathrm{C}]$ ornithine did not increase significantly at low water potential and accounted for only a small fraction of the proline deposition. Proline is also not catabolized and utilized in the root tips under low water potential. But, increased uptake was noticed suggesting increased transport of it. Removal of endosperm from the germinated seedlings decreased the proline accumulation in root tips at low water potentials in maize (Raymond and Smirnoff, 2002). They conclude that endosperm is the source of proline accumulation in the root tips of intact seedlings constituting $10 \%$ of the free amino acids released from endosperm. Labeling experiments also revealed that proline is transported from the scutellum to other parts of the seedlings and peaked in the root tips (Raymond and Smirnoff, 2002). Though the biosynthetic capacity of roots to produce proline is low, proline accumulation perhaps is regulated by the rates of its transport and utilization. A unique saltinducible proline transporter was isolated from barley (Hordeum vulgare) and found to express strongly in root cap cells under salt stress (Ueda et al., 2001). Hence, it may play a role in the root tip region when plants are exposed to salt stress. Spollen et al. (2008) analyzed the spatial distribution of transcript changes in the maize primary root elongation zone at low water potential. They concluded that different signaling and metabolic response mechanisms (wall loosening proteins in region I, and for elements of $\mathrm{ABA}$ and ethylene signaling) are involved in the response to water stress in different regions of the maize primary root elongation zone. Further, overexpression of Vigna P5CS in tobacco (Kishor et al., 1995) and Sorghum bicolor (Reddy et al., 2015) resulted in better root biomass under salt stress conditions. Proline accumulation by overexpression of P5CSF129A, a mutated version of Vigna P5CS in transgenic chickpea (Ghanti et al., 2011) also resulted in root elongation and higher root biomass both under salt and drought stress conditions. It appears therefore, proline may play a direct or indirect role in enhancing root biomass in transgenics under stress conditions perhaps by supplying necessary energy $\left[\mathrm{FADH}_{2}\right.$ and $\mathrm{NAD}(\mathrm{P}) \mathrm{H}]$ and nitrogen.

Several studies related to seed germination have suggested that oxidative pentose phosphate pathway (OPPP) is important in triggering seed germination (Botha et al., 1992; Hare and Cress, 1997). Since this pathway generates NADPH, the energy may be utilized for anabolic reactions during seed germination (Besse and Buchanan, 1997). Shetty (2004) also suggested a link between proline and OPPP. But, the molecular events leading to upregulation of OPPP and its precise role in triggering seed germination is not known completely. Hare et al. (2003) have analyzed the dehydrogenase enzymes involved in OPPP during Arabidopsis seed germination and found activation of this pathway along with fourfold increase in proline content before the emergence of the radicle. When AtP5CS1 gene was inserted in an antisense orientation into Arabidopsis, delayed emergence of radicle was noticed. Proline synthesis might replenish the $\mathrm{NADP}^{+}$pool and therefore activate OPPP. This indicates a functional link between elevated proline biosynthesis and enhanced OPPP activity and coupling of both the pathways may be important in stimulating germination.

\section{Effects of Proline Accumulation in Reproductive Tissues under Non-Stressed Conditions}

Under normal physiological (non-stressed) conditions, plants accumulate large amounts of proline during the transition to flower initiation (Chiang and Dandekar, 1995; Schwacke et al., 1999; Mattioli et al., 2009b), a common phenomenon but often an overlooked fact. Similarly, proline content in tomato flowers was 60 times higher than any other vegetative tissue and $>70 \%$ of total free amino acids was noticed in pollen grains (Schwacke et al., 1999). A wide range of plants like mature fruits of citrus (Clements and Leland, 1962), pollen grains of petunia and tomato (Zhang et al., 1982; Fujita et al., 1998), ovules of broad bean (Venekamp and Koot, 1984), inflorescences and siliques of Brassica napus (Flasinski and Rogozinska, 1985) also contain very high levels of proline. Accumulation of proline is due to upregulation of proline biosynthetic pathway gene P5CS, downregulation of ProDH and activation of a proline transporter T (ProT) in flowers (Savoure et al., 1995; Rentsch et al., 1996; Schwacke et al., 1999; Hayashi et al., 2000). Proline accumulation predominantly in reproductive tissues suggests that it may be associated with their development (Chiang and Dandekar, 1995; Schwacke et al., 1999). It is also transported to reproductive organs as has been pointed out by Rentsch et al. (1996) and Fischer et al. (1998). These findings suggest that proline may play a role during flower initiation and its subsequent development.

Overexpression of P5CS in tobacco resulted in higher accumulation of proline, and enhanced flowering with more number of flowers per plant and coflorescence (cluster of flowers) formation under stress conditions (Kishor et al., 1995). Over expression of P5CS1 and over-accumulation of proline lead to early flowering and bolting promotion during the early stages of plant development in Arabidopsis (Mattioli et al., 2008). But, during the later stages, downregulation occurred probably because of gene silencing, leading to low proline and bushy appearance of the plants. On the contrary, antisense expression of P5CS1 inhibited bolting in Arabidopsis (Nanjo et al., 1999). The p5cs1 loss-of-function mutants exhibit delay 
in flowering implying that P5CS1 plays an important role in flowering time (Mattioli et al., 2009b). They also noticed that down-regulation of P5CS1 caused reduction in proline content, impaired flowering, and stunted growth. Funck et al. (2012) made a detailed analysis of the proline biosynthetic pathway enzymes to dissect out their roles during vegetative and reproductive development of Arabidopsis. In their conditions, they found alteration in flowering time in $p 5 c s 1$ mutants, but significant flowering delay in $p 5 c s 2$ mutants. It is hypothesized that the flowering delay of the 35S-P5CS1 plants as reported by Mattioli et al. (2008) could be accounted for by the homology-based co-silencing of P5CS2 triggered by the expression of P5CS1. Samach et al. (2000) found that AtP5CS2 is a target of CONSTANS $(C O)$, a transcriptional activator associated with flowering time. Besides, rolD oncogene from Agrobacterium rhizogenes is known to encode a functional ornithine cyclodeaminase enzyme in bacteria which ultimately converts ornithine to proline (Trovato et al., 2001). The main trait induced by overexpression of rolD gene in tobacco is precocity in flower setting and a strong enhancement of the flowering potential in tobacco (Mauro et al., 1996) and tomato (Bettini et al., 2003). These experiments thus point out that proline plays a vital role during flower transition, bolting, and in changing the architecture of inflorescence (coflorescence formation). However, we are not sure about the molecular events leading exactly how proline influences flowering time and changes the inflorescence architecture in higher plants when produced in optimum concentrations.

\section{Proline Contribution to Plant Cell Wall Architecture in Root Development and Reproductive Organs}

It is a fact that proline and Hyp are the major amino acid constituents of hydrolysates of cell wall proteins. As pointed out by Kaplan and Hagemann (1991), morphogenesis takes place in plants because of differential growth of organs at the cell wall. Therefore, studies on cell walls are vital though they are highly intricate and complex. Cell wall matrix encoding proteins are enriched with proline residues which are integrated in the form of HRGPs classified into three categories: (i) moderately glycosylated EXTs; (ii) hyperglycosylated arabinogalactanproteins (AGPs); and (iii) Hyp/proline (Pro)-Rich proteins (H/PRPs).

\section{Role of Extensins in Cell Wall Modification During Root and Pollen Development}

The extracellular matrices of plants and animals contain structural glycoproteins which are important scaffolding components. Some of these glycoproteins like collagens in animals and HRGPs of plants share functions like enhancing tensile strength (Shirsat et al., 1996) and contributing tissue integrity. In all the three subgroups, proline, and Hyp residues are abundant and play an important role in cell wall development. EXTs are self-assembling amphiphiles. They are modular in nature and contain Hyp and are usually characterized by pentameric Ser-Hyp peptide repeats. EXTs exhibit $35-65 \%$ glycosylation on Ser-(Hyp $)_{n} \geq 2$ motifs and the side chains contain oligosaccharides of arabinose and galactose (Showalter, 1993; Kieliszewski and Lamport, 1994). The genome of A. thaliana encodes 63 putative EXTs with high amino acid sequence similarity between them. Out of the 63 members, some are classical, while others are EXT-like chimeras and hybrid EXTs (Lamport et al., 2011). It is not clear whether they have redundant or specific functions to carry in different cell types of various plant tissues. EXTs exhibit repetitive nature of their sequences with many post-transcriptional modifications in plant genomes like signal peptide processing in the ER, hydroxylation of proline to Hyp residues by $\mathrm{P} 4 \mathrm{H}$, $O$-glycosylation on Hyp and Ser residues with chains of up to four linear Ara residues on each Hyp by arabinosyltransferases (Velasquez et al., 2011; Ogawa-Ohnishi et al., 2013), and cross-linking of glycosylated EXTs at the tyrosine residues in the cell wall (Jackson et al., 2001; Price et al., 2003; Lamport et al., 2011; Hijazi et al., 2014) forming intra- and inter- EXT linkages (Nunez et al., 2009; Dick-Perez et al., 2011). This helps EXTs to form a three-dimensional structure of protein and to interact with pectins in the cell walls (Dick-Perez et al., 2011). The coordination of EXTs with other components and their functions during cell wall architecture and plant development are not completely known. This is mostly because of their modular nature, existence in large numbers, hydroxylation by different $\mathrm{P} 4 \mathrm{Hs}$, complex structure, and redundant expression of these proteins in the same plant tissues (Genevestigator database, https://www.genevestigator.com). EXTs can also interact with pectins and serve as templates for cell wall deposition (Cannon et al., 2008; Lamport et al., 2011). Recently, Tan et al. (2013) found an ARABINOXYLAN PECTIN ARABINOGALACTAN PROTEIN1 (APAP1) in A. thaliana which consists of pectin and arabinoxylan that are covalently linked to an AGP. They suggested a role for APAP1 proteoglycan in plant cell wall architecture and function. However, the exact coordinated control of EXTs and pectin interaction during cell wall formation is unclear.

Several groups have used root hair as a model to study the functions of EXTs (Park et al., 2011; Velasquez et al., 2011). Mutants lacking the synthesis of wall polymer in the root hair are impaired in growth (Diet et al., 2006; Ringli, 2010; Park et al., 2011; Velasquez et al., 2012). Since posttranslational modifications of HRGPs are carried out by (P4Hs), this defines the subsequent $O$-glycosylation sites in EXTs (mostly arabinosylated) and polarized growth in root hairs. O-glycans enhance the HRGP solubility and resistance to proteolytic degradation (Kieliszewski et al., 1989; Shpak et al., 2001; Lamport et al., 2011). Velasquez et al. (2011) proved that $O$-glycosylation on EXTs is essential for cell-wall assembly and root hair elongation. Root hairs develop by tip growth akin to pollen tubes, axons, and fungal hyphae. Velasquez et al. (2015) recently carried out genetic analysis related to the regulation of $\mathrm{P} 4 \mathrm{H}$. They proved that $\mathrm{P} 4 \mathrm{H} 5$ and $\mathrm{P} 4 \mathrm{H} 2$ and $\mathrm{P} 4 \mathrm{H} 13$ are pivotal for root hair tip growth. The impact of deficient proline hydroxylation on the cell wall architecture has also been shown by them. Their results demonstrate that peptidyl-proline 
hydroxylation on EXTs is required for cell wall assembly and therefore root hair elongation in Arabidopsis. The above works point out that changes in the $O$-glycosylation status impact the functions of EXTs during cell wall assembly, cell shape, and expansion. Genes that encode $\mathrm{P} 4 \mathrm{Hs}$ are also linked to hypoxia, and $\mathrm{P} 4 \mathrm{H}$ proteins are regarded as oxygen sensors under hypoxic stress. Zou et al. (2011) found out that $P 4 H$ genes are subjected to alternative splicing in roots of maize seedlings under waterlogging conditions. The diverse transcripts generated due to alternative splicing are expressed at different levels clearly indicate that $\mathrm{ZmP} 4 \mathrm{H}$ genes are under specific control by posttranscriptional regulation under waterlogging stress in maize. Hall and Cannon (2002) identified that cell wall Hyp-rich glycoprotein ROOT-SHOOT-HYPOCOTYL-DEFECTIVE (RSH) is necessary for normal embryo development in Arabidopsis. Xu et al. (2011) further found out the role of HRGPs during somatic embryogenesis of Musa sp. Their results suggest that HRGPs play a vital role in regeneration and germination of embryos during early plant development via somatic embryogenesis (Table 1). Proper localization and appropriate quantities of HRGPs seem to be critical for the formation and regeneration of somatic embryos. But, the exact mechanism by which HRGPs trigger somatic embryogenesis in callus or suspension cultures is not known. Thus, the functions proline-rich and Hyp-rich glycoproteins appear to be complex, but clearly associated with developmental regulation in different plant systems. Using protein blots and immunohistochemistry, EXTs have been found to be abundantly expressed in vivo for pollen tubes and transmitting tissues (Zhang et al., 2014). When inhibitors of HRGP were used, decreased pollen tube growth and stylar length were recorded. It appears that HRGPs especially EXTs play a critical role in the pollen tube and style cell growth. Wu et al. (2001) demonstrated that EXTs are involved in pollen recognition and fertilization. These evidences along with the works of Cannon et al. (2008), Ringli (2010), Lamport et al. (2011), and Velasquez et al. (2012) infer that cross-linked EXTs play pivotal roles during cell expansion and growth of pollen tube.

\section{Arabinogalactan Protein Involvement in Vegetative and Reproductive Growth}

Arabinogalactan proteins are Hyp-rich extracellular proteins with a high proportion of sugars (up to 90\%). Among them, arabinose and galactose are predominant residues, but minor sugars like rhamnose, fucose, glucuronic, acid, and xylose are common (Nothnagel, 1997; Showalter, 2001). AGPs are characterized by extensive glycosylation with arabinose, galactose or both and analogous to animal proteoglycans (Clarke et al., 1979; Lord and Sanders, 1992). AGPs possess repetitive Alanine (Ala) or Serine (Ser) motifs that interact with Hyp molecules. They are chemically stable, and resistant to proteolytic enzymes in their native state due to the presence of carbohydrates (Fincher et al., 1983). AGPs contain glycosylphosphatidylinositols (GPIs) which tethers them to the plasma membranes in plants (Schultz et al., 2004). The biosynthesis, structure, expressions, and functions of AGPs have been well studied and reviewed (Showalter, 2001; Ellis et al.,
2010; Tan et al., 2012; Lamport and Várnai, 2013; NguemaOna et al., 2013; Knoch et al., 2014). Cassab (1998) pointed out that carbohydrate groups (D-galactose and L-arabinose) are mostly linked by $\mathrm{O}$-glycosylation to the hydroxyl group of Ser and Hyp of the AGP backbone. However, diversity exists in the degree and pattern of $O$-glycosylation depending on the type and length of glycan chains attached to the proteins. Many studies suggest that AGPs play important roles in vegetative and reproductive growth. AGPs were observed throughout the plant kingdom, in leaves, stems, roots, floral parts, and seeds (Fincher et al., 1983; Nothnagel, 1997). They were found in plasma membranes, cell walls, intracellular multivesicular bodies, and also as secretions to intercellular spaces (Nothnagel, 1997).

Some AGPs are also implicated with redundant architectural functions of cell walls (Bosch et al., 2001). Several lines of evidence indicate that AGPs are associated with growth and differentiation in lower as well as in higher plants. For example, when inhibitors of AGP biosynthesis were used, the growth of leaf primordia was suppressed in a liverwort (Basile and Basile, 1993). Yariv reagent inhibited cell division in cell suspension cultures and roots in carrot and Arabidopsis, respectively, (Willats and Knox, 1996; Ding and Zhu, 1997). This indicated that AGPs are involved in cellular growth and development. While certain AGPs promote somatic embryogenesis in Daucus carota, (Kreuger and van Holst, 1993), and Picea abies (Egertsdotter and von Arnold, 1995), others inhibit it as noticed by Toonen et al. (1997) in carrot.

AGPs were noticed in xylem, stylar transmitting tissues and cell suspension cultures. In targeting certain AGPs to the specific cell surface locations, the role of certain sequence determinants in the carbohydrate or protein need to be determined. Their multilocation (organs, tissues, and cell-types) occurrence indicates specific functions of AGPs in different tissues like flowers and other cell types (Pennell and Roberts, 1990). Many AGPs possess a C-terminal Ole e 1 domain (pollen allergens, named after a secreted protein isolated from Olea europeae pollen; Villalba et al., 1994). Developmental and tissue specific expressions were noticed for several AGPs in different plants (Pennell et al., 1991; Chen et al., 1994; Gao and Showalter, 2000), consistent with the assumption that they play a role in plant development. Altered expression of AGP in pea plants has changed the sexual development as shown by Pennell and Roberts (1990). Some AGPs like stylar transmitting tissue-specific PRPs (TTS proteins) play vital roles in reproductive growth and development like pollen-pistil interactions (Bosch et al., 2001; Hancock et al., 2005). When the gene knockdown experiments were carried out, TTS proteins have been found important for pollen-pistil interactions. This may be possible for TTS proteins which act as cell surface adhesives (Wu et al., 2000). AGPs are also implicated in cellular signaling events (Schultz et al., 1998). They are the important components in plant exudates and gums, as shown in the case of gum arabic extracted from Acacia senegal (Serpe and Nothnagel, 1999) and used as an additive in the food industry. Type II arabinogalactans have also been found to stimulate animal immune systems and thus may be useful in medicine as well (Yamada and Kiyohara, 1999). Further, 
chimeric proteins containing AGP domains may interact with polysaccharides. In this connection, the experiments of Griffiths et al. (2014) have proved that CELLULOSE SYNTHASE5 (CESA5) and the SALT-OVERLY SENSITIVE5 (SOS5), a Fasciclin-AGP are required for mucilage adherence in seeds of $A$. thaliana. They conclude that SOS5 mediates adherence through pectin (Griffiths et al., 2014). AGPs are involved in cell-cell/intercellular communications involved in tracheary element differentiation (Motose et al., 2001) and thus AGPs are found to be associated with the differentiation of xylem and programmed cell death (Schindler et al., 1995; Gao and Showalter, 2000). Thus, AGPs perform several functions in different aspects of plant growth and development, though the underlying molecular mechanisms are not clearly known.

\section{Pursuit of Hyp/Pro-Rich Proteins (H/PRPs) in Plant Development}

Hyp/Pro-rich proteins belong to the HRGP superfamily like that of EXTs and AGPs. Based on repetitive motifs and domain organization, PRP proteins are classified into three subtypes; (a) repetitive PRPs, (b) non-repetitive PRPs, and (c) multidomain hybrid PRPs (Jose-Estanyol and Puigdomenech, 2000). Proline residues in PRPs are hydroxylated and form Hyps which are then glycosylated as mentioned earlier. But, $\mathrm{O}$-glycosylation of H/PRPs and their interactions with polysaccharides is not completely known. Twelve H/PRP proteins have been identified so far in Arabidopsis thaliana, carrot, cotton, tobacco, common bean, capsicum, and petunia (Hijazi et al., 2014). Bradley et al. (1992), and Frueauf et al. (2000) pointed out that H/PRPs may be cross-linked in cell walls, but evidence is lacking for such an assumption.

Pro-rich proteins are involved in cell elongation (Ito et al., 1998), abscission and senescence (Merkouropoulos and Shirsat, 2003). As shown in Table 1, HRGPs are essential for the structure and function of cell wall as well as plasma membranecytoskeleton continuum (Showalter, 1993; Showalter et al., 2010). It is highly interesting to note that the PRP genes show developmental stage-, organ-, tissue-, and even cell-specific expressions (Hong et al., 1989; Ye and Varner, 1991; Wyatt et al., 1992). Hong et al. (1987, 1989) reported the characterization of three developmentally regulated proline-rich cell wall protein genes from Glycine max. Marked differences were observed in the pattern of expression of PRPs in different organs like hypocotyls, roots, stems (Wyatt et al., 1992), immature embryos (Jose-Estanyol et al., 1992), immature seed coats and developing seeds and in cells that are undergoing lignifications (Vignols et al., 1999). Fowler et al. (1999) characterized and expressed four proline-rich cell wall protein genes in Arabidopsis encoding two distinct subsets of multiple domain proteins. Their studies support a model for the involvement of PRPs in specifying cell-type specific wall structures, and provide the basis for a genetic approach to dissect the function of PRPs during growth and development. While S. bicolor PRP1 (SbPRP1) mRNA is abundant in elongating regions of hypocotyl epidermal cells, $S b P R P 2$ mRNA is more expressed in phloem cells. Further, it has been found that $S b P R P 3$ mRNA is localized in the endodermoid layer of cells in the hypocotyl elongating region (Wyatt et al., 1992). Bernhardt and Tierney (2000) demonstrated that expression of AtPRP3, a proline-rich structural cell wall protein is regulated by cell-type-specific developmental pathways involved in root hair (single cells that develop by tip growth and are specialized in absorption of nutrients) formation. They noticed enhanced expression of AtPRP3/ $\beta$-glucuronidase (GUS) in roots of transgenic seedlings treated with 1-aminocyclopropane-1-carboxylic acid (ACC) or $\alpha$-naphthaleneacetic acid, two compounds known to promote root hair formation. The results indicate that AtPRP3 is regulated by developmental pathways involved in root hair formation (Bernhardt and Tierney, 2000). Overexpression of GhPRP5 in A. thaliana resulted in smaller cell size, but knock-down of GhPRP5 expression by RNA interference in cotton increased the fiber length (Xu et al., 2013). Their data suggest that this protein participates in modulating fiber development of cotton.

\section{Involvement of PRPs as Signaling Proteins in Reproductive Tissues}

Pro-rich proteins perform a whole spectrum of functions like providing structural integrity to mediate cell-cell integrations and communications (Wu et al., 2001). They are also involved and play a vital role in wall assembly and remodeling during cell growth and development (Ellis et al., 2010; Doblin et al., 2010). Gibbon et al. (1998) characterized several profilin isoforms from maize pollen and showed that their function depends on interaction with proline-rich motifs. They found out that profilin isoforms expressed in a single cell can have different effects on actin in living cells and the poly-L-proline binding function of profilin may have important consequences for the regulation of actin cytoskeletal dynamics. Two stylar transmitting tissue specific PRPs (TTS-1 and TTS-2) have also been reported in tobacco (Cheung et al., 1993). TTS-1 and TTS-2 mRNAs have been induced in floral buds, during the later stages of flower development especially when style elongation is rapid and are active at anthesis (Cheung et al., 1993). The occurrence of PRPs in transmitting tissues has been predicted to play a vital role in maintaining the structural integrity of the style. Transgenic Arabidopsis overexpressing Atext1 displayed altered inflorescence and affected stem thickening and height (Roberts and Shirsat, 2006). However, the functions of all PRPs are not completely known during different developmental stages, thus leaving a gap in our understanding of PRPs.

\section{Role of PRPs in Cell Wall Modification under Stress}

Genes that encode PRPs and the localization of these proteins in plant cell walls have been studied (Cassab and Varner, 1988; Showalter and Varner, 1989; Cassab, 1998). During growth, differentiation and also during different environmental stress conditions, cells may expand up to 200 times their original length. Water stress could result in alterations in cell volume and shape, 
as well need to deal with loss of turgor (Wakabayashi et al., 1997). Therefore, cell walls must possess tensile strength to withstand the turgor pressures. This involves very large chemical and biochemical modifications of cell wall constituents including cell wall proteins representing 1 to $2 \%$ of the genome in plants such as Arabidopsis (Somerville et al., 2004). Cell wall protein transcripts are also affected under salt, drought, and temperature stress conditions (Covarrubias et al., 1995). PRPs are insolubilized in the cell walls with the involvement of $\mathrm{H}_{2} \mathrm{O}_{2}$-mediated oxidative cross linking and it precedes the expression of transcriptiondependent defences. Though cell wall bound PRPs may play a role in the structural integrity of plant cells, participate in defence related activities, and plant cell surface interactions as pointed out by Roberts (1989), Varner and Lin (1989), interestingly, the mRNA levels of PRPs increase in response to water scarcity in higher plants (Creelman and Mullet, 1991; Colmenero-Flores et al., 1997).

Battaglia et al. (2007) characterized two proline-rich glycoproteins of 33 and $36 \mathrm{kDa}$ (p33 and p36) which are found in the cell wall soluble fraction of common bean (Phaseolus vulgaris). They observed the highest accumulation of these proteins in growing regions, predominantly in phloem tissues in response to drought indicating that cell wall modifications are induced in actively growing cells of common bean. Gothandam et al. (2010) reported a flower specific PRP from rice (OSPRP3) that is expressed during the late stages of flower development. While AtPRP3 is associated with root hair formation, overexpression of Oryza sativa PRP3 (OsPRP3) showed an increase in cold tolerance compared to the wild-type plants. They showed that this OsPRP3 enhances cell wall integrity in the cold tolerant plants. Knockout mutants displayed defects in floral organogenesis suggesting a role for PRPs in reproductive tissues. Tseng et al. (2013) reported ABA-induced expression of a family of four genes, REPETETIVE PROLINE-RICH PROTEIN $(R e P R P)$ in rice. These genes encode proline-rich glycoproteins with highly repetitive $\mathrm{PX}_{1} \mathrm{PX}_{2}$ motifs, RePRP1, and RePRP2. Their work also revealed that ABA treatment increases RePRP expression and that these proteins are localized to the plasma membrane. It has been found that knockdown of the expression of RePRP1 and RePRP2 decreases the sensitivity of roots to ABA. Thus, these experiments reveal that RePRP proteins play an essential role in $\mathrm{ABA} /$ stress regulation of root growth and development. It has also been noticed that rice RePRPs interact with arabinogalactan polysaccharide in a dosage-dependent manner (Tseng et al., 2013). Zhan et al. (2012) recently identified a PRP called SICKLE (SIC) in Arabidopsis. This protein is critical for not only development in Arabidopsis but also in imparting abiotic stress tolerance. The loss-of-function sic-1 mutants displayed reduction in plant height, delay in flowering, and abnormal inflorescence phyllotaxy. These mutants accumulated reduced levels of a subset of miRNAs and transacting siRNAs but enhanced levels of primary miRNAs than the controls. The sic-1 mutant plants are also sensitive to cold and salt stresses (Zhan et al., 2012). Therefore, it appears that SIC is a PRP associated with the biogenesis of some miRNAs and degradation of some spliced introns and is vital for plant development.

\section{Hybrid Proline-Rich Proteins (HyPRPs) are Crucial Players in Cell Elongation}

Out of the three subclasses of PRPs, one of them contains several copies of POVEKPOVXK motif (Hong et al., 1990), while the other two subclasses show a hybrid structure and are called hybrid proline-rich proteins (HyPRPs). Plant HyPRPs represent putative cell wall proteins consisting of a repetitive prolinerich N-terminal domains and a conserved C-terminal domain. They are unique to seed plants and analysis of the families from different plant species suggests rapid diversification of their sequences and expression patterns (Dvorakova et al., 2007). Their functions are not clearly known, but their occurrence indicates that they may be involved in basic cellular processes (Dvorakova et al., 2012). To understand the functions of HyPRPs, Dvorakova et al. (2012) modulated the expression of three HyPRP genes in tobacco and potato. Transgenic plants displayed cell elongation, and enhanced size of calli. HyPRPs contain two regions, and one of them is rich in proline and the other in cystein residues (Deutch and Winicov, 1995). Further, it has been shown that the third sub-class, namely NHyPRPs displays high percentage of proline residues organized in repetitive sequence motifs in C-terminus region (Castonguay et al., 1994; Menke et al., 2000). Thus, it appears that HyPRPs are important players in plant cell elongation, though the molecular mechanism is not yet elucidated.

\section{Proline-Rich Extensin-Like Receptor Kinases (PERKs)}

A family of receptor kinase-like proteins has been discovered that are involved in cell wall signaling. In A. thaliana, 11 members of the PERK family have been discovered which share some features of EXTs. Altered expression of PERK receptor kinases resulted in changes of plant growth and floral organ formation (Haffani et al., 2006). In a novel work, it has been elucidated by Bai et al. (2009) that the gene PERK4 (coding for a protein kinase located in the plasma membrane) is required for the ABA-dependent influx of $\mathrm{Ca}^{2+}$ and normal ABA sensitivity in seeds and roots of $A$ thaliana. Their experiments suggest that PERK4 is associated in ABA perception and also might interact with wall polymers. Thus, PRPs are implicated in cell wall signal transduction cascades. Further work is necessary since it is not yet clear about how many PERK proteins are associated with this process and also their additional roles if any during growth and stress tolerance.

\section{Proline Deficiency Affects the Biosynthesis of Cell Wall Matrix Proteins}

The experiments conducted by Cooper et al. (1994) demonstrated initially in tobacco that HRGPs are critical for cell morphology and osmotic stability. Strong evidence has come later from Nanjo et al. (1999) who created Arabidopsis plants with antisense P5CS. Interestingly, the transgenics displayed morphological defects in 
leaves and elongation of inflorescences. Further, they observed that protein biosynthesis was normal in proline deficient plants, but structural proteins localized in the cell walls were strikingly altered (Nanjo et al., 1999) indicating a direct evidence for a key role of proline in cell walls. Specifically, proline and Hyp contents were reduced in the cell wall preparations of Arabidopsis transgenic leaves. They pointed out that PRPs and HRGPs are responsible for morphological abnormalities observed in the leaves of antisense Arabidopsis plants since these proteins provide mechanical support for cells under stressed conditions. Such a role in cell morphology and the mechanical support for the PRPs and HRGPs also has been demonstrated by Showalter (1993) and Munoz et al. (1998). Thus, proline-related growth abnormalities are correlated well with the effects of proline on plant cell wall development. Wang et al. (2014) found out that proline deficiency causes downregulation of cyclin genes and affects general protein synthesis. However, they did not find out its effect on the cell wall proteins. It would be interesting to find out if there would be any changes that take place in H/PRPs associated with cell wall development. Several overexpressions and knockout mutations of plants containing PRPs and HRGPs are needed to demonstrate the exact role(s) of these proteins during cell wall synthesis and growth.

\section{Proline Transporters Play an Important Role in Plant Development Including Xylogenesis}

Proline transporters have been isolated from several species and three from Arabidopsis (AtProTs) so far (Rentsch et al., 1996; Schwacke et al., 1999; Fujiwara et al., 2010; Lehmann et al., 2011). Transcript levels of Arabidopsis ProT2, mangrove ProT homologues, barley HvProT as well as proline levels are elevated under salt stress (Rentsch et al., 1996; Hibino et al., 2001; Ueda et al., 2001; Waditee et al., 2002). Like in bacteria, proline transporters also act as glycinebetaine transporters in plants but with different affinities. Further, they also transport other compatible solutes like $\gamma$-aminobutyric acid (GABA) in plants (Grallath et al., 2005; Fujiwara et al., 2010). Though intracellular localization, substrate selectivity, and affinity of the three AtProTs are similar, they exhibited differential expression patterns in various plant organs indicating differential transport of these molecules. By fusing transporter genes with GUS, they demonstrated that AtProT1, AtProT2, and AtProT3 are localized in lignified tissues like phloem of all organs, epidermis, and cortex of roots and in epidermis of leaves respectively, (Grallath et al., 2005). ProT1 isolated from tomato has shown to be expressed specifically in pollen grains (Schwacke et al., 1999). Further, Lehmann et al. (2010) clearly showed a relation between uneven distribution of proline in the lower epidermis and rest of the leaf with the expression levels of AtProT3. In spite of the fact, that single, double, and triple knockout mutants of the AtProT1, AtProT2, and AtProT3 genes responsible for proline transport in plants have been isolated and characterized, these mutants did not reveal any differences when compared to wild type plants (Lehmann et al., 2011). This underlays the complex situation and therefore needs further elucidation. The functions of these transporters might be highly redundant and possibly complemented by other transporters, since transporters mediating proline uptake across the plasma membrane have also been noticed in the amino acid transporter (ATF) or amino acid/auxin permease (AAAP) and in the amino acidpolyamine-choline (APC) families (Rentsch et al., 2007). Along with glutamate, amino acid permease (AAP) family mediates proton coupled uptake of neutral amino acids such as proline (Frommer et al., 1993; Lee and Pallas, 2007; Schmidt et al., 2007). Couturier et al. (2010) isolated AAP11 from Populus trichocarpa and overexpressed it in poplar and yeast. PtAAP11 was highly expressed in differentiating xylem cells in different organs. Further, functional characterization revealed that it is a high affinity amino ATF, more particularly for proline. Therefore, the authors suggested that PtAAP11 may play an important role in xylogenesis by providing proline which is required for xylem cell wall proteins in poplar. Lysine-histidine transporter (LHT) family transports neutral and acidic amino acids including proline (Lee and Tegeder, 2004). Contrary to the AAP and LHT families, ProTs transport proline and other compatible solutes like GABA but not other proteogenic amino acids (Rentsch et al., 1996). The fact that both low and high affinity-proline transporters exist in plants implicate that they play a role in general transfer of nitrogen and also proline for its specific functions in different cells and tissues. Selective transport of proline by ProTs and also their expression analysis during drought/salt stresses or in pollen grains suggests that they play a vital role in proline homeostasis both under stress and nonstress conditions (Rentsch et al., 1996; Ueda et al., 2001; Waditee et al., 2002; Lehmann et al., 2010). The list of proline/betaine transporters and transport mutants known from different plants is shown in Table 2. In summary, proline transporters (AAPs) play an important role in plant development by providing proline as a source of nitrogen and energy which is required for lignifications, xylem differentiation and cell wall modification during plant development (as a component of plant cell wall proteins). But the exact mechanisms how proline can mediate these functions are not known.

\section{Mutations in p5cs2 and p5cr Cause Embryo Lethality and Affect Seed Development}

The amount of free proline and its metabolism modulates transition to flowering, pollen, and embryo development. Several studies found that immature/mature seeds of Vicia faba and Arabidopsis accumulate proline before ripening (Venekamp and Koot, 1984; Chiang and Dandekar, 1995; Schmidt et al., 2007). In support of it, concomitant expression of genes encoding enzymes of proline metabolism was observed in seeds (Armengaud et al., 2004; Deuschle et al., 2004; Hur et al., 2004; Szekely et al., 2008). It was noticed that P5CS2 is expressed mostly in actively dividing meristematic cells, developing tissues, callus, and cell suspension cultures. p5cs 2 mutations cause embryo abortion during late stages of seed development though both P5CS mRNAs are 
TABLE 2 | List of proline/betaine transporters and transport mutants known from different plants.

\begin{tabular}{|c|c|c|c|c|}
\hline Name of plant & $\begin{array}{l}\text { Type of } \\
\text { transporter/mutant }\end{array}$ & Affinity for proline & Tissue specific expression/function & Reference \\
\hline Amaranthus tricolor & AmtBet/ProT1 & $\begin{array}{l}\text { Low compared to } \\
\text { betaine }\end{array}$ & Not known & Yamada et al. (2011) \\
\hline Arabidopsis thaliana & AtProT1 & High & Phloem parenchyma in all organs, pollen grains & $\begin{array}{l}\text { Rentsch et al. (1996), Grallath et al. } \\
\text { (2005) }\end{array}$ \\
\hline A.thaliana & AtProT2 & High & $\begin{array}{l}\text { Root cortex, epidermis, seedling after salt and } \\
\text { drought stress }\end{array}$ & $\begin{array}{l}\text { Rentsch et al. (1996), Grallath et al. } \\
\text { (2005) }\end{array}$ \\
\hline A.thaliana & AtProT3 & High & Leaf epidermis, sepal & $\begin{array}{l}\text { Rentsch et al. (1996), Grallath et al. } \\
\text { (2005) }\end{array}$ \\
\hline Avicennia marina & AmBet/AmProT1 & High & Leaf and root after salt stress & Waditee et al. (2002) \\
\hline A. marina & AmBet/AmProT2 & High & Leaf and root after salt stress & Waditee et al. (2002) \\
\hline A. marina & AmProT3 & Not known & & Yamada et al. (2011) \\
\hline A. thaliana & raz1 mutant & - & Affected in the high affinity uptake of proline & Verbruggen et al. (1993) \\
\hline Atriplex gmelinii & AgBet/ProT1 & $\begin{array}{l}\text { Low compared to } \\
\text { betaine }\end{array}$ & Not known & Yamada et al. (2011) \\
\hline A. hortensis & AhProT1 & High & Roots & Shen et al. (2002) \\
\hline Beta vulgaris & BvBet/ProT1 & High & Xylem and phloem parenchyma cells, petiole, leaf & Yamada et al. (2009, 2011) \\
\hline Chlorella vulgaris & pup mutant & - & $\begin{array}{l}\text { Impaired in alanine, glycine, proline, and serine } \\
\text { transport }\end{array}$ & Sauer and Tanner (1985) \\
\hline Elaeis guineensis & EgProT1 & High & Not known (may be uptake into roots) & Yamada et al. (2011) \\
\hline Hordeum vulgare & HvProT1 & Not known & Root cap, cortex, stele, phloem after salt stress & $\begin{array}{l}\text { Ueda et al. (2001, 2008), Fujiwara } \\
\text { et al. (2010) }\end{array}$ \\
\hline H. vulgare & HvProT2 & High & $\begin{array}{l}\text { Leaves, root, plasma membrane, mestome sheaths, } \\
\text { lateral root caps }\end{array}$ & $\begin{array}{l}\text { Ueda et al. (2001), Fujiwara et al. } \\
\text { (2010) }\end{array}$ \\
\hline Lycopersicon esculentum & LeProT1 & High & Flowers, mature and germinating pollen & Schwacke et al. (1999) \\
\hline L. esculentum & LeProT2 & Not known & Could not be detected with RNA gel blot & Schwacke et al. (1999) \\
\hline L. esculentum & LeProT3 & Not known & Could not be detected using RNA gel blot & Schwacke et al. (1999) \\
\hline Oryza sativa & OsProT1 & Not known & All organs & Igarashi et al. (2000) \\
\hline A. thaliana & AtAAP1 & $\begin{array}{l}\text { Low in yeast High } \\
\text { in Xenopus }\end{array}$ & $\begin{array}{l}\text { Expression in the seed, root epidermis, root tip, root } \\
\text { hairs }\end{array}$ & Frommer et al. (1993) \\
\hline A. thaliana & AtAAP2 & High & Vascular bundles in leaf, root, silique, flower & $\begin{array}{l}\text { Kwart et al. (1993), Fischer et al. } \\
\text { (1998), Hirner et al. (1998) }\end{array}$ \\
\hline A. thaliana & AtAAP3 & High & Root, tip of filament and seedlings (cotyledons) & $\begin{array}{l}\text { Fischer et al. (1995), Okumoto et al. } \\
\text { (2004) }\end{array}$ \\
\hline A. thaliana & AtAAP4 & High & Stem, source leaf, sink leaf, flower & Fischer et al. (1998) \\
\hline A. thaliana & AtAAP5 & High & Source leaf, stem, flower, fruit, root & Fischer et al. (1998) \\
\hline A. thaliana & AtAAP6 & Low & Xylem parenchyma in aerial parts, sink leaf, root & $\begin{array}{l}\text { Rentsch et al. (1996), Okumoto et al. } \\
\text { (2002) }\end{array}$ \\
\hline O. sativa & OsAAPs & Not known & Not known & Tegeder and Ward (2012) \\
\hline Solanum tuberosum & StAAP1 & Not known & Source leaf & Fischer et al. (1998) \\
\hline S. tuberosum & StAAP2 & Not known & Stem & Fischer et al. (1998) \\
\hline Ricinus communis & RCAAP1 & Not known & Sink leaf, source leaf, root and seedling & Fischer et al. (1998) \\
\hline R. communis & RCAAP2 & Not known & Sink leaf, source leaf, root, and seedling & Fischer et al. (1998) \\
\hline A. thaliana & AtLHT1 & Low & $\begin{array}{l}\text { Leaf epidermis and mesophyll, root tip, stem, petals, } \\
\text { sepals }\end{array}$ & $\begin{array}{l}\text { Kwart et al. (1993), Chen and Bush } \\
\text { (1997) }\end{array}$ \\
\hline A. thaliana & AtLHT2 & Low & Tapetum, pollen & $\begin{array}{l}\text { Lee and Tegeder (2004), Foster et al. } \\
\text { (2008) }\end{array}$ \\
\hline O. sativa & OsLHTS & Not known & Not known & Tegeder and Ward (2012) \\
\hline A. thaliana & AtCAT1 & High & Flower, veins of root, leaf, silique, stem & Frommer et al. (1995) \\
\hline A. thaliana & $\begin{array}{l}\text { BASIC AMINO } \\
\text { ACID CARRIER2 }\end{array}$ & Not known & $\begin{array}{l}\text { Mitochondria Mutations in } B A A C \text { contribute to proline } \\
\text { accumulation in response to hyperosmotic stress }\end{array}$ & Toka et al. (2010) \\
\hline
\end{tabular}

detectable throughout embryonic development under warmer climate (Szekely et al., 2008). Their experiments indicated exogenous proline can rescue $p 5 c s 2$ mutants by ex vivo cultivation of developing seeds, but the mutant plants undergo aberrant development and become sterile. It has also been reported that homozygous $p 5 c s 2$ mutant plants died before the onset of flowering. Therefore, specific role of P5CS2 in reproductive development could not be analyzed (Szekely et al., 2008; 
Mattioli et al., 2009b). When Arabidopsis plants were simultaneously silenced or co-suppressed with P5CS1 and P5CS2 genes, they resulted in retarded growth, delayed flowering and reduced apical dominance (Nanjo et al., 1999; Mattioli et al., 2008). The above results indicate that Arabidopsis P5CS1 is insufficient for compensation of developmental defects caused by knockout of P5CS2. Expression of P5CS2-GFP was observed in leaf primordia, where the levels of P5CS1-GFP levels are very low. P5CS2-GFP also displayed cell-type specific subcellular localization pattern compared to P5CS1-GFP in root tips, leaves, flower organs, and embryonic cells (Szekely et al., 2008). Taken together, the data demonstrate that these two genes have non-redundant functions in plants. In A. thaliana, Funck et al. (2012) did not observe developmental defects in single mutants devoid of P5CS1 gene. $p 5 c s 1 / p 5 c s 2$ double mutants displayed pollen sterility, while egg cells are fertile. Thus, these results emphasize the role(s) of P5CS in pollen fertility. $p 5 c s 2$ T-DNA insertion lines have been described as embryo lethal or conditionally embryo lethal. It has been found that P5CS2 is not essential for sexual reproduction (Funck et al., 2012). They obtained homozygous $p 5 c s 2$ mutants that are viable and could produce fertile seeds by in vitro culture of immature mutant seeds on MS medium supplemented with $60 \mathrm{mM}$ sucrose and $2 \mathrm{mM}$ proline. This allowed generating homozygous plants that are phenotypically normal, but showed reduced growth compared to the wild type plants. Under short day and low-light conditions, these plants produced viable seeds. Funck et al. (2012) pointed out that embryo lethality in heterozygous atp $5 c s 2$ plants might be due to premature desiccation of homozygous embryos that develop more slowly than heterozygous embryos. In a contrast to $p 5 c s 1 / p 5 c s 2$ double mutants, normal pollen fertility was observed in the absence of a functional P5CR gene indicating that P5CR is not needed for pollen fertility. These results implicate that P5CS1/P5CS2 genes are crucial for pollen fertility, but not P5CR. It has been found that $p 5 \mathrm{cr}$ mutations cause embryonic lethality, however. Disruption of P5CR gene using T-DNA insertions resulted in embryo defective mutation emb2722 (Meinke et al., 2008) in Arabidopsis. They reported arrest of the embryo development after the second division of the embryo proper in $p 5 \mathrm{cr}$ mutants. All attempts to rescue putative homozygous $p 5 \mathrm{cr}$ mutant embryos in vitro by proline feeding failed. Their efforts to promote embryo development of homozygous seeds in siliques of

\section{References}

Alm, J., Ohnmeiss, T. E., Lanza, J., and Vriesenga, L. (1990). Preference of cabbage white butterflies and honey bees for nectar that contains amino acids. Oecologia 84, 53-57. doi: 10.1007/BF006 65594

Armengaud, P., Thiery, L., Buhot, N., March, G., and Savoure, A. (2004). Transcriptional regulation of proline biosynthesis in Medicago truncatula reveals developmental and environmental specific features. Physiol. Plant. 120, 442-450. doi: 10.1111/j.0031-9317.2004. 00251.x

Bai, L., Zhang, G., Zhou, Y., Zhang, Z., Wang, W., Du, Y., et al. (2009). Plasma membrane-associated proline-rich extensin-like receptor kinase 4, a novel heterozygous parents in situ by feeding the proline exogenously or induction of internal proline accumulation through salt stress were not fruitful. Thus, activities of both P5CS and P5CR enzymes have been found essential for sexual reproduction in plants.

\section{Conclusion}

Proline not only participates in protein synthesis, but regulates several important functions like osmotic adjustment and protection of proteins during stress conditions. Its multifarious functions are always enigmatic in plants. However, it is increasingly becoming evident that proline modulates a widearray of functions like regulation of cyclin genes at the transcriptional level, cell wall elongation and modifications, xylogenesis, stem elongation, root, and shoot growth, inflorescence architecture, embryo formation/seed development, and seed germination during the life cycle of a plant. Deficiency in proline biosynthesis leads to abnormal plants and cell wall defects, thus implicating its role in structural proteins. But, clear evidence is lacking for attributing several specific functions to this amino acid. We do not know yet how the genes like P5CS1, P5CS2, P5CR, OAT, PDH, and P5CDH interact with other genes and we still do not have an interactome map of the network of genes. Unless such maps are available along with their experimental validation, we cannot pinpoint the exact metabolic functions of proline other than its role in primary metabolism in protein biosynthesis.

\section{Author Contributions}

All the authors of the manuscript meet the essential criteria of the publication. All authors have read and approved the manuscript.

\section{Acknowledgments}

PK is thankful to the CSIR, New Delhi, for awarding the Emeritus Fellowship (No.21(0934)12/EMR-II). PH is grateful to the CSIR, New Delhi, for providing fellowship in the form of Senior Research Fellowship.

regulator of Ca signaling, is required for abscisic acid responses in Arabidopsis thaliana. Plant J. 60, 314-327. doi: 10.1111/j.1365-313X.2009.03956.x

Basile, D. V., and Basile, M. R. (1993). The role and control of the place-dependent suppression of cell division in plant morphogenesis and phylogeny. Mem. Torrey Botanical. Club 25, 63-68.

Battaglia, M., Solorzano, R. M., Hernandez, M., Ortiz, S. C., Gomez, B. G., Marquez, J., et al. (2007). Proline-rich cell wall proteins accumulate in growing regions and phloem tissue in response to water deficit in common bean seedlings. Planta 225, 1121-1133. doi: 10.1007/s00425-006-0423-9

Bernhardt, C., and Tierney, M. L. (2000). Expression of AtPRP3, a proline-rich structural cell wall protein from Arabidopsis, is regulated by cell-type-specific developmental pathways involved in root hair formation. Plant Physiol. 122, 705-714. doi: 10.1104/pp.122.3.705 
Besse, I., and Buchanan, B. B. (1997). Thioredoxin-linked plant and animal processes: the new generation. Bot. Bull. Acad. Sin. 38, 1-11. doi: 10.1046/j.1469-8137.1997.00784.x

Bettini, P., Michelotti, S., Bindi, D., Giannini, R., Capuana, M., and Buiatti, M. (2003). Pleiotropic effect of the insertion of the Agrobacterium rhizogenes rolD gene in tomato (Lycopersicon esculentum Mill). Theore. Appl. Genet. 107, 831-836. doi: 10.1007/s00122-003-1322-0

Bo, Z., Xiao-Ping, Z., and Zhang-Cheng, Z. (2000). Studies on pollen free proline content and seed yield of Gordonia acuminata in different communities. Acta Phytoecol. Sin. 24, 617-620.

Bosch, M., Sommer-Knudsen, J., Derksen, J., and Mariani, C. (2001). Class III pistil-specific extensin-like proteins from tobacco have characteristics of arabinogalactan proteins. Am. Soc. Plant Phys. 125, 2180-2188. doi: 10.1104/pp.125.4.2180

Botha, F. C., Potgieter, G. P., and Botha, A. M. (1992). Respiratory metabolism and gene expression during seed germination. Plant Growth Regul. 11, 211-224. doi: 10.1007/BF00024560

Bradley, D. J., Kjellbom, P., and Lamb, C. J. (1992). Elicitor-induced and wound induced oxidative cross-linking of a proline rich plant cell wall protein: a novel, rapid defense response. Cell 70, 21-30. doi: 10.1016/0092-8674(92) 90530-P

Buttery, R. G., Ling, L. C., and Juliano, B. O. (1982). 2-Acetyl-1-pyrroline: an important aroma component of cooked rice. Chem. Industry (London) 12, 958-959.

Cannon, M. C., Terneus, K., Hall, Q., Tan, L., Wang, Y., Wegenhart, B. L., et al. (2008). Self-assembly of the plant cell wall requires an extensin scaffold. Proc. Natl. Acad. Sci. U.S.A. 105, 2226-2231. doi: 10.1073/pnas.0711980105

Carter, C., Shafir, S., Yehonatan, L., Palmer, R. G., and Thornburg, R. (2006). A novel role for proline in plant floral nectars. Naturwissenschaften 93, 72-79. doi: 10.1007/s00114-005-0062-1

Cassab, G. I. (1998). Plant cell wall proteins. Annu. Rev. Plant Physiol. Plant Mol. Biol. 49, 281-309. doi: 10.1146/annurev.arplant.49.1.281

Cassab, G. I., and Varner, J. E. (1988). Cell wall proteins. Ann. Rev. Plant Physiol. Plant Mol. Biol. 39, 321-353. doi: 10.1146/annurev.pp.39.060188.001541

Castonguay, Y., Laberge, S., Nadeau, P., and Vezina, L. P. (1994). A cold induced gene from Medicago sativa encodes a bimodular protein similar to developmentally regulated protein. Plant Mol. Biol. 24, 799-804. doi: 10.1007/BF00029861

Chen, C. G., Pu, Z. Y., Moritz, R. L., Simpson, R. J., Bacic, A., Clarke, A. E., et al. (1994). Molecular cloning of a gene encoding an arabinogalactan-protein from pear (Pyrus communis) cell suspension culture. Proc. Natl. Acad. Sci. U.S.A. 91, 10305-10309. doi: 10.1073/pnas.91.22.10305

Chen, L., and Bush, D. R. (1997). LHT1, a lysine- and histidinespecific amino acid transporter in Arabidopsis. Plant Physiol. 115, 1127-1134. doi: 10.1104/pp.115.3.1127

Chen, S., Yang, Y., Shi, W., Ji, Q., He, F., Zhang, Z., et al. (2008). Badh2, encoding betaine aldehyde aldehyde dehydrogenase, inhibits the biosynthesis of 2-acetyl1-pyrroline, a major component in rice fragrance. Plant Cell 20, 1850-1861. doi: 10.1105/tpc.108.058917

Cheung, A. Y., May, B., Kawata, E. E., Gu, Q., and Wu, H.-M. (1993). Characterization of cDNAs for stylar transmitting tissue-specific proline-rich proteins in tobacco. Plant J. 3, 151-160. doi: 10.1046/j.1365-313X.1993.t01-700999.x

Chiang, H. H., and Dandekar, A. M. (1995). Regulation of proline accumulation in Arabidopsis thaliana (L.) Heynh during development and in response to desiccation. Plant Cell Environ. 18, 1280-1290. doi: 10.1111/j.13653040.1995.tb00187.x

Clarke, A., Gleeson, P., Harrison, S., and Knox, R. B. (1979). Pollenstigma interactions: identification and characterization of surface components with recognition potential (cell surface receptors/plant glycoproteins/adhesion/concanavalin A/tridacnin). Proc. Natl. Acad. Sci. U.S.A. 76, 3358-3362. doi: 10.1073/pnas.76.7.3358

Clements, R. L., and Leland, H. V. (1962). An ion-exchange study of the free amino acids in the juices of six varieties of citrus. J. Food Sci. 27, 20-25. doi: 10.1111/j.1365-2621.1962.tb00051.x

Coleman, C. E., Lopes, M. A., Gillikin, J. W., Boston, R. S., and Larkins, B. A. (1995). A defective signal peptide in the maize high lysine mutant floury 2. Proc. Natl. Acad. Sci. U.S.A. 92, 6828-6831. doi: 10.1073/pnas.92.15.6828
Colmenero-Flores, J. M., Campos, F., Garciarrubio, A., and Covarrubias, A. A. (1997). Characterization of Phaseolus vulgaris cDNA clones responsive to water deficit: identification of a novel late embryogenesis abundant-like protein. Plant Mol. Biol. 35, 393-405. doi: 10.1023/A:1005802505731

Cooper, J. B., Heuser, J. E., and Varner, J. E. (1994). 3,4-dehydroproline inhibits cell wall assembly and cell division in tobacco protoplasts. Plant Physiol. 104, 747-752. doi: 10.1104/pp.104.2.747

Couturier, J., Fay, E. D., Fitz, M., Wipf, D., Blaudez, D., and Chalot, M. (2010). PtAAP11, a high affinity amino acid transporter specifically expressed in differentiating xylem cells of poplar. J. Exp. Bot. 61, 1671-1682. doi: $10.1093 /$ jxb/erq036

Covarrubias, A. A., Ayala, J. W., Reyes, J. L., Hernandez, M., and Garciarrubio, A. (1995). Cell-wall proteins induced by water deficit in bean (Phaseolus vulgaris L.) seedlings. Plant Physiol. 107, 1119-1128. doi: 10.1104/pp.107.4.1119

Creelman, R. A., and Mullet, J. E. (1991). Water deficit modulates gene expression in growing zones of soybean seedlings. Analysis of differentially expressed cDNAs, a new $\beta$-tubulin gene, and expression of genes encoding cell wall proteins. Plant Mol. Biol. 17, 591-608. doi: 10.1007/BF00037046

Delauney, A. J., Hu, C. A. A., Kishor, P. B. K., and Verma, D. P. S. (1993). Cloning of ornithine $\delta$-aminotransferase cDNA from Vigna aconitifolia by transcomplementation in Escherichia coli and regulation of proline biosynthesis. J. Biol. Chem. 268, 18673-18678.

Delauney, A. J., and Verma, D. P. S. (1990). A soybean $\Delta 1$-pyrroline-5-carboxylate reductase gene was isolated by functional complementation in Escherichia coli and is found to be osmoregulated. Mol. Gen. Genet. 221, 299-305. doi: 10.1007/BF00259392

Delauney, A. J., and Verma, D. P. S. (1993). Proline biosynthesis and osmoregulation in plants. Plant J. 4, 215-223. doi: 10.1046/j.1365313X.1993.04020215.X

Deuschle, K., Funck, D., Forlani, G., Stransky, H., Biehl, A., Leister, D., et al. (2004). The role of $\Delta 1$-pyrroline-5-carboxylate dehydrogenase in proline degradation. Plant Cell 16, 3413-3425. doi: 10.1105/tpc.104.023622

Deuschle, K., Funck, D., Hellman, H., Daschner, K., Binder, S., and Frommer, W. B. (2001). A nuclear gene encoding mitochondrial $\Delta 1$-pyrroline-5-carboxylate dehydrrogenase and its potential role in protection from proline toxicity. Plant J. 27, 345-356. doi: 10.1046/j.1365-313X.2001.01101.x

Deutch, C. E., and Winicov, I. (1995). Post-transcriptional regulation of a salt-inducible alfalfa gene encoding a putative chimeric proline-rich cell wall protein. Plant Mol. Biol. 27, 411-418. doi: 10.1007/BF000 20194

Dick-Perez, M., Zhang, Y., Hayes, J., Salazar, A., Zabotina, O. A., and Hong, M. (2011). Structure and interactions of plant cell-wall polysaccharides by two and three-dimensional magic-angle-spinning solid-state NMR. Biochemistry 50, 989-1000. doi: 10.1021/bi101795q

Diet, A., Link, B., Seifert, G. J., Schellenberg, B., Wagner, U., Pauly, M., et al. (2006). The Arabidopsis root hair cell wall formation mutant $\operatorname{lrx} 1$ is suppressed by mutations in the RHM1 gene encoding a UDP-L-rhamnose synthase. Plant Cell 18, 1630-1641. doi: 10.1105/tpc.105.038653

Ding, L., and Zhu, J.-K. (1997). A role for arabinogalactan-proteins in root epidermal cell expansion. Planta 203, 289-294. doi: 10.1007/s004250050194

Doblin, M. S., Pettolino, F., and Bacic, A. (2010). Plant cell walls: the skeleton of the plant world. Funct. Plant Biol. 37, 357-381. doi: 10.1071/FP09279

Dvorakova, L., Cvrckova, F., and Fischer, L. (2007). Analysis of the hybrid prolinerich protein families from seven plant species suggests rapid diversification of their sequences and expression patterns. BMC Genomics 8:412. doi: 10.1186/1471-2164-8-412

Dvorakova, L., Srba, M., Opatrny, Z., and Fischer, L. (2012). Hybrid proline-rich proteins: novel players in plant cell elongation? Ann. Bot. 109, 453-462. doi: $10.1093 / \mathrm{aob} / \mathrm{mcr} 278$

Egertsdotter, U., and von Arnold, S. (1995). Importance of arabinogalactan proteins for the development of somatic embryos of Norway spruce (Picea abies). Physiol. Plant. 93, 334-345. doi: 10.1111/j.1399-3054.1995.tb02237.x

Ellis, M., Egelund, J., Schultz, C. J., and Bacic, A. (2010). Arabinogalactanproteins: key regulators at the cell surface? Plant Physiol. 153, 403-419. doi: 10.1104/pp.110.156000

Fincher, G. B., Stone, B. A., and Clarke, A. E. (1983). Arabinogalactan-proteins: structure, biosynthesis, and function. Ann. Rev. Plant Physiol. 34, 47-70. doi: 10.1146/annurev.pp.34.060183.000403 
Fischer, W. N., Andre, B., Rentsch, D., Krolkiewicz, S., Tegedar, M., Breitkreuz, K., et al. (1998). Amino acid transport in plants. Trends Plant Sci. 3, 188-195. doi: 10.1016/S1360-1385(98)01231-X

Fischer, W. N., Kwart, M., Hummel, S., and Frommer, W. B. (1995). Substrate specificity and expression profile of amino acid transporters (AAPs) in Arabidopsis. J. Biol. Chem. 270, 16315-16320. doi: 10.1074/jbc.270.27.16315

Flasinski, S., and Rogozinska, J. (1985). Effect of water deficit on proline accumulation, protein and chlorophyll content during flowering and seed formation in winter rape (Brassica napus L. var. oleifera). Acta Agrobot. 38, 11-21.

Foster, J., Lee, Y. H., and Tegeder, M. (2008). Distinct expression of members of the LHT amino acid transporter family in flowers indicates specific roles in plant reproduction. Sexual Plant Rep. 21, 143-152. doi: 10.1007/s00497-0080074-z

Fowler, T. J., Bernhardt, C., and Tierney, M. L. (1999). Characterization and expression of four proline-rich cell wall protein genes in arabidopsis encoding two distinct subsets of multiple domain proteins. Plant Physiol. 121, 1081-1091. doi: 10.1104/pp.121.4.1081

Frommer, W. B., Hummel, S., and Riesmeier, J. W. (1993). Expression cloning in yeast of a cDNA encoding a broad specificity amino acid permease from Arabidopsis thaliana. Proc. Natl. Acad. Sci. U.S.A. 90, 5944-5948. doi: 10.1073/pnas.90.13.5944

Frommer, W. B., Hummel, S., Unseld, M., and Ninnemann, O. (1995). Seed and vascular expression of a high-affinity transporter for cationic amino acids in Arabidopsis. Proc. Natl. Acad. Sci. U.S.A. 92, 12036-12040. doi: 10.1073/pnas.92.26.12036

Frueauf, J. B., Dolata, M., Leykam, J. F., Lloyd, E., Gonzales, M., Vandenbosch, K., et al. (2000). Peptides isolated from cell walls of Medicago truncatula nodules and uninfected root. Phytochem. 55, 429-438. doi: 10.1016/S00319422(00)00336-8

Fujita, T., Maggio, A., Garcia-Rios, M., Bressan, R. A., and Csonka, L. N. (1998). Comparative analysis of the regulation of expression and structures of two evolutionarily divergent genes for $\Delta 1$-pyrroline-5-carboxylate synthetase. Plant Physiol. 118, 661-674. doi: 10.1104/pp.118.2.661

Fujiwara, T., Mitsuya, S., Miyake, H., Hattori, T., and Takabe, T. (2010). Characterization of novel glycinebetaine/proline transporter gene expressed in the mestome sheath and lateral root cap cells in barley. Planta 232, 133-143. doi: 10.1007/s00425-010-1155-4

Funck, D., Eckard, S., and Muller, G. (2010). Non-redundant functions of two proline dehydrogenase isoforms in Arabidopsis. BMC Plant Biol. 10:70. doi: 10.1186/1471-2229-10-70

Funck, D., Stadelhofer, B., and Koch, W. (2008). Ornithine- $\delta$-aminotransferase is essential for arginine catabolism but not for proline biosynthesis. BMC Plant Biol. 8:40 doi: 10.1186/1471-2229-8-40

Funck, D., Winter, G., Baumgarten, L., and Forlani, G. (2012). Requirement of proline synthesis during Arabidopsis reproductive development. BMC Plant Biol. 12:191. doi: 10.1186/1471-2229-12-191

Gao, M., and Showalter, A. M. (2000). Immunolocalization of LeAGP-1, a modular arabinogalactan-protein, reveals its developmentally regulated expression in tomato. Planta 210, 865-874. doi: 10.1007/s004250050691

Gavazzi, G., Nava-Racchi, M., and Tonelli, C. (1975). A mutation causing proline requirement in Zea mays. Theor. Appl. Genet. 46, 339-345. doi: 10.1007/BF00281675

Ghanti, K. K. S., Sujata, K. G., Kumar, B. M. V., Karba, N. N., Reddy, K. J., Rao, M. S., et al. (2011). Heterologous expression of P5CS gene in chickpea enhances salt tolerance without affecting yield. Biol. Plant. 55, 634-640. doi: 10.1007/s10535-011-0161-0

Gibbon, B. C., Zonia, L. E., Kovar, D. R., Hussey, P. J., and Staiger, C. J. (1998). Pollen profiling function depends on interaction with proline-rich motifs. Plant Cell 10, 981-993. doi: 10.1105/tpc.10.6.981

Gothandam, K. M., Nalini, E., Karthikeyan, S., and Shin, J. S. (2010). OsPRP3, a flower specific proline-rich protein of rice, determines extracellular matrix structure of floral organs and its overexpression confers cold-tolerance. Plant Mol. Biol. 72, 125-135. doi: 10.1007/s11103-009-9557-z

Grallath, S., Weimar, T., Meyer, A., Gumy, C., Suter-Grotemeyer, M., Neuhaus, J., et al. (2005). The AtProT family. Compatible solute transporters with similar substrate specificity but differential expression patterns. Plant Physiol. 137, 117-126. doi: 10.1104/pp.104.055079
Griffiths, J. S., Tsai, A. Y., Xue, H., Voiniciuc, C., Šola, K., Seifert, G. J., et al. (2014). SALT-OVERLY SENSITIVE5 mediates Arabidopsis seed coat mucilage adherence and organization through pectins. Plant Physiol. 165, 991-1004. doi: 10.1104/pp.114.239400

Haffani, Y. Z., Silva-Gagliardi, N. F., Sewter, S. K., Grace Aldea, M., Zhao, Z., Nakhamchik, A., et al. (2006). Altered expression of PERK receptor kinases in Arabidopsis leads to changes in growth and floral organ formation. Plant Signal. Behav. 1, 251-260. doi: 10.4161/psb.1.5.3324

Hall, Q., and Cannon, M. (2002). The cell wall hydroxyproline-rich glycoprotein RSH is essential for normal embryo development in Arabidopsis. Plant Cell 14, 1161-1172. doi: 10.1105/tpc.010477

Hancock, C. N., Kent, L., and McClure, B. A. (2005). The stylar $120 \mathrm{kDa}$ glycoprotein is required for S-specific pollen rejection in Nicotiana. Plant J. 43, 716-723. doi: 10.1111/j.1365-313X.2005.02490.x

Hare, P. D., and Cress, W. (1997). Metabolic implications of stress induced proline accumulation in plants. Plant Growth Reg. 21, 79-102. doi: 10.1023/A:1005703923347

Hare, P. D., Cress, W. A., and Van Staden, J. (1999). Proline synthesis and degradation: a model system for elucidating stress-related signal transduction. J. Exp. Bot. 50, 413-434. doi: 10.1093/jxb/50.333.413

Hare, P. D., Cress, W. A., and Van Staden, J. (2003). A regulatory role for proline metabolism in stimulating Arabidopsis thaliana seed germination. Plant Growth Reg. 39, 41-50. doi: 10.1023/A:1021835902351

Hayashi, F., Ichino, T., Osanai, M., and Wada, K. (2000). Oscillation and regulation of proline content by $\mathrm{P} 5 \mathrm{CS}$ and $\mathrm{ProDH}$ gene expressions in the light/dark cycles in Arabidopsis thaliana. Plant Cell Physiol. 41, 1096-1101. doi: $10.1093 / \mathrm{pcp} / \mathrm{pcd} 036$

Held, M. A., Tan, L., Kamyab, A., Hare, M., Shpak, E., and Kieliszewksi, M. J. (2004). Di-isodityrosine is the intermolecular cross-link of isodityrosine-rich extensin analogs cross-linked in vitro. J. Biol. Chem. 279, 55474-55482. doi: 10.1074/jbc.M408396200

Henrissat, B., Coutinho, P. M., and Davies, G. J. (2001). A census of carbohydrateactive enzymes in the genome of Arabidopsis thaliana. Plant Mol. Biol. 47, 55-72. doi: 10.1023/A:1010667012056

Hibino, T., Meng, Y. L., Kawamitsu, Y., Uehara, N., Matsuda, N., Tanaka, Y., et al. (2001). Molecular cloning and functional characterization of two kinds of betaine-aldehyde dehydrogenase in betaine-accumulating mangrove Avicennia marina (Forsk.) Vierh. Plant Mol. Biol. 45, 353-363. doi: 10.1023/A:1006497113323

Hijazi, M., Roujol, D., Nguyen-Kim, H., Del Rocio Cisneros Castillo, L., Saland, E., Jamet, E., et al. (2014). Arabinogalactan protein 31 (AGP31), a putative network-forming protein in Arabidopsis thaliana cell walls? Ann. Bot. 114, 1087-1097. doi: 10.1093/aob/mcu038

Hirner, B., Fischer, W. N., Rentsch, D., Kwart, M., and Fommer, W. B. (1998). Developmental control of $\mathrm{H}+$ /amino acid permease gene expression during seed development of Arabidopsis. Plant J. 14, 535-544. doi: 10.1046/j.1365313X.1998.00151.x

Holding, D. R., Meeley, R. B., Hazebroek, J., Selinger, D., Gruis, F., Jung, R., et al. (2010). Identification and characterization of the maize arogenate dehydrogenase gene family. J. Exp. Bot. 61, 3663-3673. doi: 10.1093/jxb/erq179

Hong, J. C., Nagao, R. T., and Key, J. L. (1987). Characterization and sequence analysis of a developmentally regulated putative cell wall protein gene isolated from soybean. J. Biol. Chem. 262, 8367-8376.

Hong, J. C., Nagao, R. T., and Key, J. L. (1989). Developmentally regulated expression of soybean proline-rich cell wall protein genes. Plant Cell 1, 937-943. doi: $10.1105 /$ tpc.1.9.937

Hong, J. C., Nagao, R. T., and Key, J. L. (1990). Characterization of a proline-rich cell wall protein gene family of soybean. A comparative analysis. J. Biol. Chem. 265, 2470-2475.

Hu, C. A. A., Delauney, A. J., and Verma, D. P. S. (1992). A bifunctional enzyme ( $\Delta 1$-pyrroline-5-carboxylate synthetase) catalyzes the first two steps in proline biosynthesis in plants. Proc. Natl. Acad. Sci. U.S.A. 89, 9354-9358. doi: 10.1073/pnas.89.19.9354

Hur, J., Jung, K. H., Lee, C. H., and An, G. (2004). Stress-inducible OsP5CS2 gene is essential for salt and cold tolerance in rice. Plant Sci. 167, 417-426. doi: 10.1016/j.plantsci.2004.04.009

Igarashi, Y., Yoshiba, Y., Takeshita, T., Nomura, S., Otomo, J., YamaguchiShinozaki, K., et al. (2000). Molecular cloning and characterization of a cDNA 
encoding proline transporter in rice. Plant Cell Physiol. 41, 750-756. doi: $10.1093 / \mathrm{pcp} / 41.6 .750$

Ito, M., Kodama, H., Komamine, A., and Watanabe, A. (1998). Expression of extensin genes is dependent on the stage of the cell cycle and cell proliferation in suspension-cultured Catharanthus roseus cells. Plant Mol. Biol. 36, 343-351. doi: 10.1023/A:1005913818129

Jackson, P. A., Galinha, C. I., Pereira, C. S., Fortunato, A., Soares, N. C., Amancio, S. B., et al. (2001). Rapid deposition of extensin during the elicitation of grapevine callus cultures is specifically catalyzed by a 40-kilodalton peroxidase. Plant Physiol. 127, 1065-1076. doi: 10.1104/pp.010192

Jose-Estanyol, M., and Puigdomenech, P. (2000). Plant cell wall glycoproteins and their genes. Plant Physiol. Biochem. 38, 97-108. doi: 10.1016/S09819428(00)00165-0

Jose-Estanyol, M., Ruiz-Avila, L., and Puigdomenench, P. (1992). A maize embryospecific gene encodes a proline-rich and hydrophobic protein. Plant Cell 4, 413-423. doi: 10.1105/tpc.4.4.413

Kaplan, D. R., and Hagemann, W. (1991). The relationship of cell and organism in vascular plants. Bioscience 41, 693-703. doi: 10.2307/1311764

Kieliszewski, M. J., and Lamport, D. T. A. (1994). Extensin: repetitive motifs, functional sites, post-translation codes and phylogeny. Plant J. 5, 157-172. doi: 10.1046/j.1365-313X.1994.05020157.x

Kieliszewski, M. J., Leykam, J. F., and Lamport, D. T. (1989). Trypsin cleaves lysylproline in a hydroxyproline-rich glycoprotein from Zea mays. Pept. Res. 2, 246-248.

Kieliszewski, M. J., and Shpak, E. (2001). Synthetic genes for the elucidation of glycosylation codes for arabinogalactan-proteins and other hydroxyprolinerich glycoproteins. Cell Mol. Life Sci. 58, 1386-1398. doi: 10.1007/PL00000783

Kim, C. S., Gibbon, B. C., Gillikin, J. W., Larkins, B. A., Boston, R. S., and Jung, R. (2006). The maize mucronate mutation is a deletion in the $16-\mathrm{kDa}$ gammazein gene that induces the unfolded protein response. Plant J. 48, 440-451. doi: 10.1111/j.1365-313X.2006.02884.x

Kishor, P. B. K., Hong, Z., Miao, G. H., Hu, C. A. A., and Verma, D. P. S. (1995). Overexpression of $\Delta 1$-pyrroline-5-carboxylate synthetase increases proline production and confers osmotolerance in transgenic plants. Plant Physiol. 108, 1387-1394.

Kishor, P. B. K., Sangam, S., Amrutha, R. N., Laxmi, P. S., Naidu, K. R., Rao, K. R. S. S., et al. (2005). Regulation of proline biosynthesis, degradation, uptake and transport in higher plants: Its implications in plant growth and abiotic stress tolerance. Curr. Sci. 88, 424-438.

Kishor, P. B. K., and Sreenivasulu, N. (2014). Is proline accumulation per se correlated with stress tolerance or is proline homoeostasis a more critical issue? Plant Cell Environ. 37, 300-311. doi: 10.1111/pce.12157

Kiyosue, T., Yoshiba, Y., Yamaguchi-Shinozaki, K., and Shinozaki, K. (1996). A nuclear gene encoding mitochondrial proline dehydogenase, an enzyme involved in proline metabolism, is upregulated by proline but downregulated by dehydration in Arabidopsis. Plant Cell 8, 1323-1335. doi: 10.1105/tpc.8.8.1323

Knoch, E., Dilokpimol, A., and Geshi, N. (2014). Arabinogalactan proteins: focus on carbohydrate active enzymes. Front. Plant Sci. 5:198. doi: $10.3389 /$ fpls. 2014.00198

Kreuger, M., and van Holst, G. (1993). Arabinogalactan proteins are essential in somatic embryogenesis of Daucus carota L. Planta 189, 243-248. doi: 10.1007/BF00195083

Kwart, M., Hirner, B., Hummel, S., and Frommer, W. B. (1993). Differential expression of two related amino-acid transporters with differing substrate specificity in Arabidopsis thaliana. Plant J. 4, 993-1002. doi: 10.1046/j.1365313X.1993.04060993.x

Lamport, D. T. A. (1967). Hydroxyproline-O-glycosidic linkage of the plant cell wall glycoprotein extensin. Nature 216, 1322-1324. doi: 10.1038/2161322a0

Lamport, D. T. A. (1977). "Structure, biosynthesis and signifi cance of cell wall glycoproteins," in Recent Advances in Phytochemistry, 11th Edn, eds F. A. Loewus and V. C. Runeckles (New York: Plenum Press), 79-115.

Lamport, D. T. A., Kieliszewski, M. J., Chen, Y., and Cannon, M. C. (2011). Role of the extensin superfamily in primary cell wall architecture. Plant Physiol. 156, 11-19. doi: 10.1104/pp.110.169011

Lamport, D. T. A., Kieliszewski, M. J., and Showalter, A. M. (2006). Salt stress upregulates periplasmic arabinogalactan proteins: using salt stress to analyse AGP function. New Phytol. 169, 479-492. doi: 10.1111/j.14698137.2005.01591.x
Lamport, D. T. A., and Várnai, P. (2013). Periplasmic arabinogalactan glycoproteins act as a calcium capacitor that regulates plant growth and development. New Phytol. 197, 58-64. doi: 10.1111/nph.12005

Lee, J. A., and Pallas, D. C. (2007). Leucine carboxyl methyltransferase-1 is necessary for normal progression through mitosis in mammalian cells. J. Biol. Chem. 282, 30974-30984. doi: 10.1074/jbc.M704861200

Lee, Y. H., and Tegeder, M. (2004). Selective expression of a novel high-affinity transport system for acidic and neutral amino acids in the tapetum cells of Arabidopsis flowers. Plant J. 40, 60-74. doi: 10.1111/j.1365-313X.2004.02186.x

Lehmann, S., Funck, D., Szabados, L., and Rentsch, D. (2010). Proline metabolism and transport in plant development. Amino Acids 39, 949-962. doi: 10.1007/s00726-010-0525-3

Lehmann, S., Gumy, C., Blatter, E., Boeffel, S., Fricke, W., and Rentsch, D. (2011). In planta function of compatible solute transporters of the AtProT family. J. Exp. Bot. 62, 787-796. doi: 10.1093/jxb/erq320

LeRudulier, D., Strom, A. R., Dandekar, A. M., Smith, L. T., and Valentaine, R. C. (1984). Molecular biology of osmoregulation. Science 224, 1064-1068. doi: 10.1126/science.224.4653.1064

Lord, E. M., and Sanders, L. C. (1992). Roles for the extracellular matrix in plant development and pollination: a special case of cell movement in plants. Dev. Biol. 153, 16-28. doi: 10.1016/0012-1606(92)90088-X

Ma, Y., and Nelson, O. E. (1975). Amino acid composition and storage proteins in two new high-lysine mutants of maize. Cereal Chem. 52, 412-419.

MacArthur, M. W., and Thornton, J. M. (1991). Influence of proline residues on protein conformation. J. Mol. Biol. 218, 397-412. doi: 10.1016/00222836(91)90721-H

Mattioli, R., Costantino, P., and Trovato, M. (2009a). Proline accumulation in plants-Not only stress. Plant Sig. Behav. 4, 1016-1018. doi: 10.4161/psb.4.11.9797

Mattioli, R., Falascb, G., Sabatini, S., Altamura, M. M., Costantino, P., and Trovato, M. (2009b). The proline biosynthetic genes P5CS1 and P5CS2 play overlapping roles in Arabidopsis flower transition but not in embryo development. Physiol. Plant. 137, 72-85. doi: 10.1111/j.1399-3054.2009. 01261.x

Mattioli, R., Marchese, D., D’Angeli, S., Altamura, M. M., Costantino, P., and Trovato, M. (2008). Modulation of intracellular proline levels affects flowering time and inflorescence architecture in Arabidopsis. Plant Mol. Biol. 66, 277-288. doi: 10.1007/s11103-007-9269-1

Mauro, M. L, Trovato, M., De Paolis, A., Gallelli, A., Costantino, P., and Altamura, M. M. (1996). The plant oncogene rolD stimulates flowering in transgenic tobacco plants. Dev. Biol. 180, 693-700. doi: 10.1006/dbio.1996.0338

Mayer, U., and Jurgens, G. (2004). Cytokinesis: lines of division taking shape. Curr. Opin. Plant Biol. 7, 599-604. doi: 10.1016/j.pbi.2004.07.008

McCann, M. C., Bush, M., Milioni, D., Sado, P., Stacey, N. J., Catchpole, G., et al. (2001). Approaches to understanding the functional architecture of the plant cell wall. Phytochemistry 57, 811-821. doi: 10.1016/S0031-9422(01)00144-3

Meinke, D., Muralla, R., Sweeney, C., and Dickerman, A. (2008). Identifying essential genes in Arabidopsis thaliana. Trends Plant Sci. 13, 483-491. doi: 10.1016/j.tplants.2008.06.003

Menke, U., Renault, N., and Mueller-Roeber, B. (2000). StGCPRP, a potato gene strongly expressed in stomatal guard cells, defines a novel type of repetitive proline-rich proteins. Plant Physiol. 122, 677-686. doi: 10.1104/pp.122.3.677

Merkouropoulos, G., and Shirsat, A. H. (2003). The unusual Arabidopsis extensin gene Atext1 is expressed throughout plant development and is induced by a variety of biotic and abiotic stresses. Planta 217, 356-366. doi: 10.1007/s00425003-1002-y

Motose, H., Sugiyama, M., and Fukuda, H. (2001). An arabinogalactan protein(s) is a key component of a fraction that mediates local intercellular communication involved in tracheary element differentiation of Zinnia mesophyll cells. Plant Cell Physiol. 42, 129-137. doi: 10.1093/pcp/pce014

Munoz, F. J., Dopico, B., and Labrador, E. (1998). A cDNA encoding a prolinerich protein from Cicer arietinum. Changes in expression during development and abiotic stress. Physiol. Plant. 102, 582-590. doi: 10.1034/j.13993054.1998.1020413.x

Nanjo, T., Kobayashi, M., Yoshiba, Y., Sanada, Y., Wada, K., Tsukaya, H., et al. (1999). Biological functions of proline in morphogenesis and osmotolerance revealed in antisense transgenic Arabidopsis thaliana. Plant J. 18, 185-193. doi: 10.1046/j.1365-313X.1999.00438.x 
Nguema-Ona, E., Vicré-Gibouin, M., Cannesan, M. A., and Driouich, A. (2013). Arabinogalactan proteins in root-microbe interactions. Trends Plant Sci. 18, 440-449. doi: 10. 1016/j.tplants.2013.03.006

Nothnagel, E. A. (1997). Proteoglycans and related components in plant cells. Inter. Rev. Cytol. 174, 195-291. doi: 10.1016/S0074-7696(08)62118-X

Nunez, A., Fishman, M. L., Fortis, L. L., Cooke, P. H., and Hotchkiss, A. T. J. (2009). Identification of extensin protein associated with sugar beet pectin. J. Agric. Food Chem. 57, 10951-10958. doi: 10.1021/jf902162t

Ober, E. S., and Sharp, R. E. (1994). Proline accumulation in maize (Zea mays L.) primary roots at low water potentials. (I Requirement for increased levels of abscisic acid). Plant Physiol. 105, 981-987.

Ogawa-Ohnishi, M., Matsushita, W., and Matsubayashi, Y. (2013). Identification of three hydroxyproline O-arabinosyltransferases in Arabidopsis thaliana. Nat. Chem. Biol. 9, 726-730. doi: 10.1038/nchembio.1351

Okumoto, S., Koch, W., Tegeder, M., Fischer, W. N., Biehl, A., Leister, D., et al. (2004). Root phloem-specific expression of the plasma membrane amino acid proton co-transporter AAP3. J. Exp. Bot. 55, 2155-2168. doi: $10.1093 /$ jxb/erh233

Okumoto, S., Schmidt, R., Tegeder, M., Fischer, W. N., Rentsch, D., Frommer, W. B., et al. (2002). High affinity amino acid transporters specifically expressed in xylem parenchyma and developing seeds of Arabidopsis. J. Biol. Chem. 277, 45338-45346. doi: 10.1074/jbc.M207730200

Park, S., Szumlanski, A. L., Gu, F., Guo, F., and Nielsen, E. (2011). A role for CSLD3 during cell-wall synthesis in apical plasma membranes of tip-growing root-hair cells. Nat. Cell Biol. 13, 973-980. doi: 10.1038/ncb2294

Pennell, R. I., Janniche, L., Kjellbom, P., Scofield, G. N., Peart, J. M., and Roberts, K. (1991). Developmental regulation of a plasma membrane arabinogalactan protein epitope in oilseed rape flowers. Plant Cell 3, 1317-1326. doi: 10.1105/tpc.3.12.1317

Pennell, R. I., and Roberts, K. (1990). Sexual development in the pea is presaged by altered expression of arabinogalactan protein. Nature 344, 547-549. doi: $10.1038 / 344547 \mathrm{a} 0$

Pope, D. G. (1977). Relationships between hydroxyproline-containing proteins secreted into the cell wall and medium by suspension- cultured Acer pseudoplatanus cells. Plant Physiol. 59, 894-900. doi: 10.1104/pp.59.5.894

Price, N. J., Pinheiro, C., Soares, C. M., Ashford, D. A., Ricardo, C. P., and Jackson, P. A. (2003). A biochemical and molecular characterization of LEP1, an extensin peroxidase from lupin. J. Biol. Chem. 278, 41389-41399. doi: 10.1074/jbc.M304519200

Racchi, M. L., Gavazzi, G., Monti, D., and Manitto, P. (1978). An analysis of the nutritional requirements of thepromutants in Zea mays. Plant Sci. Lett. 13, 357-364. doi: 10.1016/0304-4211(78)90213-4

Raymond, M. J., and Smirnoff, N. (2002). Proline metabolism and transport in maize seedlings at low water potential. Ann. Bot. 89, 813-823. doi: $10.1093 / \mathrm{aob} / \mathrm{mcf0} 2$

Reddy, P. S., Jogeswar, G., Rasineni, G. K., Maheswari, M., Reddy, A. R., Varshney, R. K., et al. (2015). Proline over-accumulation alleviates salt stress and protects photosynthetic and antioxidant enzyme activities in transgenic sorghum [Sorghum bicolor (L.) Moench]. Plant Physiol. Biochem. 94, 104-113. doi: 10.1016/j.plaphy.2015.05.014

Rentsch, D., Hirner, B., Schmelzer, E., and Frommer, W. B. (1996). Salt-stress induced proline transporters and salt-repressed broad specificity amino acid permeases identified by suppression of a yeast amino acid permease-targeting mutant. Plant Cell 8, 1437-1446. doi: 10.1105/tpc.8.8.1437

Rentsch, D., Schmidt, S., and Tegeder, M. (2007). Transporters for uptake and allocation of organic nitrogen compounds in plants. FEBS Lett. 581, 2281-2289. doi: 10.1016/j.febslet.2007.04.013

Rhee, S. Y., Osborne, E., Poindexter, P. D., and Somerville, C. R. (2003). Microspore separation in the quartet 3 mutants of Arabidopsis is impaired by a defect in a developmentally regulated polygalacturonase required for pollen mother cell wall degradation. Plant Physiol. 133, 1170-1180. doi: 10.1104/pp.103. 028266

Rhodes, D., Handa, S., and Bressan, R. A. (1986). Metabolic changes associated with adaptation of plant cells to water stress. Plant Physiol. 82, 890-903. doi: 10.1104/pp.82.4.890

Ringli, C. (2010). The hydroxyproline-rich glycoprotein domain of the Arabidopsis LRX1 requires Tyr for function but not for insolubilization in the cell wall. Plant J. 63, 662-669. doi: 10.1111/j.1365-313X.2010.04270.x
Roberts, K. (1989). The plant extracellular matrix. Cur. Opin. Cell Biol. 1, 1020-1027. doi: 10.1016/0955-0674(89)90074-4

Roberts, K., and Shirsat, A. H. (2006). Increased extension levels in Arabidopsis affect inflorescence stem thickening and height. J. Exp. Bot. 57, 537-545. doi: 10.1093/jxb/erj036

Romanczyk, L. J. Jr., McClelland, C. A., Post, L. S., and Aitken, W. M. (1995). Formation of 2-acetyl-1-pyrroline by several Bacillus cereus strains isolated from cocoa fermentation boxes. J. Agric. Food Chem. 43, 469-475. doi: 10.1021/jf00050a040

Samach, A., Onouchi, H., Gold, S. E., Ditta, G. S., Schwarz-Sommer, Z., Yanofsky, M. F., et al. (2000). Distinct roles of CONSTANS target genes in reproductive development of Arabidopsis. Science 288, 1613-1616. doi: $10.1126 /$ science.288.5471.1613

Sauer, N., and Tanner, W. (1985). Selection and characterization of Chlorella mutants deficient in amino acid transport. Plant Physiol. 79, 760-764. doi: 10.1104/pp.79.3.760

Savoure, A., Jaoua, S., Hua, X., Ardiles, W., Montagu, M. V., and Verbruggen, N. (1995). Isolation, characterization, and chromosomal location of a gene encoding the $\Delta 1$-pyrroline-5-carboxylate synthetase in Arabidopsis thaliana. FEBS Lett. 372, 13-19. doi: 10.1016/0014-5793(95)00935-3

Schindler, T., Bergfeld, R., and Schopfer, P. (1995). Arabinogalactan proteins in maize coleoptiles: developmental relationship to cell death during xylem differentiation but not to extension growth. Plant J. 5, 157-172. doi: 10.1046/j.1365-313X.1995.07010025.x

Schmidt, R. J., Burr, F. A., and Burr, B. (1987). Transposon tagging and molecular analysis of the maize regulatory locus opaque-2. Science 238, 960-963. doi: $10.1126 /$ science. 2823388

Schmidt, R., Stransky, H., and Koch, W. (2007). The amino acid permease AAP8 is important for early seed development in Arabidopsis thaliana. Planta 226, 805-813. doi: 10.1007/s00425-007-0527-x

Schultz, C. J., Ferguson, K. L., Lahnstein, J., and Bacic, A. (2004). Posttranslational modifications of arabinogalactan-peptides of Arabidopsis thaliana: endoplasmic reticulum and glycosylphosphatidylinositol-anchor signal cleavage sites and hydroxylation of proline. J. Biol. Chem. 279, 45503-45511. doi: 10.1074/jbc.M407594200

Schultz, C., Gilson, P., Oxley, D., Youl, J., and Bacic, A. (1998). GPI-anchors on arabinogalactan-proteins: implications for signalling in plants. Trends Plant Sci. 3, 426-431. doi: 10.1016/S1360-1385(98)01328-4

Schwacke, R., Grallath, S., Breitkreuz, K. E., Stransky, H., Frommer, W. B., and Rentsch, D. (1999). LeProT1, a transporter for proline, glycine betaine and $\gamma$-aminobutyric acid in tomato pollen. Plant Cell 11, 377-391. doi: 10.1105/tpc.11.3.377

Serpe, M. D., and Nothnagel, E. A. (1999). Arabinogalactan-proteins in the multiple domains of the plant cell surface. Adv. Bot. Res. 30, 207-289. doi: 10.1016/S0065-2296(08)60229-3

Sharma, S., Shinde, S., and Verslues, P. E. (2013). Functional characterization of an ornithine cyclodeaminase-like protein of Arabidopsis thaliana. BMC Plant Biol. 13:182. doi: 10.1186/1471-2229-13-182

Sharma, S., and Verslues, P. E. (2010). Mechanisms independent of abscisic acid (ABA) or proline feedback have a predominant role in transcriptional regulation of proline metabolism during low water potential and stress recovery. Plant Cell Environ. 33, 1838-1851. doi: 10.1111/j.1365-3040.2010.02188.x

Shen, Y. G., Zhang, W. K., Yan, D. Q., Du, B. X., Zhang, J. S., and Chen, S. Y. (2002). Overexpression of proline transporter gene isolated from halophyte confers salt tolerance in Arabidopsis. Acta Bot. Sin. 44, 956-962.

Shetty, K. (2004). Role of proline-linked pentose phosphate pathway in biosynthesis of plant phenolics for functional food and environmental applications: a review. Process Biochem. 39, 789-803. doi: 10.1016/S00329592(03)00088-8

Shirsat, A. H., Wieczorek, D., and Kozbial, P. (1996). A gene for Brassica napus extensin is differentially expressed on wounding. Plant Mol. Biol. 30, 1291-1300. doi: 10.1007/BF00019559

Showalter, A. M. (1993). Structure and function of plant cell wall proteins. Plant Cell 5, 9-23. doi: 10.1105/tpc.5.1.9

Showalter, A. M. (2001). Arabinogalactan-proteins: structure, expression, and function. Cell. Mol. Life Sci. 58, 1399-1417. doi: 10.1007/PL000 00784 
Showalter, A. M., Keppler, B., Lichtenberg, J., Gu, D., and Welch, L. R. (2010). A bioinformatics approach to the identification, classification, and analysis of hydroxyproline-rich glycoproteins. Plant Physiol. 153, 485-513. doi: 10.1104/pp.110.156554

Showalter, A. M., and Varner, J. E. (1989). "Plant hydroxyproline-rich glycoproteins," in The Biochemistry of Plants, ed. M. Abraham (New York: Academic Press), 485-520.

Shpak, E., Barbar, E., Leykam, J. F., and Kieliszewski, M. J. (2001). Contiguous hydroxyproline residues direct hydroxyproline arabinosylation in Nicotiana tabacum. J. Biol. Chem. 276, 11272-11278. doi: 10.1074/jbc.M011 323200

Somerville, C., Bauer, S., Brininstool, G., Facette, M., Hamann, T., Milne, J., et al. (2004). Toward a systems approach to understanding plant cell walls. Science 306, 2206-2211. doi: 10.1126/science.1102765

Spoljarevic, M., Agic, D., Lisjak, M., Gumze, A., Wilson, I. D., Hancock, J. T., et al. (2011). The relationship of proline content and metabolism on the productivity of maize plants. Plant Sign. Behav. 6, 251-257. doi: 10.4161/psb.6.2.14336

Spollen, W. G., Tao, W., Valliiyodan, B., Chen, K., Hejlek, L. G., Kim, J. J., et al. (2008). Spatial distribution of transcript changes in the maize primary root elongation zone at low water potential. BMC Plant Biol. 8:32. doi: 10.1186/14712229-8-32

Stines, A. P., Grubb, J., Gockowiak, H., Henschke, P. A., Hoj, P. B., and van Heeswijck, R. (2000). Proline and arginine accumulation in developing berries of Vitis vinifera L. in Australian vineyards: Influence of vine cultivar, berry maturity and tissue type. Aust. J. Grape Wine Res. 6, 150-158. doi: 10.1111/j.1755-0238.2000.tb00174.x

Strizhov, N., Abraham, E., Okresz, L., Blickling, S., Zilberstein, A., Schell, J., et al. (1997). Differential expression of two P5CS genes controlling proline accumulation during salt-stress requires $\mathrm{ABA}$ and is regulated by $\mathrm{ABA} 1$, ABI1 and AXR2 in Arabidopsis. Plant J. 12, 557-569. doi: 10.1111/j.09607412.1997.00557.x

Szabados, L., and Savoure, A. (2010). Proline: a multifunctional amino acid. Trends Plant Sci. 15, 89-97. doi: 10.1016/j.tplants.2009.11.009

Szekely, G., Abraham, E., Cseplo, A., Rigo, G., Zsigmond, L., Csiszar, J., et al. (2008). Duplicated P5CS genes of Arabidopsis play distinct roles in stress regulation and developmental control of proline biosynthesis. Plant J. 53, 11-28. doi: 10.1111/j.1365-313X.2007.03318.x

Tan, L., Eberhard, S., Pattathil, S., Warder, C., Glushka, J., Yuan, C., et al. (2013). An Arabidopsis cell wall proteoglycan consists of pectin and arabinoxylan covalently linked to an arabinogalactan protein. Plant Cell 25, 270-287. doi: 10.1105/tpc.112.107334

Tan, L., Showalter, A. M., Egelund, J., Hernandez-Sanchez, A., Doblin, M. S., and Bacic, A. (2012). Arabinogalactan-proteins and the research challenges for these enigmatic plant cell surface proteoglycans. Front. Plant Sci. 3:140. doi: $10.3389 /$ fpls.2012.00140

Tegeder, M., and Ward, J. M. (2012). Molecular evolution of plant AAP and LHT amino acid transporters. Front Plant Sci. 3:21. doi: 10.3389/fpls.2012.00021

Thornburg, R. W. (2007). "Molecular biology of the Nicotiana floral nectar," in Nectaries and Nectar, eds S. W. Nicolson, M. Nepi, and E. Pacini (Heidelberg: Springer-Verlag), 265-287.

Toka, I., Planchais, S., Cabassa, C., Justin, A. M., De Vos, D., Richard, L., et al. (2010). Mutations in the hyperosmotic stress-responsive mitochondrial BASIC AMINOACID CARRIER2 enhance proline accumulation in Arabidopsis. Plant Physiol. 152, 1851-1862. doi: 10.1104/pp.109.152371

Tonelli, C., Gavazzi, G., Manzocchi, L., Di Fonzo, N., and Soave, C. (1986). Opaque 6 allelic to pro1 mutant. Maize Newslett. 60, 100.

Tonelli, C., Racchi, M. L., Viani, I., and Gavazzi, G. (1984). In vitro development of proline requiring mutant maize kernels. Atti. Assoc. Genet. Italy 30:231.

Toonen, M. A. J., Schmidt, E. D. L., Van Kammen, A., and De Vries, S. C. (1997). Promotive and inhibitory effects of diverse arabinogalactan proteins on Daucus carota L. somatic embryogenesis. Planta 203, 188-195. doi: $10.1007 / \mathrm{s} 004250050181$

Trovato, M., Maras, B., Linhares, F., and Costantino, P. (2001). The plant oncogenic rolD encodes a functional ornithine cyclodeaminase. Proc. Nat. Acad. Sci. U.S.A. 98, 13449-13453. doi: 10.1073/pnas.231320398

Tseng, I. C., Hong, C. Y., Yu, S. M., and Ho, T. H. (2013). Abscisic acid- and stressinduced highly proline-rich glycoproteins regulate root growth in rice. Plant Physiol. 163, 118-134. doi: 10.1104/pp.113.217547
Ueda, A., Shi, W., Sanmiya, K., Shono, M., and Takabe, T. (2001). Functional analysis of salt-inducible proline transporter of barley roots. Plant Cell Physiol. 42, 1282-1289. doi: 10.1093/pcp/pce166

Ueda, A., Shi, W., Shimada, T., Miyake, H., and Takabe, T. (2008). Altered expression of barley proline transporter causes different growth responses in Arabidopsis. Planta 227, 277-286. doi: 10.1007/s00425-007-0615-y

Varner, J. E., and Lin, L. S. (1989). Plant cell wall architecture. Cell 56, 231-239. doi: 10.1016/0092-8674(89)90896-9

Velasquez, S. M., Ricardi, M. M., Dorosz, J. G., Fernandez, P. V., Nadra, A. D., PolFachin, L., et al. (2011). O-glycosylated cell wall proteins are essential in root hair growth. Science 332, 1401-1403. doi: 10.1126/science.1206657

Velasquez, S. M., Ricardi, M. M., Poulsen, C. P., Oikawa, A., Dilokpimol, A., Halim, A., et al. (2015). Complex regulation of prolyl-4-hydroxylases impacts root hair expansion. Mol. Plant 8, 734-746. doi: 10.1016/j.molp.2014. 11.017

Velasquez, S. M., Salgado Salter, J., Petersen, B. L., and Estevez, J. M. (2012). Recent advances on the post-translational modifications of EXTs and their roles in plant cell walls. Front. Plant Sci. 3:93. doi: 10.3389/fpls.2012.00093

Venekamp, J. H., and Koot, J. T. M. (1984). The distribution of free amino acids, especially of proline, in the organs of field bean plants. Vicia faba L., during development in the field. J. Plant Physiol. 116, 343-349. doi: 10.1016/S01761617(84)80113-3

Verbruggen, N., and Hermans, C. (2008). Proline accumulation in plants: a review. Amino Acids 35, 753-759. doi: 10.1007/s00726-008-0061-6

Verbruggen, N., Villarroel, R., and Van Montagu, M. (1993). Osmoregulation of a pyrroline-5-carboxylate reductase gene in Arabidopsis. Plant Physiol. 103, 771-781. doi: 10.1104/pp.103.3.771

Verslues, P. E., and Sharp, R. E. (1999). Proline accumulation in maize (Zea mays L.) primary roots at low water potentials. II. Metabolic source of increased proline deposition in the elongation zone. Plant Physiol. 119, 1349-1360. doi: 10.1104/pp.119.4.1349

Vignols, F., Jose-Estanyol, M., Caparros-Ruiz, D., Rigau, J., and Puigdomenech, P. (1999). Involvement of a maize proline-rich protein in secondary cell wall formation as deduced from its specific mRNA localization. Plant Mol. Biol. 39, 945-952. doi: 10.1023/A:1006129703262

Villalba, M., Batanero, E., Monsalve, R. I., Gonzalez, M. A., Lahoz, C., and Rodriguez, R. (1994). Cloning and expression of Ole e I, the major allergen from olive tree pollen. Polymorphism analysis and tissue specificity. J. Biol. Chem. 269, 15217-15222.

Voetberg, G. S., and Sharp, R. E. (1991). Growth of maize primary root at low water potentials. III. Role of increased proline deposition in osmotic adjustment. Plant Physiol. 96, 1125-1130. doi: 10.1104/pp.96.4.1125

Waditee, R., Hibino, T., Tanaka, Y., Nakamura, T., Incharoensakdi, A., Hayakawa, S., et al. (2002). Functional characterization of betaine/proline transporters in betaine-accumulating mangrove. J. Biol. Chem. 277, 1837818382. doi: 10.1074/jbc.M112012200

Wakabayashi, K., Hoson, T., and Kamisaka, S. (1997). Changes in amounts and molecular mass distribution of cell-wall polysaccharides of wheat (Triticum aestivum L.) coleoptiles under water stress. J. Plant Physiol. 151, 33-40. doi: 10.1016/S0176-1617(97)80033-8

Wang, G., Sun, X., Wang, G., Wang, F., Gao, Q., Sun, X., et al. (2011). Opaque7 encodes an acyl-activating enzyme-like protein that affects storage protein synthesis in maize endosperm. Genetics 189, 1281-1295. doi: 10.1534/genetics.111.133967

Wang, G., Zhang, J., Wang, G., Fan, X., Sun, X., Qin, H., et al. (2014). Proline responding1 plays a critical role in regulating general protein synthesis and the cell cycle in maize. Plant Cell 26, 2582-2600. doi: 10.1105/tpc.114. 125559

Wang, Z. Y., and He, J. X. (2004). Brassinosteroid signal transduction - choices of signals and receptors. Trends Plant Sci. 9, 91-96. doi: 10.1016/j.tplants.2003.12.009

Willats, W. G. T., and Knox, J. P. (1996). A role for arabinogalactan-proteins in plant cell expansion: evidence from studies on the interaction of glucosyl Yariv reagent with seedlings of Arabidopsis thaliana. Plant J. 9, 919-925. doi: 10.1046/j.1365-313X.1996.9060919.x

Wu, H., de Graaf, B., Mariani, C., and Cheung, A. Y. (2001). Hydroxyproline-rich glycoproteins in plant reproductive tissues: structure, functions and regulation. Cell. Mol. Life Sci. 58, 1418-1429. doi: 10.1007/PL00000785 
Wu, H-M., Wong, E., Ogdahl, J., and Cheung, A. Y. (2000). A pollen tube growth-promoting arabinogalactan protein from Nicotiana alata is similar to the tobacco TTS protein. Plant J. 22, 165-176. doi: 10.1046/j.1365313x.2000.00731.x

Wyatt, R. E., Nagao, R. T., and Key, J. L. (1992). Patterns of soybean proline-rich protein gene expression. Plant Cell. 4, 99-110. doi: 10.1105/tpc. 4.1.99

Xu, C., Takc, T., Burbach, C., Menzel, D., and Samaj, J. (2011). Developmental localization and the role of hydroxyproline rich glycoproteins during somatic embryogenesis of banana (Musa spp. AAA). BMC Plant Biol. 11:38. doi: 10.1186/1471-2229-11-38

Xu, W. L., Zhang, D. J., Wu, Y. F., Qin, L. X., Huang, G. Q., Li, J., et al. (2013). Cotton PRP5 gene encoding a proline-rich protein is involved in fiber development. Plant Mol. Biol. 82, 353-365. doi: 10.1007/s11103-013-0066-8

Xue, X., Liu, A., and Hua, X. (2009). Proline accumulation and transcriptional regulation of proline biosynthesis and degradation in Brassica napus. BMB Rep. 42, 28-34. doi: 10.5483/BMBRep.2009.42.1.028

Yamada, H., and Kiyohara, H. (1999). "Complement-activating polysaccharides from medicinal herbs," in Immunomodulatory Agents from Plants, ed. H. Wagner (Reinach: Birkhäuser Basel), 161-202. doi: 10.1007/978-3-03488763-2_7

Yamada, N., Promden, W., Yamane, K., Tamagake, H., Hibino, T., Tanaka, Y., et al. (2009). Preferential accumulation of betaine uncoupled to choline monooxygenase in young leaves of sugar beet-importance of long-distance translocation of betaine under normal and salt stressed conditions. J. Plant Physiol. 166, 2058-2070. doi: 10.1016/j.jplph.2011.03.007

Yamada, N., Sakakibara, S., Tsutsumi, K., Waditee, R., Tanaka, Y., and Takabe, T. (2011). Expression and substrate specificity of betaine/proline transporters suggest a novel choline transport mechanism in sugar beet. J. Plant Physiol. 168, 1609-1616. doi: 10.1016/j.jplph.2011.03.007

Ye, Z. H., Song, Y. R., Marcus, A., and Varner, J. E. (1991). Comparative localization of three classes of cell wall proteins. Plant J. 1, 175-183. doi: 10.1111/j.1365313X.1991.00175.x
Ye, Z. H., and Varner, J. E. (1991). Tissue-specific expression of cell wall proteins in developing soybean tissues. Plant Cell 3, 23-37. doi: 10.1105/tpc.3.1.23

Zhan, X., Wang, B., Li, H., Liu, R., Kalia, R. K., Zhu, J. K., et al. (2012). Arabidopsis proline-rich protein important for development and abiotic stress tolerance is involved in microRNA biogenesis. Proc. Nat. Acad. Sci. U.S.A. 109, 1819818203. doi: 10.1073/pnas.1216199109

Zhang, H. Q., Croes, A., and Linskens, H. (1982). Protein synthesis in germinating pollen of Petunia: role of proline. Planta 154, 199-203. doi: 10.1007/BF00387864

Zhang, X., Ma, H., Qi, H., and Zhao, J. (2014). Roles of hydroxyproline-rich glycoproteins in the pollen tube and style cell growth of tobacco (Nicotiana tabacum L.). J. Plant Physiol. 171, 1036-1045. doi: 10.1016/j.jplph.2014. 02.010

Zhao, Z. D., Tan, L., Showalter, A. M., Lamport, D. T. A., and Kieliszewski, M. J. (2002). Tomato LeAGP-1 arabinogalactan-protein purified from transgenic tobacco corroborates the Hyp contiguity hypothesis. Plant J. 31, 431-444. doi: 10.1046/j.1365-313X.2002.01365.x

Zou, X., Jiang, Y., Zheng, Y., Zhang, M., and Zhang, Z. (2011). Prolyl 4hydroxylase genes are subjected to alternative splicing in roots of maize seedlings under waterlogging. Ann. Bot. 108, 132-1335. doi: 10.1093/aob/ $\operatorname{mcr} 223$

Conflict of Interest Statement: The authors declare that the research was conducted in the absence of any commercial or financial relationships that could be construed as a potential conflict of interest.

Copyright (c) 2015 Kavi Kishor, Hima Kumari, Sunita and Sreenivasulu. This is an open-access article distributed under the terms of the Creative Commons Attribution License (CC BY). The use, distribution or reproduction in other forums is permitted, provided the original author(s) or licensor are credited and that the original publication in this journal is cited, in accordance with accepted academic practice. No use, distribution or reproduction is permitted which does not comply with these terms. 


\title{
Role of proline and GABA in sexual reproduction of angiosperms
}

\author{
Marco Biancucci ${ }^{1}$, Roberto Mattioli ${ }^{1}$, Giuseppe Forlani ${ }^{2}$, Dietmar Funck ${ }^{3}$, \\ Paolo Costantino ${ }^{1}$ and Maurizio Trovato ${ }^{1 *}$
}

${ }^{1}$ Department of Biology and Biotechnology, Sapienza University of Rome, Roma, Italy, ${ }^{2}$ Department of Life Science and Biotechnology, University of Ferrara, Ferrara, Italy, ${ }^{3}$ Department of Biology, University of Konstanz, Konstanz, Germany

\section{OPEN ACCESS}

Edited by:

Matthew Gilliham,

University of Adelaide, Australia

Reviewed by:

Naser A. Anjum,

University of Aveiro, Portugal Matthew Gilliham,

University of Adelaide, Australia

Paul E. Vers/ues,

Academia Sinica, Taiwan

*Correspondence:

Maurizio Trovato,

Department of Biology and Biotechnology, Sapienza University of Rome, P.le A. Moro 5, 00185 Rome,

Italy

maurizio.trovato@uniroma1.it

Specialty section:

This article was submitted to Plant Physiology,

a section of the journal Frontiers in Plant Science

Received: 26 April 2015

Accepted: 17 August 2015 Published: 04 September 2015

Citation:

Biancucci M, Mattioli R, Forlani G,

Funck $D$, Costantino $P$ and Trovato $M$ (2015) Role of proline and GABA in sexual reproduction of angiosperms.

Front. Plant Sci. 6:680. doi: 10.3389/fp/s.2015.00680
Two glutamate derivatives, proline and $\gamma$-aminobutyric acid (GABA), appear to play pivotal roles in different aspects of sexual reproduction in angiosperms, although their precise function in plant reproduction and the molecular basis of their action are not yet fully understood. Proline and GABA have long been regarded as pivotal amino acids in pollen vitality and fertility. Proline may constitute up to $70 \%$ of the free amino acid pool in pollen grains and it has been recently shown that Arabidopsis mutants affected in the first and rate-limiting step in proline synthesis produce aberrant and infertile pollen grains, indicating that proline synthesis is required for pollen development and fertility. Concerning GABA, a large body of evidence points to this glutamate derivative as a key determinant of post-pollination fertilization. Intriguingly, proline has also been associated with pollination, another aspect of sexual reproduction, since honeybees were reported to show a strong preference for proline-enriched nectars. In this review, we survey current knowledge on the roles of proline and GABA in plant fertility, and discuss future perspectives potentially capable to improve our understanding on the functions of these amino acids in pollen development, pollination, and pollen tube guidance.

Keywords: proline, GABA, pollen, anthers, P5CS1, P5CS2, Arabidopsis, sexual plant reproduction

\section{Setting the Scene: Microsporogenesis and Microgametogenesis}

Higher plants cycle between a diploid sporophytic generation and a haploid gametophytic generation. While spending most of their lifespan as diploid individuals, plants rely on their haploid gametophytic generation for sexual reproduction. Cells committed to sexual reproduction undergo meiosis in specialized organs of the flower, called stamens and pistils, and, after a limited number of mitoses, develop as mature haploid gametophytes. The male gametophyte, also referred to as micro-gametophyte or pollen grain, develops in the pollen sac of the anther passing through two sequential developmental phases, called microsporogenesis and microgametogenesis (Figure 1).

In early stages of pollen development, microsporogenesis takes place within the anthers, where archeosporal cells divide periclinally to generate an outer parietal layer and an inner sporogenous layer. The outer layer undergoes both periclinal and anticlinal divisions, to generate concentric layers that differentiate into the endothecium, middle layer, and the tapetum. The inner layer gives rise to the microsporocytes, also known as pollen mother cells or meiocytes, which divide by meiosis to form a tetrad, consisting of four haploid microspores embedded in a joint callose coat. The microspores are eventually released from the tetrad as single-celled haploid microspores, by the action of a $ß(1-3)$ glucanase (callase) secreted by the tapetum. In addition to producing hydrolytic enzymes for callose degradation, the tapetum supplies nutrients and metabolites to the developing 


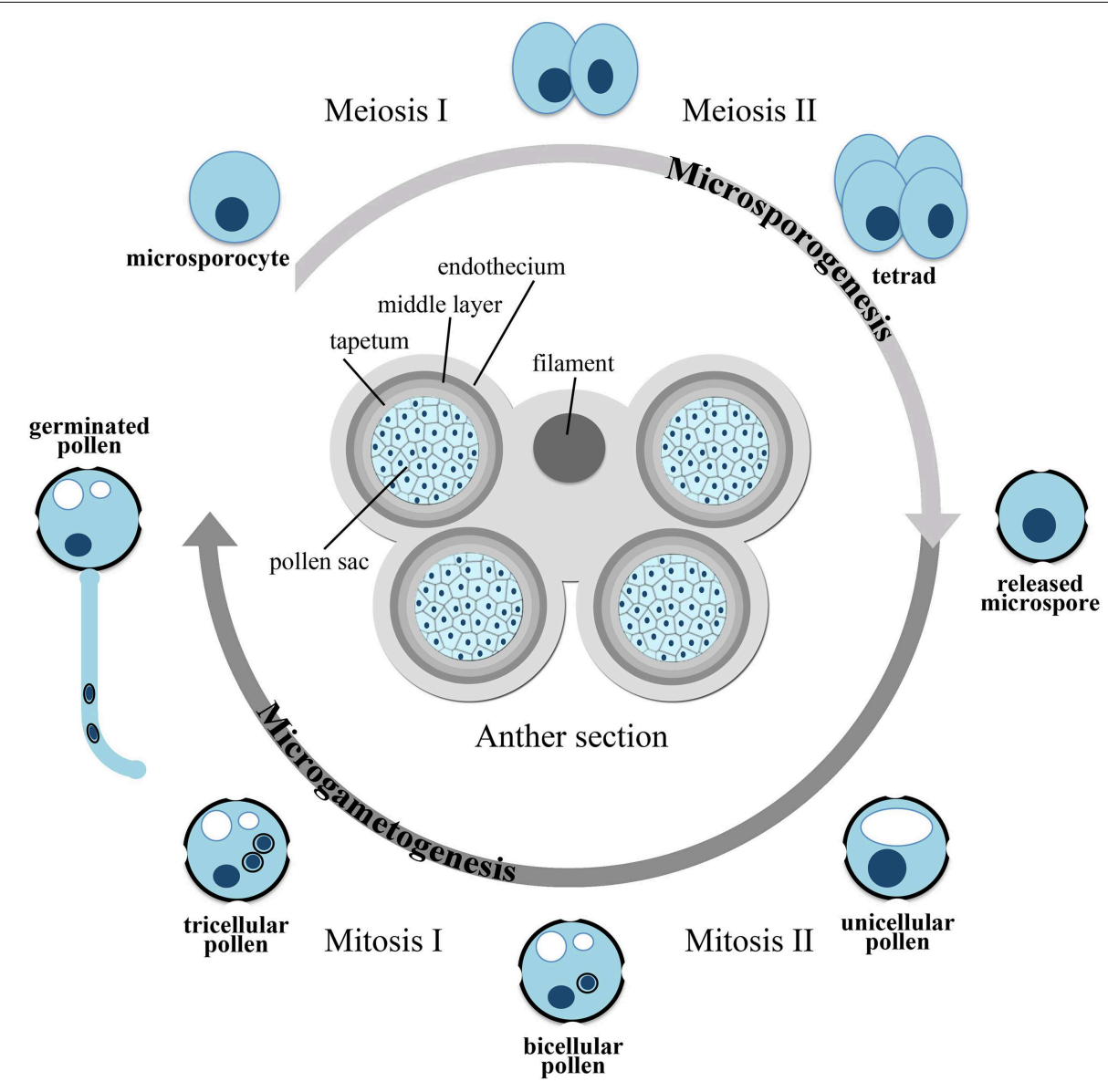

FIGURE 1 | Scheme of microsporogenesis and microgametogenesis.

microspores and plays an essential role in pollen development, as first shown by Mariani et al. (1990), who reported that a genetic ablation of the tapetal cells results in male sterility. In early stages of pollen development, before meiosis takes place, all the layers of the anther are physically interconnected with the tapetum by plasmodesmata, through which all nutrients and metabolites can be freely exchanged. After tetrad separation, the microspores start to build an extremely durable extracellular layer, the exine, to which the tapetum contributes the major building material, the sporopollenin. However, at the end of microspore development, by the time of pollen mitosis I (PM I), the tapetum starts a process of progressive vacuolization and degeneration, and from this point onward the developing microspores have to rely on endogenous resources. The series of events leading from unicellular microspores to mature bior tri-cellular pollen grains, depending on species, is known as microgametogenesis (Figure 1). During this phase the unicellular microspores, generated by callose degradation of the tetrads, begin a process of expansion and vacuolization usually leading to the formation of a large vacuole. The nucleus undergoes PM I, an asymmetric mitotic division, which generates a pollen grain with a larger vegetative cell and a smaller generative cell. The generative cell subsequently separates from the pollen grain wall and gets internalized into the cytoplasm of the vegetative cell. In some plant families (e.g., Cruciferae and Gramineae), the generative cell divides once more by mitosis (PM II) to form two sperm cells in the mature, tri-cellular pollen grain. In many angiosperm families, however, the pollen grain is released from the anther after the first mitotic division as a bi-cellular pollen grain and PM II occurs after pollen germination, when the pollen tube is already growing through the female pistil. The two generative cells are delivered through the pollen tube to the embryo sac within the ovule to fertilize the egg cell and the central cell (double fertilization). At the mature stage, the pollen is covered by the resistant exine coat and is highly dehydrated, probably to withstand even harsh environmental conditions during the transfer to a pistil. Rehydration of the pollen happens on the stigma surface of a compatible pistil and leads to germination and growth of the pollen tube.

\section{Proline and Pollen Development: A Historical Perspective}

Beside the well-established role of proline in protein synthesis, this amino acid is known to participate to the set of responses 
put in place by cells to respond to, and protect from different types of stresses, particularly salt and drought stress (for a review see Hayat et al., 2012). Indeed, accumulation of proline under stress is a well-documented phenomenon in a large number of different organisms, including protozoa, eubacteria, marine invertebrates, algae, and plants (Trovato et al., 2008). In higher plants, however, proline has also been shown to participate in plant development, accumulating under non-stressed conditions in specific developmental phases, particularly in reproductive organs and structures, such as flowers, anthers and pollen grains. The first hints of the special role played by proline in pollen development and function came from qualitative and quantitative analyses of free amino acid content made by independent laboratories in several plant species. As early as 1948, Auclair and Jamieson reported a high, although not precisely quantified, content of free proline in the pollen grains of dandelion (Taraxum officinale) and willow (Salix spp.) (Auclair and Jamieson, 1948). The first quantitative assessment of proline content in pollen was probably performed by Bathurst (1954), who analyzed the free amino acidic content in the pollen grains of four species of grasses, finding the presence of 17 amino acids, of which proline was the most highly represented accounting for $1.65 \%$ of pollen dry weight. Since these initial reports, several other authors (including but not limited to Vansuyt et al., 1979; Venekamp and Koot, 1988; Mutters et al., 1989; Walton et al., 1991; Chiang and Dandekar, 1995; Schwacke et al., 1999) confirmed these findings and reported strong accumulation of proline in floral organs, siliques, and pollen grains of different plant species under unstressed physiological conditions. As evidenced in Arabidopsis by Chiang and Dandekar (1995), the content of proline is low during the vegetative phase, but starts to accumulate dramatically in the reproductive phase, just after floral transition. These authors reported that proline accumulates in Arabidopsis reproductive tissues, such as flowers and seeds, to up to $26 \%$ of the total free amino acid pool while in vegetative tissues, such as rosette leaves and roots, it only represents $1-3 \%$ of the total free amino acids. As pointed out by the authors, proline accumulation in reproductive organs might be correlated to the levels of desiccation tolerance, as most of the tissues enriched in proline, such as pollen grains, seeds and embryos, are naturally dehydrated (Chiang and Dandekar, 1995). In this case proline might function as a compatible osmolyte protecting cells from the detrimental effects of dehydration, and both developmental and environmental responses might be part of a common strategy against drought and water stress. Although there is no general agreement, as yet, on the actual role of proline in development, it is largely accepted that pollen grains are the plant structures with the highest proline concentration. In a study conducted in the anthers of devil's trumpet (Datura metel), a Solanaceous species, Sangwan (1978) analyzed the changes in the amino acid content occurring in pollen throughout development and found the presence of a limited number of free amino acids (proline, glutamic acid, aspartic acid, threonine, serine, and alanine), of which proline was the most abundant and the only one to show a consistent increment during pollen development. Similarly, during in vitro germination of Petunia pollen, Hong-Qi et al. (1982) reported that proline was the most abundant amino acid, accounting for $55 \%$ of the total amino acid pool and exceeding by $70 \%$ the proline incorporated in proteins (Hong-Qi et al., 1982). In the most striking case, reported by Schwacke et al. (1999), the content of free proline in tomato flowers was found to be 60 -fold higher than in any other organ analyzed. Within the floral organs, most of the free proline was found in pollen, where it represented over $70 \%$ of total free amino acids.

\section{Expression Pattern of Proline Metabolic and Transporter Genes}

In higher plants, proline is mainly synthesized in the cytoplasm from glutamate (Figure 2), which is converted into glutamic-semialdehyde (GSA) by the bi-functional enzyme $\delta^{1}$-pyrroline-5-carboxylate (P5C) synthetase (P5CS). GSA spontaneously cyclizes into $\mathrm{P} 5 \mathrm{C}$, which, in turn, is reduced to proline by the action of $\mathrm{P} 5 \mathrm{C}$ reductase (P5CR). Alternative routes of proline synthesis are possible from ornithine via ornithine $\delta$-aminotransferase $(\delta$-OAT) and, possibly, ornithine $\alpha$-aminotransferase $(\alpha$-OAT), but these alternative pathways have been shown to have no or little relevance in proline synthesis (Funck et al., 2008). The enzyme catalyzing the first and rate-limiting step of proline synthesis in higher plants, P5CS, is coded for in Arabidopsis by P5CS1 and P5CS2, two paralog genes sharing extensive sequence homology (Strizhov et al., 1997). Proline catabolism takes place in mitochondria, where proline is oxidized to glutamate by the sequential action of proline dehydrogenase (ProDH) and pyrroline-5-carboxylate dehydrogenase (P5CDH) (Figure 2). In most plant species several abiotic stresses, particularly salt and drought stress, induce accumulation of high levels of proline. At molecular level stress-induced proline accumulation is mainly due to the increased synthesis and reduced degradation of proline, accounted for by the contemporaneous upregulation of P5CS1, and downregulation of ProDH (Verbruggen and Hermans, 2008; Szabados and Savouré, 2010). Proline accumulation, however, also occurs under non-stressed conditions in specific developmental phases (Trovato et al., 2008), and is likely controlled by different regulatory conditions. In non-stressed conditions, the pattern of expression of P5CS1 and P5CS2 is complex and conflicting data have been reported from different groups. High expression of P5CS1 was initially detected in Arabidopsis flowers by Savouré et al. (1995) and Yoshiba et al. (1995) by Northern blot analysis. The existence of a second gene coding for P5CS that is subjected to differential regulation, was subsequently assessed by Strizhov et al. (1997), who found high P5CS1 expression, and, respectively, low P5CS2 expression in differentiated tissues of Arabidopsis, such as roots, stems, leaves and flowers. Though, the expression of P5CS2 was high in dividing cells from callus and cell cultures (Strizhov et al., 1997). In contrast, similar levels of both P5CS1 and P5CS2 transcripts were reported in flowers and embryos by Mattioli et al. (2009), and in embryos by Székely et al. (2008). However, although P5CS1 and P5CS2 transcripts may share a similar pattern of expression in embryos, only homozygous $p 5 c s 2$ mutants are embryo-lethal while $p 5 c s 1$ mutants are not, indicating that the 


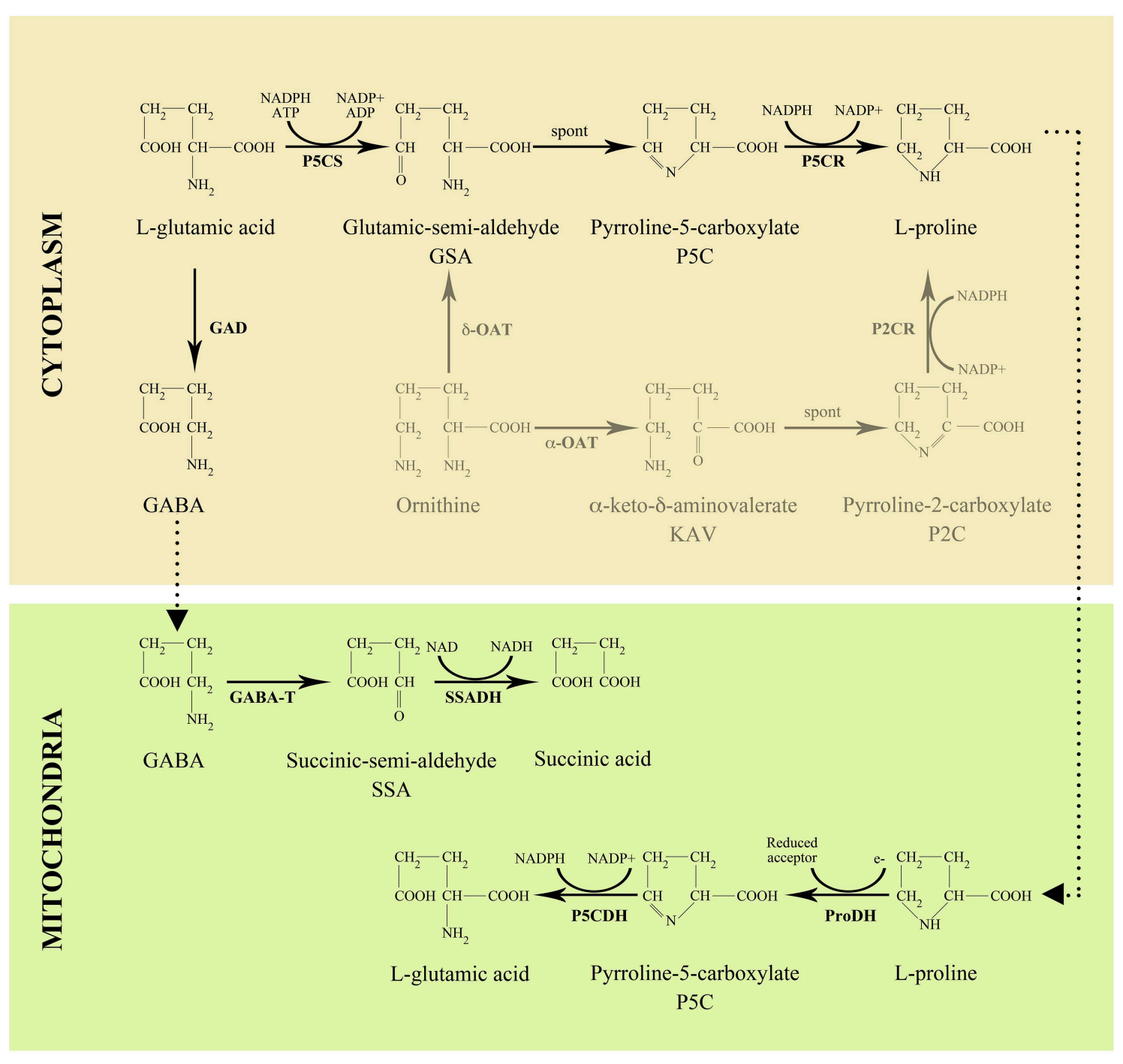

FIGURE 2 | Pathway of proline and GABA metabolism in higher plants.

embryo-lethality of $p 5 c s 2$ mutants is not caused by differential transcriptional regulation of the two P5CS genes. According to Székely et al. (2008), the striking difference between P5CS1 and P5CS2 in embryo development could be accounted for by a different sub-cellular localization of P5CS1 and P5CS2 proteins in embryonic cells. As observed by these authors, P5CS1-GFP exhibited a peculiar dotted pattern in embryonic cells, in contrast with the uniform cytoplasmic distribution of P5CS2-GFP. By high-resolution confocal laser scanning microscopy, P5CS1-GFP turned out to be sequestered into so far uncharacterized subcellular bodies, suggesting that the sequestered P5CS1 could be a non-functional enzyme unable to complement proline deficiency in homozygous $p 5 c s 2$ embryos. Consistent with transcript detection by in situ-hybridization, P5CS-GFP was also detected in inflorescence meristems, flower primordia, flower buds and anthers, although experiments with reporter genes should always be interpreted with caution.
Another strong confirmation of the importance of proline in the reproductive phase of angiosperms came from the observation that the genes coding for metabolic enzymes and transport proteins of the proline pathway (Figure 2) tend to be highly expressed in reproductive organs. Rentsch et al. (1996) reported that Proline Transporter 1 (ProT1), a gene coding for a specific proline transporter belonging to the amino acid permease (AAP) gene family, was highly expressed in Arabidopsis flowers, and was downregulated in the course of flower development, suggesting a role of ProT1 in flowers. Similarly Schwacke et al. (1999) reported that LeProT1, a tomato homolog of ProT1, was highly expressed in tomato flowers, as revealed by Northern blot analysis. The expression of LeProT1 increased steadily during flower development, and reached a peak at anther dehiscence. Moreover, through in situ hybridization performed on dissected flowers, these authors found that the expression of LeProT1 is restricted to anthers, consistent with the high concentration 
of proline measured in pollen grains. More recently Grallath et al. (2005) and Lehmann et al. (2011) reported that AtPro1, as well as AtPro3, a new member of the AtProT gene family, were strongly expressed in flowers, especially in mature pollen, suggesting that AtProT1 is important for flower development. In addition, AtProT1 expression was detected in the phloem or phloem parenchyma cells throughout the whole plant, indicating a possible role in long-distance proline transport. Interestingly, Grallath et al. (2005) also described that, contrary to previous reports, all ProT amino acid transporters could transport $\gamma$-aminobutyric acid (GABA), in addition to proline, although with lower efficiency. This finding raises the possibility that GABA as well as proline might be transported to floral tissues through the phloem.

Surprisingly, deletion of the gene ProT1 in Arabidopsis had no effect on the composition of the free amino acid pool in mature pollen, while it affected the contents of free glutamate and arginine, but not proline or GABA in germinating pollen (Lehmann et al., 2011). In addition, in spite of the altered concentrations of glutamate and arginine, pollen germination of prot1-1 mutants was normal, possibly because of a functional complementation by ProT3. Unfortunately, amino acid analyses of double or triple prot mutant pollen have not been reported yet.

The evidence that the variation of glutamate and arginine was only seen in germinating pollen from prot1-1 mutants may suggest that proline transport is especially or uniquely active during pollen germination. This hypothesis is consistent with independent pollen transcriptome analyses, which detected high ProT1 transcript abundance during late stages of pollen maturation, just before pollen germination (Honys and Twell, 2004; Bock et al., 2006). The variation of the amino acid composition of prot1-1 germinating pollen relative to wild type-with glutamate and arginine showing a lower and higher concentration, respectively-is more difficult to interpret. According to Shelp et al. (1999) in vitro germination and subsequent harvesting is stressful to germinating pollen and causes a raise of proline and GABA. Accordingly, we may speculate that under stress conditions, ProT3 may not be able to compensate for the lack of ProT1 in germinating prot1-1 pollen, causing a compensative induction of endogenous proline synthesis and, in turn, depletion of glutamate and increase of arginine.

In Arabidopsis, the genes coding for the enzymes mediating proline catabolism are also highly expressed in reproductive organs, and both ProDH and $\mathrm{P} 5 \mathrm{CDH}$ are more expressed in floral tissues, especially pollen, than in vegetative tissues. In 8week-old Arabidopsis plants expressing the gene uidA under the control of the ProDH promoter, GUS expression was observed in pollen, in mature stigmas with pollen, in stigma of immature siliques, in ovules, and in the abscission zone of sepals and petals (Nakashima et al., 1998). Similarly, strong GUS expression was detected in pollen grains of P5CDH-GUS transgenic Arabidopsis by Deuschle et al. (2004). In spite of the high level of ProDH and $\mathrm{P} 5 \mathrm{CDH}$ transcription driven in pollen by their respective promoters, the actual activities of these enzymes have not been analyzed to date, and their putative function in pollen remains unknown.

\section{New Insights in the Role of Proline in Pollen Development}

Recently, Arabidopsis mutants defective in proline biosynthetic enzymes were found to produce aberrant and infertile pollen grains, providing genetic and molecular support to the idea that proline is required for pollen development (Funck et al., 2012; Mattioli et al., 2012). T-DNA insertional mutants have been characterized for both P5CS1 (Mattioli et al., 2008; Székely et al., 2008) and P5CS2 (Székely et al., 2008; Mattioli et al., 2009), and their phenotypes provided information about the specific functions of P5CS1 and P5CS2 that have been summarized previously (Szabados and Savouré, 2010).

Homozygous mutants for $p 5 c s 2$ are embryo lethal under normal cultivation conditions (Székely et al., 2008; Mattioli et al., 2009), although Funck et al. (2012) were able to generate viable and fertile homozygous $p 5 c s 2$ mutants by in vitro cultivation of immature mutant seeds supplemented with exogenous proline and sucrose. In contrast, $p 5 c s 1$ p $5 c s 2$ double mutants, homozygous for both $p 5 c s 1$ and $p 5 c s 2$ mutant alleles, were not vital and could not be isolated. However, by using heterozygous double mutants, two groups independently reported defects in pollen development and fertility associated with proline deficiency in presumably double homozygous pollen (Funck et al., 2012; Mattioli et al., 2012). Both groups found that in reciprocal crosses between plants homozygous for $p 5 c s 1$ and heterozygous for $p 5 c s 2$ ( $p 5 c s 1 p 5 c s 2 / P 5 C S 2$ ) and wild type, the $p 5 c s 2$ mutant allele was not transmitted to the offspring when the proline-deficient $p 5 c s 1 p 5 c s 2 / P 5 C S 2$ plant was used as a pollen donor, but was detected in almost half of the progeny when p5cs 1 p5cs 2/P5CS2 flowers were used as a female. In addition, the dominant sulfadiazine resistance marker, linked to the $p 5 c s 2$ allele, was found to segregate in a 1:1 ratio, as expected in a gametophytic mutation, and the siliques of the self-pollinated population were found to be devoid of aberrant embryos, as expected if a gametophytic defect should prevent the formation of homozygous embryos (Funck et al., 2012; Mattioli et al., 2012). Morphological and functional analysis of the mutant pollen revealed, at least from stage 11 of pollen development, a population of small, progressively degenerating pollen grains, representing about half of the total pollen population. The aberrant pollen grains appeared misshaped, shriveled and no longer viable, as revealed by orcein staining, DAPI analysis, Alexander's staining, and in vitro pollen germination assays. As expected for a mutant carrying mutations in both the genes encoding P5CS, the partial double mutant $p 5 c s 1$ p5cs2/P5CS2 accumulates very little proline, on average one fourth as much as a wild type, and this proline shortage is the likely cause of the developmental defects of pollen grains from $p 5 c s 1$ p5cs2/P5CS2 mutants. Accordingly, the content of free proline measured in pollen grains from $p 5 c s 1 p 5 c s 2 / P 5 C S 2$ mutants was $105 \pm 23 \mathrm{ng}$, roughly corresponding to $15 \mathrm{pg} /$ pollen of proline, compared to $336 \pm 31 \mathrm{ng}$, roughly corresponding to $48 \mathrm{pg} / \mathrm{pollen}$, measured in pollen grains from Col-0 wild type (Mattioli et al., 2012). Importantly, $10 \mu \mathrm{M}$ L-proline, supplemented in planta to developing anthers of $p 5 c s 1$ p $5 c s 2 / P 5 C S 2$ mutants, significantly complemented the morphological and physiological defects of 
pollen grains from $p 5 c s 1$ p5cs2/P5CS2 mutants (Mattioli et al., 2012). Surprisingly, pollen carrying a mutation in the single copy of P5CR, the gene coding for the second and final step in proline biosynthesis, did not show developmental or fertility defects (Funck et al., 2012). Although proline is clearly required for pollen development and fertility, the relative role of proline coming from, or synthesized in, gametophytic (i.e., pollen grains) and sporophytic (i.e., tapetum) tissues is yet to be clarified. Intriguingly the presence of the p5cs2 mutant allele was detected, by PCR analysis, also in the wild type-like population of pollen grains from $p 5 c s 1 p 5 c s 2 / P 5 C S 2$ mutants (Mattioli et al., 2012), suggesting that the phenotypic aberrations observed in pollen grains from $p 5 c s 1$ p5cs2/P5CS2 flowers, may not, or not only, have a genetic basis, which prompts an investigation of the relevance of proline coming from either sporophytic or gametophytic tissues. Equally unknown, at present, is the role of proline in pollen development. Several authors (Chiang and Dandekar, 1995; Székely et al., 2008) have proposed that proline accumulation may function to protect macromolecular structures in pollen grains during dehydratation, essentially because proline is a well-known compatible osmolyte and because a mature pollen grain is a highly dehydrated structure. Although this is a likely hypothesis, it still lacks a formal demonstration, and other functions, such as energy source or signaling molecule, are equally possible and are being actively investigated.

\section{Proline and Pollination: To Bee or Not to Bee}

Intriguingly, proline has been also associated with pollination, another aspect of sexual reproduction of angiosperms, since honeybees were reported to show a strong preference for prolineenriched nectars (Carter et al., 2006; Bertazzini et al., 2010). To ensure efficient pollination, numerous angiosperms depend upon pollinators, usually birds or flying insects, to transfer the mature gametophyte from the dehisced anther to a receptive stigma. Pollinators are attracted to pollen grains by the use of specialized strategies co-evolved with prevalent pollinator species. In most of the cases flowers have evolved bright and vivid colors, fragrant scents and sweet and nourishing nectars to attract insects, particularly butterflies and honeybees. Once a pollinator lands on a flower, during its daily foraging activity, pollen grains get stuck on the pollinator to be transported from flower to flower to accomplish a high degree of cross-pollination. However, many of the pollinating insects also actively collect the nutrientand proline-rich pollen itself, especially to support their own reproduction and growth. By collecting a significant proportion of the pollen, the pollinators force the plants to produce pollen in large excess over the need for pollination.

Key to pollination activity is nectar, an aqueous sugar solution mainly derived from phloem sap and synthesized in specialized cells, located at the base of the ovary and known as nectaries. Within these specialized floral cells, phloem sap is enzymatically processed and transformed into nectar. A relevant fraction of the sucrose present in the phloem, for instance, is usually hydrolyzed to glucose and fructose. However, not all the components of the phloem sap are modified and in several instances nectar composition simply reflects the composition of phloem sap, as in the case of proline, which mainly derives from the proline present in phloem sap and actively transported from vegetative to reproductive tissues.

The molecular and genetic basis of the dynamic interaction between pollinators and proline content in nectar is unknown, but it might be mediated by pollination signals similarly to the coordination between pollination and nectar production, which has been shown to be coordinated either by jasmonic acid (Heil, 2004; Radhika et al., 2010) or auxin (Bender et al., 2013). Regardless of environmental cues, nectar production and secretion are under strict developmental control to ensure coordination between pollen shed and nectar secretion and to minimize energy waste. It has been calculated that nectar production consumes up to $37 \%$ of a plant's available energy (Pyke, 1991). Consistently a large number of genes involved in regulating nectar composition and secretion are timely expressed in nectaries or adjacent tissues. As an example, immature nectaries are devoid of invertase and accumulate starch in early developmental phases. Around anthesis CELL WALL INVERTASE 4 (AtCWINV4), becomes strongly induced in nectaries allowing them to accumulate the glucose and fructose necessary for nectar production (Ruhlmann et al., 2010). Accordingly, because proline homeostasis derives from a finetuning among proline synthesis, degradation and transport, the production of nectars enriched in proline likely implies a complex developmental and tissue-specific regulation of proline metabolism. Even if proline is predominantly delivered by the phloem sap, a contribution of local proline synthesis in the nectaries from glutamate cannot be role rule out. In support of this possibility, microarray and RNA sequencing data from Arabidopsis nectaries revealed significant expression of P5CS2 in mature nectaries (Kram et al., 2009; Bender et al., 2013).

Honeybees, the most efficient and wide-spread pollinators on a world scale, use nectar as their primary carbohydrate, amino acid and energy source, to provide energy for flight, colony maintenance, general daily activities and nutrient storage for the winter. There is an intimate relationship between nectar and honeybees as, on one hand, nectar is essential for honeybee's life and, on the other hand, plants need the foraging activity of honeybees for cross-pollination, and eventually species survival. The attractiveness of nectars for honeybees has important practical implications since a more attractive nectar will induce bees to show a higher fidelity to flowers of the same species and more cross-fertilized seeds will be produced, increasing, in turn, crop productivity (Allen-Wardell et al., 1998). The importance of pollinator-driven cross-fertilization might be questioned for the survival of autogamous plant species, such as Arabidopsis. However, despite the advantages of self-pollination over cross-pollination in some ecological niches, occasional cross-pollination is still advantageous even in autogamous species, most of which are facultative autogamous plants (Goodwillie et al., 2005).

In addition, the quality and quantity of the honey, which bees produce from nectar, is critically dependent on the nectars produced by different plant species. Not all nectars, however, 
are equally attracting and nutritive, and marked differences exist among nectars from different plant species, which deeply orient honeybee's foraging activities to selected plant species. Nectar composition is extremely variable and is mainly composed of sugars, especially sucrose, glucose and fructose (Baker and Baker, 1973; Jackson and Nicolson, 2002), as well as of a number of other compounds including proteins, lipids, organic acids, alkaloids, phenolics, terpens, flavonoids, vitamins, and amino acids (Ball, 2007). Nectars also contain specific plant-defense proteins that are thought to protect the gynoecium from microbial invasion brought about by pollinators and wind (Peumans et al., 1997; Carter et al., 1999; Carter and Thornburg, 2000, 2004; Thornburg et al., 2003; Naqvi et al., 2005). Although the amino acid pool is a minor component of the nectar sap, ranging from 0.002 to $4.8 \%$ of organic matter (Gardener and Gillman, 2001), it is of relevant biological importance, at least for insects, and it has been shown that nectars from plants pollinated by insects have higher amino acid concentration than nectars from plants pollinated by birds (Baker and Baker, 1986). In addition, the quality and quantity of nectar's amino acids are believed to enhance insect longevity and fecundity (Mevi-Schutz and Erhardt, 2005). Proline, in particular, has been shown to be the most abundant amino acid in the nectar of many angiosperms (Gardener and Gillman, 2002; Kaczorowski et al., 2005; Carter et al., 2006; Terrab et al., 2007). Furthermore, it has been reported that honeybees exhibit a strong preference toward nectars rich in the amino acid proline. According to Carter et al. (2006), honeybees prefer proline-rich nectars over nectars lacking or poor in proline, and can specifically perceive and track proline-rich nectars or sugar solutions supplied with proline. The honeybees' ability to "taste" proline relies on the stimulation of the salt cells of labellar chemosensory cells, and equivalent cells are found on the sensory apparatus of further insect taxa, including hymenoptera and lepidoptera (Hansen et al., 1998; Gardener and Gillman, 2002). The strong preference that honeybees seem to grant to proline-rich nectars may rely on the energy property of this amino acid, which has been proposed as the main fuel employed by honeybees during the earliest or more expensive stages of flight (Micheu et al., 2000). Indeed, the complete oxidation of one molecule of proline yields 21 ATP molecules, almost as many as the 24 ATP molecules obtained from complete oxidation of one molecule of glucose, but obtained with no initial ATP consumption (Micheu et al., 2000). Bertazzini et al. (2010) have confirmed and extended these reports by analyzing, by dual choice feeding tests, the preference of forager honeybees for specific amino acids. They found that honeybees prefer artificial nectar containing proline over artificial nectar containing only sugars. On the contrary, nectars containing either alanine or serine gave neutral or negative responses. Importantly, when honeybees were given the choice between nectars supplemented with different amino acids, proline was preferred above both alanine and serine, and alanine above serine. While sugars (or generally energy and organic carbon) are usually not a limiting resource for plant growth, the nitrogen contained in amino acids usually is. More detailed studies will be required to reveal whether plants enrich their nectar in proline to make it especially nutritious and thus attractive for insects, or whether they try to cheat on the insects by specifically enriching proline as the amino acid that insects can best detect in the nectar.

\section{GABA and Pollen Tube Guidance}

Another glutamate derivative critically important for plant reproduction is GABA, a non-proteinogenic amino acid ubiquitously present in plants and implicated in $\mathrm{pH}$ regulation, plant development and pathogen defense (Shelp et al., 1999). GABA is also regarded as a stress molecule involved in the metabolic responses of plants to either abiotic stresses (Mazzucotelli et al., 2006; Michaeli and Fromm, 2015), such as low light (Michaeli et al., 2011) and salt (Renault et al., 2013), or pathogen attack (Forlani et al., 2014).

GABA is directly produced from glutamate in the cytosol by the action of glutamate decarboxylase (GAD; Figure 2). GABA degradation takes place in the mitochondrion where it is converted to succinic acid by the sequential action of GABA transaminase (GABA-T) and succinic semialdehyde dehydrogenase (SSADH) (Shelp et al., 1999). A large body of evidence points to GABA as a molecule involved, at least in some species, in pollen tube growth and guidance. In flowering plants, after pollen grains are deposited on the stigma surface of a diploid female pistil, the pollen starts germination and begins to elongate a pollen tube, a cytoplasmic protrusion through which the two male sperm cells are polarly transported to the elongating tip and finally delivered to the embryo sack. Unlike animal sperms and spermatocytes of primitive plants, such as bryophytes, pollen grains from higher plants have lost their flagella during evolution, and cannot swim anymore, relying completely on pollen tube elongation and intracellular transport to reach the embryo sac and fulfill fertilization. The elongation of the pollen tube starts at a receptive stigma, continues along the style through several different cell layers, and is eventually guided to an ovule micropyle, to enter the ovule. Once the tip of the pollen tube has reached the target, the two sperm cells are released to fuse with the embryo sac, and eventually produce a diploid zygote and the triploid endosperm nucleus. As inferred by the number of mutants affecting pollen guidance (Higashiyama and Takeuchi, 2015), this developmental process is critical for plant reproduction, and of unexpected complexity. Although a simple mechanical guidance has been reported in some species (Higashiyama and Takeuchi, 2015), pollen tubes are usually guided to their target by a number of signal and attractant molecules, such as $\mathrm{Ca}^{++}$(Iwano et al., 2014), lipids (Wolters-Arts et al., 1998), pollen coat proteins (Mayfield et al., 2001), arabinogalactans (Wu et al., 2000; Suárez et al., 2013), pectins (Mollet et al., 2000), secreted cystein-rich proteins (Okuda et al., 2009), or brassinosteroids (Vogler et al., 2014). Consistently, transcriptomic analyses carried out both in Arabidopsis thaliana and in Torenia fourneri pollen tubes have discovered an extraordinarily high number of genes involved in pollen tube elongation, and, above all, that pollen tubes modify gene expression profiles during their growth along the pistil (Qin et al., 2009; Okuda et al., 2013).

A novel role for GABA in pollen tube growth and guidance was found out by Palanivelu et al. (2003) by characterizing the 
A. thaliana POP2 gene, coding for a GABA-T. Previously, the pop2 mutation was observed to cause a late-stage defect in the elongation of the pollen tube, which bypassed the micropyle and thus failed to hit the ovule cells (Wilhelmi and Preuss, 1996). Consistently with a role of POP2 in GABA degradation, pop2 flowers exhibited GABA accumulation-as much as 60 fold higher in pop2-1 flowers than in wild type flowers-leading to infertility because pop 2 pollen tube growth was arrested or misguided in $p \circ p 2$ pistils. Fertility rate and floral GABA levels were found inversely correlated across different pop2 alleles (Palanivelu et al., 2003) suggesting that the infertility of the pop2 homozygous mutants might be associated with an aberrant accumulation of GABA along the style. Interestingly, however, wild type pollen was able to fertilize flowers of pop2 mutants, indicating that the egg and other embryo sac cells are functional. The higher success rate of wild type over pop2 mutant pollen additionally indicates that GABA processing by GABA-T within the pollen tube is essential for guidance toward the embryo sack. By a combination of enzymatic and immunohistochemical methods, a GABA gradient, with increasing concentrations from stigma to micropyle, was identified in wild type pistils, but not in pop2 pistils that showed steadily high levels of GABA all along the pistil. In addition, in in vitro germination assays, a large excess of exogenous GABA was inhibitory to pollen tube growth, although at low concentrations GABA had a stimulatory effect (Palanivelu et al., 2003). For wild type, GABA concentrations ranging from 1 to $10 \mathrm{mM}$ stimulated pollen tube elongation, while higher concentrations were inhibitory. In contrast, pop2 pollen tubes were stimulated at GABA concentrations as low as $1-10 \mu \mathrm{M}$, and were inhibited at concentrations higher than $10 \mu \mathrm{M}$ (Palanivelu et al., 2003). The establishment of a correct GABA gradient, therefore, is necessary to assist fertilization by guiding the growing pollen tube from the stigma surface to the ovule micropyle, in a way that resembles the guidance function of GABA in animal systems, where the migrating neuronal precursor cells of spinal and cortical neurons reach their target destinations with the help of GABA receptors and $\mathrm{Ca}^{++}$signaling (Behar et al., 2001).

As in animals, the mechanism of GABA guidance of pollen tube seems in fact to rely on $\mathrm{Ca}^{++}$signaling. Yu et al. (2014) measured the dynamics of $\mathrm{Ca}^{++}$fluxes, by means of whole-cell voltage-clamp experiments and non-invasive micromeasurement technology, and proposed that the influx of $\mathrm{Ca}^{++}$increased in pollen tubes in response to exogenous GABA. In addition, since the $\mathrm{Ca}^{++}$responses to GABA were specifically blocked by $\mathrm{Gd}^{3+}$, a specific inhibitor of $\mathrm{Ca}^{++}$-permeable channels, the authors concluded that signaling triggered by exogenous GABA affects pollen tube growth by modulating putative $\mathrm{Ca}^{++}$-permeable membrane channels.

Recently, the activity of an aluminum-activated malate transporter in wheat (TaALMT1, Ramesh et al., 2015), belonging to a large family of plant anion channels/transporters (ALMT) was shown to be regulated by GABA. ALMTs of six plant species were regulated by GABA, confirming that GABA acts as a signaling molecule by regulating ion fluxes across cell membranes in plants, as it does in animals. Ramesh et al. (2015) demonstrated that malate flux through TaALMT1 is stimulated by anions and inhibited by GABA leading to increased or reduced root growth, respectively, most probably mediated by changes in the membrane potential. Interestingly, malate flux and, in turn, root growth were specifically inhibited by muscimol-a GABA analog working as a specific agonist of mammalian $\mathrm{GABA}_{\mathrm{A}}$ receptors (Olsen and Sieghart, 2008). In addition, the effect of muscimol was attenuated by treatment with bicuculline-a well-known competitive antagonist of GABA binding to mammalian $\mathrm{GABA}_{\mathrm{A}}$ receptors (Olsen and Sieghart, 2008).

Overall these results indicate that GABA exerts at least some of its multiple physiological effects in plants, including pollen tube guidance and root growth, through modulation of ALMT activity, and that GABA can finally be regarded as a genuine signaling molecule in plant, as it is in animals. Accordingly Ramesh et al. (2015) provided evidence that also pollen tube elongation and guidance depend on GABA-gated activity of ALMTs, since muscimol reduced A. thaliana and Vitis vinifera pollen tube elongation in vitro, while bicuculline antagonized muscimol regulation.

\section{Conclusions and Future Perspectives}

The overall picture that emerges from this review is the surprising and specific importance of proline and GABA in aspects of plant reproduction as important as pollen development, pollination, and pollen tube guidance. Egg cell development seems much more tolerant to disturbances in proline and GABA metabolism, potentially because the embryo sack is in closer contact to the parental tissue. For each one developmental process, however, much work has still to be done and we have more open questions than precise answers available. For instance, during pollen development and fertilization the importance of proline synthesized within the male gametophyte, from unicellular microspore to tricellular pollen, compared to proline that may be provided by sporophytic tissues, is still unclear. In addition, the precise function of proline in pollen development and fertility remains, as yet, elusive.

With regard to the role of proline in pollination, by contrast, it is generally believed that the high energetic value of proline has been the underlying reason for the actual preference of honeybees for proline-rich nectars. It remains to be seen, however, how strong this preference is, and its ecologic and agronomic impact on crops and cultivations.

As to the role of GABA in pollen guidance, in spite of the recent advances of our knowledge on GABA signaling, further work is still needed in diverse areas of research. A major field of interest will be the identification and characterizations of novel activators, agonists and antagonists of plant ALMTs as well as of second messengers and downstream genes, to hopefully improve our knowledge of GABA signaling and to explore the possibility of manipulating GABA-mediated developmental processes. Equally important would be the crystallization of the ALMT receptor either free or bound to GABA or GABA analogs to understand the action of these drugs and the underlying mechanism of GABA signaling. Finally, the question emerges whether or not the functions of the glutamate-derived amino 
acids proline and GABA in reproduction may be interconnected. Further work is needed to address these fascinating questions.

\section{Author Contributions}

All authors have contributed significantly and have approved the final manuscript.

\section{References}

Allen-Wardell, G., Bernhardt, P., Bitner, R., Burquez, A., Buchmann, S., and Cane, J. (1998). The potential consequences of pollinator declines on the conservation of biodiversity and stability of food crop yields. Conserv. Biol. 12, 8-17. doi: 10.1046/j.1523-1739.1998.97154.x

Auclair, J. L., and Jamieson, C. A. (1948). A qualitative analysis of amino acids in pollen collected by bees. Science 108, 357-358. doi: 10.1126/science.108.2805.357

Baker, H. G., and Baker, I. (1973). Amino acids in nectar and their evolutionary significance. Nature 241, 543-545. doi: 10.1038/241543b0

Baker, H. G., and Baker, I. (1986). The occurrence and significance of amino acids in floral nectar. Plant Syst. Evol. 151, 175-186. doi: 10.1007/BF02430273

Ball, D. W. (2007). The chemical composition of honey. J. Chem. Educ. 84, 1643-1646. doi: 10.1021/ed084p1643

Bathurst, N. O. (1954). The amino acids of grass pollen. J. Exp. Bot. 5, 253-256. doi: $10.1093 / \mathrm{jxb} / 5.2 .253$

Behar, T. N., Smith, S. V., Kennedy, R. T., McKenzie, J. M. M., Maric, I., and Barker, J. L. (2001). $\mathrm{GABA}_{\mathrm{B}}$ receptors mediate motility signals for migrating embryonic cortical cells. Cereb. Cortex 11, 744-753. doi: 10.1093/cercor/ 11.8.744

Bender, R. L., Fekete, M. L., Klinkenberg, P. M., Hampton, M., Bauer, B., Malecha, M., et al. (2013). PIN6 is required for nectary auxin response and short stamen development. Plant J. 74, 893-904. doi: 10.1111/tpj.12184

Bertazzini, M., Medrzycki, P., Bortolotti, L., Maistrello, L., and Forlani, G. (2010). Amino acid content and nectar choice by forager honeybees (Apis mellifera $\mathrm{L}$.). Amino Acids 39, 315-318. doi: 10.1007/s00726-010-0474-x

Bock, K. W., Honys, D., Ward, J. M., Padmanaban, S., Nawrocki, E. P., Hirschi, K. D., et al. (2006). Integrating membrane transport with male gametophyte development and function through transcriptomics. Plant Physiol. 140, 1151-1168. doi: 10.1104/pp.105.074708

Carter, C., Graham, R. A., and Thornburg, R. W. (1999). Nectarin I is a novel germin-like protein expressed in the nectar of Nicotiana sp. Plant Mol. Biol. 41, 207-216. doi: 10.1023/A:1006363508648

Carter, C., Shafir, S., Yehonatan, L., Palmer, R. G., and Thornburg, R. (2006). A novel role for proline in plant floral nectars. Naturwiss 93, 72-79. doi: 10.1007/s00114-005-0062-1

Carter, C., and Thornburg, R. W. (2000). Tobacco Nectarin I: purification and characterization as a germin-like manganese superoxide dismutase implicated in the defense of floral reproductive tissues. J. Biol. Chem. 275, 36726-36733. doi: 10.1074/jbc.M006461200

Carter, C., and Thornburg, R. W. (2004). Is the nectar redox cycle a floral defense against microbial attack? Trends Plant Sci. 9, 320-324. doi: 10.1016/j.tplants.2004.05.008

Chiang, H. H., and Dandekar, A. M. (1995). Regulation of proline accumulation in Arabidopsis during development and in response to dessication. Plant Cell Environ. 18, 1280-1290. doi: 10.1111/j.1365-3040.1995.tb00187.x

Deuschle, K., Funck, D., Forlani, G., Stransky, H., Biehl, A., Leister, D., et al. (2004). The role of $\delta^{1}$-pyrroline-5-carboxylate dehydrogenase in proline degradation. Plant Cell 16, 3413-3425. doi: 10.1105/tpc.104.023622

Forlani, G., Bertazzini, M., and Giberti, S. (2014). Differential accumulation of $\gamma$-aminobutyric acid in elicited cells of two rice cultivars showing contrasting sensitivity to the blast pathogen. Plant Biol. 16, 1127-1132. doi: $10.1111 / \mathrm{plb} .12165$

Funck, D., Stadelhofer, B., and Koch, W. (2008). Ornithine- $\delta$-aminotransferase is essential for arginine catabolism but not for proline biosynthesis. BMC Plant Biol. 8:40. doi: 10.1186/1471-2229-8-40

\section{Acknowledgments}

This work was partially supported by research grants from the Ministero dell'Istruzione, Università e Ricerca, Progetti di Ricerca di Interesse Nazionale, from Sapienza Università di Roma to PC, and by grants from Università La Sapienza to MT.

Funck, D., Winter, G., Baumgarten, L., and Forlani, G. (2012). Requirement of proline synthesis during Arabidopsis reproductive development. BMC Plant Biol. 12:191. doi: 10.1186/1471-2229-12-191

Gardener, M. C., and Gillman, M. P. (2001). Analyzing variability in nectar amino acids: composition is less important than concentration. J. Chem. Ecol. 27, 2545-2558. doi: 10.1023/A:1013687701120

Gardener, M. C., and Gillman, M. P. (2002). The taste of nectar-a neglected area of pollination. Oikos 98, 552-557. doi: 10.1034/j.1600-0706.2002.980322.x

Goodwillie, C., Kalisz, S., and Eckert, C. G. (2005). The evolutionary enigma of mixed mating systems in plants: occurrence, theoretical explanations, and empirical evidence. Annu. Rev. Ecol. Evol. Syst. 36, 47-79. doi: 10.1146/annurev.ecolsys.36.091704.175539

Grallath, S., Weimar, T., Meyer, A., Gumy, C., Suter-Grotemeyer, M., Neuhaus, J.-M., et al. (2005). The AtProT family. Compatible solute transporters with similar substrate specificity but differential expression patterns. Plant Physiol. 137, 117-126. doi: 10.1104/pp.104.055079

Hansen, K., Wacht, S., Seebauer, H., and Schnuch, M. (1998). New aspects of chemoreception in flies. Ann. N.Y. Acad. Sci. 855, 143-147. doi: 10.1111/j.17496632.1998.tb10556.x

Hayat, S., Hayat, Q., Alyemeni, M. N., Wani, A. S., Pichtel, J., and Ahmad, A. (2012). Role of proline under changing environments. Plant Signal. Behav. 7 , 1456-1466. doi: 10.4161/psb.21949

Heil, M. (2004). Induction of two indirect defences benefits Lima bean (Phaseolus lunatus, Fabaceae) in nature. J. Ecol. 92, 527-536. doi: 10.1111/j.00220477.2004.00890.x

Higashiyama, T., and Takeuchi, H. (2015). The mechanism and key molecules involved in pollen tube guidance. Annu. Rev. Plant Biol. 66, 393-413. doi: 10.1146/annurev-arplant-043014-115635

Hong-Qi, Z., Croes, A. F., and Linskens, H. F. (1982). Protein synthesis in germinating pollen of Petunia: role of proline. Planta 154, 199-203. doi: 10.1007/BF00387864

Honys, D., and Twell, D. (2004). Transcriptome analysis of haploid male gametophyte development in Arabidopsis. Genome Biol. 5:R85. doi: 10.1186/gb-2004-5-11-r85

Iwano, M., Igarashi, M., Tarutani, Y., Kaothien-Nakayama, P., Nakayama, H., Moriyama, H., et al. (2014). A pollen coat- inducible autoinhibited $\mathrm{Ca}^{2+}$-ATPase expressed in stigmatic papilla cells is required for compatible pollination in the Brassicaceae. Plant Cell 26, 636-649. doi: $10.1105 /$ tpc.113.121350

Jackson, S., and Nicolson, S. W. (2002). Xylose as a nectar sugar: from biochemistry to ecology. Comp. Biochem. Physiol. 131, 613-620. doi: 10.1016/S10964959(02)00028-3

Kaczorowski, R. L., Gardener, M. C., and Holtsford, T. P. (2005). Nectar traits in Nicotiana section Alatae (Solanaceae) in relation to floral traits, pollinators and mating system. Am. J. Bot. 92, 1270-1283. doi: 10.3732/ajb.92.8.1270

Kram, B. W., Xu, W. W., and Carter, C. J. (2009). Uncovering the Arabidopsis thaliana nectary transcriptome: investigation of differential gene expression in floral nectariferous tissues. BMC Plant Biol. 9:92. doi: 10.1186/1471-2229-9-92

Lehmann, S., Gumy, C., Blatter, E., Boeffel, S., Fricke, W., and Rentsch, D. (2011). In planta function of compatible solute transporters of the AtProT family. J. Exp. Bot. 62, 787-796. doi: 10.1093/jxb/erq320

Mariani, C., De Beuckeleer, M., Truettner, J., Leemans, J., and Goldberg, R. B. (1990). Induction of male sterility in plants by a chimeric ribonuclease gene. Nature 357, 737-741. doi: 10.1038/347737a0

Mattioli, R., Biancucci, M., Lonoce, C., Costantino, P., and Trovato, M. (2012). Proline is required for male gametophyte development in Arabidopsis. BMC Plant Biol. 12:236. doi: 10.1186/1471-2229-12-236 
Mattioli, R., Falasca, G., Sabatini, S., Altamura, M. M., Costantino, P., and Trovato, M. (2009). The proline biosynthetic genes P5CS1 and P5CS2 play overlapping roles in Arabidopsis flower transition but not in embryo development. Physiol. Plant. 137, 72-85. doi: 10.1111/j.1399-3054.2009.01261.x

Mattioli, R., Marchese, D., D’Angeli, S., Altamura, M. M., Costantino, P., and Trovato, M. (2008). Modulation of intracellular proline levels affects flowering time and inflorescence architecture in Arabidopsis. Plant Mol. Biol. 66, 277-288. doi: 10.1007/s11103-007-9269-1

Mayfield, J. A., Fiebig, A., Johnstone, S. E., and Preuss, D. (2001). Gene families from the Arabidopsis thaliana pollen coat proteome. Science 292, 2482-2485. doi: $10.1126 /$ science. 1060972

Mazzucotelli, E., Tartari, A., Cattivelli, L., and Forlani, G. (2006). Metabolism of $\gamma$-aminobutyric acid during cold acclimation and freezing and its relationship to frost tolerance in barley and wheat. J. Exp. Bot. 57, 3755-3766. doi: 10.1093/jxb/erl141

Mevi-Schutz, J., and Erhardt, A. (2005). Amino acids in nectar enhance butterfly fecundity: a long-awaited link. Am. Nat. 165, 411-420. doi: 10.1086/429150

Michaeli, S., Fait, A., Lagor, K., Nunes-Nesi, A., Grillich, N., Yellin, A., et al. (2011). A mitochondrial GABA permease connects the GABA shunt and the TCA cycle, and is essential for normal carbon metabolism. Plant J. 67, 485-498. doi: 10.1111/j.1365-313X.2011.04612.x

Michaeli, S., and Fromm, H. (2015). Closing the loop on the GABA shunt in plants: are GABA metabolism and signaling entwined? Front. Plant Sci. 6:419. doi: 10.3389/fpls.2015.00419

Micheu, S., Crailsheim, K., and Leonhard, B. (2000). Importance of proline and other amino acids during honeybee flight (Apis mellifera carnica POLLMANN). Amino Acids 18, 157-175. doi: 10.1007/s007260050014

Mollet, J. C., Park, S. Y., Nothnagel, E. A., and Lord, E. M. (2000). A lily stylar pectin is necessary for pollen tube adhesion to an in vitro stylar matrix. Plant Cell 12, 1737-1750. doi: 10.1105/tpc.12.9.1737

Mutters, R. G., Ferreira, L. G. R., and Hall, A. E. (1989). Proline content of the anthers and pollen of heat-tolerant and heat-sensitive cowpea subjected to different temperatures. Crop Sci. 29, 1497-1500. doi: 10.2135/cropsci1989.0011183X002900060036x

Nakashima, K., Satoh, R., Kiyosue, T., Yamaguchi-Shinozaki, K., and Shinozaki, K. (1998). A gene encoding proline dehydrogenase is not only induced by proline and hypoosmolarity, but is also developmentally regulated in the reproductive organs of Arabidopsis. Plant Physiol. 118, 1233-1241. doi: 10.1104/pp.118.4.1233

Naqvi, S. M. S., Harper, A., Carter, C., Ren, G., Guirgis, A., York, W. S., et al. (2005). Nectarin IV, a potent endoglucanase inhibitor secreted into the nectar of ornamental tobacco plants. Isolation cloning and characterization. Plant Physiol. 139, 1389-1400. doi: 10.1104/pp.105.065227

Okuda, S., Suzuki, T., Kanaoka, M., Mori, H., Sasaki, N., and Higashiyama, T. (2013). Acquisition of LURE-binding activity at the pollen tube tip of Torenia fournieri. Mol. Plant 6, 1074-1090. doi: 10.1093/mp/sst050

Okuda, S., Tsutsui, H., Shiina, K., Sprunck, S., Takeuchi, H., Yui, R., et al. (2009). Defensin-like polypeptide LUREs are pollen tube attractants secreted from synergid cells. Nature 458, 357-361. doi: 10.1038/nature07882

Olsen, R. W., and Sieghart, W. (2008). International Union of Pharmacology LXX. Subtypes of $\gamma$-aminobutyric acidA receptors: classification on the basis of subunit composition, pharmacology, and function. Update. Pharmacol. Rev. 60, 243-260. doi: 10.1124/pr.108.00505

Palanivelu, R., Brass, L., Edlund, A. F., and Preuss, D. (2003). Pollen tube growth and guidance is regulated by POP2, an Arabidopsis gene that controls GABA levels. Cell 114, 47-59. doi: 10.1016/S0092-8674(03)00479-3

Peumans, W. J., Smeets, K., Van Nerum, K., Van Leuven, F., and Van Damme, E. J. M. (1997). Lectin and alliinase are the predominant proteins in nectar from leek (Allium porrum L.) flowers. Planta 201, 298-301. doi: 10.1007/s0042500 50070

Pyke, G. H. (1991). What does it cost a plant to produce floral nectar? Nature 350, 58-59. doi: 10.1038/350058a0

Qin, Y., Leydon, A. R., Manziello, A., Pandey, R., Mount, D., Denic, S., et al. (2009). Penetration of the stigma and style elicits a novel transcriptome in pollen tubes, pointing to genes critical for growth in a pistil. PLOS Genet. 5:e1000621. doi: 10.1371/journal.pgen.1000621

Radhika, V., Kost, C., Boland, W., and Heil, M. (2010). The role of jasmonates in floral nectar secretion. PLOS ONE 5:e9265. doi: 10.1371/journal.pone.0009265
Ramesh, S. A., Tyerman, S. D., Xu, B., Bose, J., Kaur, S., Conn, V., et al. (2015). GABA signalling modulates plant growth by directly regulating the activity of plant-specific anion transporters. Nat Commun. 6, 7879. doi: $10.1038 /$ ncomms 8879

Renault, H., El Amrani, A., Berger, A., Mouille, G., Soubigou-Taconnat, L., Bouchereau, A., et al. (2013). $\gamma$-aminobutyric acid transaminase deficiency impairs central carbon metabolism and leads to cell wall defects during salt stress in Arabidopsis roots. Plant Cell Environ. 36, 1009-1018. doi: $10.1111 /$ pce. 12033

Rentsch, D., Hirner, B., Schmelzer, H., and Frommer, W. (1996). Salt stress-induced proline transport and salt stress-repressed broad specificity amino acid permeases ldentified by suppression of a yeast amino acid permease-targeting mutant. Plant Cell 8, 1437-1446. doi: 10.1105/tpc. 8.8.1437

Ruhlmann, J. M., Kram, B. W., and Carter, C. J. (2010). CELL WALL INVERTASE 4 is required for nectar production in Arabidopsis. J. Exp. Bot. 61, 395-404. doi: 10.1093/jxb/erp309

Sangwan, R. S. (1978). Change in the amino-acid content during male gametophyte formation of Datura metel in situ. Theor. Appl. Genet. 52, 221-225. doi: 10.1007/BF00273893

Savouré, A., Jaoua, S., Hua, X. J., Ardiles, W., Van Montagu, M., and Verbruggen, N. (1995). Isolation and characterization, and chromosomal location of a gene encoding the $\delta^{1}$-pyrroline- 5-carboxylate synthetase in Arabidopsis. FEBS Lett 372, 13-19. doi: 10.1016/0014-5793(95) 00935-3

Schwacke, R., Grallath, S., Breitkreuz, K. E., Stransky, H., Frommer, W. B., and Rentsch, D. (1999). LeProT1, a transporter for proline, glycine betaine, and $\gamma$-amino butyric acid in tomato pollen. Plant Cell 11, 377-391. doi: $10.2307 / 3870867$

Shelp, B. J., Bown, A. W., and McLean, M. D. (1999). Metabolism and functions of gamma-aminobutyric acid. Trends Plant Sci. 4, 446-452. doi: 10.1016/S13601385(99)01486-7

Strizhov, N., Ábrahám, E., Ökrész, L., Blickling, S., Zilberstein, A., Schell, J., et al. (1997). Differential expression of two P5CS genes controlling proline accumulation during salt-stress requires $\mathrm{ABA}$ and is regulated by $\mathrm{ABA} 1$, ABI1 and AXR2 in Arabidopsis. Plant J. 12, 557-569. doi: 10.1111/j.09607412.1997.00557.x

Suárez, C., Zienkiewicz, A., Castro, A. J., Zienkiewicz, K., Majewska-Sawka, A. and Rodríguez-García, M. I. (2013). Cellular localization and levels of pectins and arabinogalactan proteins in olive (Olea europaea L.) pistil tissues during development: implications for pollen-pistil interaction. Planta 237, 305-319. doi: $10.1007 / \mathrm{s} 00425-012-1774-\mathrm{Z}$

Szabados, L., and Savouré, A. (2010). Proline: a multifunctional amino acid. Trends Plant Sci. 15, 89-97. doi: 10.1016/j.tplants.2009.11.009

Székely, G., Ábrahám, E., Cséplo, Á., Rigo, G., Zsigmond, L., Csiszár, J., et al. (2008). Duplicated P5CS genes of Arabidopsis play distinct roles in stress regulation and developmental control of proline biosynthesis. Plant J. 53, 11-28. doi: 10.1111/j.1365-313X.2007.03318.x

Terrab, A., Garcia-Castano, J. L., Romero, J. M., Berjano, R., De Vega, C., and Talavera, S. (2007). Analysis of amino acids in nectar from Silene colorata Poiret (Caryophyllaceae). Bot. J. Linn. Soc. 155, 49-56. doi: 10.1111/j.10958339.2007.00673.x

Thornburg, R. W., Carter, C., Powell, A., Mittler, R., Rizhsky, L., and Horner, H. T. (2003). A major function of the tobacco floral nectary is defense against microbial attack. Plant Syst. Evol. 238, 211-218. doi: 10.1007/s00606-0030282-9

Trovato, M., Mattioli, R., and Costantino, P. (2008). Multiple roles of proline in plant stress tolerance and development. Rend. Lincei 19, 325-346. doi: 10.1007/s12210-008-0022-8

Vansuyt, G., Vallee, J. C., and Prevost, J. (1979). La pyrroline-5-carboxylate réductase et la proline déhydrogénase chez Nicotiana tabacum var. Xanthi n.c. en fonction de son développement. Physiol. Veg. 19, 95-105.

Venekamp, J. H., and Koot, J. T. M. (1988). The sources of free proline and asparagine in field bean plants, Vicia faba L., during and after a short period of water withholding. J. Plant Physiol. 32, 102-109. doi: 10.1016/S01761617(88)80192-5

Verbruggen, N., and Hermans, C. (2008). Proline accumulation in plants: a review. Amino Acids 35, 753-759. doi: 10.1007/s00726-008-0061-6 
Vogler, F., Schmalzl, C., Englhart, M., Bircheneder, M., and Sprunck, S. (2014). Brassinosteroids promote Arabidopsis pollen germination and growth. Plant Reprod. 27, 153-167. doi: 10.1007/s00497-014-0247-x

Walton, E. F., Clark, C. J., and Boldingh, H. L. (1991). Effect of hydrogen cyanamide on amino acid profiles in kiwifruit buds during bud-break. Plant Physiol. 97, 1256-1259. doi: 10.1104/pp.97.3.1256

Wilhelmi, L. K., and Preuss, D. (1996). Self-sterility in Arabidopsis due to defective pollen tube guidance. Science 274, 1535-1537. doi: $10.1126 /$ science.274.5292.1535

Wolters-Arts, M., Lush, W. M., and Mariani, C. (1998). Lipids are required for directional pollen-tube growth. Nature 392, 818-821. doi: 10.1038/33929

Wu, H. M., Wong, E., Ogdahl, J., and Cheung, A. Y. (2000). A pollen tube growth- promoting arabinogalactan protein from Nicotiana alata is similar to the tobacco TTS protein. Plant J. 22, 165-176. doi: 10.1046/j.1365313x.2000.00731.x

Yoshiba, Y., Kiyosue, T., Katagiri, T., Ueda, H., Wada, K., Harada, Y., et al. (1995). Correlation between the induction of a gene for $\delta^{1}$-pyrroline-5carboxylate synthetase and the accumulation of proline in Arabidopsis under osmotic stress. Plant J. 7, 751-760. doi: 10.1046/j.1365-313X.1995. 07050751.x

Yu, G. H., Zou, J., Feng, J., Peng, X. B., Wu, J. Y., Wu, Y. L., et al. (2014). Exogenous $\gamma$-aminobutyric acid affects pollen tube growth via modulating putative $\mathrm{Ca}^{2+}$-permeable membrane channels and is coupled to negative regulation on glutamate decarboxylase. J. Exp. Bot. 12, 3235-3248. doi: 10.1093/jxb/eru171

Conflict of Interest Statement: The authors declare that the research was conducted in the absence of any commercial or financial relationships that could be construed as a potential conflict of interest.

Copyright (c) 2015 Biancucci, Mattioli, Forlani, Funck, Costantino and Trovato. This is an open-access article distributed under the terms of the Creative Commons Attribution License (CC BY). The use, distribution or reproduction in other forums is permitted, provided the original author(s) or licensor are credited and that the original publication in this journal is cited, in accordance with accepted academic practice. No use, distribution or reproduction is permitted which does not comply with these terms. 


\title{
How and why does tomato accumulate a large amount of GABA in the fruit?
}

\author{
Mariko Takayama and Hiroshi Ezura* \\ The Ezura Laboratory, Graduate School of Life and Environmental Sciences, University of Tsukuba, Tsukuba, Japan
}

Gamma-aminobutyric acid (GABA) has received much attention as a health-promoting functional compound, and several GABA-enriched foods have been commercialized. In higher plants, GABA is primarily metabolized via a short pathway called the GABA shunt. The GABA shunt bypasses two steps (the oxidation of $\alpha$-ketoglutarate to succinate) of the tricarboxylic acid (TCA) cycle via reactions catalyzed by three enzymes: glutamate decarboxylase, GABA transaminase, and succinic semialdehyde dehydrogenase. The GABA shunt plays a major role in primary carbon and nitrogen metabolism and is an integral part of the TCA cycle under stress and non-stress conditions. Tomato is one of the major crops that accumulate a relatively high level of GABA in its fruits. The GABA levels in tomato fruits dramatically change during fruit development; the GABA levels increase from flowering to the mature green stage and then rapidly decrease during the ripening stage. Although GABA constitutes up to $50 \%$ of the free amino acids at the mature green stage, the molecular mechanism of GABA accumulation and the physiological function of GABA during tomato fruit development remain unclear. In this review, we summarize recent studies of GABA accumulation in tomato fruits and discuss the potential biological roles of GABA in tomato fruit development.

Keywords: GABA, GABA shunt, tomato, fruit, metabolism Hiroshi Ezura, The Ezura Laboratory, Graduate School of Life and Environmental Sciences, University of Tsukuba, Tennodai 1-1-1, Tsukuba, Ibaraki 305-8572, Japan ezura@gene.tsukuba.ac.jp

Specialty section: This article was submitted to Plant Physiology, a section of the journal Frontiers in Plant Science

Received: 29 April 2015 Accepted: 23 July 2015 Published: 10 August 2015

Citation:

Takayama M and Ezura H (2015) How and why does tomato accumulate a large amount of GABA in the fruit?

Front. Plant Sci. 6:612.

doi: 10.3389/fp/s.2015.00612

\section{Introduction}

Gamma-aminobutyric acid (GABA), a four-carbon non-proteinogenic amino acid, is widely found in animals, plants and bacteria. In humans, GABA functions as an inhibitory neurotransmitter in the central nervous system (Owens and Kriegstein, 2002). It has also been reported that GABA is effective at reducing blood pressure, inducing relaxation and enhancing immunity when administered orally (Inoue et al., 2003; Abdou et al., 2006). Thus, GABA has received much attention as a healthpromoting functional compound, and several GABA-enriched foods have been commercialized. In higher plants, GABA is primarily metabolized via a short pathway called the GABA shunt, which bypasses two steps (the oxidation of $\alpha$-ketoglutarate to succinate) of the tricarboxylic acid (TCA) cycle (Satya-Narayan and Nair, 1990; Bouché and Fromm, 2004; Figure 1). In this pathway, GABA is synthesized from glutamate in a reaction catalyzed by the enzyme glutamate decarboxylase (GAD) and subsequently catabolized to succinate through two consecutive reactions catalyzed by GABA transaminase (GABA-T) and succinic semialdehyde dehydrogenase (SSADH). Previous studies have suggested that the GABA shunt is involved in multiple physiological responses, such as the regulation of cytosolic $\mathrm{pH}$, maintenance of carbon/nitrogen balance, defense against insects, protection from oxidative stress, and production of energy (Bouché and Fromm, 2004; Fait et al., 2008). 


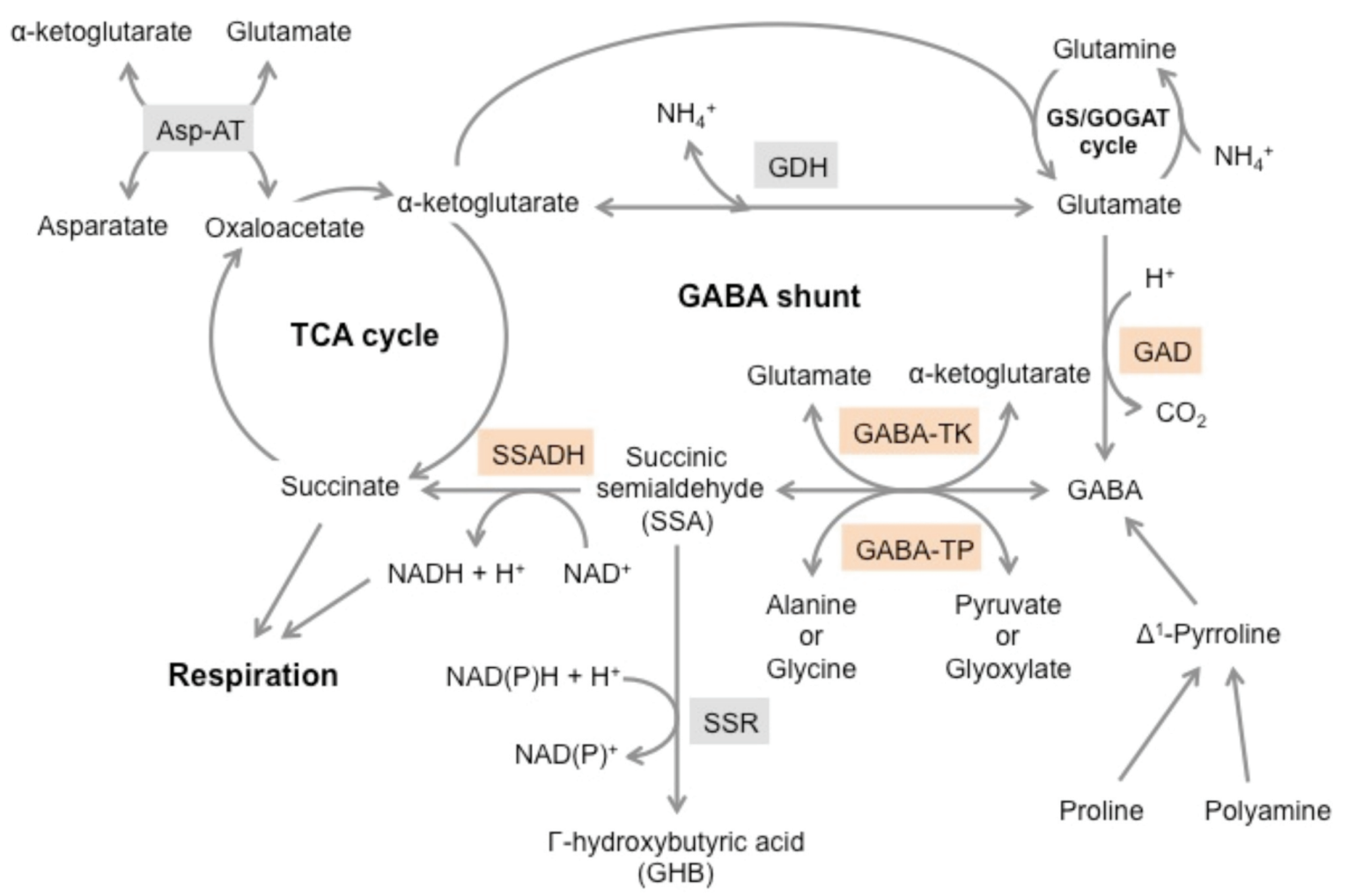

FIGURE 1 | GABA metabolism and related pathways. GAD, glutamate decarboxylase; GABA-TK, $\alpha$-ketoglutarate-dependent GABA transaminase; GABA-TP, pyruvate-dependent GABA transaminase; SSA, succinic semialdehyde; SSADH, succinic semialdehyde dehydrogenase; GDH, glutamate dehydrogenase; SSR, succinic semialdehyde reductase; Asp-AT, aspartate aminotransferase.
Moreover, the GABA level rapidly increases in plant tissues subjected to diverse stimuli, including heat shock, mechanical stimulation, hypoxia, and phytohormones (Shelp et al., 1999).

Tomato (Solanum lycopersicum) is a major crop produced worldwide. Tomato fruits are a significant food resource and have been considered an experimental model for studying the physiology, development and ripening of fleshy fruits (Steinhauser et al., 2010; Osorio et al., 2011). Tomatoes accumulate a relatively high level of GABA in the fruits (Matsumoto et al., 1997). In several cultivated tomatoes, drastic changes in GABA levels have been observed during fruit development; the GABA level increases to the mature green stage and subsequently rapidly decreases during the ripening stage (Rolin et al., 2000; Carrari et al., 2006; Akihiro et al., 2008; Saito et al., 2008; Osorio et al., 2011). In cherry tomatoes, GABA is reported to constitute up to $50 \%$ of the free amino acids at the mature green stage (Rolin et al., 2000). Despite the large accumulation, the molecular mechanism of GABA accumulation and the physiological function of this amino acid during tomato fruit development remain elusive. Elucidating these topics would help us to gain a better understanding of plant physiology, particularly in fruits. In this review, we summarize recent studies concerning GABA accumulation in tomato fruits and discuss the potential biological roles of GABA in tomato fruit development.

\section{GABA Biosynthesis}

In plants, GABA is primarily synthesized via the cytosolic enzyme $\mathrm{GAD}$, which catalyzes the irreversible conversion of glutamate to GABA and $\mathrm{CO}_{2}$ (Figure 1). A plant GAD gene was first isolated from Petunia hybrida (Baum et al., 1993), and subsequently, several GAD homologues have been identified in various plant species (Ling et al., 1994; Snedden et al., 1995; Turano and Fang, 1998; Yevtushenko et al., 2003). Unlike its counterparts in animals and bacteria, most plant GADs possess a calcium/calmodulin $\left(\mathrm{Ca}^{2+} / \mathrm{CaM}\right)$ binding domain (CaMBD) at the C-terminus. In vitro studies have shown that GAD activity is stimulated through a low $\mathrm{pH}$ or the binding of $\mathrm{Ca}^{2+} / \mathrm{CaM}$ to the CaMBD at physiological pH (Snedden et al., 1996; Gut et al., 2009). In addition, transgenic studies revealed that the removal of the CaMBD increased GABA accumulation in plants (Baum et al., 1996; Akama and Takaiwa, 2007). Thus, it is considered that the CaMBD acts as a negative regulator/autoinhibitory domain in the absence of $\mathrm{Ca}^{2+} / \mathrm{CaM}$, and the negative regulation is relieved through the binding of $\mathrm{Ca}^{2+} / \mathrm{CaM}$.

In tomato, GAD gene was first cloned in 1995. Gallego et al. (1995) isolated ERT D1, a gene encoding a putative GAD protein, from a cDNA library of the pericarp of cv. "Ailsa Craig." Similar to other plant GADs, ERT D1 protein contained a putative CaMBD. It was also revealed that ERT D1 mRNA levels peaked at the 
beginning of fruit ripening (Gallego et al., 1995). Subsequently, Kisaka et al. (2006) isolated GAD-19, a gene encoding another GAD protein, from tomato roots. The antisense suppression of this gene in tomato plants resulted in the production of fruits with decreased levels of GAD mRNA. However, the GABA level in these fruits was not significantly decreased compared with the WT levels, although increased levels of total free amino acids (particularly glutamate, which is the precursor to GABA) were observed (Kisaka et al., 2006). Subsequently, Akihiro et al. (2008) isolated three GAD genes, designated SlGAD1, SlGAD2, and SlGAD3, from the immature fruits of cv. "Micro-Tom." Because the amino acid sequences of SlGAD1 and ERT D1 are precisely identical, $S I G A D 1$ is considered an allele of ERT D1 (Akihiro et al., 2008). However, neither SlGAD2 nor SlGAD3 share precisely identical sequences with $G A D-19$, although blast database searches indicate that SlGAD2 has the highest homology to GAD-19 (95\% identity and $98 \%$ similarity in amino acid sequences). Among the three SlGADs isolated from cv. "MicroTom," SlGAD2 and SlGAD3 appear to play a major role in GABA production in tomato fruits, as the expression levels of SlGAD2 and $S G A D 3$ are positively correlated with the GABA accumulation during fruit development (Akihiro et al., 2008). Additionally, transgenic tomato plants, in which SlGAD2 or SlGAD3 was specifically suppressed, accumulated a significantly decreased level of GABA in the fruits, whereas SlGAD1-suppressed plants produced fruits with normal levels of GABA (Takayama et al., 2015). Moreover, in triple SlGADs-suppressed plants, the fruit GABA level decreased to less than $10 \%$ of the WT level (Takayama et al., 2015), suggesting that the main route of GABA biosynthesis in tomato fruits is the decarboxylation of glutamate via GAD enzymes under normal growth conditions.

Enhanced GABA accumulation in tomato fruits has been observed in plants grown under salinity conditions or in fruits stored under $10 \% \mathrm{CO}_{2}$ or under low $\mathrm{O}_{2}$ conditions after harvesting (Deewatthanawong et al., 2010; Yin et al., 2010; Mae et al., 2012). Although the expression levels of SlGAD2 and SlGAD3 were not enhanced in the fruits under salinity conditions (Yin et al., 2010), those in fruits stored under $10 \% \mathrm{CO}_{2}$ or low $\mathrm{O}_{2}$ conditions were up-regulated (Deewatthanawong et al., 2010; Mae et al., 2012). These results suggest that SlGAD2 and SlGAD3 are responsive to some types of stresses. It has been suggested that stress-induced GABA accumulation in plant cells reflects increases in cytosolic $\mathrm{H}^{+}, \mathrm{Ca}^{2+}$ or glutamate levels, as these factors stimulate GAD activity (Shelp et al., 1999). However, in tomato, stress-induced GAD activity might also be regulated at the transcriptional level. Although it is reported that GABA can also be formed from polyamines or proline via a $\Delta^{1}$-pyrroline intermediate formation in response to abiotic stresses (Flores and Filner, 1985; Shelp et al., 2012; Yang et al., 2013; Signorelli et al., 2015), the contribution of these pathways in tomato fruits is still unclear.

\section{GABA Catabolism}

In many organisms, GABA is first converted to SSA via a transamination reaction through GABA-T (Figure 1). According to substrate specificity, the GABA-T enzyme can be divided into two types: $\alpha$-ketoglutarate-dependent GABA-T (GABA-TK) and pyruvate-dependent GABA-T (GABA-TP). The former uses $\alpha$ ketoglutarate as an amino group acceptor to generate glutamate, whereas the latter uses pyruvate to generate alanine (Bouché and Fromm, 2004). It is clear that GABA-TP also has glyoxylatedependent GABA-T (GABA-TG) activity, which uses glyoxylate as an amino group acceptor to generate glycine (Clark et al., 2009a,b; Shimajiri et al., 2013; Trobacher et al., 2013). GABATK is exclusively utilized in bacteria, yeast, fungi and mammals (Satya-Narayan and Nair, 1990). However, both GABA-TK and GABA-TP activities have been detected in plant crude extracts (Shelp et al., 1995; Van Cauwenberghe and Shelp, 1999; Bartyzel et al., 2003), although only the GABA-TP gene has been isolated from plants (Van Cauwenberghe et al., 2002). Tomato is one of the species exhibiting both GABA-TK and GABATP activities. Although most previously investigated plants have shown lower GABA-TK activity than GABA-TP activity, Akihiro et al. (2008) detected a significantly higher level of GABA-TK activity in tomato fruits after the breaker stage. Comparison analyses between ordinary and GABA rich cultivars revealed a negative correlation between GABA contents and GABA-TK activity during fruit development (Akihiro et al., 2008). Similar trends were also observed in the tomato fruits stored under low $\mathrm{O}_{2}$ conditions, in which GABA levels were increased compared with those in fruits stored under control (air) conditions (Mae et al., 2012). These observations suggest that GABA-TK plays a major role in catabolism in tomato fruits. However, Clark et al. (2009b) presented a different view, as no GABA-TK activity was detected in assays using the cell-free extracts from the fruits of cv. "Micro-Tom," which is the same cultivar used in Akihiro et al. (2008). Moreover, Clark et al. (2009b) observed higher levels of GABA-TP activity in tomato fruits. Thus, these authors noted the possibility that the previous study detected artificial GABA-TK activity and concluded that pyruvate/glyoxylatedependent GABA-T activity probably accounts for the GABA catabolism observed in tomato fruits. Currently, three GABA$\mathrm{T}$ genes, designated $S l G A B A-T 1, S l G A B A-T 2$, and $S l G A B A-T 3$, have been isolated from tomato cv. "Micro-Tom" (Akihiro et al., 2008; Clark et al., 2009b). Although the encoded proteins are localized to distinct subcellular compartments [i.e., mitochondrion (SlGABA-T1), cytosol (SlGABA-T2), or plastid (SlGABA-T3)], all three isoforms are characterized as GABA-TPs, which exhibit pyruvate/glyoxylate-dependent GABAT activity (Clark et al., 2009b). To clarify the physiological function of these SIGABA-T isoforms in tomato fruits, Koike et al. (2013) conducted loss-of-function analyses using RNA interference (RNAi) transgenic lines with suppressed SlGABA$T$ genes. In this study, increased GABA accumulation was observed in the fruits of SlGABA-T1-suppressed lines (1.3-2.0 times higher in mature green fruits and 6.8-9.2 times higher in red fruits), whereas almost no correlation was observed between the GABA content and the expressions of SlGABAT2 and SlGABA-T3 (Koike et al., 2013). Considering that the enzymatic activity of SIGABA-T1 is highest among the three isoforms in tomato fruits (Clark et al., 2009b), Koike et al. (2013) concluded that pyruvate- and glyoxylate-dependent SIGABA-T1 is the essential isoform for GABA reduction in the ripening fruits. 
In plants, GABA-derived SSA is catabolized via the $\mathrm{NAD}^{+}$. dependent enzyme SSADH, which oxidizes SSA to succinate concomitantly with $\mathrm{NADH}$ production in mitochondria (Breitkreuz and Shelp, 1995; Busch and Fromm, 1999; Figure 1). Alternatively, SSA can also be catabolized to $\gamma$-hydroxybutyric acid (GHB) through enzymes with SSA reductase (SSR) activity (Breitkreuz et al., 2003; Hoover et al., 2007; Simpson et al., 2008; Figure 1). The former pathway provides substrates (succinate and $\mathrm{NADH}$ ) for the mitochondrial respiratory machinery, which produces ATP as a final product (Bouché and Fromm, 2004). It is also known that SSADH activity is highly sensitive to the energy status in mitochondria (Busch and Fromm, 1999). Thus, under stress conditions in which the $\mathrm{NAD}^{+}: \mathrm{NADH}$ ratio is low, SSADH activity would be inhibited, resulting in the accumulation of SSA and feedback inhibition of GABA-T (Busch and Fromm, 1999; Van Cauwenberghe and Shelp, 1999). However, the pathway from SSA to GHB is stimulated under stress conditions and likely functions in stress tolerance through the detoxification of SSA (Breitkreuz et al., 2003; Allan et al., 2008). In tomato, one SSADH gene (SlSSADH) and two SSR genes (SlSSR1, SISSR2) have been isolated (Akihiro et al., 2008). SISSADH is expressed in fruits at all developmental stages, and the expression of this gene is poorly correlated with the GABA contents (Akihiro et al., 2008). However, the expression of SISSR1 gene is slightly higher in red fruits than in breaker fruits, whereas SISSR2 expression is higher in breaker fruits compared with red fruits (Deewatthanawong et al., 2010). However, the biochemical properties of the encoded proteins and their contribution to GABA accumulation in tomato fruits remain unclear.

\section{The Potential Role of GABA Metabolism in Tomato Plants}

In plants, GABA metabolism is involved in a wide range of physiological processes. For example, pop2, an Arabidopsis GABA-T-deficient mutant, is defective in the guidance and growth of pollen tubes (Palanivelu et al., 2003; Renault et al., 2011). Arabidopsis SSADH-deficient mutants exhibit severe dwarfism and necrotic lesions under the standard light conditions (Bouché et al., 2003). Additionally, these mutants exhibit the enhanced accumulation of reactive oxygen intermediates and cell death under environmental stresses (Bouché et al., 2003). Another Arabidopsis SSADH-deficient mutant, enf1, forms both abaxialized and adaxialized leaves (Toyokura et al., 2011). Notably, the abnormal phenotypes observed in the two different studies of ssadh mutants (Bouché et al., 2003; Toyokura et al., 2011) are both suppressed through an additional mutation in GABA-T, suggesting that these phenotypes reflect the accumulation of SSA or close derivatives, such as GHB (Ludewig et al., 2008; Toyokura et al., 2011). In tomatoes, several abnormalities have also been observed when GABA metabolism is altered. For example, $S I G A B A-T 1$-suppressed plants exhibited severe infertility, and both $S I G A B A-T 1-$ and $S l G A B A-$ T3-suppressed plants exhibited dwarf phenotypes (Koike et al., 2013). Moreover, SISSADH-suppressed plants show a dwarf phenotype, curled leaves and enhanced ROS accumulation under normal conditions (Bao et al., 2014). Interestingly, when tomato seedlings were grown under salt stress (200 mM NaCl), SISSADHsuppressed plants exhibited significantly higher shoot biomass levels and increased chlorophyll contents and photosynthetic rates compared with control plants (Bao et al., 2014). However, SlGADs-suppressed plants and SlGABA-Ts-suppressed plants are more sensitive to salt stress, resulting in reduced biomass and the total collapse of tissue (Bao et al., 2014). These observations indicate that GABA shunt is involved in salt stress tolerance in tomato plants. Moreover, GABA shunt has been implicated in resistance against Botrytis cinerea, as GABA shunt genes are upregulated in the leaves of the $B$. cinerea-resistant mutant, sitiens, and the exogenous application of GABA decreases susceptibility to $B$. cinerea in wild-type leaves (Seifi et al., 2013).

As described above, effects of impaired GABA metabolism on tomato plants have been increasingly reported. However, little is known about the function of GABA and the metabolism of this amino acid in fruits. Previous studies have suggested that GABA production during fruit development might contribute to the regulation of cellular $\mathrm{pH}$ (Rolin et al., 2000). During tomato fruit development, organic acids are continuously synthesized from unloaded sucrose, coupled with proton production. Overaccumulation of protons would cause an intracellular acidification, but the intracellular $\mathrm{pH}$ is probably regulated by ATP-driven proton pumps that extrude intracellular protons out of the cytoplasm, or by the proton-consuming decarboxylation of organic acids. Because GAD reaction requires protons, it might act as a sink for excess protons, preventing intracellular acidification (Rolin et al., 2000; Figure 2A). Moreover, the GAD reaction also promotes glutamate transport. In cherry tomatoes, glutamate is translocated through phloem sap and unloaded in fruits. The unloaded glutamate is subsequently transported symplastically or taken up through a proton symport mechanism across the membrane. In the latter transport mechanism, glutamate and protons are cotransported into the cytosol, thereby promoting cytoplasmic acidosis and the depolarization of the plasma membrane. Thus, continuous GABA accumulation during fruit development reflects the continuous GAD reaction, which potentially maintains glutamate transport through the consumption of excess protons (Snedden et al., 1992; Rolin et al., 2000). In addition, accumulated GABA in tomato fruits functions as an energy source, as ${ }^{14} \mathrm{C}$-labeled $\mathrm{CO}_{2}$ was discharged from fruits fed ${ }^{14} \mathrm{C}$-labeled $\mathrm{GABA}$, indicating that GABA is utilized as a substrate for respiration (Yin et al., 2010; Figure 2B). Indeed, GABA shunt also functions as an alternative pathway for the production of succinate (the substrate for respiration) in tomato leaves when the enzyme of the TCA cycle is impaired (Studart-Guimarães et al., 2007). However, recent findings suggest that GABA metabolism has little effect on tomato fruit development under normal conditions, as the fruits of RNAi transgenic plants targeting the three SlGADs exhibited normal development, although the enzymatic activity of GAD and the GABA content in fruits were dramatically decreased (Takayama et al., 2015). Similarly, RNAi transgenic plants targeting SlGABA$T$ also produced normal fruits, although the GABA levels in red fruits were 6.8-9.2 times higher than those in wild-type controls (Koike et al., 2013). Therefore, GABA metabolism 


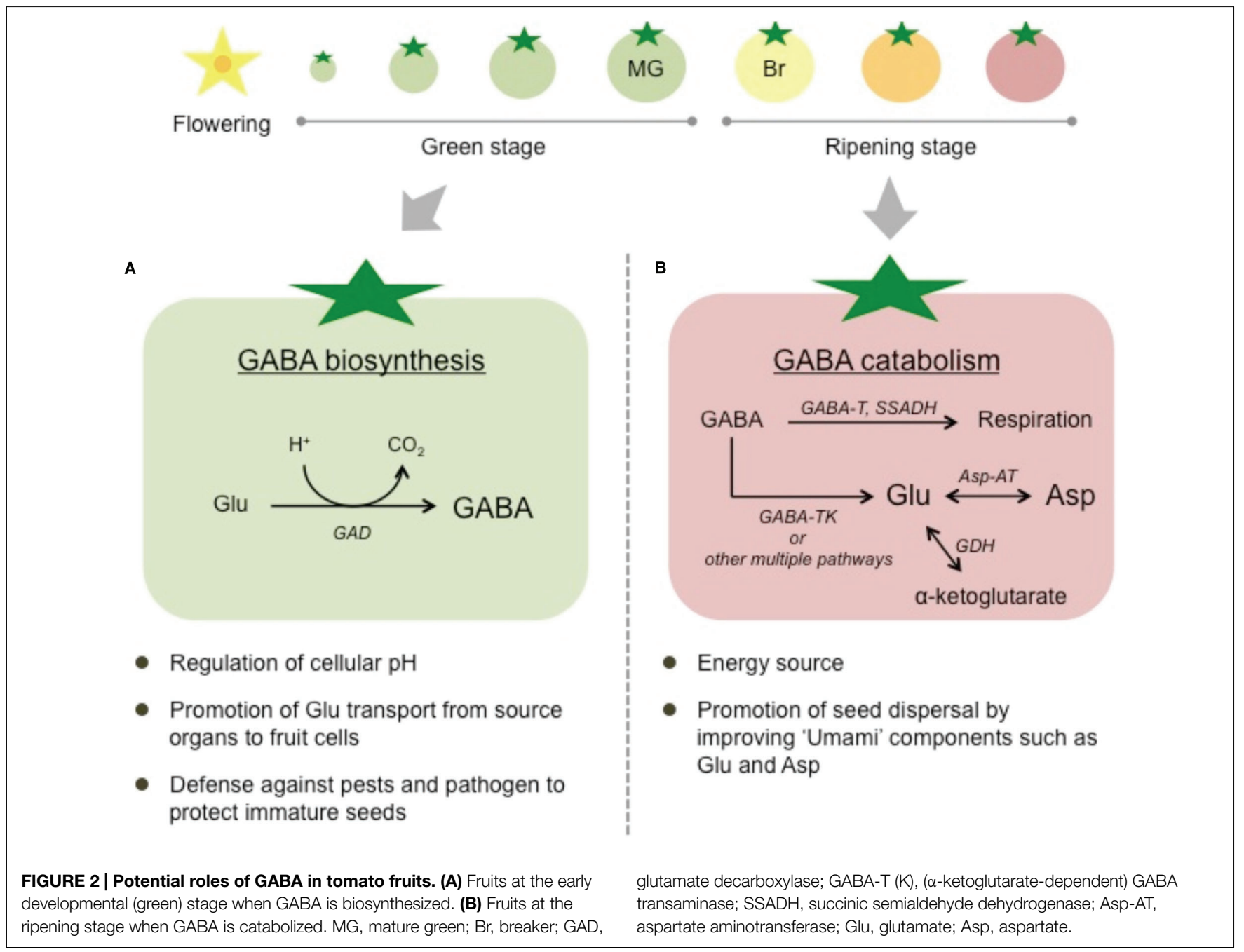

in tomato fruits might be involved in stress tolerance, similar to other plants. Another possibility is that GABA contributes to tomato seed dispersal through changes in the amino acid composition during fruit development. Because GABA functions in defense against pests and pathogens (Bown et al., 2006; Seifi et al., 2013), GABA accumulation in fruits at the early developmental stage might protect immature seeds (Figure 2A). However, the GABA levels in fruits rapidly decline during the ripening stage, when seeds have already matured. In parallel, the levels of glutamate and/or aspartate, which provide the "Umami taste," dramatically increase during the ripening stage. These changes in the amino acid composition might attract insects and animals, resulting in successful seed dispersal (Figure 2B). The increases in glutamate and/or aspartate during fruit ripening have been well characterized in various cultivars (Rolin et al., 2000; Akihiro et al., 2008; Koike et al., 2013). The increase in glutamate probably reflects the increase in glutamate dehydrogenase (GDH) and GABA-TK activities during the ripening stage and the decreased consumption of glutamate through GAD, which is almost undetectable in ripe fruits (Sorrequieta et al., 2010; Ferraro et al., 2015; Figure 1). On the other hand, aspartate is synthesized from glutamate through aspartate aminotransferase
(Figure 1). In GABA-rich cultivars, lower levels of glutamate and aspartate have been observed in ripening fruits (Akihiro et al., 2008), suggesting that GABA catabolism contributes to the accumulation of glutamate and glutamate-derived aspartate in ripening fruits. Furthermore, Snowden et al. (2015) recently identified a tonoplast-localized glutamate/aspartate/GABA exchanger (SICAT9) in tomato fruits. As overexpression of the SlCAT9 gene strongly influences the accumulation of glutamate, aspartate, and GABA during tomato fruit development, it is suggested that the intracellular transport of amino acids between vacuole and cytosol is also a major determinant of their accumulation in ripening fruits (Snowden et al., 2015). Although the pathway involving the conversion from GABA to glutamate remains uncertain, GABA catabolism might play a crucial role in the determination of tomato fruit taste during ripening.

\section{Acknowledgments}

This research was funded in part by the Research and Development Program for New Bio-industry initiatives (BRAIN) to HE. We also thank all members our laboratory for helpful discussions throughout the work. 


\section{References}

Abdou, A. M., Higashiguchi, S., Horie, K., Kim, M., Hatta, H., and Yokogoshi, H. (2006). Relaxation and immunity enhancement effects of gammaaminobutyric acid (GABA) administration in humans. Biofactors 26, 201-208. doi: 10.1002/biof.5520260305

Akama, K., and Takaiwa, F. (2007). C-terminal extension of rice glutamate decarboxylase (OsGAD2) functions as an autoinhibitory domain and overexpression of a truncated mutant results in the accumulation of extremely high levels of GABA in plant cells. J. Exp. Bot. 58, 2699-2707. doi: $10.1093 /$ jxb/erm 120

Akihiro, T., Koike, S., Tani, R., Tominaga, T., Watanabe, S., Iijima, Y., et al. (2008). Biochemical mechanism on GABA accumulation during fruit development in tomato. Plant Cell Physiol. 49, 1378-1389. doi: 10.1093/pcp/pcn113

Allan, W. L., Simpson, J. P., Clark, S. M., and Shelp, B. J. (2008). Gammahydroxybutyrate accumulation in Arabidopsis and tobacco plants is a general response to abiotic stress: putative regulation by redox balance and glyoxylate reductase isoforms. J. Exp. Bot. 59, 2555-2564. doi: 10.1093/jxb/ern122

Bao, H., Chen, X., Lv, S., Jiang, P., Feng, J., Fan, P., et al. (2014). Virus-induced gene silencing reveals control of reactive oxygen species accumulation and salt tolerance in tomato by $\gamma$-aminobutyric acid metabolic pathway. Plant Cell Environ. 38, 600-613. doi: 10.1111/pce.12419

Bartyzel, I., Pelczar, K., and Paszkowski, A. (2003). Functioning of the $\gamma$ aminobutyrate pathway in wheat seedlings affected by osmotic stress. Biol. Plant. 47, 221-225. doi: 10.1023/B:BIOP.0000022255.01125.99

Baum, G., Chen, Y., Arazi, T., Takatsuji, H., and Fromm, H. (1993). A plant glutamate decarboxylase containing a calmodulin binding domain. Cloning, sequence, and functional analysis. J. Biol. Chem. 268, 19610-19617.

Baum, G., Lev-Yadun, S., Fridmann, Y., Arazi, T., Katsnelson, H., Zik, M., et al. (1996). Calmodulin binding to glutamate decarboxylase is required for regulation of glutamate and GABA metabolism and normal development in plants. EMBO J. 15, 2988-2996.

Bouché, N., Fait, A., Bouchez, D., Møller, S. G., and Fromm, H. (2003). Mitochondrial succinic-semialdehyde dehydrogenase of the $\gamma$-aminobutyrate shunt is required to restrict levels of reactive oxygen intermediates in plants. Proc. Natl. Acad. Sci. U.S.A. 100, 6843-6848. doi: 10.1073/pnas.1037532100

Bouché, N., and Fromm, H. (2004). GABA in plants: just a metabolite? Trends Plant Sci. 9, 110-115. doi: 10.1016/j.tplants.2004.01.006

Bown, A. W., MacGregor, K. B., and Shelp, B. J. (2006). Gamma-aminobutyrate: defense against invertebrate pests? Trends Plant Sci. 11, 424-427. doi: 10.1016/j.tplants.2006.07.002

Breitkreuz, K. E., Allan, W. L., Van Cauwenberghe, O. R., Jakobs, C., Talibi, D., André, B., et al. (2003). A novel $\gamma$-hydroxybutyrate dehydrogenase. Identification and expression of an Arabidopsis cDNA and potential role under oxygen deficiency. J. Biol. Chem. 278, 41552-41556. doi: 10.1074/jbc.M305717200

Breitkreuz, K. E., and Shelp, B. J. (1995). Subcellular compartmentation of the 4aminobutyrate shunt in protoplasts from developing soybean cotyledons. Plant Physiol. 108, 99-103.

Busch, K. B., and Fromm, H. (1999). Plant succinic semialdehyde dehydrogenase. Cloning, purification, localization in mitochondria, and regulation by adenine nucleotides. Plant Physiol. 121, 589-597. doi: 10.1104/pp.121.2.589

Carrari, F., Baxter, C., Usadel, B., Urbanczyk-Wochniak, E., Zanor, M., NunesNesi, A., et al. (2006). Integrated analysis of metabolite and transcript levels reveals the metabolic shifts that underlie tomato fruit development and highlight regulatory aspects of metabolic network behavior. Plant Physiol. 142, 1380-1396. doi: $10.1104 / \mathrm{pp} .106 .088534$

Clark, S. M., Di Leo, R., Dhanoa, P. K., Van Cauwenberghe, O. R., Mullen, R. T., and Shelp, B. J. (2009a). Biochemical characterization, mitochondrial localization, expression, and potential functions for an Arabidopsis $\gamma$-aminobutyrate transaminase that utilizes both pyruvate and glyoxylate. J. Exp. Bot. 60, 1743-1757. doi: 10.1093/jxb/erp044

Clark, S. M., Di Leo, R., Van Cauwenberghe, O. R., Mullen, R. T., and Shelp, B. J. (2009b). Subcellular localization and expression of multiple tomato $\gamma$ aminobutyrate transaminases that utilize both pyruvate and glyoxylate. J. Exp. Bot. 60, 3255-3267. doi: 10.1093/jxb/erp161

Deewatthanawong, R., Rowell, P., and Watkins, C. B. (2010). $\gamma$-Aminobutyric acid (GABA) metabolism in $\mathrm{CO}_{2}$ treated tomatoes. Postharvest Biol. Technol. 57, 97-105. doi: 10.1016/j.postharvbio.2010.03.007
Fait, A., Fromm, H., Walter, D., Galili, G., and Fernie, A. R. (2008). Highway or byway: the metabolic role of the GABA shunt in plants. Trends Plant Sci. 13, 14-19. doi: 10.1016/j.tplants.2007.10.005

Ferraro, G., D’Angelo, M., Sulpice, R., Stitt, M., and Valle, E. M. (2015). Reduced levels of NADH-dependent glutamate dehydrogenase decrease the glutamate content of ripe tomato fruit but have no effect on green fruit or leaves. J. Exp. Bot. 66, 3381-3389. doi: 10.1093/jxb/erv150

Flores, H. E., and Filner, P. (1985). Polyamine catabolism in higher plants: characterization of pyrroline dehydrogenase. Plant Growth Regul. 3, 277-291. doi: 10.1007/BF00117586

Gallego, P. P., Whotton, L., Picton, S., Grierson, D., and Gray, J. E. (1995). A role for glutamate decarboxylase during tomato ripening: the characterization of a cDNA encoding a putative glutamate decarboxylase with a calmodulin-binding site. Plant Mol. Biol. 27, 1143-1151. doi: 10.1007/BF00020887

Gut, H., Dominici, P., Pilati, S., Astegno, A., Petoukhov, M. V., Svergun, D. I., et al. (2009). A common structural basis for $\mathrm{pH}$ - and calmodulin-mediated regulation in plant glutamate decarboxylase. J. Mol. Biol. 392, 334-351. doi: 10.1016/j.jmb.2009.06.080

Hoover, G. J., Van Cauwenberghe, O. R., Breitkreuz, K. E., Clark, S. M., Merrill, A. R., and Shelp, B. J. (2007). Characteristics of an Arabidopsis glyoxylate reductase: general biochemical properties and substrate specificity for the recombinant protein, and developmental expression and implications for glyoxylate and succinic semialdehyde metabolism in planta. Can. J. Bot. 85, 883-895. doi: 10.1139/B07-081

Inoue, K., Shirai, T., Ochiai, H., Kasao, M., Hayakawa, K., Kimura, M., et al. (2003). Blood-pressure-lowering effect of a novel fermented milk containing gammaaminobutyric acid (GABA) in mild hypertensives. Eur. J. Clin. Nutr. 57, 490-495. doi: 10.1038/sj.ejcn.1601555

Kisaka, H., Kida, T., and Miwa, T. (2006). Antisense suppression of glutamate decarboxylase in tomato (Lycopersicon esculentum L.) results in accumulation of glutamate in transgenic tomato fruits. Plant Biotechnol. 23, 267-274. doi: 10.5511/plantbiotechnology.23.267

Koike, S., Matsukura, C., Takayama, M., Asamizu, E., and Ezura, H. (2013). Suppression of $\gamma$-aminobutyric acid (GABA) transaminases induces prominent GABA accumulation, dwarfism and infertility in the tomato (Solanum lycopersicum L.). Plant Cell Physiol. 54, 793-807. doi: 10.1093/pcp/pct035

Ling, V., Snedden, W. A., Shelp, B. J., and Assmann, S. M. (1994). Analysis of a soluble calmodulin binding protein from fava bean roots: identification of glutamate decarboxylase as a calmodulin-activated enzyme. Plant Cell 6, 1135-1143. doi: 10.1105/tpc.6.8.1135

Ludewig, F., Hüser, A., Fromm, H., Beauclair, L., and Bouché, N. (2008). Mutants of GABA transaminase (POP2) suppress the severe phenotype of succinic semialdehyde dehydrogenase (ssadh) mutants in Arabidopsis. PLoS ONE 3:e3383. doi: 10.1371/journal.pone.0003383

Mae, N., Makino, Y., Oshita, S., Kawagoe, Y., Tanaka, A., Aoki, K., et al. (2012). Accumulation mechanism of $\gamma$-aminobutyric acid in tomatoes (Solanum lycopersicum L.) under low $\mathrm{O}_{2}$ with and without $\mathrm{CO}_{2}$. J. Agric. Food Chem. 60, 1013-1019. doi: 10.1021/jf2046812

Matsumoto, Y., Ohno, K., and Hiraoka, Y. (1997). Studies on the utilization of functional food materials containing high levels of gamma-aminobutyric acid (Part1). Ehime Kougi Kenkyu Houkoku (In Japanese). 35, 97-100.

Osorio, S., Alba, R., Damasceno, C. M., Lopez-Casado, G., Lohse, M., Zanor, M. I., et al. (2011). Systems biology of tomato fruit development: combined transcript, protein, and metabolite analysis of tomato transcription factor (nor, rin) and ethylene receptor (Nr) mutants reveals novel regulatory interactions. Plant Physiol. 157, 405-425. doi: 10.1104/pp.111.175463

Owens, D. F., and Kriegstein, A. R. (2002). Is there more to GABA than synaptic inhibition? Nat. Rev. Neurosci. 3, 715-727. doi: 10.1038/nrn919

Palanivelu, R., Brass, L., Edlund, A. F., and Preuss, D. (2003). Pollen tube growth and guidance is regulated by POP2, an Arabidopsis gene that controls GABA levels. Cell 114, 47-59. doi: 10.1016/S0092-8674(03)00479-3

Renault, H., El Amrani, A., Palanivelu, R., Updegraff, E. P., Yu, A., Renou, J. P., et al. (2011). GABA accumulation causes cell elongation defects and a decrease in expression of genes encoding secreted and cell wall-related proteins in Arabidopsis thaliana. Plant Cell Physiol. 52, 894-908. doi: 10.1093/pcp/ pcr041

Rolin, D., Baldet, P., Just, D., Chevalier, C., Biran, M., and Raymond, P. (2000). NMR study of low subcellular $\mathrm{pH}$ during the development of cherry tomato fruit. Aust. J. Plant Physiol. 27, 61-69. doi: 10.1071/pp99051 
Saito, T., Matsukura, C., Sugiyama, M., Watahiki, A., Ohshima, I., Iijima, Y., et al. (2008). Screening for $\gamma$-aminobutyric acid (GABA)-rich tomato varieties. J. Japan. Soc. Hort. Sci. 77, 242-250. doi: 10.2503/jjshs1.77.242

Satya-Narayan, V., and Nair, P. M. (1990). Metabolism, enzymology and possible roles of 4-aminobutyrate in higher plants. Phytochemistry 29, 367-375. doi: 10.1016/0031-9422(90)85081-P

Seifi, H. S., Curvers, K., De Vleesschauwer, D., Delaere, I., Aziz, A., and Höfte, M. (2013). Concurrent overactivation of the cytosolic glutamine synthetase and the GABA shunt in the ABA-deficient sitiens mutant of tomato leads to resistance against Botrytis cinerea. New Phytol. 199, 490-504. doi: 10.1111/nph.12283

Shelp, B. J., Bown, A. W., and McLean, M. D. (1999). Metabolism and functions of gamma-aminobutyric acid. Trends Plant Sci. 4, 446-452. doi: 10.1016/S13601385(99)01486-7

Shelp, B. J., Bozzo, G. G., Trobacher, C. P., Zarei, A., Deyman, K. L., and Brikis, C. J. (2012). Hypothesis/review: contribution of putrescine to 4-aminobutyrate (GABA) production in response to abiotic stress. Plant Sci. 193-194, 130-135. doi: 10.1016/j.plantsci.2012.06.001

Shelp, B. J., Walton, C. S., Snedden, W. A., Tuin, L. G., Oresnik, I. J., and Layzell, D. B. (1995). Gaba shunt in developing soybean seeds is associated with hypoxia. Physiol. Plant. 94, 219-228. doi: 10.1111/j.1399-3054.1995.tb05304.x

Shimajiri, Y., Ozaki, K., Kainou, K., and Akama, K. (2013). Differential subcellular localization, enzymatic properties and expression patterns of $\gamma$-aminobutyric acid transaminases (GABA-Ts) in rice (Oryza sativa). J. Plant Physiol. 170, 196-201. doi: 10.1016/j.jplph.2012.09.007

Signorelli, S., Dans, P. D., Coitiño, E. L., Borsani, O., and Monza, J. (2015). Connecting proline and $\gamma$-aminobutyric acid in stressed plants through non-enzymatic reactions. PLoS ONE 10:e0115349. doi: 10.1371/ journal.pone.0115349

Simpson, J. P., Di Leo, R., Dhanoa, P. K., Allan, W. L., Makhmoudova, A., Clark, S. M., et al. (2008). Identification and characterization of a plastid-localized Arabidopsis glyoxylate reductase isoform: comparison with a cytosolic isoform and implications for cellular redox homeostasis and aldehyde detoxification. J. Exp. Bot. 59, 2545-2554. doi: 10.1093/jxb/ern123

Snedden, W. A., Arazi, T., Fromm, H., and Shelp, B. J. (1995). Calcium/calmodulin activation of soybean glutamate decarboxylase. Plant Physiol. 108, 543-549.

Snedden, W. A., Chung, I., Pauls, R. H., and Bown, A. W. (1992). Proton/l-glutamate symport and the regulation of intracellular $\mathrm{pH}$ in isolated mesophyll cells. Plant Physiol. 99, 665-671. doi: 10.1104/pp.99.2.665

Snedden, W. A., Koutsia, N., Baum, G., and Fromm, H. (1996). Activation of a recombinant petunia glutamate decarboxylase by calcium/calmodulin or by a monoclonal antibody which recognizes the calmodulin binding domain. J. Biol. Chem. 271, 4148-4153. doi: 10.1074/jbc.271.8.4148

Snowden, C. J., Thomas, B., Baxter, C. J., Smith, J. A., and Sweetlove, L. J. (2015). A tonoplast Glu/Asp/GABA exchanger that affects tomato fruit amino acid composition. Plant J. 81, 651-660. doi: 10.1111/tpj.12766

Sorrequieta, A., Ferraro, G., Boggio, S. B., and Valle, E. M. (2010). Free amino acid production during tomato fruit ripening: a focus on l-glutamate. Amino Acids 38, 1523-1532. doi: 10.1007/s00726-009-0373-1

Steinhauser, M., Steinhauser, D., Koehl, K., Carrari, F., Gibon, Y., Fernie, A. R., et al. (2010). Enzyme activity profiles during fruit development in tomato cultivar and Solanum pennellii. Plant Physiol. 153, 80-98. doi: 10.1104/pp.110. 154336
Studart-Guimarães, C., Fait, A., Nunes-Nesi, A., Carrari, F., Usadel, B., and Fernie, A. R. (2007). Reduced expression of succinyl-coenzyme A ligase can be compensated for by up-regulation of the $\gamma$-aminobutyrate shunt in illuminated tomato leaves. Plant Physiol. 145, 626-639. doi: 10.1104/pp.107. 103101

Takayama, M., Koike, S., Kusano, M., Matsukura, C., Saito, K., Ariizumi, T., et al. (2015). Tomato glutamate decarboxylase genes SlGAD2 and SIGAD3 play key roles in regulating $\gamma$-aminobutyric acid levels in tomato (Solanum lycopersicum). Plant Cell Physiol. doi: 10.1093/pcp/pcv075 [Epub ahead of print].

Toyokura, K., Watanabe, K., Oiwaka, A., Kusano, M., Tameshige, T., Tatematsu, K., et al. (2011). Succinic semialdehyde dehydrogenase is involved in the robust pattering of Arabidopsis leaves along the adaxial-abaxial axis. Plant Cell Physiol. 52, 1340-1353. doi: 10.1093/pcp/pcr079

Trobacher, C. P., Clark, S. M., Bozzo, G. G., Mullen, R. T., DeEll, J. R., and Shelp, B. J. (2013). Catabolism of GABA in apple fruit: subcellular localization and biochemical characterization of two $\gamma$-aminobutyrate transaminases. Postharvest Biol. Technol. 75, 106-113. doi: 10.1016/j.postharvbio.2012.08.005

Turano, F. J., and Fang, T. K. (1998). Characterization of two glutamate decarboxylase cDNA clones from Arabidopsis. Plant Physiol. 117, 1411-1421. doi: 10.1104/pp.117.4.1411

Van Cauwenberghe, O. R., Makhmoudova, A., McLean, M. D., Clark, S., and Shelp, B. J. (2002). Plant pyruvate-dependent gamma-aminobutyrate transaminase: identification of an Arabidopsis cDNA and its expression in Escherichia coli. Can. J. Bot. 80, 933-941. doi: 10.1139/b02-087

Van Cauwenberghe, O. R., and Shelp, B. J. (1999). Biochemical characterization of partially purified gaba:pyruvate transaminase from Nicotiana tabacum. Phytochemistry 52, 575-581. doi: 10.1016/S0031-9422(99)00301-5

Yang, R., Guo, Q., and Gu, Z. (2013). GABA shunt and polyamine degradation pathway on $\gamma$-aminobutyric acid accumulation in germinating fava bean (Vicia faba L.) under hypoxia. Food Chem. 136, 152-159. doi: 10.1016/ j.foodchem.2012.08.008

Yevtushenko, D. P., McLean, M. D., Peiris, S., Van Cauwenberghe, O. R., and Shelp, B. J. (2003). Calcium/calmodulin activation of two divergent glutamate decarboxylases from tobacco. J. Exp. Bot. 54, 2001-2002. doi: $10.1093 /$ jxb/erg210

Yin, Y. G., Tominaga, T., Iijima, Y., Aoki, K., Shibata, D., Ashihara, H., et al. (2010). Metabolic alterations in organic acids and $\gamma$-aminobutyric acid in developing tomato (Solanum lycopersicum L.) fruits. Plant Cell Physiol. 51, 1300-1314. doi: $10.1093 / \mathrm{pcp} / \mathrm{pcq} 090$

Conflict of Interest Statement: The authors declare that the research was conducted in the absence of any commercial or financial relationships that could be construed as a potential conflict of interest.

Copyright (C) 2015 Takayama and Ezura. This is an open-access article distributed under the terms of the Creative Commons Attribution License (CC BY). The use, distribution or reproduction in other forums is permitted, provided the original author(s) or licensor are credited and that the original publication in this journal is cited, in accordance with accepted academic practice. No use, distribution or reproduction is permitted which does not comply with these terms. 


\section{OPEN ACCESS}

Edited by: Dietmar Funck,

University of Konstanz, Germany

Reviewed by: Helena Storchova, Institute of Experimental Botany Academy of Sciences of the Czech

Republic, Czech Republic

Ewa Sobieszczuk-Nowicka,

Adam Mickiewicz University, Poland

*Correspondence: Donald F. Becker, Redox Biology Center, Department of Biochemistry, University of Nebraska-Lincoln, N258 Beadle Center, Lincoln, NE 68588, USA dbecker3@unl.edu

Specialty section: This article was submitted to Plant Physiology,

a section of the journal Frontiers in Plant Science

Received: 14 May 2015 Accepted: 06 July 2015 Published: 22 July 2015

Citation:

Zhang L and Becker DF (2015) Connecting proline metabolism and signaling pathways in plant senescence.

Front. Plant Sci. 6:552 doi: $10.3389 / \mathrm{fp} / \mathrm{s} .2015 .00552$

\section{Connecting proline metabolism and signaling pathways in plant senescence}

\author{
Lu Zhang and Donald F. Becker* \\ Redox Biology Center, Department of Biochemistry, University of Nebraska-Lincoln, Lincoln, NE, USA
}

The amino acid proline has a unique biological role in stress adaptation. Proline metabolism is manipulated under stress by multiple and complex regulatory pathways and can profoundly influence cell death and survival in microorganisms, plants, and animals. Though the effects of proline are mediated by diverse signaling pathways, a common theme appears to be the generation of reactive oxygen species (ROS) due to proline oxidation being coupled to the respiratory electron transport chain. Considerable research has been devoted to understand how plants exploit proline metabolism in response to abiotic and biotic stress. Here, we review potential mechanisms by which proline metabolism influences plant senescence, namely in the petal and leaf. Recent studies of petal senescence suggest proline content is manipulated to meet energy demands of senescing cells. In the flower and leaf, proline metabolism may influence ROS signaling pathways that delay senescence progression. Future studies focusing on the mechanisms by which proline metabolic shifts occur during senescence may lead to novel methods to rescue crops under stress and to preserve post-harvest agricultural products.

Keywords: proline, proline dehydrogenase, ${ }^{1} \Delta$-pyrroline-5-carboxylate synthetase, plant senescence, reactive oxygen species

\section{Introduction}

Proline metabolism involves the interconversion of proline and glutamate, a process linked to cellular energetics directly via the respiratory electron transport chain. Proline in higher plants is synthesized from glutamate and ornithine (Fichman et al., 2014). Glutamate-derived proline requires the bifunctional enzyme ${ }^{1} \Delta$-pyrroline-5-carboxylate (P5C) synthetase (P5CS), which catalyzes a twostep reaction requiring ATP and NADPH to generate glutamate- $\gamma$-semialdehyde (GSA; Figure 1). GSA spontaneously cyclizes to P5C which is then reduced to proline in a NADPH dependent reaction catalyzed by P5C reductase (P5CR) (P5CR; At5g14800; Liang et al., 2013). In higher plant, P5CS, the rate-limiting enzyme of proline biosynthesis, has two isoforms, P5CS1 (At2g39800) and P5CS2 (At3g55610). P5CS1, localized in the chloroplast, is responsible for stress-induced proline synthesis (Liu et al., 2012a) whereas P5CS2, localized in the cytosol, is important for embryo development (Szekely et al., 2008; Funck et al., 2012). Ornithine-derived proline requires ornithine$\delta$-aminotransferase, which converts ornithine into GSA (Funck et al., 2008; Fichman et al., 2014).

The oxidation of proline to glutamate involves two mitochondrial enzymes, the flavin-dependent proline dehydrogenase $(\mathrm{PRODH})$ and $\mathrm{NAD}^{+}$-dependent $\mathrm{P} 5 \mathrm{C}$ dehydrogenase $(\mathrm{P} 5 \mathrm{CDH})(\mathrm{P} 5 \mathrm{CDH}$; At5g62530; Figure 1; Liang et al., 2013). PRODH catalyzes the rate-determining step of proline catabolism and in plants exists as two isoforms, PRODH1 (At3g30775) and PRODH2 (At5g38710). A search of available plant genomes indicates that the two PRODH isoforms are commonly 

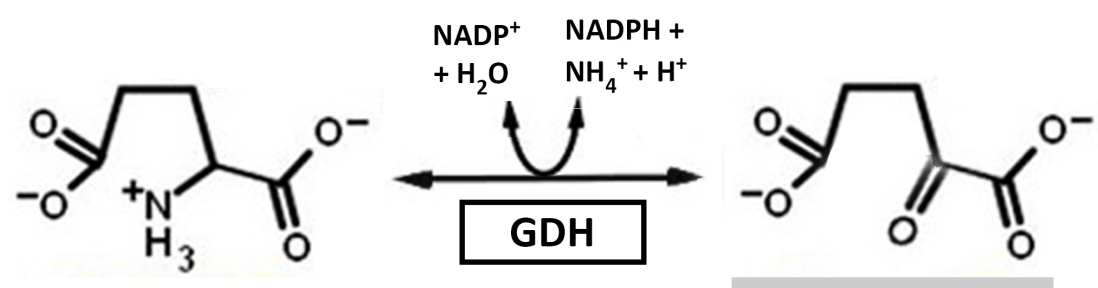

Glutamate

$\alpha$-Ketoglutarate

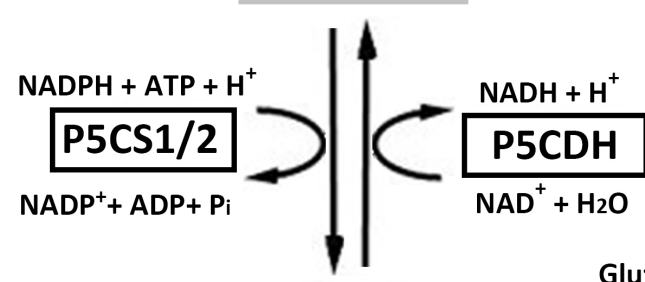

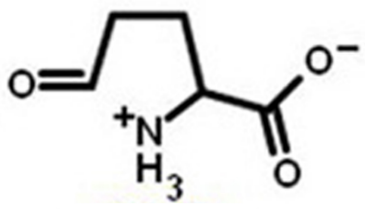

GSA<smiles>CC(=O)O</smiles>

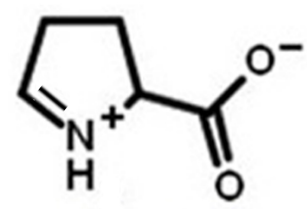

P5C
Glutamate $\alpha$-Ketoglutarate

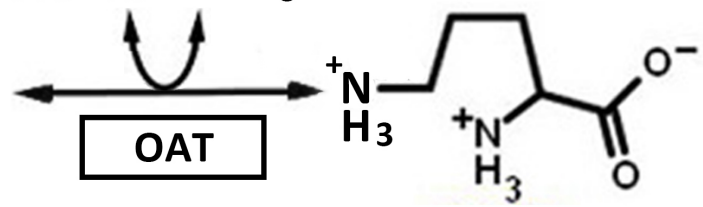

Ornithine
FIGURE 1 | Proline metabolic pathways in higher plants. In the biosynthesis pathway, ornithine and glutamate can be converted to glutamate- $\gamma$-semialdehyde (GSA) by ornithine- $\delta$-aminotransferase (OAT) and ${ }^{1} \Delta$-pyrroline-5-carboxylate (P5C) synthetase (P5CS), respectively. GSA can then spontaneously cyclize to P5C by losing one molecule of $\mathrm{H}_{2}$ O. P5C is the substrate for P5C reductase (P5CR), which catalyzes the last step in proline synthesis. In the catabolic pathway, proline

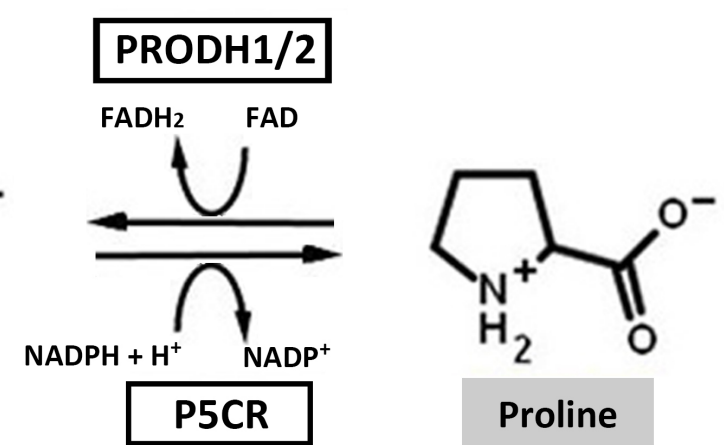

dehydrogenase $(\mathrm{PRODH})$ and $\mathrm{P} 5 \mathrm{C}$ dehydrogenase $(\mathrm{P} 5 \mathrm{CDH})$ catalyze the oxidation of proline to glutamate. Electrons from reduced flavin $\left(F A D H_{2}\right)$ are transferred to the respiratory electron transport chain to regenerate oxidized flavin (FAD) and complete the PRODH catalytic cycle. Glutamate dehydrogenase $(\mathrm{GDH})$ interconverts glutamate and $\alpha$-ketoglutarate, which enters the tricarboxylic acid cycle. Higher plants harbor two isoforms of P5CS and PRODH. found in higher plants. PRODH1 is ubiquitously expressed in plant whereas $P R O D H 2$ expression is localized to the vasculature (Funck et al., 2010). Proline oxidation yields approximately 30 ATP due to coupling with the mitochondrial electron transport chain and glutamate entering the tricarboxylic acid cycle as $\alpha$ ketoglutarate (Verbruggen and Hermans, 2008).

Besides its proteogenic function, proline has roles in energy utilization (Hare and Cress, 1997), reactive oxygen species (ROS) generation (Donald et al., 2001; Szekely et al., 2008; Liu et al., 2012b), programmed cell death (PCD; Donald et al., 2001; Liu et al., 2012b), unfolded protein response (Liang et al., 2014), cell reprogramming and development (Pistollato et al., 2010; Funck et al., 2012; D’Aniello et al., 2015), stress resistance (Strizhov et al., 1997; Krishnan et al., 2008; Szekely et al., 2008; Szabados and Savoure, 2010), and aging (Zarse et al., 2012; Pang and Curran, 2014). In plants, proline metabolism has been proposed to provide stress protection by helping maintain $\mathrm{NADPH} / \mathrm{NADP}^{+}$balance, GSH levels, and during pathogen infection, drive the oxidative 
burst of the hypersensitive response (HR; Miller et al., 2009; Ben Rejeb et al., 2014). Of interest to us are mechanisms by which proline influences the senescence process. Here, we review evidence for proline metabolism having a role in plant petal and leaf senescence.

\section{Proline in Senescing Petals: Energy Depletion and ROS Accumulation}

Senescence is initiated in flowers by natural aging, pollination, and detachment, and culminates in PCD (Rogers, 2012, 2013). Petals, one of the non-productive organs of flowers undergoing senescence (Rogers, 2012), are not photosynthetic (Rogers, 2013). Petals commonly serve as nutrient sinks during development and typically exhibit energy depletion during late stages of senescence (Rogers, 2013). Here, we discuss proline metabolism in the senescing petal of cut flowers.

Studies of flowers have revealed that proline metabolism may have several impacts on petal senescence. A 14-fold increase in proline content was found in petals of cut roses (Rosa hybrid) during senescence (Kumar et al., 2009). In rose petals, increased activity of P5CS and PRODH was observed during senescence progression. P5CS activity was generally higher than PRODH at different stages of senescence (Kumar et al., 2009). The elevated P5CS activity indicates that higher proline content was primarily due to biosynthesis from glutamate (Kumar et al., 2009). Glutamate can be generated by coordinated glutamine synthetase (GS) and glutamine oxoglutarate aminotransferase activity at early stages of senescence and, at late-senescence, by glutamate dehydrogenase (GDH; Figure 1; Kumar et al., 2009). The rise in proline content during senescence may be triggered by lower water potential as senescing rose petals were found to have decreased water potential and elevated levels of the stress hormone abscisic acid (ABA; Kumar et al., 2008), a signaling molecule that induces proline biosynthesis during stress (Savoure et al., 1997; Strizhov et al., 1997). It has not yet been fully explored whether increased proline content facilitates petal senescence or is only a consequence of senescence. Interestingly, at complete flower senescence proline levels dropped by $50 \%$ suggesting that loss of endogenous proline correlates with the end of senescence and termination of vase life. Exogenous application of proline to petals was thus suggested as a possible approach for extending vase life (Kumar et al., 2009).

Because flowers are heterotrophic, increased PRODH activity likely unleashes proline as a fuel source for ATP production during petal senescence. In cut tulips (Tulipa gesneriana), ATP content in petals drops dramatically at day one of flower opening (Azad et al., 2008). Sucrose supplementation, which helps maintain ATP levels, increases vase life indicating the importance of ATP production (Azad et al., 2008). Evidence supporting utilization of proline during senescence was shown by treatment of carnations (Dianthus caryophyllus) with aminooxyacetic acid, an inhibitor of ethylene biosynthesis (Yakimova et al., 1997). Aminooxyacetic acid extended vase life, which correlated with a $40 \%$ decrease in endogenous proline content of senescing petals compared to untreated flowers (Yakimova et al., 1997). Also, application of the plant hormone salicylic acid (SA) to lisianthus flowers (Eustoma grandiflorum Mariachi) doubled the vase life, which correlated with a 75\% reduction in proline content (Kazemi et al., 2011). It is possible that the lower proline content observed with SA treatment is due to increased PRODH activity in the petals. In leaves of Arabidopsis, exogenous application of SA activates transcription of PRODH1 (Cecchini et al., 2011). Whether SA also increases PRODH activity in flower petals needs to be confirmed. Application of $5 \mathrm{mM}$ proline extended the vase life of Rosa hybrida by 30\%, resulting in higher endogenous proline content and PRODH levels (Kumar et al., 2010). Altogether, the results from different studies suggest that proline catabolism and exogenous proline treatment may delay petal senescence by preventing depletion of ATP.

The benefit of proline catabolism during energy-depleted conditions is well known in other organisms and, in worms has been linked to the aging process. For example, in the Caenorhabditis elegans daf-2 mutant, AAK-2 (AMP-activated kinase, AMPK) upregulates $P R O D H$, which is proposed to facilitate lifespan extension by replenishing ATP levels and generating ROS as a signaling molecule to induce antioxidant defenses (Zarse et al., 2012). The AMPK homolog in plants is SnRK1 (sucrose-non-fermenting-1-related protein kinase-1), which regulates members of the S1 basic leucine zipper (bZIP) transcription family, such as bZIP1, bZIP11, and bZIP53, under low sugar or energy conditions (Polge and Thomas, 2007; Tome et al., 2014). bZIP11 is expressed more abundantly in petals (Hummel et al., 2009) relative to bZIP1 and dZIP53 (Llorca et al., 2014). Overexpression of bZIP11 resulted in decreased proline content consistent with upregulation of proline catabolism (Hanson et al., 2008). In Arabidopsis, expression of $P R O D H 2$ in the vascular tissue and abscission zone of petals is regulated by bZIP11 (Hanson et al., 2008) whereas PRODH1 expression is induced by bZIP1 and bZIP53 (Dietrich et al., 2011). Thus, in response to low sucrose, expression levels of $d Z I P 1, b Z I P 11$, and $b Z I P 53$ increase, resulting in higher expression of $P R O D H 1$ and $P R O D H 2$ thereby increasing proline catabolic flux (Funck et al., 2010; Llorca et al., 2014).

Is regulation of PRODH by bZIPs relevant to senescence? A role for SnRK1 and bZIPs in senescence is supported by different studies. For example, disruption of SnRK1 was shown to accelerate senescence progression in moss (Physcomitrella patens, Polge and Thomas, 2007). The expression of bZIP1 and bZIP53 in Arabidopsis are upregulated during dark-induced leaf senescence (Dietrich et al., 2011). PRODH1, regulated by bZIP1 and bZIP53, is the dominant isoform in flowers under most conditions (Funck et al., 2010). Therefore, bZIP1, bZIP53, and PRODH1, may have important roles in the mechanism by which SnRK1 influences the senescence of cut flowers. More direct evidence is needed to establish whether PRODH1 and PRODH2 have a critical role in senescence delay via the SnRK1 signaling pathway.

Besides energy depletion, another important factor of senescence is the accumulation of oxidative damage in aging tissue. Studies have shown that ROS accumulation plays a vital role in flower senescence as reviewed by Rogers (2012). ROS appears to facilitate the aging process and is necessary for petal senescence. $\mathrm{H}_{2} \mathrm{O}_{2}$ was found to buildup in daylilies (Hemerocallis) during senescence progression (Chakrabarty 
et al., 2009). In Tulipa gesneriana, rising $\mathrm{H}_{2} \mathrm{O}_{2}$ levels followed the appearance of senescence markers (protease activity and cytochrome c) at the end of flower opening (Azad et al., 2008). To alleviate the oxidative burden in snapdragon (Antirrhinum majus) petals, ascorbic acid was applied as an exogenous antioxidant resulting in a $20 \%$ longer vase life relative to untreated flowers (Abdulrahman et al., 2012).

Proline is known to protect against oxidative stress in many organisms including fungi (Chen and Dickman, 2005; Chen et al., 2006), bacteria (Zhang et al., 2015), plants (Szabados and Savoure, 2010; Sorkheh et al., 2012), and to animals (Natarajan et al., 2012; Zarse et al., 2012). It is still controversial, however, whether protection is due to proline directly scavenging ROS. Proline is expected to efficiently react with ${ }^{\bullet} \mathrm{OH}$ (Signorelli et al., 2014) and was suggested to scavenge ${ }^{1} \mathrm{O}_{2}$ (Alia et al., 2001). Another study, however, reported that proline does not quench ${ }^{1} \mathrm{O}_{2}$ in plants (Signorelli et al., 2013) and recently, proline was found not to directly scavenge $\mathrm{H}_{2} \mathrm{O}_{2}$ (Zhang et al., 2015). In bacteria and mammalian cells, $\mathrm{PRODH}$ is necessary for prolinemediated adaptation to oxidative stress (Natarajan et al., 2012; Zhang et al., 2015). Proline catabolism generates $\mathrm{H}_{2} \mathrm{O}_{2}$ as a by-product thereby activating antioxidant signaling pathways. Proline oxidation induces the OxyR regulon of Escherichia coli (Zhang et al., 2015), Akt pathway in human cells (Natarajan et al., 2012), and the MAPK pathway (Okuyama et al., 2010) in the C. elegans daf-2 mutant (Zarse et al., 2012). Thus, although the signaling pathways vary, $\mathrm{H}_{2} \mathrm{O}_{2}$ production by proline catabolism seems to be a conserved mechanism by which proline influences antioxidant defenses.

Could proline metabolism influence ROS accumulation during senescence? Interestingly, relative to untreated roses, proline treatment sustained $\mathrm{Mn}$-dependent superoxide dismutase (MnSOD) activity, the dominant SOD in petals, resulting in twofold lower levels of superoxide anion radicals at each stage of petal senescence (Kumar et al., 2010). In plant, MAPK cascades respond to ROS and regulate antioxidant signaling pathways (Pitzschke et al., 2009; Sinha et al., 2011). MAPK cascades consist of three kinases: MAPK kinase kinases (MEKKs), MAPK kinases (MKKs) and MAPKs (MPKs; Sinha et al., 2011). $\mathrm{H}_{2} \mathrm{O}_{2}$ activates ROS scavenging enzymes by initiating a phosphorylation cascade involving MKK4/5 and MPK3/6 (Kovtun et al., 2000). MKK4 and MKK5 are known to be necessary for flower organ abscission in Arabidopsis (Cho et al., 2008). Tandem RNAi knockdown of both $M K K 4$ and MKK5 showed a defect in petal abscission, although individual RNAi knockdown of either gene had a normal phenotype (Cho et al., 2008). MPK3 and MPK6 were also required for petal abscission (Cho et al., 2008). Future studies are needed to understand whether ROS triggers the phosphorylation of MKK4/5 and MPK3/6 during petal senescence thereby promoting petal abscission.

Little is known about the relationship between PRODH and the MAPK pathway in plants, however, hypoosmotic stress was observed to induce a similar pattern of MPK20 and PRODH expression in Arabidopsis, suggesting a link between PRODH and MPK20 (Moustafa et al., 2008). A recent genomic study showed MPK20 was highly induced by $\mathrm{H}_{2} \mathrm{O}_{2}$ stress in cotton (Gossypium raimondii, Zhang et al., 2014). Is it plausible that ROS generated by proline metabolism induces MPK20 during petal senescence? Stronger evidence is available for linking proline biosynthesis with MAPK signaling, namely MKK4. Overexpression of ZmMKK4, a gene from Zea mays, in tobacco or Arabidopsis resulted in elevated P5CS2 activity (Kong et al., 2011a), leading to increased proline content and tolerance to hyperosmotic stress (Kong et al., 2011a,b). In line with the ROS sensing role of the MAPK signaling pathway, overexpression of $Z m M K K 4$ resulted in higher peroxidase activity and lower ROS levels (Kong et al., 2011a,b). Whether MKK4 also upregulates P5CS during flower senescence needs to be determined.

\section{Proline in Leaf Senescence: Upregulation of Proline Catabolism and Hormone-Induced Pathways}

Leaf senescence, a developmentally regulated PCD, is agedependent and induced by environmental signals (drought, detachment or darkness; Smart, 1994; van Doorn and Woltering, 2004). The role of proline metabolism in leaf senescence shares some similarities with petals but also has unique features (Price et al., 2008). Proline levels increase proportionally with leaf age in excised leaf segments and are an indicator of leaf senescence (Wang et al., 1982; Mondal et al., 1985). Unlike flowers, leaf generates energy via photosynthesis which is maintained during late stages of senescence (Smart, 1994; Kotakis et al., 2014). Thus, proline is not needed as an energy source during leaf senescence.

What are the relevant mechanisms of proline metabolism in leaf senescence? One observation is that during natural leaf aging proline catabolism appears to be upregulated via increased expression of $\mathrm{PRODH} 2$ and $\mathrm{P} 5 \mathrm{CDH}$. In Arabidopsis thaliana and Brassica napus (rapeseed), $\mathrm{PRODH} 2$ expression was strongly induced in the course of natural leaf aging (Funck et al., 2010; Faes et al., 2015), whereas PRODH1 expression was moderately upregulated (Funck et al., 2010). P5CDH expression was also reported to increase in older leaves of Arabidopsis (Deuschle et al., 2004). Why proline catabolism would be upregulated during natural leaf senescence remains largely unresolved since there does not seem to be a significant energy deficit as mentioned above. Proline degradation, however, may assist nitrogen recycling in the phloem from old leaves to sink organs (Faes et al., 2015) which would be consistent with the stronger expression of $\mathrm{PRODH} 2$ in vascular tissues at senescence. Cytosolic GS1 and GDH are also induced during leaf senescence, apparently to facilitate nitrogen recycling (Masclaux-Daubresse et al., 2005). In fact, proline treatment of Arabidopsis was observed to induce expression of GS1 and GDH (Masclaux-Daubresse et al., 2005), indicating that proline catabolism provides glutamate as a substrate for GS1 and GDH. Thus, proline may be needed for nitrogen cycling during leaf senescence.

The possibility of PRODH1 being involved in plant hormone induced senescence is inferred from a recent study showing proline metabolism is regulated by phosphatidylinositol-3,4,5triphosphate dependent kinase (PI3K; Leprince et al., 2015). 


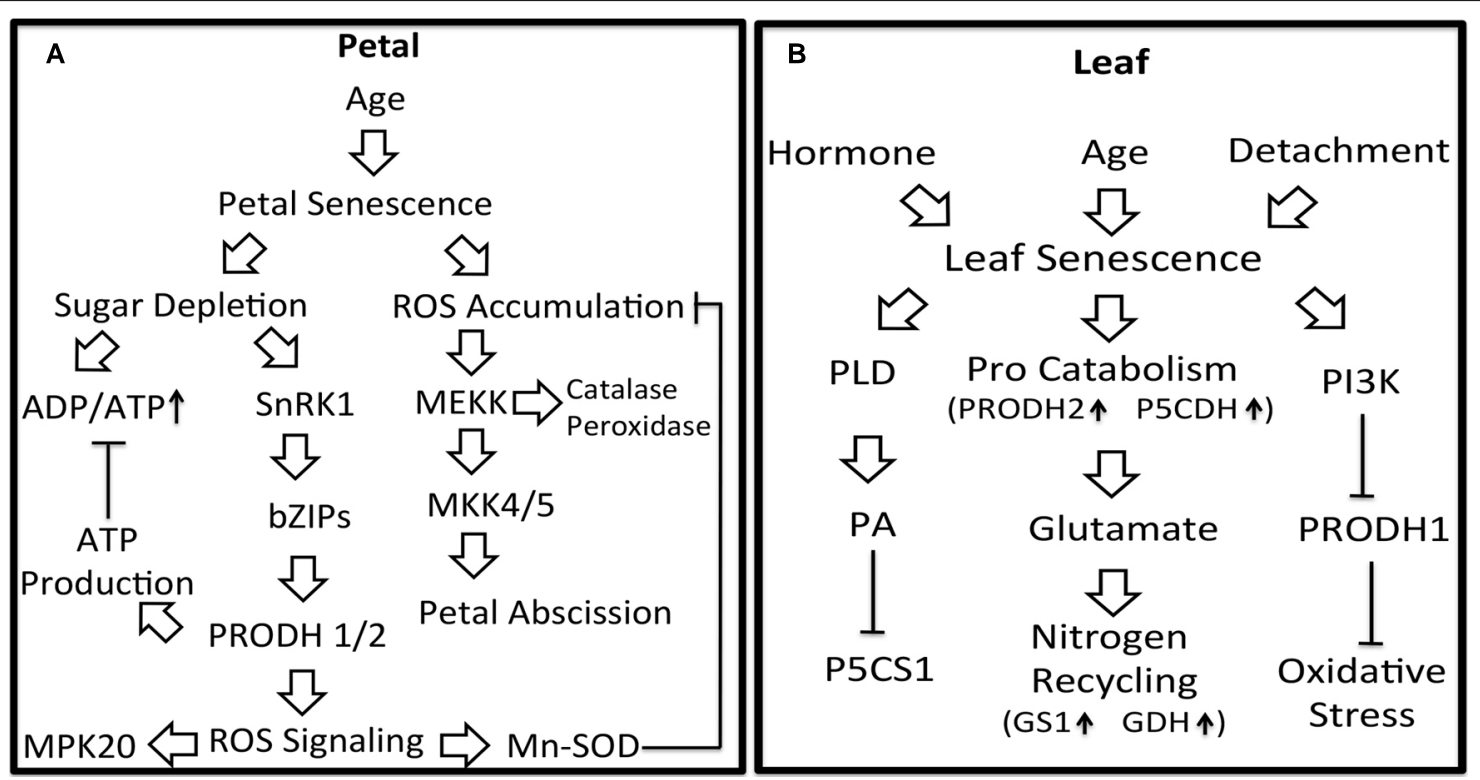

FIGURE 2 | Potential linkages between proline metabolism and signaling pathways in petal and leaf senescence. Petal senescence (A): Aging induced petal senescence results in ROS accumulation and sugar depletion. SnRK1, activated in response to depleted sugar, is proposed to induce PRODH1/2 expression via bZIP1, bZIP11, and bZIP53. Upregulation of $P R O D H 1 / 2$ expression would be predicted to generate ATP thereby attenuating increases in ADP/ATP. PRODH activity is also expected to generate ROS as a by-product, possibly leading to activation of MPK2O and increased Mn-SOD activity. Enhanced Mn-SOD activity would help diminish accumulated ROS and oxidative damage during petal senescence. Activation of MAPK pathways by ROS would induce expression of antioxidant enzymes and petal abscission. Leaf senescence (B): Leaf senescence can be induced by plant hormones, age and detachment. During age-related senescence, the expression of $P R O D H 2$ and $\mathrm{P} 5 \mathrm{CDH}$ are induced, suggesting a higher flux of proline catabolism and more glutamate available for nitrogen recycling. In response to $\mathrm{H}_{2} \mathrm{O}_{2}$ during hormone-induced senescence, $\mathrm{PI} 3 \mathrm{~K}$ may down-regulate $\mathrm{PRODH} 1$ resulting in less ROS signaling and adaptation to oxidative stress. Also during hormone-induced senescence, phospholipase D (PLD) and its product phosphatidic acid (PA) inhibit P5CS1 expression.
Under salt stress, inhibition of PI3K by LY294002 resulted in lower P5CS1 and higher PRODH1 expression and, decreased proline content in Arabidopsis (Leprince et al., 2015). PRODH1 expression was also higher in a pi3k-hemizygous Arabidopsis mutant (Leprince et al., 2015). Thus, PI3K appears to repress PRODH1 expression. The mechanism by which plant hormones such as methyl jasmonic-acid (JA; Hung et al., 2006) and ABA (Hung and Kao, 2005) induce senescence, involves PI3K signaling and $\mathrm{H}_{2} \mathrm{O}_{2}$. Inhibiting PI3K activity with LY294002 aborted $\mathrm{H}_{2} \mathrm{O}_{2}$ production and delayed JA induced-senescence in rice leaves (Hung et al., 2006). Phosphatidylinositol-3-phosphate (PI3P), the product of $\mathrm{PI} 3 \mathrm{~K}$, has also been shown to be necessary for ABAinduced $\mathrm{H}_{2} \mathrm{O}_{2}$ production and senescence (Hung and Kao, 2005). Thus, $\mathrm{PI} 3 \mathrm{~K}$ and PI3P promote plant hormone-induced senescence via $\mathrm{H}_{2} \mathrm{O}_{2}$ production. Repression of $P R O D H 1$ by $\mathrm{PI} 3 \mathrm{~K}$ could potentially increase oxidative stress burden as discussed above due to loss of proline-mediated ROS signaling and decreased antioxidant defense.

Additional evidence for proline metabolism being involved in plant hormone-induced senescence is from studies of lipid degradation. Lipid degradation is generally upregulated during leaf senescence due to loss of cellular membrane integrity (Lim et al., 2007). Phospholipase D (PLD) catalyzes the production of membrane-bound phosphatidic acid (PA) from phospholipid (Kolesnikov et al., 2012). PLD activity has been shown to gradually increase at senescence in castor oil plant (Ricinus communis) leaves (Ryu and Wang, 1995). Plants harbor three isoforms of PLDs: PLD $\alpha, \operatorname{PLD} \beta$ and PLD $\delta$ (Kolesnikov et al., 2012). Antisense suppression of $P L D \alpha$, the most abundant PLD, slowed down ABAand ethylene-induced senescence of detached Arabidopsis leaves, while there was no effect on natural aging leaf senescence (Fan et al., 1997). The suppression of $P L D \delta$, which is upregulated during senescence, also delays ABA-promoted senescence in Arabidopsis (Jia et al., 2013). PLD appears to be a negative regulator of P5CS1 as inhibition of PA signaling by 1-butanol was observed to increase P5CS1 expression in Arabidopsis (Thiery et al., 2004) and Thellungiella halophila (Ghars et al., 2012). Because senescence-specific degradation of Calvin cycle enzymes has been shown to lower $\mathrm{NADP}^{+}$levels in chloroplasts (Zhang et al., 2012) increased P5CS1 expression may help maintain $\mathrm{NADPH} / \mathrm{NADP}^{+}$balance (Liang et al., 2013). Whether regulation of P5CS1 by PA signaling is important during hormone-induced leaf senescence needs to be determined.

\section{Summary and Future Direction}

The various roles of proline metabolism in energetics, ROS signaling, and cellular processes continue to unfold (Ben Rejeb et al., 2014). Proline metabolic ROS production appears to be a general phenomenon in diverse organisms potentially impacting cellular processes such as aging and plant senescence. An excellent example of PRODH mediated ROS production in plants is from 
studies of Pseudomonas syringae pathogen induced HR (Cecchini et al., 2011). In the late stages of $\mathrm{HR}, P R O D H$ and P5CDH expression becomes uncoupled, thereby enabling proline/P5C cycling via PRODH and P5CR, leading to an oxidative burst (Cecchini et al., 2011; Monteoliva et al., 2014).

The molecular pathways by which proline metabolism is regulated during plant senescence are summarized in Figure 2. The ability of proline to delay senescence in petal and leaf tissues is likely due to protection against oxidative stress that occurs in the aging tissue. In leaf, proline may help redistribute nitrogen to younger tissue whereas in flowers, proline helps counter energy shortages. Further insights into how proline metabolism impacts petal and leaf senescence will require

\section{References}

Abdulrahman, Y. A., Ali, S. F., and Faizi, H. S. (2012). Effect of sucrose and ascorbic acid concentrations on vase life of snapdragon (Antirrhinum Majus L.) cut flowers. Int. J. Pure Appl. Sci. Technol. 13, 32-41.

Alia, Mohanty, P., and Matysik, J. (2001). Effect of proline on the production of singlet oxygen. Amino Acids 21, 195-200. doi: 10.1007/s007260170026

Azad, A. K., Ishikawa, T., Ishikawa, T., Sawa, Y., and Shibata, H. (2008). Intracellular energy depletion triggers programmed cell death during petal senescence in tulip. J. Exp. Bot. 59, 2085-2095. doi: 10.1093/jxb/ern066

Ben Rejeb, K., Abdelly, C., and Savoure, A. (2014). How reactive oxygen species and proline face stress together. Plant Physiol. Biochem. 80, 278-284. doi: 10.1016/j.plaphy.2014.04.007

Cecchini, N. M., Monteoliva, M. I., and Alvarez, M. E. (2011). Proline dehydrogenase contributes to pathogen defense in Arabidopsis. Plant Physiol. 155, 1947-1959. doi: 10.1104/pp.110.167163

Chakrabarty, D., Verma, A. K., and Datta, S. K. (2009). Oxidative stress and antioxidant activity as the basis of senescence in Hemerocallis (day lily) flowers. J. Hortic. For. 1, 113-119.

Chen, C., and Dickman, M. B. (2005). Proline suppresses apoptosis in the fungal pathogen Colletotrichum trifolii. Proc. Natl. Acad. Sci. U.S.A. 102, 3459-3464. doi: 10.1073/pnas.0407960102

Chen, C. B., Wanduragala, S., Becker, D. F., and Dickman, M. B. (2006). Tomato QM-Like protein protects Saccharomyces cerevisiae cells against oxidative stress by regulating intracellular proline levels. Appl. Environ. Microbiol. 72, 4001-4006. doi: 10.1128/Aem.02428-05

Cho, S. K., Larue, C. T., Chevalier, D., Wang, H., Jinn, T. L., Zhang, S., et al. (2008). Regulation of floral organ abscission in Arabidopsis thaliana. Proc. Natl. Acad. Sci. U.S.A. 105, 15629-15634. doi: 10.1073/pnas.0805539105

D’Aniello, C., Fico, A., Casalino, L., Guardiola, O., Di Napoli, G., Cermola, F., et al. (2015). A novel autoregulatory loop between the Gcn2-Atf4 pathway and LProline metabolism controls stem cell identity. Cell Death Differ. 22, 1094-1105. doi: $10.1038 /$ cdd.2015.24

Deuschle, K., Funck, D., Forlani, G., Stransky, H., Biehl, A., Leister, D., et al. (2004). The role of $\Delta^{1}$-pyrroline-5-carboxylate dehydrogenase in proline degradation. Plant Cell 16, 3413-3425. doi: 10.1105/tpc.104.023622

Dietrich, K., Weltmeier, F., Ehlert, A., Weiste, C., Stahl, M., Harter, K., et al. (2011). Heterodimers of the Arabidopsis transcription factors bZIP1 and bZIP $_{53}$ reprogram amino acid metabolism during low energy stress. Plant Cell 23, 381-395. doi: 10.1105/tpc.110. 075390

Donald, S. P., Sun, X. Y., Hu, C. A., Yu, J., Mei, J. M., Valle, D., et al. (2001). Proline oxidase, encoded by 553 -induced gene- 6 , catalyzes the generation of proline-dependent reactive oxygen species. Cancer Res. 61, 1810-1815.

Faes, P., Deleu, C., Ainouche, A., Le Caherec, F., Montes, E., Clouet, V., et al. (2015). Molecular evolution and transcriptional regulation of the oilseed rape proline dehydrogenase genes suggest distinct roles of proline catabolism during development. Planta 241, 403-419. doi: 10.1007/s00425-014-2189-9

Fan, L., Zheng, S., and Wang, X. (1997). Antisense suppression of phospholipase $\mathrm{D} \alpha$ retards abscisic acid- and ethylene-promoted senescence of postharvest Arabidopsis leaves. Plant Cell 9, 2183-2196. doi: 10.1105/tpc.9.12.2183 additional studies that connect proline with plant senescence signaling pathways. Exploration of the linkages between proline metabolism and important pathways of plant senescence such as MAPK signaling, the SnRK1-bZIP pathway and PI3K signaling will be valuable targets for future study. Better understanding of proline metabolism in senescing leaves may uncover novel strategies for preserving post-harvest flowers and delaying stressinduced leaf senescence.

\section{Acknowledgment}

This research was supported in part by grants GM079393 and P30GM103335 from the National Institutes of Health.

Fichman, Y., Gerdes, S. Y., Kovacs, H., Szabados, L., Zilberstein, A., and Csonka, L. N. (2014). Evolution of proline biosynthesis: enzymology, bioinformatics, genetics, and transcriptional regulation. Biol. Rev. Camb. Philos. Soc. doi: 10.1111/brv.12146 [Epub ahead of print].

Funck, D., Eckard, S., and Muller, G. (2010). Non-redundant functions of two proline dehydrogenase isoforms in Arabidopsis. BMC Plant Biol. 10:70. doi: 10.1186/1471-2229-10-70

Funck, D., Stadelhofer, B., and Koch, W. (2008). Ornithine- $\delta$-aminotransferase is essential for arginine catabolism but not for proline biosynthesis. BMC Plant Biol. 8:40. doi: 10.1186/1471-2229-8-40

Funck, D., Winter, G., Baumgarten, L., and Forlani, G. (2012). Requirement of proline synthesis during Arabidopsis reproductive development. BMC Plant Biol. 12:191. doi: 10.1186/1471-2229-12-191

Ghars, M. A., Richard, L., Lefebvre-De Vos, D., Leprince, A. S., Parre, E., Bordenave, M., et al. (2012). Phospholipases C and D modulate proline accumulation in Thellungiella halophila/Salsuginea differently according to the severity of salt or hyperosmotic stress. Plant Cell Physiol. 53, 183-192. doi: 10.1093/pcp/ pcr164

Hanson, J., Hanssen, M., Wiese, A., Hendriks, M. M., and Smeekens, S. (2008). The sucrose regulated transcription factor bZIP11 affects amino acid metabolism by regulating the expression of ASPARAGINE SYNTHETASE1 and PROLINE DEHYDROGENASE2. Plant J. 53, 935-949. doi: 10.1111/j.1365313X.2007.03385.x

Hare, P. D., and Cress, W. A. (1997). Metabolic implications of stressinduced proline accumulation in plants. Plant Growth Regul. 21, 79-102. doi: 10.1023/A:1005703923347

Hummel, M., Rahmani, F., Smeekens, S., and Hanson, J. (2009). Sucrose-mediated translational control. Ann. Bot. 104, 1-7. doi: 10.1093/aob/mcp086

Hung, K. T., Hsu, Y. T., and Kao, C. H. (2006). Hydrogen peroxide is involved in methyl jasmonate-induced senescence of rice leaves. Physiol. Plant. 127, 293-303. doi: 10.1111/J.1399-3054.2006.00662.X

Hung, K. T., and Kao, C. H. (2005). Phosphatidylinositol 3-phosphate is required for abscisic acid-induced hydrogen peroxide production in rice leaves. Plant Growth Regul. 45, 95-101. doi: 10.1007/S10725-005-1434-4

Jia, Y., Tao, F., and Li, W. (2013). Lipid profiling demonstrates that suppressing Arabidopsis phospholipase D $\delta$ retards ABA-promoted leaf senescence by attenuating lipid degradation. PLoS ONE 8:e65687. doi: 10.1371/journal.pone.0065687

Kazemi, M., Aran, M., and Zamani, S. (2011). Extending the vase life of lisianthus (Eustoma grandiflorum mariachi. cv. blue) with different preservative. Am. J. Plant Physiol. 6, 167-175. doi: 10.3923/ajpp.2011.167.175

Kolesnikov, Y. S., Nokhrina, K. P., Kretynin, S. V., Volotovski, I. D., Martinec, J., Romanov, G. A., et al. (2012). Molecular structure of phospholipase D and regulatory mechanisms of its activity in plant and animal cells. Biochem. (Mosc.) 77, 1-14. doi: 10.1134/S0006297912010014

Kong, X. P., Pan, J. W., Zhang, M. Y., Xing, X., Zhou, Y., Liu, Y., et al. (2011a). $Z m M K K 4$, a novel group $\mathrm{C}$ mitogen-activated protein kinase kinase in maize (Zea mays), confers salt and cold tolerance in transgenic Arabidopsis. Plant Cell Environ. 34, 1291-1303. doi: 10.1111/J.1365-3040.2011.02329.X

Kong, X. P., Sun, L. P., Zhou, Y., Zhang, M. Y., Liu, Y., Pan, J. W., et al. (2011b). $Z m M K K 4$ regulates osmotic stress through reactive oxygen species scavenging 
in transgenic tobacco. Plant Cell Rep. 30, 2097-2104. doi: 10.1007/S00299-0111116-9

Kotakis, C., Kyzeridou, A., and Manetas, Y. (2014). Photosynthetic electron flow during leaf senescence: evidence for a preferential maintenance of photosystem I activity and increased cyclic electron flow. Photosynthetica 52, 413-420. doi: 10.1007/S11099-014-0046-5

Kovtun, Y., Chiu, W. L., Tena, G., and Sheen, J. (2000). Functional analysis of oxidative stress-activated mitogen-activated protein kinase cascade in plants. Proc. Natl. Acad. Sci. U.S.A. 97, 2940-2945. doi: 10.1073/pnas.97.6.2940

Krishnan, N., Dickman, M. B., and Becker, D. F. (2008). Proline modulates the intracellular redox environment and protects mammalian cells against oxidative stress. Free Radic. Biol. Med. 44, 671-681. doi: 10.1016/j.freeradbiomed.2007.10.054

Kumar, N., Pal, M., Singh, A., Sairam, R. K., and Srivastava, G. C. (2010). Exogenous proline alleviates oxidative stress and increase vase life in rose (Rosa hybrida $\mathrm{L}$ 'Grand Gala'). Sci. Hortic. 127, 79-85. doi: 10.1016/J.Scienta.2010.09.009

Kumar, N., Pal, M., and Srivastava, G. C. (2009). Proline metabolism in senescing rose petals (Rosa hybrida L. 'First Red'). J. Hortic. Sci. Biotech. 84, 536-540.

Kumar, N., Srivastava, G. C., and Dixit, K. (2008). Hormonal regulation of flower senescence in roses (Rosa hybrida L.). Plant Growth Regul. 55, 65-71. doi: 10.1007/S10725-008-9259-6

Leprince, A. S., Magalhaes, N., De Vos, D., Bordenave, M., Crilat, E., Clement, G., et al. (2015). Involvement of Phosphatidylinositol 3-kinase in the regulation of proline catabolism in Arabidopsis thaliana. Front. Plant Sci. 5:772. doi: 10.3389/Fpls.2014.00772

Liang, X., Dickman, M. B., and Becker, D. F. (2014). Proline biosynthesis is required for endoplasmic reticulum stress tolerance in Saccharomyces cerevisiae. J. Biol. Chem. 289, 27794-27806. doi: 10.1074/jbc.M114.562827

Liang, X., Zhang, L., Natarajan, S. K., and Becker, D. F. (2013). Proline mechanisms of stress survival. Antioxid. Redox Signal. 19, 998-1011. doi: 10.1089/ars.2012.5074

Lim, P. O., Kim, H. J., and Nam, H. G. (2007). Leaf senescence. Annu. Rev. Plant Biol. 58, 115-136. doi: 10.1146/annurev.arplant.57.032905.105316

Liu, P., Sun, F., Gao, R., and Dong, H. (2012a). RAP2.6L overexpression delays waterlogging induced premature senescence by increasing stomatal closure more than antioxidant enzyme activity. Plant Mol. Biol. 79, 609-622. doi: 10.1007/s11103-012-9936-8

Liu, W., Le, A., Hancock, C., Lane, A. N., Dang, C. V., Fan, T. W., et al. (2012b). Reprogramming of proline and glutamine metabolism contributes to the proliferative and metabolic responses regulated by oncogenic transcription factor c-MYC. Proc. Natl. Acad. Sci. U.S.A 109, 8983-8988. doi: $10.1073 /$ pnas.1203244109

Llorca, C. M., Potschin, M., and Zentgraf, U. (2014). bZIPs and WRKYs: two large transcription factor families executing two different functional strategies. Front. Plant Sci. 5:169. doi: 10.3389/Fpls.2014.00169

Masclaux-Daubresse, C., Carrayol, E., and Valadier, M. H. (2005). The two nitrogen mobilisation- and senescence-associated GS1 and GDH genes are controlled by $\mathrm{C}$ and N metabolites. Planta 221, 580-588. doi: 10.1007/s00425-004-1468-2

Miller, G., Honig, A., Stein, H., Suzuki, N., Mittler, R., and Zilberstein, A. (2009). Unraveling $\Delta^{1}$-pyrroline-5-carboxylate-proline cycle in plants by uncoupled expression of proline oxidation enzymes. J. Biol. Chem. 284, 26482-26492. doi: 10.1074/jbc.M109.009340

Mondal, W. A., Dey, B. B., and Choudhuri, M. A. (1985). Proline accumulation as a reliable indicator of monocarpic senescence in rice cultivars. Experientia 41, 346-348. doi: 10.1007/Bf02004497

Monteoliva, M. I., Rizzi, Y. S., Cecchini, N. M., Hajirezaei, M. R., and Alvarez, M. E. (2014). Context of action of proline dehydrogenase (ProDH) in the hypersensitive response of Arabidopsis. BMC Plant Biol. 14:21. doi: 10.1186/1471-2229-14-21

Moustafa, K., Lefebvre-De Vos, D., Leprince, A. S., Savoure, A., and Laurie 'Re, C. (2008). Analysis of the Arabidopsis mitogen-activated protein kinase families: organ specificity and transcriptional regulation upon water stresses. Sch. Res. Exch. 143658. doi: 10.3814/2008/143656

Natarajan, S. K., Zhu, W. D., Liang, X. W., Zhang, L., Demers, A. J., Zimmerman, M. C., et al. (2012). Proline dehydrogenase is essential for proline protection against hydrogen peroxide-induced cell death. Free Radic. Bio. Med. 53, 1181-1191. doi: 10.1016/J.Freeradbiomed.2012.07.002

Okuyama, T., Inoue, H., Ookuma, S., Satoh, T., Kano, K., Honjoh, S., et al. (2010). The ERK-MAPK pathway regulates longevity through SKN-1 and insulin-like signaling in Caenorhabditis elegans. J. Biol. Chem. 285, 30274-30281. doi: 10.1074/Jbc.M110.146274

Pang, S., and Curran, S. P. (2014). Adaptive capacity to bacterial diet modulates aging in C. elegans. Cell Metab. 19, 221-231. doi: 10.1016/j.cmet.2013.12.005

Pistollato, F., Persano, L., Rampazzo, E., and Basso, G. (2010). L-Proline as a modulator of ectodermal differentiation in ES cells. Focus on "L-Proline induces differentiation of ES cells: a novel role for an amino acid in the regulation of pluripotent cells in culture. Am. J. Physiol. Cell Physiol. 298, C979-C981. doi: 10.1152/ajpcell.00072.2010

Pitzschke, A., Djamei, A., Bitton, F., and Hirt, H. (2009). A major role of the MEKK1-MKK1/2-MPK4 pathway in ROS signalling. Mol. Plant 2, 120-137. doi: $10.1093 / \mathrm{mp} / \mathrm{ssn} 079$

Polge, C., and Thomas, M. (2007). SNF1/AMPK/SnRK1 kinases, global regulators at the heart of energy control? Trends Plant Sci. 12, 20-28. doi: 10.1016/J.Tplants.2006.11.005

Price, A. M., Aros Orellana, D. F., Salleh, F. M., Stevens, R., Acock, R., BuchananWollaston, V., et al. (2008). A comparison of leaf and petal senescence in wallflower reveals common and distinct patterns of gene expression and physiology. Plant Physiol. 147, 1898-1912. doi: 10.1104/pp.108.120402

Rogers, H. J. (2012). Is there an important role for reactive oxygen species and redox regulation during floral senescence? Plant Cell Environ. 35, 217-233. doi: 10.1111/J.1365-3040.2011.02373.X

Rogers, H. J. (2013). From models to ornamentals: how is flower senescence regulated? Plant Mol. Biol. 82, 563-574. doi: 10.1007/s11103-012-9968-0

Ryu, S. B., and Wang, X. (1995). Expression of phospholipase D during castor bean leaf senescence. Plant Physiol. 108, 713-719.

Savoure, A., Hua, X. J., Bertauche, N., Van Montagu, M., and Verbruggen, N. (1997). Abscisic acid-independent and abscisic acid-dependent regulation of proline biosynthesis following cold and osmotic stresses in Arabidopsis thaliana. Mol. Gen. Genet. 254, 104-109. doi: 10.1007/s004380050397

Signorelli, S., Arellano, J. B., Melo, T. B., Borsani, O., and Monza, J. (2013). Proline does not quench singlet oxygen: evidence to reconsider its protective role in plants. Plant Physiol. Biochem. 64, 80-83. doi: 10.1016/j.plaphy.2012.12.017

Signorelli, S., Coitino, E. L., Borsani, O., and Monza, J. (2014). Molecular mechanisms for the reaction between $\mathrm{OH}$ radicals and proline: insights on the role as reactive oxygen species scavenger in plant stress. J. Phys. Chem. B 118, 37-347. doi: 10.1021/jp407773u

Sinha, A. K., Jaggi, M., Raghuram, B., and Tuteja, N. (2011). Mitogen-activated protein kinase signaling in plants under abiotic stress. Plant Signal. Behav. 6, 196-203. doi: 10.4161/psb.6.2.14701

Smart, C. M. (1994). Gene expression during leaf senescence. New Phytol. 126, 419-448. doi: 10.1111/j.1469-8137.1994.tb04243.x

Sorkheh, K., Shiran, B., Khodambashi, M., Rouhi, V., Mosavei, S., and Sofo, A. (2012). Exogenous proline alleviates the effects of $\mathrm{H}_{2} \mathrm{O}_{2}$-induced oxidative stress in wild almond species. Russ. J. Plant Physiol. 59, 788-798. doi: $10.1134 /$ S1021443712060167

Strizhov, N., Abraham, E., Okresz, L., Blickling, S., Zilberstein, A., Schell, J., et al. (1997). Differential expression of two P5CS genes controlling proline accumulation during salt-stress requires $\mathrm{ABA}$ and is regulated by $\mathrm{ABA} 1$, ABI1 and AXR2 in Arabidopsis. Plant J. 12, 557-569. doi: 10.1046/j.1365313X.1997.00537.x

Szabados, L., and Savoure, A. (2010). Proline: a multifunctional amino acid. Trends Plant Sci. 15, 89-897. doi: 10.1016/j.tplants.2009.11.009

Szekely, G., Abraham, E., Cseplo, A., Rigo, G., Zsigmond, L., Csiszar, J., et al. (2008). Duplicated P5CS genes of Arabidopsis play distinct roles in stress regulation and developmental control of proline biosynthesis. Plant J. 53, 11-28. doi: 10.1111/j.1365-313X.2007.03318.x

Thiery, L., Leprince, A. S., Lefebvre, D., Ali Ghars, M., Debarbieux, E., and Savoure, A. (2004). Phospholipase D is a negative regulator of proline biosynthesis in Arabidopsis thaliana. J. Biol. Chem. 279, 14812-14818. doi: 10.1074/Jbc.M308456200

Tome, F., Nagele, T., Adamo, M., Garg, A., Marco-Ilorca, C., Nukarinen, E., et al. (2014). The low energy signaling network. Front. Plant Sci. 5:353. doi: 10.3389/Fpls.2014.00353

van Doorn, W. G., and Woltering, E. J. (2004). Senescence and programmed cell death: substance or semantics? J. Exp. Bot. 55, 2147-2153. doi: $10.1093 / \mathrm{Jxb} / \mathrm{Erh} 264$

Verbruggen, N., and Hermans, C. (2008). Proline accumulation in plants: a review. Amino Acids 35, 753-759. doi: 10.1007/s00726-008-0061-6 
Wang, C. Y., Cheng, S. H., and Kao, C. H. (1982). Senescence of rice leaves. 7. Proline accumulation in senescing excised leaves. Plant Physiol. 69, 1348-1349. doi: 10.1104/Pp.69.6.1348

Yakimova, E., Atanassova, B., and Kapchina-Toteva, V. (1997). Longevity and some metabolic envents in postharvest spray-SPRAY-carnation (D. Caryophyllus $F$. sapry, Hort) Flowers. Bulg. J. Plant. Physiol. 23, 57-65.

Zarse, K., Schmeisser, S., Groth, M., Priebe, S., Beuster, G., Kuhlow, D., et al. (2012). Impaired insulin/IGF1 signaling extends life span by promoting mitochondrial L-proline catabolism to induce a transient ROS signal. Cell Metab. 15, 451-465. doi: 10.1016/j.cmet.2012.02.013

Zhang, L., Alfano, J. R., and Becker, D. F. (2015). Proline metabolism increases katG expression and oxidative stress resistance in Escherichia coli. J. Bacteriol. 197, 431-440. doi: 10.1128/JB.02282-14

Zhang, X., Wang, L., Xu, X., Cai, C., and Guo, W. (2014). Genome-wide identification of mitogen-activated protein kinase gene family in Gossypium raimondii and the function of their corresponding orthologs in tetraploid cultivated cotton. BMC Plant Biol. 14:345. doi: 10.1186/s12870-014-0345-9
Zhang, Z., Li, G., Gao, H., Zhang, L., Yang, C., Liu, P., et al. (2012). Characterization of photosynthetic performance during senescence in stay-green and quick-leaf-senescence Zea mays L. inbred lines. PLoS ONE 7:e42936. doi: 10.1371/journal.pone. 0042936

Conflict of Interest Statement: The authors declare that the research was conducted in the absence of any commercial or financial relationships that could be construed as a potential conflict of interest.

Copyright (C) 2015 Zhang and Becker. This is an open-access article distributed under the terms of the Creative Commons Attribution License (CC BY). The use, distribution or reproduction in other forums is permitted, provided the original author(s) or licensor are credited and that the original publication in this journal is cited, in accordance with accepted academic practice. No use, distribution or reproduction is permitted which does not comply with these terms. 


\title{
Dynamic proline metabolism: importance and regulation in water limited environments
}

\author{
Govinal B. Bhaskara, Tsu-Hao Yang and Paul E. Verslues* \\ Institute of Plant and Microbial Biology, Academia Sinica, Taipei, Taiwan
}

Drought-induced proline accumulation observed in many plant species has led to the hypothesis that further increases in proline accumulation would promote drought tolerance. Here we discuss both previous and new data showing that proline metabolism and turnover, rather than just proline accumulation, functions to maintain growth during water limitation. Mutants of $\Delta^{1}$-Pyrroline-5-Carboxylate Synthetase 1 (P5CS1) and Proline Dehydrogenase $1(P D H 1)$, key enzymes in proline synthesis and catabolism respectively, both have similar reductions in growth during controlled soil drying. Such results are consistent with patterns of natural variation in proline accumulation and with evidence that turnover of proline can act to buffer cellular redox status during drought. Proline synthesis and catabolism are regulated by multiple cellular mechanisms, of which we know only a few. An example of this is immunoblot detection of P5CS1 and PDH1 showing that the Highly ABA-induced (HAl) protein phosphatase 2Cs (PP2Cs) have different effects on P5CS1 and PDH1 protein levels despite having similar increases in proline accumulation. Immunoblot data also indicate that both P5CS1 and PDH1 are subjected to unknown post-translational modifications.

Correspondence:

Paul E. Verslues,

Institute of Plant and Microbial

Biology, Academia Sinica,

No. 128 Section 2 Academia Road, Nankang District, Taipei 11529,

Taiwan

paulv@gate.sinica.edu.tw

Specialty section: This article was submitted to Plant Physiology,

a section of the journal

Frontiers in Plant Science

Received: 07 April 2015

Accepted: 16 June 2015

Published: 25 June 2015

Citation:

Bhaskara GB, Yang T-H and Verslues PE (2015) Dynamic proline

metabolism: importance and regulation in water limited

environments.

Front. Plant Sci. 6:484.

doi: 10.3389/fp/s.2015.00484

Keywords: proline, drought, P5CS1, proline dehydrogenase, protein phosphatase 2C, natural variation, post-translational modification, Arabidopsis thaliana

Free proline can accumulate to high levels in drought-stressed plants. For example, tissue proline levels in excess of $100 \mathrm{mM}$ have been reported in the root growth zone of maize seedlings exposed to low water potential (Voetberg and Sharp, 1991; Ober and Sharp, 1994; Verslues and Sharp, 1999). Proline has chemical properties, including high solubility and zwitterionic structure, common to protective compatible solutes (Yancey et al., 1982). Given that proline is likely excluded from the vacuole, even relatively low bulk tissue levels of proline can indicate osmotically significant levels of proline in the cytoplasm and organelles (Bussis and Heineke, 1998). Why proline, rather than other metabolites, accumulates to high levels as well as how proline metabolism may be modified to improve drought tolerance are long standing questions in plant stress biology (Lehmann et al., 2010; Szabados and Savouré, 2010; Verslues and Sharma, 2010; Kavi Kishor and Sreenivasulu, 2014).

Proline is synthesized from glutamate by the action of two enzymes, $\Delta^{1}$-pyrroline-5carboxylate synthetase (P5CS) and $\Delta^{1}$-pyrroline-5-carboxylate reductase (P5CR). Conversely, proline catabolism to glutamate occurs via proline dehydrogenase (PDH) and $\Delta^{1}$-pyrroline-5carboxylate dehydrogenase (P5CDH; Szabados and Savouré, 2010; Verslues and Sharma, 2010). Together proline synthesis and catabolism form a cycle the halves of which are separated by compartmentation and, possibly, tissue specific location (Szabados and Savouré, 2010; Verslues and Sharma, 2010). P5CS1 (AT2G39800) and PDH1 (AT3G30775) gene expression patterns suggest that proline synthesis is high and proline catabolism suppressed in photosynthetic tissue during stress 
while proline catabolism continues at high rate in the root and shoot meristematic regions (Sharma et al., 2011).

Expression of Arabidopsis P5CS1 is induced by various types of abiotic stress including drought (Savoure et al., 1995; Yoshiba et al., 1995, 1997, 1999; Peng et al., 1996). This, as well as restricted proline accumulation in $p 5 c s 1$ mutants, increased proline accumulation of P5CS1 overexpression plants, and study of enzymatic properties of P5CS1 indicated that P5CS1 may be a rate limiting enzyme for proline accumulation (Kavi Kishor et al., 1995; Zhang et al., 1995; Szekely et al., 2008). Reduced expression of Arabidopsis $P D H 1$ is also thought to be needed for droughtinduced proline accumulation (Kiyosue et al., 1996; Yoshiba et al., 1997; Miller et al., 2005; Sharma et al., 2011). P5CS1 and PDH1 expression, along with other observations (for example Voetberg and Sharp, 1991; Ober and Sharp, 1994) made it clear that proline metabolism is highly regulated and proline accumulation during drought is not a symptom of stress injury nor a result of passive accumulation caused by growth reduction.

Regulation of proline metabolism under stress has been linked to abscisic acid (Savoure et al., 1997; Strizhov et al., 1997; Abraham et al., 2003); although ABA alone cannot duplicate drought-induced proline accumulation (Sharma and Verslues, 2010). Other data indicate a link of proline metabolism to cellular redox status. Study of P5CR activity found that its regulation by proline and chloride ions differed depending on whether NADH or NADPH was used as the co-factor (Giberti et al., 2014). This observation is consistent with proline metabolism having a special effect on NADP/NADPH ratio (Sharma et al., 2011). Studies of natural variation in proline accumulation also indicate an influence of redox sensitive enzymes including thioredoxins (Verslues et al., 2014) and mitochondrial NAD dehydrogenases (Lovell et al., 2015).

Identification of the key genes in proline metabolism prompted a wave of studies that sought to overexpress P5CS1 (or its orthologs from other plant species) to increase proline and enhance drought tolerance (for example: Kavi Kishor et al., 1995; Zhu et al., 1998; Sawahel and Hassan, 2002; Su and Wu, 2004; Molinari et al., 2007). Some studies also sought to increase stress tolerance by further suppressing $\mathrm{PDH} 1$ expression (Nanjo et al., 1999; Tateishi et al., 2005). Several studies claimed success in increasing drought tolerance; however, the methods used to evaluate drought tolerance varied greatly and often relied on counting plant survival after rapid severe dehydration rather than on monitoring responses to less severe water limitation which may be more informative (Skirycz et al., 2011; Claeys et al., 2014). Whether or not modification of proline metabolism may be used to engineer drought tolerance, and how such modification should be done, remains uncertain.

The above examples illustrate how experimental design and interpretation have been influenced by the "more is better" view of proline accumulation whereby increasing proline, no matter how it is done, should lead to better drought tolerance. This view is based on the transcriptional up-regulation of P5CS1 and decreased expression of $\mathrm{PDH} 1$ during drought stress as well as hypotheses that proline turnover under stress is low and that proline accumulation is cell autonomous and isolated from other metabolic pathways (Verslues and Sharma, 2010). It also implies that transcriptional regulation of $P 5 C S 1$ and $P D H 1$ are main determinants of proline accumulation. We propose that these ideas need to be critically examined and present some evidence that support a more dynamic view of proline metabolism during drought and suggest the existence of multiple layers of regulation.

\section{More is not Always Better: Natural Variation as well as $p 5 c s 1$ and $p d h 1$ Mutants Suggest a More Complex Relationship between Proline Accumulation and Drought Tolerance}

Sharma et al. (2011) found that both p5cs1-4 and pdh1-2 mutants had similar reductions in growth when transferred from normal media to low water potential PEG-infused agar $(-0.7 \mathrm{MPa}$ and $-1.2 \mathrm{MPa}$ ). Under these conditions, $p 5 c s 1$ mutants have reduced proline accumulation while $p d h 1$ mutants have increased proline, particularly in the root. Exogenous proline could restore growth of $p 5 c s 1$ mutants but not $p d h 1$ mutants, indicating that proline catabolism was required to maintain growth. Furthermore, high $P D H 1$ expression in meristematic tissue, reduced root tip oxygen consumption in $p d h 1-2$, and altered NADP/NADPH all indicated an effect of proline catabolism on redox status and growth (Sharma et al., 2011).

The stress experiments in Sharma et al. (2011) were performed on PEG-infused agar plates. To confirm that these results are applicable to different developmental stages and to drought stress more broadly, we performed controlled soil drying experiments where wild type and mutants were grown together in the same pots to ensure exposure to the same degree of soil drying (Figure 1A). Partial re-watering was performed midway through the drying cycle to equalize water content between the pots and lengthen the exposure to moderate water limitation. Growth data for mutants was normalized to wild type grown in the same pot. Soil water potential was in the range of -0.6 to $-0.8 \mathrm{MPa}$ for most of the drying cycle before decreasing to approximately $-1.2 \mathrm{MPa}$ by the end of the experiment.

Growth of wild type was reduced approximately 25 percent by the water limitation (data not shown) and proline content increased nearly 20-fold (Figure 1B). Proline accumulation of p5cs1-4 was less than that of $p d h 1-2$; however, both $p 5 c s 1-4$ and pdh1-2 had a similar 15 percent growth reduction in the soil drying treatment (Figures 1B,C). Neither p5cs1-4 nor pdh1-2 differed from wild type in the well watered control. There was no difference in relative water content (Figure 1B) indicating that none of the genotypes was more or less dehydrated then the others. The similar growth reduction in p5cs 1-4 and pdh1-2 despite different levels of proline accumulation argue against the level of proline accumulation itself being the main determinant of drought tolerance. Instead, it may be hypothesized that both p5cs1-2 and pdh1-2 have reduced metabolic flux through the cycle of proline synthesis and catabolism and this may be a key factor limiting their growth. We note that proline level in the soil grown plants was less than that of seedlings. This was likely because of the gradual stress imposition and later developmental stage. More mature plants have greater portion of highly vacuolated cells in 


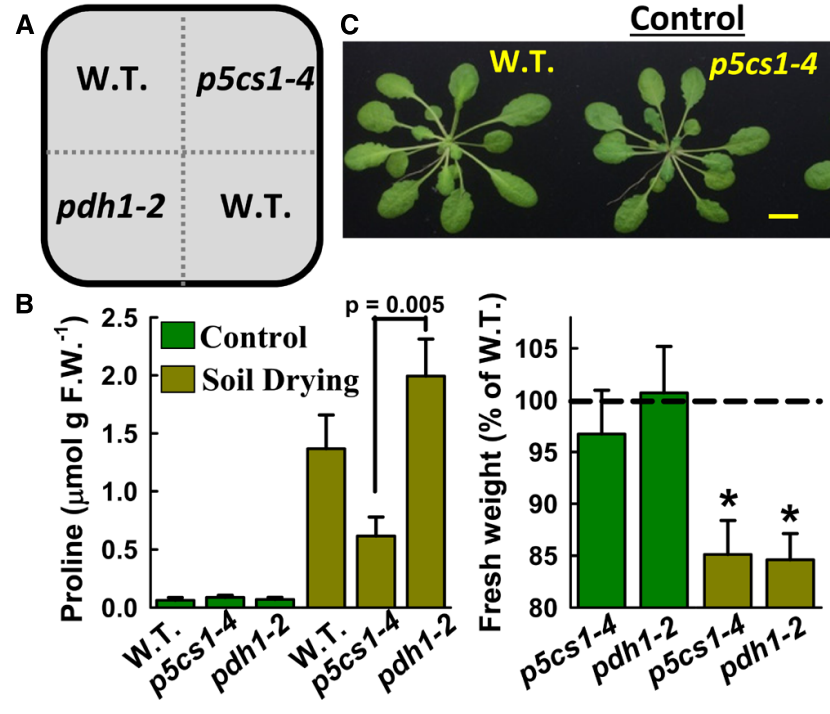

FIGURE 1 | Mutants of P5CS1 and PDH1 have similar growth reductions during soil drying. (A) Arrangement of genotypes in pots used for soil drying. Two plants were grown in each sector and rosettes harvested at the end of the drying cycle. Twelve to fifteen replicate pots were used for each treatment. (B) Proline content, fresh weight, dry weight and relative water content of plants kept under well water conditions or subjected to controlled soil drying. The fresh
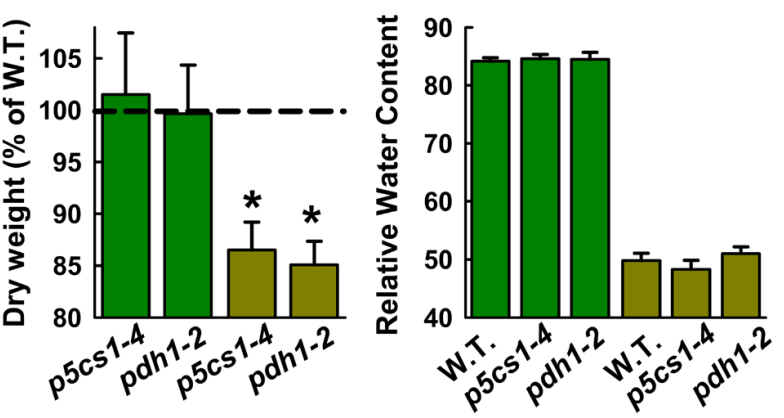

weight and dry measurements are relative to Columbia wild type, indicated by the dashed line. Asterisks $\left(^{\star}\right)$ indicate significant differences from wild type by one-sided $t$-test $(p \leq 0.05)$. Error bars indicate standard errors $(n=5-9$ for proline, $n=15-18$ for fresh and dry weights and relative water content). Data are combined from two independent experiments. (C) Representative rosettes of each genotype from the well watered control and soil drying treatments. which proline accumulates in the relatively small volume of the cytoplasm and organelles.

Another set of data relevant to the more-is-better question arises from the 10 -fold variation in low water potential-induced proline accumulation among Arabidopsis accessions (Kesari et al., 2012; Verslues et al., 2014). Interestingly, comparing proline accumulation to climate data from accession sites of origin indicated that accessions from generally drier regions had lower proline accumulation (Kesari et al., 2012). Local adaptation is well established in Arabidopsis with accession from dry regions differing in many aspects of their response to water limitation (De Marais et al., 2013; Juenger, 2013). Thus, in the accessions examined so far, adaptation to drier climate seems not to involve increased proline accumulation. This may seem to be at odds with the drought sensitivity of $p 5 c s 1-4$ and $p d h 1-2$. However, the combined data indicate that while proline accumulation contributes to drought tolerance, accessions that habitually face drought have other metabolic adjustments such that high levels of proline accumulation are not needed. It must also be kept in mind that we do not know if higher or lower proline accumulation correlates with higher or lower flux through proline synthesis and catabolism. As a caveat: the relationship of proline to climate across many accessions is compelling but we recommend due caution in interpretation as the exact microenvironment an accession has adapted to cannot be known and whether some accessions rely on drought escape (such by accelerated flowering at the onset of drought) rather than tolerance of low water potentials is also not clear.

The Shahdara (Sha, also called Shakdara) accession is an interesting example of natural variation in metabolism and drought response. It has been proposed to be a drought tolerant accession (Bouchabke et al., 2008; however, see discussion in Trontin et al., 2011 for questions of Sha's origin) and is a low proline accumulator mainly because of alternative splicing at the P5CS1 locus (Kesari et al., 2012). A profile of major metabolites in Sha showed reduced levels of all glutamate family amino acids as well as several major organic acids. In contrast, other amino acids, particularly leucine and isoleucine, had greater drought-induced accumulation in Sha (Sharma et al., 2013). Is this pattern true across a larger number of accessions? Is the lower proline accumulation of Sha indicative of lower (or higher) flux through proline synthesis and catabolism? Do such differences represent a different metabolic strategy of drought tolerance in Sha compared to accessions with higher proline accumulation? Another interesting example is the accession Pt0 which is essentially a naturally occurring P5CS1 mutant as it has extreme low levels of P5CS1 transcript and protein and has extreme low level of proline accumulation similar to $p 5 c s 1$ 4 (Kesari et al., 2012). Is Pt-0 more drought sensitive or does it employ a different metabolic strategy for drought tolerance that makes proline accumulation uneccessary? Answering these questions as well as determining the underlying genetic control of metabolic drought responses is of substantial interest.

\section{Regulatory Diversity: Protein Phosphatase 2C (PP2C) Mutants Illustrate Multiple Mechanisms Leading to Increased Proline Accumulation}

Transcription of P5CS1 and PDH1 is affected oppositely by drought stress in most plant tissues (see example in Figure 2A). 

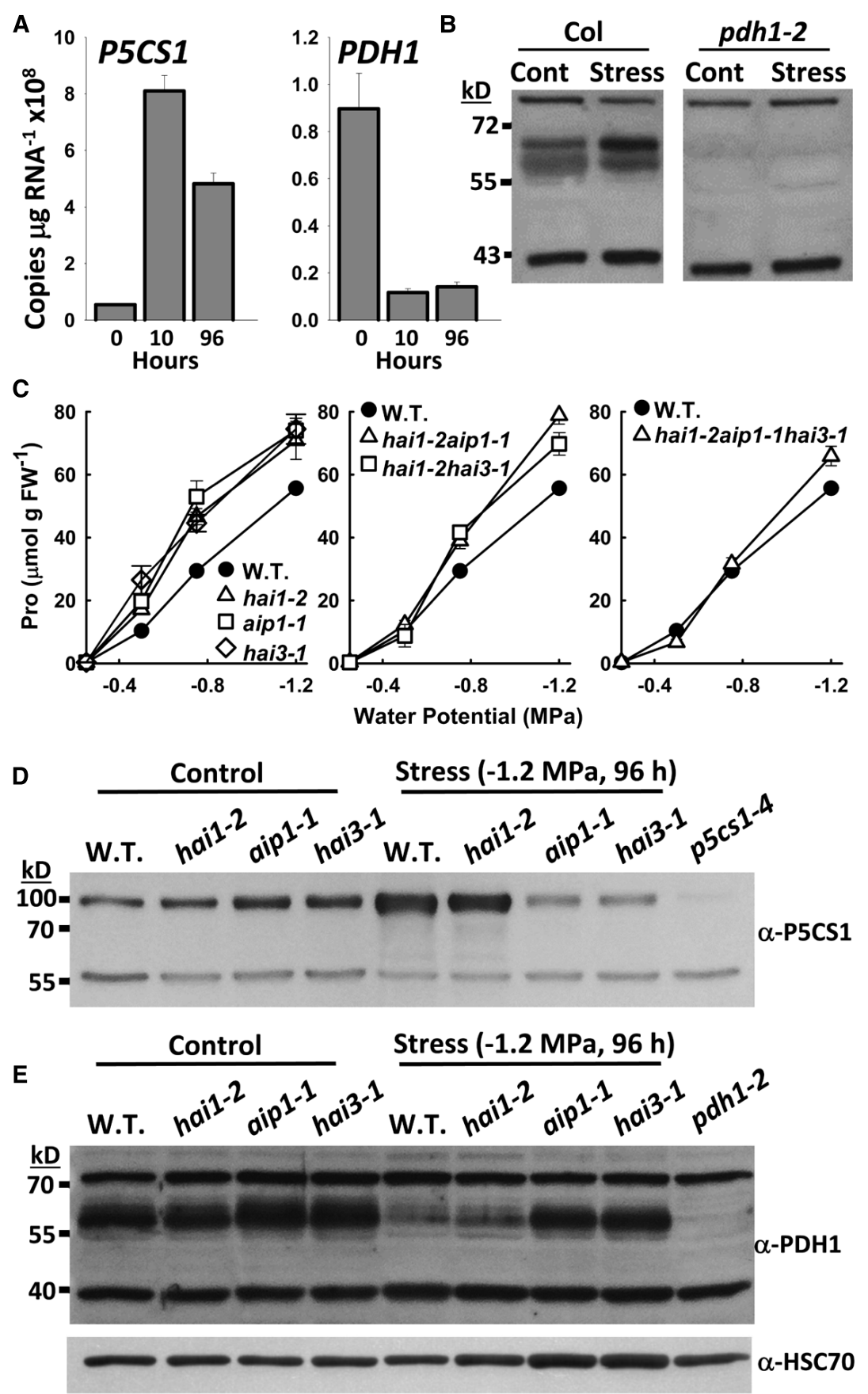

FIGURE 2 | Immunoblotting reveals differential regulation of P5CS1 and PDH1 protein levels by the Highly ABA Induced (HAl) protein phosphatase $2 \mathrm{Cs}$ as well as P5CS1 and PDH1 post-translational modification. (A) Gene expression of P5CS1 and PDH1 at the indicated times after transfer of seedlings from control media $(-0.25 \mathrm{MPa})$ to PEG-infused agar plates $(-1.2 \mathrm{MPa})$. Note that data for seedlings kept at $-0.25 \mathrm{MPa}$ was collected but did not show substantial change in expression and has thus been omitted for clarity. Data are re-plotted from Sharma and Verslues (2010). (B) Immunoblot detection of PDH1 in seedlings of Columbia wild type or pdh 1-2 under either unstressed conditions (Control) or $24 \mathrm{~h}$ after transfer to $-1.2 \mathrm{MPa}$ (Stress). All samples were on the same gel and blotted to the same membrane but intervening lanes have been removed for clarity. Non-specific bands at approximately 80 and $40 \mathrm{kD}$ indicate equal loading.
(C) Proline contents of hai mutants at $96 \mathrm{~h}$ after transfer to PEG-infused agar plates of a range of low water potential severities. Data are replotted from Bhaskara et al. (2012). (D) Immunoblot detection of P5CS1 in Columbia wild type or hai mutants under control and stress $(-1.2 \mathrm{MPa}, 96 \mathrm{~h})$ conditions. An additional lane of $p 5 c s 1-4$ (control) was included to verify specificity of the antisera. $50 \mu \mathrm{g}$ of protein was loaded per lane. The non-specific band at $56 \mathrm{kD}$ indicates equal loading. (E) Immunoblot detection of PDH1. The same samples and blotting conditions were used as in D but with pdh1-2 (grown under control conditions) included to verify antisera specificity. Non-specific bands at 80 and $40 \mathrm{kD}$ indicate equal loading. As an additional check of loading, the blot was stripped and reprobed with anti HSC 70. This blot was also reprobed with anti-P5CS1 which gave the same pattern of P5CS1 protein accumulation as seen in (D). 
While the transcriptional regulation of P5CS1 and PDH1 is consistent with accumulation of proline, it is only the first level of regulation. Improvements in proteomics have made it clear that protein levels do not always match transcript levels and the mismatch can be most extreme for transcriptionally downregulated genes (Vélez-Bermúdez and Schmidt, 2014). This may be the case at least transiently for PDH1 as immunoblots using PDH1 antisera developed in our laboratory show that PDH1 remains high at $24 \mathrm{~h}$ after stress treatment (Figure 2B) even though $\mathrm{PDH} 1$ expression was dramatically down regulated by $10 \mathrm{~h}$ (Figure 2A). This was consistent with previous observations of PDH1 (Parre et al., 2007). High level of PDH1 present at the same time that proline levels are increasing rapidly have been observed (Kaplan et al., 2007; Schertl et al., 2014) and imply either post-translation regulation of PDH1 activity or sequestering of proline away from $\mathrm{PDH} 1$, such as by limited proline transport into the mitochondria. We also previously noted a decrease in P5CS1 gene expression with no change in P5CS1 protein abundance in Arabidopsis Histidine Kinase1 (AHK1) mutants (Kumar et al., 2013).

Bhaskara et al. (2012), showed that mutants of three Clade A PP2Cs, Highly ABA-Induced 1 (HAI1), HAI2 (also known as AKT-Interacting Phosphatase1, AIP1) and HAI3, had increased proline accumulation at low water potential. Curiously, hail2aip1-1 and hai1-2hai3-1 double mutants had a reduced proline phenotype compared to the single mutants and the high proline phenotype was abolished in a hai1-2aip1-1hai3-1 triple mutant (Figure 2C; Bhaskara et al., 2012). The reason for this was unclear until we examined P5CS1 and PDH1 protein levels: aip11 and hai3-1 lack both the low water potential-induced increase in P5CS1 as well as the decrease in PDH1 (Figures 2D,E). Conversely, hai1-2 has similar P5CS1 and PDH1 protein levels as wild type. Bhaskara et al. (2012) noted that HAI1 had different interaction with the PYL ABA receptors than AIP1 or HAI3, implying a different substrate specificity, and also had substantial effect on gene expression patterns. Thus, we can speculate that HAI1 affects proline accumulation indirectly through changes in regulatory gene expression while AIP1 and HAI2 may affect proline more directly by regulation of P5CS1 and PDH1 expression or protein stability. Possibly, the gene expression changes in hail-2 allow wild type levels of proline accumulation even when $\mathrm{P} 5 \mathrm{CS} 1$ and $\mathrm{PDH} 1$ protein levels are reduced by mutation of $A I P 1$ or HAI3.

The immunoblots indicated that both P5CS1 and PDH1 have unknown post-translational modifications. For P5CS1, its apparent molecular weight of $90-95 \mathrm{kD}$ is heavier than its predicted molecular weight of $77.8 \mathrm{kD}$ (Figure 2D; Kesari et al., 2012). For PDH1, only a small portion of the protein runs at the expected molecular weight of $55 \mathrm{kD}$ while the rest is seen as a broad band or combination of bands from 57 to $65 \mathrm{kD}$ (Figure 2E) similar to previous results (Schertl et al., 2014). The nature of these post-translational modifications is unknown. Redox sensitive modification is one possibility, especially for PDH1 based on its role in electron transport (Servet et al., 2012; Schertl et al., 2014). Other types of modification are possible for P5CS1 where the single band and relatively large shift in molecular weight may be more consistent with sumoylation, glycosylation, or multiple phosphorylation. Whether or not post-translational modification affects $\mathrm{P} 5 \mathrm{CS} 1$ or PDH1 activity or localization is of interest for future research.

\section{Future Perspectives}

Several lines of evidence indicate that more proline is not always better for drought tolerance. Rather, the amount of proline that accumulates is dependent on metabolic context and the activity of a number of other metabolic and signaling pathways. How then do we determine the contribution of proline to drought resistance? One point to consider is whether higher or lower proline accumulation is indicative of higher or lower flux through proline synthesis and catabolism and whether the flux and turnover of proline play a key role in drought resistance (see Sharma et al., 2011; Kavi Kishor and Sreenivasulu, 2014 for further discussion). Thus we need to understand the connections of proline metabolism to other metabolic pathways and cellular redox status. Analysis of natural variation through metabolite profiling, and quantitative genetics approaches such as genome wide association analysis and quantitative trait loci mapping can reveal how proline metabolism fits into different drought resistance strategies. The different metabolite profile of Sha discussed above is one example. Also promising are studies of proline metabolism enzymes themselves including localization, which is still unclear for P5CS1, interacting proteins and posttranslational modification.

The broad natural variation in proline accumulation indicates that the optimal level of proline accumulation is dependent on species and genotype. This needs to be taken into account in transgenic approaches that seek to modify proline metabolism to improve drought tolerance. Use of stress-inducible promoters may be valuable, but perhaps even more important is to control the tissue specificity of modifications to proline synthesis or catabolism (Sharma et al., 2011). Such experiments should be accompanied by careful analysis of drought physiology, including longer term assays using moderate levels of drought stress where effects of proline metabolism on growth are more relevant to crop productivity and likely to be different than the effects of proline on survival of severe stress.

\section{Materials and Methods}

\section{Soil Drying Experiments}

A standard potting mix was combined with $25 \%$ Turface (Turface MVP, Profile Products LLC, USA) to improve porosity and consistency of drying. Seeds of four genotypes were planted in sectors (two plants per sector) of $8 \mathrm{~cm} \times 8 \mathrm{~cm} \times 10 \mathrm{~cm}(\mathrm{~L} \times \mathrm{W} \times \mathrm{H})$ plastic pots (Figure 1A) and grown in a short day chamber $(8 \mathrm{~h}$ light period, $25 \mathrm{C}$, light intensity of $100-120 \mu \mathrm{mol} \mathrm{m}^{-2} \mathrm{sec}^{-1}$ ). Hyponex nutrient solution ( $1 \mathrm{~g} \mathrm{liter}^{-1}$ ) was supplied once per week. On 18 day after planting, pots were watered to saturation, allowed to drain and weighed. Water was withheld for 12 days (leading to 50-60 percent reduction in pot weight) and then each pot re-watered to 75 percent of the initial pot weight by injecting water into the middle of the pot with a syringe. The pots were allowed to dry another 8-10 day until pot weight again reached 
50-60 percent of the starting weight. Representative rosettes were then photographed and the rest used for measurements of fresh weight, fully hydrated weight and dry weight. Proline was quantified on samples of either whole rosettes (well watered control) or the eighth and ninth leaf (soil drying) using ninhydrin assay (Bates et al., 1973).

\section{P5CS1 and PDH1 Protein Blotting}

Seedling growth and stress treatment were performed as previously described (Bhaskara et al., 2012). Protein extraction was carried out as described in Martinez-Garcia et al. (1999) using approximately $100 \mathrm{mg}$ of tissue. Protein contents were measured by BCA assay (Pierce). For each sample, $50 \mu \mathrm{g}$ of total protein was resolved on $10 \%$ SDS PAGE gels and immunoblotting performed

\section{References}

Abraham, E., Rigo, G., Szekely, G., Nagy, R., Koncz, C., and Szabados, L. (2003). Light-dependent induction of proline biosynthesis by abscisic acid and salt stress is inhibited by brassinosteroid in Arabidopsis. Plant Mol. Biol. 51, 363-372. doi: 10.1023/A:1022043000516

Bates, L. S., Waldren, R. P., and Teare, I. D. (1973). Rapid determination of free proline for water stress studies. Plant Soil 39, 205-207. doi: 10.1007/ BF00018060

Bhaskara, G. B., Nguyen, T. T., and Verslues, P. E. (2012). Unique drought resistance functions of the Highly ABA-Induced clade A protein phosphatase 2Cs. Plant Physiol. 160, 379-395. doi: 10.1104/pp.112.202408

Bouchabke, O., Chang, F. Q., Simon, M., Voisin, R., Pelletier, G., and DurandTardif, M. (2008). Natural variation in Arabidopsis thaliana as a tool for highlighting differential drought responses. PLOS ONE 3:e1705. doi: 10.1371/journal.pone.0001705

Bussis, D., and Heineke, D. (1998). Acclimation of potato plants to polyethylene glycol-induced water deficit. II. Contents and subcellular distribution of organic solutes. J. Exp. Bot. 49, 1361-1370. doi: 10.1093/jxb/49.325.1361

Claeys, H., Van Landeghem, S., Dubois, M., Maleux, K., and Inze, D. (2014). What is stress? Dose-response effects in commonly used in vitro stress assays. Plant Physiol. 165, 519-527. doi: 10.1104/pp.113.234641

De Marais, D. L., Hernandez, K. M., and Juenger, T. E. (2013). Genotypeby-environment interaction and plasticity: exploring genomic responses of plants to the abiotic environment. Annu. Rev. Ecol. Evol. Syst. 44, 5-29. doi: 10.1146/annurev-ecolsys-110512-135806

Giberti, S., Funck, D., and Forlani, G. (2014). $\Delta^{1}$-pyrroline-5-carboxylate reductase from Arabidopsis thaliana: stimulation or inhibition by chloride ions and feedback regulation by proline depend on whether NADPH or NADH acts as cosubstrate. New Phytol. 202, 911-919. doi: 10.1111/nph.12701

Juenger, T. E. (2013). Natural variation and genetic constraints on drought tolerance. Curr. Opin. Plant Biol. 16, 274-281. doi: 10.1016/j.pbi.2013.02.001

Kaplan, F., Kopka, J., Sung, D. Y., Zhao, W., Popp, M., Porat, R., et al. (2007). Transcript and metabolite profiling during cold acclimation of Arabidopsis reveals an intricate relationship of cold-regulated gene expression with modifications in metabolite content. Plant J. 50, 967-981. doi: 10.1111/j.1365313X.2007.03100.x

Kavi Kishor, P. B., Hong, Z., Miao, G. H., Hu, C. A. A., and Verma, D. P. S. (1995). Overexpression of $\Delta^{1}$-pyrroline-5-carboxylate synthase increases proline production and confers osmotolerance in transgenic plants. Plant Physiol. 108, 1387-1394.

Kavi Kishor, P. B., and Sreenivasulu, N. (2014). Is proline accumulation per se correlated with stress tolerance or is proline homeostasis a more critical issue? Plant Cell Environ. 37, 300-311. doi: 10.1111/pce.12157

Kesari, R., Lasky, J. R., Villamor, J. G., Marais, D. L. D., Chen, Y. J. C., Liu, T. W., et al. (2012). Intron-mediated alternative splicing of Arabidopsis P5CS1 and its association with natural variation in proline and climate adaptation. Proc. Natl. Acad. Sci. U.S.A. 109, 9197-9202. doi: 10.1073/pnas.1203433109 serum raised against P5CS1 (Kesari et al., 2012) and PDH1. PDH1 antisera generation and immunoblot procedures were essentially identical to those described in Kesari et al. (2012).

\section{Author Contributions}

$\mathrm{PV}$ conceived research and wrote the manuscript with assistance from GB. GB performed all experiments except generation of PDH1 antisera and some immunoblots which were performed by TY. All authors approved the manuscript.

\section{Acknowledgments}

This work was funded by Academia Sinica and the Taiwan Ministry of Science and Technology.

Kiyosue, T., Yoshiba, Y., Yamaguchi-Shinozaki, K., and Shinozaki, K. (1996). A nuclear gene encoding mitochondrial proline dehydrogenase, an enzyme involved in proline metabolism, is upregulated by proline but downregulated by dehydration in Arabidopsis. Plant Cell 8, 1323-1335. doi: 10.1105/tpc.8.8.1323

Kumar, M. N., Jane, W. N., and Verslues, P. E. (2013). Role of the putative osmosensor Arabidopsis histidine kinasel in dehydration avoidance and low water potential response. Plant Physiol. 161, 942-953. doi: 10.1104/pp.112.209791

Lehmann, S., Funck, D., Szabados, L., and Rentsch, D. (2010). Proline metabolism and transport in plant development. Amino Acids 39, 949-962. doi: 10.1007/s00726-010-0525-3

Lovell, J. T., Mullen, J. L., Lowry, D. B., Awole, K., Richards, J. H., Sen, S., et al. (2015). Exploiting differential gene expression and epistasis to discover candidate genes for drought-associated QTLs in Arabidopsis thaliana. Plant Cell 27, 969-983. doi: 10.1105/tpc.15.00122

Martinez-Garcia, J. F., Monte, E., and Quail, P. H. (1999). A simple, rapid and quantitative method for preparing Arabidopsis protein extracts for immunoblot analysis. Plant J. 20, 251-257. doi: 10.1046/j.1365-313x.1999.00579.x

Miller, G., Stein, H., Honig, A., Kapulnik, Y., and Zilberstein, A. (2005). Responsive modes of Medicago sativa proline dehydrogenase genes during salt stress and recovery dictate free proline accumulation. Planta 222, 70-79. doi: 10.1007/s00425-005-1518-4

Molinari, H. B. C., Marur, C. J., Daros, E., de Campos, M. K. F., de Carvalho, J., Bespalhok, J. C., et al. (2007). Evaluation of the stress-inducible production of proline in transgenic sugarcane (Saccharum spp.): osmotic adjustment, chlorophyll fluorescence and oxidative stress. Physiol. Plant 130, 218-229. doi: 10.1111/j.1399-3054.2007.00909.x

Nanjo, T., Kobayashi, M., Yoshiba, Y., Kakubari, Y., Yamaguchi-Shinozaki, K., and Shinozaki, K. (1999). Antisense suppression of proline degradation improves tolerance to freezing and salinity in Arabidopsis thaliana. FEBS Lett. 461, 205-210. doi: 10.1016/S0014-5793(99)01451-9

Ober, E. S., and Sharp, R. E. (1994). Proline accumulation in maize (Zea mays L.) primary roots at low water potentials. 1. Requirement for increased levels of abscisic acid. Plant Physiol. 105, 981-987.

Parre, E., Ghars, M. A., Leprince, A. S., Thiery, L., Lefebvre, D., Bordenave, M., et al. (2007). Calcium signaling via phospholipase $C$ is essential for proline accumulation upon ionic but not nonionic hyperosmotic stresses in Arabidopsis. Plant Physiol. 144, 503-512. doi: 10.1104/pp.106.095281

Peng, Z., Lu, Q., and Verma, D. P. S. (1996). Reciprocal regulation of $\Delta^{1}$ pyrroline-5-carboxylate synthetase and proline dehydrogenase genes controls proline levels during and after osmotic stress in plants. Mol. Gen. Genet. 253, 334-341.

Savoure, A., Hua, X. J., Bertauche, N., VanMontagu, M., and Verbruggen, N. (1997). Abscisic acid-independent and abscisic acid-dependent regulation of proline biosynthesis following cold and osmotic stresses in Arabidopsis thaliana. Mol. Gen. Genet. 254, 104-109. doi: 10.1007/s004380050397

Savoure, A., Jaoua, S., Hua, X. J., Ardiles, W., Vanmontagu, M., and Verbruggen, N. (1995). Isolation, characterization and chromosomal location of a gene encoding 
the $\Delta^{1}$-pyrroline-5-carboxylate synthetase in Arabidopsis thaliana. FEBS Lett. 372, 13-19. doi: 10.1016/0014-5793(95)00935-3

Sawahel, W. A., and Hassan, A. H. (2002). Generation of transgenic wheat plants producing high levels of the osmoprotectant proline. Biotech. Lett. 24, 721-725. doi: 10.1023/A:1015294319114

Schertl, P., Cabassa, C., Saadallah, K., Bordenave, M., Savoure, A., and Braun, H. P. (2014). Biochemical characterization of proline dehydrogenase in Arabidopsis mitochondria. FEBS J. 281, 2794-2804. doi: 10.1111/febs.12821

Servet, C., Ghelis, T., Richard, L., Zilberstein, A., and Savoure, A. (2012). Proline dehydrogenase: a key enzyme in controlling cellular homeostasis. Front. Biosci. 17:3947. doi: $10.2741 / 3947$

Sharma, S., Lin, W. D., Villamor, J. G., and Verslues, P. E. (2013). Divergent low water potential response in Arabidopsis thaliana accessions Landsberg erecta and Shahdara. Plant Cell Environ. 36, 994-1008. doi: 10.1111/pce. 12032

Sharma, S., and Verslues, P. E. (2010). Mechanisms independent of ABA or proline feedback have a predominant role in transcriptional regulation of proline metabolism during low water potential and stress recovery. Plant Cell Environ. 33, 1838-1851. doi: 10.1111/j.1365-3040.2010.02188.x

Sharma, S., Villamor, J. G., and Verslues, P. E. (2011). Essential role of tissue-specific proline synthesis and catabolism in growth and redox balance at low water potential. Plant Physiol. 157, 292-304. doi: 10.1104/pp.111.183210

Skirycz, A., Vandenbroucke, K., Clauw, P., Maleux, K., De Meyer, B., Dhondt, S., et al. (2011). Survival and growth of Arabidopsis plants given limited water are not equal. Nat. Biotech. 29, 212-214. doi: 10.1038/nbt.1800

Strizhov, N., Abraham, E., Okresz, L., Blickling, S., Zilberstein, A., Schell, J., et al. (1997). Differential expression of two P5CS genes controlling proline accumulation during salt-stress requires $\mathrm{ABA}$ and is regulated by $\mathrm{ABA1}$, ABI1 and AXR2 in Arabidopsis. Plant J. 12, 557-569. doi: 10.1111/j.09607412.1997.00557.x

$\mathrm{Su}$, J., and $\mathrm{Wu}, \mathrm{R}$. (2004). Stress-inducible synthesis of proline in transgenic rice confers faster growth under stress conditions than that with constitutive synthesis. Plant Sci. 166, 941-948. doi: 10.1016/j.plantsci.2003.12.004

Szabados, L., and Savouré, A. (2010). Proline: a multifunctional amino acid. Trends Plant Sci. 15, 89-97. doi: 10.1016/j.tplants.2009.11.009

Szekely, G., Abraham, E., Cselo, A., Rigo, G., Zsigmond, L., Csiszar, J., et al. (2008). Duplicated P5CS genes of Arabidopsis play distinct roles in stress regulation and developmental control of proline biosynthesis. Plant J. 53, 11-28. doi: 10.1111/j.1365-313X.2007.03318.x

Tateishi, Y., Nakagawa, T., and Esaka, M. (2005). Osmotolerance and growth stimulation of transgenic tobacco cells accumulating free proline by silencing proline dehydrogenase expression with double-stranded RNA interference technique. Physiol. Plant 125, 224-234. doi: 10.1111/j.1399-3054.2005.00553.x

Trontin, C., Tisne, S., Bach, L., and Loudet, O. (2011). What does Arabidopsis natural variation teach us (and does not teach us) about adaptation in plants? Curr. Opin. Plant Biol. 14, 225-231. doi: 10.1016/j.pbi.2011.03.024

Vélez-Bermúdez, I. C., and Schmidt, W. (2014). The conundrum of discordant protein and mRNA expression. Are plants special? Front. Plant Sci. 5:619. doi: $10.3389 /$ fpls.2014.00619
Verslues, P. E., Lasky, J. R., Juenger, T. E., Liu, T. W., and Kumar, M. N. (2014). Genome-wide association mapping combined with reverse genetics identifies new effectors of low water potential-induced proline accumulation in Arabidopsis. Plant Physiol. 164, 144-159. doi: 10.1104/pp.113.224014

Verslues, P. E., and Sharma, S. (2010). Proline metabolism and its implications for plant-environment interaction. Arabidopsis Book 8, e0140. doi: 10.1199/ tab.0140

Verslues, P. E., and Sharp, R. E. (1999). Proline accumulation in maize (Zea mays L.) primary roots at low water potentials. II. Metabolic source of increased proline deposition in the elongation zone. Plant Physiol. 119, 1349-1360. doi: 10.1104/pp.119.4.1349

Voetberg, G. S., and Sharp, R. E. (1991). Growth of the maize primary root at low water potentials. 3. Role of increased proline deposition in osmotic adjustment. Plant Physiol. 96, 1125-1130. doi: 10.1104/pp.96.4.1125

Yancey, P. H., Clark, M. E., Hand, S. C., Bowlus, R. D., and Somero, G. N. (1982). Living with water stress: evolution of osmolyte systems. Science 217, 1214-1222. doi: $10.1126 /$ science. 7112124

Yoshiba, Y., Kiyosue, T., Katagiri, T., Ueda, H., Mizoguchi, T., YamaguchiShinozaki, K., et al. (1995). Correlation between the induction of a gene for $\Delta^{1}$-pyrroline-5-carboxylate synthetase and the accumulation of proline in Arabidopsis thaliana under osmotic stress. Plant J. 7, 751-760. doi: 10.1046/j. 1365-313X.1995.07050751.x

Yoshiba, Y., Kiyosue, T., Nakashima, K., Yamaguchi-Shinozaki, K., and Shinozaki, K. (1997). Regulation of levels of proline as an osmolyte in plants under water stress. Plant Cell Physiol. 38, 1095-1102. doi: 10.1093/oxfordjournals. pcp.a029093

Yoshiba, Y., Nanjo, T., Miura, S., Yamaguchi-Shinozaki, K., and Shinozaki, K. (1999). Stress-responsive and developmental regulation of $\Delta^{1}$-pyrroline-5carboxylate synthetase 1 (P5CS1) gene expression in Arabidopsis thaliana. Biochem. Biophys. Res. Commun. 261, 766-772. doi: 10.1006/bbrc.1999.1112

Zhang, C. S., Lu, Q., and Verma, D. P. S. (1995). Removal of feedback inhibition of $\Delta^{1}$-pyrroline-5-carboxylate synthetase, a bifunctional enzyme catalyzing the first two steps of proline biosynthesis in plants. J. Biol. Chem. 270, 20491-20496. doi: $10.1074 /$ jbc.270.35.20491

Zhu, B. C., Su, J., Chan, M. C., Verma, D. P. S., Fan, Y. L., and Wu, R. (1998) Overexpression of a $\Delta^{1}$-pyrroline-5-carboxylate synthetase gene and analysis of tolerance to water- and salt-stress in transgenic rice. Plant Sci. 139, 41-48. doi: $10.1016 /$ S0168-9452(98)00175-7

Conflict of Interest Statement: The authors declare that the research was conducted in the absence of any commercial or financial relationships that could be construed as a potential conflict of interest.

Copyright (c) 2015 Bhaskara, Yang and Verslues. This is an open-access article distributed under the terms of the Creative Commons Attribution License (CC BY). The use, distribution or reproduction in other forums is permitted, provided the original author(s) or licensor are credited and that the original publication in this journal is cited, in accordance with accepted academic practice. No use, distribution or reproduction is permitted which does not comply with these terms. 


\section{OPEN ACCESS}

Edited by:

Dietmar Funck

University of Konstanz, Germany

Reviewed by:

Mick Ayliffe,

CSIRO Plant Industry, Australia

Baoshan Chen,

Gaungxi University, China

Donald Becker

University of Nebraska-Lincoln, USA

*Correspondence:

María E. Alvarez,

Centro de Investigaciones en Química

Biológica de Córdoba CIQUIBIC,

UNC-CONICET, Departamento de

Química Biológica, Facultad de

Ciencias Químicas, Universidad

Nacional de Córdoba, Haya de la

Torre y Medina Allende, Ciudad

Universitaria, X5000HUA Córdoba,

Argentina

malena@mail.fcq.unc.edu.ar

${ }^{\dagger}$ These authors have contributed equally to this work.

Specialty section:

This article was submitted to

Plant Physiology,

a section of the journal

Frontiers in Plant Science

Received: 30 April 2015

Accepted: 10 July 2015

Published: 28 July 2015

Citation:

Rizzi YS, Monteoliva MI, Fabro G, Grosso CL, Laróvere LE and Alvarez

ME (2015) P5CDH affects the

pathways contributing to Pro synthesis after ProDH activation by biotic and abiotic stress conditions.

Front. Plant Sci. 6:572.

doi: $10.3389 /$ fpls.2015.00572

\section{P5CDH affects the pathways} contributing to Pro synthesis after ProDH activation by biotic and abiotic stress conditions

\author{
Yanina S. Rizzi ${ }^{1}$, Mariela I. Monteoliva ${ }^{1+}$, Georgina Fabro ${ }^{1 t}$, Carola L. Grosso ${ }^{2}$, \\ Laura E. Laróvere ${ }^{2}$ and María E. Alvarez ${ }^{1 *}$ \\ ${ }^{1}$ Centro de Investigaciones en Química Biológica de Córdoba CIQUIBIC, UNC-CONICET, Departamento de Química \\ Biológica, Facultad de Ciencias Químicas, Universidad Nacional de Córdoba, Córdoba, Argentina, ${ }^{2}$ Centro de Estudio de las \\ Metabolopatías Congénitas, Hospital de Niños de la Santísima Trinidad, Cátedra de Clínica Pediátrica, Facultad de Ciencias \\ Médicas, Universidad Nacional de Córdoba, Córdoba, Argentina
}

Plants facing adverse conditions usually alter proline (Pro) metabolism, generating changes that help restore the cellular homeostasis. These organisms synthesize Pro from glutamate (Glu) or ornithine (Orn) by two-step reactions that share $\Delta^{1}$ pyrroline-5-carboxylate (P5C) as intermediate. In the catabolic process, Pro is converted back to Glu using a different pathway that involves Pro dehydrogenase (ProDH), P5C dehydrogenase (P5CDH), and P5C as intermediate. Little is known about the coordination of the catabolic and biosynthetic routes under stress. To address this issue, we analyzed how P5CDH affects the activation of Pro synthesis, in Arabidopsis tissues that increase ProDH activity by transient exposure to exogenous Pro, or infection with Pseudomonas syringae pv. tomato. Wild-type (Col-0) and p5cdh mutant plants subjected to these treatments were used to monitor the Pro, Glu, and Orn levels, as well as the expression of genes from Pro metabolism. Col-0 and $p 5 c d h$ tissues consecutively activated ProDH and Pro biosynthetic genes under both conditions. However, they manifested a different coordination between these routes. When external Pro supply was interrupted, wild-type leaves degraded Pro to basal levels at which point Pro synthesis, mainly via Glu, became activated. Under the same condition, p5cdh leaves sustained ProDH induction without reducing the Pro content but rather increasing it, apparently by stimulating the Orn pathway. In response to pathogen infection, both genotypes showed similar trends. While Col-0 plants seemed to induce both Pro biosynthetic routes, $p 5 c d h$ mutant plants may primarily activate the Orn route. Our study contributes to the functional characterization of $\mathrm{P} 5 \mathrm{CDH}$ in biotic and abiotic stress conditions, by revealing its capacity to modulate the fate of P5C, and prevalence of Orn or Glu as Pro precursors in tissues that initially consumed Pro.

Keywords: $\Delta 1$ pyrroline-5-carboxylate dehydrogenase (P5CDH), proline dehydrogenase (ProDH), exogenous proline treatment, hypersensitive response, Pro, Orn, Glu, cell death 


\section{Introduction}

Proline (Pro) metabolism is intimately associated with stress adaptation. Most plants accumulate Pro under drought, salinity, extreme temperatures, UV radiation, or pathogen infection. The levels reached by this compound depend on the plant species and the type of stress (Verslues and Sharma, 2010; Liang et al., 2013). Once stress is released Pro undergoes oxidative degradation, providing nitrogen, and energy for the recovery process. Stress-induced Pro accumulation is often accompanied by increased synthesis and reduced catabolism (Verslues and Sharma, 2010), but could also coexist with activation of both processes (Fabro et al., 2004; Kaplan et al., 2007). The Pro metabolic changes occurring under stress help alleviate cell damage in different ways. Due to its chemical nature, this amino acid can function as osmolyte, ROS scavenger and protein chaperone. Beyond this, its synthesis and degradation generates an extensive transport of intermediates and cofactors between different subcellular compartments, helping to adjust the cytosolic pH and cellular redox status (Liang et al., 2013; Ben Rejeb et al., 2014). Moreover, the Pro synthesized in shoots can be transported to roots for subsequent degradation, sustaining root growth under unfavorable conditions (Sharma et al., 2011). Interestingly, stress tolerance does not necessarily require Pro accumulation and even more, Pro degradation is needed for plant defense activation under some adverse conditions. This has been observed in Arabidopsis tissues developing the Hypersensitive Response (HR) triggered by the bacterial pathogen Pseudomonas syringae pv. tomato DC3000 AvrRpm1 (Pst-AvrRpm1). In this case, the limiting enzyme in Pro catabolism potentiates the oxidative burst and cell death associated to the HR program (Cecchini et al., 2011). Similarly, low doses of exogenous Pro activate the catabolic route, improving tolerance to salt, UV radiation, and temperature stress by alleviating cytoplasmic acidosis and redox alterations (Hayat et al., 2012).

Most enzymes of Pro metabolism are well characterized at the biochemical level, but connections between anabolic and catabolic processes have not been thoroughly studied, particularly under circumstances where initial Pro degradation contributes to stimulate plant defenses. The Pro catabolic pathway takes place in mitochondria, where Pro is oxidized to glutamic acid (Glu) in two steps (Verslues and Sharma, 2010) (Figure 1). First, Pro dehydrogenase (ProDH) transforms Pro into $\Delta 1$ pyrroline-5-carboxylate (P5C), and then P5C dehydrogenase $(\mathrm{P} 5 \mathrm{CDH})$ converts the tautomeric form of $\mathrm{P} 5 \mathrm{C}$ (glutamate-semialdehyde; GSA) into Glu. In turn, Glu can be used as precursor of Pro synthesis at cytosol and plastids. There, P5C synthase (P5CS) reduces Glu to GSA/P5C, and P5C reductase (P5CR) converts P5C into Pro (Funck et al., 2012). ProDH and P5CS control the rate-limiting steps in

Abbreviations: Glu, glutamic acid; GSA, glutamate-semialdehyde; HPLC, high performance liquid chromatography; HR, hypersensitive response; OAT, ornithine $\delta$-aminotransferase; Orn, ornithine; $\mathrm{P} 5 \mathrm{C}, \Delta 1$ pyrroline-5-carboxylate; $\mathrm{P} 5 \mathrm{CDH}$, $\Delta 1$ pyrroline-5-carboxylate dehydrogenase; P5CR, $\Delta 1$ pyrroline-5-carboxylate reductase; P5CS, $\Delta 1$ pyrroline-5-carboxylate synthase; Pro, proline; ProDH, proline dehydrogenase; Pst-AvrRpm1, Pseudomonas syringae pv. tomato DC3000 AvrRpm1; ROS, reactive oxygen species.

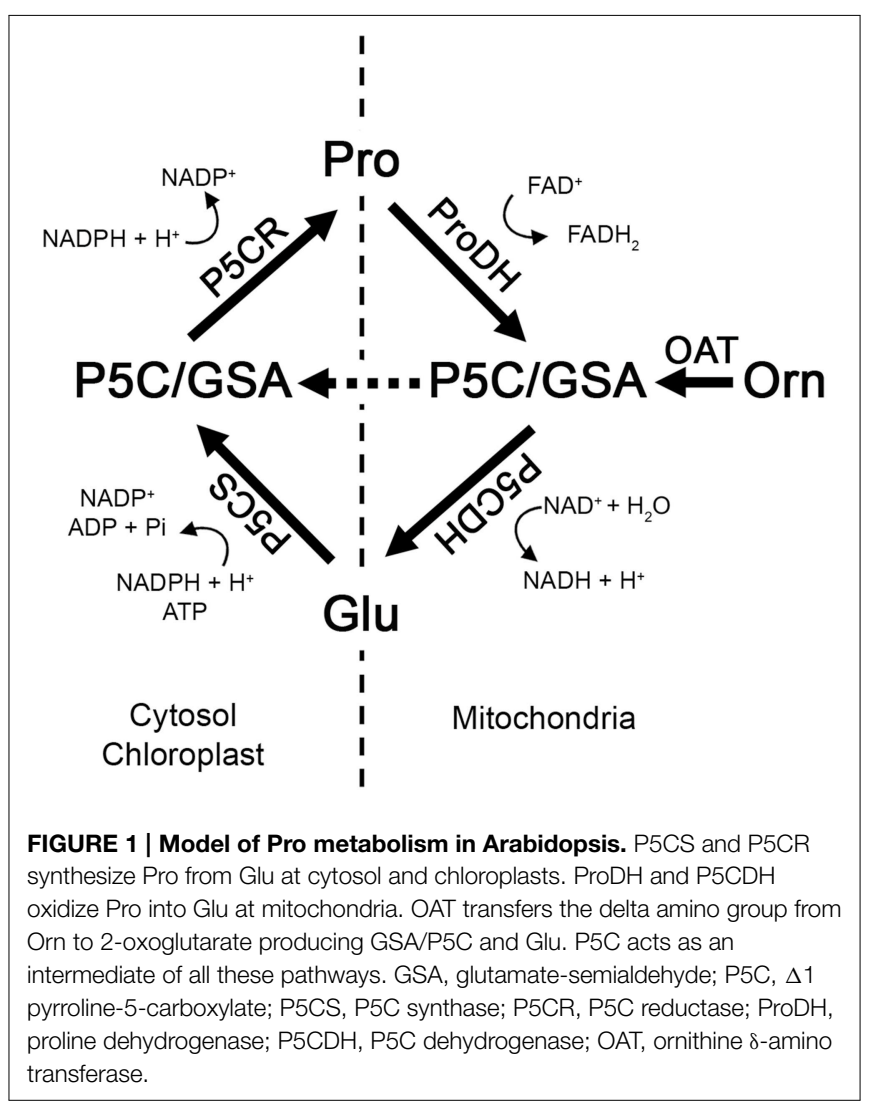

Pro-Glu interconversion. Arabidopsis contains two isoforms of these enzymes, which may function under different conditions (Funck et al., 2010; Verslues and Sharma, 2010). In addition, plants can synthesize Pro from ornithine (Orn). This route is initiated in mitochondria, where ornithine $\delta$-amino transferase (OAT) mediates transamination of Orn into GSA/P5C (Verslues and Sharma, 2010; Liang et al., 2013). Mitochondrial P5C is a common product of ProDH and OAT activities, and may be either transformed into Glu by $\mathrm{P} 5 \mathrm{CDH}$ initiating the Glu pathway (Funck et al., 2008), or transferred to cytosol to generate Pro by P5CR (Miller et al., 2009). Then, coordination of P5CDH and P5CR determines the use of P5C under stress (Figure 1).

Enzymes controlling Pro metabolism are regulated at different levels (Fichman et al., 2014; Kavi Kishor and Sreenivasulu, 2014). In general, OAT, P5CS, and ProDH are subject to transcriptional control, whereas P5CR and $\mathrm{P} 5 \mathrm{CDH}$ may be regulated at both, transcriptional and post-transcriptional levels. P5CS displays allosteric inhibition by endproduct and is affected by alternative splicing. $\mathrm{P} 5 \mathrm{CDH}$ is post-transcriptionally regulated by cis antisense transcripts, and P5CR is sensitive to redox regulation (Giberti et al., 2014). Coordination of synthesis and catabolism is much more complex and has been less studied under both, physiological and stress conditions. In some cases both routes may be activated together. P5CS (Savoure et al., 1995), ProDH (Nakashima et al., 1998), and P5CDH (Deuschle et al., 2004) may be induced in parallel in reproductive organs. Similarly, P5CS2, P5CR, and ProDH1 increase their 
expression in meristematic tissues such as root tip, shoot apex, and inflorescences (Kavi Kishor and Sreenivasulu, 2014). In addition, under some stress conditions genes from synthesis and catabolism are simultaneously induced (Fabro et al., 2004; Kaplan et al., 2007; Sharma and Verslues, 2010; Senthil-Kumar and Mysore, 2012). Recently, a shortcut connecting synthesis and catabolism by coupling ProDH and P5CR activities (Pro/P5C cycle) was suggested to operate under stress (Miller et al., 2009; Lv et al., 2011; Monteoliva et al., 2014). However, it is worth noting that the function of this cycle is sustained in the exclusive location of $\mathrm{P} 5 \mathrm{CDH}$ and $\mathrm{P} 5 \mathrm{CR}$ in mitochondria and cytosol, respectively, as well as in the existence of P5C transporters in plants.

As observed, Pro metabolism is subject to a complex control and its alteration may have different consequences. The GluPro conversion consumes NADPH in cytosol and chloroplasts and may thus alleviate photoinhibition, as well as repression of the oxidative pentose phosphate pathway. The Pro-Glu pathway provides reducing equivalents for mitochondrial oxidative phosphorylation (Verslues and Sharma, 2010; Liang et al., 2013). Therefore, Pro-Glu interconversion couples oxidation of the cytosolic NADPH/NADP ${ }^{+}$pool with mitochondrial activity. In turn, the Pro/P5C cycle shuttles reductants into mitochondria and promotes accumulation of reactive oxygen species (ROS) probably due to hyper-activation of ProDH (Ben Rejeb et al., 2014). Given this scenario, the activity of P5CDH seems to be crucial to define the fate of P5C (synthesis of Glu or Pro), the degree of Pro oxidation, and the level of ROS. Moreover, $\mathrm{P} 5 \mathrm{CDH}$ can provide plasticity to turn on or off synthesis and degradation routes at different stages of stress. Despite this, the effects of this enzyme on the coordination of Pro metabolic pathways have been poorly studied.

Plants that trigger Pro consumption as a first response to stress, may replenish the amino acid pool by synthesis or transport. While Glu is considered the main source of Pro under stress, the Orn pathway provides Pro at early developmental stages and under high nitrogen conditions where it allows transfer of nitrogen from Arg to other amino acids (Verslues and Sharma, 2010; Liang et al., 2013). In addition, Orn can also be used to synthesize Pro under hostile conditions. $N$. plumbaginifolia plants over-expressing Arabidopsis OAT increase Pro levels in response to osmotic stress (Roosens et al., 2002). Curiously, different effects have been reported for this enzyme under salinity. Radish cotyledons activate OAT by treatment with $\mathrm{NaCl}(100-250 \mathrm{mM})$ in a dose-dependent manner, and reduce salt-induced Pro accumulation (six-fold) in response to OAT inhibitors (Hervieu et al., 1995). OAT activation also accompanies Pro accumulation (12-fold) in cashew leaves treated with 100-400 mM NaCl (Da Rocha et al., 2012). In contrast, the absence of OAT does not affect Pro increase (fourfold) in Arabidopsis plants treated with 25-100 mM NaCl (Funck et al., 2008). Interestingly, although OAT may synthesize Pro under some particular adverse conditions, it is unknown how this is achieved (via P5CDH/P5CS/P5CR or P5CR). Similarly, the traits that determine the use of Glu or Orn as Pro precursors, and the role of $\mathrm{P} 5 \mathrm{CDH}$ on coordination of the different Pro pathways under stress deserve further characterization. The exhaustive study of Pro metabolism under these circumstances may contribute to identify metabolic and physiological features associated to stress tolerance.

In the present work, we evaluated whether Glu and Orn are used to regenerate Pro in tissues that initially consume Pro as a result of Pro supply, or Pst-AvrRpm1 infection. Using wildtype and $p 5 c d h$ mutant plants we assessed how $\mathrm{P} 5 \mathrm{CDH}$ affects the catabolic and biosynthetic pathways under these conditions. To this end, we monitored the expression of Pro metabolic genes and the levels of Pro, Orn, and Glu at different stages of plant treatment. Our results indicated that both plants trigger Pro synthesis after initial Pro degradation apparently activating different metabolic circuits.

\section{Materials and Methods}

\section{Plant Growth and Pathogen Infection}

Arabidopsis Col-0 (wild-type) and p5cdh mutant (Salk_021026) seeds were obtained from ABRC (Arabidopsis Biological Research Center, Ohio, USA). Plants were grown on GM-agar medium (GM with $30 \mathrm{mM}$ sucrose) for 10 days, and then transferred to soil for 6-8 weeks in growth chamber under $12 / 12$ light-dark photoperiod at $22^{\circ} \mathrm{C}$. Pst-AvrRpm1 was grown in King's B agar medium ( $2 \%$ proteose peptone, $1 \%$ glycerol, $1.5 \mathrm{~g} \mathrm{l}^{-1} \mathrm{~K} 2 \mathrm{HPO} 4,1 \%$ agar $)$ with kanamycin $(50 \mathrm{mg} / \mathrm{ml})$ and rifampicin $(100 \mathrm{mg} / \mathrm{ml})$, and used to infiltrate leaves at $1 \times 10^{7}$ $\mathrm{cfu} / \mathrm{ml}$ as described by Cecchini et al. (2011).

\section{Pro Feeding Assay}

Leaves of 6-8 weeks old Col-0 and $p 5 c d h$ plants were excised with a razor blade and transferred to eppendorf tubes containing $300 \mu \mathrm{l}$ of 20 or $50 \mathrm{mM}$ Pro solution (Sigma-Aldrich, St Louis) avoiding formation of air bubbles, and maintained under the growth conditions indicated above. To determine Pro uptake we measured the volume of feeding solution remaining in test tubes at $0,2,6,10$, and $24 \mathrm{~h}$ post-incubation (4 replicates per genotype). Tubes containing no leaves were used to evaluate and discount evaporation. Aliquots $(5 \mu \mathrm{l})$ extracted at 0,6 , and $24 \mathrm{~h}$ were used to determine Pro content (Bates et al., 1973). After feeding, leaves were rinsed with distilled water and transferred to wet chamber without water uptake.

\section{Cell Death Analysis}

Trypan blue staining (Pavet et al., 2005) was used to detect dead cells in leaves fed for $24 \mathrm{~h}$ with water (control) or Pro solutions $(50,100$, or $150 \mathrm{mM})$.

\section{Quantification of Amino Acids by HPLC}

HPLC was used to quantify Pro, Glu, and Orn in leaf extracts after derivatization with 6-aminoquinolyl- $N$-hydroxysuccinimidyl carbamate as reported by Monteoliva et al. (2014). Amino acids separation was carried out using a Zorbax C18 Plus column $(100 \times 4.6 \mathrm{~mm}, 3.5 \mu \mathrm{m})$ on a quaternary HPLC system (Hewlett Packard series 1100) with: (A) sodium acetate buffer $140 \mathrm{mM}$ pH 5.8 and $7 \mathrm{mM}$ triethylamine; (B) acetonitrile and (C) water. The gradient elution was $0.01 \mathrm{~min} 100 \% \mathrm{~A} ; 0.50 \mathrm{~min} 99 \% \mathrm{~A}+$ $1 \% \mathrm{~B} ; 27.5 \min 91 \% \mathrm{~A}+9 \% \mathrm{~B} ; 28.50 \min 89 \% \mathrm{~A}+11 \% \mathrm{~B}$; $44.50 \min 82 \% \mathrm{~A}+18 \% \mathrm{~B} ; 47.5 \min 60 \% \mathrm{~B}+40 \% \mathrm{C} ; 50.5 \mathrm{~min}$ 
$100 \%$ A. The equipment was coupled to a fluorescent detector (excitation at $300 \mathrm{~nm}$ and emission at $400 \mathrm{~nm}$ ). Retention times and quantification were determined using external standards. Values are expressed as nmol/g FW.

\section{Gene Expression}

cDNA was synthesized from $2 \mu \mathrm{g}$ RNA as previously described (Cambiagno et al., 2015). Semi-quantitative PCR was developed with primers and conditions indicated in Supplementary Table 3. GapC was used as housekeeping gene to control equal amount of cDNA in each reaction. qPCR was performed with Hot Start DNA Polymerase (Biodynamics), SYBR green dye, and dNTPs. Primers were used at the annealing temperature indicated in Supplementary Table 3. Reactions were developed as follows: $10 \mathrm{~min}$ at $95^{\circ} \mathrm{C}$; 40 cycles of $35 \mathrm{~s}$ at $95^{\circ} \mathrm{C}, 30 \mathrm{~s}$ of annealing, and $35 \mathrm{~s}$ at $72^{\circ} \mathrm{C}$. Reaction efficiency (E) was in the range $81-91 \%$ for all analyzed genes, including UBQ5 (At3g62250) used as internal control. Relative transcript levels (RTL) were calculated using the Pfaffl equation as follows: RTL = $\mathrm{E}_{\text {target }}-\Delta \Delta \mathrm{Ct}$ (treated, basal) $/ \mathrm{E}_{U B Q 5}-\Delta \Delta \mathrm{Ct}$ (treated, basal).

\section{Protein Levels and Prodh Activity}

Western blots were performed with polyclonal antibodies as previously described (Monteoliva et al., 2014). In vitro ProDH activity was determined by the method reported in Monteoliva et al. (2014) with two minor modifications: (i) grinding was performed with an automated tissue disruptor; (ii) ${ }^{14} \mathrm{C}$-Pro (NEC Perkin Elmer; $266 \mathrm{mCi} / \mathrm{mmol}$ ) was used as substrate. Two aliquots per sample ( \pm T4C; L-thiazolidine-4-carboxylic acid) were used. In the activities here reported the values of T4C-treated aliquots have been subtracted.

\section{Results}

\section{Pro Uptake in Detached Leaves}

We wondered if $\mathrm{P} 5 \mathrm{CDH}$ affects Pro metabolism in tissues transiently exposed to Pro. To test this, wild-type (Col-0) and $p 5 c d h$ tissues were treated with Pro solutions (feeding phase) and then deprived of the amino acid (recovery phase). These treatments would initially activate ProDH (Kiyosue et al., 1996; Satoh et al., 2002), and then promote Pro consumption and eventually re-activation of Pro synthesis.

The studies were conducted on detached leaves of analogous developmental stage that were excised from plants of similar age (6-8 weeks). This model, at difference of whole plants, allows studying homogeneous responses unaffected by transport of substances between organs. In the feeding phase Pro was supplied through the leaf petiole, and in the recovery phase leaves were maintained in a humid chamber without water uptake. The experiments described below differ in the concentration of Pro solutions, and the periods of feeding or recovery.

To start characterizing the experimental system, we assessed Pro uptake in both genotypes. For this purpose, aliquots of the Pro solutions used to feed individual leaves were taken at 2, 6, 10 , and $24 \mathrm{~h}$ post treatment (hpt) to determine Pro content. In addition, volume solution was checked at these stages. Pro 20 and $50 \mathrm{mM}$ solutions conserved their original concentration at 24 hpt $(20.2 \pm 0.2$ or $49.9 \pm 1.9 \mathrm{mM}$ Pro, respectively). Sustained volume reduction was detected in all assays, and was faster for incubations with $20 \mathrm{mM}$ Pro (Figure 2A). Solution volumes were identical in tubes holding different genotypes, analyzed at the same time point and Pro concentration. Hence, in this assay Col- 0 and $p 5 c d h$ leaves showed no differences in Pro uptake.

The estimated amino acid content at 0,6 and $24 \mathrm{hpt}$ with each Pro solution is informed on Figure 2A. Based on these values, and the average weight of a leaf $(30 \mathrm{mg})$, we estimated that individual leaves could incorporate up to 400 or $900 \mathrm{nmol}$ Pro at 24 hpt when fed with 20 or $50 \mathrm{mM}$ Pro, respectively.

In our hands, Col-0 and $p 5 c d h$ leaves did not show significant damage at $24 \mathrm{hpt}$ with $50 \mathrm{mM}$ Pro (Figure 2B). Nevertheless, higher Pro concentrations (100 or $150 \mathrm{mM}$ Pro) triggered perivascular cell death in both genotypes. Curiously, cell death was less pronounced on $p 5 c d h$ than wild-type leaves.

\section{Changes in Pro Levels During Feeding and Recovery}

Next, we used a colorimetric assay (Bates et al., 1973) to quantify the free Pro content in leaves. Leaves exposed to Pro (20 or $50 \mathrm{mM}, 24 \mathrm{~h}$ ) and transferred to humid chamber $(6 \mathrm{~h})$, were analyzed at basal condition (basal), $24 \mathrm{~h}$ post-feeding (P-24h), and 4 and $6 \mathrm{~h}$ of recovery (R-4h, R-6h) (Figure 3A).

At the stage P-24h, Col-0 leaves increased 14 or 27 times the basal Pro levels, reaching $2.9 \pm 0.1$ or $5.8 \pm 0.6 \mu \mathrm{mol}$ Pro/g FW in response to 20 or $50 \mathrm{mM}$ Pro, respectively (Figure 3A). At the same stage, $p 5 c d h$ leaves increased 27 or 41 times the amino acid content $(6.0 \pm 0.2$ or $11.0 \pm 1.7 \mu \mathrm{mol}$ Pro/g FW for 20 or $50 \mathrm{mM}$ Pro, respectively), almost doubling the levels of Col-0 leaves.

Then, Pro was higher in leaves exposed to higher Pro concentrations, and for a given solution was greater in $p 5 c d h$ than Col-0 leaves. The fact that Pro uptake was similar in both leaves (Figure 2A), but Pro leaf content was higher in $p 5 c d h$ (Figure 3A) suggested that during feeding Pro may be consumed more efficiently in Col-0. Supporting this possibility, differences in tissue Pro were manifested during recovery, when Col-0 sharply reduced the Pro content (half levels at R-6h vs P-24h), while $p 5 c d h$ did not consume it, and even manifested a slight trend to increase the amino acid level (Figure 3A).

To test if ProDH was activated at the feeding phase, leaves were incubated with $20 \mathrm{mM}$ Pro for only $2 \mathrm{~h}$, and then transferred to humid chamber (Figure 3B). Samples obtained at basal condition, $2 \mathrm{~h}$ post-feeding ( $\mathrm{P}-2 \mathrm{~h}$ ), and 2 and $24 \mathrm{~h}$ of recovery (R2h, R-24h), were used to determine Pro content and ProDH genes expression. At P-2h, Pro increased at the same extent in Col-0 and $p 5 c d h$ leaves ( 6 fold compared to basal condition), reaching $1.2 \pm 0.1$ or $1.4 \pm 0.1 \mu \mathrm{mol} / \mathrm{g} \mathrm{FW}$, respectively (Figure 3B), and ProDH1 and ProDH2 genes displayed similar activation. During recovery, Col-0 leaves initially lost ProDH2 induction (R-2h), and then ProDH1 activation (R-24h). In parallel, these leaves reduced Pro to almost recover its basal content at R-24h $(0.219 \pm 0.041$ Pro/g FW). In contrast, $p 5 c d h$ leaves maintained ProDH1 and ProDH2 activation until R-24h, showing no significant reduction in the Pro content ( $14 \%$ at R-24h vs. P-2h) (Figure 3B).

Consequently, $2 \mathrm{~h}$ treatment with Pro $20 \mathrm{mM}$ was sufficient to induce ProDH1 and ProDH 2 in Col-0 and $p 5 c d h$ leaves, but insufficient to generate differential Pro content in these genotypes. Since this kind of differences were manifested at $24 \mathrm{~h}$ 


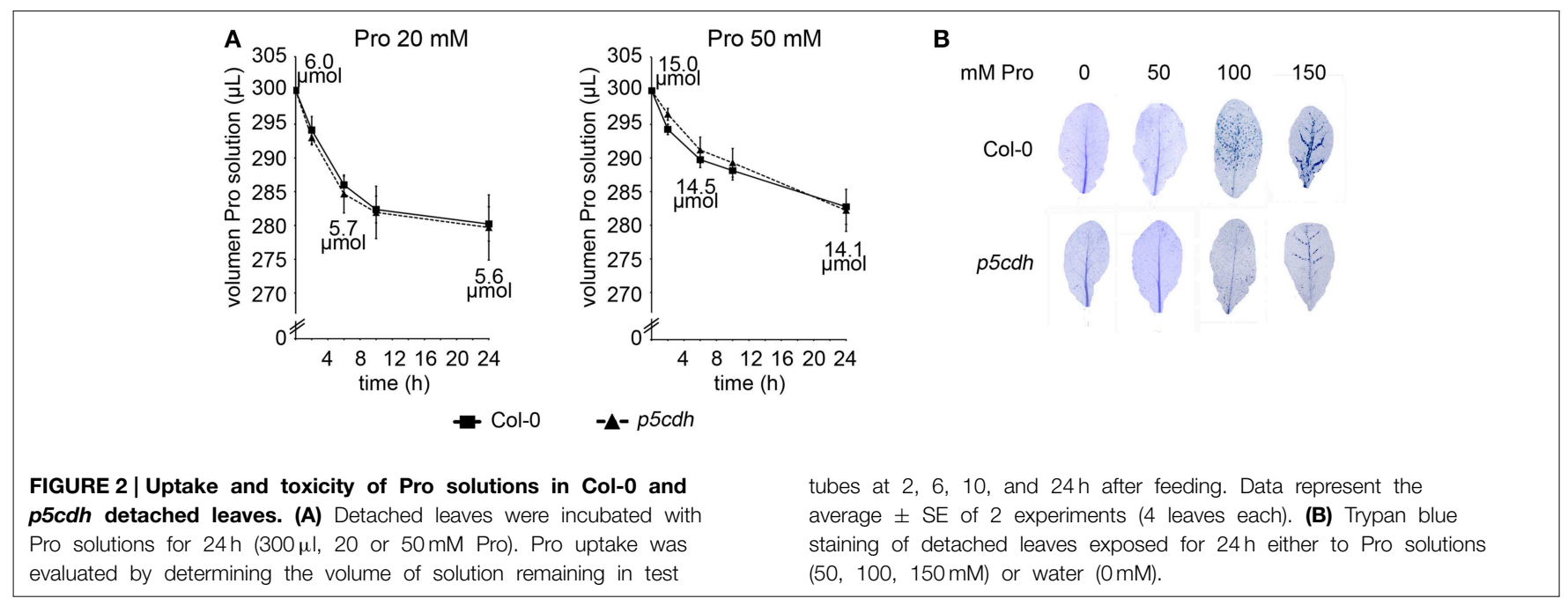

post feeding (P-24h, Pro 20 or $50 \mathrm{mM}$; Figure 3A), it is likely that they result from processes not yet triggered at $2 \mathrm{~h}$ post feeding.

\section{Metabolic Alterations Triggered by Exogenous Pro}

$p 5 c d h$ leaves seem to accumulate Pro after being deprived of the amino acid (R-6h vs. P-24h; Figure 3A). To test if this response was strengthened along recovery, we preserved the feeding period $(24 \mathrm{~h})$ and extended the recovery phase $(24 \mathrm{~h})$ using incubations with $20 \mathrm{mM}$ Pro. $p 5 c d h$ and Col-0 leaves were analyzed in parallel at basal, P-24h, R-4h, R-6h, and R-24h stages (Figure 4A). To comprehensively assess the Pro metabolic routes altered under this condition, we evaluated amino acids content and expression of genes of Pro metabolism (ProDH, P5CDH, P5CS, P5CR, and OAT).

We conducted HPLC studies to unequivocally determine the leaf Pro content. By adapting this assay we also quantified Glu and Orn in the same samples. Pro, Orn and Glu values are reported in Supplementary Table 1, by indicating significant differences along treatment for each compound in the same genotype. All amino acids altered their levels on at least one condition. To better visualize these changes, we expressed the fold change of a compound in the test sample (P-24h, R-6h, R-24h) relative to control sample (basal) (Figure 4A).

Changes in Pro levels detected by HPLC assays were consistent with those previously observed by colorimetric reactions. That is, at P-24h $p 5 c d h$ contained higher Pro levels than Col- 0 samples and during recovery, $p 5 c d h$ accumulated Pro while Col- 0 reduced it. The Pro increase occurred in $p 5 c d h$ during recovery was intensified along this phase (Figure $\mathbf{4 A}$ ).

On the other hand, Glu was not significantly altered in Col-0 leaves, but was clearly reduced in $p 5 c d h$ at all phases analyzed (reduction of $81 \%$ at $\mathrm{P}-24 \mathrm{~h}$, and $65 \%$ at $\mathrm{R}-24 \mathrm{~h}$, compared to basal levels). As Glu is a central intermediate for synthesis of amino acids, this reduction may derive from its conversion into other derivatives different from Pro, such as Arg, GABA, Gln, or inhibition of the routes that replenish this amino acid (Forde and Lea, 2007).
Curiously, Orn increased in Col- 0 and $p 5 c d h$ leaves after feeding (3.5 and 2.9 times, respectively, compared to basal condition). Latter, during recovery, Orn was reduced to half values in Col- 0 , while increased in $p 5 c d h$ ( 2.3 fold at R-24h, compared to P-24h) (Supplementary Table 1, Figure 4A). In summary, the genotypes kept their trends to either consume $($ Col- 0$)$ or accumulate $(p 5 c d h)$ Pro, and these variations were accompanied by similar changes in Orn (reduction in Col-0, increase in $p 5 c d h$ ), but did not correlate with changes in Glu levels.

As mentioned, we conducted gene expression analysis with the same set of samples (Figure 4B). Col-0 leaves accumulated ProDH1 and $\mathrm{ProDH} 2$ transcripts at $\mathrm{P}-24 \mathrm{~h}$, and reverted gene induction at R-6h, when Pro doubled its initial content (Supplementary Table 1). Then, these leaves activated $P 5 \mathrm{CDH}$ (R-4h), and P5CS1 (R-6h), displaying maximal P5CS1 expression at R-24h when Pro recovered its basal level (Figure 4B, Supplementary Table 1). Moreover, at P-24h, R-6h, and R-24h, $P 5 C R$ and $O A T$ were induced in these samples. These results suggest that once Col-0 leaves stimulate Pro synthesis after consuming the amino acid incorporated from the medium involving P5CS and OAT, and P5CR activities. At this condition, the synthesis of Pro from Glu and Orn, converging on P5C, could help maintain the Pro basal level. Our experimental scheme did not inform whether this route would generate accumulation of Pro at longer times.

The $p 5 c d h$ leaves exhibited a different behavior. ProDH1 and $\mathrm{ProDH} 2$ were also induced at $\mathrm{P}-24 \mathrm{~h}$, but reinforced their activation along recovery, reaching maximal expression at R24h (Figure 4B). Thus, ProDH activation accompanied Pro increase in $p 5 c d h$ tissues (Figure 4; Supplementary Table 1). From feeding onwards, P5CS1 became repressed and P5CS2 maintained basal expression. Besides this, OAT and P5CR was induced at $\mathrm{P}-24 \mathrm{~h}$ or $\mathrm{R}-4 \mathrm{~h}$, respectively. Thus, the Pro increase detected in $p 5 c d h$ leaves would not derive from the P5CS pathway. Alternatively, this increase might result from consecutive transformation of Orn into P5C and Pro, by OAT and P5CR. 
A
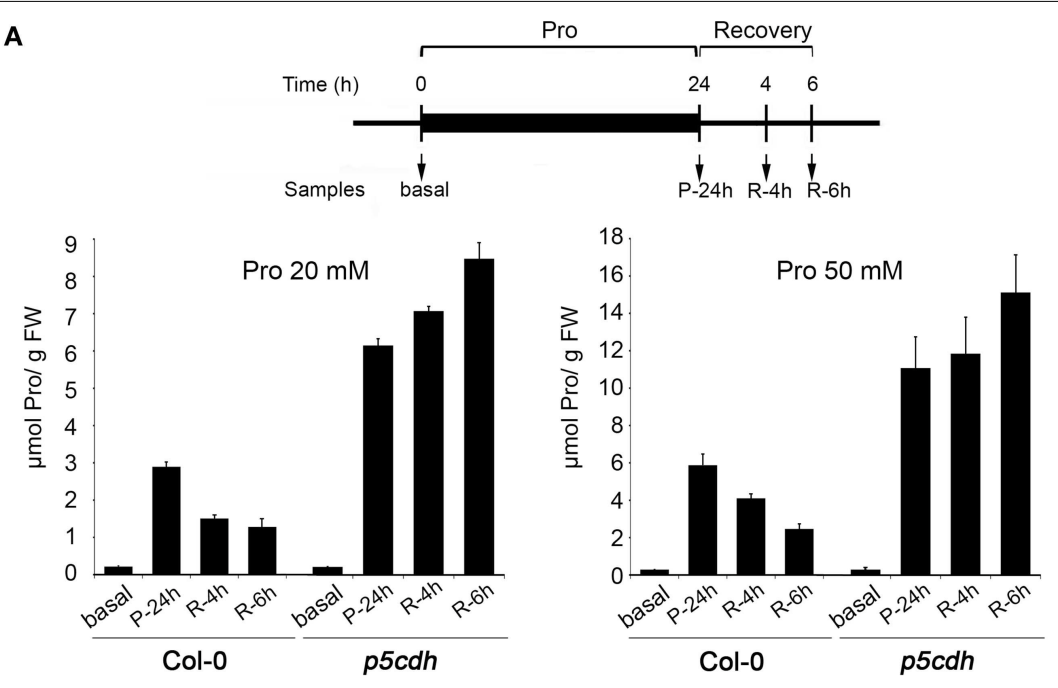

B
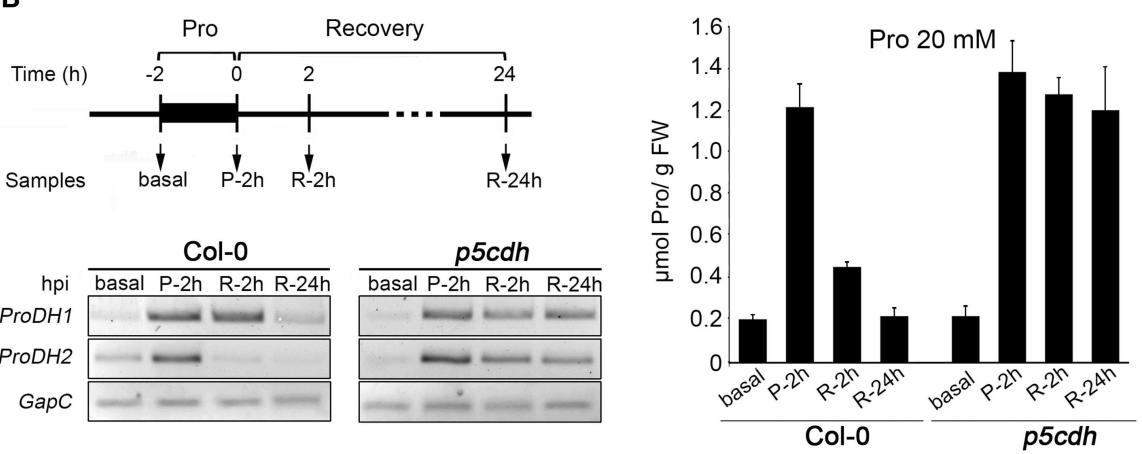

FIGURE 3 | Pro levels and ProDH expression in wild-type and p5cdh detached leaves treated with Pro. (A) Leaves were fed with 20 or $50 \mathrm{mM}$ Pro during $24 \mathrm{~h}$, and then deprived of the amino acid for $6 \mathrm{~h}$ (recovery) to evaluate samples at different stages of treatment (top). Graphics compare the Pro levels in both genotypes (bottom). (B) Leaves fed with $20 \mathrm{mM}$ Pro for $2 \mathrm{~h}$, and deprived of the amino acid for $24 \mathrm{~h}$, were used to determine Pro levels and $\mathrm{ProDH}$ expression at different stages (top). Gene expression was studied by sq-RT-PCR using GapC as internal control (bottom). Pro was quantified by colorimetric assays (right). In each case, one representative experiment from three independent assays is shown. Data represents the average \pm SE of 3 leaves per time point. Pro content displayed significant differences $(p<0.001$ by $t$-test) between treated samples and basal condition in both genotypes, except for the R-24h, Col-0 sample that was similar to control (B).
RT-qPCR assays confirmed the expression pattern of P5CS1, P5CS2, P5CR, and OAT genes at basal, P-24h and R-24h stages in Col-0 and $p 5 c d h$ leaves (Supplementary Figure 1). The quantification of ProDH activity (Monteoliva et al., 2014) in Col0 and $p 5 c d h$ samples at basal and P-24h stages, indicated that Pro treatment activates the enzyme to similar extent in both plants (4.1 and 3.7 fold for Col-0 and $p 5 c d h$, respectively) (Figure 4C). This was consistent with the increase in the ProDH content detected by Western blot in Col- 0 and $p 5 c d h$ samples at P-24h (Figure 4D). In addition, we observed that Col-0 leaves also increased $\mathrm{P} 5 \mathrm{CDH}$ at this stage.

\section{Metabolic Alterations Triggered by Pathogen Infection}

Arabidopsis plants infected with Pst-AvrRpm1 initially activate ProDH and later induce P5CS expression accumulating Pro, suggesting that early Pro consumption stimulates the Glu biosynthetic pathway (Fabro et al., 2004; Cecchini et al., 2011; Monteoliva et al., 2014). To investigate how P5CDH affects the second process, we analyzed the behavior of wild-type and $p 5 c d h$ plants $72 \mathrm{~h}$ post infection (hpi) with Pst-AvrRpm1. In addition, we assessed if OAT could mediate Pro synthesis under this condition. These issues, as well as the overall expression of Pro metabolism genes have not been evaluated under this condition.

Healthy and infected leaves were used to quantify Pro, Orn, and Glu by HPLC, and assess the expression of Pro metabolism genes. Pathogen treatment similarly affected the amino acids level in both plants, where Pro content was doubled, Orn increased 3.6 times and Glu was reduced (47\% in Col-0 and $37 \%$ in $p 5 c d h$ ) (Supplementary Table 2; Figure 5A). This indicated that $\mathrm{P} 5 \mathrm{CDH}$ was not required to adjust Pro, Orn, and Glu levels at late stages of HR.

We confirmed that Col-0 plants induced ProDH and P5CS genes at $72 \mathrm{hpi}$, as previously reported (Fabro et al., 2004), increasing as well the expression of $O A T$ (Figure 5B). Conversely, although infected $p 5 c d h$ plants activated ProDH and OAT expression, they slightly repressed the expression of P5CS. 
A
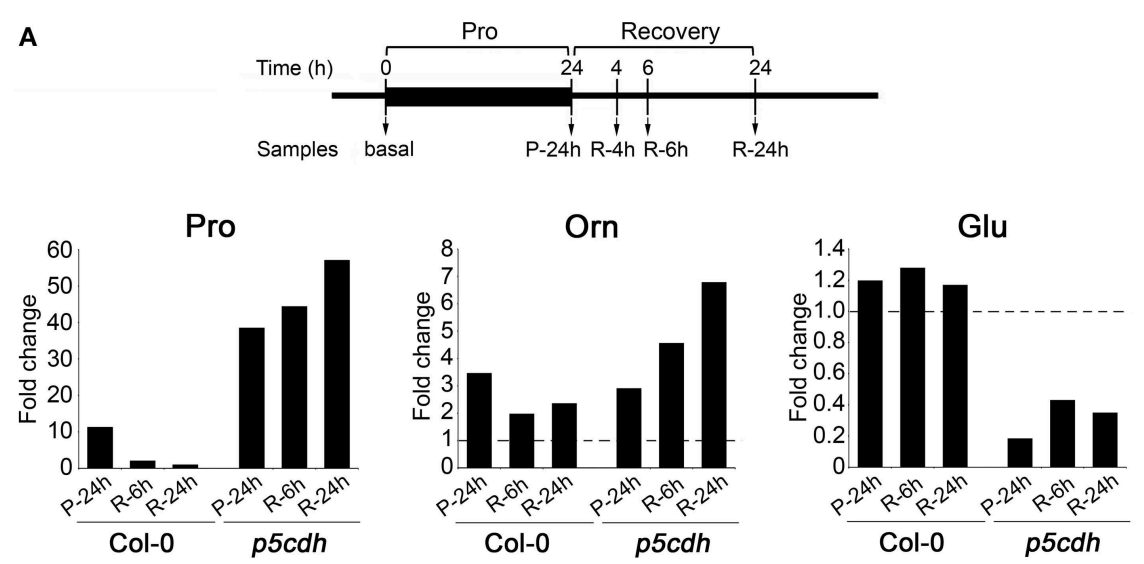

B
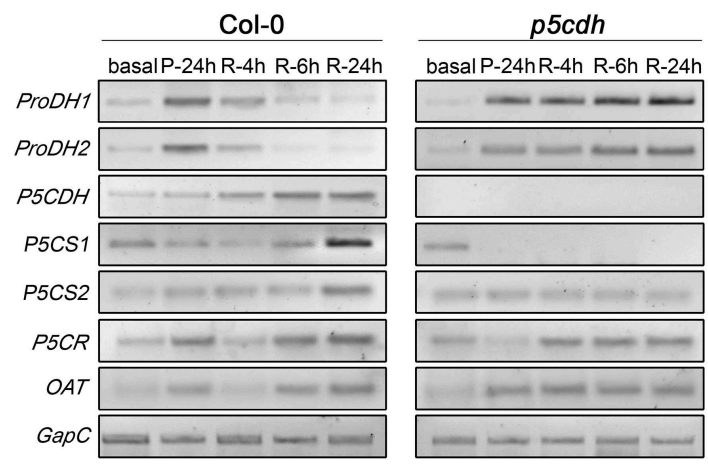

C

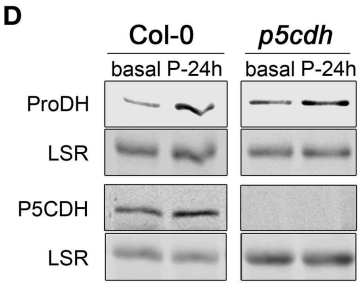

FIGURE 4 | Metabolic changes induced by exogenous Pro in detached leaves of wild-type and $p 5 c d h$ plants. (A) Leaves were incubated with $20 \mathrm{mM}$ Pro ( $24 \mathrm{~h}$ ) and then deprived from the amino acid ( $24 \mathrm{~h}$ ) to take samples at different treatment stages (top). Pro, Glu, and Orn levels were determined by HPLC (Supplementary Table 1) and the ratio between their content in treated and basal conditions was depicted (bottom).
(B) Expression of genes of Pro metabolism evaluated as described in Figure 3B. (C) ProDH activity in total extracts of leaf samples taken at basal and $\mathrm{P}-24 \mathrm{~h}$ stages. Values are expressed as nmol of Pro $\mathrm{min}^{-1} \mathrm{mg}$ protein $^{-1}$. Each value is mean \pm SE of two biological replicates. (D) $\mathrm{ProDH}$ and P5CDH content at basal and P-24h conditions examined by Western blot with polyclonal antibodies. LSR, large subunit of rubisco.
Consequently, in advanced stages of HR wild plants could use Glu as Pro precursor while the mutant, lacking P5CS induction, would not stimulate this particular pathway. The activation of $O A T$ occurring in both plants suggests that $\mathrm{P} 5 \mathrm{CDH}$ is not required to generate $\mathrm{P} 5 \mathrm{C}$ from Orn during the interaction of Arabidopsis with Pst-AvrRpm1.

\section{Discussion}

\section{Responses of Detached Leaves to Exogenous Pro}

Most studies evaluating the effect of exogenous Pro in Arabidopsis, supply the amino acid by the root (Kiyosue et al.,
1996; Nakashima et al., 1998; Nanjo et al., 1999, 2003; Hellmann et al., 2000; Mani et al., 2002; Satoh et al., 2002; Deuschle et al., 2004; Miller et al., 2009; Funck et al., 2010; Sharma and Verslues, 2010). Root and shoot can differentially perceive, transport, or metabolize the amino acid, and moreover, leaves from distinct developmental stages can regulate Pro metabolism in alternative manners (Lehmann et al., 2010; Kavi Kishor and Sreenivasulu, 2014). Unlike this, our experimental model uses leaves of similar age and developmental stage that synchronously assimilate Pro and can trigger more homogeneous responses. Then, detached leaves could be useful for detection of slight changes that might go unnoticed in the whole plant system. Under the conditions tested here, Col-0 and $p 5 c d h$ leaves 


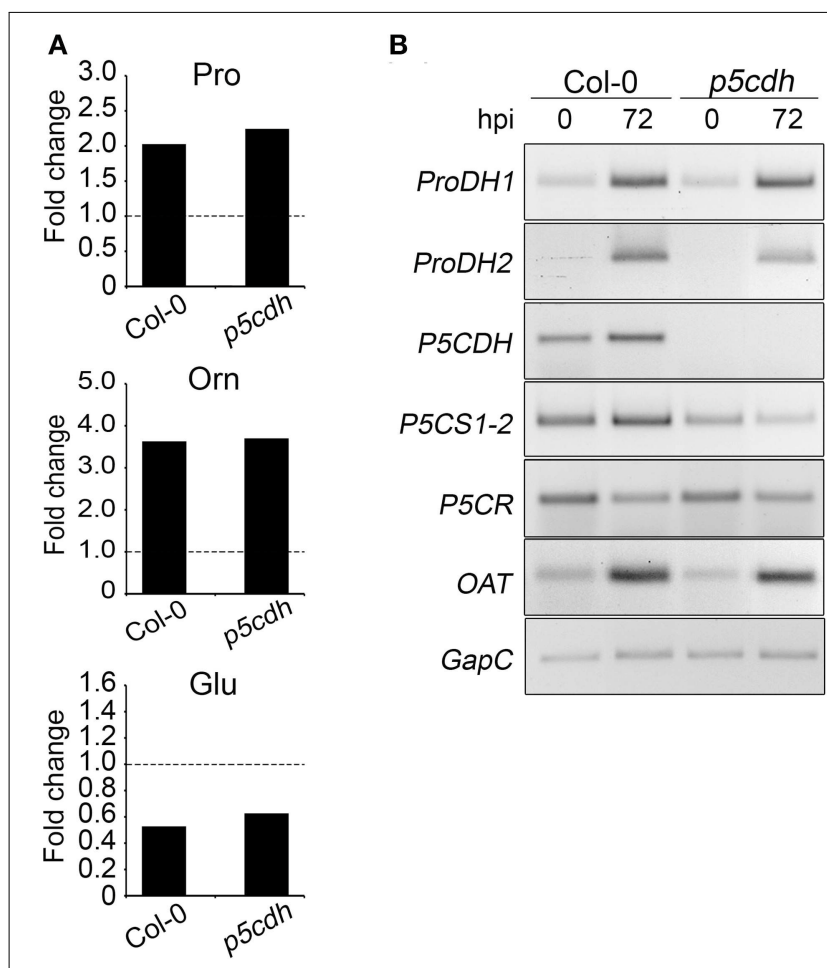

FIGURE 5 | Metabolic changes induced by Pst-AvrRpm1 infection in wild-type and $\mathbf{p 5 c d h}$ plants. (A) HPLC was used to determine the Pro, Glu, or Orn content in uninfected and infected tissues (72 h post-infection, hpi) (Supplementary Table 2). Fold changes of amino acid in infected and basal conditions are here informed. (B) Expression of genes of Pro metabolism evaluated at basal condition and $72 \mathrm{hpi}$ as described in Figure 3B.

assimilated Pro in similar way without saturating their absorption capacity, as the amino acid content increased with longer incubation periods or more concentrated solutions. Therefore, the responses here described likely correspond to tissues that actively incorporate and metabolize exogenous Pro. In support of this, Pro consumption was already detected at the feeding phase in Col-0 leaves.

Detached leaves fed for $24 \mathrm{~h}$ with $50 \mathrm{mM}$ Pro accumulated similar amino acid levels (5.8 and $11.0 \mu \mathrm{mol} / \mathrm{g} \mathrm{FW}$ for Col0 and $p 5 c d h$, respectively) than whole plants exposed $48 \mathrm{~h}$ to $100 \mathrm{mM}$ Pro (10 and $35 \mu \mathrm{mol} / \mathrm{g}$ FW for Col- 0 and $p 5 c d h$ respectively; Miller et al., 2009). These leaves did not manifest significant lesions under the above conditions, although they induced cell death in perivascular tissues in response to higher Pro concentrations (100-150 mM). Pro also induced leaf cell death in plants sprayed for 3 consecutive days with $20 \mathrm{mM}$ solution (Deuschle et al., 2004), indicating that in photosynthetic tissues its toxicity is independent of the route of entry (phloem or epidermis). Curiously, Pro treatment produced less damage in $p 5 c d h$ than Col-0 leaves (Figure 2B). As the mutant accumulated higher Pro content, then Pro levels and cell death symptoms would not be directly correlated. The finding of more cell death in the mutant contrasts with the effect reported for the amino acid supplied by root to seedlings of these genotypes (Deuschle et al., 2004; Funck et al., 2010). Therefore, we cannot discard that $p 5 c d h$ plants exhibit dissimilar sensitivity to exogenous Pro in roots and leaves.

\section{Pro Metabolism Changes Induced by Exogenous Pro}

Col-0 and $p 5 c d h$ leaves treated with exogenous Pro displayed similar ProDH activation (Figure 4C) accompanied by gene induction (Figure 4B) and protein accumulation (Figure 4D). This increase in ProDH activity (3-4 fold) was similar to that reported for Arabidopsis cells treated with $50 \mathrm{mM}$ Pro during $21 \mathrm{~h}$ (4-5 fold; Schertl et al., 2014). However, this treatment triggers different metabolic changes in both plants (Figures 4A,B, 6). Wild-type leaves consumed the excess of Pro, to then increase the P5CS1 expression and apparently stimulate the synthesis of Pro from Glu. In contrast, $p 5 c d h$ leaves maintained ProDH and P5CR induction, apparently activating the Pro/P5C cycle. Curiously, these leaves accumulated Pro after being removed from the feeding solution. This response did not seem to be caused by massive protein degradation as it was not accompanied by tissue damage or general rise in free amino acids. Such Pro increase was manifested after prolonged feeding (P$24 \mathrm{~h}$ but not $\mathrm{P}-2 \mathrm{~h}$ ), and was accentuated throughout the recovery phase (R-24h vs. R-6h). The activation of OAT, but not P5CS, detected under this condition suggested that Pro may derive from Orn (Figure 4B). As the mutant cannot use P5C in the catabolic pathway, it is possible that OAT provides $\mathrm{P} 5 \mathrm{C}$ into the biosynthetic route leading to Pro accumulation (Figure 6). The $p 5 c d h$ tissues that induced OAT (Figure 4A) accumulate Pro (Supplementary Table 1). Moreover, in vitro assays suggested that OAT activity was not inhibited by high Pro levels (Sekhar et al., 2007). Note that the Orn concentration found at P-24h in $p 5 c d h$ leaves was 100 times lower than that of Pro, and thereafter Orn and Pro increased together (Supplementary Table 1). This suggests that Orn accumulation corresponds to the amino acid pool that feeds Pro synthesis. In this case, Pro and Orn may not increase in similar manner, since their synthesis and degradation could be altered differently (i.e., greater increase in Orn to Pro conversion, than in Pro degradation). Parallel increases of Orn and Pro have been also observed in cashew plants treated with $\mathrm{NaCl}$, which activated OAT and curiously repressed P5CDH (Da Rocha et al., 2012). It is important to note that our results are insufficient to establish that $p 5 c d h$ leaves synthesize Pro from Orn during recovery. Additional studies will be required to formally demonstrate this assumption. In addition, it is not clear whether Col-0 leaves synthesize Pro from Orn at the recovery phase, since they reduce the Orn pool and slightly increase the OAT transcripts. We currently ignore which amino acid may act as Orn precursor during the recovery phase. Since Col- 0 and $p 5 c d h$ leaves activate the expression of the ARG1 (arginase) gene at R$24 \mathrm{~h}$ (Supplementary Figure 1), it is possible that Arg is used to this end.

A curious result was the repression of P5CS1 caused by Pro treatment in $p 5 c d h$ leaves (Figure 4B). This response may not be caused by Pro accumulation $(6 \mu \mathrm{mol} / \mathrm{g}$ FW Pro; Figure 3A), since Pro-treated Col-0 leaves reaching similar amino acid content did not reduce P5CS1 expression (not shown). Furthermore, $p 5 c d h$ seedlings repressed P5CS during 


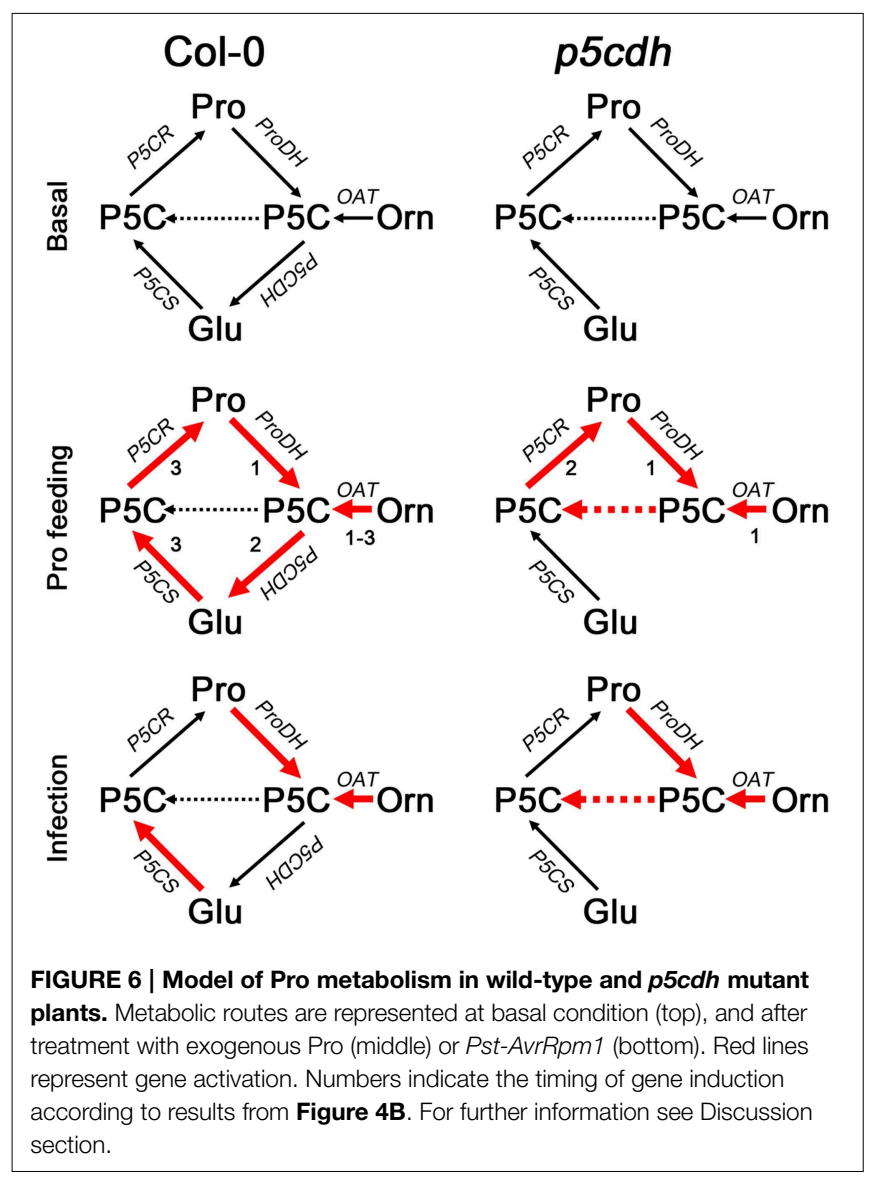

recovery from drought (Miller et al., 2009), suggesting that the absence of $\mathrm{P} 5 \mathrm{CDH}$ would somehow alter transcriptional regulation of P5CS1 under stress.

\section{Pro Metabolism Changes Induced by Infection with Pst-AvrRpm1}

Arabidopsis plants infected with Pst-AvrRpm1 initially activate ProDH, to then induce P5CS and accumulate Pro (Fabro et al., 2004; Cecchini et al., 2011; Monteoliva et al., 2014). To learn more about the Pro metabolic routes that become altered at late stages of infection, we compared the behavior of $p 5 c d h$ and Col-0 plants. At 72 hpi both genotypes modified the Pro, Orn, and Glu levels in similar way, but they used slightly different alternatives to generate these changes.

Both plants activated $O A T$ and increased Orn and Pro. As described before, this may suggest that Orn is used as a Pro precursor. The rise in Orn taking place in infected tissues may derive from activation of arginase, which generates Orn from Arg, as the enzyme is enriched in the mitochondrial fraction of Arabidopsis tissues infected with Pst-AvrRpm1 (Jones et al., 2006). Interestingly, $N$. benthamiana tissues infected with Pseudomonas syringae pv. tomato T1 may also synthesize Pro from Orn, since they require $O A T$ for normal development of HR (Senthil-Kumar and Mysore, 2012). Here again, the notion that OAT feeds Pro synthesis in $p 5 c d h$ infected leaves is consistent with activation of the Pro/P5C cycle in these tissues (Monteoliva et al., 2014).

Reduction of Glu accompanies Pro increase in both plants, but is only associated with induction of P5CS gene in wild plant, suggesting that this reduction may not necessarily reflect the activation of this pathway.

Therefore, Col- 0 and $p 5 c d h$ plants treated with Pst-AvrRpm 1 would combine differently the anabolic and catabolic routes to generate similar Pro increases. One possibility is that both biosynthetic routes (P5CS and OAT) and the complete catabolic route (ProDH, P5CDH) are combined in Col-0, while one anabolic route (OAT) and partial Pro oxidation work in $p 5 c d h$ (Figure 6).

In summary, both experimental systems enable ProDH induction and apparently subsequent Pro synthesis, triggering less pronounced amino acid changes at late infection stages than in response to Pro treatment. $p 5 c d h$ tissues seem to maintain ProDH participating in the Pro/P5C cycle, while feeding Pro synthesis with the P5C derived from OAT activity. In contrast, wild-type tissues seem to use OAT and/or P5CS for Pro synthesis and $\mathrm{ProDH}$ and $\mathrm{P} 5 \mathrm{CDH}$ for Pro catabolism. These results demonstrate that under stress $\mathrm{P} 5 \mathrm{CDH}$ may indeed affect the fate of P5C, and the routes of Pro synthesis (OAT or P5CS), while the absence of this enzyme could reduce the ability of plants to induce P5CS expression.

\section{Acknowledgments}

We thank Mohammad-Reza Hajirezaei (Leibniz Institute of Plant Genetics and Crop Plant Research (IPK), Molecular Plant Nutrition, Germany) for providing us with AQC, and helping us with the design of HPLC assays. This work was supported by grants from Agencia Nacional de Promoción Científica y Tecnológica, Argentina (PICT 2012-2117) and Secretaría de Ciencia y Tecnología, Universidad Nacional de Córdoba to MEA. YR is a CONICET fellow, GF and MEA are senior Career Investigators of CONICET.

\section{Supplementary Material}

The Supplementary Material for this article can be found online at: http://journal.frontiersin.org/article/10.3389/fpls.2015. 00572

Supplementary Table 1 | Amino acid content in detached leaves from wild-type and $p 5 c d h$ plants treated with exogenous Pro.

Supplementary Table 2 | Amino acid content in wild-type and $p 5 c d h$ plants infected with Pst-AvrRpm1.

Supplementary Table 3 | Primers and conditions used for RT-sqPCR and RT-qPCR.

Supplementary Figure 1 | Expression level of Pro metabolism genes in wild-type and p5cdh leaves treated with $20 \mathrm{mM}$ Pro determined by RT-qPCR. The samples used in this study are those shown in Figure 4B. Relative transcript levels of different Pro metabolism genes (A-E) were calculated as described in Materials and Methods. Letters indicate statistically significant differences respect to the basal sample (a: $p<0.05$; b: $p<0.01$, by $t$-test). 


\section{References}

Bates, L. S., Waldren, R. P., and Teare, I. D. (1973). Rapid determination of free proline for water-stress studies. Plant Soil 39, 205-207. doi: 10.1007/BF00018060

Ben Rejeb, K., Abdelly, C., and Savoure, A. (2014). How reactive oxygen species and proline face stress together. Plant Physiol. Biochem. 80, 278-284. doi: 10.1016/j.plaphy.2014.04.007

Cambiagno, D. A., Lonez, C., Ruysschaert, J. M., and Alvarez, M. E. (2015). The synthetic cationic lipid diC14 activates a sector of the Arabidopsis defense network requiring endogenous signaling components. Mol. Plant Pathol. doi: 10.1111/mpp.12252. [Epub ahead of print].

Cecchini, N. M., Monteoliva, M. I., and Alvarez, M. E. (2011). Proline dehydrogenase contributes to pathogen defense in Arabidopsis. Plant Physiol. 155, 1947-1959. doi: 10.1104/pp.110.167163

Da Rocha, I. M., Vitorello, V. A., Silva, J. S., Ferreira-Silva, S. L., Viegas, R. A., Silva, E. N., et al. (2012). Exogenous ornithine is an effective precursor and the $\delta$-ornithine amino transferase pathway contributes to proline accumulation under high $\mathrm{N}$ recycling in salt-stressed cashew leaves. J. Plant Physiol. 169, 41-49. doi: 10.1016/j.jplph.2011.08.001

Deuschle, K., Funck, D., Forlani, G., Stransky, H., Biehl, A., Leister, D., et al. (2004). The role of $\Delta 1$-pyrroline-5-carboxylate dehydrogenase in proline degradation. Plant Cell 16, 3413-3425. doi: 10.1105/tpc.104.023622

Fabro, G., Kovacs, I., Pavet, V., Szabados, L., and Alvarez, M. E. (2004). Proline accumulation and AtP5CS2 gene activation are induced by plant-pathogen incompatible interactions in Arabidopsis. Mol. Plant Microbe Interact. 17, 343-350. doi: 10.1094/MPMI.2004.17.4.343

Fichman, Y., Gerdes, S. Y., Kovacs, H., Szabados, L., Zilberstein, A., and Csonka, L. N. (2014). Evolution of proline biosynthesis: enzymology, bioinformatics, genetics, and transcriptional regulation. Biol. Rev. Camb. Philos. Soc. doi: 10.1111/brv.12146. [Epub ahead of print].

Forde, B. G., and Lea, P. J. (2007). Glutamate in plants: metabolism, regulation, and signalling. J. Exp. Bot. 58, 2339-2358. doi: 10.1093/jxb/erm121

Funck, D., Eckard, S., and Muller, G. (2010). Non-redundant functions of two proline dehydrogenase isoforms in Arabidopsis. BMC Plant Biol. 10:70. doi: 10.1186/1471-2229-10-70

Funck, D., Stadelhofer, B., and Koch, W. (2008). Ornithine- $\delta$-aminotransferase is essential for arginine catabolism but not for proline biosynthesis. BMC Plant Biol. 8:40. doi: 10.1186/1471-2229-8-40

Funck, D., Winter, G., Baumgarten, L., and Forlani, G. (2012). Requirement of proline synthesis during Arabidopsis reproductive development. BMC Plant Biol. 12:191. doi: 10.1186/1471-2229-12-191

Giberti, S., Funck, D., and Forlani, G. (2014). Delta1-Pyrroline-5-carboxylate reductase from Arabidopsis thaliana: stimulation or inhibition by chloride ions and feedback regulation by proline depend on whether NADPH or NADH acts as co-substrate. New Phytol. 202, 911-919. doi: 10.1111/nph.12701

Hayat, S., Hayat, Q., Alyemeni, M. N., Wani, A. S., Pichtel, J., and Ahmad, A. (2012). Role of proline under changing environments: a review. Plant Signal. Behav. 7, 1456-1466. doi: 10.4161/psb.21949

Hellmann, H., Funck, D., Rentsch, D., and Frommer, W. B. (2000). Hypersensitivity of an Arabidopsis sugar signaling mutant toward exogenous proline application. Plant Physiol. 123, 779-789. doi: 10.1104/pp.123.2.779

Hervieu, F., Le Dily, F., Huault, C., and Billard, J. P. (1995). Contribution of ornithine aminotransferase to proline accumulation in $\mathrm{NaCl}$-treated radish cotyledons. Plant Cell Environ. 18, 205-210. doi: 10.1111/j.13653040.1995.tb00354.x

Jones, A. M., Thomas, V., Bennett, M. H., Mansfield, J., and Grant, M. (2006). Modifications to the Arabidopsis defense proteome occur prior to significant transcriptional change in response to inoculation with Pseudomonas syringae. Plant Physiol. 142, 1603-1620. doi: 10.1104/pp.106.086231

Kaplan, F., Kopka, J., Sung, D. Y., Zhao, W., Popp, M., Porat, R., et al. (2007). Transcript and metabolite profiling during cold acclimation of Arabidopsis reveals an intricate relationship of cold-regulated gene expression with modifications in metabolite content. Plant J. 50, 967-981. doi: 10.1111/j.1365313X.2007.03100.x

Kavi Kishor, P. B., and Sreenivasulu, N. (2014). Is proline accumulation per se correlated with stress tolerance or is proline homeostasis a more critical issue? Plant Cell Environ. 37, 300-311. doi: 10.1111/pce.12157
Kiyosue, T., Yoshiba, Y., Yamaguchi-Shinozaki, K., and Shinozaki, K. (1996). A nuclear gene encoding mitochondrial proline dehydrogenase, an enzyme involved in proline metabolism, is upregulated by proline but downregulated by dehydration in Arabidopsis. Plant Cell 8, 1323-1335. doi: 10.1105/tpc.8. 8.1323

Lehmann, S., Funck, D., Szabados, L., and Rentsch, D. (2010). Proline metabolism and transport in plant development. Amino Acids 39, 949-962. doi: $10.1007 /$ s00726-010-0525-3

Liang, X., Zhang, L., Natarajan, S. K., and Becker, D. F. (2013). Proline mechanisms of stress survival. Antioxid. Redox Signal. 19, 998-1011. doi: 10.1089/ars.2012.5074

Lv, W. T., Lin, B., Zhang, M., and Hua, X. J. (2011). Proline accumulation is inhibitory to Arabidopsis seedlings during heat stress. Plant Physiol. 156, 1921-1933. doi: 10.1104/pp.111.175810

Mani, S., Van De Cotte, B., Van Montagu, M., and Verbruggen, N. (2002). Altered levels of proline dehydrogenase cause hypersensitivity to proline and its analogs in Arabidopsis. Plant Physiol. 128, 73-83. doi: 10.1104/pp. 010572

Miller, G., Honig, A., Stein, H., Suzuki, N., Mittler, R., and Zilberstein, A. (2009). Unraveling $\Delta$ 1-pyrroline-5-carboxylate-proline cycle in plants by uncoupled expression of proline oxidation enzymes. J. Biol. Chem. 284, 26482-26492. doi: 10.1074/jbc.M109.009340

Monteoliva, M. I., Rizzi, Y. S., Cecchini, N. M., Hajirezaei, M. R., and Alvarez M. E. (2014). Context of action of proline dehydrogenase (ProDH) in the Hypersensitive Response of Arabidopsis. BMC Plant Biol. 14:21. doi: 10.1186/1471-2229-14-21

Nakashima, K., Satoh, R., Kiyosue, T., Yamaguchi-Shinozaki, K., and Shinozaki, K. (1998). A gene encoding proline dehydrogenase is not only induced by proline and hypoosmolarity, but is also developmentally regulated in the reproductive organs of Arabidopsis. Plant Physiol. 118, 1233-1241. doi: 10.1104/pp.118.4.1233

Nanjo, T., Fujita, M., Seki, M., Kato, T., Tabata, S., and Shinozaki, K. (2003). Toxicity of free proline revealed in an arabidopsis T-DNA-tagged mutant deficient in proline dehydrogenase. Plant Cell Physiol. 44, 541-548. doi: $10.1093 / \mathrm{pcp} / \mathrm{pcg} 066$

Nanjo, T., Kobayashi, M., Yoshiba, Y., Kakubari, Y., Yamaguchi-Shinozaki, K., and Shinozaki, K. (1999). Antisense suppression of proline degradation improves tolerance to freezing and salinity in Arabidopsis thaliana. FEBS Lett. 461, 205-210. doi: 10.1016/S0014-5793(99)01451-9

Pavet, V., Olmos, E., Kiddle, G., Mowla, S., Kumar, S., Antoniw, J., et al. (2005). Ascorbic acid deficiency activates cell death and disease resistance responses in Arabidopsis. Plant Physiol. 139, 1291-1303. doi: 10.1104/pp.105. 067686

Roosens, N., Bitar, F., Loenders, K., Angenon, G., and Jacobs, M. (2002). Overexpression of ornithine- $\delta$-aminotransferase increases proline biosynthesis and confers osmotolerance in transgenic plants. Mol. Breed. 9, 73-80. doi: 10.1023/A:1026791932238

Satoh, R., Nakashima, K., Seki, M., Shinozaki, K., and Yamaguchi-Shinozaki, K. (2002). ACTCAT, a novel cis-acting element for proline- and hypoosmolarityresponsive expression of the $\mathrm{ProDH}$ gene encoding proline dehydrogenase in Arabidopsis. Plant Physiol. 130, 709-719. doi: 10.1104/pp.009993

Savoure, A., Jaoua, S., Hua, X. J., Ardiles, W., Van Montagu, M., and Verbruggen, N. (1995). Isolation, characterization, and chromosomal location of a gene encoding the $\Delta 1$-pyrroline-5-carboxylate synthetase in Arabidopsis thaliana. FEBS Lett. 372, 13-19. doi: 10.1016/0014-5793(95)00935-3

Schertl, P., Cabassa, C., Saadallah, K., Bordenave, M., Savouré, A., and Braun, H.-P. (2014). Biochemical characterization of proline dehydrogenase in Arabidopsis mitochondria. FEBS J. 281, 2794-2804. doi: 10.1111/febs.12821

Sekhar, P. N., Amrutha, R. N., Sangam, S., Verma, D. P., and Kishor, P. B. (2007). Biochemical characterization, homology modeling and docking studies of ornithine $\delta$-aminotransferase- an important enzyme in proline biosynthesis of plants. J. Mol. Graph. Model. 26, 709-719. doi: 10.1016/j.jmgm.2007. 04.006

Senthil-Kumar, M., and Mysore, K. S. (2012). Ornithine- $\delta$-aminotransferase and proline dehydrogenase genes play a role in non-host disease resistance by regulating pyrroline-5-carboxylate metabolism-induced hypersensitive response. Plant Cell Environ. 35, 1329-1343. doi: 10.1111/j.1365-3040.2012.02492.x 
Sharma, S., and Verslues, P. E. (2010). Mechanisms independent of abscisic acid (ABA) or proline feedback have a predominant role in transcriptional regulation of proline metabolism during low water potential and stress recovery. Plant Cell Environ. 33, 1838-1851. doi: 10.1111/j.1365-3040.2010.02188.x

Sharma, S., Villamor, J. G., and Verslues, P. E. (2011). Essential role of tissuespecific proline synthesis and catabolism in growth and redox balance at low water potential. Plant Physiol. 157, 292-304. doi: 10.1104/pp.111.183210

Verslues, P. E., and Sharma, S. (2010). Proline metabolism and its implications for plant-environment interaction. Arabidopsis Book 8:e0140. doi: 10.1199/tab.0140
Conflict of Interest Statement: The authors declare that the research was conducted in the absence of any commercial or financial relationships that could be construed as a potential conflict of interest.

Copyright (c) 2015 Rizzi, Monteoliva, Fabro, Grosso, Laróvere and Alvarez. This is an open-access article distributed under the terms of the Creative Commons Attribution License (CC BY). The use, distribution or reproduction in other forums is permitted, provided the original author(s) or licensor are credited and that the original publication in this journal is cited, in accordance with accepted academic practice. No use, distribution or reproduction is permitted which does not comply with these terms. 


\title{
Role of proline and
}

\section{pyrroline-5-carboxylate metabolism in plant defense against invading pathogens}

\author{
Aarzoo Qamar', Kirankumar S. Mysore ${ }^{2}$ and Muthappa Senthil-Kumar ${ }^{1 *}$ \\ ${ }^{1}$ National Institute of Plant Genome Research, New Delhi, India, ${ }^{2}$ Plant Biology Division, The Samuel Roberts Noble \\ Foundation, Ardmore, OK, USA
}

OPEN ACCESS

Edited by:

Dietmar Funck,

University of Konstanz, Germany

Reviewed by:

Paul E. Vers/ues, Academia Sinica, Taiwan

Yasuhiro Ishiga,

University of Tsukuba, Japan

*Correspondence: Muthappa Senthil-Kumar, National Institute of Plant Genome Research, Aruna Asaf Ali Marg, P. O. Box 10531, New Delhi 110067, India skmuthappa@nipgr.ac.in

Specialty section: This article was submitted to Plant Physiology, a section of the journal Frontiers in Plant Science

Received: 29 April 2015

Accepted: 22 June 2015

Published: 06 July 2015

Citation:

Qamar A, Mysore KS and Senthil-Kumar M (2015) Role of proline and pyrroline-5-carboxylate metabolism in plant defense against

invading pathogens.

Front. Plant Sci. 6:503.

doi: 10.3389/fpls.2015.00503
Pyrroline-5-carboxylate (P5C) is an intermediate product of both proline biosynthesis and catabolism. Recent evidences indicate that proline-P5C metabolism is tightly regulated in plants, especially during pathogen infection and abiotic stress. However, role of P5C and its metabolism in plants has not yet been fully understood. Studies indicate that P5C synthesized in mitochondria has a role in both resistance $(R)$-gene-mediated and non-host resistance against invading pathogens. Proline dehydrogenase and delta-ornithine amino transferase-encoding genes, both involved in P5C synthesis in mitochondria are implicated in defense response of Nicotiana benthamiana and Arabidopsis thaliana against bacterial pathogens. Such defense response is proposed to involve salicylic acid-dependent pathway, reactive oxygen species (ROS) and hypersensitive response (HR)-associated cell death. Recently HR, a form of programmed cell death (PCD), has been proposed to be induced by changes in mitochondrial P5C synthesis or the increase in P5C levels per se in plants inoculated with either a host pathogen carrying suitable avirulent (Avr) gene or a non-host pathogen. Consistently, A. thaliana mutant plants deficient in P5C catabolism showed HR like cell death when grown in external P5C or proline supplemented medium. Similarly, yeast and plant cells under oxidative stress were shown to increase ROS production and PCD due to increase in P5C levels. Similar mechanism has also been reported as one of the triggers for apoptosis in mammalian cells. This review critically analyzes results from various studies and enumerates the pathways for regulation of P5C levels in the plant cell, especially in mitochondria, during pathogen infection. Further, mechanisms regulating P5C- mediated defense responses, namely $\mathrm{HR}$ are outlined. This review also provides new insights into the differential role of proline-P5C metabolism in plants exposed to pathogen infection.

Keywords: P5C, proline, ROS, oxidative burst, hypersensitive response, plant defense, non-host resistance 


\section{Introduction}

Plant defense against invading pathogen involves complex responses that culminate either in plant susceptibility or resistance. Virulent pathogen colonizes on host plant by surpassing the plant resistance mechanism. Most virulent pathogens have capability to manipulate host gene expression patterns to directly benefit their fitness and cause susceptibility of host plant. However, avirulent pathogen infection provokes $R$-gene mediated resistance owing to recognition of avirulent factor by resistance protein (R-protein). This recognition leads to hypersensitive response (HR)-cell death or other defense responses. Further, a form of defense called non-host resistance is responsible for conferring immunity to a plant species against all races of a potential pathogen (Senthil-Kumar and Mysore, 2013). Non-host resistance is classified into type I and type II resistance (Mysore and Ryu, 2004). Type I resistance does not produce any visible symptom but type II resistance shows HR- cell death as shown in $R$-gene mediated resistance (SenthilKumar and Mysore, 2013; see Supplementary Table S1 for terminologies).

These plant defense responses can be systematically categorized in two tiers (Jones and Dangl, 2006) namely, pathogen-associated molecular patterns (PAMPs) triggered immunity (PTI) and effector triggered immunity (ETI). The first tier of immunity, PTI involves perception of pathogen due to PAMPs by plant receptors called pattern recognition receptors (PRRs; Bigeard et al., 2015). This recognition leads to defense response in plant. Some pathogens surpass this tier and are not recognized by plant. Such pathogens release effectors in to the cell to suppress plant defense and acquire nutrition. Then the plant executes second tier of immunity, ETI by which plant recognizes effectors of pathogen by some resistance proteins (R-protein) and prevent pathogen infection (Jones and Dangl, 2006; see Supplementary Table S1 for terminologies). In the past a number of studies had been carried out to unravel the complex resistance mechanisms and susceptibility contributing factors in plants. Recent studies proposed a new line of thinking to explain a part of defense response through proline and 1-pyrroline-5-carboxylic acid (P5C) metabolism.

Proline is a multi-functional imino acid which confers tolerance to plants against abiotic stresses (Hare and Cress, 1997; Lehmann et al., 2010; Szabados and Savoure, 2010) and has been correlated to plant defense against pathogens (Fabro et al., 2004; Cecchini et al., 2011; Senthil-Kumar and Mysore, 2012). Plant accumulates proline by increasing its synthesis and reducing catabolism under abiotic stresses (Kishor et al., 2005; Verbruggen and Hermans, 2008; Supplementary File S1). Proline content was also increased during plant defense against pathogen in Arabidopsis thaliana (Fabro et al., 2004; Verslues and Sharma, 2010). However, proline per se has not been shown to play a role in defense against pathogen infection. Recent studies have shown that proline catabolism is enhanced during early stages of plant defense against invading pathogens (Cecchini et al., 2011). Based on the evidences from recent studies (Hellmann et al., 2000; Hu et al., 2007; Nishimura et al., 2012; Lee et al., 2013), we speculate that $\mathrm{P} 5 \mathrm{C}$, an intermediate imino acid in proline metabolism, plays important role in plant defense. So far, the role of $\mathrm{P} 5 \mathrm{C}$ in plant defense against pathogens is not compiled and discussed in the literature. This review focuses on the role of $\mathrm{P} 5 \mathrm{C}$ and its metabolism in plant-pathogen relations and attempts to infuse new thoughts in attributing relevance of P5C metabolism in plants under pathogen infection.

\section{P5C and Its Metabolism}

P5C, an N-substituted imino acid containing imino and carboxyl functional groups (IUPAC, 1997), is an intermediate not only in proline biosynthesis but also in its catabolism (Figure 1; Supplementary Table S2). P5C is synthesized from glutamate by pyrroline-5-carboxylate synthase (P5CS; Hu et al., 1992) and then converted to proline by pyrroline-5-carboxylate reductase (P5CR; Szoke et al., 1992; Hare and Cress, 1997) in cytosol and plastids. Proline is transported into mitochondria by membrane located transporters for its catabolism. Proline dehydrogenase (ProDH) catalyzes conversion of proline to $\mathrm{P} 5 \mathrm{C}$, which is then converted to glutamate by pyrroline-5-carboxylate dehydrogenase (P5CDH) in mitochondria (Elthon and Stewart, 1981; Hare and Cress, 1997). In addition to proline catabolism by ProDH (Boggess et al., 1978; Kiyosue et al., 1996), catabolism of arginine to ornithine by arginase (ARG; Goldraij and Polacco, 2000) and later transamination of ornithine by delta-ornithine amino transferase ( $\delta$ OAT) also synthesizes P5C (Delauney et al., 1993; Roosens et al., 1998; Sekhar et al., 2007; Funck et al., 2008; Stránská et al, 2008; Supplementary Table S2; Figure 1). P5C remains in rapid equilibrium with glutamate semi-aldehyde (GSA; Vogel and Davis, 1952). This equilibrium is $\mathrm{pH}$ dependent and $\mathrm{P} 5 \mathrm{C}$ form is favored over GSA at physiological pH of around 7.0 (Lewis et al., 1993; Bearne and Wolfenden, 1995).

\section{Mitochondrial ROS Accumulation and Cell Death}

In plant cells, mitochondria is one of the major sites for the production of reactive oxygen species (ROS) namely superoxide radicals and hydroxyl radicals (Lam et al., 2001; Moller, 2001; Bailey-Serres and Mittler, 2006; Sharma et al., 2012). Electrons are derived from metabolic reducing equivalents [NAD (P) $\mathrm{H}_{2}$ and FAD $(\mathrm{P}) \mathrm{H}_{2}$ ] and fed into mitochondrial electron transport chain (mETC) through complex I (NADH-ubiquinone oxidoreductase) and/or II (succinate-coenzyme Q reductase) and later transferred to oxygen via terminal oxidase to produce water (Moller, 2001; Arora et al., 2002; Turrens, 2003; Murphy, 2009; Schertl and Braun, 2014). But these carriers can also pass electron directly to oxygen to form superoxide which later produces other forms of ROS and this contributes to cell death (Fredlund, 1996; Lam et al., 2001). A localized cell death known as HR that prevents progression of pathogen infection is an important component of plant defense (Alvarez et al., 1998; Lam et al., 2001). Proline catabolism pathway mediated by ProDH enzyme in mitochondria is one of the sources of mitochondrial ROS during avirulent pathogen infection in A. thaliana (Cecchini 


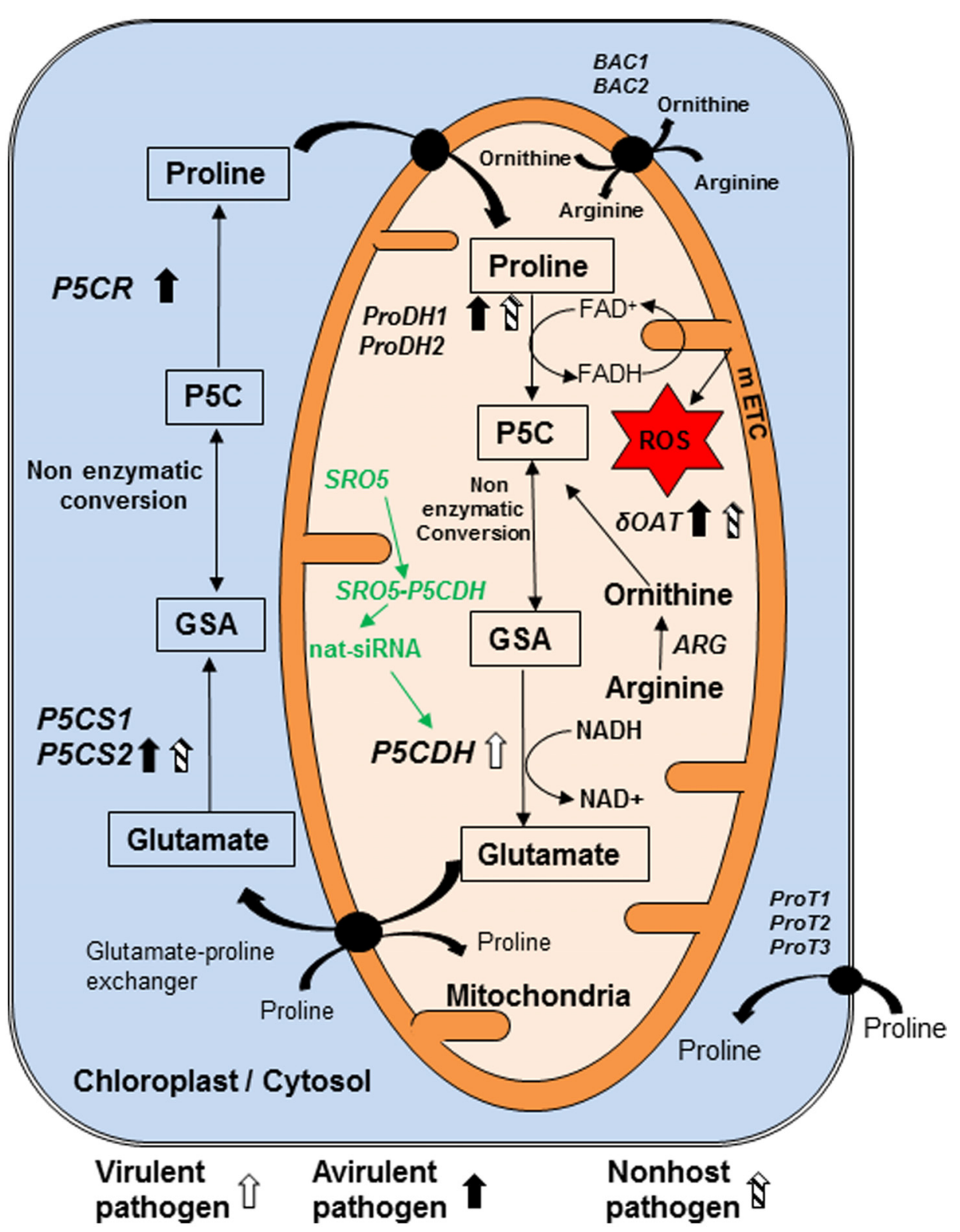

FIGURE 1| Model showing genes and pathways possibly involved in synthesis and catabolism of P5C in plant cell and their regulation in response to pathogen infection. Pyrroline 5-carboxylate (P5C) is the intermediate product of both biosynthesis and catabolism of proline. It is synthesized in mitochondria during catabolism of proline by enzyme proline dehydrogenase (ProDH1/2). We speculate that like their counterparts from bacteria and yeast, this enzyme reduces FAD+ to FADH and increases electron flow in mitochondrial electron transport chain (mETC). Arginine is converted into ornithine by arginase (ARG) enzyme. Another enzyme delta-ornithine amino transferase (SOAT) convert ornithine to P5C in mitochondria. P5C is catabolized by pyrroline 5-carboxylate dehydrogenase $(\mathrm{P} 5 \mathrm{CDH})$ in mitochondria into glutamate. In addition, P5C is synthesized in cytosol and chloroplast, from glutamate by pyrroline 5-carboxylate synthase 1 and 2 (P5CS1, P5CS2) and converted to proline by pyrroline 5-carboxylate reductase (P5CR). P5C and glutamate semi aldehyde (GSA) are non-enzymatically inter-convertible forms. Virulent pathogen infection in plants increases transcript accumulation of ProDH1. Avirulent pathogen infection increases transcript accumulation of P5CS2 and ProDH1. Non-host pathogen infection increases transcript accumulation of $\delta O A T$ as well as PrODH1 and P5CS2. Three transporters located on plasma membrane are ProT1, ProT2 and ProT3. Arginine is imported into mitochondria directly and/or in exchange of ornithine by basic amino acid career (BAC1) and BAC2. $P 5 C D H$ gene is down regulated post transcriptionally by natural siRNAs from similar to RCD one-5 (SRO5) genes. No direct evidence available for this pathway shown in green color during plant-pathogen interaction and this information is speculated based on evidence available under salt stress. Compounds shown in rectangle are important component of metabolism of P5C and line arrows indicates the direction of synthesis. Curved arrows show the transport of compounds. Transporters shown in circle are present on membrane. Block thick arrows show upregulation of genes, in which white arrow indicates virulent pathogen, dark arrows represent avirulent pathogen and striped arrows indicate non-host pathogen. ROS, reactive oxygen species. This model is predominantly based on information from Arabidopsis thaliana literature (Ayliffe et al., 2002; Borsani et al., 2005; Miller et al., 2009; Verslues and Sharma, 2010; Cecchini et al., 2011; Senthil-Kumar and Mysore, 2012). 
et al., 2011) and the ROS produced at the infection site leads to oxidative burst thereby initiates HR (Lamb and Dixon, 1997).

There are two schools of thoughts regarding ROS production by $\mathrm{P} 5 \mathrm{C}$ metabolism in mitochondria. First one emphasizes the involvement of proline-P5C cycle (Miller et al., 2009) and the extended pathway involving proline-P5C-glutamate in ROS generation. According to proline-P5C cycle, $\mathrm{P} 5 \mathrm{C}$ synthesized due to proline catabolism in mitochondria is likely to be transported through an unknown transporter into the cytosol where P5CR enzyme converts it into proline. The proline thus formed is transported back to mitochondria where it consistently acts as a substrate for ProDH enzyme (Miller et al., 2009). In some cases, pathogen infection increased the ProDH transcript level and enhanced proline catabolism triggered proline-P5C-proline cycle between mitochondria and cytosol (Cecchini et al., 2011; Monteoliva et al., 2014). This cycle can increase the transfer of reducing equivalents to mitochondria and alter $\mathrm{NADP}^{+} / \mathrm{NADPH}$ ratio in the cytosol (Rejeb et al., 2014). This condition potentially impacts the redox sensitive pathways such as defense-associated oxidative pentose phosphate pathway and eventually produces ROS (Hare and Cress, 1997). ProDH is located in inner membrane of mitochondria toward matrix where it oxidizes proline into $\mathrm{P} 5 \mathrm{C}$ and increases electron flow leading to the production of another batch of ROS (Tanner, 2008; Wanduragala et al., 2010; Cecchini et al., 2011; Supplementary Figure S2). In Trypanosoma cruzi parasite, TcProDH mutation resulted in resistance to oxidative imbalances and ProDH enzyme activity was implicated in ROS production in this study (Paes et al., 2013). However, occurrence of proline-P5C cycle is not conclusively proven. Presence of a $\mathrm{P} 5 \mathrm{C}$ transporter at the mitochondrial membrane has only been suggested (Miller et al., 2009) but not yet identified. Similarly the role of genes encoding the mitochondrial transporters that are needed for proline cycle are not well studied. Apart from proline-P5C cycle described above, cyclic metabolisms of proline also occur by other pathways. For example, the extended pathway involving glutamate formation in mitochondria and its cycling back to cytosol for proline synthesis can also be a source of ROS production. However, till date this pathway has not been studied under plant-pathogen interaction.

The second school of thought suggests the role for $\mathrm{P} 5 \mathrm{C}$ per se in ROS production. Exogenous application of P5C led to apoptosis in human tumor cells through oxidative burst (Maxwell and Davis, 2000). In yeast, overexpression of proline utilization (PUT1) gene, which mediates the conversion of proline to $\mathrm{P} 5 \mathrm{C}$, induced cell death (Chen et al., 2006). The put2 (PUT2 gene mediates the conversion of $\mathrm{P} 5 \mathrm{C}$ to glutamate) mutant grown in proline supplemented minimal medium inhibited yeast growth due to higher ROS production (Deuschle et al., 2001). Similarly, put2 mutant of a fungal pathogen (Cryptococcus neoformans) generated high amount of mitochondrial superoxide radicals and showed cell death in proline supplemented medium (Lee et al., 2013). In addition, overexpression of ProDH induced P5C dependent mitochondria-mediated apoptosis in colorectal cancer cells ( $\mathrm{Hu}$ et al., 2007). Proline-induced cell death in yeast was also shown to be due to increased P5C levels in mitochondria (Nomura and Takagi, 2004). Nishimura et al.
(2012) reported that accumulation of P5C is responsible for mitochondrial ROS production and hence cell death in yeast. Similarly, A. thaliana $p 5 c d h$ mutant, which cannot catabolize P5C, and ProDH overexpression lines accumulated P5C in mitochondria and were hypersensitive to exogenous application of proline, ornithine, and arginine (Deuschle et al., 2004). The enhanced catabolism of proline due to increased expression of AtProDH in the $p 5 c d h$ mutant plants led to P5C accumulation with concomitant cell death (Deuschle et al., 2004). Also, AtP5CDH overexpressed plants did not show cell death but wildtype $A$. thaliana plants grown on MS medium supplemented with P5C showed hypersensitivity (Miller et al., 2009). These evidences suggest the role for $\mathrm{P} 5 \mathrm{C}$ per se in ROS generation and eventually cell death (Supplementary Figure S2). P5C spray on wild-type $A$. thaliana leaves resulted in HR like cell death with concomitant callose accumulation (Deuschle et al., 2004). Similarly, proline hypersensitivity in wild-type A. thaliana leaves manifested as necrosis and browning symptoms were attributed to increased cellular P5C (Hellmann et al., 2000). Taken together, these studies indicate that proline-P5C cycle and/or P5C per se can contribute to HR to confine pathogen growth in plants.

\section{Role of P5C Metabolism During Plant-Avirulent Pathogen Interactions}

HR occurs during interaction of plant with either a host pathogen carrying avirulent (Avr) gene, for which plant contains corresponding $R$ gene, or a non-host pathogen (Ausubel et al., 1995; Boyes et al., 1998; Mysore and Ryu, 2004; Senthil-Kumar and Mysore, 2013). Role of P5C metabolism under both type of resistance are discussed in this section. Pseudomonas syringae pv tomato DC3000 (causal agent of bacterial spec disease in tomato) carrying AvrRpm1 or AvrRpt2 gene produces AvrRPM1 or AvrRPT2 effector protein, respectively. These are recognized by resistance to $P$. syringae maculicola 1 (RPM1) or resistance to P. syringae 2 (RPS2) protein, respectively, in host plant through RPM interacting protein (RIN4) (Mackey et al., 2002; Axtell and Staskawicz, 2003). This recognition leads to changes in prolineP5C metabolism (Cecchini et al., 2011; Monteoliva et al., 2014) and up regulation of various defense genes and ROS production culminating in HR-cell death (Torres et al., 2006). External proline application, that can provoke $\mathrm{P} 5 \mathrm{C}$ accumulation induces pathogenesis related $1(P R 1)$ gene expression in $A$. thaliana (Chen et al., 2011). These recent studies highlight the role of P5C and its metabolism in inducing defense responses, including HR during plant-pathogen interactions.

Fabro et al. (2004) reported the accumulation of AtP5CS2 transcripts and increased proline content prior to $\mathrm{HR}$ in A. thaliana plants infected with avirulent pathogens ( $P$. syringae pv tomato DC3000 AvrRpt2 and P. syringae pv tomato DC3000 AvrRpm1). Cecchini et al. (2011) reported upregulation of AtProDH1 and AtProDH2 genes in A. thaliana at the inoculation site of avirulent pathogen ( $P$. syringae pv tomato DC3000 AvrRpm1). In addition, avirulent pathogen inoculation led to increased ProDH protein content and enzyme activity and was 
suggested to be responsible for oxidative burst and HR (Cecchini et al., 2011). Consistently, AtProDH silenced A. thaliana plants inoculated with avirulent pathogen compromised ROS production (Cecchini et al., 2011). AtProDH gene transcript expression was modulated by exogenous application of salicylic acid (SA, Cecchini et al., 2011). Authors in this study proposed that SA-mediated signaling plays a role in the induction of AtProDH1, but not AtProDH2, expression likely through nonexpressor of $P R$ genes 1 (NPR1) and SA induction-deficient 2 (SID2) during early stages of avirulent pathogen infection in A. thaliana. Proline was shown to induce SA via non-responsive to disease resistance 1 (NDR1)-dependent pathway (Vlot et al., 2009; Chen et al., 2011). In green bean (Phaseolus vulgaris), Rhizoctonia solani (causal agent of seed rot and damping off in bean) infection induced proline accumulation in SA-dependent manner (Ayoubi and Soleimani, 2014). Taken together, these studies implicate a role for AtP5CS2 and AtProDH1 genes in regulating the proline and $\mathrm{P} 5 \mathrm{C}$ under Avr- $\mathrm{R}$ interaction. The defense responses involving these genes are likely part of ETI and involve SA.

Further, AtP5CDH gene transcription was downregulated in $A$. thaliana upon inoculation with $P$. syringae pv. tomato DC3000 AvrRpm1. Consistently in $p 5 c d h$ mutant of A. thaliana, slight increase in $\mathrm{P} 5 \mathrm{C}$ level was observed in the $\mathrm{HR}$ region upon inoculation by P. syringae pv. tomato DC3000 AvrRpm1 (Monteoliva et al., 2014). This scenario facilitate decrease in degradation of $\mathrm{P} 5 \mathrm{C}$ and hence results in high accumulation of P5C (Supplementary Figures S1 and S2). Notably, slight increase in concentration of $\mathrm{P} 5 \mathrm{C}$ due to induction of $\mathrm{P} 5 \mathrm{C}$ biosynthesis by avirulent pathogen inoculation had been shown to provoke HR (Hellmann et al., 2000; Nishimura et al., 2012). It is likely that $A t P 5 C D H$-mediated P5C regulation is also a part of ETI.

Similar to avirulent pathogens, non-host pathogen infection also involves $\mathrm{HR}$ and other defenses likely mediated by P5C metabolism (Senthil-Kumar and Mysore, 2012, 2013). Recently, virus-induced gene silencing (VIGS)-based forward genetics screen identified the role for ProDH1 and $\delta O A T$ in nonhost resistance (Senthil-Kumar and Mysore, 2012). Silencing of NbSOAT and NbProDH1 genes compromised non-host resistance against $P$. syringae pv. tomato $\mathrm{T} 1$ in Nicotiana benthamiana. Further, A. thaliana $\delta$ oat and $p 5 c d h$ mutant plants lacked oxidative burst and compromised non-host resistance against P. syringae pv. tabaci (Senthil-Kumar and Mysore, 2012). These mutant plants grown in Murashige and Skoog (MS) medium externally supplemented with P5C showed higher ROS content as compared to those grown in non-P5C medium. Taken together, this study suggested the possible involvement of P5C synthesis step in non-host resistance, possibly as a part of both PTI and ETI.

\section{Role of P5C Metabolism During Plant-Virulent Pathogen Interaction}

Flax (Linum usitatissimum) plant infected with the Melampsora lini (causal agent of flax rust), an obligate biotrophic fungal pathogen, induced the FIS1 (flax inducible sequence 1) gene at the pathogen infection site (Ayliffe et al., 2002). Interestingly, FIS1 gene showed 73\% nucleotide homology with the A. thaliana AtP5CDH gene (Mitchell et al., 2006). Similarly, $N$. benthamiana plants infected with virulent pathogen (P. syringae pv. tabaci) showed upregulation of $\mathrm{NbP5CDH}$ gene expression (Senthil-Kumar and Mysore, 2012). It is possible that virulent pathogens enhance the catabolism of $\mathrm{P} 5 \mathrm{C}$ by upregulating AtP5CDH-mediated step (Figure 2; Supplementary Figure $\mathrm{S} 1$ ). In $A$. thaliana, infection by virulent pathogen (P. syringae pv. tomato DC3000) did not lead to upregulation of ProDH gene (Cecchini et al., 2011). Similarly, N. benthanmiana plants also did not show upregulation of $\mathrm{NbProDH} 1$ and $\mathrm{NbProDH} 2$ gene expression at $P$. syringae pv. tabaci infection site (Senthil-Kumar and Mysore, 2012). This indicates that plants attempt to maintain proline levels in the cells undergoing disease induced cell death. This is consistent with previous studies that indicated the role of proline in reducing cell death in yeast (Chen et al., 2006), fungus (Chen and Dickman, 2005), and plants (Banu et al., 2009). Proline provides protection against $\mathrm{H}_{2} \mathrm{O}_{2}$-induced cell death and apoptosis in mammalian cell culture (Krishnan et al., 2008). Taken together, these studies demonstrated that proline, not $\mathrm{P} 5 \mathrm{C}$, is likely to play a role in regulating diseaseinduced cell death (Figure 2). In addition to bacterial and fungal pathogens, virus infection was shown to influence proline-P5C metabolism. For example, rice plants infected with Brome mosaic virus (BMV) or Rice tungro virus led to proline accumulation (Mohanty and Sridhar, 1982; Xu et al., 2008). However, the positive role of proline in cell death regulation is not examined in these studies.

\section{Coordinated Regulation of Proline-P5C Metabolism During Plant Defense}

High proline accumulation in non-stressed plants has been shown to negatively impact plant growth and also cause cell death (Hellmann et al., 2000; Chen et al., 2011). However, when these plants are subjected to stress the toxicity due to proline accumulation is reduced. The proline content increases upon pathogen infection (Fabro et al., 2004), but exogenous proline application to non-stressed plants cause increase in its catabolism (Verslues and Sharma, 2010). Proline itself at low concentrations is known to reduce the cell death, but $\mathrm{P} 5 \mathrm{C}$ metabolism provokes cell death (Deuschle et al., 2004; Banu et al., 2009). These evidences indicate the complex but finetuned regulation of proline-P5C metabolism during pathogen infection (Figure 2). It is possible that in virulent pathogen infected cells, proline acts as a positive regulator of cell death as part of basal immune response to decrease the severity of disease development. It is also possible that the un-infected cells surrounding the HR developing cells upon inoculation with avirulent pathogen increase proline levels to prevent runaway cell death (Senthil-Kumar and Mysore, 2012). In contrary, either proline-P5C cycle or P5C accumulation per se triggers the defense cell death (HR) upon avirulent pathogen infection. In order to bring a fine-tuned regulation of proline-P5C pathway 


\section{Compatible interaction}

\section{Incompatible interaction}

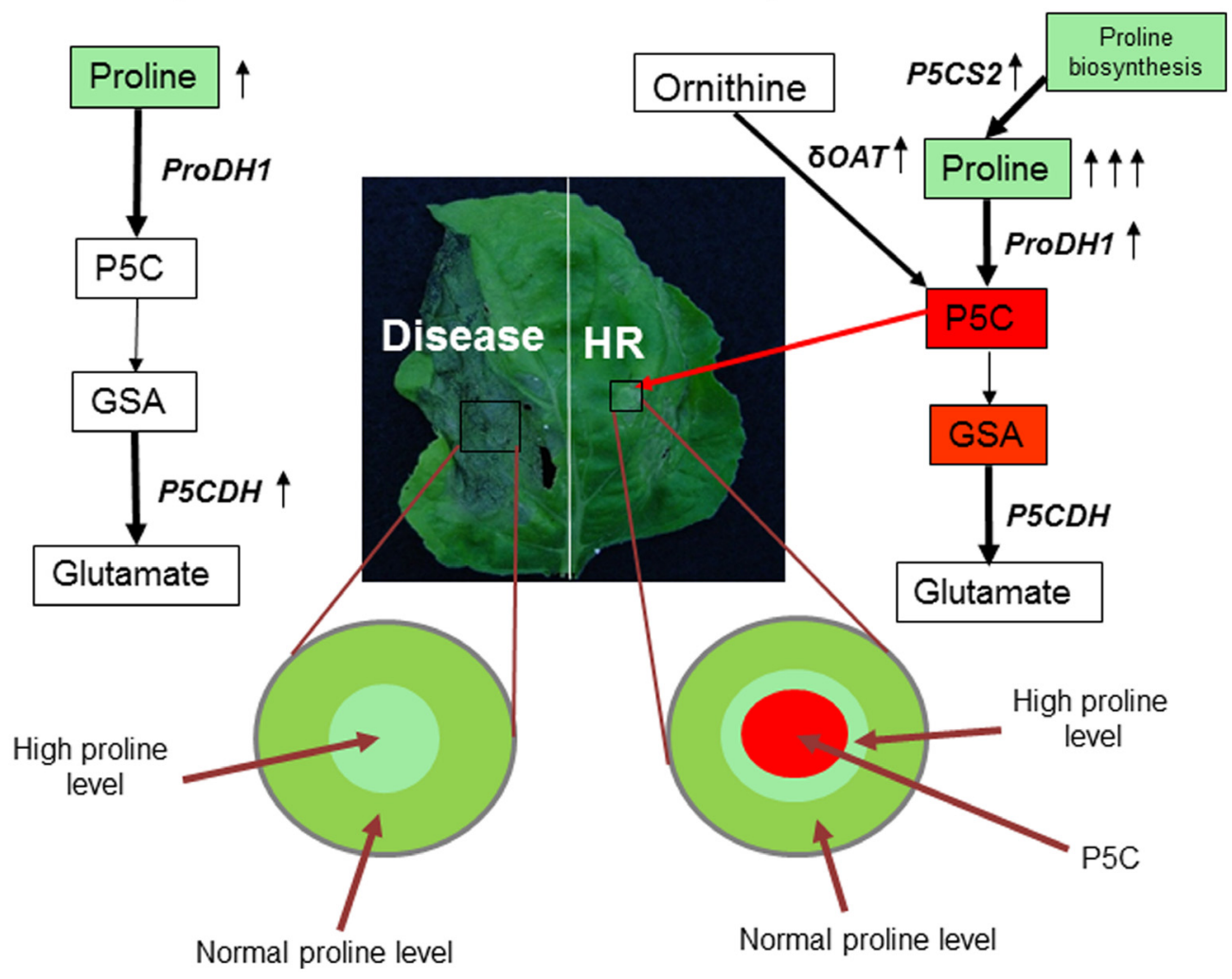

FIGURE 2 | Model for coordinated regulation of proline-P5C metabolism under pathogen infection. Virulent pathogen (example as shown here for Pseudomonas syringae pv. tabaci in Nicotiana benthamiana) infection leads to disease on host plant (left). However, non-host (example as shown here for $P$. syringae pv. tomato $\mathrm{T} 1$ in $N$. benthamiana) [and avirulent, not shown in the picture] pathogen infection on plant leads to defense reaction, including HR at infection site (right). Pathways leading to P5C biosynthesis and catabolism in mitochondria are highlighted. Plants increase P5C content or ProDH-mediated step and produce ROS to defend against pathogen by upregulating ProDH and $\delta O A T$ gene transcription. In addition, mild increase in proline in the tissues surrounding the HR cell death prevents runaway HR to non-infected cells. However, virulent pathogens decrease P5C levels in mitochondria by upregulating P5CDH-mediated step. Also proline content moderately increases in the infected cells possibly to decrease the rate of disease progression as a part of basal defense response. Proline is known to prevent or delay the cell death. During this regulation, proline synthesis can also play a role in controlling proline levels (shown under resistant reaction). Red rectangle box depicts pro cell death compound; green rectangle depicts compound that delays cell death; arrow depicts upregulation of transcripts. the plant must regulate the genes involved in defense through a systematic network. We speculate that this network may involve a tight regulation at the promoters. Yet another possibility of post transcriptional regulation could involve micro RNAs (miRNAs). In potato, miRNAs targeting proline metabolism genes were predicted and their role in regulation was verified (Yang et al., 2013). We speculate that proline metabolism genes can be regulated by microRNAs under pathogen attack as well.

Additionally, expression of proline metabolism genes is differentially regulated by drought, salinity, and abscisic acid suggesting that these genes play a specific role in the control of proline synthesis (Supplementary File S1). This indicates the coordinated regulation of proline-P5C metabolism under abiotic stress as well. Considering literature information available on their role under abiotic stresses, we comprehensively illustrated this information together with the regulation under plant-pathogen interaction. Both proline and P5C content under various abiotic stresses changes and regulation of genes involved in different steps of proline catabolism can be correlated to their levels under pathogen stress.

\section{Concluding Remarks}

P5C plays a role in HR against invading pathogen and its levels or metabolism could possibly be manipulated by virulent pathogen. To know the importance of its metabolism in plant defense, it is necessary to understand the regulation of genes involved in its pathway at transcriptional and post transcriptional level. Bioinformatic analysis and experimental validation of promoter region of all proline-P5C metabolism genes will be useful for understanding the regulatory components. In addition, studies to understand post transcriptional modifications, 
cofactor binding, protein interactors and activity of all proteins involved in the pathway during plant-pathogen interaction are required.

It is still not clear whether the changes in proline-P5C metabolism are primarily regulated by ETI or PTI or both. Systematic studies that will exclusively use PAMPs, effectors and specific triggers for innate immunity is needed to delineate the defense pathway that provoke changes in P5C metabolism. In addition to this, the downstream defense pathway activated by $\mathrm{P} 5 \mathrm{C}$ that culminates in $\mathrm{HR}$ or other defense responses is also not well understood. Studies using externally supplied purified P5C and genetically manipulated plants with altered P5C levels under a specific defense inducer can provide useful information. Non-host plant-pathogen interaction can be used as model to study both defense involving $\mathrm{HR}$ and others. This is because both type-I (HR not involved) and type-II (involving HR) defenses can be tested using the same plant species.

More studies are needed to understand whether P5C content in mitochondria, suppression of its catabolism or its transport plays a role in plant defense. The contribution of cytosolic P5C in pathogen induced plant defense is also not yet studied. In order to understand whether the level of P5C plays any role in triggering $\mathrm{HR}$, it is necessary to accurately estimate the P5C content. However, currently available methods do not provide organelle specific accurate estimation of this compound. For

\section{References}

Alvarez, M. E., Pennell, R. I., Meijer, P. J., Ishikawa, A., Dixon, R. A., and Lamb, C. (1998). Reactive oxygen intermediates mediate a systemic signal network in the establishment of plant immunity. Cell 92, 773-784. doi: 10.1016/S00928674(00)81405-1

Arora, A., Sairam, R. K., and Srivastava, G. C. (2002). Oxidative stress and antioxidative system in plants. Curr. Sci. 82, 1227-1238.

Ausubel, F. M., Katagiri, F., Mindrinos, M., and Glazebrook, J. (1995). Use of Arabidopsis thaliana defense-related mutants to dissect the plant response to pathogens. Proc. Natl. Acad. Sci. U.S.A. 92, 4189-4196. doi: 10.1073/pnas.92.10.4189

Axtell, M. J., and Staskawicz, B. J. (2003). Initiation of RPS2-specified disease resistance in Arabidopsis is coupled to the AvrRpt2-directed elimination of RIN4. Cell 112, 369-377. doi: 10.1016/S0092-8674(03) 00036-9

Ayliffe, M. A., Roberts, J. K., Mitchell, H. J., Zhang, R., Lawrence, G. J., Ellis, J. G., et al. (2002). A plant gene up-regulated at rust infection sites. Plant Physiol. 129, 169-180. doi: 10.1104/pp.010940

Ayoubi, N., and Soleimani, M. J. (2014). Possible effects of pathogen inoculation and salicylic acid pre-treatment on the biochemical changes and proline accumulation in green bean. Arch. Phytopathol. Plant Prot. 48, 212-222. doi: $10.1080 / 03235408.2014 .884826$

Bailey-Serres, J., and Mittler, R. (2006). The roles of reactive oxygen species in plant cells. Plant Physiol. 141, 311. doi: 10.1104/pp.104.900191

Banu, M. N. A., Hoque, M. A., Watanabe-Sugimoto, M., Matsuoka, K., Nakamura, Y., Shimoishi, Y., et al. (2009). Proline and glycinebetaine induce antioxidant defense gene expression and suppress cell death in cultured tobacco cells under salt stress. J. Plant Physiol. 166, 146-156. doi: 10.1016/j.jplph.2008.03.002

Bearne, S. L., and Wolfenden, R. (1995). Glutamate gamma-semialdehyde as a natural transition state analogue inhibitor of Escherichia coli glucosamine6-phosphate synthase. Biochemistry 34, 11515-11520. doi: 10.1021/bi00 $036 \mathrm{a} 026$ example, colorimetric method of $\mathrm{P} 5 \mathrm{C}$ estimation by $\mathrm{O}$-aminobenzaldehyde assay is not sensitive enough to estimate P5C content in vivo. Hence, development of suitable P5C estimation method is important.

Since proline is well known to play a positive role during drought stress and also during stress recovery, it will be interesting to study the plant's interaction with virulent and avirulent pathogens under these two conditions. Further, studies on proline pathway gene regulation under the combined drought and pathogen infection will be useful.

\section{Acknowledgments}

This work was supported by the core funding to MS-K's lab from National Institute of Plant Genome Research, New Delhi. AQ thanks the University Grant Commission (UGC), New Delhi for a junior research fellowship award (Ref. No. 23/12/2012(ii) EUV). Authors also thank Mr. Mehanathan Muthamilarasan and Dr. Prachi Pandey for critical reading of the manuscript.

\section{Supplementary Material}

The Supplementary Material for this article can be found online at: http://journal.frontiersin.org/article/10.3389/fpls.2015.00503

Bigeard, J., Colcombet, J., and Hirt, H. (2015). Signaling mechanisms in pattern-triggered immunity (PTI). Mol. Plant 8, 521-539. doi: 10.1016/j.molp.2014.12.022

Boggess, S. F., Koeppe, D. E., and Stewart, C. R. (1978). Oxidation of proline by plant mitochondria. Plant Physiol. 62, 22-25. doi: 10.1104/pp.62.1.22

Borsani, O., Zhu, J., Verslues, P. E., Sunkar, R., and Zhu, J.-K. (2005). Endogenous siRNAs derived from a pair of natural cis-antisense transcripts regulate salt tolerance in Arabidopsis. Cell 123, 1279-1291. doi: 10.1016/j.cell.2005. 11.035

Boyes, D. C., Nam, J., and Dangl, J. L. (1998). The Arabidopsis thaliana RPM1 disease resistance gene product is a peripheral plasma membrane protein that is degraded coincident with the hypersensitive response. Proc. Natl. Acad. Sci. U.S.A. 95, 15849-15854. doi: 10.1073/pnas.95.26. 15849

Cecchini, N. M., Monteoliva, M. I., and Alvarez, M. E. (2011). Proline dehydrogenase contributes to pathogen defense in Arabidopsis. Plant Physiol. 155, 1947-1959. doi: 10.1104/pp.110.167163

Chen, C., and Dickman, M. B. (2005). Proline suppresses apoptosis in the fungal pathogen Colletotrichum trifolii. Proc. Natl. Acad. Sci. U.S.A. 102, 3459-3464. doi: 10.1073/pnas.0407960102

Chen, C., Wanduragala, S., Becker, D. F., and Dickman, M. B. (2006). Tomato QMlike protein protects Saccharomyces cerevisiae cells against oxidative stress by regulating intracellular proline levels. Appl. Environ. Microbiol. 72, 4001-4006. doi: 10.1128/AEM.02428-05

Chen, J., Zhang, Y., Wang, C., Lü, W., Jin, J. B., and Hua, X. (2011). Proline induces calcium-mediated oxidative burst and salicylic acid signaling. Amino Acids 40, 1473-1484. doi: 10.1007/s00726-010-0757-2

Delauney, A., Hu, C., Kishor, P. K., and Verma, D. (1993). Cloning of ornithine d-aminotransferase cDNA by trans-complementation in Escherichia coli and regulation of proline biosynthesis. J. Biol. Chem. 268, 1867318678.

Deuschle, K., Funck, D., Forlani, G., Stransky, H., Biehl, A., Leister, D., et al. (2004). The role of $\Delta 1$-pyrroline-5-carboxylate dehydrogenase in proline degradation. Plant Cell 16, 3413-3425. doi: 10.1105/tpc.104.023622 
Deuschle, K., Funck, D., Hellmann, H., Daschner, K., Binder, S., and Frommer, W. B. (2001). A nuclear gene encoding mitochondrial $\Delta$ 1-pyrroline-5carboxylate dehydrogenase and its potential role in protection from proline toxicity. Plant J. 27, 345-355. doi: 10.1046/j.1365-313X.2001.01101.x

Elthon, T. E., and Stewart, C. R. (1981). Submitochondrial location and electron transport characteristics of enzymes involved in proline oxidation. Plant Physiol. 67, 780-784. doi: 10.1104/pp.67.4.780

Fabro, G., Kovacs, I., Pavet, V., Szabados, L., and Alvarez, M. E. (2004). Proline accumulation and AtP5CS2 gene activation are induced by plant-pathogen incompatible interactions in Arabidopsis. Mol. Plant Microbe Interact. 17, 343-350. doi: 10.1094/MPMI.2004.17.4.343

Fredlund, K. M. (1996). NAD $(P) H$ Dehydrogenases in Plant Mitochondria. Ph.D. Thesis, Lund University, Lund.

Funck, D., Stadelhofer, B., and Koch, W. (2008). Ornithine- $\delta$-aminotransferase is essential for arginine catabolism but not for proline biosynthesis. BMC Plant Biol. 8:40. doi: 10.1186/1471-2229-8-40

Goldraij, A., and Polacco, J. C. (2000). Arginine degradation by arginine in mitochondria of soybean seedling cotyledons. Planta 4, 652-658. doi: 10.1007/s004250050056

Hare, P. D., and Cress, W. A. (1997). Metabolic implications of stressinduced proline accumulation in plants. Plant Growth Regul. 21, 79-102. doi: 10.1023/A:1005703923347

Hellmann, H., Funck, D., Rentsch, D., and Frommer, W. B. (2000). Hypersensitivity of an Arabidopsis sugar signaling mutant toward exogenous proline application. Plant Physiol. 123, 779-789. doi: 10.1104/pp.123.2.779

$\mathrm{Hu}$, C. A., Delauney, A. J., and Verma, D. P. (1992). A bifunctional enzyme ( $\Delta$ 1-pyrroline-5-carboxylate synthetase) catalyzes the first two steps in proline biosynthesis in plants. Proc. Natl. Acad. Sci. U.S.A. 89, 9354-9358. doi: 10.1073/pnas.89.19.9354

Hu, C. A. A., Donald, S. P., Yu, J., Lin, W. W., Liu, Z., Steel, G., et al. (2007). Overexpression of proline oxidase induces proline-dependent and mitochondria-mediated apoptosis. Mol. Cell. Biochem. 295, 85-92. doi: 10.1007/s11010-006-9276-6

IUPAC. (1997). “Compendium of Chemical Terminology," in The Gold Book, 2nd Edn, eds A. D. McNaught and A. Wilkinson (Oxford: Blackwell Scientific Publications).

Jones, J. D., and Dangl, J. L. (2006). The plant immune system. Nature 444, 323-329. doi: 10.1038/nature05286

Kishor, P. K., Sangam, S., Amrutha, R. N., Laxmi, P. S., Naidu, K. R., Rao, K. R. S. S., et al. (2005). Regulation of proline biosynthesis, degradation, uptake and transport in higher plants: its implications in plant growth and abiotic stress tolerance. Curr. Sci. 88, 424-438.

Kiyosue, T., Yoshiba, Y., Yamaguchi-Shinozaki, K., and Shinozaki, K. (1996). A nuclear gene encoding mitochondrial proline dehydrogenase, an enzyme involved in proline metabolism, is upregulated by proline but downregulated by dehydration in Arabidopsis. Plant Cell 8, 1323-1335. doi: 10.1105/tpc.8. 8.1323

Krishnan, N., Dickman, M. B., and Becker, D. F. (2008). Proline modulates the intracellular redox environment and protects mammalian cells against oxidative stress. Free Radic. Biol. Med. 44, 671-681. doi: 10.1016/j.freeradbiomed.2007.10.054

Lam, E., Kato, N., and Lawton, M. (2001). Programmed cell death, mitochondria and the plant hypersensitive response. Nature 411, 848-853. doi: $10.1038 / 35081184$

Lamb, C., and Dixon, R. A. (1997). The oxidative burst in plant disease resistance. Annu. Rev. Plant Physiol. Plant Mol. Biol. 48, 251-275. doi: 10.1146/annurev.arplant.48.1.251

Lee, I. R., Lui, E. Y., Chow, E. W., Arras, S. D., Morrow, C. A., and Fraser, J. A. (2013). Reactive oxygen species homeostasis and virulence of the fungal pathogen Cryptococcus neoformans requires an intact proline catabolism pathway. Genetics 194, 421-433. doi: 10.1534/genetics.113.150326

Lehmann, S., Funck, D., Szabados, L., and Rentsch, D. (2010). Proline metabolism and transport in plant development. Amino Acids 39, 949-962. doi: 10.1007/s00726-010-0525-3

Lewis, M. L., Rowe, C. J., Sewald, N., Sutherland, J. D., Wilson, E. J., and Wright, M. C. (1993). The effect of $\mathrm{pH}$ on the solution structure of delta-1-pyrroline-2carboxylic acid as revealed by NMR and electrospray mass spectroscopy. Bioorg. Med. Chem. Lett. 3, 1193-1196. doi: 10.1016/S0960-894X(00)80313-3
Mackey, D., Holt, B. F. III., Wiig, A., and Dangl, J. L. (2002). RIN4 interacts with Pseudomonas syringae type III effector molecules and is required for RPM1mediated resistance in Arabidopsis. Cell 108, 743-754. doi: 10.1016/S00928674(02)00661-X

Maxwell, S. A., and Davis, G. E. (2000). Differential gene expression in p53mediated apoptosis-resistant vs. apoptosis-sensitive tumor cell lines. Proc. Natl. Acad. Sci. U.S.A. 97, 13009-13014. doi: 10.1073/pnas.230445997

Miller, G., Honig, A., Stein, H., Suzuki, N., Mitler, R., and Zilberstein, A. (2009). Unraveling delta1-pyrroline-5-carboxylate-proline cycle in plants by uncoupled expression of proline oxidation enzymes. J. Biol. Chem. 289, 26482-26492. doi: 10.1074/jbc.M109.009340

Mitchell, H. J., Ayliffe, M. A., Rashid, K. Y., and Pryor, A. J. (2006). A rust-inducible gene from flax (fis1) is involved in proline catabolism. Planta 223, 213-222. doi: 10.1007/s00425-005-0079-x

Mohanty, S. K., and Sridhar, R. (1982). Physiology of rice tungro virus disease: proline accumulation due to infection. Physiol. Plant. 56, 89-93. doi: 10.1111/j.1399-3054.1982.tb04904.x

Moller, I. M. (2001). Plant mitochondria and oxidative stress: electron transport, NADPH turnover, and metabolism of reactive oxygen species. Annu. Rev. Plant Physiol. Plant Mol. Biol. 52, 561-591. doi: 10.1146/annurev.arplant. 52.1.561

Monteoliva, M. I., Rizzi, Y. S., Cecchini, N. M., Hajirezaei, M. R., and Alvarez, M. E. (2014). Context of action of proline dehydrogenase (ProDH) in the hypersensitive response of Arabidopsis. BMC Plant Biol. 14:21. doi: 10.1186/1471-2229-14-21

Murphy, M. P. (2009). How mitochondria produce reactive oxygen species. Biochem. J. 417, 1-13. doi: 10.1042/BJ20081386

Mysore, K. S., and Ryu, C. M. (2004). Nonhost resistance: how much do we know? Trends Plant Sci. 9, 97-104. doi: 10.1016/j.tplants.2003.12.005

Nishimura, A., Nasano, R., and Takagi, H. (2012). The proline metabolism intermediate (delta) 1-pyrroline-5-carboxylate directly inhibits the mitochondrial respiration in budding yeast. FEBS Lett. 586, 2411-2416. doi: 10.1016/j.febslet.2012.05.056

Nomura, M., and Takagi, H. (2004). Role of the yeast acetyltransferase Mpr1 in oxidative stress: regulation of oxygen reactive species caused by a toxic proline catabolism intermediate. Proc. Natl. Acad. Sci. U.S.A. 101, 12616-12621. doi: 10.1073/pnas.0403349101

Paes, L. S., Mantilla, B. S., Zimbres, F. M., Pral, E. M. F., Melo, P. D., Tahara, E. B., et al. (2013). Proline dehydrogenase regulates redox state and respiratory metabolism in Trypanosoma cruzi. PLoS ONE 8:e69419. doi: 10.1371/journal.pone.0069419

Rejeb, K. B., Abdelly, C., and Savouré, A. (2014). How reactive oxygen species and proline face stress together. Plant Physiol. Biochem. 80, 278-284. doi: 10.1016/j.plaphy.2014.04.007

Roosens, N. H., Thu, T. T., Iskandar, H. M., and Jacobs, M. (1998). Isolation of the ornithine- $\delta$-aminotransferase cDNA and effect of salt stress on its expression in Arabidopsis thaliana. Plant Physiol. 117, 263-271. doi: 10.1104/pp.117.1.263

Schertl, P., and Braun, H. P. (2014). Respiratory electron transfer pathways in plant mitochondria. Front. Plant Sci. 5:163. doi: 10.3389/fpls.2014.00163

Sekhar, P. N., Amrutha, R. N., Sangam, S., Verma, D. P. S., and Kishor, P. K. (2007). Biochemical characterization, homology modeling and docking studies of ornithine $\delta$-aminotransferase-an important enzyme in proline biosynthesis of plants. J. Mol. Graph. Model. 26, 709-719. doi: 10.1016/j.jmgm.2007. 04.006

Senthil-Kumar, M., and Mysore, K. S. (2012). Ornithine-delta-aminotransferase and proline dehydrogenase genes play a role in non-host disease resistance by regulating pyrroline-5-carboxylate metabolism-induced hypersensitive response. Plant Cell Environ. 35, 1329-1343. doi: 10.1111/j.1365-3040.2012.02492.x

Senthil-Kumar, M., and Mysore, K. S. (2013). Nonhost resistance against bacterial pathogens: retrospectives and prospects. Annu. Rev. Phytopathol. 51, 407-427. doi: 10.1146/annurev-phyto-082712-102319

Sharma, P., Jha, A. B., Dubey, R. S., and Pessarakli, M. (2012). Reactive oxygen species, oxidative damage, and antioxidative defense mechanism in plants under stressful conditions. J. Bot. 2012, 1-26. doi: 10.1155/2012/217037

Stránská, J., Kopečný, D., Tylichová, M., Snégaroff, J., and Šebela, M. (2008). Ornithine $\delta$-aminotransferase: an enzyme implicated in salt tolerance in higher plants. Plant Signal. Behav. 3, 929-935. doi: 10.4161/psb.6771 
Szabados, L., and Savoure, A. (2010). Proline: a multifunctional amino acid. Trends Plant Sci. 15, 89-97. doi: 10.1016/j.tplants.2009.11.009

Szoke, A., Miao, G. H., Hong, Z., and Verma, D. P. (1992). Subcellular Location of delta 1-pyrroline-5-carboxylate reductase in root/nodule and leaf of soybean. Plant Physiol. 99, 1642-1649. doi: 10.1104/pp.99.4.1642

Tanner, J. J. (2008). Structural biology of proline catabolism. Amino Acids 35, 719-730. doi: 10.1007/s00726-008-0062-5

Torres, M. A., Jones, J. D., and Dangl, J. L. (2006). Reactive oxygen species signaling in response to pathogens. Plant Physiol. 141, 373-378. doi: 10.1104/pp.106.079467

Turrens, J. F. (2003). Mitochondrial formation of reactive oxygen species. J. Physiol. 552, 335-344. doi: 10.1113/jphysiol.2003.049478

Verbruggen, N., and Hermans, C. (2008). Proline accumulation in plants: a review. Amino Acids 35, 753-759. doi: 10.1007/s00726-008-0061-6

Verslues, P. E., and Sharma, S. (2010). Proline metabolism and its implications for plant-environment interaction. Arabidopsis Book 8:e0140. doi: 10.1199/ tab. 0140

Vlot, A. C., Dempsey, D. M. A., and Klessig, D. F. (2009). Salicylic acid, a multifaceted hormone to combat disease. Annu. Rev. Phytopathol. 47, 177-206. doi: 10.1146/annurev.phyto.050908.135202

Vogel, H. J., and Davis, B. D. (1952). Glutamic $\gamma$-semi aldehyde and $\Delta 1$-pyrroline5-carboxylic acid, intermediates in the biosynthesis of proline. J. Am. Chem. Soc. 74, 109-112. doi: 10.1021/ja01121a025
Wanduragala, S., Sanyal, N., Liang, X., and Becker, D. F. (2010). Purification and characterization of Putlp from Saccharomyces cerevisiae. Arch. Biochem. Biophys. 498, 136-142. doi: 10.1016/j.abb.2010.04.020

Xu, P., Chen, F., Mannas, J. P., Feldman, T., Sumner, L. W., and Roossinck, M. J. (2008). Virus infection improves drought tolerance. New Phytol. 180, 911-921. doi: 10.1111/j.1469-8137.2008.02627.x

Yang, J., Zhang, N., Ma, C., Qu, Y., Si, H., and Wang, D. (2013). Prediction and verification of microRNAs related to proline accumulation under drought stress in potato. Comput. Biol. Chem. 46, 48-54. doi: 10.1016/j.compbiolchem.2013.04.006

Conflict of Interest Statement: The Review Editor Yasuhiro Ishiga declares that, despite having collaborated and co-authored manuscripts with the authors, Mysore and Senthil-Kumar, the review process was conducted objectively. The authors declare that the research was conducted in the absence of any commercial or financial relationships that could be construed as a potential conflict of interest.

Copyright (C) 2015 Qamar, Mysore and Senthil-Kumar. This is an open-access article distributed under the terms of the Creative Commons Attribution License (CC BY). The use, distribution or reproduction in other forums is permitted, provided the original author(s) or licensor are credited and that the original publication in this journal is cited, in accordance with accepted academic practice. No use, distribution or reproduction is permitted which does not comply with these terms. 


\title{
Closing the loop on the GABA shunt in plants: are GABA metabolism and signaling entwined?
}

\author{
Simon Michaeli ${ }^{\dagger}$ and Hillel Fromm* \\ Department of Molecular Biology and Ecology of Plants, Faculty of Life Sciences, Tel Aviv University, Tel Aviv, Israel
}

\section{OPEN ACCESS}

Edited by:

Giuseppe Forlani,

University of Ferrara, Italy

Reviewed by:

Vasileios Fotopoulos,

Cyprus University of Technology,

Cyprus

Wayne Snedden

Queen's University, Canada

*Correspondence: Hillel Fromm,

Department of Molecular Biology and Ecology of Plants, Faculty of Life Sciences, Tel Aviv University, Haim Levanon Street, Tel Aviv 69978,

Israel

hillelf@tauex.tau.ac.il

${ }^{\dagger}$ Present address:

Simon Michaeli,

Biochimie et Physiologie Moléculaire des Plantes, Institut de Biologie Intégrative des Plantes,

UMR5004 CNRS/INRA/UM2/SUPAgro,

2 Place Viala, 34060 Montpellier cedex 2, France

Specialty section:

This article was submitted to Plant Physiology,

a section of the journal Frontiers in Plant Science

Received: 28 March 2015 Accepted: 25 May 2015

Published: 09 June 2015

Citation:

Michaeli S and Fromm H (2015) Closing the loop on the GABA shunt in plants: are GABA metabolism

and signaling entwined?

Front. Plant Sci. 6:419.

doi: 10.3389/fp/s.2015.00419
$\gamma$-Aminobutyric acid (GABA) is a non-proteinogenic amino acid that is found in uniand multi-cellular organisms and is involved in many aspects of plant life cycle. GABA metabolism occurs by the action of evolutionary conserved enzymes that constitute the GABA shunt, bypassing two steps of the TCA cycle. The central position of GABA in the interface between plant carbon and nitrogen metabolism is well established. In parallel, there is evidence to support a role for GABA as a signaling molecule in plants. Here we cover some of the recent findings on GABA metabolism and signaling in plants and further suggest that the metabolic and signaling aspects of GABA may actually be inseparable.

Keywords: $\gamma$-Aminobutyric acid, GABA shunt, glutamate decarboxylase, tricarboxylic acids, Arabidopsis, stress

\section{GABA is Associated with Primary Nitrogen and Carbon Metabolism and is Tightly Linked to the TCA Cycle}

$\gamma$-Aminobutyric acid (GABA) is a four-carbon (C) non-proteinogenic amino acid (AA) that was first discovered in plants over half a century ago (Steward et al., 1949). GABA was later revealed in mammalian brain (Roberts and Frankel, 1950) and soon main interest in GABA shifted to animals when it was shown to play a major role in neurotransmission (Roberts et al., 1960). GABA has since been investigated in several organisms including bacteria, fungi, plants and animals (Bouché et al., 2003b). In both eukaryotes and bacteria GABA is a significant component of the free AA pool (Shelp et al., 1999) and the enzymes involved in GABA metabolism are conserved (Metzer and Halpern, 1990; Tillakaratne et al., 1995; Bown and Shelp, 1997; Kumar et al., 2000). Similar enzymes (e.g., glutamate decarboxylase) are found also in archaea, however their roles in GABA metabolism are questionable (Tomita et al., 2014). Interest in plant GABA increased mainly following observations of rapid elevation of its levels under abiotic stresses. Nevertheless, the roles of GABA under these conditions is not clear (Kinnersley and Turano, 2000; Fait et al., 2008). Recent combined genetics and physiological studies of the GABA shunt indicate that its function is required for proper growth in response to abiotic stresses such as low light (Michaeli et al., 2011) and salt (Renault et al., 2013).

$\gamma$-Aminobutyric acid is metabolized via a short pathway known as the GABA shunt (Figure 1), which bypasses two steps of the tricarboxylic acid cycle (TCAC). GABA is mainly produced by the irreversible reaction of the cytosolic enzyme glutamate decarboxylase (GAD; EC 4.1.1.15) that consumes a proton and releases $\mathrm{CO}_{2}$ (Baum et al., 1993; Fait et al., 2008). However, GABA synthesis may also occur via polyamine (putrescine and spermidine) degradation (Fait et al., 2008; Shelp et al., 2012a) and possibly by a non-enzymatic reaction from proline under oxidative stress (Signorelli et al., 2015). GABA catabolism occurs in the mitochondrial matrix of multicellular organisms by the action of GABA transaminase (GABA-T; EC 2.6.1.19) to produce succinic semi-aldehyde (SSA) with the possible participation of several amino acceptors such as $\alpha$-ketoglutarate (AKG), pyruvate or glyoxylate (Clark et al., 2009a; Shelp et al., 2012b). Subsequently, SSA is converted by another 


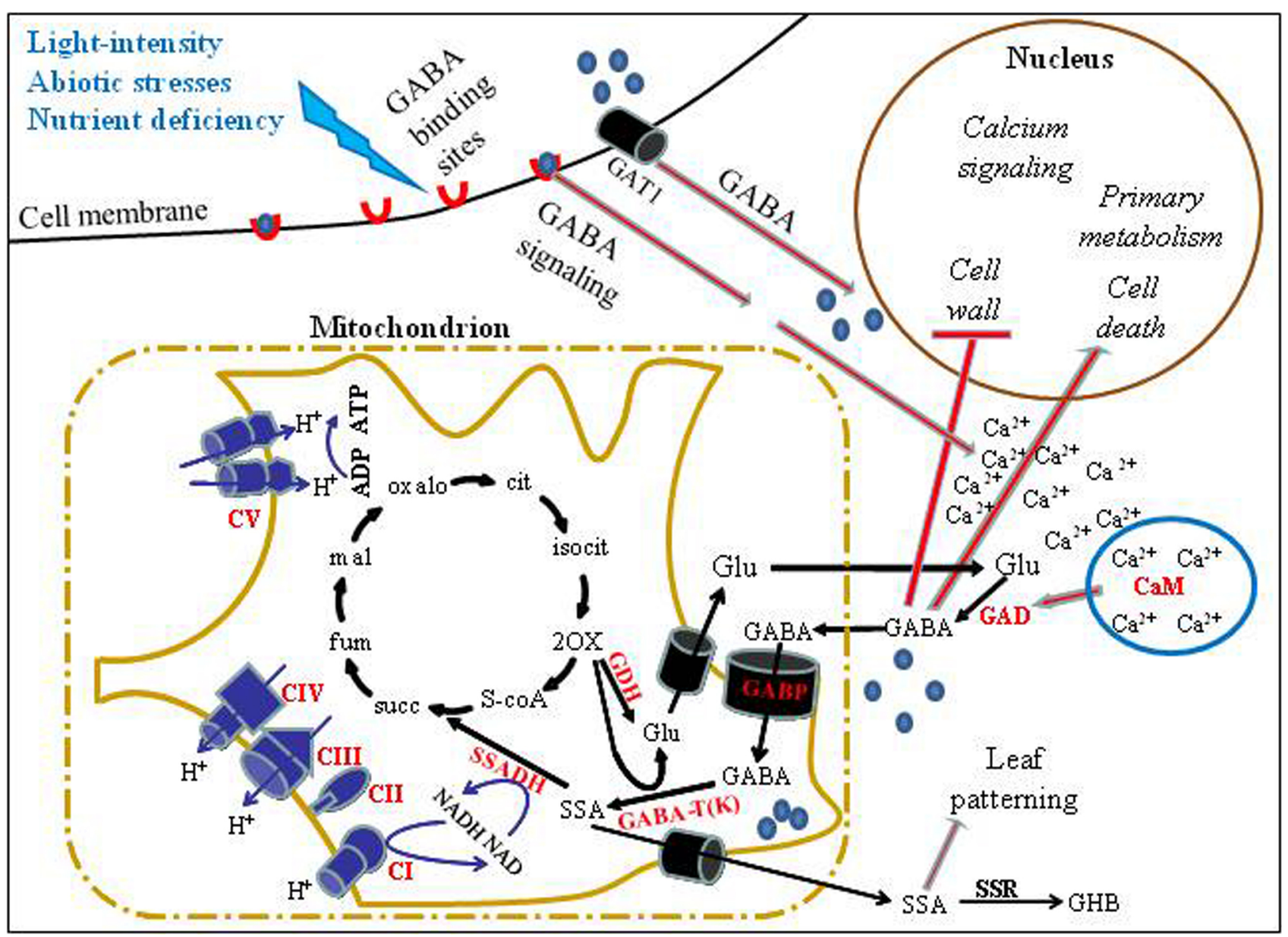

FIGURE 1 | A schematic model of GABA transport, metabolism and signaling in plant cells. External stimuli, such as abiotic stresses and light deficiency, regulate the expression of GABA shunt-associated genes. Such stimuli may further result in increased levels of GABA, enabling its attachment to cell-surface binding sites that generate transient $\mathrm{Ca}^{2+}$ increase and transport into cells via high affinity GABA transporters (e.g., GAT1; Meyer et al., 2006). Consequentially, GAD may be activated via a $\mathrm{Ca}^{2+} / \mathrm{CaM}$ complex (Baum et al., 1993). This increase in intracellular GABA may induce the expression of several signaling and metabolism-associated genes while repressing other genes such as genes associated with cell wall-modifications. Depending on the environmental conditions, a significant proportion of cytosolic GABA may enter mitochondria through the GABA permease, AtGABP (Michaeli et al., 2011), for catabolism by GABA-T and SSADH, resulting in succinate formation to feed the TCAC and mitochondrial ETC. Alternatively, the toxic intermediate, SSA, may be transported out of the mitochondrion to form GHBA via the enzyme SSR (GHBDH). We note that recently a tonoplast Glu/Asp/GABA exchanger in tomato fruit has been reported (Snowden et al., 2015), which is not included in the presented model. Enzyme names are in bold and red, whereas the reactions they perform are indicated as black arrows. Red-filled lines indicate a regulatory effect. Blue spheres denote GABA, red crescents denote GABA receptors. Abbreviations: GDH, Glutamate dehydrogenase; succ, succinate; fum, fumarate; Mal, malate; oxalo, oxaloacetate; cit, citrate; isocit, isocitrate; 2-OX, 2-oxoglutarate ( $\alpha$-ketoglutarate); Glu, glutamate; $\mathrm{Cl}, \mathrm{Cll}, \mathrm{CIII}, \mathrm{CIV}$, and CV, complexes I, II, III, IV and V of the mitochondrial ETC, Respectively. mitochondrial enzyme, SSA dehydrogenase (SSADH; EC 1.2.1.16) to succinate that acts both as an electron donor to the mitochondrial electron transport chain (ETC) and as a component of the TCAC (Bouché et al., 2003a; Shelp et al., $2012 b$ ). Alternatively, SSA can be converted to $\gamma$-hydroxybutyric acid (GHBA) through a GHB dehydrogenase (GHBDH) that was reported in animals and plants (Andriamampandry et al., 1998; Breitkreuz et al., 2003) and more recently in E. coli (Saito et al., 2009). Because AKG may serve as a precursor of glutamate and subsequently of GABA, this metabolic pathway may be viewed as bypassing two enzymatic steps of the TCAC (Figure 1), AKG dehydrogenase (AKGDH) and Succinyl Co-A ligase (SCOAL), thus termed the GABA shunt (Bown and Shelp, 1997).
Direct functional association of the GABA shunt and the TCAC was demonstrated in transgenic tomato plants with reduced activity of SCOAL (Studart-Guimaraes et al., 2007) and in potato slices treated with a specific chemical inhibitor of AKGDH (Araújo et al., 2008). Both enzymes are bypassed by the GABA shunt and in both cases elevated flux through the GABA shunt compensated for the lost activity of these TCAC enzymes. Reciprocally, mutants of the Arabidopsis GABP (mitochondrial GABA transporter) resulted in reduced uptake of GABA into mitochondria and increased TCAC activity (Michaeli et al., 2011). Much data accumulated regarding plant TCAC in illuminated leaves (Tcherkez et al., 2009; Sweetlove et al., 2010; Araújo et al., 2011a) where it seems to "lose" its classical cyclic metabolic form. 
Evidence suggests that citrate, stored from the TCAC activity of the previous night, is mainly shuttled for $\mathrm{N}$ assimilation (Tcherkez et al., 2009; Sweetlove et al., 2010). Interestingly, the GABA shunt, as one of the bridges between $\mathrm{N}$ and $\mathrm{C}$ metabolism, is significantly active in illuminated Xanthium strumarium (cocklebur) leaves as demonstrated by the high metabolic flux through it. On the other hand, a metabolic flux from AKG to succinate through the TCAC was almost undetectable (Tcherkez et al., 2009; Figure 1). In other words, the GABA shunt is apparently the major source for succinate in leaves at day time, contrary to the night-type heterotrophic plant metabolism where TCAC functions in its classical cyclic form (Sweetlove et al., 2010).

An interesting transcriptional co-response was demonstrated with Arabidopsis genes that encode the GABA shunt pathway (GAD2 and SSADH) and other primary $\mathrm{C}$ metabolism associated genes, providing further support for the association of GABA with primary $\mathrm{C}$ metabolism in plants at both the metabolome and transcriptome levels (Fait et al., 2008). It should be noted that the genes encoding the mitochondrial GABA permease, AtGABP and the GABA catabolic enzyme SSADH are also highly coexpressed in response to stress (Michaeli et al., 2011). Changes in the cellular distribution and quantity of GABA were shown also to affect levels of several AAs in different plant species (Baum et al., 1996; Michaeli et al., 2011; Deleu et al., 2013; Shimajiri et al., 2013a; Batushansky et al., 2014; Snowden et al., 2015) and even in seeds (Fait et al., 2011). As a molecule synthesized mainly from glutamate, yet intimately associated with the TCAC, GABA is continuously suggested to be an important component in the balance between $\mathrm{C}$ and nitrogen $(\mathrm{C}: \mathrm{N})$ pools of plant cells (Fait et al., 2008; Renault, 2013; Batushansky et al., 2014).

\section{Effects of GABA on Plant Development, Organ Patterning, Interaction with Pathogens and Fertility}

A signaling role for GABA in plants is continuously suggested and discussed (Bouché et al., 2003b; Bouché and Fromm, 2004; Häusler et al., 2014) and was especially highlighted following the discovery of the interesting role of GABA in pollen tube growth and guidance (Palanivelu et al., 2003) and by identifying genes that encode putative receptors that possess domains, which are structurally homologous to mammalian $\mathrm{GABA}_{B}$ receptors (Lacombe et al., 2001). Also, the role of GABA in regulating expression of several genes was reported: ACC-synthase (Kathiresan et al., 1997), arginine-decarboxylase (Turano et al., 1997), 14-3-3 (Lancien and Roberts, 2006), and genes encoding secreted and cell wall associated proteins (Renault et al., 2011). Consistent with a role in ACC-Synthase regulation, exogenously applied GABA was shown to induce ethylene emission in Stellaria longipes and sunflower (Kathiresan et al., 1997, 1998). Nevertheless, in our opinion, the most compelling evidence suggesting that GABA is a signaling molecule in plants is the detection of Quantum dot (QD)-mediated GABA binding sites on the surface of plant pollen and somatic protoplasts, implying the existence of GABA receptors. Importantly, the binding of the QD-GABA probes caused transient elevation in intracellular
$\mathrm{Ca}^{2+}$ levels (Yu et al., 2006; Figure 1), which is known to induce signaling pathways in plants (Galon et al., 2010; Batistič and Kudla, 2012; Fromm and Finkler, 2015). Notably, it was recently shown that this increase in $\mathrm{Ca}^{2+}$ levels is the result of the modulation of $\mathrm{Ca}^{2+}$-permeable channels to enable proper pollen tube growth, further supporting the suggestion that the requirement for a GABA gradient along pollen tube is related to its signaling role rather than to a metabolic one (Yu et al., 2014).

Furthermore, a role for GABA as a signal between plants and bacteria is already well established (Shelp et al., 2006). GABA that accumulates in wounded plant tissues restricts quorum sensing of Agrobacterium tumefaciens by regulating expression of the bacterial attKLM operon (Chevrot et al., 2006). It was shown that plant GAD is responsible for the accumulation of the GABA signal that is transported into the bacteria via the bacterial Bra $\mathrm{ABC}$ transporter. Interestingly, plants with an ectopically functional GAD, which lacks the $\mathrm{Ca}^{2+} /$ Calmodulin $\left(\mathrm{Ca}^{2+} / \mathrm{CaM}\right)$ binding domain, exhibited increased resistance to Agrobacterium infection (Chevrot et al., 2006). Increased GABA shunt activity was more recently also associated with tomato resistance to Botrytis cinerea (Seifi et al., 2013). In pepper (Capsicum annuum), the Xanthomonas campestris pv. vesicatoria effector, AvrBsT, induces hypersensitive cell death. Investigation of this effector revealed that it interacts with a pepper arginine decarboxylase $(\mathrm{CaADC} 1)$ and their joint expression is accompanied by polyamine, nitric oxide and hydrogen peroxide bursts. Treatment of pepper with GABA significantly reduced growth of avirulent Xanthomonas campestris, suggesting a role for GABA in cell death promotion (Kim et al., 2013). This is consistent with an older report exhibiting GABA as a modulator of soybean (Glycine max) arginine decarboxylase, ultimately affecting polyamine biosynthesis (Turano et al., 1997). The association of GABA and cell death was also demonstrated in Arabidopsis seeds expressing a constitutively active GAD where up-regulation of cell-death-associated genes was detected. Among the over-represented categories of genes expressed in the hyperaccumulating GABA seeds were those involved in $\mathrm{Ca}^{2+}$. mediated signaling, redox and cysteine proteases (Fait et al., 2011). The over representation of $\mathrm{Ca}^{2+}$-signaling associated genes is intriguing in light of the transient change in $\mathrm{Ca}^{2+}$ levels caused by applying GABA to protoplasts (Yu et al., 2006). Furthermore, mutation in GABA-T (which results in GABA accumulation) was shown also to suppress plant response to E-2-Hexenal, a herbivore and pathogen associated $\mathrm{C}_{6}$-volatile. This volatile was shown to increase GABA levels (Mirabella et al., 2007).

$\gamma$-Aminobutyric acid accumulation also results in the down regulation of genes associated with cell-wall modifications (Renault et al., 2011; Batushansky et al., 2014). Cell wall modification are correlated with developmental changes which are exerted on plants by environmental constraints (Roppolo and Geldner, 2012). Indeed, the association of increased GABA levels with altered plant development was demonstrated extensively. Ectopically active GAD (lacking its $\mathrm{Ca}^{2+} / \mathrm{CaM}$ regulated autoinhibitory domain) in transgenic tobacco results in developmental abnormalities that include shortened and more branched plants. Young developing leaves exhibited a delay in greening and were narrower than normal and the plants lacked 
pollen. Histological analysis revealed shorter cells in the stem cortex parenchyma of these lines, which could in part explain the short plant phenotype (Baum et al., 1996). These observations are consistent with more recent evidence from GABA-T mutants displaying cell elongation defects and dwarfism in Arabidopsis and tomato (Renault et al., 2011; Koike et al., 2013), especially under salt stress (Renault et al., 2013). Immuno-localization of GABA in pine seedlings suggests a role for GABA in vascular development (Molina-Rueda et al., 2015). Moreover, Arabidopsis ssadh mutants are significantly dwarfed, a phenotype that can be rescued by a second mutation in $G A B A-T$, which encodes the enzyme that functions upstream of SSADH in the mitochondrial GABA catabolism process (Ludewig et al., 2008). Interestingly, SSADH was implicated in Arabidopsis patterning along the abaxial/adaxial axis and these patterning defects were recovered in an ssadh/gaba-t double mutant (Toyokura et al., 2011). These authors suggested that it is the SSA intermediate between GABA and succinate that is mediating this patterning process (Toyokura et al., 2011, 2012).

In conclusion, GABA levels can readily be affected by activating plant GAD (which is tightly regulated by $\mathrm{Ca}^{2+} / \mathrm{CaM}$ ), by suppressing its catabolism (through suppression of GABA-T), by exogenous application of GABA or by interrupting the cellular $\mathrm{C}: \mathrm{N}$ balance (through suppressing of primary metabolism related enzymes such as TCAC enzymes). Such changes in GABA levels result in plant responses at the metabolic, transcriptional and developmental levels, which are difficult to explain solely within a metabolic context. Although there is yet no direct evidence for a signaling role of GABA in plants, it's impossible to ignore the numerous evidences that imply the existence of such signaling pathways.

\section{Coupling of GABA Metabolism and Signaling Under Nutrient Starvation}

In order to obtain further insight into natural modulation of GABA levels, we discuss the current knowledge regarding GABA metabolism under plant starvation in comparison to metabolism in starved animals. We suggest that the metabolic and signaling functions of GABA evolved to be functionally entwined.

Metabolic profiling of Arabidopsis mutants impaired in enzymes that are essential for respiration under extended darkinduced $\mathrm{C}$ starvation revealed an increase in the content of several AAs associated with starvation-induced protein degradation (Araújo et al., 2010). On the other hand, the levels of GABA and of the TCAC intermediate, succinate, increased significantly in these mutants although both metabolites are not the product of protein degradation. The authors suggested that an increase in GABA shunt activity produces mitochondrial succinate to maintain respiration in these mutants (Araújo et al., 2010). This was also apparent in a different study that employed metabolic and transcriptional analyses to decipher mechanisms mediating the Arabidopsis response to dark-induced starvation. GABA was once again highlighted as the major non-proteinogenic AA with a tight correlation to succinate levels (Caldana et al., 2011). Notably, at the transcriptional level, starving plants exhibited significant reduced expression of TCAC-associated SCOAS genes in parallel to a significant increase in $S S A D H$ expression, implying an induction of the GABA shunt bypass under these circumstances (Caldana et al., 2011). Though succinate accumulation in starved plants may be explained by its use as an electron donor in the respiratory chain, the accumulation of GABA, if not merely a metabolic side effect, awaits explanation. One possibility is that the accumulation of GABA is a prerequisite for its signaling function. Let's look at an example for such a metabo-signaling coupling of GABA under low energy status in an organism with a well-established signaling role of GABA.

In mice, the brain is responsible for inducing a hunger sensation under low (or no) food availability that will result in a behavioral response of seeking and consuming food (Atasoy et al., 2012; Wu et al., 2012). An interesting report demonstrates how specific neurons (AgRP neurons of the arcuate nucleus) modulate feeding behavior in mice by providing GABAergic input into mice brainstem (the parabrachial nucleus; $\mathrm{PBN}$ ) and how inactivation of GABA biosynthesis (by inactivating murine Gad1) in the arcuate nucleus, or block of $\mathrm{GABA}(\mathrm{A})$ receptors in the $\mathrm{PBN}$, promotes mice anorexia (Wu et al., 2009). This elegantly shows how GABA neurotransmission participates in the maintenance of energy homeostasis of the whole organism (feeding behavior) and how it is directly affected by GABA biosynthesis through the action of Gad1 (Dietrich and Horvath, 2009; Wu et al., 2009).

As autotrophic organisms, the two main aspects of plant "feeding" are photosynthesis and nutrient uptake by the root. GABA is one of few metabolites whose metabolic path is divided between the cytosol and the mitochondrion matrix. The possibility to target mitochondrial metabolism as a means to enhance photosynthesis was already discussed (Nunes-Nesi et al., 2011; Araújo et al., 2014) and shown (Araújo et al., 2011b). Notably, in silico analysis of optimal photosynthesis in cyanobacteria highlighted the importance of the GABA shunt, combined with an incomplete TCAC during autotrophic metabolism (Nogales et al., 2012). Remarkably, some GABA-T isoforms of rice and tomato were shown to localize in plastids (Clark et al., 2009b; Shimajiri et al., 2013b). This suggests a role for GABA or its derivatives in plastid-associated functions. Indeed exogenously applied GABA resulted in increased photosynthesis parameters is muskmelon seedlings, mainly under hypoxia stress (Xia et al., 2011).

Nevertheless, in order to more accurately compare animal feeding with plant "feeding" it is more prudent to refer also to nutrient uptake by plant roots. Two reports suggest a signaling role for GABA in nitrate uptake in Brassica napus roots (Beuve et al., 2004) and more recently in Arabidopsis thaliana (Barbosa et al., 2011). An earlier report described how GABA enhances significantly the growth of Lemna minor plants by increasing mineral consumption. In contrast, isomers of GABA such as 3-aminobutyric acid and 2-aminobutyric acid inhibited plant growth. Remarkably, GABA mediated promotion of Lemna growth was inhibited by bicuculline and picrotoxin, which are competitive and non-competitive antagonists of GABA receptors in the mammalian central nerve system, respectively. Consistent with these findings, Baclofen, a known GABA agonist in animals, significantly increased GABA mediated promotion of Lemna growth suggesting the existence of GABA receptors in plants that 
participate in nutrient uptake and eventually affect plant growth (Kinnersley and Lin, 2000). Moreover, a role for GABA in the up-regulation of nodule activity was also suggested (Sulieman and Schulze, 2010) followed by evidences for a role of GABA in increasing the efficiency of symbiotic $\mathrm{N}_{2}$ fixation in legumes (Sulieman, 2011). Thus, it seems that GABA levels increase during plant starvation and energetically demanding stresses. Importantly, GABA seems to induce plant responses that may aid in replenishing the energetic supply, very similar to the action of GABA in mammals where it modulates feeding behavior. The already mentioned effect of GABA on exerting cell-elongation arrest may be important in parallel in order to save cellular energy. Thus, the combined GABA associated effects of modulating photosynthesis and nutrient uptake, in parallel to growth arrest, may be pivotal to ensure plant survival under energetically demanding stresses.

\section{Concluding Remarks}

$\gamma$-Aminobutyric acid is a major metabolic component in the interface between $\mathrm{C}$ and $\mathrm{N}$ metabolism. As such, its levels are "sensitive" to the availability of both essential elements, which makes it an excellent sensor for the energetic state of the cell. Thus, GABA seems ideal to serve as a signal that participates in a pathway that "instructs" the organism whether to try and gain more energy, or whether to initiate processes to

\section{References}

Andriamampandry, C., Siffert, J. C., Schmitt, M., Garnier, J. M., Staub, A., Muller, C., et al. (1998). Cloning of a rat brain succinic semialdehyde reductase involved in the synthesis of the neuromodulator $\gamma$-hydroxybutyrate. Biochem. J. 334, 43-50.

Araújo, W. L., Ishizaki, K., Nunes-Nesi, A., Larson, T. R., Tohge, T., Krahnert, I., et al. (2010). Identification of the 2-hydroxyglutarate and isovaleryl-CoA dehydrogenases as alternative electron donors linking lysine catabolism to the electron transport chain of Arabidopsis mitochondria. Plant Cell 22, 1549-1563. doi: $10.1105 /$ tpc. 110.075630

Araújo, W. L., Nunes-Nesi, A., and Fernie, A. (2014). On the role of plant mitochondrial metabolism and its impact on photosynthesis in both optimal and sub-optimal growth conditions. Photosyn. Res. 119, 141-156. doi: 10.1007/s11120-013-9807-4

Araújo, W. L., Nunes-Nesi, A., Nikoloski, Z., Sweetlove, L. J., and Fernie, A. R. (2011a). Metabolic control and regulation of the tricarboxylic acid cycle in photosynthetic and heterotrophic plant tissues. Plant Cell Environ. 35, 1-21. doi: 10.1111/j.1365-3040.2011.02332.x

Araújo, W. L., Nunes-Nesi, A., Osorio, S., Usadel, B. R., Fuentes, D., Nagy, R. K., et al. (2011b). Antisense inhibition of the iron-sulphur subunit of succinate dehydrogenase enhances photosynthesis and growth in tomato via an organic acid-mediated effect on stomatal aperture. Plant Cell 23, 600-627. doi: 10.1105/tpc.110.081224

Araújo, W. L., Nunes-Nesi, A., Trenkamp, S., Bunik, V. I., and Fernie, A. R. (2008). Inhibition of 2-oxoglutarate dehydrogenase in potato tuber suggests the enzyme is limiting for respiration and confirms its importance in nitrogen assimilation. Plant Physiol. 148, 1782-1796. doi: 10.1104/pp.108.126219

Atasoy, D., Betley, J. N., Su, H. H., and Sternson, S. M. (2012). Deconstruction of a neural circuit for hunger. Nature 488, 172-177. doi: 10.1038/nature11270

Barbosa, J. M., Singh, N. K., Cherry, J. H., and Locy, R. D. (2011). Nitrate uptake and utilization is modulated by exogenous $\gamma$-aminobutyric acid in Arabidopsis thaliana seedlings. Plant Physiol. Biochem. 48, 443-450. doi: 10.1016/j.plaphy.2010.01.020 cope with excess energy. Also, it seems that GABA is a major alternative pathway to which $\mathrm{C}$ skeleton is directed depending on the environment, growth stage, and tissue specificity. These reports summarized here suggest that from an evolutionary perspective, GABA metabolism predated signaling, and that mechanistically, GABA metabolism underlies its signaling functions. This is consistent with a work that integrated metabolomics with transcript and enzyme activity profiling of plants undergoing diurnal cycles. The authors concluded that “...correlation between metabolites and transcripts are due to regulation of gene expression by metabolites, rather than metabolites being changed as a consequence of a change in gene expression" (Gibon et al., 2006). Future research should shed light on the manner by which physiological increase in GABA levels, either by GAD induction, repression of catabolism or increased flux in the direction of the GABA shunt, affect plant resilience and development. Identification of the cell-surface localized GABA receptors, as well as identifying components that participate in GABA-mediated gene control is essential to this end.

\section{Acknowledgments}

We thank Dr. Oren Tzfadia (VIB Ghent, Belgium) for critical reading of the manuscript and fruitful input. We apologize to colleagues whose work we could not cite due to space limitations.

Batistič, O., and Kudla, J. (2012). Analysis of calcium signaling pathways in plants. Biochim. Biophys. Acta 1820, 1283-1293. doi: 10.1016/j.bbagen.2011.10.012

Batushansky, A., Kirma, M., Grillich, N., Toubiana, D., Pham, P. A., Balbo, I., et al. (2014). Combined transcriptomics and metabolomics of Arabidopsis thaliana seedlings exposed to exogenous GABA suggest its role in plants is predominantly metabolic. Mol. Plant 7, 1065-1068. doi: 10.1093/mp/ ssu017

Baum, G., Chen, Y., Arazi, T., Takatsuji, H., and Fromm, H. (1993). A plant glutamate decarboxylase containing a calmodulin binding domain. Cloning, sequence, and functional analysis. J. Biol. Chem. 268, 19610-19617

Baum, G., Lev-Yadun, S., Fridmann, Y., Arazi, T., Katsnelson, H., Zik, M., et al. (1996). Calmodulin binding to glutamate decarboxylase is required for regulation of glutamate and GABA metabolism and normal development in plants. EMBO J. 15, 2988-2996

Beuve, N., Rispail, N., Laine, P., Cliquet, J. B., Ourry, A., and Le Deunff, E. (2004). Putative role of $\gamma$-aminobutyric acid (GABA) as a long-distance signal in up-regulation of nitrate uptake in Brassica napus L. Plant Cell Environ. 27, 1035-1046. doi: 10.1111/j.1365-3040.2004.01208.x

Bouché, N., Fait, A., Bouchez, D., Moller, S. G., and Fromm, H. (2003a). Mitochondrial succinic-semialdehyde dehydrogenase of the $\gamma$-aminobutyrate shunt is required to restrict levels of reactive oxygen intermediates in plants. Proc. Natl. Acad. Sci. U.S.A. 100, 6843-6848. doi: 10.1073/pnas. 1037532100

Bouché, N., Lacombe, B., and Fromm, H. (2003b). GABA signaling: a conserved and ubiquitous mechanism. Trends Cell Biol. 13, 607-610. doi: 10.1016/j.tcb.2003.10.001

Bouché, N., and Fromm, H. (2004). GABA in plants: just a metabolite? Trends Plant Sci. 9, 110-115. doi: 10.1016/j.tplants.2004.01.006

Bown, A. W., and Shelp, B. J. (1997). The metabolism and functions of $\gamma$ aminobutyric acid. Plant Physiol. 115, 1-5.

Breitkreuz, K. E., Allan, W. L., Van Cauwenberghe, O. R., Jakobs, C., Talibi, D., Andre, B., et al. (2003). A novel $\gamma$-hydroxybutyrate dehydrogenase. J. Biol. Chem. 278, 41552-41556. doi: 10.1074/jbc.M305717200

Caldana, C., Degenkolbe, T., Cuadros-Inostroza, A., Klie, S., Sulpice, R., Leisse, A., et al. (2011). High-density kinetic analysis of the metabolomic and 
transcriptomic response of Arabidopsis to eight environmental conditions. Plant J. 67, 869-884. doi: 10.1111/j.1365-313X.2011.04640.x

Chevrot, R., Rosen, R., Haudecoeur, E., Cirou, A., Shelp, B. J., Ron, E., et al. (2006). GABA controls the level of quorum-sensing signal in Agrobacterium tumefaciens. Proc. Natl. Acad. Sci. U.S.A. 103, 7460-7464. doi: 10.1073/pnas.0600313103

Clark, S. M., Di Leo, R., Dhanoa, P. K., Van Cauwenberghe, O. R., Mullen, R. T., and Shelp, B. J. (2009a). Biochemical characterization, mitochondrial localization, expression, and potential functions for an Arabidopsis $\gamma$-aminobutyrate transaminase that utilizes both pyruvate and glyoxylate. J. Exp. Bot. 60, 1743-1757. doi: 10.1093/jxb/erp044

Clark, S. M., Di Leo, R., Van Cauwenberghe, O. R., Mullen, R. T., and Shelp, B. J. (2009b). Subcellular localization and expression of multiple tomato $\gamma$ aminobutyrate transaminases that utilize both pyruvate and glyoxylate. J. Exp. Bot. 60, 3255-3267. doi: 10.1093/jxb/erp161

Deleu, C., Faes, P., Niogret, M.-F. O., and Bouchereau, A. (2013). Effects of the inhibitor of the $\gamma$-aminobutyrate-transaminase, vinyl- $\gamma$-aminobutyrate, on development and nitrogen metabolism in Brassica napus seedlings. Plant Physiol. Biochem. 64, 60-69. doi: 10.1016/j.plaphy.2012.12.007

Dietrich, M. O., and Horvath, T. L. (2009). GABA keeps up an appetite for life. Cell 137, 1177-1179. doi: 10.1016/j.cell.2009.06.002

Fait, A., Fromm, H., Walter, D., Galili, G., and Fernie, A. R. (2008). Highway or byway: the metabolic role of the GABA shunt in plants. Trends Plant Sci. 13, 14-19. doi: 10.1016/j.tplants.2007.10.005

Fait, A., Nesi, A. N., Angelovici, R., Lehmann, M., Pham, P. A., Song, L., et al. (2011). Targeted enhancement of glutamate-to- $\gamma$-aminobutyrate conversion in Arabidopsis seeds affects carbon-nitrogen balance and storage reserves in a development-dependent manner. Plant Physiol. 157, 1026-1042. doi: 10.1104/pp.111.179986

Fromm, H., and Finkler, A. (2015). Repression and de-repression of gene expression in the plant immune response: the complexity of modulation by $\mathrm{Ca}^{2+}$ and calmodulin. Mol. Plant 8, 671-673. doi: 10.1016/j.molp.2015.01.019

Galon, Y., Finkler, A., and Fromm, H. (2010). Calcium-regulated transcription in plants. Mol. Plant 3, 653-669. doi: 10.1093/mp/ssq019

Gibon, Y., Usadel, B., Blaesing, O., Kamlage, B., Hoehne, M., Trethewey, R., et al. (2006). Integration of metabolite with transcript and enzyme activity profiling during diurnal cycles in Arabidopsis rosettes. Genome Biol. 7, 1-23. doi: 10.1186/gb-2006-7-8-r76

Häusler, R. E., Ludewig, F., and Krueger, S. (2014). Amino acids-a life between metabolism and signaling. Plant Sci. 229, 225-237. doi: 10.1016/j.plantsci.2014.09.011

Kathiresan, A., Miranda, J., Chinnappa, C. C., and Reid, D. M. (1998). $\gamma$ aminobutyric acid promotes stem elongation in Stellaria longipes: the role of ethylene. Plant Growth Regul. 26, 131-137. doi: 10.1023/A:1006107815064

Kathiresan, A., Tung, P., Chinnappa, C. C., and Reid, D. M. (1997). $\gamma$-aminobutyric acid stimulates ethylene biosynthesis in sunflower. Plant Physiol. 115, 129-135.

Kim, N. H., Kim, B. S., and Hwang, B. K. (2013). Pepper arginine decarboxylase is required for polyamine and $\gamma$-aminobutyric acid signaling in cell death and defense response. Plant Physiol. 162, 2067-2083. doi: 10.1104/pp.113.217372

Kinnersley, A. M., and Lin, F. (2000). Receptor modifiers indicate that 4aminobutyric acid (GABA) is a potential modulator of ion transport in plants. Plant Growth Regul. 32, 65-76. doi: 10.1023/a:1006305120202

Kinnersley, A. M., and Turano, F. J. (2000). $\gamma$ aminobutyric acid (GABA) and plant responses to stress. Crit. Rev. Plant Sci. 19, 479-509. doi: 10.1080/07352680091139277

Koike, S., Matsukura, C., Takayama, M., Asamizu, E., and Ezura, H. (2013). Suppression of $\gamma$-aminobutyric acid (GABA) transaminases induces prominent GABA accumulation, dwarfism and infertility in the tomato (Solanum lycopersicum L.). Plant Cell Physiol. 54, 793-807. doi: 10.1093/pcp/pct035

Kumar, S., Punekar, N. S., Satyanarayan, V., and Venkatesh, K. V. (2000). Metabolic fate of glutamate and evaluation of flux through the 4-aminobutyrate (GABA) shunt in Aspergillus niger. Biotechnol. Bioeng. 67, 575-584. doi: 10.1002/(SICI)1097-0290(20000305)67:5<575::AID-BIT8>3.0.CO;2-L

Lacombe, B., Becker, D., Hedrich, R., Desalle, R., Hollmann, M., Kwak, J. M., et al. (2001). The identity of plant glutamate receptors. Science 292, 1486-1487. doi: $10.1126 /$ science.292.5521.1486b

Lancien, M., and Roberts, M. R. (2006). Regulation of Arabidopsis thaliana 14-3-3 gene expression by $\gamma$-aminobutyric acid. Plant Cell Environ. 29, 1430-1436. doi: 10.1111/j.1365-3040.2006.01526.x
Ludewig, F., Hüser, A., Fromm, H., Beauclair, L., and Bouché, N. (2008). Mutants of GABA transaminase (POP2) suppress the severe phenotype of succinic semialdehyde dehydrogenase (ssadh) Mutants in Arabidopsis. PLoS ONE 3:e3383. doi: 10.1371/journal.pone.0003383

Metzer, E., and Halpern, Y. S. (1990). In vivo cloning and characterization of the gabCTDP gene cluster of Escherichia coli K-12. J. Bacteriol. 172, 3250-3256.

Meyer, A., Eskandari, S., Grallath, S., and Rentsch, D. (2006). AtGAT1, a high affinity transporter for $\gamma$-aminobutyric acid in Arabidopsis thaliana. J. Biol. Chem. 281, 7197-7204. doi: 10.1074/jbc.M510766200

Michaeli, S., Fait, A., Lagor, K., Nunes-Nesi, A., Grillich, N., Yellin, A., et al. (2011). A mitochondrial GABA permease connects the GABA shunt and the TCA cycle, and is essential for normal carbon metabolism. Plant J. 67, 485-498. doi: 10.1111/j.1365-313X.2011.04612.x

Mirabella, R., Rauwerda, H., Struys, E. A., Jakobs, C., Triantaphylides, C., Haring, M. A., et al. (2007). The Arabidopsis her1 mutant implicates GABA in E-2-hexenal responsiveness. Plant J. 53, 197-213. doi: 10.1111/j.1365-313X.2007.03323.x

Molina-Rueda, J. S., Pascual, M. N., Pissarra, J., and Gallardo, F. (2015). A putative role for $\gamma$-aminobutyric acid (GABA) in vascular development in pine seedlings. Planta 241, 257-267. doi: 10.1007/s00425-014-2157-4

Nogales, J., Gudmundsson, S., Knight, E. M., Palsson, B. O., and Thiele, I. (2012). Detailing the optimality of photosynthesis in cyanobacteria through systems biology analysis. Proc. Natl. Acad. Sci. U.S.A. 109, 2678-2683. doi: 10.1073/pnas.1117907109

Nunes-Nesi, A., Araújo, W. L., and Fernie, A. R. (2011). Targeting mitochondrial metabolism and machinery as a means to enhance photosynthesis. Plant Physiol. 155, 101-107. doi: 10.1104/pp.110.163816

Palanivelu, R., Brass, L., Edlund, A. F., and Preuss, D. (2003). Pollen tube growth and guidance is regulated by POP2, an Arabidopsis gene that controls GABA levels. Cell 114, 47-59. doi: 10.1016/S0092-8674(03)00479-3

Renault, H. (2013). Fiat lux! Phylogeny and bioinformatics shed light on GABA functions in plants. Plant Signal. Behav. 8, e24274. doi: 10.4161/psb.24274

Renault, H., El Amrani, A., Berger, A., Mouille, G., Soubigou-Taconnat, L., Bouchereau, A., et al. (2013). $\gamma$-aminobutyric acid transaminase deficiency impairs central carbon metabolism and leads to cell wall defects during salt stress in Arabidopsis roots. Plant Cell Environ. 36, 1009-1018. doi: 10.1111/pce.12033

Renault, H., El Amrani, A., Palanivelu, R., Updegraff, E. P., Yu, A. S., Renou, J.-P., et al. (2011). GABA accumulation causes cell elongation defects and a decrease in expression of genes encoding secreted and cell wall-related proteins in Arabidopsis thaliana. Plant Cell Physiol. 52, 894-908. doi: 10.1093/pcp/ pcr041

Roberts, E., Eidelberg, E., Carl, C. P., and John, R. S. (1960). Metabolic and neurophysiological roles of $\gamma$-aminobutyric acid. Int. Rev. Neurobiol. 2, 279-332.

Roberts, E., and Frankel, S. (1950). $\gamma$-aminobutyric acid in brain: its formation from glutamic-acid. J. Biol. Chem. 187, 55-63.

Roppolo, D., and Geldner, N. (2012). Membrane and walls: who is master, who is servant? Curr. Opin. Plant Biol. 15, 608-617. doi: 10.1016/j.pbi.2012.09.009

Saito, N., Robert, M., Kochi, H., Matsuo, G., Kakazu, Y., Soga, T., et al. (2009). Metabolite profiling reveals YihU as a novel hydroxybutyrate dehydrogenase for alternative succinic semialdehyde metabolism in Escherichia coli. J. Biol. Chem. 284, 16442-16451. doi: 10.1074/jbc.M109.002089

Seifi, H. S., Curvers, K., De Vleesschauwer, D., Delaere, I., Aziz, A., and Höfte, M. (2013). Concurrent overactivation of the cytosolic glutamine synthetase and the GABA shunt in the ABA-deficient sitiens mutant of tomato leads to resistance against Botrytis cinerea. New Phytol. 199, 490-504. doi: 10.1111/nph.12283

Shelp, B. J., Bown, A. W., and Faure, D. (2006). Extracellular $\gamma$-aminobutyrate mediates communication between plants and other organisms. Plant Physiol. 142, 1350-1352. doi: 10.1104/pp.106.088955

Shelp, B. J., Bown, A. W., and Mclean, M. D. (1999). Metabolism and functions of $\gamma$-aminobutyric acid. Trends Plant Sci. 4, 446-452. doi: 10.1016/S13601385(99)01486-7

Shelp, B. J., Bozzo, G. G., Trobacher, C. P., Zarei, A., Deyman, K. L., and Brikis, C. J. (2012a). Hypothesis/review: contribution of putrescine to 4-aminobutyrate (GABA) production in response to abiotic stress. Plant Sci. 193-194, 130-135. doi: 10.1016/j.plantsci.2012.06.001

Shelp, B. J., Mullen, R. T., and Waller, J. C. (2012b). Compartmentation of GABA metabolism raises intriguing questions. Trends Plant Sci. 17, 57-59. doi: 10.1016/j.tplants.2011.12.006

Shimajiri, Y., Oonishi, T., Ozaki, K., Kainou, K., and Akama, K. (2013a). Genetic manipulation of the $\gamma$-aminobutyric acid (GABA) shunt in rice: 
overexpression of truncated glutamate decarboxylase (GAD2) and knockdown of $\gamma$-aminobutyric acid transaminase (GABA-T) lead to sustained and high levels of GABA accumulation in rice kernels. Plant Biotechnol. J. 11, 594-604. doi: $10.1111 /$ pbi. 12050

Shimajiri, Y., Ozaki, K., Kainou, K., and Akama, K. (2013b). Differential subcellular localization, enzymatic properties and expression patterns of $\gamma$-aminobutyric acid transaminases (GABA-Ts) in rice (Oryza sativa). J. Plant Physiol. 170, 196-201. doi: 10.1016/j.jplph.2012.09.007

Signorelli, S., Dans, P. D., Coitiño, E. L., Borsani, O., and Monza, J. (2015). Connecting proline and $\gamma$-aminobutyric acid in stressed plants through non-enzymatic reactions. PLoS ONE 10:e0115349. doi: 10.1371/journal.pone.0115349

Snowden, C. J., Thomas, B., Baxter, C. J., Smith, J. A. C., and Sweetlove, L. J. (2015). A tonoplast Glu/Asp/GABA exchanger that affects tomato fruit amino acid composition. Plant J. 81, 651-660. doi: 10.1111/tpj.12766

Steward, F. C., Thompson, J. F., and Dent, C. E. (1949). $\gamma$-aminobutyric acid: a constituent of the potato tuber? Science 110, 439-440.

Studart-Guimaraes, C., Fait, A., Nunes-Nesi, A., Carrari, F., Usadel, B., and Fernie, A. R. (2007). Reduced expression of succinyl-coenzyme A ligase can be compensated for by up-regulation of the $\gamma$-aminobutyrate shunt in illuminated tomato leaves. Plant Physiol. 145, 626-639. doi: 10.1104/pp.107.103101

Sulieman, S. (2011). Does GABA increase the efficiency of symbiotic $\mathrm{N}_{2}$ fixation in legumes? Plant Signal. Behav. 6, 32-36. doi: 10.4161/psb.6.1.14318

Sulieman, S., and Schulze, J. (2010). Phloem-derived $\gamma$-aminobutyric acid (GABA) is involved in upregulating nodule $\mathrm{N}_{2}$ fixation efficiency in the model legume Medicago truncatula. Plant Cell Environ. 33, 2162-2172. doi: 10.1111/j.13653040.2010.02214.x

Sweetlove, L. J., Beard, K. F. M., Nunes-Nesi, A., Fernie, A. R., and Ratcliffe, R. G. (2010). Not just a circle: flux modes in the plant TCA cycle. Trends Plant Sci. 15, 462-470. doi: 10.1016/j.tplants.2010.05.006

Tcherkez, G., Mahé, A., Gauthier, P., Mauve, C., Gout, E., Bligny, R., et al. (2009). In folio respiratory fluxomics revealed by $13 \mathrm{C}$ isotopic labeling and $\mathrm{H} / \mathrm{D}$ isotope effects highlight the noncyclic nature of the tricarboxylic acid "cycle" in illuminated leaves. Plant Physiol. 151, 620-630. doi: 10.1104/pp.109. 142976

Tillakaratne, N. J. K., Medina-Kauwe, L., and Gibson, K. M. (1995). $\gamma$-aminobutyric acid (GABA) metabolism in mammalian neural and nonneural tissues. Comp. Biochem. Physiol. A Physiol. 112, 247-263.

Tomita, H., Yokooji, Y., Ishibashi, T., Imanaka, T., and Atomi, H. (2014). An archaeal glutamate decarboxylase homolog functions as an aspartate decarboxylase and is involved in $\beta$-alanine and coenzyme A biosynthesis. $J$. Bacteriol. 196, 1222-1230. doi: 10.1128/JB.01327-3

Toyokura, K., Hayashi, M., Nishimura, M., and Okada, K. (2012). Adaxial-abaxial patterning: a novel function of the GABA shunt. Plant Signal. Behav. 7, 705-707. doi: $10.4161 /$ psb.20346

Toyokura, K., Watanabe, K., Oiwaka, A., Kusano, M., Tameshige, T., Tatematsu, K., et al. (2011). Succinic semialdehyde dehydrogenase is involved in the robust patterning of Arabidopsis leaves along the adaxial-abaxial axis. Plant Cell Physiol. 52, 1340-1353. doi: 10.1093/pcp/pcr079

Turano, F. J., Kramer, G. F., and Wang, C. Y. (1997). The effect of methionine, ethylene, and polyamine catabolic intermediates on polyamine accumulation in detached soybean leaves. Physiol. Plant. 101, 510-518. doi: 10.1111/j.13993054.1997.tb01031.x

Wu, Q., Boyle, M. P., and Palmiter, R. D. (2009). Loss of GABAergic signaling by AgRP neurons to the parabrachial nucleus leads to starvation. Cell 137, 1225-1234. doi: 10.1016/j.cell.2009.04.022

Wu, Q., Clark, M. S., and Palmiter, R. D. (2012). Deciphering a neuronal circuit that mediates appetite. Nature 483, 594-597. doi: 10.1038/nature10899

Xia, Q.-P., Gao, H.-B., and Li, J.-R. (2011). Effects of $\gamma$-aminobutyric acid on the photosynthesis and chlorophyll fluorescence parameters of muskmelon seedlings under hypoxia stress. Chin. J. Appl. Ecol. 22, 999-1006.

Yu, G.-H., Zou, J., Feng, J., Peng, X. B., Wu, J.-Y., Wu, Y.-L., et al. (2014). Exogenous $\gamma$-aminobutyric acid (GABA) affects pollen tube growth via modulating putative $\mathrm{Ca}^{2+}$-permeable membrane channels and is coupled to negative regulation on glutamate decarboxylase. J. Exp. Bot. 65, 3235-3248. doi: 10.1093/jxb/ eru171

Yu, G., Liang, J., He, Z., and Sun, M. (2006). Quantum dot-mediated detection of $\gamma$-aminobutyric acid binding sites on the surface of living pollen protoplasts in tobacco. Chem. Biol. 13, 723-731. doi: 10.1016/j.chembiol.2006.05.007

Conflict of Interest Statement: The authors declare that the research was conducted in the absence of any commercial or financial relationships that could be construed as a potential conflict of interest.

Copyright (c) 2015 Michaeli and Fromm. This is an open-access article distributed under the terms of the Creative Commons Attribution License (CC BY). The use, distribution or reproduction in other forums is permitted, provided the original author(s) or licensor are credited and that the original publication in this journal is cited, in accordance with accepted academic practice. No use, distribution or reproduction is permitted which does not comply with these terms 


\section{OPEN ACCESS}

Edited by:

Giuseppe Forlani,

University of Ferrara, Italy

Reviewed by:

Sakiko Okumoto,

Virginia Polytechnic Institute and State

University, USA

Wayne Snedden,

Queen's University, Canada

*Correspondence:

Aaron Fait,

Laboratory of Plant Metabolism, The

French Associates Institute for

Agriculture and Biotechnology of

Drylands, The Jacob Blaustein Institutes for Desert Research, The Ben-Gurion University of the Negev. Villa 1, Midreshet Ben-Gurion 84990,

Beer Sheva, Israel fait@bgu.ac.il

Specialty section

This article was submitted to Plant Physiology, a section of the journal

Frontiers in Plant Science

Received: 14 May 2015 Accepted: 11 September 2015 Published: 29 September 2015

Citation:

Batushansky A, Kirma M, Grillich N,

Pham PA, Rentsch D, Galili G,

Fernie AR and Fait A (2015) The transporter GAT1 plays an important

role in GABA-mediated

carbon-nitrogen interactions in Arabidopsis. Front. Plant Sci. 6:785.

doi: 10.3389/fpls.2015.00785

\section{The transporter GAT1 plays an important role in GABA-mediated carbon-nitrogen interactions in Arabidopsis}

\author{
Albert Batushansky ${ }^{1}$, Menny Kirma ${ }^{2}$, Nicole Grillich ${ }^{3}$, Phuong A. Pham ${ }^{3}$, Doris Rentsch ${ }^{4}$, \\ Gad Galili ${ }^{2}$, Alisdair R. Fernie ${ }^{3}$ and Aaron Fait ${ }^{1 *}$ \\ ${ }^{1}$ The Albert Katz International School for Desert Studies, The Jacob Blaustein Institutes for Desert Research, Ben-Gurion \\ University of the Negev, Midreshet Ben-Gurion, Beer Sheva, Israel, ${ }^{2}$ Department of Plant Science, Weizmann Institute of \\ Science, Rehovot, Israel, ${ }^{3}$ Central Metabolism Group, Willmitzer Department, Max-Planck Institute of Molecular Plant \\ Physiology, Potsdam-Golm, Germany, ${ }^{4}$ Department of Biology, Institute of Plant Sciences, University of Bern, Bern, \\ Switzerland
}

Glutamate derived $\gamma$-aminobutyric acid (GABA) is synthetized in the cytosol prior to delivery to the mitochondria where it is catabolized via the TCA cycle. GABA accumulates under various environmental conditions, but an increasing number of studies show its involvement at the crossroad between $\mathrm{C}$ and $\mathrm{N}$ metabolism. To assess the role of GABA in modulating cellular metabolism, we exposed seedlings of $A$. thaliana GABA transporter gat1 mutant to full nutrition medium and media deficient in $\mathrm{C}$ and $\mathrm{N}$ combined with feeding of different concentrations $(0.5$ and $1 \mathrm{mM})$ of exogenous GABA. GC-MS based metabolite profiling showed an expected effect of medium composition on the seedlings metabolism of mutant and wild type alike. That being said, a significant interaction between GAT1 deficiency and medium composition was determined with respect to magnitude of change in relative amino acid levels. The effect of exogenous GABA treatment on metabolism was contingent on both the medium and the genotype, leading for instance to a drop in asparagine under full nutrition and low $\mathrm{C}$ conditions and glucose under all tested media, but not to changes in GABA content. We additionally assessed the effect of GAT1 deficiency on the expression of glutamate metabolism related genes and genes involved in abiotic stress responses. These results suggest a role for GAT1 in GABA-mediated metabolic alterations in the context of the $\mathrm{C}-\mathrm{N}$ equilibrium of plant cells.

Keywords: GABA, glutamate, carbon nitrogen metabolism, GAT1, GC-MS profiling

\section{Introduction}

$\gamma$-Aminobutyric acid (GABA) was first extracted from plants in the middle of the previous century (Steward et al., 1949). Due to its intermediate position between glutamate and the TCA cycle, the GABA shunt is intimately connected to the $\mathrm{C}-\mathrm{N}$ balance and partitioning of the plant and a central role of GABA metabolism at the junction of carbon (C) and nitrogen (N) metabolism was recently emphasized (Fait et al., 2008, 2011). Studies have shown that the key regulatory enzyme of GABA biosynthesis is glutamate decarboxylase (GAD) whose overexpression in transgenic plants significantly reduced the levels of glutamate (Baum et al., 1996; Bouché and Fromm, 2004). This observation suggests that GABA could participate in the regulation of $\mathrm{N}$ metabolism and uptake (Forde, 2002) yet raised questions concerning the transport of GABA both within the plant and 
within the plant cell. That being said and despite the fact that the biosynthesis and metabolism of this compound are relatively well-characterized (Baum et al., 1993; Shelp et al., 1999), the transport of GABA is less well-researched.

Evidence of the enhanced growth of Arabidopsis supplied with GABA previously suggested the existence of GABA transporters, confirmed by the identification of proline transporter 2 (ProT2) as a quaternary transporter which mediates the influx of both GABA and proline with preference to the latter substrate (Breitkreuz et al., 1999). The first GABA specific transporter in plants, GAT1, was isolated from the cell membrane of Arabidopsis thaliana (Meyer et al., 2006). In contrast to ProT2 GAT1 does not transport proline but shows high affinity to GABA compared to other substrates such as alanine and $\beta$ alanine (Meyer et al., 2006). Only recently was the transport of GABA to the mitochondria also, at least, partially elucidated by the isolation of a GABA-permease (GABP), localized to the mitochondrial membrane (Michaeli et al., 2011). In spite of these recent findings the functional role of GABA transport and its involvement in modulation of C-N metabolism remains elusive. Here we studied the metabolism and gene expression of the Arabidopsis mutant gat1, lacking the GABA transporter GAT1, under different C-N media and when supplemented with exogenous GABA.

\section{Materials and Methods}

\section{Chemicals}

All chemicals were purchased from Sigma-Aldrich Israel Ltd. (Jerusalem, Israel) with the exception of $N$-methyl- $N$ [trimethylsilyl]-trifluoroacetamide (Macherey-Nagel $\mathrm{GmbH}$ \& Co. KG, Düren, Germany).

\section{Plant Material and Growing Conditions}

Arabidopsis thaliana ecotype Wassilewskija gat1 lines were isolated from pools of the lines created and published by Patrick J. Krysan and coauthors (Krysan et al., 1996) using the gene specific primer (T23G18-R3) 5'GATGGGAATTGTGCCAAAACC- $3^{\prime}$ and T-DNA primer (F-LB) 5'-GATGCACTCGAAATCAGCCAATTTTAGAC-3'. The mutation was validated by the results of RT-PCR that did not reveal any AtGAT1 mRNA in the ws gat1 T-DNA insertion line, while after the same number of cycles in wild type plants the transcript were amplified. The seeds of the mutants lacking GAT1 in the ws background were sterilized with $50 \%$ commercial bleach for $5 \mathrm{~min}$, and then three times washed with distilled water. After sterilization seeds were sown in the semiliquid sterile full nutrition (FN) media consisting of $2 \mathrm{mM} \mathrm{KNO}_{3}$, $1 \mathrm{mM} \mathrm{NH} \mathrm{NHO}_{3}, 1 \mathrm{mM}$ Gln, $3 \mathrm{mM} \quad \mathrm{KH}_{2} \mathrm{PO}_{4} / \mathrm{K}_{2} \mathrm{HPO}_{4}, \mathrm{pH}$ $5.8,4 \mathrm{mM} \mathrm{CaCl}_{2}, 1 \mathrm{mM} \mathrm{MgSO}_{4}, 2 \mathrm{mM} \mathrm{K}_{2} \mathrm{SO}_{4}, 3 \mathrm{mM}$ MES, $\mathrm{pH} 5.8,0.5 \%(\mathrm{w} / \mathrm{v})$ sucrose, and microelements (i.e., $40 \mathrm{mM}$ $\mathrm{Na}_{2}$ FeEDTA, $60 \mathrm{mM} \mathrm{H}_{3} \mathrm{BO}_{3}, 14 \mathrm{mM} \mathrm{MnSO}$, $1 \mathrm{mM} \mathrm{ZnSO}_{4}$, $0.6 \mathrm{mM} \mathrm{CuSO}_{4}, 0.4 \mathrm{mM} \mathrm{NiCl}_{2}, 0.3 \mathrm{mM} \mathrm{HMoO}_{4}, 20 \mathrm{mM} \mathrm{CoCl}_{2}$ ). The seedling were incubated in $25 \mathrm{ml}$ of FN sterile liquid cultures (250 $\mathrm{ml}$ Erlenmeyer glass flasks) on orbital shakers with constant speed, uniform fluorescent light $( \pm 50 \mu \mathrm{E}$ in the flask) and temperature $\left(21 \pm 1^{\circ} \mathrm{C}\right)$ at 18:6 $\mathrm{h}(\mathrm{L}: \mathrm{D})$ for 14 days. Thereafter the seedlings were transferred to new flasks with different freshly prepared media: FN, C, and $\mathrm{N}$ deficiency (12 flasks per media) for additional $24 \mathrm{~h}$. The medium for $\mathrm{C}$ deficiency had the same composition as $\mathrm{FN}$ but without $0.5 \%(\mathrm{w} / \mathrm{v})$ sucrose; the medium for $\mathrm{N}$ deficiency contained $0.1 \mathrm{mM} \mathrm{KNO}_{3}, 50 \mu \mathrm{M} \mathrm{NH}_{4} \mathrm{NO}_{3}$, no Gln, and $3 \mathrm{mM} \mathrm{KCl}$, and all other components as in FN media (Scheible et al., 2004; Osuna et al., 2007). Then, GABA at 0.5 and $1 \mathrm{mM}$ concentrations was added to each medium (four flasks per concentration). Following $6 \mathrm{~h}$ after GABA addition seedlings from each flask were collected, damped, separately homogenized in liquid nitrogen and kept at $-80^{\circ} \mathrm{C}$ until further analysis.

\section{Metabolite Extraction and Analysis}

Metabolite extraction from $100 \mathrm{mg}$ of Arabidopsis seedlings followed an established protocol (Lisec et al., 2006). $100 \mu \mathrm{l}$ of the extract were dried in a vacuum concentrator (Eppendorf Concentrator Plus). Ahead of injection, samples were derivatized by adding $40 \mu \mathrm{l}$ of fresh solution of methoxyaminhydrochloride in pyridine $\left(20 \mathrm{mg} \mathrm{ml}^{-1}\right)$; (Lisec et al., 2006). Following derivatization, samples were transferred to GC-MS auto-sampler glass vials. Chromatographic separation was carried out on a Thermo Scientific DSQ II GC/MS using a FactorFour Capillary VF-5 ms column.

\section{Metabolite Annotation and Data Analysis}

Chromatograms were analyzed using TagFinder04 software 1.0 (Luedemann et al., 2008). The mass spectra were compared to an available standard library from Golm database (Hummel et al., 2007). Principal component analysis (PCA) and tests of significance were performed on the data sets obtained from metabolite profiling with the software package tMEV (Saeed et al., 2003). Prior to the analysis, data were normalized to the exact weight of each sample and internal standard (ribitol) and Log-transformed. Pairwise Student's $t$-test with confidence interval 95\% was used to compare two genotypes within each media. Differences between treatments were tested for significance by One-Way ANOVA with confidence interval 95\% and Bonferroni correction.

\section{Total RNA Extraction and Gene Expression Analysis}

Based on the results of metabolite analysis, RNA extraction was carried out on seedlings from the different treatments with and without $1 \mathrm{mM}$ GABA only (skipping intermediate GABA concentration), using the standard TRIzol (Invitrogen) protocol. Whole transcriptome analysis was performed using microarray technology according to the standard Affymetrix protocols (available online http://www.affymetrix.com/support/ technical/manuals.affx) on Arabidopsis Genome ATH1 Array chip. Each test was performed on two biological replicates. Briefly, on the first stage first-strand cDNA was synthesized from RNA sample and, then was converted into a double stranded cDNA (dsDNA). The reaction employs DNA polymerase and RNase to simultaneously degrade the RNA and synthesize second-strand cDNA. Labeling, hybridization, scanning, and data extraction were performed by Affymetrix protocols. Analysis of transcriptomic data was done using Partek Genome Suite 
software (Partek); (www.partek.com). Pre-processing was carried out using the Robust Microarray Averaging (RMA) algorithm (Irizarry et al., 2003) and then Two-Way ANOVA was performed. False discovery rate (FDR) method was used to decrease the falseeffect of multiple comparisons. Differentially expressed genes were chosen according to $p_{F D R}<0.05$ and $\geq 4$ times foldchange. Functional annotation, overrepresentation analysis and metabolic pathways annotation was performed by The Database for Annotation, Visualization and Integrated Discovery (DAVID) resource (http://david.abcc.ncifcrf.gov).

\section{Results}

The analysis of central metabolites by GC-MS showed that of the 44 uniquely and unequivocally annotated metabolites 59, 61, and $63 \%$ changed significantly in the gat 1 seedlings compared to the wild type under $\mathrm{FN}$, low $\mathrm{C}$, low $\mathrm{N}$ conditions respectively (Table 1). Furthermore, comparing the levels of metabolites of plants grown in the different media showed that the vast majority of the significantly changed metabolites are common to two or all three of the tested media (Figure 1).

The quantitative analysis of the differences between gat 1 and $w s$ revealed a common pattern of change for the majority of the carboxylic acids and sugars across plants grown in all tested media. The abundance of most of these metabolites was lower in gat 1 compared to $w s$ with exception of lactate, benzoate, maltose, and rhamnose, which accumulated significantly in the mutant (Figure 2). By contrast, the effect of GAT1 deficiency on amino acid metabolism was dependent on the media. Specifically the patterns of change in AA content differ under low $\mathrm{N}$ compared to $\mathrm{FN}$ and low $\mathrm{C}$ (Figure 2). A significant decrease was detected in gat 1 compared to $w s$ for methionine, glutamine, asparagine, arginine, ornithine under $\mathrm{FN}$, glycine under low $\mathrm{C}$, and alanine under both media. By contrast, a significant increase (from weak of methionine level to very strong of glutamine level) of these AAs was measured under low N. Unexpectedly, increased levels of GABA were measured in gat 1 under and $\mathrm{C}$ and $\mathrm{N}$ - deficient media compared to $w s$, while no changes in the non-protein amino acid were detected between the two genotypes under FN (Table 1, Figure 2).

\section{The Opposite Response of Amino Acid Metabolism and the Metabolism of Carboxylic Acids and Sugars to $\mathbf{C}$ and $\mathbf{N}$ Limitation was Detected for both gat1 and ws Seedlings}

When compared to ws (Batushansky et al., 2014), the gat1 genotype showed similar trends in the metabolite response to medium compositions. Compared to FN medium, low $\mathrm{N}$ affected specifically the metabolism of amino acids (Supplementary Figure 2). The level of most of amino acids of both genotypes decreased under low $\mathrm{N}$ in comparison to the $\mathrm{FN}$ medium. Specifically, the level of alanine, arginine, asparagine, glutamine and ornithine was reduced to 4-8 times as compared to FN medium. Nevertheless, the magnitude of decrease differed between the genotypes (Figure 2), namely gat1 maintained a higher level of amino acids than ws under low N. Unexpectedly, the aromatic amino acids (AAA) tyrosine, phenylalanine kept
TABLE 1 | Metabolites that level changed significantly in the gat1 samples compared to ws under the tested conditions according to the Student's $t$-test $(n=4)$ with confidence interval $95 \%$.

\begin{tabular}{|c|c|c|c|c|c|}
\hline FN medium & $t$-value & $\begin{array}{l}\text { Low C } \\
\text { medium }\end{array}$ & $t$-value & $\begin{array}{l}\text { Low } \mathbf{N} \\
\text { medium }\end{array}$ & $t$-value \\
\hline Glu & 3.4 & Glu & 3.5 & Glu & 4.4 \\
\hline Asn & 4.3 & Asn & 8.8 & Asn & 11.0 \\
\hline Met & 4.0 & Lys & 3.7 & Lys & 9.6 \\
\hline Ala & 5.2 & Met & 2.7 & Ala & 6.5 \\
\hline Ornithine & 6.6 & Ala & 5.6 & $\beta$-Ala & 6.8 \\
\hline Gln & 4.3 & $\beta$-Ala & 6.0 & Ornithine & 6.8 \\
\hline Tyr & 2.6 & Gly & 6.0 & Gly & 10.0 \\
\hline Arg & 6.9 & Tyr & 4.0 & Gln & 24.3 \\
\hline Maltose & 9.0 & Arg & 11.2 & Tyr & 5.7 \\
\hline Malate & 27.9 & Sucrose & 17.9 & Arg & 10.9 \\
\hline $\begin{array}{l}\text { Malic } \\
\text { acid-2-methyl }\end{array}$ & 6.3 & Maltose & 5.5 & Maltose & 14.3 \\
\hline Fumarate & 10.2 & Glycerol & 22.6 & Glycerol & 12.6 \\
\hline Citrate & 15.4 & Inositol & 8.0 & Malate & 2.9 \\
\hline Glyceric acid & 5.9 & Malate & 14.1 & Fumarate & 16.6 \\
\hline Succinate & 22.2 & $\begin{array}{l}\text { Malic } \\
\text { acid-2-methyl }\end{array}$ & 7.4 & Glyceric acid & 9.8 \\
\hline $\begin{array}{l}\text { Dehydroascorbic } \\
\text { acid }\end{array}$ & 5.9 & Fumarate & 11.0 & Succinate & 3.1 \\
\hline Melibiose & 4.5 & Citrate & 22.6 & $\begin{array}{l}\text { Dehydroascorbic } \\
\text { acid }\end{array}$ & 17.0 \\
\hline Spermine & 2.6 & Glyceric-acid & 4.1 & Lactate & 4.7 \\
\hline $\begin{array}{l}\text { Adenosine-5- } \\
\text { MP }\end{array}$ & 9.3 & Succinate & 7.2 & Adenine & 5.8 \\
\hline Benzoic acid & 6.2 & $\begin{array}{l}\text { Dehydroascorbic } \\
\text { acid }\end{array}$ & 7.0 & GABA & 18.1 \\
\hline Nicotinic acid & 5.9 & GABA & 5.2 & Spermine & 5.1 \\
\hline Rhamnose & 10.6 & $\begin{array}{l}\text { Adenosine-5- } \\
\text { MP }\end{array}$ & 3.1 & $\begin{array}{l}\text { Adenosine } \\
-5-M P\end{array}$ & 17.0 \\
\hline Fructose & 3.4 & Benzoic acid & 4.4 & Benzoic acid & 4.9 \\
\hline $\begin{array}{l}\text { Glucaric acid- } \\
\text { 1,4-lactone }\end{array}$ & 4.2 & Nicotinic acid & 4.1 & $\begin{array}{l}\text { Glucaric acid- } \\
\text { 1,4-lactone }\end{array}$ & 16.7 \\
\hline Turanose & 9.0 & Glucose-6P & 3.6 & Rhamnose & 13 \\
\hline \multirow[t]{3}{*}{ Palatinose } & 4.5 & Fructose & 3.3 & Turanose & 4.9 \\
\hline & & Glucose & 3.7 & Kestose & 2.9 \\
\hline & & & & Glucose & 11.1 \\
\hline
\end{tabular}

a similar level under $\mathrm{C}$ and $\mathrm{N}$ deficiency (Supplementary Figure 1). Relatedly, the AAA-precursor shikimate was measured at comparable levels under low $\mathrm{N}$ and FN (Supplementary Figure 2).

Low $C$ induced changes in the content of TCA cycle intermediates and sugars (Supplementary Figures 2, 3). Specifically the levels of malate, citrate, succinate, and lactate in low $\mathrm{C}$ medium were 25 and $12.5 \%$ lower than in FN medium (Supplementary Figure 2); the levels of the primary sugars sucrose, glucose, and fructose decreased to $25 \%$ their level in FN (Supplementary Figure 3). These changes were very close to those in ws (Batushansky et al., 2014), demonstrating a general similarity of the response to different medium composition of the mutant and WT. It should be noted that low $\mathrm{C}$ also affected 


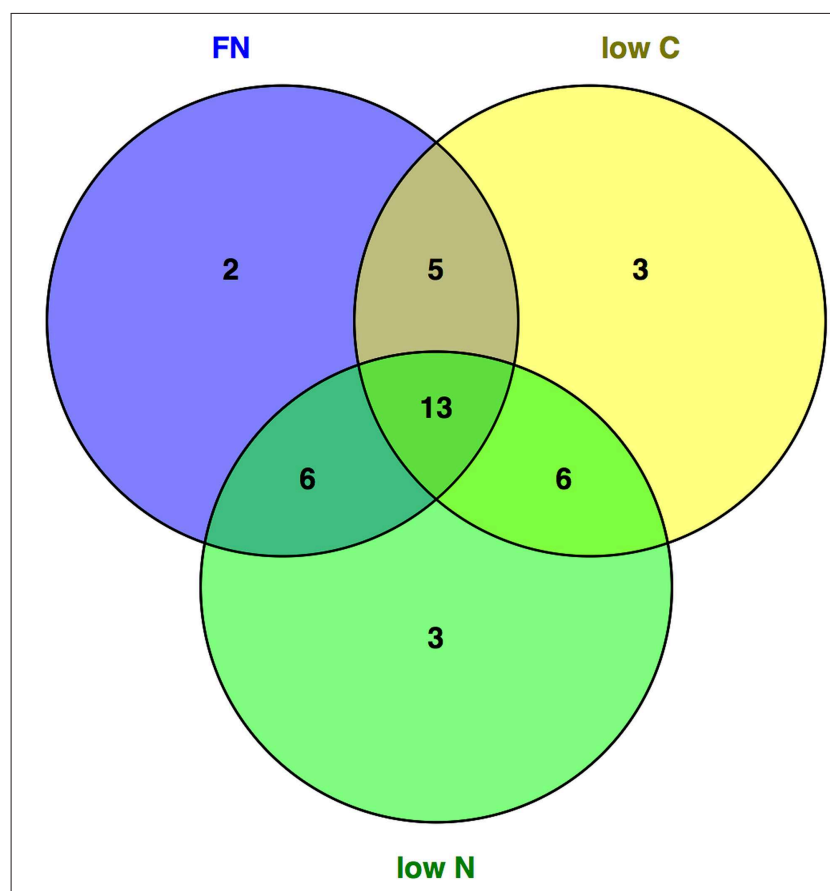

FIGURE 1 | General and medium-specific metabolite response of gat compared to ws. Metabolites that changed significantly $(p<0.05)$ in gat1 compared to ws across media were plotted using a Venn Diagram to identify communal and medium specific response.

the content of some of amino acids. However, in contrast to the effect on the levels of carboxylic acids and sugars, the effect on amino acids content was only moderate (Supplementary Figure 1). Exceptionally among the amino acids, under low $C$ serine and ornithine levels increased 1.5-3 times in comparison with the FN medium (Supplementary Figure 1).

\section{The Global Effect of Exogenous GABA on Seedling Metabolism under $\mathbf{C}$ - and $\mathbf{N}$-limiting Conditions}

The metabolic dataset was next analyzed by principle component analysis (PCA). The first three PCs explained more than $89 \%$ of total variance within the metabolite dataset among samples (Figure 3). The distribution of the samples across the 1st principal component (Figure 3A), which accounted for $65.9 \%$ of total variance, generates distinct groups according to the plant growing media and reflects the underlying differences in metabolism similarly to WT (Supplementary Figure 4). By extracting the eigenvalues we identified those metabolites that mostly affected the separation between samples on the 1st, 2nd, and 3rd components (Supplementary Table 1). Interestingly, glutamine was one of the most affected metabolites accordingly to first two components, demonstrating its high sensitivity to media composition. Together with glutamine other N-rich compounds asparagine, arginine, ornithine, and spermidine contributed to the high variability among the samples under different conditions (Supplementary Table 1). Alanine, glycine, lysine, saccharic acids, glucose, threonine, glycerol, and proline had the strongest contribution to the distribution of the samples across 3rd component accounting less than $6 \%$ of total variance (Figure 3B, Supplementary Table 1). Metabolic differences were identified between the samples supplemented with GABA (1 mM) from untreated samples and those supplemented with GABA $0.5 \mathrm{mM}$ under nutrient-limited conditions (see dispersion of samples on $\mathrm{PC}_{2}$ and $\mathrm{PC}_{3}$ in Figures $\mathbf{3 A}, \mathbf{B}$ ). Under $\mathrm{FN}$ separation was observed between GABA treated samples (either 0.5 or $1 \mathrm{mM}$ ) and non-treated (Figure 3). To statistically validate the implications derived from the PCA, the dataset was subjected to One-Way analysis of variance (ANOVA), with confidence interval 95\% and Bonferroni correction. The effect of GABA on metabolism was visibly less specific to the culture medium as compared to the results obtained for the wild type (Batushansky et al., 2014). The effect of different concentrations of exogenous GABA on the metabolites level was significant for nine compounds (20\%) under FN and low C media and for four compounds under low N medium (Supplementary Table 2).

Endogenous GABA was not affected in content by the addition of exogenous GABA in the mutants, but it increased in the ws (Batushansky et al., 2014) demonstrating the role of GAT1 in GABA influx into the cell. The level of asparagine significantly dropped in content (to 50\%) under both FN and low $\mathrm{C}$ media with supplemental GABA (Figure 4). The level of alanine also changed consistently under both media in response to GABA, increasing in a range from $2-3$ fold $(0.5 \mathrm{mM}$ GABA) to 3-4 times (1 mM GABA). GABA treated seedlings strongly accumulated glycerol (4-6 fold increased) under FN (Figure 4A) while it decreased under low $\mathrm{C}$ medium by $50 \%$ (Figure $4 \mathrm{~B}$ ) and under low N (Figure 4C). Citrate and lactate significantly changed under low $\mathrm{C}$, but if citrate slightly decreased in presence of $1 \mathrm{mM}$ GABA, the level of lactate increased 1.5-fold under the same conditions (Figure 4B). The samples under low $\mathrm{N}$ supplied with $1 \mathrm{mM}$ GABA showed strong decrease of glycine (Figure 4C). On the contrary, the changes in the level of the GABA precursor glutamate were detected only under FN medium supplied with $1 \mathrm{mM}$ GABA where glutamate slightly, but unexpectedly, decreased (Figure 4A). Last, a drop in the relative content of $\beta$-glucose under all tested media supplied with both GABA concentrations was observed (Figure 4, Supplementary Table 2). Taken together the GABA dependent metabolic response in contrast to previously published results on $w s$ (Batushansky et al., 2014) was mostly irrespective of biochemical class of the affected metabolites and medium compositions.

\section{Whole-genome Transcript Analysis of gat1 and Exogenous GABA}

Considering the broadly similar effect of medium composition on metabolic classes between gat1 and ws, gene expression of both genotypes was compared under FN conditions only. The results, when filtered as described in Materials and methods, showed a significant change in expression of 796 genes (393 up- and 403 down-regulated) in gat1 plants compared to ws (Supplementary Data 1). Following DAVID enrichment analysis and taking into consideration only an enrichment score higher than 2 (Huang da et al., 2009) five clusters for up-regulated and six clusters for down-regulated genes were 


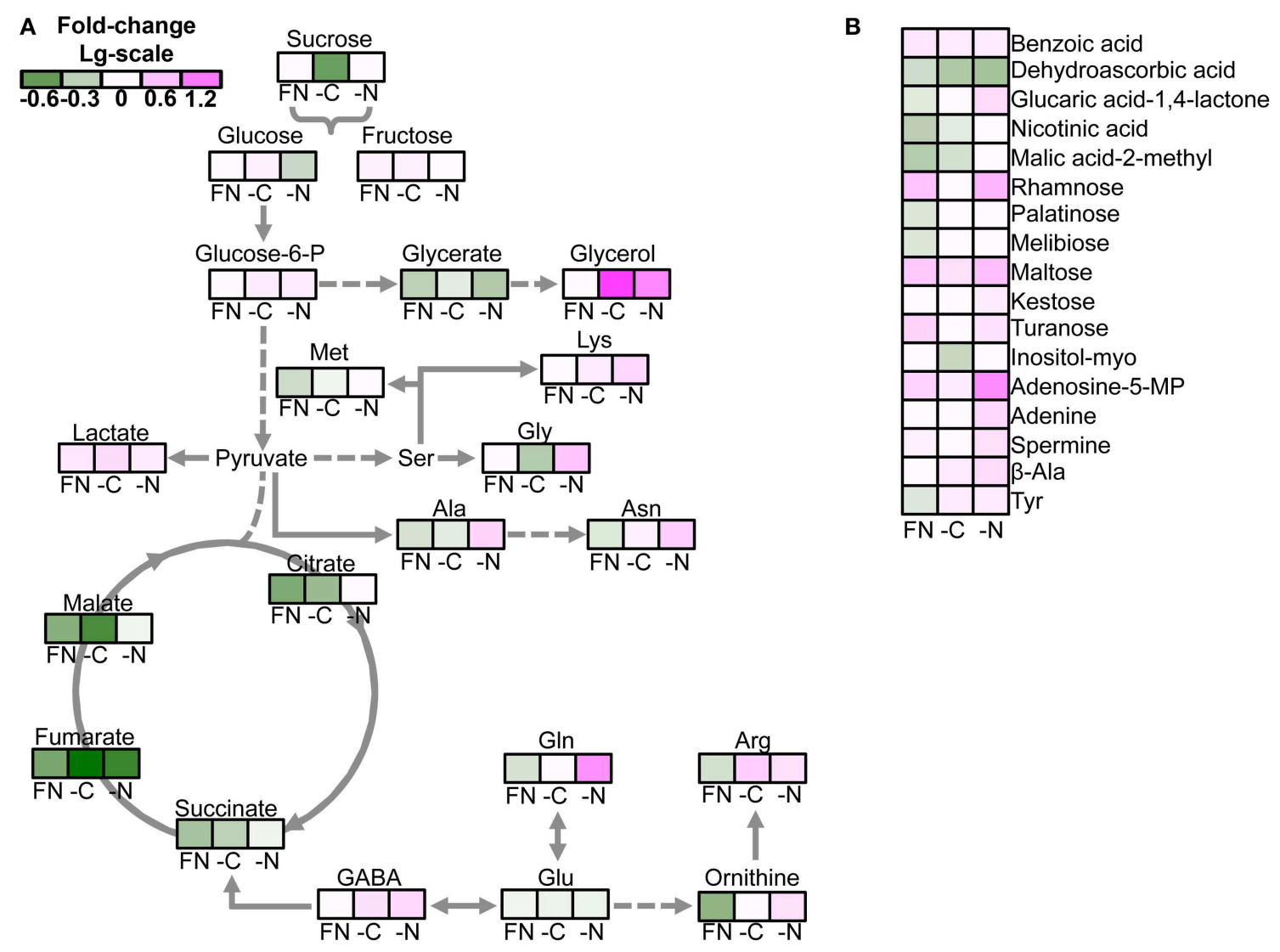

FIGURE 2 | Effect of media on the metabolite profile of gat1 compared to ws. The false color heat-map of the fold-changes in the levels of metabolite between gat1 and ws under FN, low C, and N media represented in the view of major metabolic pathways (A) and the rest of metabolites (B). Only significant changes between the genotypes according to the Student's $t$-test with confidence interval $95 \%$ are presented. Data are fold-change of means $(n=4)$ in Log ${ }_{10}$-scale.
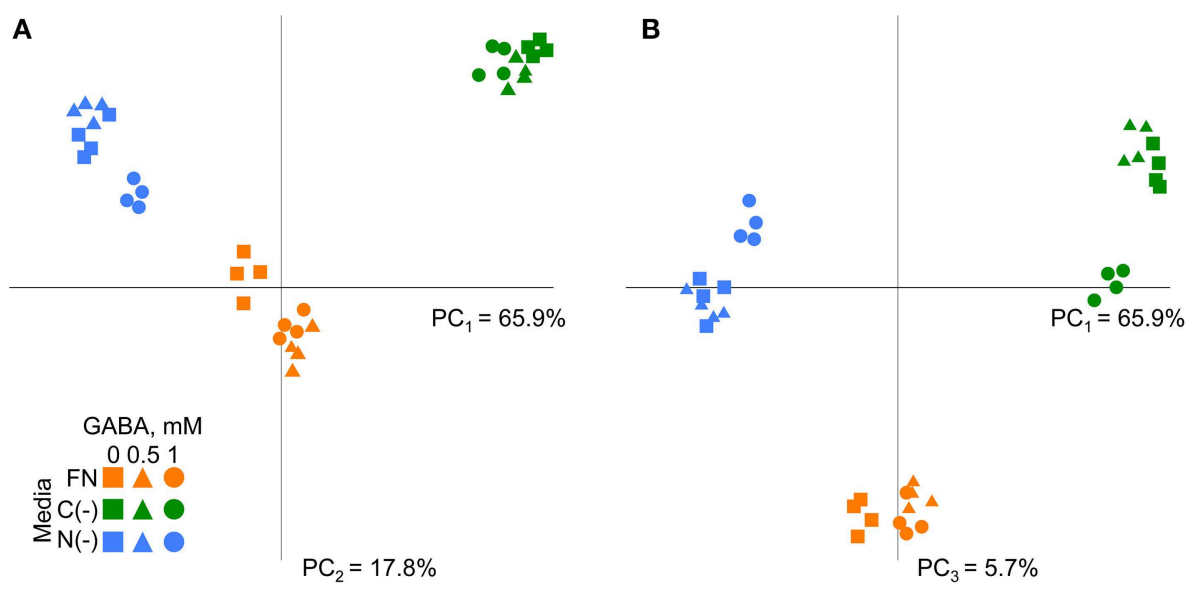

FIGURE 3 | Principal component analysis of metabolic profiles of Arabidopsis gat1 genotype under different conditions. (A) First principal component $\left(\mathrm{PC}_{1}\right)$ and second principal component $\left(\mathrm{PC}_{2}\right)$ are plotted on the axes. (B) First principal component $\left(\mathrm{PC}_{1}\right)$ and third principal component $\left(\mathrm{PC}_{3}\right)$ are plotted on the axes. Variance explained by each component is indicated on the plot.

selected based on the GOTERM database (Supplementary Data 2 ). These results revealed that up-regulated genes in gat 1 were mainly involved in the response to abiotic stimuli such as heat and light. However, more interestingly, we additionally identified up-regulation of CaM-binding transcription activator 1 (At5g09410) and glutamate dehydrogenase 2 (At5g07440), 
A

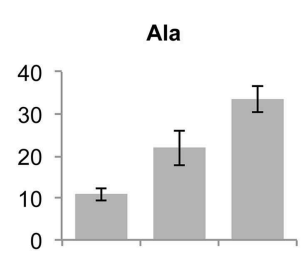

Ornithine

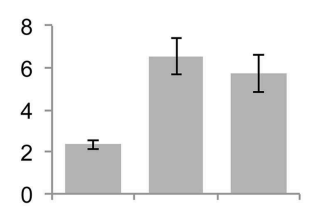

Glucaric acid-1,4-lactone

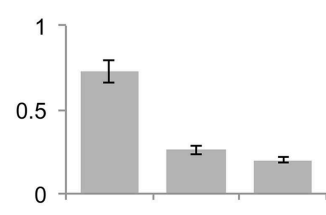

B

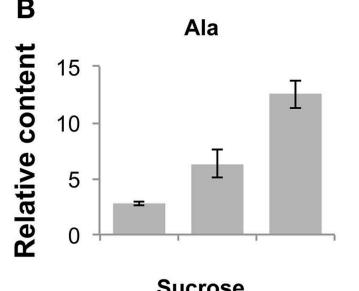

Sucrose

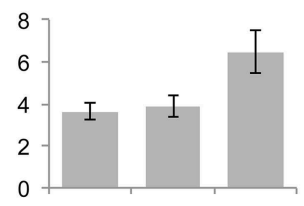

Lactate

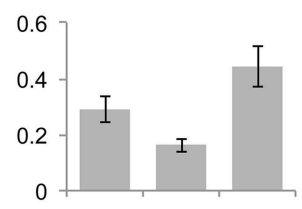

C

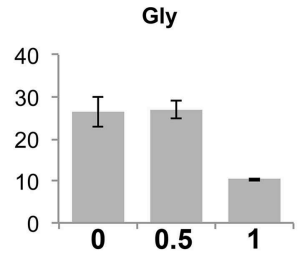

Arg

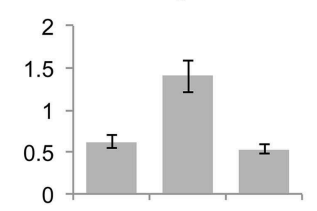

Maltose
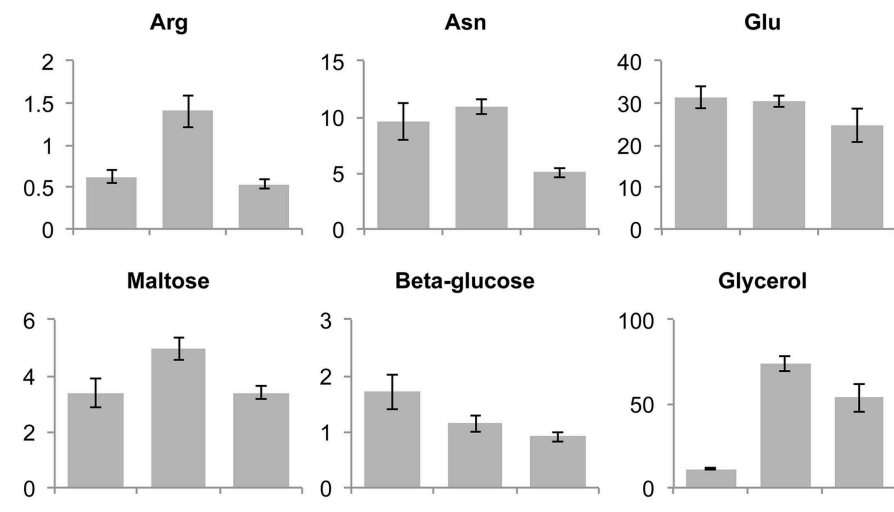

Glycerol

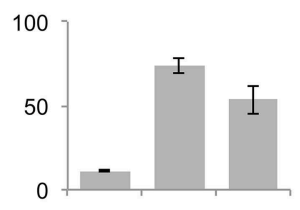


aminotransferase (At2g30970), chorismate mutase (At3g29200) and cytochrome P450 (At2g34770). We additionally tested the effect of $1 \mathrm{mM}$ exogenous GABA on gene expression levels in gat1 under low $\mathrm{C}$ deficiency. Results from this experiment revealed a significant difference in expression of 136 genes (62 up- and 74 down-regulated). Following functional annotation we identified that the up-regulated genes in the samples treated with exogenous GABA mostly belonged to chloroplastic activity and abiotic stimulus gene classes (Figures 5, 6, Supplementary Data 3), whilst down-regulated genes were involved in the response to endogenous stimulus, mostly ethylene-responsive transcriptional factors (At1g28370, At3g20310, At1g77640); (Figure 5, Supplementary Data 4).

\section{Discussion}

Several amino acids permeases have been isolated in Arabidopsis, whose main substrates were glutamate, glutamine and aspartate, with low or no affinity to GABA (Fischer et al., 2002; Meyer et al., 2006). The first plant transporter with high affinity to GABA was identified by Meyer et al. (2006). Despite the authors did not demonstrate a direct flux of GABA via GAT1 protein in plant cell, its role in GABA transport was shown in yeast by complementation strategy. Moreover, gat1 expression was detected in plant tissues particularly under conditions associated with wounding or senescence and it was in correlation with GABA concentration (Meyer et al., 2006). In spite of an increasing number of studies suggesting a pivotal role of GABA in $\mathrm{C}-\mathrm{N}$ metabolism, the functional significance of its transport in respect to central cellular metabolism has yet to be addressed. Here the metabolism of the plasma membrane localized GABA transporter deficient mutant was investigated under FN and nutrient deficient media. Changes caused by both $\mathrm{C}$ - and $\mathrm{N}$-deficiency in comparison with FN medium in gat 1 were similar in trend but significantly sharper in magnitude when compared to those in ws (Figure 2), suggesting a significant role
A

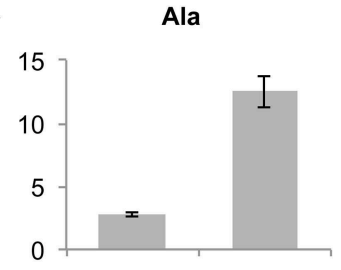

Beta-glucose
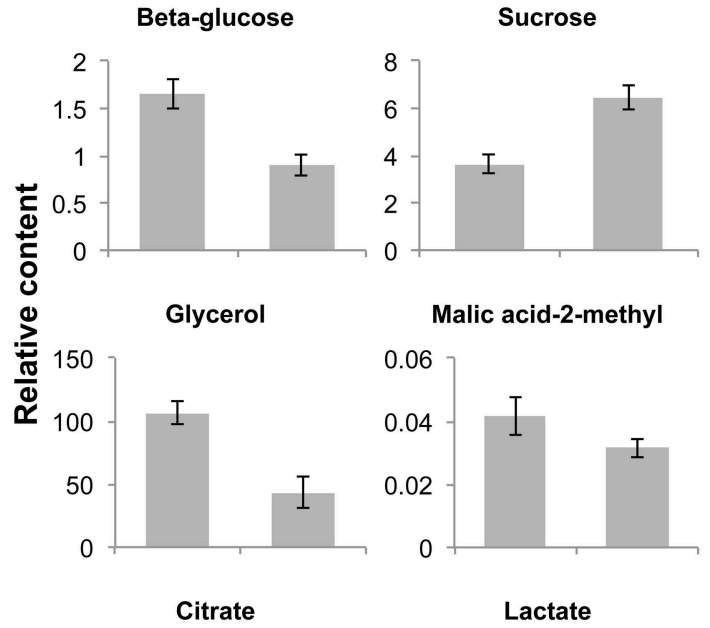

Lactate

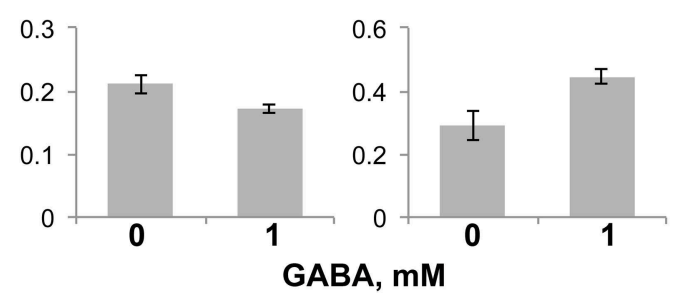

B

Thioredoxin-like 3, chloroplastic

Mitochondrial cytochrome b-like protein AtMg00590

ATP synthase subunit 9, mitochondrial

AT3G27690

Lipid phosphate phosphatase 1

Lupeol synthase 1

AT3G28340

Pyruvate kinase

AT3G47540

Alpha-glucan water dikinase 1, chloroplastic

Phosphoglucan, water dikinase, chloroplastic

Chloroplastic homoserine dehydrogenase 1

AT1G76400

AT1G69220

AT1G18160

AT3G20830

CBL-interacting serine/threonine-protein kinase 1

AT3G16420; AT5G54490

AT1G09070

Serine carboxypeptidase-like 48

AT5G19120

RING-H2 finger protein ATL1R

RING-H2 finger protein ATL5H

AT5G51750

AT4G03510

AT2G29630

Calcium-transporting ATPase 4, plasma membrane-type

FIGURE 5 | Significantly changed metabolite content and gene expression levels in the gat1 samples in respect to 1 mM GABA treatment under low C medium. Bar graphs $(\mathbf{A})$ represent a relative content of metabolites ( $Y$ axis) after normalization by fresh weight and standard ribitol. Data are mean $(n=4) \pm S D$.

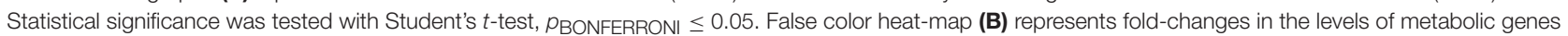
expression between $1 \mathrm{mM}$ GABA-treated samples and untreated samples under low $\mathrm{C}$ medium. Only significantly changed $\left(p_{\mathrm{FDR}} \leq 0.05\right.$, 4 times fold-change) and functionally annotated metabolic genes are presented. 


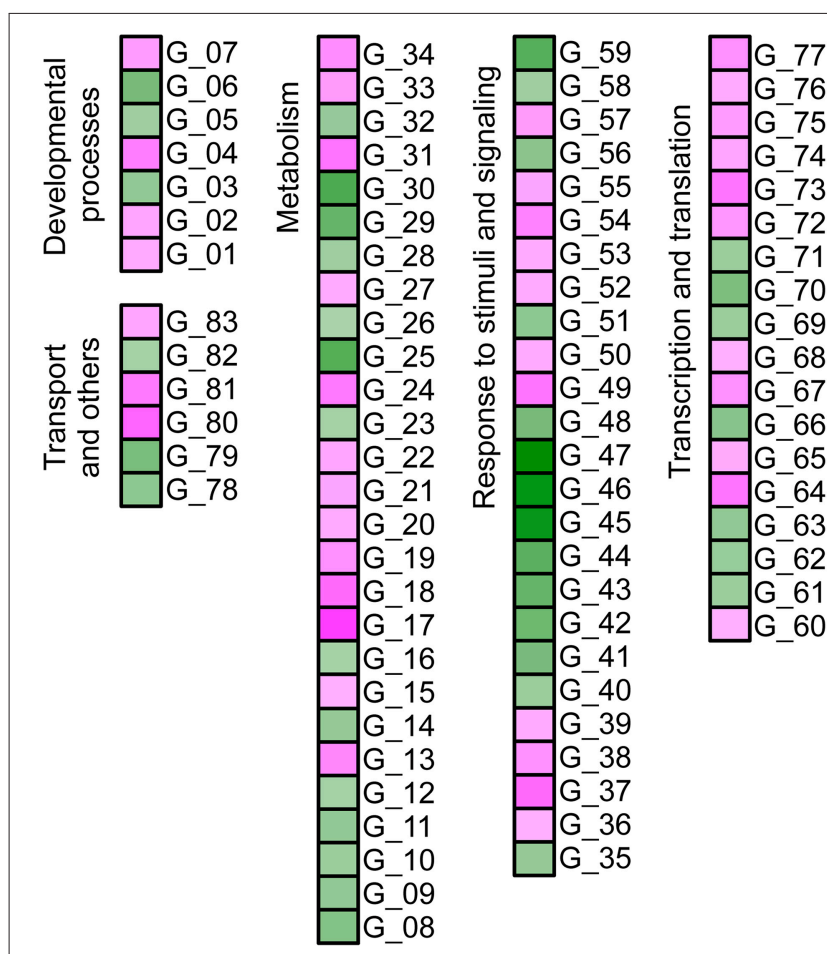

FIGURE 6 | Effect of $1 \mathrm{mM}$ exogenous GABA on gene expression profile of gat1 samples under low $\mathbf{C}$ conditions. Only significantly changed ( $p_{\mathrm{FDR}} \leq 0.05,4$ times fold-change) and functionally annotated genes are presented (see Supplementary Data 5 for details).

of the GABA transporter under $\mathrm{C} / \mathrm{N}$ deficiency in the seedling metabolism. Our results find support in one of the few research exploring GABA effect on plant metabolism: in Brassica napus GABA treatment induced significant changes in the expression of BnNrt2, suggesting that it could act as a putative long-distance inter-organ signal molecule in plants in respect to $\mathrm{N}$ metabolism (Beuve et al., 2004).

Generally, C- and N-limiting conditions resulted in decreased content of most metabolites in comparison with FN medium, but the degree of this decline varied. Low $\mathrm{N}$ induced an expected reduction in the content of most amino acids. These data support previous works. For example Urbanczyk-Wochniak and Fernie showed a wide impact of nitrate deficiency on metabolism, whose main effect was a decrease in the level of amino and carboxylic acids (Scheible et al., 2004; Urbanczyk-Wochniak and Fernie, 2005). The effect of low $\mathrm{N}$ on glutamine and asparagine-AA with the central role in $\mathrm{N}$ assimilation, storage, and transport within the plant (Coruzzi and Bush, 2001)in gat 1 was even greater compared to ws. Specifically under low $\mathrm{N}$ gat1 showed higher content for asparagine, glutamine, arginine, lysine, ornithine compared to ws. The endogenous content of GABA in gat 1 was also significantly higher than that in ws under low $\mathrm{N}$ and low $\mathrm{C}$ conditions and at comparable level under FN medium (Figure 2). In striking contrast, the content of glutamate, while maintaining relatively high (10 time higher than glutamine) in both gat1 and ws, was lower in the mutant under all media. The different response of the closely related glutamate and glutamine to the medium conditions can be thus attributed to glutamate central position in amino acid metabolism (Stitt et al., 2002) or to the reversibility of amino acid transferase reactions as suggested by Forde and Lea (2007). Taken together, these metabolic data could suggest that the glutamate conversion to GABA is shifted toward the production of the other amino acids. A limited export of GABA could lead to its accumulation in the cell; enhanced transamination reactions of GABA would follow and contribute to other amino acids. Furthermore GABA-induced feedback inhibition of glutamate decarboxylase (Porter and Martin, 1984) would eventually lead to glutamate repartitioning to different amino acids.

In contrast to a decreased amino acid pool under $\mathrm{N}$ limitation we measured an accumulation of malate in both genotypes compared to FN. Malate accumulation under N deficiency was already observed (Pasqualini et al., 2001) and it could be attributed to a transient storage of $\mathrm{C}$ (Recht et al., 2014). However, when comparing gat1 to ws, a higher GABA to glutamate ratio (in the first) was accompanied by lower abundance of virtually all TCA cycle intermediates, namely succinate, malate, citrate, and fumarate. The up-regulation of glutamate dehydrogenase (GDH) in the mutant and increased pool of free amino acids likely supports the hypothesis of a repartitioning of $2 \mathrm{OG}$ to glutamate-derived amino acids, emphasizing the link between GABA-glutamate metabolism and GDH, "one of the key checkpoints controlling the C-N status of the plant" (Tercé-Laforgue et al., 2004). Under the proposed scenario of a limited GABA efflux inducing glutamatederived amino acids biosynthesis and cataplerotic TCA cycle activity, gat 1 is suggested to modulate $\mathrm{C}-\mathrm{N}$ metabolism. A very recent report found that GABA modulates plant growth by directly regulating the activity of another transporter, a plantspecific anion transporters for malate (Ramesh et al., 2015). Accumulation of GABA decreased malate efflux by direct binding to the ALMT transporter. This is the first report showing a direct effect of GABA as a signaling molecule mediating cellular metabolism, ending more than a decade-long debate. In our study, malate did not accumulate in the mutant under any media in spite of GABA endogenous accumulation. However, when exogenous GABA was added to the medium, in the WT genotype enhanced influx of GABA led to its accumulation under low $\mathrm{C}$ conditions, and it was accompanied with further decrease in malate and citrate content (compared to FN). In the gat1 mutant, GABA feeding caused no changes in GABA content under low $\mathrm{C}$, very likely due to limited GABA influx. The absence of the one specific GABA transporter can be partly compensated by other quaternary transporters (i.e., two known amino acid transporters, amino acid permease 3 , AAP3, and the proline transporters 2, ProT2, potentially transport GABA). However, in plants like in animals and bacteria, related compounds like proline compete with GABA and inhibit its transport via-for instance-ProT2. Supplemented GABA to gat1 seedlings led to a significant and unexpected alteration in $\beta$-glucose and glycerol (lines of evidence in mammals suggest GABA inhibits glucagon secretion and glucose in blood, (Bailey et al., 2007). GABA treatment in gat 1 mutant also significantly affected the regulation of pyruvate kinase, an enzyme involved in the production of 
pyruvate from PEP, that is the entry point between the glycolysis and the TCA cycle. Furthermore, we detected a decrease of malic acid-2-methyl, substrate for pyruvate production, and citrate following $1 \mathrm{mM}$ GABA feeding (Figure 5). Thus a role of GABA in the regulation of $C$ metabolism is very likely and suggested by (i) previous studies in transgenic Arabidopsis plants with a deregulated GAD which showed alterations in $\mathrm{N}$ to $\mathrm{C}$ ratio in Arabidopsis seeds (Fait et al., 2011) and by (ii) the effect of GABA on the metabolism of ws Arabidopsis seedlings under $\mathrm{C}$ deficiency (Batushansky et al., 2014) or when grown in darkness (Araújo et al., 2010). Having said that, surprisingly the malate content in the mutant under low $\mathrm{C}$ did not change when exogenous GABA was applied. Under the very recent scenario proposed by Ramesh, we would suggest that increased extracellular GABA (by the feeding treatment) bound to the ALMT site, which by convention are extracellular preventing malate efflux. It should be noted that this is a hypothesis prompted by the very recent findings by Ramesh (Ramesh et al., 2015). While tempting, in our study we did not distinguish between intra and extracellular partitioning of metabolites and the ALMT binding site location needs validation. Future studies will need to test the validity of this hypothesis.

\section{References}

Araújo, W. L., Ishizaki, K., Nunes-Nesi, A., Larson, T. R., Tohge, T., Krahnert, I., et al. (2010). Identification of the 2-Hydroxyglutarate and Isovaleryl-CoA Dehydrogenases as alternative electron donors linking lysine catabolism to the electron transport chain of Arabidopsis Mitochondria. Plant Cell 22, 1549-1563. doi: 10.1105/tpc.110.075630

Bailey, S. J., Ravier, M. A., and Rutter, G. A. (2007). Glucose-Dependent Regulation of $\gamma$-Aminobutyric Acid (GABAA) receptor expression in mouse pancreatic Islet $\alpha$-Cells. Diabetes 56, 320-327. doi: 10.2337/db06-0712

Batushansky, A., Kirma, M., Grillich, N., Toubiana, D., Pham, P. A., Balbo, I., et al. (2014). Combined transcriptomics and metabolomics of Arabidopsis thaliana seedlings exposed to exogenous GABA suggest its role in plants is predominantly metabolic. Mol. Plant 7, 1065-1068. doi: 10.1093/mp/ssu017

Baum, G., Chen, Y., Arazi, T., Takatsuji, H., and Fromm, H. (1993). A plant glutamate decarboxylase containing a calmodulin binding domain. Cloning, sequence, and functional analysis. J. Biol. Chem. 268, 19610-19617.

Baum, G., Lev-Yadun, S., Fridmann, Y., Arazi, T., Katsnelson, H., Zik, M., et al. (1996). Calmodulin binding to glutamate decarboxylase is required for regulation of glutamate and GABA metabolism and normal development in plants. EMBO J. 15, 2988-2996.

Beuve, N., Rispail, N., Laine, P., Cliquet, J. B., Ourry, A., and Le Deunff, E. (2004). Putative role of $\gamma$-aminobutyric acid (GABA) as a long-distance signal in up-regulation of nitrate uptake in Brassica napus L. Plant Cell Environ. 27, 1035-1046. doi: 10.1111/j.1365-3040.2004.01208.x

Bouché, N., and Fromm, H. (2004). GABA in plants: just a metabolite? Trends Plant Sci. 9, 110-115. doi: 10.1016/j.tplants.2004.01.006

Breitkreuz, K. E., Shelp, B. J., Fischer, W. N., Schwacke, R., and Rentsch, D. (1999). Identification and characterization of GABA, proline and quaternary ammonium compound transporters from Arabidopsis thaliana. FEBS Lett. 450, 280-284. doi: 10.1016/S0014-5793(99)00516-5

Coruzzi, G., and Bush, D. R. (2001). Nitrogen and carbon nutrient and metabolite signaling in plants. Plant Physiol. 125, 61-64. doi: 10.1104/pp.125.1.61

Fait, A., Fromm, H., Walter, D., Galili, G., and Fernie, A. R. (2008). Highway or byway: the metabolic role of the GABA shunt in plants. Trends Plant Sci. 13, 14-19. doi: 10.1016/j.tplants.2007.10.005

Fait, A., Nesi, A. N., Angelovici, R., Lehmann, M., Pham, P. A., Song, L., et al. (2011). Targeted enhancement of glutamate-to-gamma-aminobutyrate
Last, among the 393 up-regulated genes in the mutant a few are involved in phenylpropanoid metabolism, including chorismate mutase of the shikimate pathway. The link between GABA and secondary metabolism is unclear, however a targeted enhancement of glutamate decarboxylase in Arabidopsis seeds was previously shown to lead to the upregulation of genes of the shikimate pathway and tryptophan metabolism (Fait et al., 2011). Future studies should try to address the link between the GABA shunt and the shikimate metabolism.

To conclude, the present study on the effects of GABA feeding in different media on Arabidopsis gat1 mutant seedlings suggest a GABA mediated regulation of $\mathrm{C}$ to $\mathrm{N}$ metabolism possibly by the newly discovered ALMT. Further studies are needed to dissect between the metabolic and signaling role of GABA in mediating changes in cellular metabolism.

\section{Supplementary Material}

The Supplementary Material for this article can be found online at: http://journal.frontiersin.org/article/10.3389/fpls.2015. 00785

conversion in Arabidopsis seeds affects carbon-nitrogen balance and storage reserves in a development-dependent manner. Plant Physiol. 157, 1026-1042. doi: 10.1104/pp.111.179986

Fischer, W. N., Loo, D. D., Koch, W., Ludewig, U., Boorer, K. J., Tegeder, M., et al. (2002). Low and high affinity amino acid $\mathrm{H}+$-cotransporters for cellular import of neutral and charged amino acids. Plant J. 29, 717-731. doi: 10.1046/j.1365313X.2002.01248.x

Forde, B. G., and Lea, P. J. (2007). Glutamate in plants: metabolism, regulation, and signalling. J. Exp. Bot. 58, 2339-2358. doi: 10.1093/jxb/erm121

Forde, B. G. (2002). Local and long-range signaling pathways regulating plant responses to nitrate. Annu. Rev. Plant Biol. 53, 203-224. doi: 10.1146/annurev.arplant.53.100301.135256

Huang da, W., Sherman, B. T., and Lempicki, R. A. (2009). Systematic and integrative analysis of large gene lists using DAVID bioinformatics resources. Nat. Protoc. 4, 44-57. doi: 10.1038/nprot.2008.211

Hummel, J., Selbig, J., Walther, D., and Kopka, J. (2007). “The Golm Metabolome Database: a database for GC-MS based metabolite profiling," in Metabolomics, eds J. Nielsen and M. Jewett (Berlin Heidelberg: Springer), 75-95.

Irizarry, R. A., Hobbs, B., Collin, F., Beazer-Barclay, Y. D., Antonellis, K. J., Scherf, U., et al. (2003). Exploration, normalization, and summaries of high density oligonucleotide array probe level data. Biostatistics 4, 249-264. doi: 10.1093/biostatistics/4.2.249

Krysan, P. J., Young, J. C., Tax, F., and Sussman, M. R. (1996). Identification of transferred DNA insertions within Arabidopsis genes involved in signal transduction and ion transport. Proc. Natl. Acad. Sci. U.S.A. 93, 8145-8150. doi: 10.1073/pnas.93.15.8145

Lisec, J., Schauer, N., Kopka, J., Willmitzer, L., and Fernie, A. R. (2006). Gas chromatography mass spectrometry-based metabolite profiling in plants. Nat. Protoc. 1, 387-396. doi: 10.1038/nprot.2006.59

Luedemann, A., Strassburg, K., Erban, A., and Kopka, J. (2008). TagFinder for the quantitative analysis of gas chromatography-mass spectrometry (GCMS)-based metabolite profiling experiments. Bioinformatics 24, 732-737. doi: 10.1093/bioinformatics/btn023

Meyer, A., Eskandari, S., Grallath, S., and Rentsch, D. (2006). AtGAT1, a high affinity transporter for gamma-aminobutyric acid in Arabidopsis thaliana. J. Biol. Chem. 281, 7197-7204. doi: 10.1074/jbc.M510766200

Michaeli, S., Fait, A., Lagor, K., Nunes-Nesi, A., Grillich, N., Yellin, A., et al. (2011) A mitochondrial GABA permease connects the GABA shunt and the TCA 
cycle, and is essential for normal carbon metabolism. Plant J. 67, 485-498. doi: 10.1111/j.1365-313X.2011.04612.x

Osuna, D., Usadel, B., Morcuende, R., Gibon, Y., Bläsing, O. E., Höhne, M., et al. (2007). Temporal responses of transcripts, enzyme activities and metabolites after adding sucrose to carbon-deprived Arabidopsis seedlings. Plant J. 49, 463-491. doi: 10.1111/j.1365-313X.2006.02979.x

Pasqualini, S., Ederli, L., Piccioni, C., Batini, P., Bellucci, M., Arcioni, S., et al. (2001). Metabolic regulation and gene expression of root phosphoenolpyruvate carboxylase by different nitrogen sources. Plant Cell Environ. 24, 439-447. doi: 10.1046/j.1365-3040.2001.00692.x

Porter, T. G., and Martin, D. L. (1984). Evidence for feedback regulation of glutamate decarboxylase by gamma-aminobutyric acid. J. Neurochem. 43, 1464-1467. doi: 10.1111/j.1471-4159.1984.tb05409.x

Ramesh, S. A., Tyerman, S. D., Xu, B., Bose, J., Kaur, S., Conn, V., et al. (2015). GABA signalling modulates plant growth by directly regulating the activity of plant-specific anion transporters. Nat. Commun. 6:7879. doi: 10.1038/ncomms8879

Recht, L., Töpfer, N., Batushansky, A., Sikron, N., Zarka, A., Gibon, Y., et al. (2014). Metabolite profiling and integrative modeling reveal metabolic constraints for carbon partitioning under nitrogen-starvation in the green alga haematococcus pluvialis. J. Biol. Chem. 289, 30387-30403. doi: 10.1074/jbc.M114.555144

Saeed, A. I., Sharov, V., White, J., Li, J., Liang, W., Bhagabati, N., et al. (2003). TM4: a free, open-source system for microarray data management and analysis. Biotechniques 34, 374-378.

Scheible, W. R., Morcuende, R., Czechowski, T., Fritz, C., Osuna, D., PalaciosRojas, N., et al. (2004). Genome-wide reprogramming of primary and secondary metabolism, protein synthesis, cellular growth processes, and the regulatory infrastructure of Arabidopsis in response to nitrogen. Plant Physiol. 136, 2483-2499. doi: 10.1104/pp.104.047019
Shelp, B. J., Bown, A. W., and McLean, M. D. (1999). Metabolism and functions of gamma-aminobutyric acid. Trends Plant Sci. 4, 446-452. doi: 10.1016/S13601385(99)01486-7

Steward, F., Thompson, J., and Dent, C. (1949). $\gamma$-Aminobutyric acid: a constituent of the potato tuber? Science, 439-440.

Stitt, M., Müller, C., Matt, P., Gibon, Y., Carillo, P., Morcuende, R., et al. (2002). Steps towards an integrated view of nitrogen metabolism. J. Exp. Bot. 53, 959-970. doi: 10.1093/jexbot/53.370.959

Tercé-Laforgue, T., Dubois, F., Ferrario-Méry, S., De Crecenzo, M. A., Sangwan, R., and Hirel, B. (2004). Glutamate dehydrogenase of tobacco is mainly induced in the cytosol of phloem companion cells when ammonia is provided either externally or released during photorespiration. Plant Physiol. 136, 4308-4317. doi: $10.1104 /$ pp. 104.047548

Urbanczyk-Wochniak, E., and Fernie, A. R. (2005). Metabolic profiling reveals altered nitrogen nutrient regimes have diverse effects on the metabolism of hydroponically-grown tomato (Solanum lycopersicum) plants. J. Exp. Bot. 56, 309-321. doi: 10.1093/jxb/eri059

Conflict of Interest Statement: The authors declare that the research was conducted in the absence of any commercial or financial relationships that could be construed as a potential conflict of interest.

Copyright (c) 2015 Batushansky, Kirma, Grillich, Pham, Rentsch, Galili, Fernie and Fait. This is an open-access article distributed under the terms of the Creative Commons Attribution License (CC BY). The use, distribution or reproduction in other forums is permitted, provided the original author(s) or licensor are credited and that the original publication in this journal is cited, in accordance with accepted academic practice. No use, distribution or reproduction is permitted which does not comply with these terms. 


\section{Suppressor mutations in the Glutamine Dumper1 protein dissociate disturbance in amino acid transport from other characteristics of the Gdu1D phenotype}

\section{OPEN ACCESS}

Edited by:

Dietmar Funck,

University of Konstanz, Germany

Reviewed by:

John M. Ward

University of Minnesota, USA

Dominique Loqué,

Lawrence Berkeley National

Laboratory, USA

*Correspondence:

Guillaume Pilot,

Department of Plant Pathology,

Physiology and Weed Science,

Virginia Polytechnic Institute and State

University, 511 Latham Hall,

220 AG Quad Lane, Blacksburg,

VA 24061, USA gpilot@vt.edu

Specialty section:

This article was submitted to

Plant Physiology,

a section of the journa

Frontiers in Plant Science

Received: 30 April 2015

Accepted: 17 July 2015

Published: 04 August 2015

Citation:

Yu S, Pratelli R, Denbow C and Pilot $G$

(2015) Suppressor mutations

in the Glutamine Dumper1 protein dissociate disturbance in amino acid transport from other characteristics

of the Gdu1D phenotype.

Front. Plant Sci. 6:593.

doi: 10.3389/fp/s.2015.00593

\section{Shi Yu, Réjane Pratelli, Cynthia Denbow and Guillaume Pilot*}

Department of Plant Pathology, Physiology and Weed Science, Virginia Polytechnic Institute and State University, Blacksburg, VA, USA

Intracellular amino acid transport across plant membranes is critical for metabolic pathways which are often split between different organelles. In addition, transport of amino acids across the plasma membrane enables the distribution of organic nitrogen through the saps between leaves and developing organs. Amino acid importers have been studied for more than two decades, and their role in this process is well-documented. While equally important, amino acid exporters are not wellcharacterized. The over-expression of GDU1, encoding a small membrane protein with one transmembrane domain, leads to enhancement of amino acid export by Arabidopsis cells, glutamine secretion at the leaf margin, early senescence and size reduction of the plant, possibly caused by the stimulation of amino acid exporter(s). Previous work reported the identification of suppressor mutations of the GDU1 over-expression phenotype, which affected the GDU1 and LOG2 genes, the latter encoding a membrane-bound ubiquitin ligase interacting with GDU1. The present study focuses on the characterization of three additional suppressor mutations affecting GDU1. Size, phenotype, glutamine transport and amino acid tolerance were recorded for recapitulation plants and over-expressors of mutagenized GDU1 proteins. Unexpectedly, the over-expression of most mutated GDU1 led to plants with enhanced amino acid export, but failing to display secretion of glutamine and size reduction. The results show that the various effects triggered by GDU1 over-expression can be dissociated from one another by mutagenizing specific residues. The fact that these residues are not necessarily conserved suggests that the diverse biochemical properties of the GDU1 protein are not only born by the characterized transmembrane and VIMAG domains. These data provide a better understanding of the structure/function relationships of GDU1 and may enable modifying amino acid export in plants without detrimental effects on plant fitness.

Keywords: Arabidopsis, suppressor screening, ethyl methanesulfonate, structure-function, amino acid transporter, glutamine transport 


\section{Introduction}

Amino acids are critical metabolites in plants which fulfill several roles, in addition to being the constituting blocks of proteins. Amino acids are used as precursors for the synthesis of many secondary metabolites, like flavonoids (Falcone Ferreyra et al., 2012), alkaloids (Ziegler and Facchini, 2008), and glucosinolates (de Kraker and Gershenzon, 2011), which are metabolites critical for interaction of the plant with the environment (attraction, defense, and protection). Amino acids, especially Gln and Asn, also serve as essential carriers for organic nitrogen throughout the plant, being transported through the xylem and phloem saps between leaves, roots, storage organs, and meristems (Tegeder, 2014). Transport in the plant is mediated at the cell level by membrane proteins with specialized functions. Amino acid importers and exporters mediate transport of amino acids in opposite directions: importers mediate transport toward the cytosol, exporters mediate transport out from the cytosol, and respectively from or to the apoplasm, vacuoles or intracellular vesicles.

Characterized amino acid importers belong to the APC superfamily (Amino Acid Polyamine transporter; Vastermark et al., 2014), composed of 63 members in Arabidopsis (Tegeder and Rentsch, 2010). Importers utilize energy of the proton gradient across membranes to import amino acids against their concentration gradient, and are involved in many roles, like uptake from the soil, import into the phloem, phloem-xylem exchange, and transport into the embryo (Tegeder, 2014). Much less is known about amino acid exchangers and exporters. One member of the CAT subfamily has recently been described as an amino acid exchanger in tomato (Snowden et al., 2015). Two APC members have been described as possible amino acid exporters: CAT8 (Yang et al., 2010) and BAT1/GABP1 (Dundar and Bush, 2009; Michaeli et al., 2011). Finally, one gene belonging to the Drug and Metabolite Transporter superfamily (Jack et al., 2001), SIAR1, has been shown to unequivocally mediate amino acid export from plant cells (Ladwig et al., 2012). The family SIAR1 belongs to contains 47 members with only one other gene characterized, the auxin transporter WAT1 (Ranocha et al., 2013).

The existence of processes controlling the activity of amino acid transporters was evidenced by the discovery of the GDU1 gene, encoding an 158 amino acid protein, with a single transmembrane domain (Pilot et al., 2004). Two domains are conserved among GDU proteins: a membrane domain, and a cytosolic 19 amino acid-long region, called the VIMAG domain (Pilot et al., 2004; see Figure 1). Over-expression of GDU1, for instance in the gdu1-1D mutant, leads to a complex phenotype characterized by reduced plant size; early leaf senescence; crystallization of Gln at the leaf margins; increased amino acid content in leaves, apoplasm, and xylem and phloem saps; tolerance to exogenously supplied amino acids; and notably enhanced amino acid export from cells, while amino acid import remains unaffected (Pilot et al., 2004; Pratelli and Pilot, 2007; Pratelli et al., 2010). This complex phenotype (called Gdu1D) can almost entirely be explained by enhanced amino acid export from cells: this phenomenon would increase amino acid content in the apoplasm and phloem and xylem saps, and prevent absorption of Gln from the xylem in the leaf, which then is excreted by the hydathodes (Pilot et al., 2004). Size reduction likely comes from the induced disturbance in nitrogen metabolism. The fact that over-expression of GDU1 in Nicotiana tabacum and GDU1-homologs in Arabidopsis leads to a Gdu1D-similar phenotype suggests that the proteins of this family have a conserved function in plants, related to the regulation of amino acid export (Pratelli and Pilot, 2006; Pratelli et al., 2010). Nevertheless, the precise function of GDU1 in this process remains unknown.

Attempts to understand the function of GDU1 in the plant led to the identification of the ubiquitin ligase LOG2 in a yeasttwo-hybrid screening. GDU1 and LOG2 localize at the plasma membrane and are able to co-immunoprecipitate when expressed in Nicotiana benthamiana (Pratelli et al., 2012). Decrease in LOG2 expression suppressed the Gdu1D phenotype, indicating that LOG2 activity is necessary for the development of the phenotype (Pratelli et al., 2012). A mammalian homolog of LOG2 is the mahogunin protein (MGRN1;45\% similarity at the protein level), involved in the regulation of the activity and trafficking of membrane proteins and in degrading aggregated proteins (Jiao et al., 2009; Perez-Oliva et al., 2009; Gunn et al., 2013; Chhangani et al., 2014). Despite coming from different organisms, MGRN1 and LOG2 were found to have several overlapping functional properties, notably the ability of the mammalian protein to partially complement the loss of the plant LOG2 in Arabidopsis (Guerra et al., 2013). It is hypothesized that LOG2 and GDU1 are involved in the regulation of the activity or trafficking of amino acid exporters, such as, when GDU1 is over-expressed, the exporters are more active at the plasma membrane (Pratelli et al., 2012; Guerra et al., 2013).

An ethyl methanesulfonate (EMS) screening was previously performed to isolate suppressor mutations of the GdulD phenotype. For this purpose, two GDU1 over-expressing lines ( $g d u 1-5 D$ and $g d u 1-6 D$, created by introducing a GDU1over-expressing construct into wild type plants) were EMSmutagenized, and screened for progenies that grew similarly to the wild type. These suppressor lines hence over-express a mutant GDU1 protein in addition to the endogenous GDU1 protein. Two suppressor mutants, loss of gdu1-1 and 2-1 (log1-1 and $\log 2-1)$ were isolated and characterized (Pratelli and Pilot, 2006; Pratelli et al., 2012). $\log 1-1$ carries a G100R substitution in the conserved VIMAG domain of GDU1, which abolishes the interaction with LOG2 (Pratelli et al., 2012). The $\log 2-1$ is a R12K substitution in LOG2, whose effect has not been determined at the biochemical level (Pratelli et al., 2012). Another mutation, $\log 2-3$, was isolated from the same screening, and is a nonsense mutation in LOG2 (R15stop; Pratelli and Pilot, unpublished data). These three $\log$ mutations led to plants that were phenotypically indistinguishable from the wild type when grown on soil and on amino acidcontaining media. From the same screening, we isolated three additional $\log 1$ mutations, whose characterization is reported here. 


\section{Results}

\section{Identification of Three New Mutations Suppressing the Gdu1D Phenotype}

The pipeline previously described by Pratelli and Pilot (2006) was used to isolate new Gdu1D suppressor mutants: visual screening of M2 plants from the EMS mutagenesis of the GDU1 overexpressors $g d u 1-5 D$ and $g d u 1-6 D$, and confirmation of continued GDU1 mRNA over-accumulation. Out of a total of 110,000 M2 seeds screened, three additional suppressor mutations were isolated that nearly restored the wild type size of the plants (Supplementary Figure S1), and suppressed the early senescence and Gln secretion. Analysis of the phenotype of F2 plants from crosses of the mutants with $g d u 1-6 D$ and the wild type Col-7 showed that the three new mutations were recessive and segregated in accordance with an intragenic mutation in GDU1 (data not shown). Similar to $\log 1-1$, the corresponding mutants came from the mutagenesis of the over-expressor $g d u 1$ $6 D$ (Pratelli and Pilot, 2006), and were named $\log 1-2, \log 1-3$, and $\log 1-4$. Sequencing of the GDU1 CDS in the T-DNA construct leading to its over-expression revealed that the mutations in $\log 1-2, \log 1-3$, and $\log 1-4$ corresponded to G202A, G119A, and C95T mutations (numbered from the ATG), respectively, in the GDU1 DNA sequence. These mutations led to E68K, G40D, and S32L substitutions, respectively, in the GDU1 protein sequence (Figure 1). Because these mutations suppressed the Gdu1D visual phenotype (plant size, Gln secretion, and early senescence), it was hypothesized that they affected GDU1 protein function. The $\log 1$ mutants were hence hypothesized to over-express inactive GDU1 proteins and the characterization of the mutations was thus a way to better understand GDU1's structure-function relationships.

Amino acid uptake of GDU1 over-expressing lines was shown to be reduced, while efflux was enhanced (Pratelli et al., 2010). It was expected that the suppressor lines would display similar uptake and efflux as wild type plants, because of their wild type phenotype on soil. To test this hypothesis, Gln uptake of the suppressor lines was analyzed and compared to the wild type and the parental line $g d u 1-6 D$. Since this assay was not performed in the $\log 1-1$ plants (Pratelli and Pilot, 2006), they were included in the present study. The $\log 1-1, \log 1-2, \log 1$ 3 , and $\log 1-4$ suppressor mutants showed uptake and efflux similar to one another, but surprisingly different from both the wild type and $g d u 1-6 D$ : the four $\log 1$ mutants had an uptake about $60 \%$ lower than the wild type (Figure 2A) and an efflux twice as large as the wild type (Figure 2B). As a comparison, Gln uptake of $g d u 1-6 D$ was $75 \%$ lower and the efflux was three times larger than the wild type. The intermediate phenotype was clearly visible when uptake and efflux were plotted on the same graph (Figure 2C). This result suggests that these recessive suppressor mutations decrease GDU1 overall activity and thus lead to a milder phenotype. It has indeed been shown that the strength of the GdulD phenotype depends on the GDU1 expression level and that the plants are small and display early senescing leaves only when GDU1 is overexpressed 10 times or more than the wild type (Pilot et al., 2004).

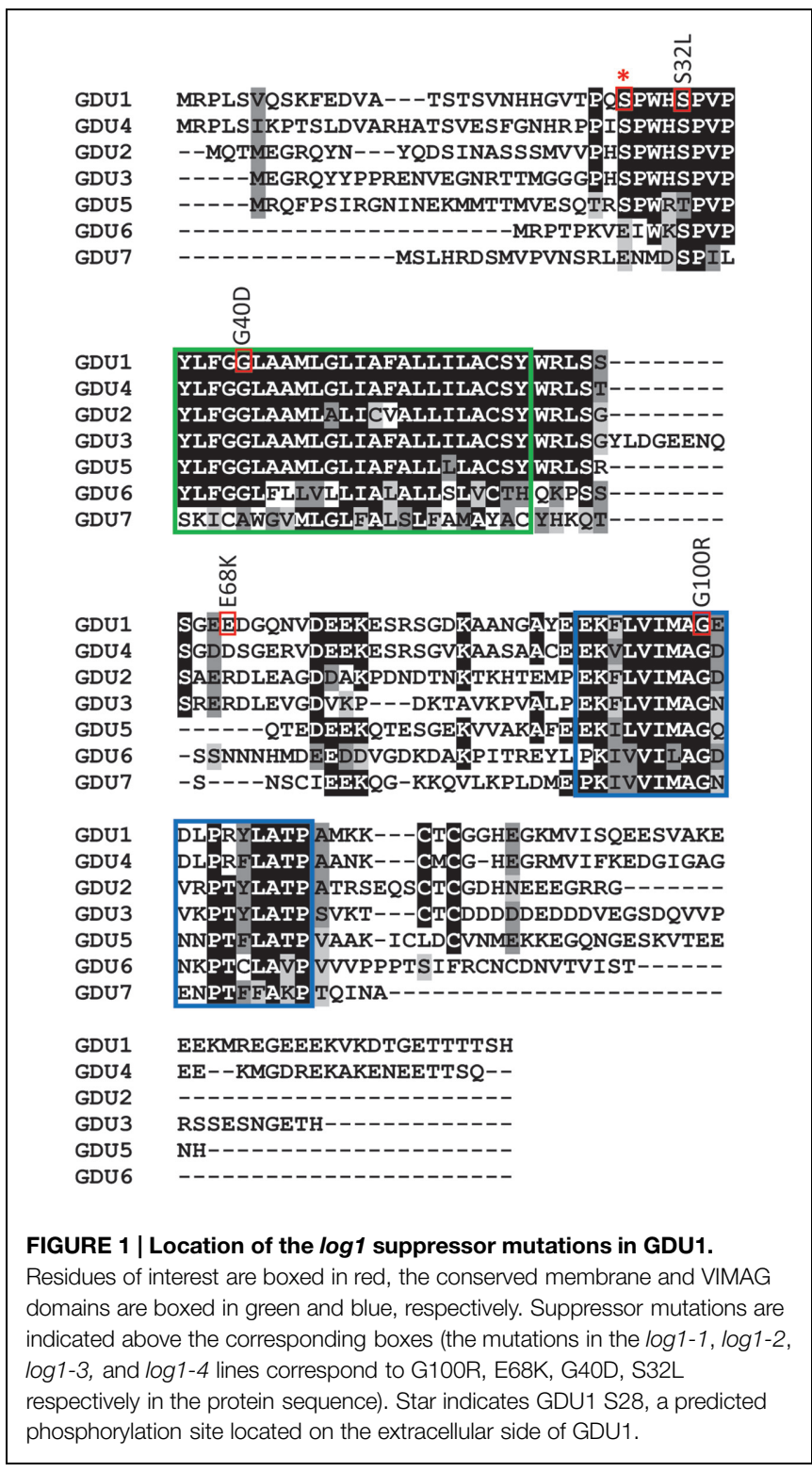

\section{Characterization of Plants Over-Expressing the GDU1 Variant Proteins}

Despite two different attempts, no suitable antibody could be raised against GDU1. The $g d u 1-5 D$ and $g d u 1-6 D$ plants and the corresponding log suppressor mutants express an un-tagged GDU1 protein, preventing any quantitation of the GDU1 protein accumulation. In order to test for any effect of the suppressor mutations on the accumulation of the GDU1 protein, wild type GDU1, the three GDU1 variants, G100R GDU1 (corresponding to the $\log 1-1$ suppressor mutant; Pratelli and Pilot, 2006) and a GDU1 protein lacking the VIMAG domain ( $\triangle$ VIMAG) were fused with the HA tag, placed under the control of the CaMV $35 \mathrm{~S}$ promoter, and expressed in $N$. benthamiana. The $\triangle$ VIMAG, G100R, E68K, and S32L mutations did not affect protein accumulation, while the G40D mutation led to a consistent reduction of about $30 \%$ in protein accumulation 
A

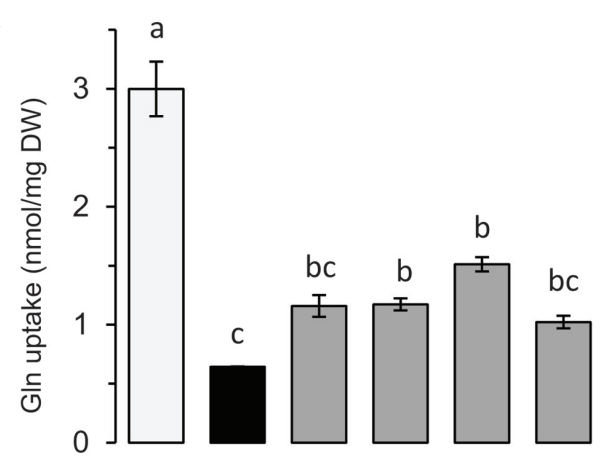

B

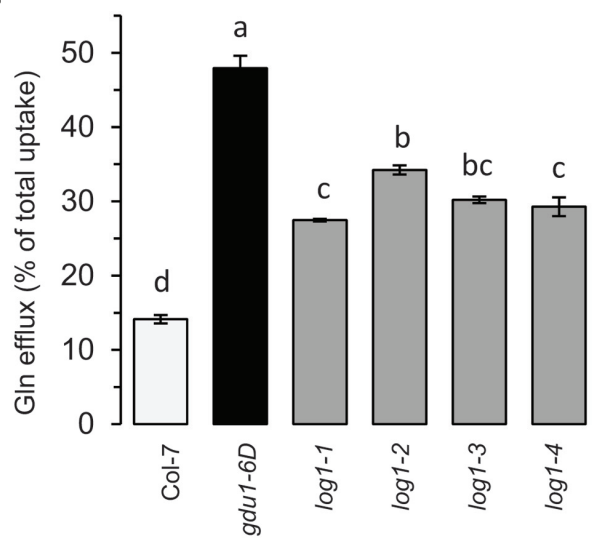

C

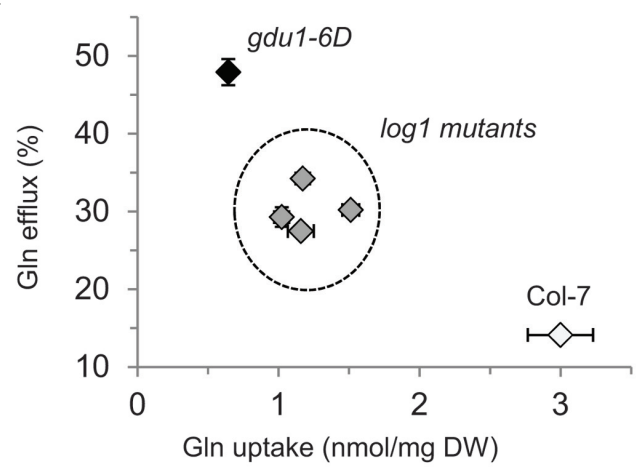

FIGURE 2 | GIn uptake and efflux analyses of the log1 suppressor mutants. (A) Gln uptake and (B) Gln efflux of 2 week-old plantlets. Efflux is expressed as the percentage of total Gln uptake. (C) Graph constructed by compiling the results of (A,B). The wild type Col-7 and gdu1-6D are indicated as white and black bars and symbols respectively. Error bars are SEM $(n=3)$; values with same letters are not statistically different, as determined by ANOVA using Tukey's HSD, $p<0.05$.

in this system (Figure 3). The sub-cellular localization of these five GDU1 variants was determined by expression of the GFP-tagged proteins (GFP positioned in C-terminal) in $N$. benthamiana leaves and observed by confocal microscopy. Similar to GDU1, the proteins localized to the plasma membrane and in compartments that could correspond to endosomes, similar to the wild type GDU1 protein (Pratelli et al., 2012). Interestingly, the fluorescence of these compartments was stronger for the $\triangle$ VIMAG, G100R and G40D GDU1 proteins,

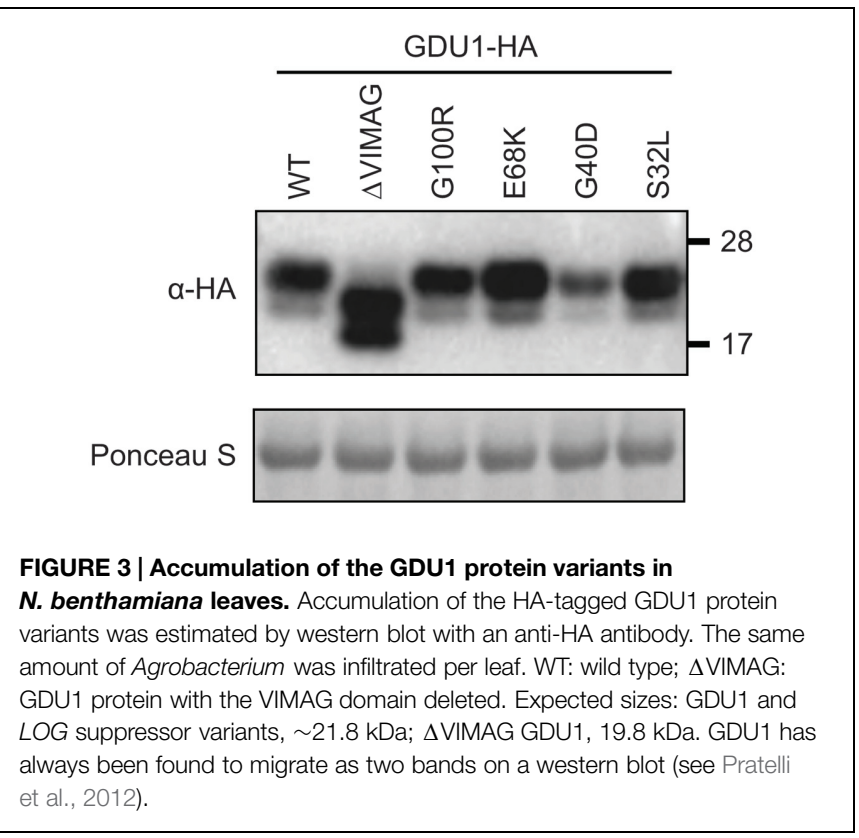

suggesting that the proteins located more in these structures than the other GDU1 proteins (Supplementary Figure S2), possibly revealing some effect of the mutations on the protein properties.

To study the ability of the proteins to lead to the Gdu1D phenotype, the HA-fusion constructs were used to transform Arabidopsis and create recapitulation lines. The accumulation of the proteins in lines that segregated 3:1 for the kanamycin resistance was tested by western blot (Figure 4B). G40D GDU1HA accumulated at a lower level than GDU1-HA, in good agreement with the $N$. benthamiana results. Surprisingly, S32L GDU1-HA did not accumulate to the GDU1-HA levels in both tested lines, but was still present at high levels. In all cases, the rosette sizes of the plants expressing the GDU1 suppressor variants were identical to the wild type. The plants overexpressing GDU1-HA showed a $\sim 45 \%$ reduction in rosette diameter compared to the wild type and the empty vectortransformed plants (Figure 4A). Only the GDU1-HA overexpressors displayed Gln secretion crystals, typical of the GdulD phenotype. The size reduction of the GDU1-HA lines was less than for the original $g d u 1-1 D$ over-expressor ( $~ 60 \%$; Figure 4A), and could be attributed to the difference in the construct used or the presence of the HA tag, which might slightly interfere with the protein stability or activity.

Since the $\log 1$ mutants showed intermediate Gln uptake and efflux between the wild type and the gdu1-6D mutant, Gln transport by the recapitulation lines was studied. G40D and S32L GDU1-HA over-expressing plants behaved similarly to the corresponding $\log 1-3$ and $\log 1-4$ suppressor mutants: the uptake was reduced by $\sim 50 \%$ while the efflux was increased by $\sim 50-100 \%$. On the contrary, E68K GDU1-HA overexpressors transported Gln similarly to the GDU1-HA plants, differently from the $\log 1-2$ plants that they were supposed to recapitulate (Figures 5A,B). This discrepancy between the Gln uptake and efflux of the $\log 1-2$ suppressor mutant and 


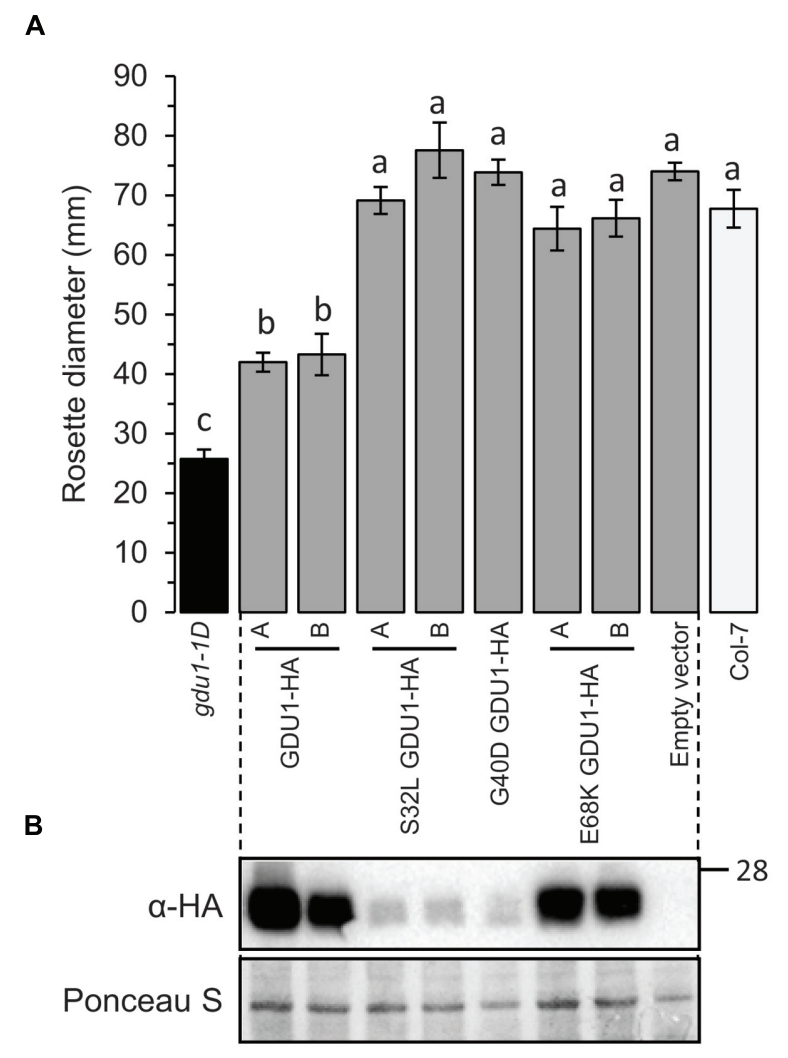

FIGURE 4 | Analysis of plants over-expressing the GDU1 variants. (A) Rosette diameter of 4 week-old Arabidopsis. Error bars are SEM of 6-8 plants; statistical significance determined by ANOVA using Tukey's HSD $p<0.01$. (B) Accumulation of the HA-tagged GDU1 proteins in each line was estimated by western blot with an anti-HA antibody. The wild type Col-7 and gdu1-1D lines are indicated as white and black bars; these lines do not express any tagged protein and were not tested by western blotting.

E68K GDU1-HA over-expressors was unexpected and is not completely explained. It is possible that the presence of the HA tag interferes with the function of the mutated protein, similar to what was observed with the wild type GDU1 (the GDU1-HA over-expression line exhibits a weaker phenotype than the original $g d u 1-1 D$; Figure 4A). These data prove that the intermediate phenotype of the $\log 1-3$ and $\log 1-4$ mutants is caused by the over-expression of variant GDU1 proteins endowed with reduced functionality.

\section{Testing the GDU1 Variants for Interaction with LOG2}

The suppression of the GdulD phenotype by the G100R mutation (in the $\log 1-1$ mutant; Pratelli and Pilot, 2006) was explained by the loss of interaction with LOG2, the ubiquitin ligase necessary for the development of the GdulD phenotype (Pratelli et al., 2012). The G100R mutation affects the Gly100 residue of the VIMAG domain that is conserved in all GDU proteins examined so far. This Gly to Arg mutation (Figure 1) is supposed to either affect the folding of the VIMAG domain or create a steric clash at the interface surface between GDU1 and
A

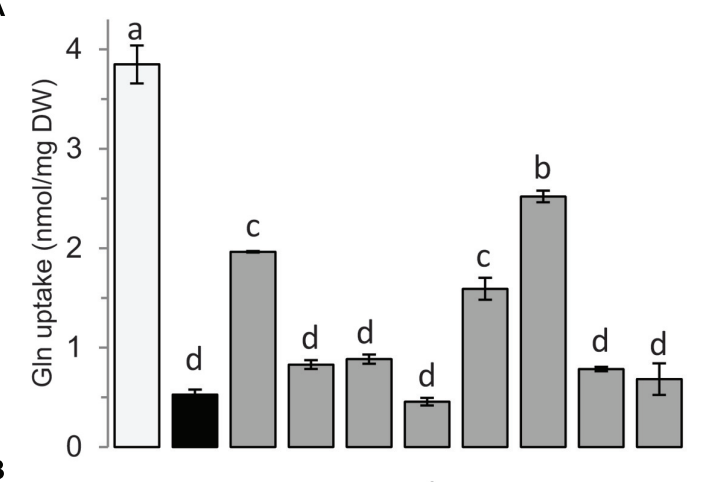

B
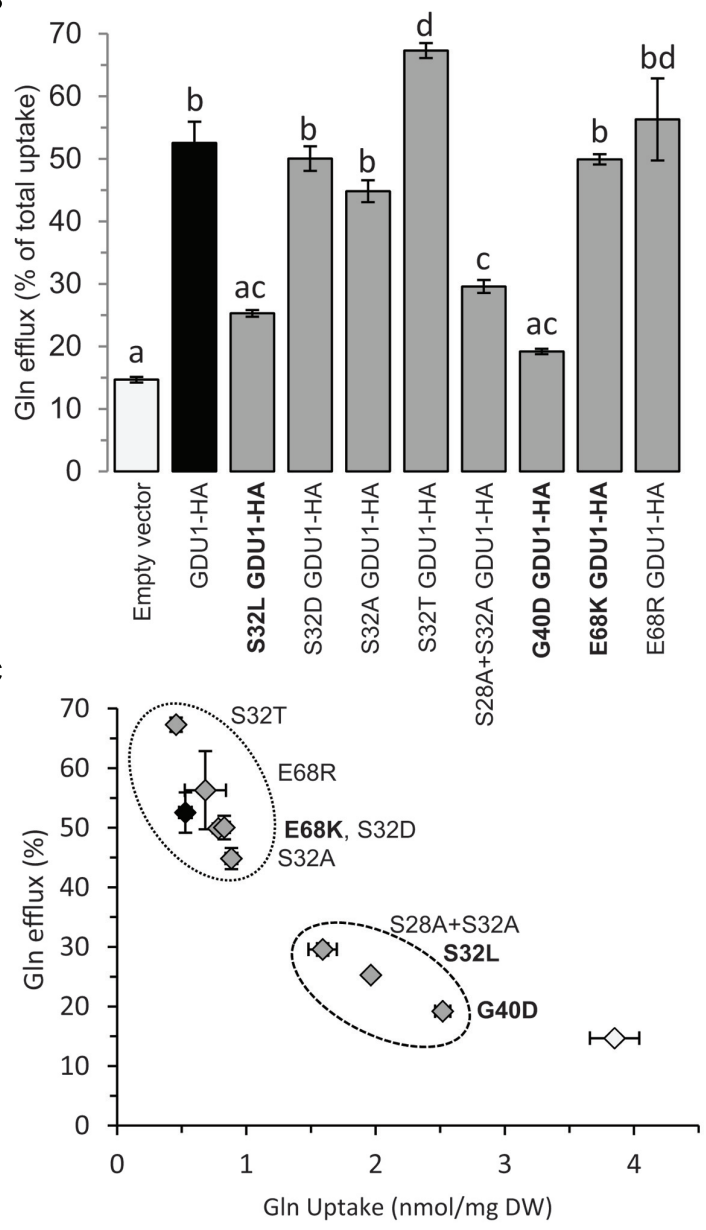

FIGURE 5 | GIn uptake and efflux analyses of plants over-expressing the GDU1 variants. (A) Gln uptake and (B) Gln efflux of 2 week-old plantlets. Efflux is expressed as a percentage of total Gln uptake. (C) Graph compiling the results of $(\mathbf{A}, \mathbf{B})$. Recapitulation lines to the $\log 1-2, \log 1-3$, and $\log 1-4$ mutants are indicated in bold; the empty vector- and GDU1-over-expressing lines are indicated as white and black bars and symbols respectively. Error bars are SEM $(n=3)$; statistical significance determined by ANOVA using Tukey's HSD $p<0.05$.

LOG2 caused by changes in residue charge and/or size. Because the three other $\log 1$ mutants displayed a similar phenotype as $\log 1-1$, we tested if any of the corresponding mutations would 
affect the GDU1-LOG2 interaction, which would explain the suppression of the Gdu1D phenotype.

The LOG2 and GDU1 proteins were fused with the Myc or the HA tag respectively, expressed in $N$. benthamiana leaves and immunoprecipitated using $\mathrm{cMyc}$ agarose beads. The $\triangle \mathrm{VIMAG}$ GDU1 protein, shown to be unable to interact with LOG2 (Pratelli et al., 2012) and to lead to the GdulD phenotype when over-expressed (Pratelli and Pilot, 2006), was used as a negative control (Figure 6). GDU1-HA, E68K GDU1-HA, and S32L GDU1-HA could be co-purified with LOG2-Myc, but G40D GDU1-HA did not co-immunoprecipitate (Figure 6). The G40D mutation, in addition to decreasing protein abundance (Figure 3), thus seems to prevent the interaction of GDU1 with LOG2, which could explain the Gdu1D suppressor effect. On the contrary, the Gdu1D suppressor effect of the other variants cannot be explained by an inability to interact with LOG2.

\section{Site Directed Mutagenesis to Understand the S32L Mutation}

Ser32 is highly conserved among GDU proteins, being sometimes replaced by Thr only, a residue with similar chemical properties (in $13 \%$ of the $\sim 100$ analyzed sequences from 20 plant species, including lower plants, conifers, monocots, and dicots; data

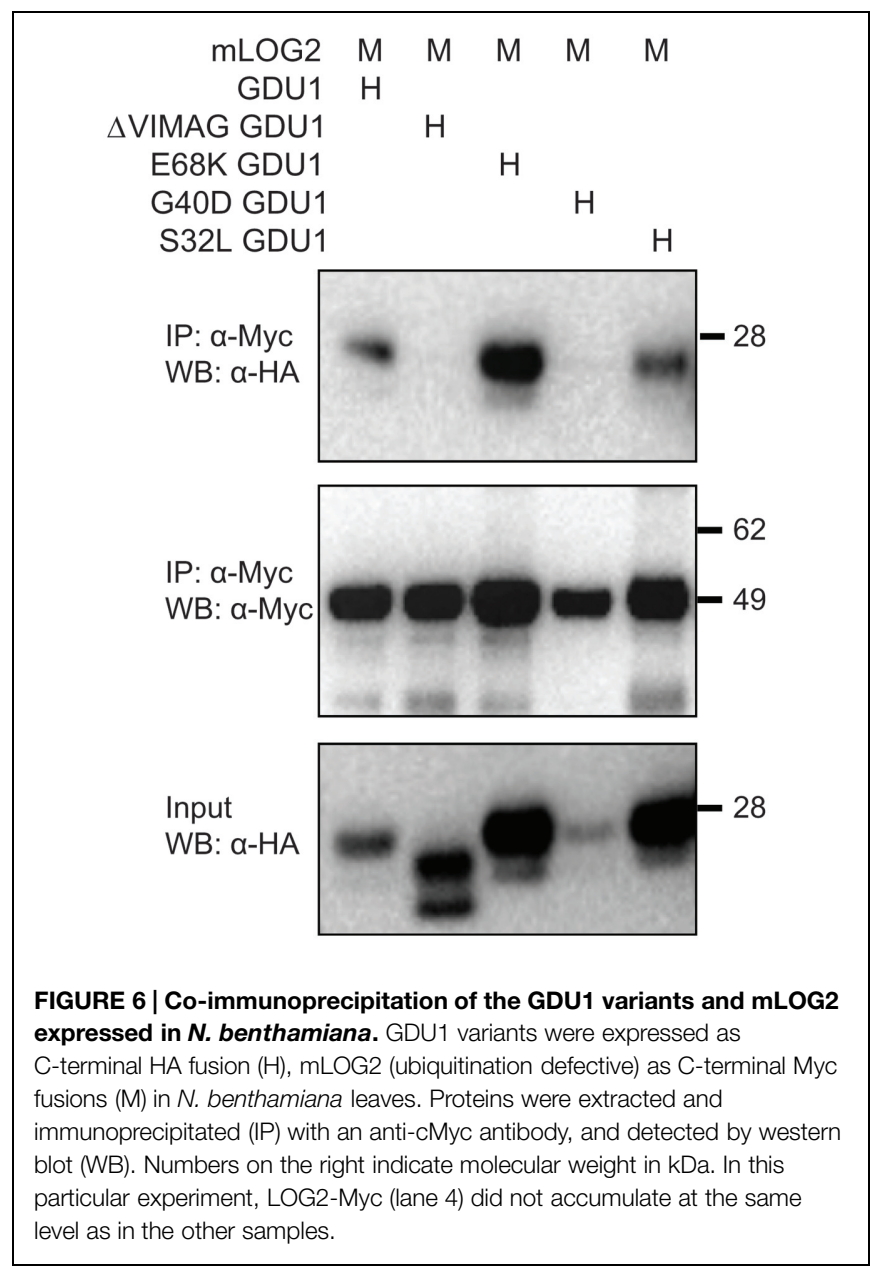

not shown). Running prediction algorithms such as PhosPhAT (Heazlewood et al., 2008; Durek et al., 2010) and PlantPhos (Lee et al., 2011) suggested that Ser32 can be phosphorylated (Supplementary Figure S3) despite the fact that this part of the protein is supposed to be extra-cellular. The PhosPhAT tool also predicted that Ser28, next to Ser32, can be phosphorylated. We hypothesized that these two Ser can be phosphorylated, and that this phosphorylation is important for GDU1 function. Ser32 was mutagenized to Thr, Asp, and Ala to test for (1) the importance of the Ser vs. Thr in GDU1 function, the effect of (2) mimicking and (3) suppressing phosphorylation at this site respectively. Ser 28 and Ser32 were also mutagenized to Ala at the same time to ensure that lack of Ser32 phosphorylation could not be complemented by phosphorylation of Ser28. The mutagenized GDU1 proteins were stably expressed in Arabidopsis in fusion with the HA tag. The size of the plants, protein accumulation, and Gln transport were determined as above.

Western blotting confirmed that all lines expressed the GDU1 protein variants (Figure $7 \mathbf{B}$ ). The size and the phenotype of

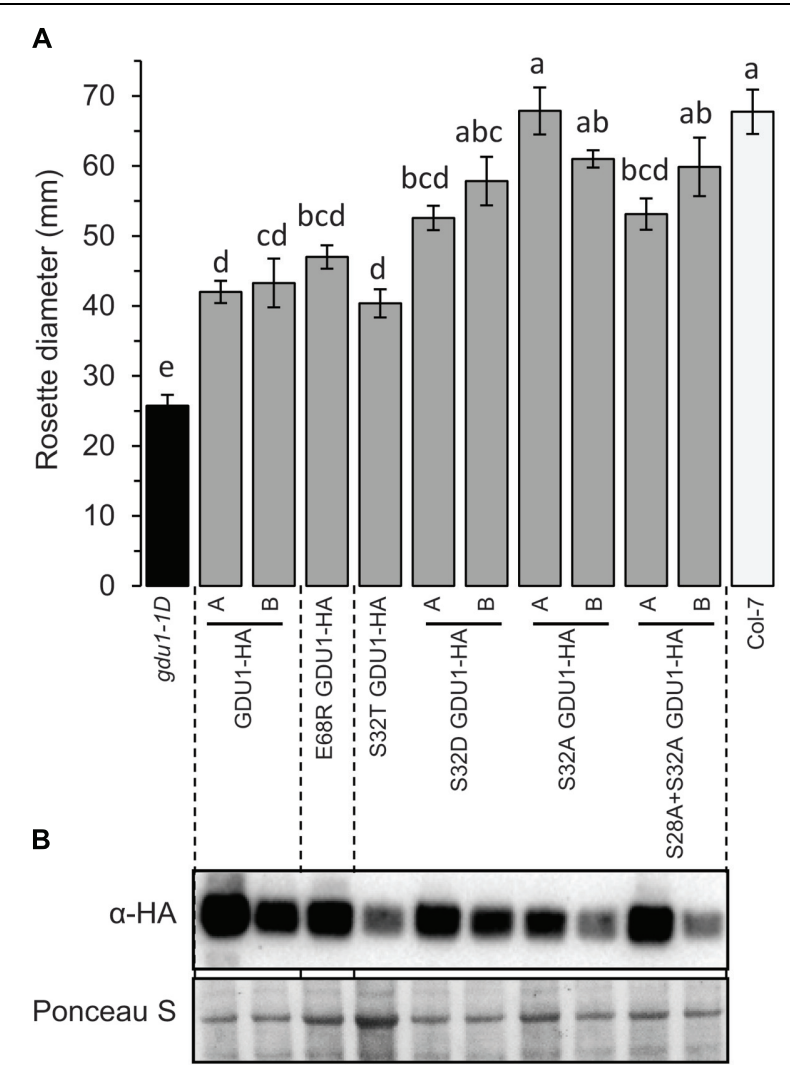

FIGURE 7 | Analysis of plants over-expressing the GDU1 variants. (A) Rosette diameter of 4 week-old Arabidopsis. Plants were grown the same time as plants in Figure 1 (the values for gdu1-1D, GDU1-HA and Col-7 are the same as in Figure 1). Error bars are SEM of 6-8 plants; statistical significance determined by ANOVA using Tukey's HSD $p<0.01$.

(B) Accumulation of the HA-tagged GDU1 proteins in each line was estimated by western blot with an anti-HA antibody. The wild type Col-7 and gdu1-1D lines are indicated as white and black bars; these lines do not express any tagged protein and were not tested by western blotting. 
the plants over-expressing S32T GDU1 was identical to the GDU1-HA over-expressors, in terms of size, Gln secretion and leaf senescence (Figures 7A and 8), suggesting that S32T GDU1 is fully functional. Over-expression of the S32D, S32A, and $\mathrm{S} 28 \mathrm{~A}+\mathrm{S} 32 \mathrm{~A}$ mutant proteins led to plants with slightly reduced size (down to $80 \%$ of the wild type), but never as much as the GDU1 or S32T GDU1 over-expressors. On the other hand, Gln transport analyses showed that the plants overexpressing the S32D, S32A, and S32T variants displayed uptake and efflux similar to the plants over-expressing the wild type GDU1 (Figure 5C). It is worth noting plants over-expressing the S32T variant displayed a higher efflux than the GDU1-HA overexpressor (Figure 5B), suggesting that this protein is more active than GDU1. Interestingly, when the GDU1 protein bearing the double mutation S28A+S32A was over-expressed, Gln uptake and efflux were very similar to the S32L mutation, present in the log 1-4 mutant. No difference between the effect of the S32A and S32D mutations was detected by this assay.

\section{A E68R Mutation Leads to an Almost Fully Functional GDU1}

We tested whether the suppressor effect of the E68K mutation came from the change in charge (negative to positive) or from

\begin{tabular}{|c|c|c|c|c|c|c|}
\hline & $\begin{array}{c}\text { Transport } \\
\text { group }^{\mathrm{a}}\end{array}$ & $\begin{array}{l}\text { Plant size } \\
(\mathrm{mm})\end{array}$ & $\begin{array}{c}\text { GDU1 } \\
\text { phenotype }^{c}\end{array}$ & $\begin{array}{l}\text { Am } \\
\text { tol } \\
\text { Leu }\end{array}$ & $\begin{array}{l}\text { ino ac } \\
\text { eranc } \\
\text { Phe }\end{array}$ & $\begin{array}{l}\text { cid } \\
e^{d} \\
\varnothing\end{array}$ \\
\hline Col7 & WT & 68 & & 1 & 1 & 4 \\
\hline $\mathrm{HA}$ & WT & 74 & & 1 & 1 & 4 \\
\hline gdu1-1D & GDU1 & 26 & yes & 4 & 4 & 4 \\
\hline GDU1-HA & GDU1 & $43^{b}$ & yes & 4 & 4 & 4 \\
\hline $\log 1-1$ & $\log 1$ & - & & 1 & 1 & 4 \\
\hline $\log 1-2$ & $\log 1$ & - & & 2.5 & 4 & 4 \\
\hline E68K GDU1-HA & GDU1 & $65^{b}$ & & 3 & 4 & 4 \\
\hline E68R GDU1-HA & GDU1 & 47 & & 3 & 3 & 4 \\
\hline $\log 1-3$ & $\log 1$ & - & & 2 & 1.5 & 4 \\
\hline G40D GDU1-HA & $\log 1$ & 74 & & 1 & 1 & 4 \\
\hline $\log 1-4$ & $\log 1$ & - & & 2 & 2 & 4 \\
\hline S32L GDU1-HA & $\log 1$ & $73^{b}$ & & 1 & 1 & 4 \\
\hline S32T GDU1-HA & GDU1 & 40 & yes & 4 & 4 & 4 \\
\hline S32D GDU1-HA & GDU1 & $55^{b}$ & & 2 & 2.5 & 4 \\
\hline S32A GDU1-HA & GDU1 & $64^{b}$ & & 2.5 & 4 & 4 \\
\hline S28,32A GDU1-HA & $\log 1$ & $57^{b}$ & & 1 & 1 & 4 \\
\hline
\end{tabular}

FIGURE 8 | Summary of uptake, size, phenotype, and amino acid tolerance of the $\log 1$ suppressor mutants and of lines over-expressing the GDU1 variants. ${ }^{a}$ WT: uptake and efflux similar to the wild type; GDU1: uptake and efflux similar to gdu1-1D; log1: intermediate between gdu1-1D and the wild type (see Figures 2 and $\mathbf{5}$ ). ' Value is the average of two lines (see Figures $\mathbf{4}$ and 7); "-" indicates that the line was not grown in this experiment. ' GIn secretion and early senescence. ${ }^{\mathrm{d}}$ Growth was scored according to the growth of the wild type (set to 1) and the gdu1-1D mutant (set to 4 ) in the corresponding growth condition; wild type and gdu1-1D plants grew equally well on the medium lacking amino acids (Ø); average of two experiments (the pictures of the plants from one experiment are displayed in Supplementary Figure S4). the specific substitution to a Lys residue. For this purpose, Glu68 was mutagenized to Arg (a positively charged amino acid), and the variant protein expressed in Arabidopsis. This protein accumulated at the same level as the wild type protein in the over-expression lines (Figure 7B), and the corresponding plants had a size similar to one over-expressing the wild type GDU1 (Figure 7A) but did not display the GdulD phenotype, i.e., secretion and early senescence (Figure 8). Transport assays showed that the plants over-expressing E68R behaved essentially similar to a GDU1 over-expressor (Figure 5), showing that the Arg at this position has little effect on the protein function in this assay.

\section{Analysis of Amino Acid Tolerance of the Suppressed Mutants and of Over-Expressors of the GDU1 Variants}

Some amino acids, when supplied at high concentration, have been shown to inhibit cell and plant growth (Bonner et al., 1992; Lee et al., 2007; Pratelli and Pilot, 2007).

Another characteristic of the Gdu1D phenotype is the tolerance of GDU1 over-expressors to toxic concentrations of amino acids (Pratelli and Pilot, 2007; Pratelli et al., 2010). The effect of high concentration of amino acids was used as a supplemental assay to characterize the phenotype of the $\log 1$ suppressors, the recapitulation lines and the over-expressors of the GDU1 variants. The presence, absence or strength of the Gdu1D phenotype was tested by growing the plants on $10 \mathrm{mM}$ Leu and Phe, shown to be particularly toxic to wild type plants (Pratelli and Pilot, 2007). All lines grew equally on the control medium (Figure 8 and Supplementary Figure S4), but showed remarkable differences on the Leu- and Phe-supplemented media. The $\log 1-1, \log 1-3$, and $\log 1-4$ mutants behaved similarly to the wild type, while $\log 1-2$ behaved intermediately between gdu1-1D and the wild type, with strong tolerance on Phe but not Leu. Both E68K and E68R GDU1 over-expressors were fairly tolerant to Leu and Phe, in good accordance with the Gln transport assays, reminiscent of the GdulD phenotype. The recapitulation line over-expressing G40D GDU1 was as sensitive as the wild type. The S32L and S28A+3S2A GDU1 over-expressors behaved similarly to the wild type; the S32T GDU1 over-expressor looked like the gdu1-1D mutant; and the S32D and S32A mutants showed an intermediate phenotype between the wild type and the $g d u 1-1 D$ mutant (Figure 8 and Supplementary Figure S4).

\section{Discussion}

\section{Suppressed Mutants Show Only Partially Abolished Gln Uptake}

A total of four mutants were found $(\log 1-1, \log 1-2, \log 1-3$, and $\log 1-4)$, all carrying recessive mutations, hence expected to behave as loss-of-functions, that suppressed the GdulD phenotype caused by the over-expression of the GDU1 protein. These suppressor lines over-express a mutant GDU1 protein in addition to the endogenous GDU1 protein. The thorough characterization of these lines presented in this study led to 
unexpected observations. The size of all the mutants was similar to the wild type and the early senescence observed in the leaves of $g d u 1-1 D$ and $g d u 1-6 D$ was absent. However, analysis of Gln uptake showed that the mutations did not suppress the Gdu1D phenotype completely (Figure 2), with uptake and efflux of the mutants being intermediate between the wild type and the parental line gdu1-6D. Interestingly, the suppressor mutation in $\log 2-1$, which affects the ubiquitin ligase LOG2, interactor of GDU1 (Pratelli et al., 2012), similarly does not completely suppress all characteristics of the Gdu1D phenotype: the wild type size is restored, the amino acid susceptibility is abolished, the plants do not secrete Gln anymore, but amino acid export is lowered to an intermediate level between the wild type and the parent (Pratelli and Pilot, unpublished data).

These mutations were obtained from a visual screening which led to the isolation of non-secreting plants of wild type size, possibly introducing a bias toward finding mutations that not necessarily affect transport, but rather toward ones that affect plant size and Gln secretion. The fact that the mutations in the $\log 1-1, \log 1-3$, and $\log 2$ suppressor mutants abolish the Gdu1D phenotype partially, in a similar way suggest that they suppress the same component of the phenotype, e.g., the one involving LOG2, leaving other components intact. This implies that the over-expression of GDU1 leads to several independent effects, some characterized by decreased size, induction of early senescence and amino acid tolerance, and others characterized by increased amino acid export.

The exact role of GDU1 is not known but two proteins interacting with it have been identified: LOG2 and its homolog LUL1, two membrane-associated ubiquitin ligases (Pratelli et al., 2012). It is proposed that GDU1 and LOG2/LUL1 form a complex stable enough to be co-immunoprecipitated, and involved in ubiquitination of yet unknown target(s) with a role in the regulation of amino acid transport (Pratelli et al., 2012). Loss of interaction with, or loss of expression of LOG2 is a simple explanation for the absence of the Gdu1D phenotype in the $\log 1-1$ and $\log 1-3$ mutants, over-expressors of a VIMAG domaindeleted GDU1 (Pratelli and Pilot, 2006) or the $\log 2$ mutants (Pratelli et al., 2012). The fact that the loss of this interaction leads to a similar phenotype as the $\log 1-2$ and $\log 1-4$ mutants is striking, since the corresponding mutations in GDU1 (S32L and $\mathrm{E} 68 \mathrm{~K}$ ) do not appear to affect the interaction with LOG2 (Figure 6). However, it is possible that the functional properties of the GDU1-LOG2 complexes resulting from the interaction of S32L and E68K GDU1 and LOG2 are affected by these mutations, explaining the resulting phenotype of the corresponding plants.

The active structure of GDU1 is not known, and a possible structure could be a multimer, either with itself or other GDU proteins (seven GDU proteins are present in the Arabidopsis genome). In this case, the mutated proteins in the suppressor mutants might assemble with the endogenous wild type proteins to form hetero-multimers, which could have retained only some functions of the original multimers while being less active. This hypothesis could explain the attenuated phenotype of the plants, in terms of uptake, size or amino acid tolerance, and the diversity of the phenotypes of the plants over-expressing the various GDU1 variants (Figure 8). It has then to be postulated that GDU1 has diverse functions, and that each suppressor mutation affects them differently. These functions remain to be determined.

\section{The G40D Mutation Might Affect GDU1 Protein Stability and/or Function}

The G40D suppressor mutation in GDU1 has two effects on the protein's properties: it does not accumulate at the same level as the other proteins when expressed in $N$. benthamiana leaves (Figure 3), and it does not co-immunoprecipitate with LOG2 (Figure 6). This mutation is located in the transmembrane domain, and affects one of the conserved residues of this domain. In about $100 \mathrm{GDU}$ proteins from higher plants, Gly40 is sometimes replaced by Ala (8\%), Ser (4\%), Leu (1\%), or Val (1\%), but never by Asp (data not shown). Gly is a very common residue in membrane helices, involved in the formation of a glycine zipper in helix packing, thus enabling helix-helix interactions (Javadpour et al., 1999; Kim et al., 2005). Mutations of such important Gly residues led to abolition of transport (G114A in DctA from Sinorhizobium meliloti; Trainer et al., 2007) or loss of dimer interaction in EmrE from Escherichia coli (Elbaz et al., 2008), supposedly by disruption of the helix structure or ability to interact. The amount of sequence conservation of the GDU helix across different species is rare for a membrane protein, and one explanation could be that it is involved in interaction with membrane helices from other proteins. In this context, the G40D mutation likely affects such interaction, and may be the reason for the Gdu1D suppressor effect on the Gdu1D phenotype. Alternatively, the presence of a charged residue (Asp) inside the hydrophobic membrane helix could destabilize the protein, making it more difficult to be integrated in the lipid bilayer. This could explain the lower accumulation of the protein: membrane proteins that have folding problems are typically degraded by the cellular quality control system (Nagy and Sanders, 2004; Houck and Cyr, 2012). While the localization of the G40D GDU1 protein was not dramatically changed in N. benthamiana leaves, this does not preclude that GDU1 and its variants are addressed to different membrane subdomains. Such a hypothesis is supported by the fact that G40D GDU1 does not interact with LOG2: these proteins would not interact if G40D GDU1 and LOG2 are targeted to different membrane subdomains. Changes in subcellular distribution have indeed been observed for mutations affecting transmembrane Gly residues (Rosnoblet et al., 2013). Nevertheless, the fact that the over-expression of G40D GDU1 in wild type plants affects amino acid transport (Figure 5C) supports the idea that the protein still retained at least one of its functions, the one affecting amino acid transport.

\section{Putative Role of the External Ser28 and Ser32}

The suppressor mutation in $\log 1-4$ affected Ser32, which is predicted to be phosphorylated, together with Ser28 (Supplementary Figure S3). It is not clear if these residues can be phosphorylated in vivo, because they are expected to lie in the extracellular region of the GDU1 protein. Based on the identification of bona fide extracellular phosphoproteins with no transmembrane domain by a proteomic analysis of the extracellular matrix of Arabidopsis cell suspension cultures, an extracellular phosphorylation network has been suggested 
(Ndimba et al., 2003). ATP has been found in the extracellular medium, and is supposed to be involved in phosphorylation of extracellular proteins, as well as in intercellular signaling or during pathogen recognition (Ndimba et al., 2003; Chivasa et al., 2005). The fact that the mutation of the conserved Ser32 to Leu suppresses the Gdu1D phenotype led to the hypothesis that phosphorylation is important in GDU1 function. To test this hypothesis, Ser32 was mutagenized to Asp and Ala (to mimic or suppress phosphorylation, respectively) or Thr (another phosphorylation site), and over-expressed in plants. None of these mutations affected the functional properties of the GDU1 proteins as related to its effects on amino acid transport, since the over-expressors displayed similar Gln transport as GDU1 over-expressors (Figure 5). On the contrary, while S32T GDU1 over-expressors were small and tolerated amino acids, S32A and S32D over-expressors had near wild type size (Figure 7) and were somewhat susceptible to amino acids, suggesting that the S32T mutation does not alter GDU1 functional properties. S32A and S32D thus dissociated Gln uptake/export from the size and amino acid tolerance (Figure 8), showing that there is no direct relationship between these characteristics of the phenotype. The fact that their effect is similar is unexpected since these mutations typically lead to opposite phenotypes in terms of phosphorylation.

The double S28A+S32A mutation created a protein with functional properties very similar to the suppressor S32L mutation, and similar to the $\log 1$ mutants (Figures 2, 5, 7, and 8), different from the single S32A mutation. Ser28 and Ser32 thus seem to have redundant functions. In the hypothesis that these Ser are extra-cellular phosphorylation sites, mutagenesis of Ser32 individually would not have any effect, as observed; and only mutagenesis of the two Ser at the same time would lead to a phenotype. This hypothesis could be tested by mutagenizing Ser28 to Asp and Ala, which is expected to lead to a similar phenotype as the S32D and S32A over-expressing plants respectively. It can also be tested by mutagenizing S28 and S32 to Asp at the same time, the expected outcome of which would be a hyperactive GDU1 protein, and lead to plants with a phenotype reverse of the S28A+S32A over-expressing plants. In this hypothesis, the S32L mutation would prevent the phosphorylation of Ser28 and Ser32, possibly by affecting binding to a protein kinase, and would explain the observed similarity of the effect of S32L with S28A+S32A. If these Ser are not phosphorylation sites, these residues have a role in the function of the protein, which will require further investigation.

\section{Substitution of Glu68 by a Positive Residue Affects GDU1 Protein Function}

The effect of the suppressor mutation E68K has been investigated based on the hypothesis that it locally changes the charge of the protein from negative to positive. The E68R mutation was expected to lead to a non-functional GDU1, which, when expressed in plants, would lead to the same effect as the E68K GDU1 from the log1-2 mutant. Plants over-expressing E68R GDU1 were smaller, and Gln transport was similar to the GDU1 over-expressors, suggesting that this variant GDU1 protein retained much of GDU1 functionality. The main difference with the $g d u 1-1 D$ over-expressor is that these plants did not secrete Gln or display early senescence.

Surprisingly, the phenotype of the recapitulation line E68K GDU1 did not recapitulate the $\log 1-2$ suppressor mutant. Amino acid transport in these plants was similar to the gdu1-1D mutant, and not intermediate between this mutant and the wild type as expected from the analysis of the $\log 1-2$ suppressor mutant (Figures 2 and 5). The E68K GDU1 over-expressing plants displayed a phenotype similar to the plants over-expressing the E68R mutant, except that the size of the plants was similar to the wild type. It is not explained why the E68K GDU1-HA overexpressors do not behave as the log 1-2 plants and will need further investigation. As suggested earlier, the addition of an HA tag to the protein could affect the function of the mutant protein and explain this discrepancy. It has also to be noted that the $\log 1-2$ mutant is tolerant to external amino acids, similar to the gdu1$1 D$ mutant (Figure 8), but does not display size reduction, Gln secretion or early senescence.

These results suggest that the mutation of Glu68 to Lys, a positively charged residue, affects protein function in a way that the over-expressing plants display enhanced Gln transport but no dramatic growth alterations. Glu68 is not conserved in GDU sequences, and is sometimes replaced by Arg in GDU2 and GDU3 (Figure 1). Yet GDU2 and GDU3 over-expressors display the typical Gdu1D phenotype (small plants secreting Gln; Pratelli et al., 2010), showing that the function of the GDU2 and GDU3 proteins is not very different from GDU1, despite this difference in sequence.

\section{Conclusion and Opportunities for Crop Engineering}

This work showed that all specific mutations in GDU1 can dissociate the various components of the GdulD phenotype. In some cases, the amino acid transport is affected in plants over-expressing mutant GDU1 proteins as in $g d u 1-1 D$, but these plants grow similarly to the wild type (S32A, E68K, and S32D). In the other cases, the transport is less affected, and the plants show a wild type phenotype (G40D, S32L) or close to wild type phenotype $(\mathrm{S} 28 \mathrm{~A}+\mathrm{S} 32 \mathrm{~A})$. The residues important for each function of GDU1 are not necessarily born by conserved residues, or domains, like the membrane or VIMAG domains. The use of the wild type GDU1 protein as a tool to modify amino acid export was impaired by the associated effects on plant fitness in Arabidopsis (Pilot et al., 2004) and tobacco (Pratelli and Pilot, 2006). The mutant proteins described here could be used to modify amino acid transport, and in particular export, in the plant without affecting plant fitness. Targeting amino acid export in plants would be another strategy to modify allocation of amino acids between the plant organs, and ultimately control protein content in reserve organs, like seeds and roots.

\section{Materials and Methods}

\section{Plant Material and Growth}

Arabidopsis thaliana (ecotype Col-7) lines were grown under $120 \mu \mathrm{E} / \mathrm{m}^{2} / \mathrm{s}, 22^{\circ} \mathrm{C}, 16 \mathrm{~h}$ light $/ 8 \mathrm{~h}$ dark on soil (Mix of Sunshine 
Mix 1 and Pro-mix HP at a 1:1 ratio) and were watered from below with $300 \mathrm{mg} / \mathrm{l}$ Miracle-Gro Fertilizer [24/8/16\% (w/w) N/P/K; Scotts, Marysville, OH, USA]. Rosette diameter of about eight plants from each line was measured with a ruler about 4 weeks after sowing. Arabidopsis thaliana were transformed by the floral dip method (Clough and Bent, 1998) using Agrobacterium tumefaciens GV3101 (pMP90). For transient expression of proteins in $N$. benthamiana, young leaves of 5-week-old plants were infiltrated with a suspension of Agrobacterium tumefaciens carrying the constructs of interest and the silencing suppressor p19 (Voinnet et al., 2003) according to (Batoko et al., 2000), with the following modifications. The bacteria were grown overnight in LB supplemented with appropriate antibiotics, washed twice in $10 \mathrm{mM} \mathrm{MgCl}_{2}, 100 \mu \mathrm{M}$ acetosyringone, and diluted to final $\mathrm{OD}_{600}$ of 0.05 in the same solution before infiltration in $N$. benthamiana leaves. Amino acid tolerance experiments were performed as described (Pratelli and Pilot, 2006): plants were grown for 10 days in long days conditions, on half-strength MS medium, supplemented with $0.5 \%$ sucrose, and $10 \mathrm{mM}$ of indicated amino acids.

\section{Cloning and Constructs}

Primer sequences used for cloning are listed in Supplementary Table S1. The $\log 1$ variants of the GDU1 gene were cloned by PCR from genomic DNA and cloned into pDONR Zeo by the Gateway technology. The Kunkel method was used for site directed mutagenesis (Kunkel et al., 1991), from sequences cloned by Gateway cloning in the pDONR Zeo Gateway vector, containing the $\mathrm{fl}$ replication origin (Lalonde et al., 2010). Mutagenized inserts were sequenced, and transferred by Gateway cloning (Life Technologies) to the binary vector pPWHTkan and pPWGTkan, derivative of pJHA212K (Yoo et al., 2004; Pratelli and Pilot, unpublished data). pPWHTkan carries in this order the CaMV $35 \mathrm{~S}$ promoter, the Gateway cassette, a double HA tag sequence and the terminator of the small subunit of the Rubisco from pea (Pisum sativum; accession no. X00806). pPWGTkan carries similar parts except that the HA tag is replaced by the enhanced GFP sequence.

\section{Western Blotting and Co-immunoprecipitation}

Protein extraction and western blotting were performed as previously described, with the following modifications ( $\mathrm{Yu}$ and Pilot, 2014): Leaves from each line (selected on kanamycin for

\section{References}

Batoko, H., Zheng, H. Q., Hawes, C., and Moore, I. (2000). A Rab1 GTPase is required for transport between the endoplasmic reticulum and golgi apparatus and for normal golgi movement in plants. Plant Cell 12, 2201-2218. doi: 10.1105/tpc.12.11.2201

Bonner, C. A., Rodrigues, A., Miller, J. A., and Jensen, R. A. (1992). Amino acids are general growth inhibitors of Nicotiana silvestris in tissue culture. Physiol. Plant. 84, 319-328. doi: 10.1111/j.1399-3054.1992.tb04671.x

Chhangani, D., Nukina, N., Kurosawa, M., Amanullah, A., Joshi, V., Upadhyay, A., et al. (2014). Mahogunin ring finger 1 suppresses misfolded polyglutamine aggregation and cytotoxicity. Biochim. Biophys. Acta 1842, 1472-1484. doi: 10.1016/j.bbadis.2014.04.014
7 days, and transferred to soil and grown for three more weeks) were collected for protein extraction and western blot. Five hundred $\mathrm{mg}$ of leaves were ground with $1 \mathrm{ml}$ of extraction buffer composed of $50 \mathrm{mM}$ Tris- $\mathrm{HCl}, \mathrm{pH} 7.3,150 \mathrm{mM} \mathrm{NaCl}$, $10 \mathrm{mM} \mathrm{MgCl} 2,10 \mathrm{mM}$ DTT, 0.5\% Nonidet P-40, and $1 \mathrm{X}$ Complete Protease Inhibitors (Roche) on ice. Homogenates were centrifuged at $14,000 \mathrm{~g}$ at $4^{\circ} \mathrm{C}$ for $15 \mathrm{~min}$. Protein concentration of the supernatant was quantified by Bradford reagent. Twenty $\mu \mathrm{g}$ of total proteins were analyzed by SDSPAGE (4-12\% polyacrylamide MES gel; Life Technologies) and western blotting. Proteins were transferred on a nitrocellulose membrane (GE Healthcare) and detected using anti-HA (clone 3F10; Roche Diagnostics; 1:5,000) primary antibody, antirat (Thermo Scientific) secondary antibody, and the ECLPrime western-blotting detection system (GE Healthcare). Co-immunoprecipitation experiments were performed from $N$. benthamiana infiltrated leaves as described (Pratelli et al., 2012).

\section{Amino Acid Uptake}

Amino acid uptakes in plants were performed as described (Pratelli et al., 2010), from segregating T2 seeds selected on kanamycin before growing in liquid medium and uptake.

\section{Author Contributions}

RP and GP designed and performed the suppressor screening. SY and RP characterized the suppressor mutants. SY and CD characterized the recapitulation lines and the mutagenized GDU1 proteins. SY and GP designed the experiments and wrote the paper.

\section{Acknowledgment}

This work was supported by the National Science Foundation (MCB1052048 to GP).

\section{Supplementary Material}

The Supplementary Material for this article can be found online at: http://journal.frontiersin.org/article/10.3389/fpls.2015.00593

Chivasa, S., Ndimba, B. K., Simon, W. J., Lindsey, K., and Slabas, A. R. (2005). Extracellular ATP functions as an endogenous external metabolite regulating plant cell viability. Plant Cell 17, 3019-3034. doi: 10.1105/tpc.105.036806

Clough, S. J., and Bent, A. F. (1998). Floral dip: a simplified method for Agrobacterium-mediated transformation of Arabidopsis thaliana. Plant J. 16, 735-743. doi: 10.1046/j.1365-313x.1998.00343.x

de Kraker, J. W., and Gershenzon, J. (2011). From amino acid to glucosinolate biosynthesis: protein sequence changes in the evolution of methylthioalkylmalate synthase in Arabidopsis. Plant Cell 23, 38-53. doi: 10.1105/tpc.110.079269

Dundar, E., and Bush, D. R. (2009). BAT1, a bidirectional amino acid transporter in Arabidopsis. Planta 229, 1047-1056. doi: 10.1007/s00425-0090892-8 
Durek, P., Schmidt, R., Heazlewood, J. L., Jones, A., Maclean, D., Nagel, A., et al. (2010). PhosPhAt: the Arabidopsis thaliana phosphorylation site database. An update. Nucleic Acids Res. 38, D828-D834. doi: 10.1093/nar/gkp810

Elbaz, Y., Salomon, T., and Schuldiner, S. (2008). Identification of a glycine motif required for packing in EmrE, a multidrug transporter from Escherichia coli. J. Biol. Chem. 283, 12276-12283. doi: 10.1074/jbc.M710338200

Falcone Ferreyra, M. L., Rius, S. P., and Casati, P. (2012). Flavonoids: biosynthesis, biological functions, and biotechnological applications. Front. Plant Sci. 3:222. doi: $10.3389 /$ fpls.2012.00222

Guerra, D. D., Pratelli, R., Kraft, E., Callis, J., and Pilot, G. (2013). Functional conservation between mammalian MGRN1 and plant LOG2 ubiquitin ligases. FEBS Lett. 587, 3400-3405. doi: 10.1016/j.febslet.2013.08.045

Gunn, T. M., Silvius, D., Bagher, P., Sun, K., and Walker, K. K. (2013). MGRN1dependent pigment-type switching requires its ubiquitination activity but not its interaction with TSG101 or NEDD4. Pigment Cell Melanoma Res. 26, 263-268. doi: 10.1111/pcmr.12059

Heazlewood, J. L., Durek, P., Hummel, J., Selbig, J., Weckwerth, W., Walther, D., et al. (2008). PhosPhAt: a database of phosphorylation sites in Arabidopsis thaliana and a plant-specific phosphorylation site predictor. Nucleic Acids Res. 36, D1015-D1021. doi: 10.1093/nar/gkm812

Houck, S. A., and Cyr, D. M. (2012). Mechanisms for quality control of misfolded transmembrane proteins. Biochim. Biophys. Acta 1818, 1108-1114. doi: 10.1016/j.bbamem.2011.11.007

Jack, D. L., Yang, N. M., and Saier, M. H. Jr. (2001). The drug/metabolite transporter superfamily. Eur. J. Biochem. 268, 3620-3639. doi: 10.1046/j.14321327.2001.02265.x

Javadpour, M. M., Eilers, M., Groesbeek, M., and Smith, S. O. (1999). Helix packing in polytopic membrane proteins: role of glycine in transmembrane helix association. Biophys. J. 77, 1609-1618. doi: 10.1016/S0006-3495(99)77009-8

Jiao, J., Sun, K., Walker, W. P., Bagher, P., Cota, C. D., and Gunn, T. M. (2009). Abnormal regulation of TSG101 in mice with spongiform neurodegeneration. Biochim. Biophys. Acta 1792, 1027-1035. doi: 10.1016/j.bbadis.2009.08.009

Kim, S., Jeon, T. J., Oberai, A., Yang, D., Schmidt, J. J., and Bowie, J. U. (2005). Transmembrane glycine zippers: physiological and pathological roles in membrane proteins. Proc. Natl. Acad. Sci. U.S.A. 102, 14278-14283. doi: 10.1073/pnas.0501234102

Kunkel, T. A., Bebenek, K., and Mcclary, J. (1991). Efficient site-directed mutagenesis using uracil-containing DNA. Methods Enzymol. 204, 125-139. doi: 10.1016/0076-6879(91)04008-C

Ladwig, F., Stahl, M., Ludewig, U., Hirner, A. A., Hammes, U. Z., Stadler, R., et al. (2012). Siliques are Red1 from Arabidopsis acts as a bidirectional amino acid transporter that is crucial for the amino acid homeostasis of siliques. Plant Physiol. 158, 1643-1655. doi: 10.1104/pp.111.192583

Lalonde, S., Sero, A., Pratelli, R., Pilot, G., Chen, J., Sardi, M. I., et al. (2010). A membrane protein/signaling protein interaction network for Arabidopsis version AMPv2. Front. Physiol. 1:24. doi: 10.3389/fphys.2010. 00024

Lee, T. Y., Bretana, N. A., and Lu, C. T. (2011). PlantPhos: using maximal dependence decomposition to identify plant phosphorylation sites with substrate site specificity. BMC Bioinformatics 12:261. doi: 10.1186/1471-210512-261

Lee, Y. H., Foster, J., Chen, J., Voll, L. M., Weber, A. P., and Tegeder, M. (2007). AAP1 transports uncharged amino acids into roots of Arabidopsis. Plant J. 50, 305-319. doi: 10.1111/j.1365-313X.2007.03045.x

Michaeli, S., Fait, A., Lagor, K., Nunes-Nesi, A., Grillich, N., Yellin, A., et al. (2011). A mitochondrial GABA permease connects the GABA shunt and the TCA cycle and is essential for normal carbon metabolism. Plant J. 67, 485-498. doi: 10.1111/j.1365-313X.2011.04612.x

Nagy, J. K., and Sanders, C. R. (2004). Destabilizing mutations promote membrane protein misfolding. Biochemistry 43, 19-25. doi: 10.1021/bi035918s

Ndimba, B. K., Chivasa, S., Hamilton, J. M., Simon, W. J., and Slabas, A. R. (2003). Proteomic analysis of changes in the extracellular matrix of Arabidopsis cell suspension cultures induced by fungal elicitors. Proteomics 3, 1047-1059. doi: 10.1002/pmic. 200300413

Perez-Oliva, A. B., Olivares, C., Jimenez-Cervantes, C., and Garcia-Borron, J. C. (2009). Mahogunin ring finger-1 (MGRN1) E3 ubiquitin ligase inhibits signaling from melanocortin receptor by competition with Galphas. J. Biol. Chem. 284, 31714-31725. doi: 10.1074/jbc.M109.028100

Pilot, G., Stransky, H., Bushey, D. F., Pratelli, R., Ludewig, U., Wingate, V. P., et al. (2004). Overexpression of GLUTAMINE DUMPER1 leads to hypersecretion of glutamine from hydathodes of Arabidopsis leaves. Plant Cell 16, 1827-1840. doi: 10.1105/tpc.021642

Pratelli, R., Guerra, D. D., Yu, S., Wogulis, M., Kraft, E., Frommer, W. B., et al. (2012). The ubiquitin E3 ligase LOSS OF GDU2 is required for GLUTAMINE DUMPER1-induced amino acid secretion in Arabidopsis. Plant Physiol. 158, 1628-1642. doi: 10.1104/pp.111.191965

Pratelli, R., and Pilot, G. (2006). The plant-specific VIMAG domain of Glutamine Dumper1 is necessary for the function of the protein in Arabidopsis. FEBS Lett. 580, 6961-6966. doi: 10.1016/j.febslet.2006.11.064

Pratelli, R., and Pilot, G. (2007). Altered amino acid metabolism in glutamine dumper1 plants. Plant Signal. Behav. 2, 182-184. doi: 10.4161/psb.2.3.3972

Pratelli, R., Voll, L. M., Horst, R. J., Frommer, W. B., and Pilot, G. (2010). Stimulation of nonselective amino acid export by glutamine dumper proteins. Plant Physiol. 152, 762-773. doi: 10.1104/pp.109.151746

Ranocha, P., Dima, O., Nagy, R., Felten, J., Corratge-Faillie, C., Novak, O., et al. (2013). Arabidopsis WAT1 is a vacuolar auxin transport facilitator required for auxin homoeostasis. Nat. Commun. 4:2625. doi: 10.1038/ncomms3625

Rosnoblet, C., Legrand, D., Demaegd, D., Hacine-Gherbi, H., De Bettignies, G., Bammens, R., et al. (2013). Impact of disease-causing mutations on TMEM165 subcellular localization, a recently identified protein involved in CDG-II. Hum. Mol. Genet. 22, 2914-2928. doi: 10.1093/hmg/ddt146

Snowden, C. J., Thomas, B., Baxter, C. J., Smith, J. A., and Sweetlove, L. J. (2015). A tonoplast Glu/Asp/GABA exchanger that affects tomato fruit amino acid composition. Plant J. 81, 651-660. doi: 10.1111/tpj.12766

Tegeder, M. (2014). Transporters involved in source to sink partitioning of amino acids and ureides: opportunities for crop improvement. J. Exp. Bot. 65, 1865-1878. doi: 10.1093/jxb/eru012

Tegeder, M., and Rentsch, D. (2010). Uptake and partitioning of amino acids and peptides. Mol. Plant 3, 997-1011. doi: 10.1093/mp/ssq047

Trainer, M. A., Yurgel, S. N., and Kahn, M. L. (2007). Role of a conserved membrane glycine residue in a dicarboxylate transporter from Sinorhizobium meliloti. J. Bacteriol. 189, 2160-2163. doi: 10.1128/JB.01247-06

Vastermark, A., Wollwage, S., Houle, M. E., Rio, R., and Saier, M. H. Jr. (2014). Expansion of the APC superfamily of secondary carriers. Proteins 82 , 2797-2811. doi: 10.1002/prot.24643

Voinnet, O., Rivas, S., Mestre, P., and Baulcombe, D. (2003). An enhanced transient expression system in plants based on suppression of gene silencing by the p19 protein of tomato bushy stunt virus. Plant J. 33, 949-956. doi: 10.1046/j.1365313X.2003.01676.x

Yang, H., Bogner, M., Stierhof, Y. D., and Ludewig, U. (2010). $\mathrm{H}^{+}$-independent glutamine transport in plant root tips. PLoS ONE 5:e8917. doi: 10.1371/journal.pone.0008917

Yoo, B. C., Kragler, F., Varkonyi-Gasic, E., Haywood, V., Archer-Evans, S., Lee, Y. M., et al. (2004). A systemic small RNA signaling system in plants. Plant Cell 16, 1979-2000. doi: 10.1105/tpc.104.023614

Yu, S., and Pilot, G. (2014). Testing the efficiency of plant artificial microRNAs by transient expression in Nicotiana benthamiana reveals additional action at the translational level. Front. Plant Sci. 5:622. doi: 10.3389/fpls.2014.00622

Ziegler, J., and Facchini, P. J. (2008). Alkaloid biosynthesis: metabolism and trafficking. Ann. Rev. Plant Biol. 59, 735-769. doi: 10.1146/annurev.arplant.59.032607.092730

Conflict of Interest Statement: The authors declare that the research was conducted in the absence of any commercial or financial relationships that could be construed as a potential conflict of interest.

Copyright (c) 2015 Yu, Pratelli, Denbow and Pilot. This is an open-access article distributed under the terms of the Creative Commons Attribution License (CC BY). The use, distribution or reproduction in other forums is permitted, provided the original author(s) or licensor are credited and that the original publication in this journal is cited, in accordance with accepted academic practice. No use, distribution or reproduction is permitted which does not comply with these terms. 


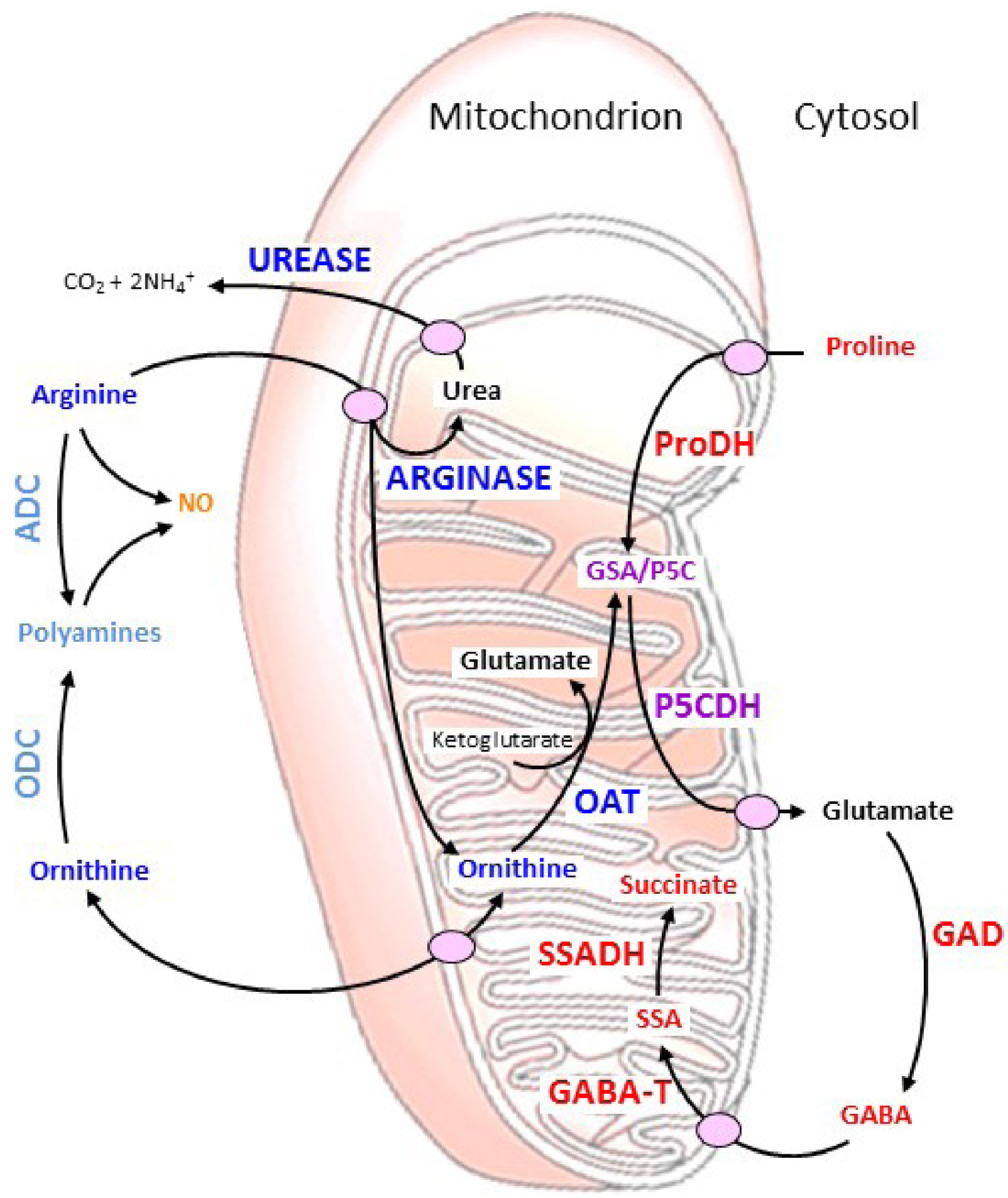

Catabolic connections between glutamate-derived amino acids. The key enzymes of amino acid catabolism are are localised in mitochondria. ADC: arginine decarboxylase, ODC: ornithine decarboxylase, NO: nitric oxide, ProDH: proline dehydrogenase, GSA: glutamate semialdehyde, P5C: pyrroline-5-carboxylate, P5CDH: P5C dehydrogenase, OAT: ornithine-d-aminotransferase, GAD: glutamate decarboxylase, GABA-T: GABA transaminase, SSA: succinate semialdehyde, SSADH: SSA dehydrogenase.

Figure modified from: Forlani G, Bertazzini M, Zarattini M and Funck D (2015) Functional characterization and expression analysis of rice $\delta 1$-pyrroline-5-carboxylate dehydrogenase provide new insight into the regulation of proline and arginine catabolism. Front. Plant Sci. 6:591. doi: 10.3389/fpls.2015.00591 


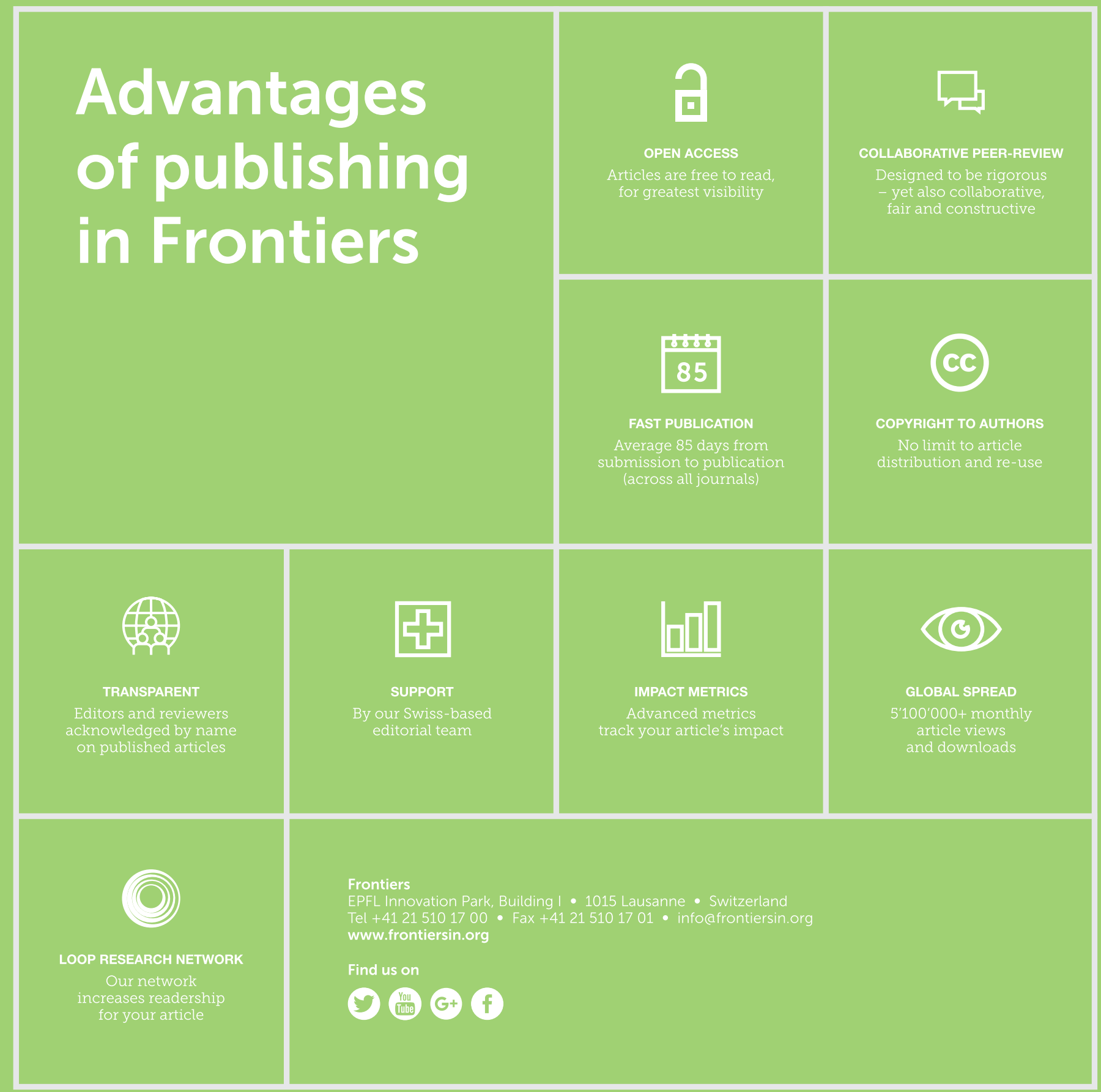

